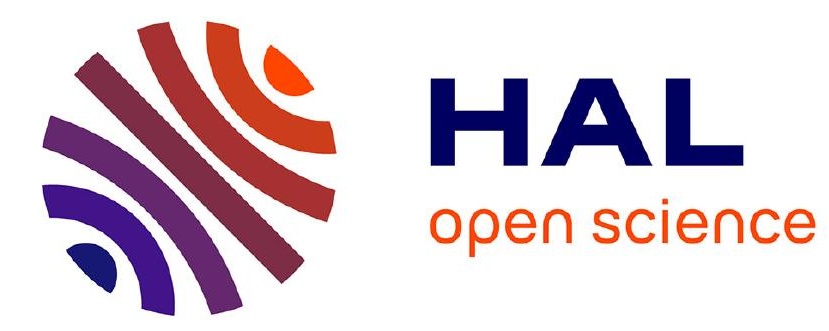

\title{
Canine reference intervals for the Sysmex XT-2000iV hematology analyzer
}

Nathalie Bourgès-Abella, Didier Concordet, . Geffré Anne, Braun Jean-Pierre \& Trumel Catherine

\section{- To cite this version:}

Nathalie Bourgès-Abella, Didier Concordet, . Geffré Anne, Braun Jean-Pierre \& Trumel Catherine. Canine reference intervals for the Sysmex XT-2000iV hematology analyzer. Veterinary Clinical Pathology, 2011, 40 (3), pp.303-315. 10.1111/j.1939-165X.2011.00333.x . hal-02645844

\section{HAL Id: hal-02645844 \\ https://hal.inrae.fr/hal-02645844}

Submitted on 29 May 2020

HAL is a multi-disciplinary open access archive for the deposit and dissemination of scientific research documents, whether they are published or not. The documents may come from teaching and research institutions in France or abroad, or from public or private research centers.
L'archive ouverte pluridisciplinaire HAL, est destinée au dépôt et à la diffusion de documents scientifiques de niveau recherche, publiés ou non, émanant des établissements d'enseignement et de recherche français ou étrangers, des laboratoires publics ou privés. 
\%PDF -1.3

\%äãÏò

$88 \odot$ obj

$<<$

/Linearized 1.0

1090

$/ H \quad\left[\begin{array}{ll}1415 & 538\end{array}\right]$

/L 454222

/E 47467

$/ \mathrm{N} 13$

/T 452418

$>>$

endobj

8850

$000000001500000 \mathrm{n}$

$000000133000000 \mathrm{n}$

$000000195300000 \mathrm{n}$

$000000220000000 \mathrm{n}$

$000000239900000 \mathrm{n}$

$000000262300000 \mathrm{n}$

$000000287200000 \mathrm{n}$

$000000368000000 \mathrm{n}$

$0000000405900000 \mathrm{n}$

$000000435400000 \mathrm{n}$

$000000696200000 \mathrm{n}$

$000000746200000 \mathrm{n}$

$000000776800000 \mathrm{n}$

$000001016700000 \mathrm{n}$

$000001077800000 \mathrm{n}$

$000001126200000 \mathrm{n}$

$000001625300000 \mathrm{n}$

$000001644200000 \mathrm{n}$

$000001686900000 \mathrm{n}$

$000001724700000 \mathrm{n}$

$000002123200000 \mathrm{n}$

$000002200500000 \mathrm{n}$

$000002257100000 \mathrm{n}$

$000002963600000 \mathrm{n}$

$000003027000000 \mathrm{n}$

$000003065700000 \mathrm{n}$

$0000003582800000 \mathrm{n}$

$000003599800000 \mathrm{n}$

$000003622000000 \mathrm{n}$

$000003641900000 \mathrm{n}$

$000003684800000 \mathrm{n}$

$000003709100000 \mathrm{n}$

$000003787900000 \mathrm{n}$

$000003816600000 \mathrm{n}$

$000003824900000 \mathrm{n}$

$000003855500000 \mathrm{n}$

$000003881100000 \mathrm{n}$

$0000004021700000 \mathrm{n}$

$000004031300000 \mathrm{n}$

$000004041000000 \mathrm{n}$

$000004056400000 \mathrm{n}$

$000004138300000 \mathrm{n}$

$000004227800000 \mathrm{n}$

$000004317800000 \mathrm{n}$

$000004405100000 \mathrm{n}$

$000004483700000 \mathrm{n}$

$000004573900000 \mathrm{n}$

$000004662500000 \mathrm{n}$

$000000141500000 \mathrm{n}$ 


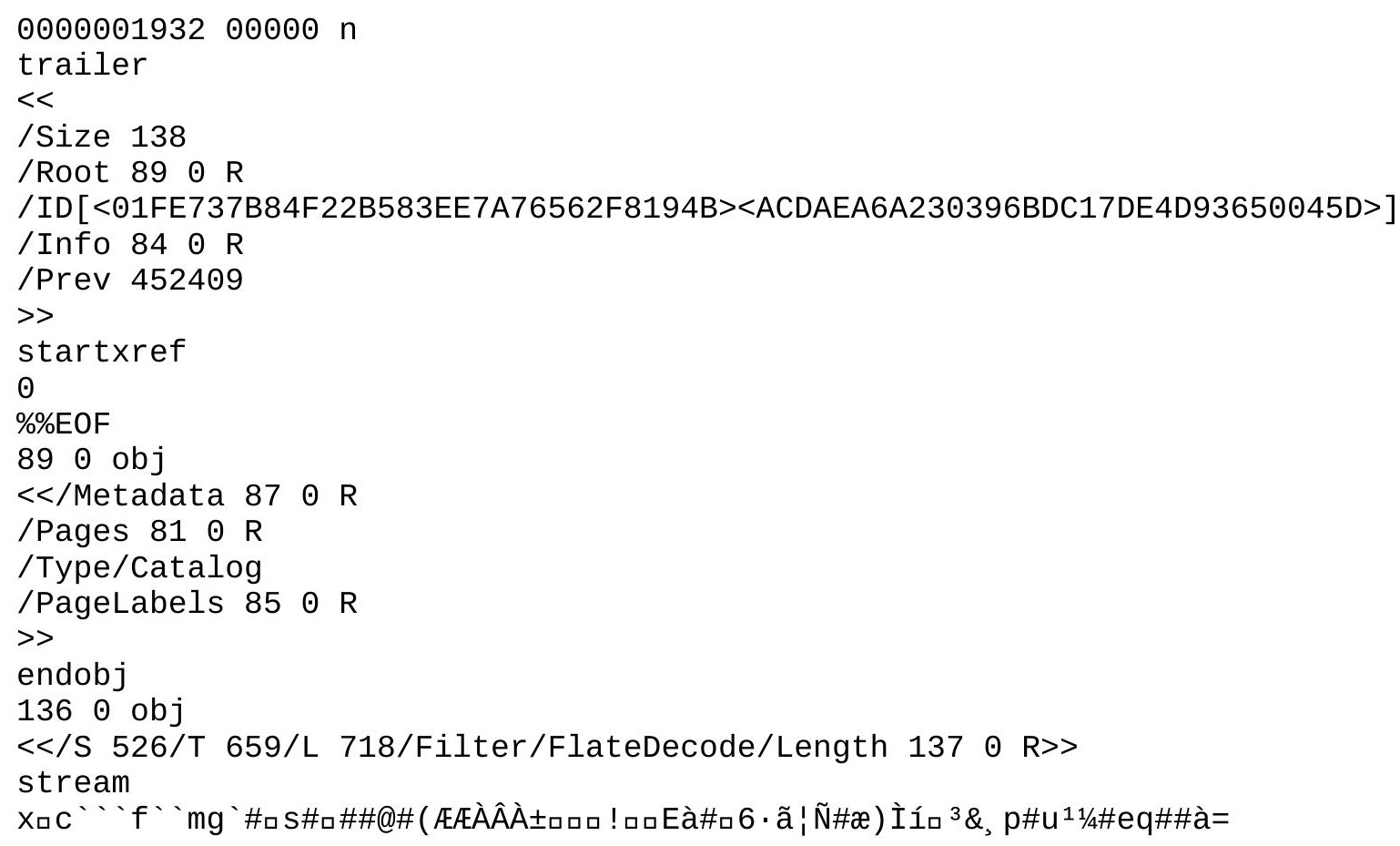




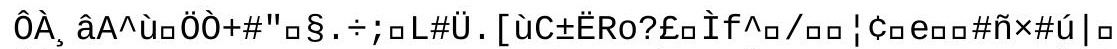

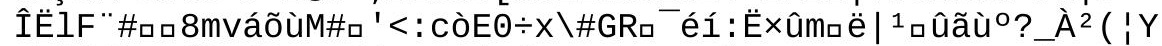

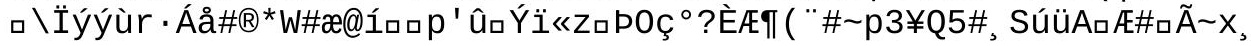




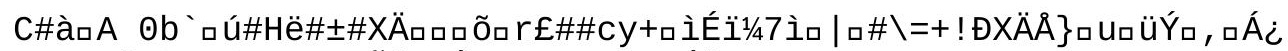

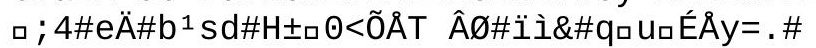

KØ4a\#8 
Ôı\&\#, ’ ®Pnìryp@Ç\#ÉaN 


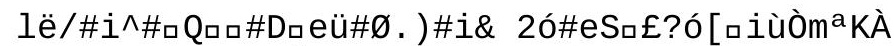

$\mathrm{B} \# \emptyset 囚 \# \mathrm{Q} \# \odot 8 \# \# \# \mathrm{RA \hat {a }}$

endstream

endobj

$137 \odot$ obj

425

endobj

$9 \odot \odot$ obj

$<<$

/Type/Page

/Resources $91 \odot \mathrm{R}$

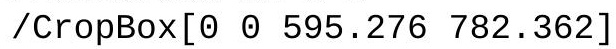

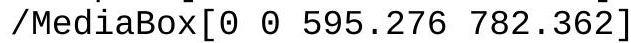

/Rotate $\odot$

/Annots $\left[\begin{array}{lll}127 & \odot & R\end{array}\right.$

]

/Parent $82 \odot \mathrm{R}$

/Contents [128 $\odot \mathrm{R}$

$129 \odot \mathrm{R}$

$130 \odot \mathrm{R}$

$131 \odot \mathrm{R}$

$132 \odot \mathrm{R}$

$133 \odot \mathrm{R}$

$134 \odot \mathrm{R}$

$135 \odot \mathrm{R}$

]

/Thumb $66 \odot \mathrm{R}$

$>>$

endobj

910 obj

$<</$ Font $<</ F 192 \odot \mathrm{R}$

$/ \mathrm{F} 295 \odot \mathrm{R}$

/F3 $98 \quad \odot \mathrm{R}$

/F4 $1010 \mathrm{R}$

/F5 $105 \odot \mathrm{R}$

/F6 108 ○ $R$

/F7 $1110 \mathrm{R}$

/F8 $11400 \mathrm{R}$

/F9 117 ○ R

/F10 $122 \odot \mathrm{R}$

$>>$

/Procset [/PDF

/Text

]

/ExtGState $<</ G S 2125 \odot \mathrm{R}$

$>>$

$>>$

endobj

$92 \odot$ obj

$<$ </Subtype/Type1

/FontDescriptor $93 \odot \mathrm{R}$

/LastChar 83

/Widths [718

$\odot$

614

0

552

$\odot$

718

718

281

0

$\odot$

500 
/BaseFont/KJMHBM+AdvFrutiger -R

/Firstchar 65

/Encoding/WinAnsiEncoding

/Type/Font

$>$

endobj

$93 \odot$ obj

$<</$ StemV 0

/FontName/KJMHBM+AdvFrutiger - R

/FontFile3 $94 \odot \mathrm{R}$

/Flags 32

/Descent - 221

/FontBBox [-166

$-239$

1000

802

]

/Ascent 750

/CapHeight 698

/XHeight 510

/Type/FontDescriptor

/ItalicAngle $\odot$

/CharSet (/space/0/R/I/G/N/A/L/E/S/C/H)

$>>$

endobj

$94 \odot$ obj

$<</$ Subtype/Type1C

/Length 722

/Filter/FlateDecode

>stream

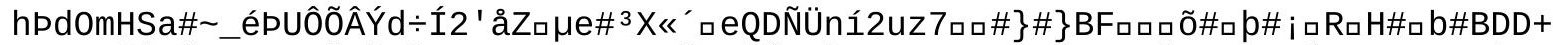

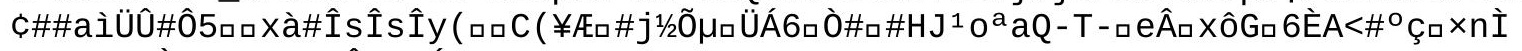

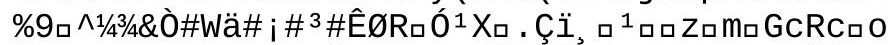

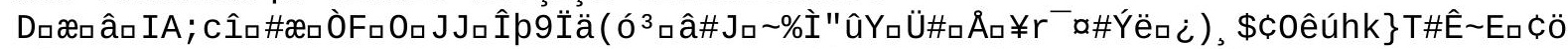

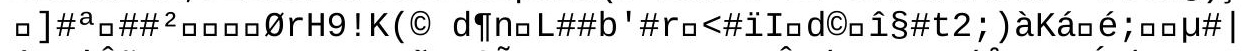

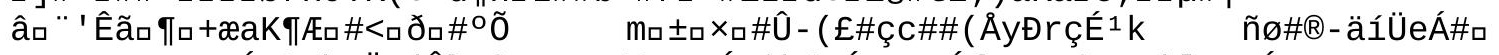

æù»ßロ \#Pù $>\mathrm{q}(<*$

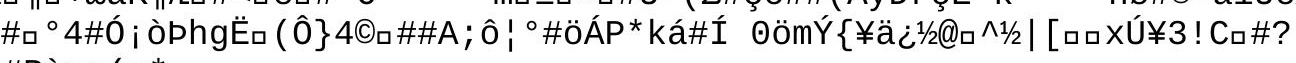

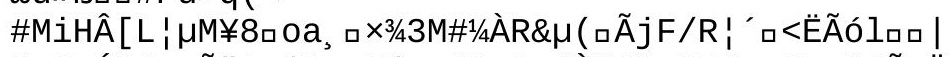

1/2a\#; É Ẽ\#U

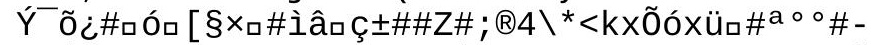

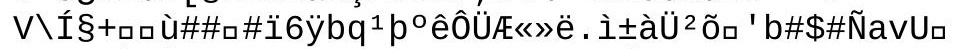

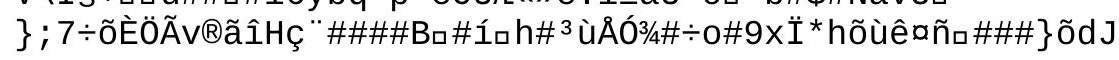

endstream

endobj

$95 \odot$ obj

$<</$ Subtype/Type1

/FontDescriptor $96 \odot \mathrm{R}$

/LastChar 122

/Widths [281

$\odot$

0

572

0 

]

/BaseFont/KJMHBN+AdvCgse

/Firstchar 45

/Encoding/WinAnsiEncoding

/Type/Font

$>$

endobj

$96 \odot$ obj

$<</$ StemV $\odot$

/FontName/KJMHBN+AdvCgse

/FontFile3 $97 \odot \mathrm{R}$

/Flags 32

/Descent - 228

/FontBBox [-197

$-218$

1000

791

]

/Ascent 734

/CapHeight 667

/XHeight 521

/Type/FontDescriptor

/ItalicAngle $\odot$

I

CharSet (/space/c/a/n/i/e/r/f/c/t/v/l/s/o/h/S/y/m/x/X/T/hyphen/two/zero/V/g/z/I/d $/ \mathrm{U} / \mathrm{M} / \mathrm{R} / \mathrm{D})$

$>>$

endobj

$97 \odot$ obj

$<</$ Subtype/Type1C

/Length 2521

/Filter/FlateDecode

>stream

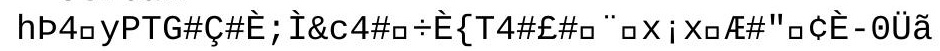




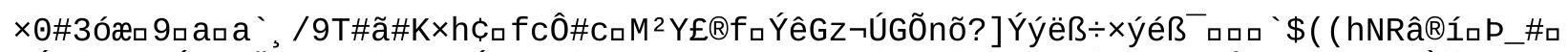

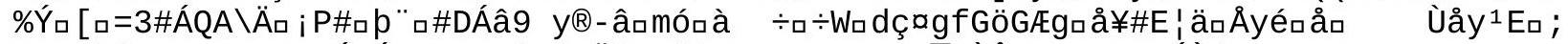

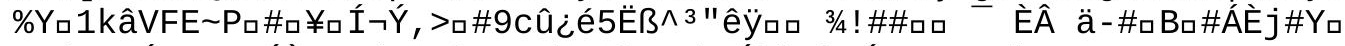

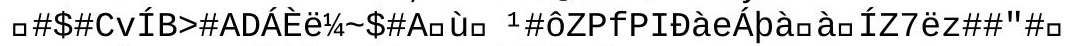

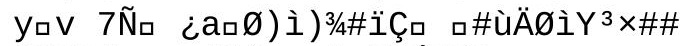

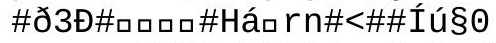

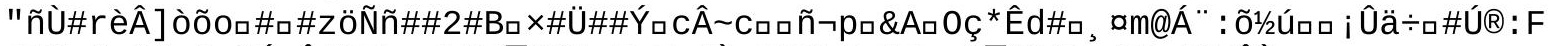

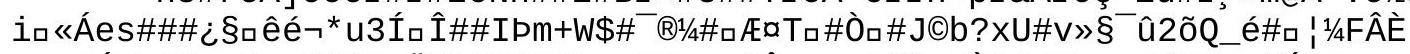

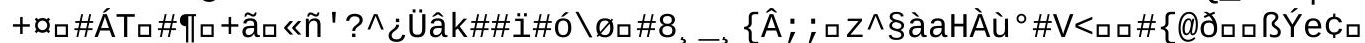

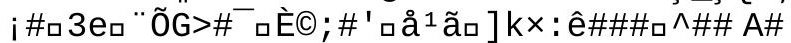




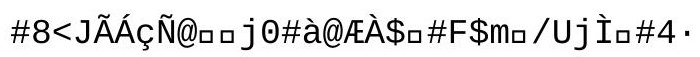




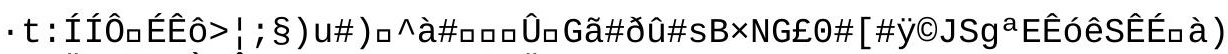

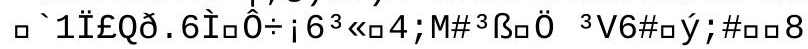




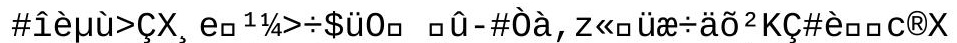

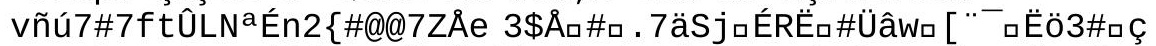

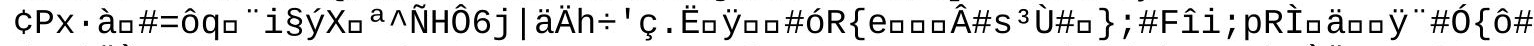

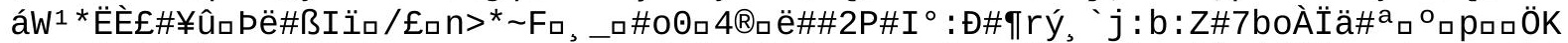

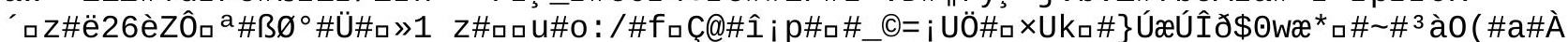

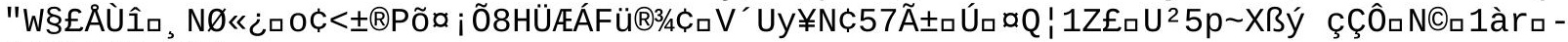
Ö\#K) ) \RঃUÔ̂̂

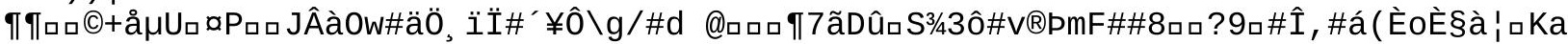

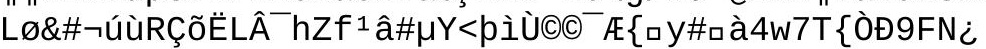




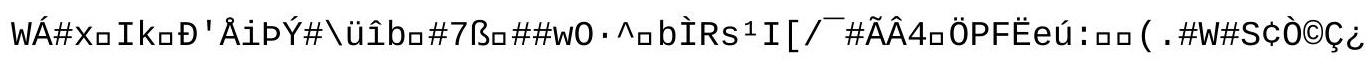




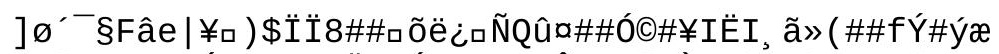

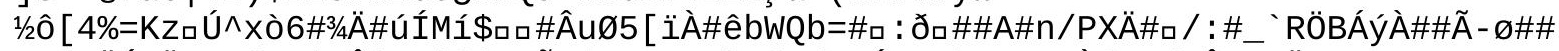

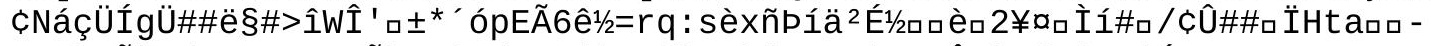

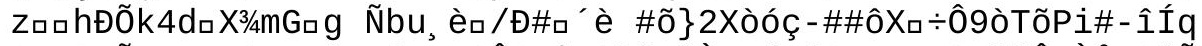

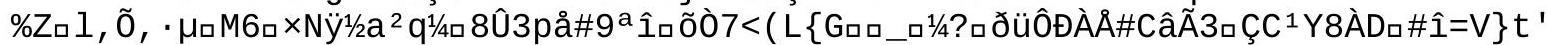
\#aÜó\#pÄpвqв\#ùOR\#nY

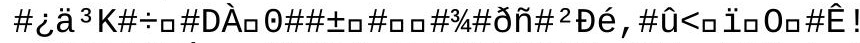

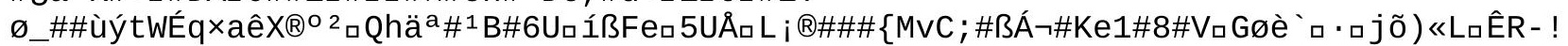

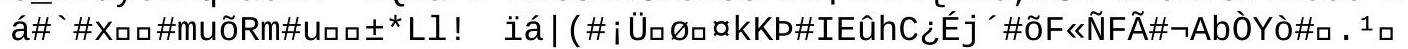


Äò>3/4çC\#ñ \#SCD\#i 


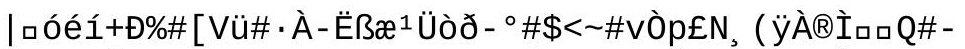

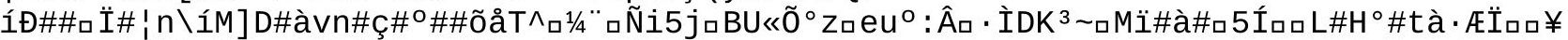

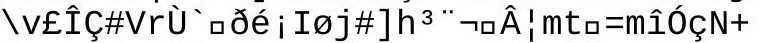

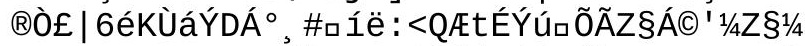

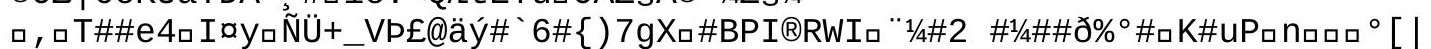

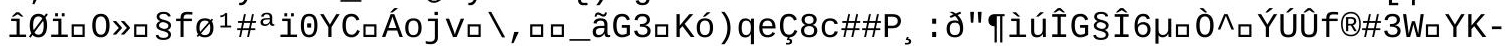

Áç ${ }^{\circ} \mathrm{E} 3 / g \# \# \# \neg F \_E$

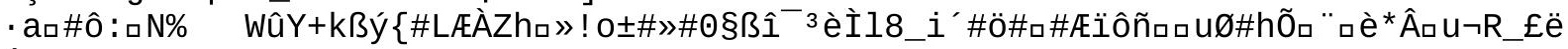

ó 


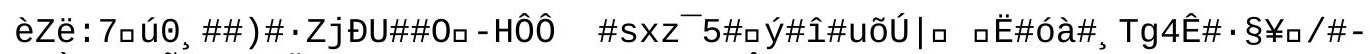

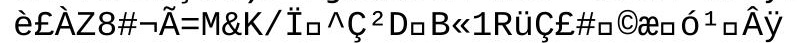

$\odot \# ! \backslash ß \#$

\section{endstream \\ endobj}

$98 \odot$ obj

$<</$ Subtype/Type1

/FontDescriptor $99 \odot \mathrm{R}$

/LastChar 180

/Widths [270

270

$\odot$

$\odot$

541

541

0

0

$\odot$

$\odot$

$\odot$

$\odot$

0

0

0

$\odot$

0

0

$\odot$

666

593

593

656

0

0

635

0

$\odot$

333

$\odot$

0

677

0

572

0

$\odot$

0

520

0

$\odot$

$\odot$

$\odot$

0

0

$\odot$

$\odot$

0

$\odot$

$\odot$ 



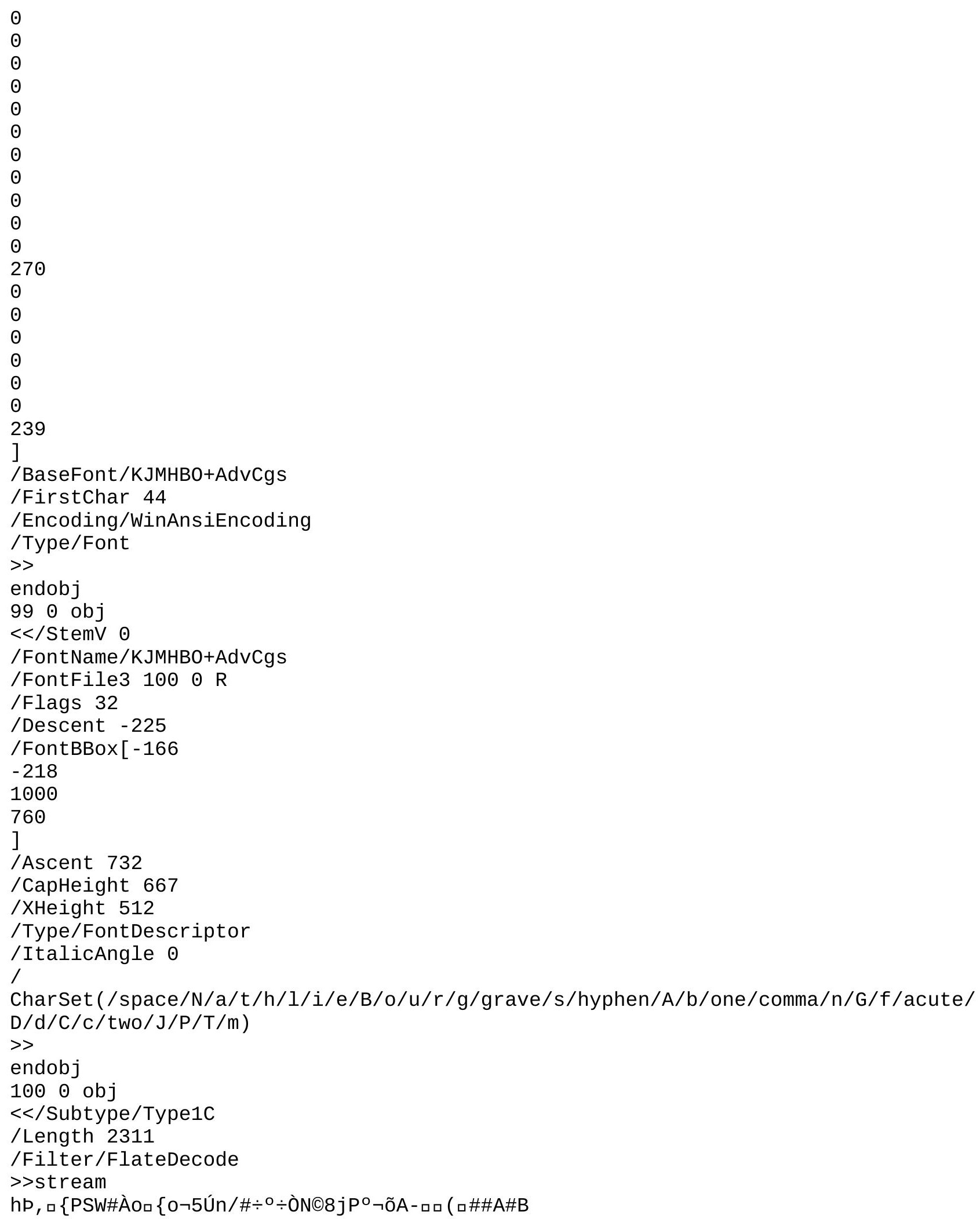




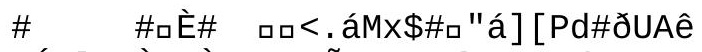

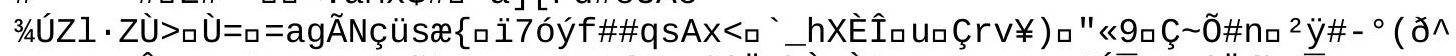

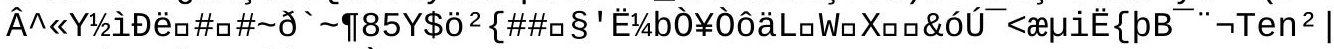
C VıôdaxRï̈̈é - \#7lyWøû`aç<È

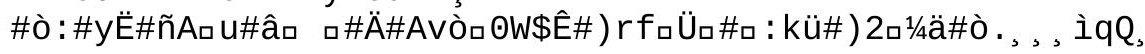




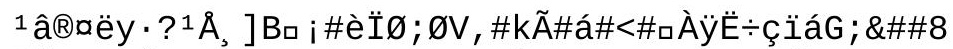

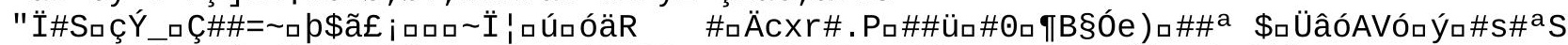

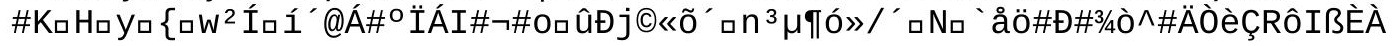


$5 ; \square \#_{\square} \wedge " \hat{I}_{\square} 1 Y^{1} Y^{1} 9 \#_{\square} \Theta \#_{\square}=\square q A^{-} \#_{\square} \hat{I}^{\circ} \vee A ́ \#_{\square}<$ 
6 - ú i ¿bı@\#

Ú॰ロ@\#\}\#t\#@û\#\#Öロ6ロëíロiU\%0̃\%|Á9n 
ロ\#SÙ\# ฉÔロÖâa aZp=\{ÏĖ $\} \#, \# M A ̀ \mu 4 L Y o ̂ \# F$ 


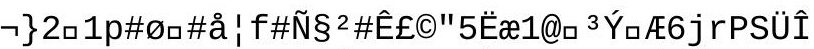

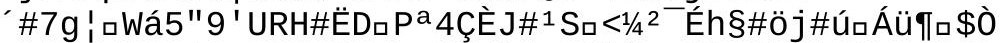

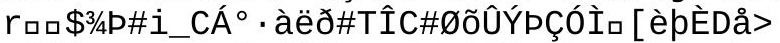

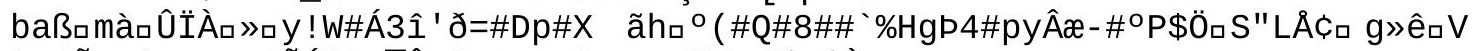

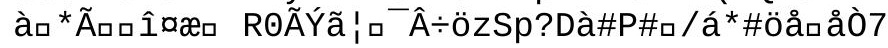


,\#On\#\#p+.aUj\#Sロ\#\}

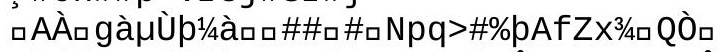

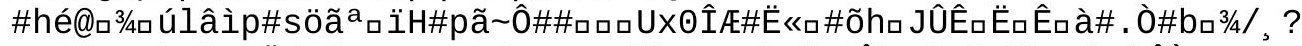

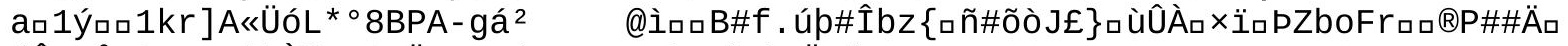

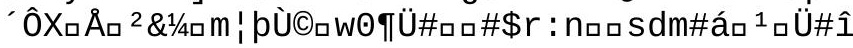


\%॰ë-úAp 


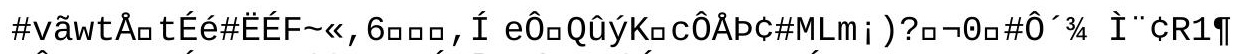

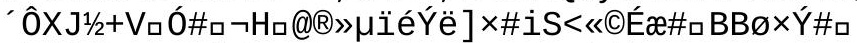

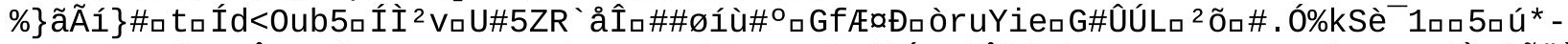

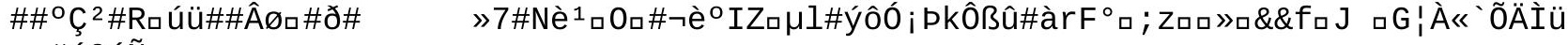
ฉם\#é@óñ 
úqtbë\#

®घ́ 37 
口\#

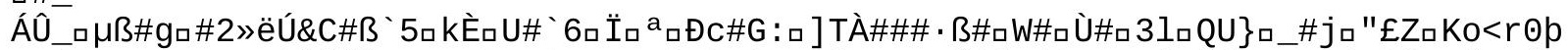

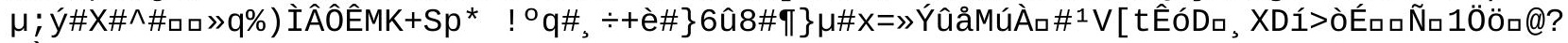
KÀ

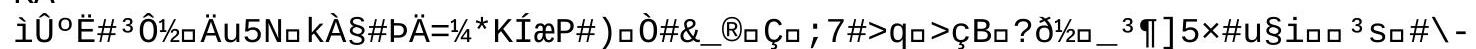

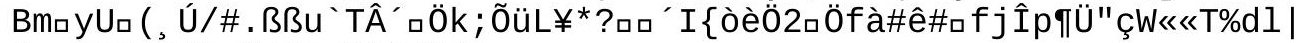

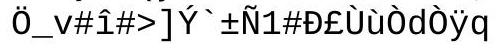


ð\#PßÜüåFêhT\#] ' Ë1ESq

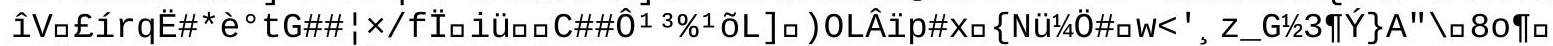

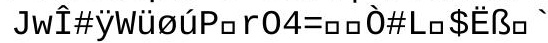




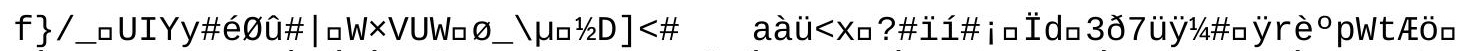

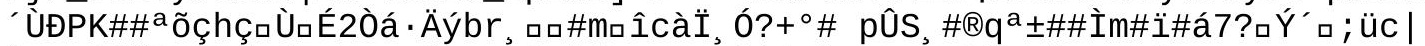

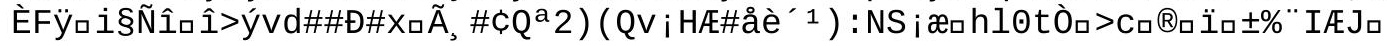






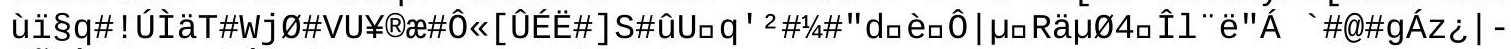

\$ÑàA ; ð>æÿ

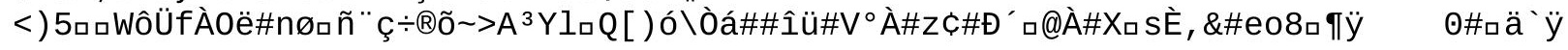

endstream

endobj

1010 obj

$<</$ Subtype/Type1

/FontDescriptor $102 \odot \mathrm{R}$

/LastChar 180

/Widths [458

0

218

0

$\odot$

0

0

520

0

$\odot$

260

260

$\odot$

0

260

260

260

260

520

520

520

520

520

520

520

520

520

520

260

260

○

520

0

$\odot$

833

625

572

583

625

489

479

604

635

239

0

531

458

812

645

666

552

0

552 



\section{$\odot$}

0

0

500

0

0

$\odot$

$\odot$

$\odot$

0

$\odot$

$\odot$

0

218

$\odot$

$\odot$

$\odot$

$\odot$

$\odot$

$\odot$

$\odot$

0

$\odot$

0

0

0

260

0

0

$\odot$

$\odot$

$\odot$

0

218

]

/BaseFont/KJMHBP+AdvCgsl

/Firstchar 30

/Encoding $104 \odot \mathrm{R}$

/Type/Font

$>$

endobj

$102 \odot$ obj

$<</$ StemV $\odot$

/FontName/KJMHBP+AdvCgsl

/FontFile3 $103 \odot \mathrm{R}$

/Flags 32

/Descent - 224

/FontBBox [-156

$-218$

1000

739

]

/Ascent 731

/CapHeight 667

/XHeight 508

/Type/FontDescriptor

/ItalicAngle 0

/

CharSet (/space/one/D/e/p/a/r/t/m/n/o/f/S/l/A/i/c/c/s/d/two/U/M/R/eight/P/h/y/g/T /X/E/acute/comma/I/N/V/u/F/B/grave/hyphen/b/three/seven/six/four/zero/colon/peri $\mathrm{od} / \mathrm{at} / \mathrm{v} / \mathrm{o} / \mathrm{slash} / \mathrm{j} / \mathrm{nine} / \mathrm{five/X/parenleft/parenright/endash/percent/G/K/Y/k/L/W/W/}$ $\mathrm{H} / \mathrm{z} / \mathrm{quoteright/q/equal/fi/semicolon)}$

$>$

endobj 
$103 \odot$ obj

$<</$ Subtype/Type1C

/Length 4903

/Filter/FlateDecode

>sstream

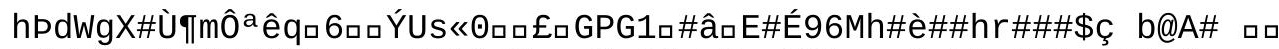

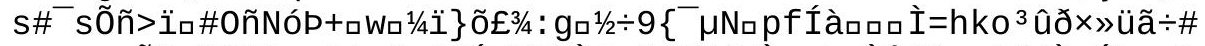

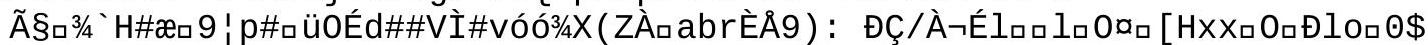

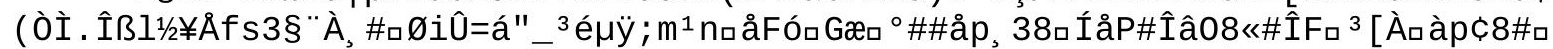

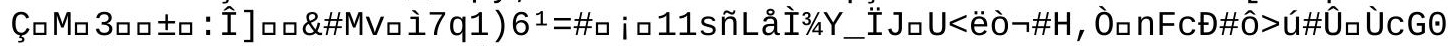

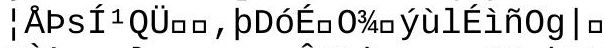

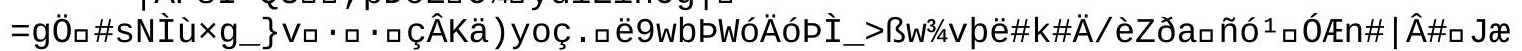




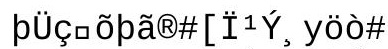

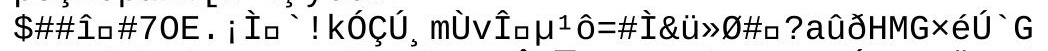

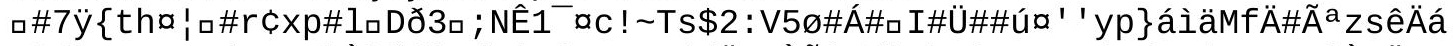

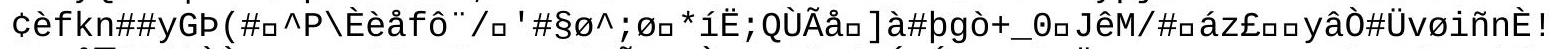

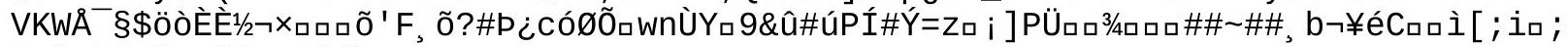

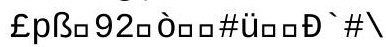

$\square X \hat{A} \# \Theta \#$

口À9`\#Xù\#|\#Ò ( $k \# \# \# ;$; øòõWà

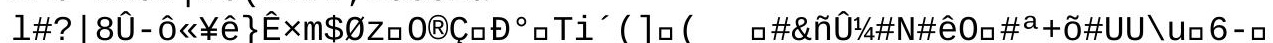

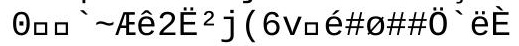

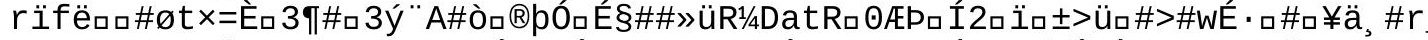

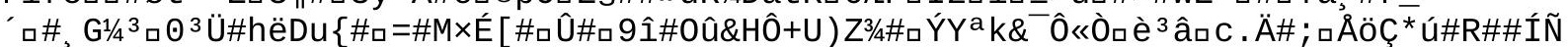

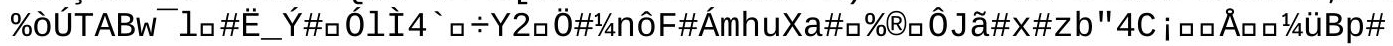




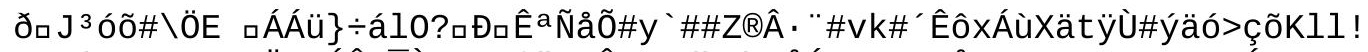

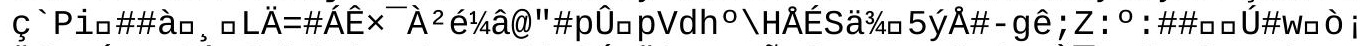

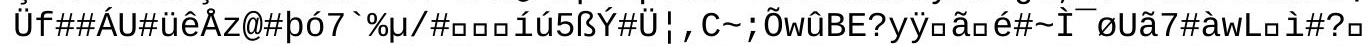

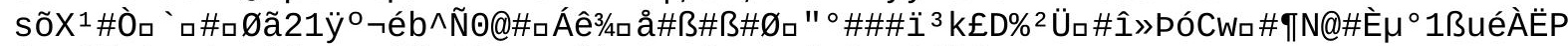

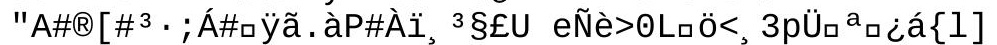

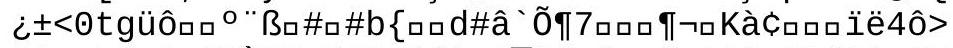

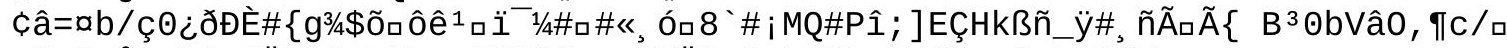

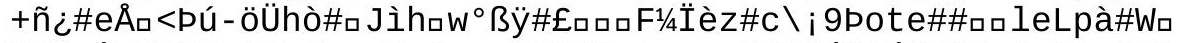

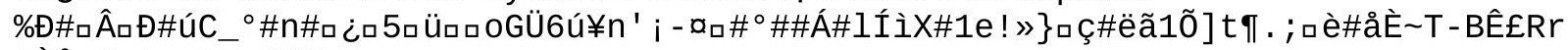

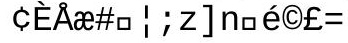




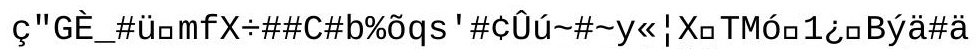

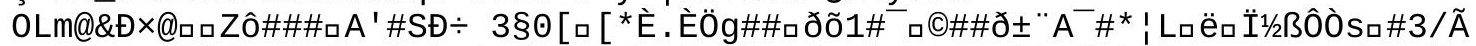

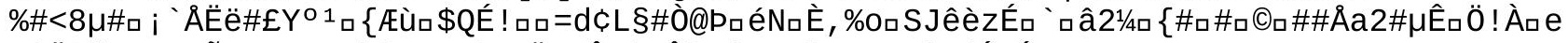

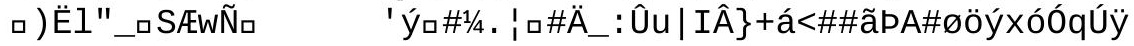




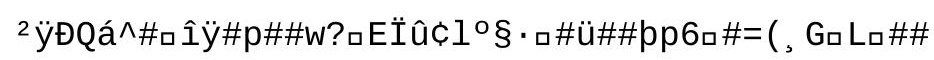


Ìzpzë

\}

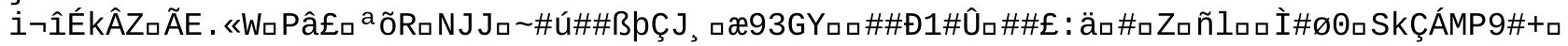




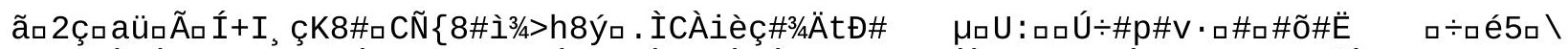

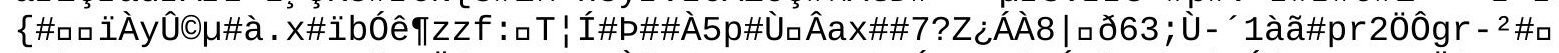

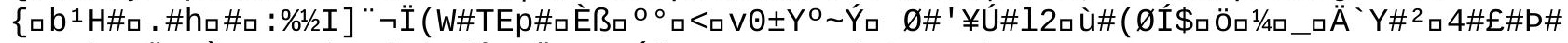

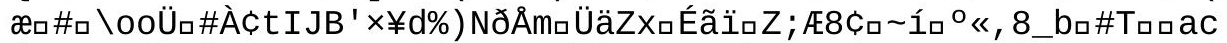

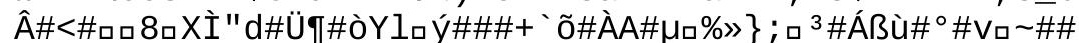

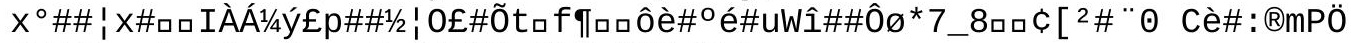

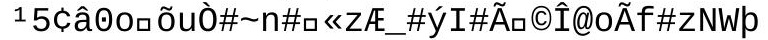

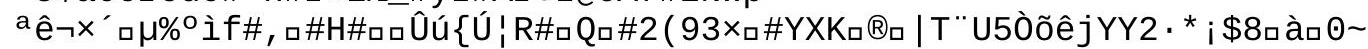

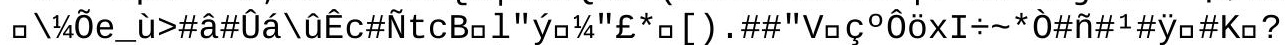

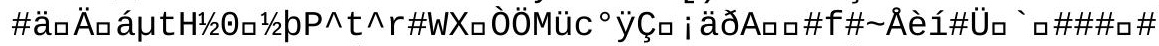

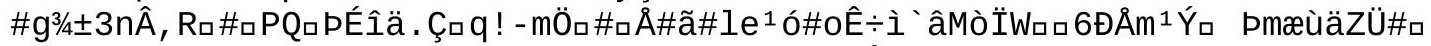

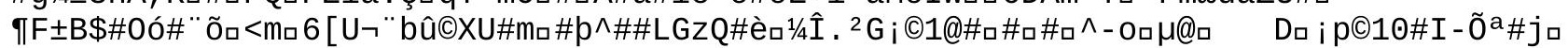
$F^{a} d_{\square} \varnothing^{a}$

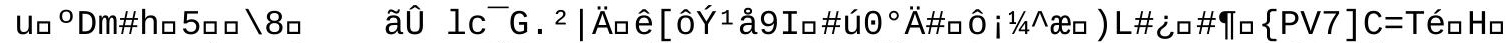

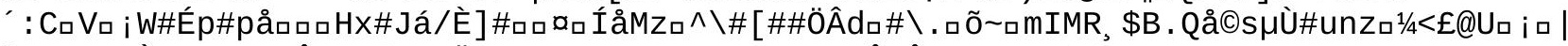

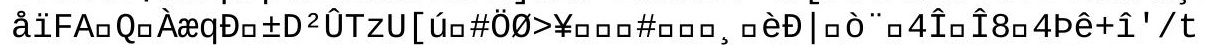

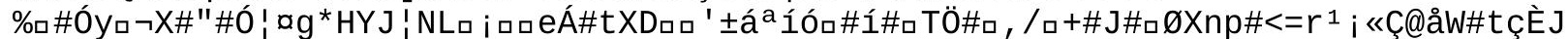
'\#- ) ĖÔUÒÓð\#0̃ $80 \cdot$ yofç\#n

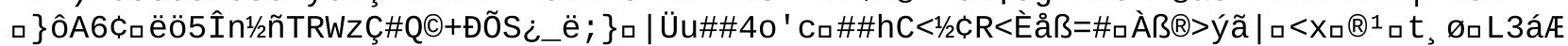

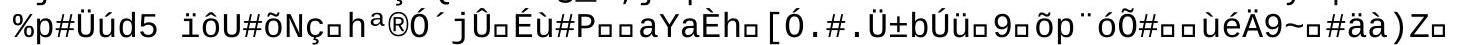

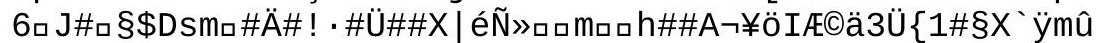
, \#Z<\\#E' 'CÍOFhÿ6aaZ

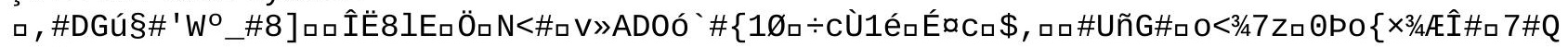
$\mathrm{Y} \square \mathrm{C}\urcorner$

Òıฉ@ae; ฉÉ

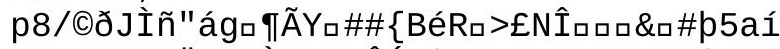

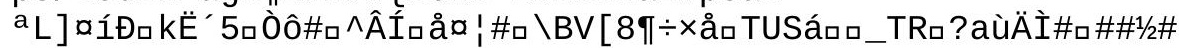

$\hat{\imath q}) £$

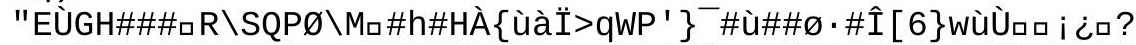

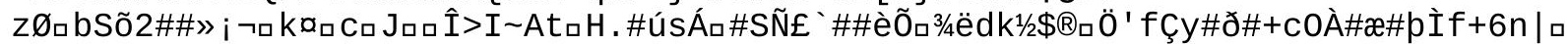

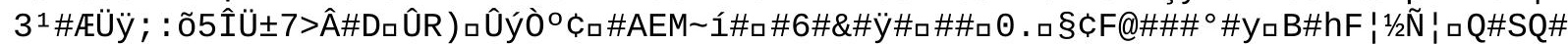
口/Úロव \}ðê_aÜ_\#\#EI\$

yt $\div$ NÇoë^òèñ̃ $a_{\square} \#: Y \neg \square$

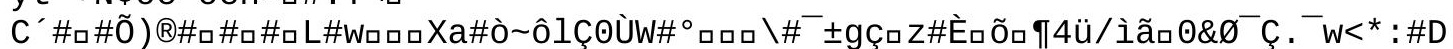

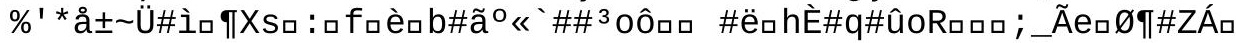

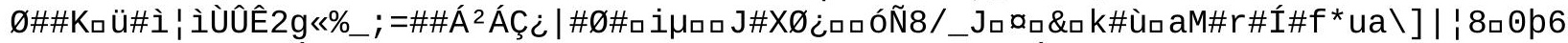

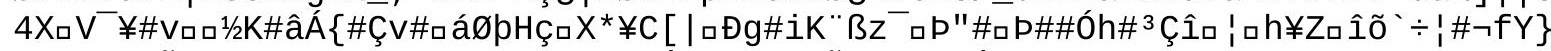

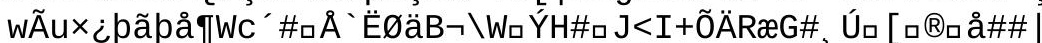

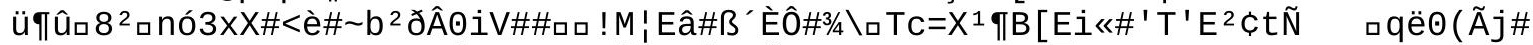

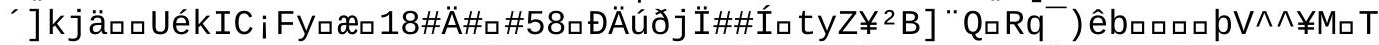

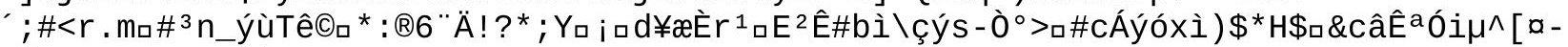

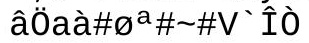




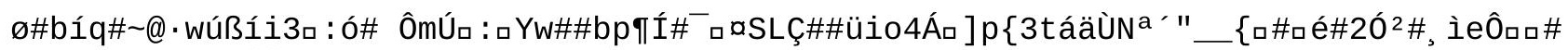

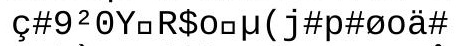

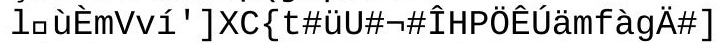

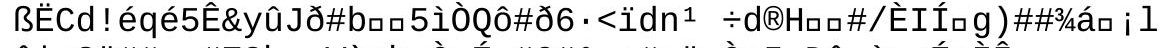

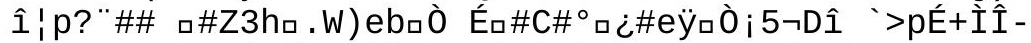

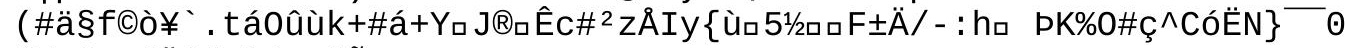

'Vঃ\#ロu\#Öå\#, líı ÖÃ 
, \#U) $\mu \# \# \square W a q u \Delta S d E ̈ E ̈ \times e q$

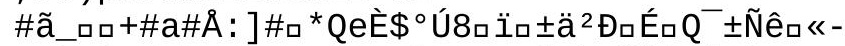

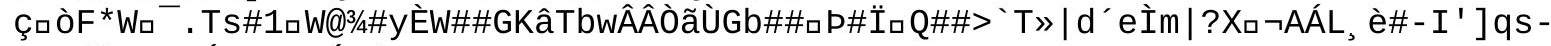
5ä\#ýlQJ\#2Ýø̈̈\#@\#Úロó i

endstream

endobj

104 o obj

$<</$ Differences [19

/Lslash

/lslash

/minus

/fraction

/breve

/caron

/dotlessi

/dotaccent

/hungarumlaut

/ogonek

/ring

$/ \mathrm{fi}$

$/ \mathrm{fl}$

]

/BaseEncoding/WinAnsiEncoding

/Type/Encoding

$>>$

endobj

$105 \odot$ obj

$<</$ Subtype/Type1

/FontDescriptor $106 \odot \mathrm{R}$

/LastChar 121

/Widths [281

281

281

0

552

552

552

552

552

552

552

552

552

0

281

0

0

$\odot$

$\odot$

0

$\odot$

708

625

604

0

541

541

$\odot$

687

302

$\odot$

614

489 


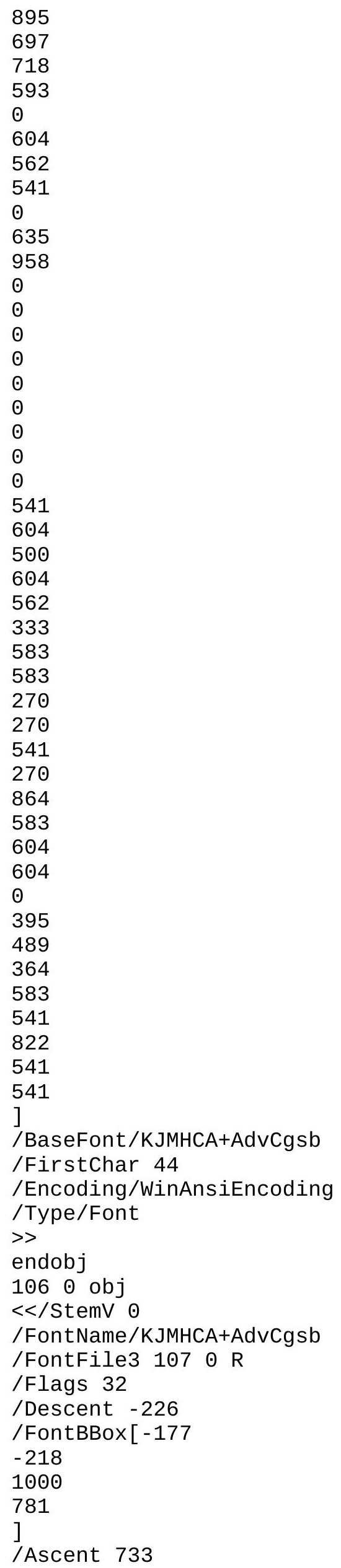


/CapHeight 667

/XHeight 517

/Type/FontDescriptor

/ItalicAngle $\odot$

I

CharSet (/space/K/e/y/W/o/r/d/s/C/p/n/c/B/a/k/g/u/colon/0/b/j/t/i/v/M/h/R/l/V/P/I /S/N/zero/two/seven/five/hyphen/six/three/eight/x/f/H/m/A/L/T/one/period/E/comma $/ \mathrm{F} / \mathrm{four} / \mathrm{W}$ )

$>$

endobj

$107 \odot$ obj

$<</$ Subtype/Type1C

/Length 3897

/Filter/FlateDecode

>stream

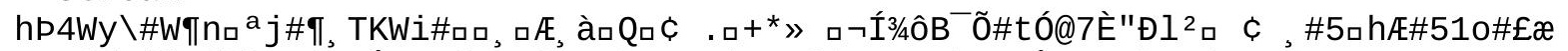

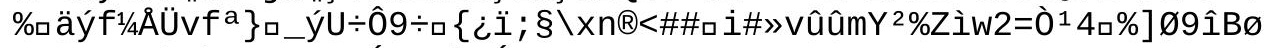
LpgpD=\#\#L\#ロÓKçxløÁsuq

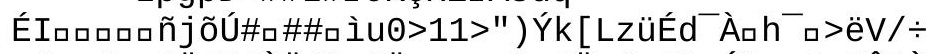

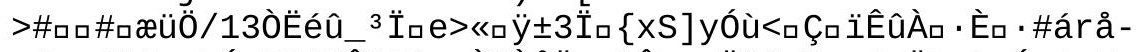

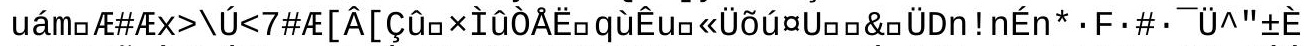

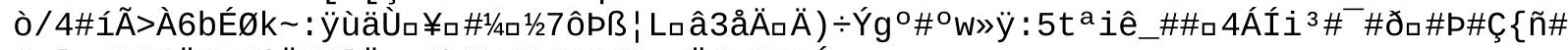

\#g] . AENäDÄÇP\#' İ̃̃XlÖ. qõ \ùP\#áü ; \#\#ào Ü\#S3/4D\#Á,

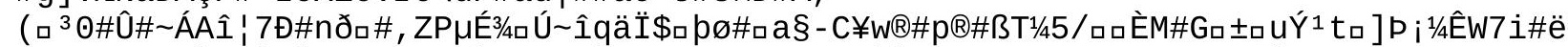
-

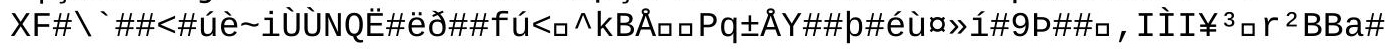




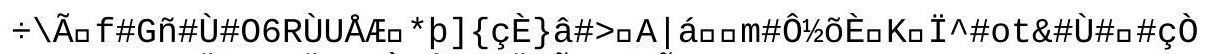

Ï£úÖ*m\#9Ü¥ロ

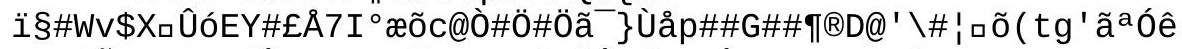

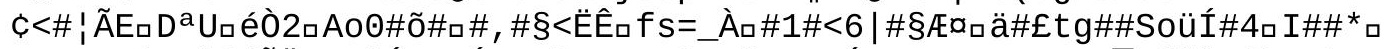

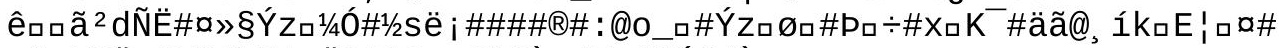

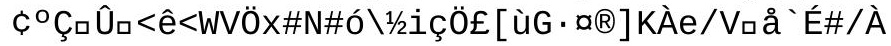

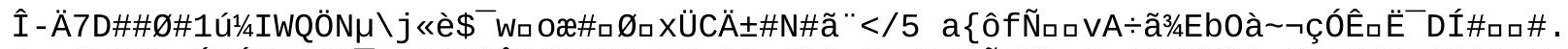

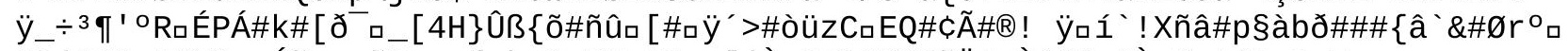

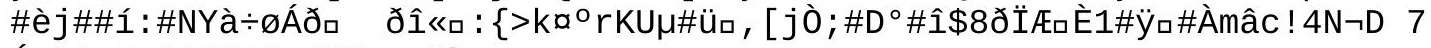
Ío ${ }^{1} \#$ aĐq ${ }^{\circ} \# \# \#$ è\#, \#Y\#ロp, \#\} 


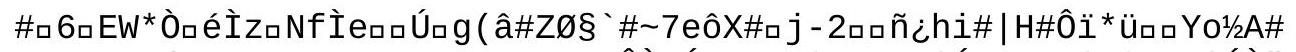

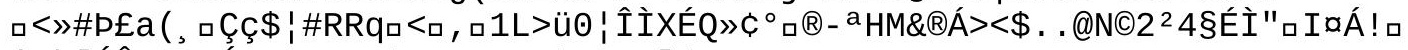

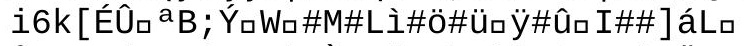

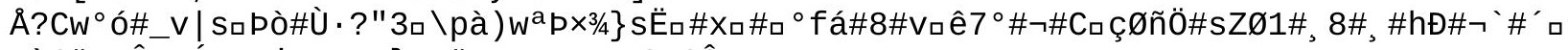

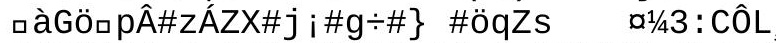

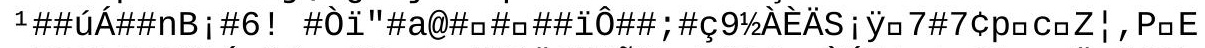

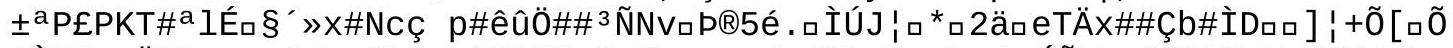

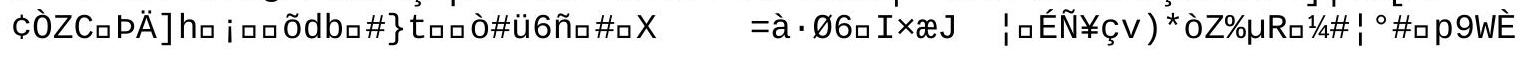




\section{ð}

- ÕTkZ)M§đTV£çWCëUvêì SnW\#Èt İð\#\#ÁÄ

ð\#\#Сも\#\#а̀\#x\#"\#ça!\#ð \#\#\#Ç\#

fal

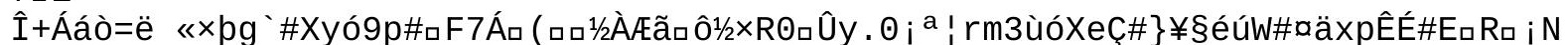

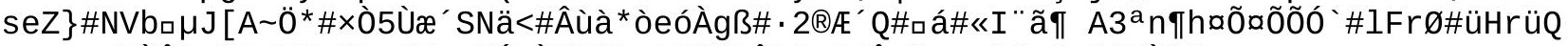

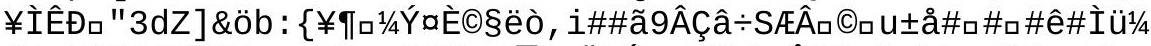

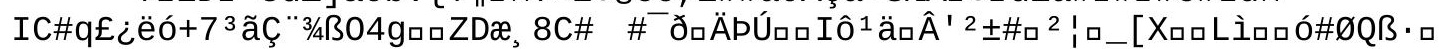

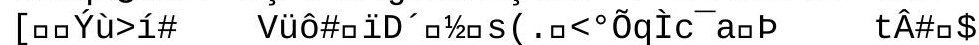

q\#_rò̀r $<\mathrm{Ia}_{\square} \mathrm{a}$

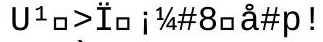

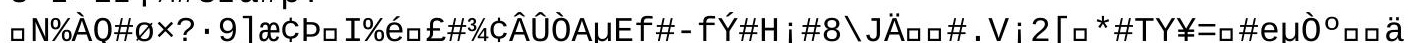

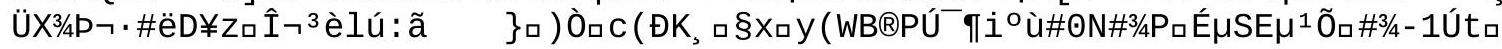

Ç£ ¿̇॰\# 


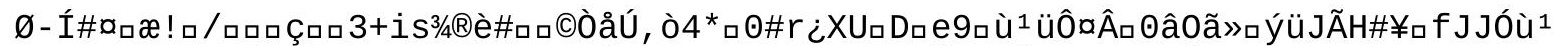

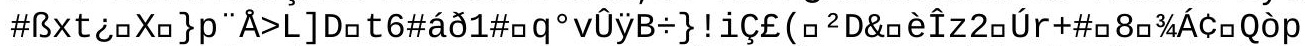

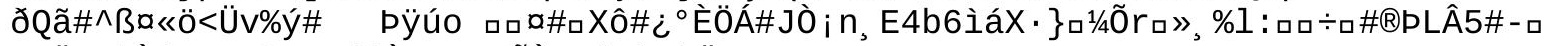

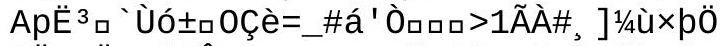

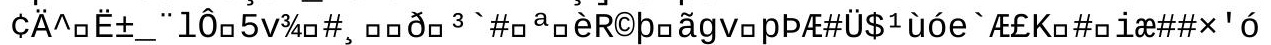

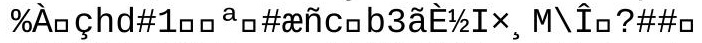

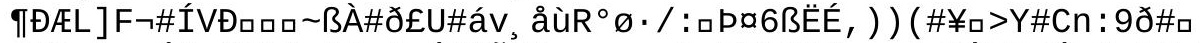

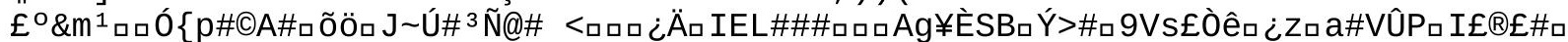

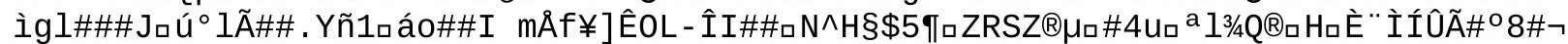

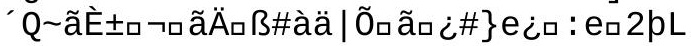




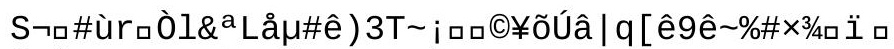

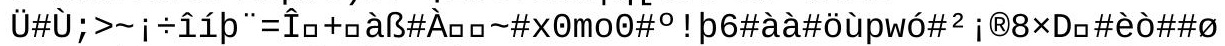

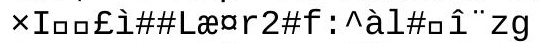

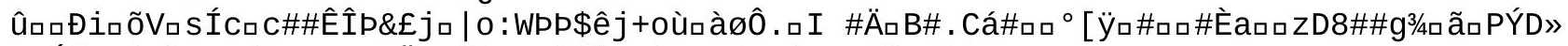

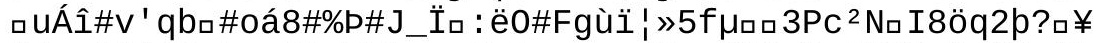

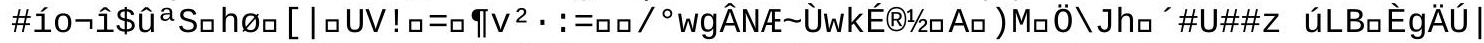

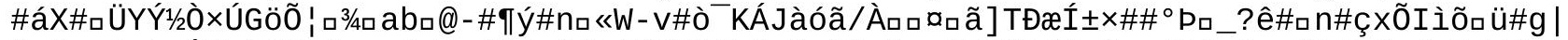

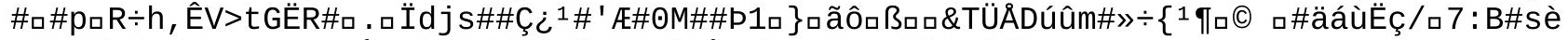

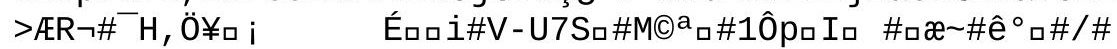




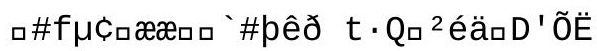




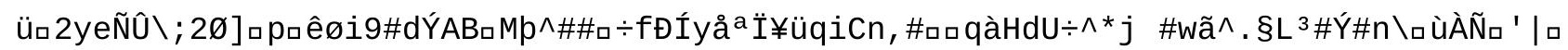

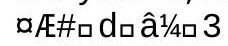

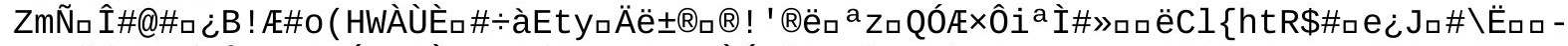

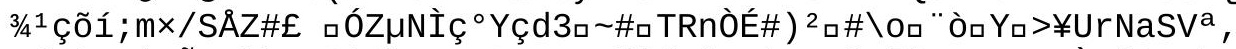

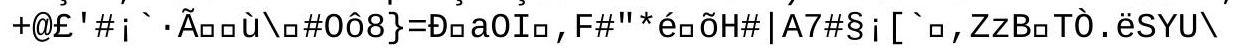


Ñ\&p\#ロ (Xí 
2\#ý\#h2ロ7õ\#̂̂ロI : Ii ${ }^{2} H I ̈ u "$ ô lòtöÀ\#đV\#ý@=ð\# 


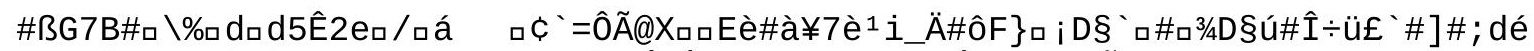

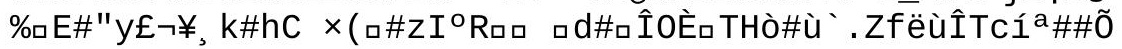


Xìırîıâò, ö\#p\#\#\#\#ÛXÝı

endstream

endobj

$108 \odot$ obj

$<</$ Subtype/Type1

/FontDescriptor $109 \odot \mathrm{R}$

/LastChar 252

/Widths [1000

718

$\odot$

385

385

$\odot$

0

281

385

281

385

552

552

552

552

552

552

552

552

552

552

281

281

0

604

0

500

0

718

666

666

718

614

552

718

718

333

447

718

552

947

718

760

552

0

666

552

552

718

666

1000

666

614

552

385

○ 


/BaseFont/KJMHCB+AdvMeridien-R

/Firstchar 37

/Encoding/MacRomanEncoding

/Type/Font

$>>$

endobj

$109 \odot$ obj

$<</$ StemV $\odot$

/FontName/KJMHCB+AdVMeridien-R

/FontFile3 $110 \odot \mathrm{R}$

/Flags 34

/Descent - 217

/FontBBox [-145

$-208$

1000

729

]

/Ascent 698

/CapHeight 634

/XHeight 460

/Type/FontDescriptor

/ItalicAngle $\odot$

I

CharSet (/space/T/h/e/l/a/s/r/hyphen/b/d/S/y/m/x/X/two/zero/i/V/t/o/g/n/z/c/u/v/p /comma/fi/f/period/w/I/F/C/L/B/one/three/parenleft/P/parenright/R/E/0/equal/nine /eight/semicolon/quoteright/D/H/W/A/N/five/M/seven/slash/four/G/k/fl/six/j/endas $\mathrm{h} / \mathrm{q} / \mathrm{colon} / \mathrm{K} / \mathrm{J} / \mathrm{dieresis} /$ quoteleft/percent/U/bracketleft/bracketright/emdash/Y/acu te/cedilla/ampersand/question/Z)

$>$

endobj

$110 \odot$ obj 
$<</$ Subtype/Type1C

/Length 6977

/Filter/FlateDecode

>stream

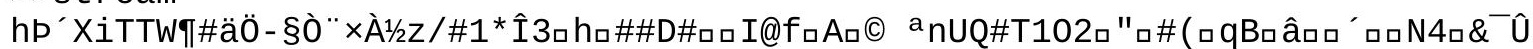




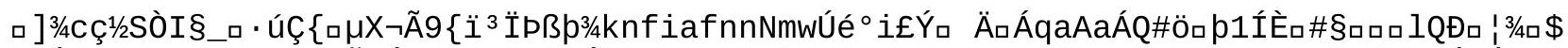

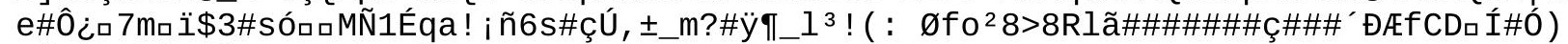
$\pm I_{\square}{ }^{\prime} q p \backslash " \wedge y ́ U ̀ 3 M_{a} \varnothing \notin ß \& .8 \$$ 
\#ロ\#\#2 ฉロÓ\#

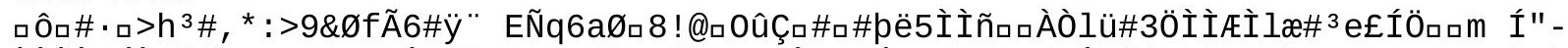

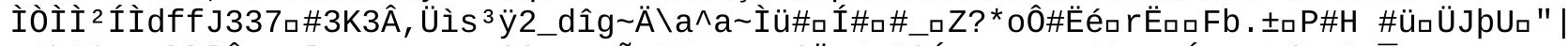

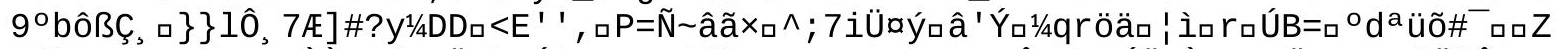

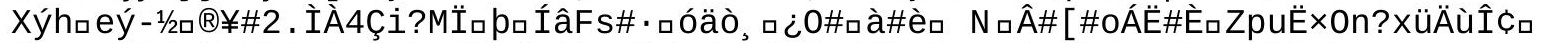

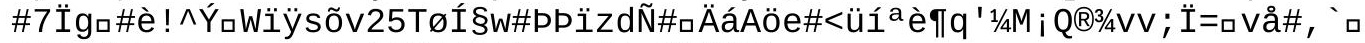

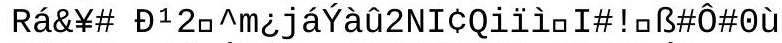

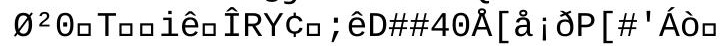

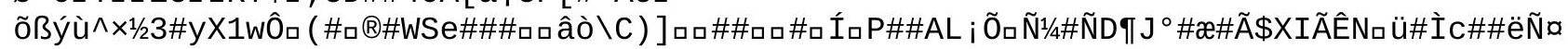
$¥ \mathrm{~h} \%^{2}$ £Mûëñ ${ }^{\circ}[$

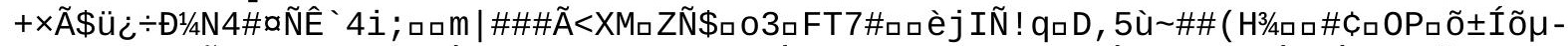

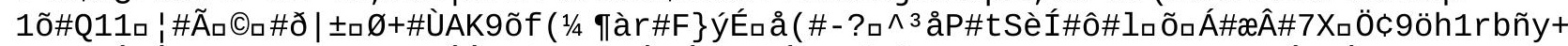

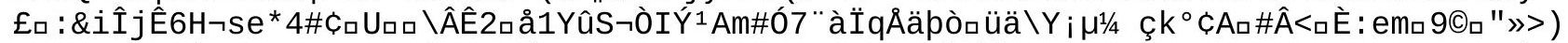

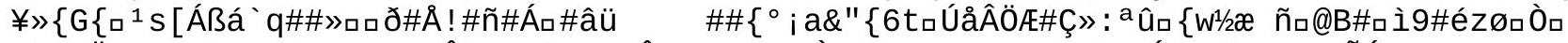

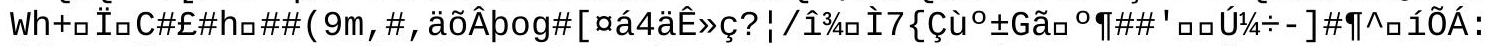

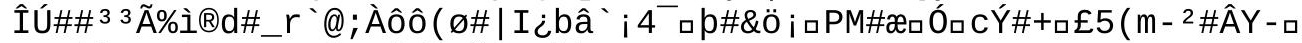

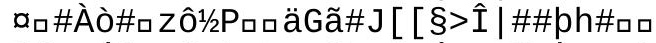

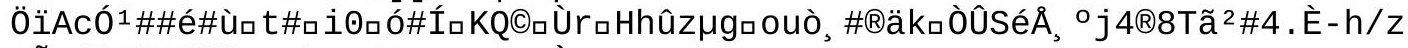

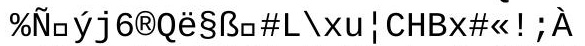

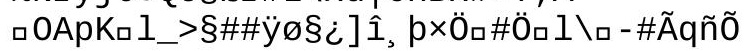


$\# ¥ \mathrm{y} \#$ Y'» 
è\#«Đ®ehç" ' $K ")+x_{\square} 1 \% \AA ̊ J e \quad$ w\#Ü\#CÈ · à

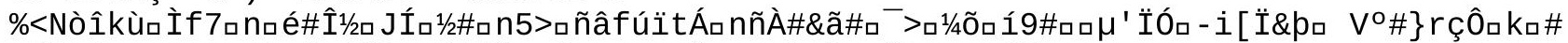

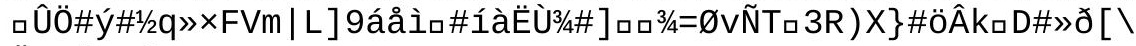

Ö\#æ"\#4ñ «ם? 


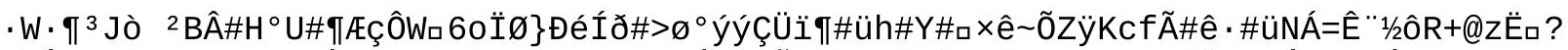

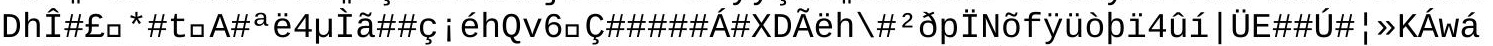

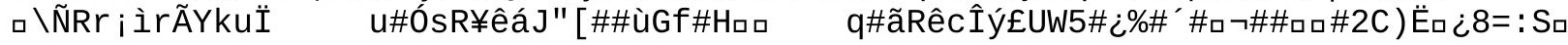
$\% 4 n_{\text {a }}$

\%\#ロ

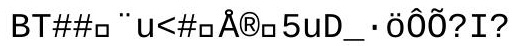

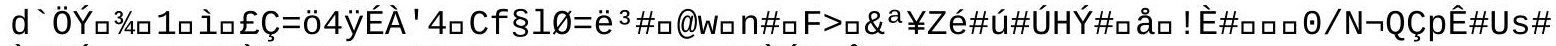

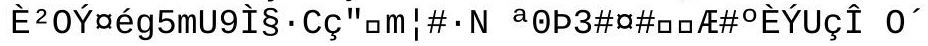




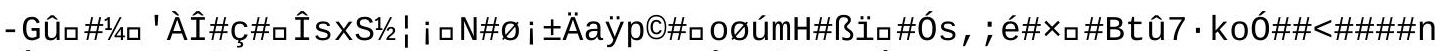

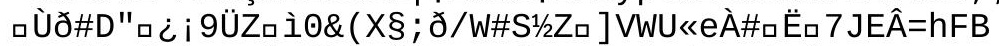

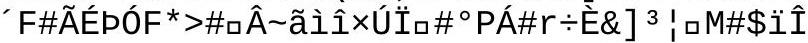




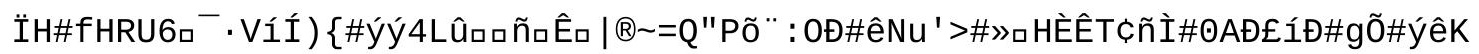




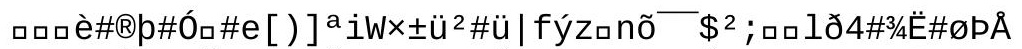

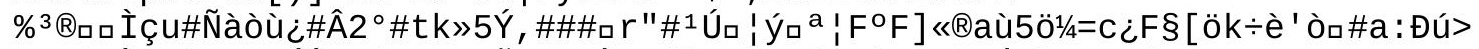

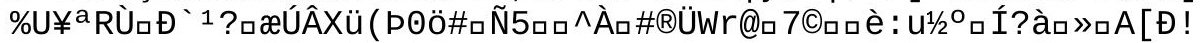




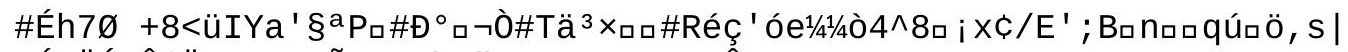

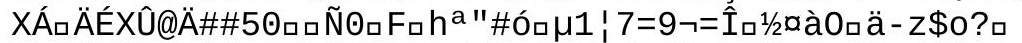

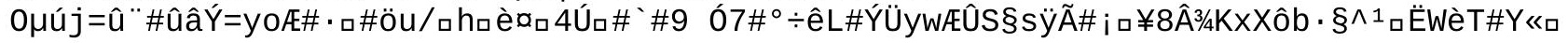
aWaè!

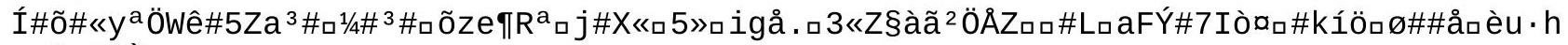
g. 'æü"Uे 


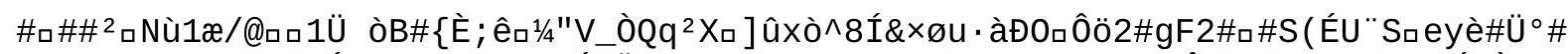
p\#伊范

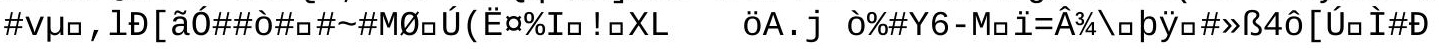


AÄ6"๑Y॰íÁ · «\#ãxò̂̂WUðÅ«* Øåa\#xý\#_?·\# 


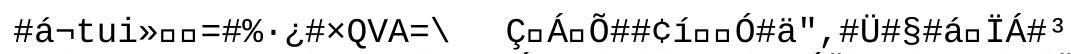

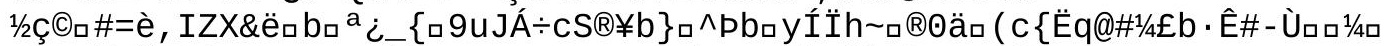

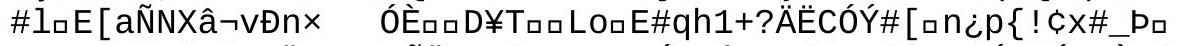
ðxé\#ðロnÄ4e\#²5

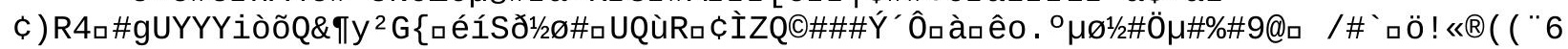




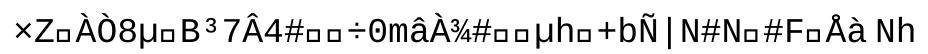




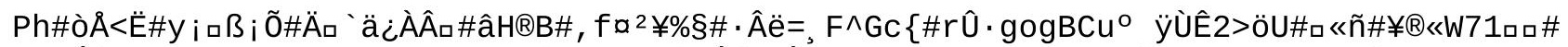

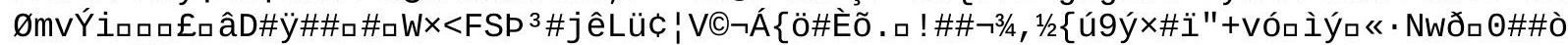

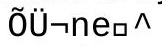
$\wedge$

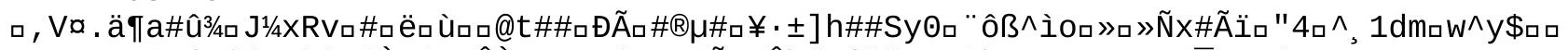

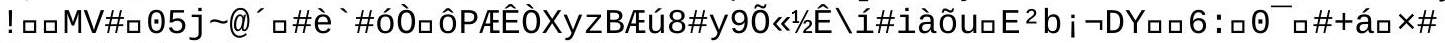

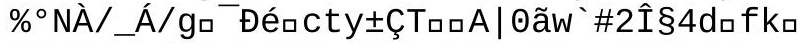

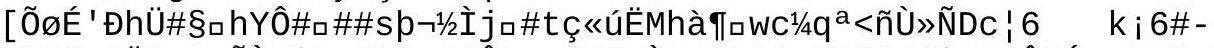

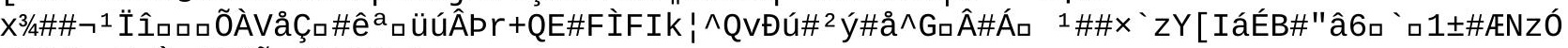

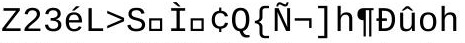

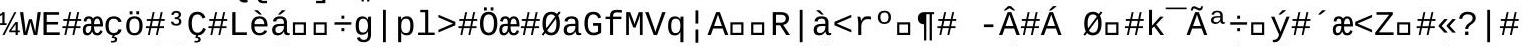

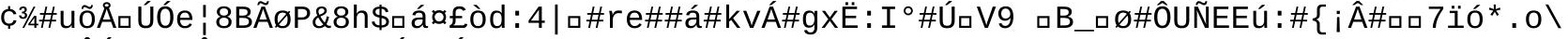

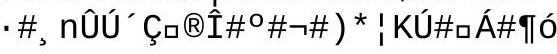




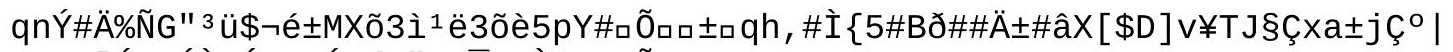
æôC\#] Ú^xÚÀâÍIDxúz $\left\{\right.$ a $^{\prime} \mathrm{J} \#^{-}$a\#Ò\# LBÑ

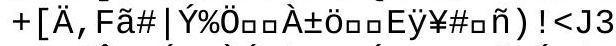

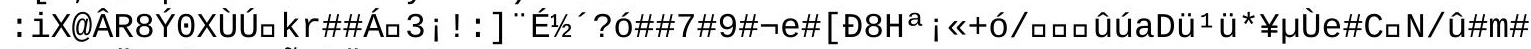
CEdp॰Ä\#ロ ÕI\#DVÃÆhÜ \%W*

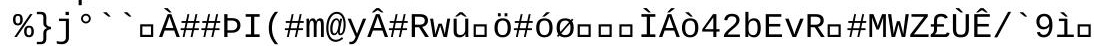

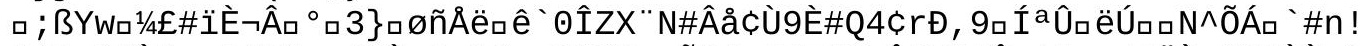

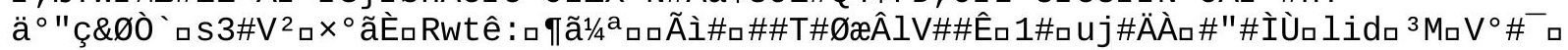

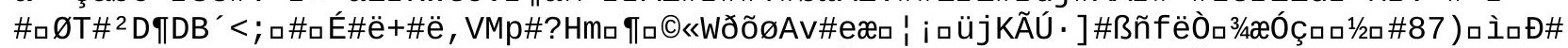

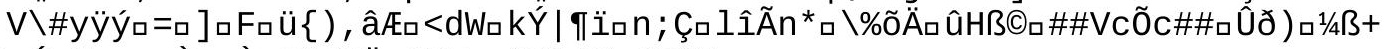

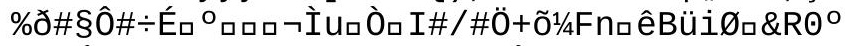

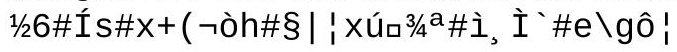




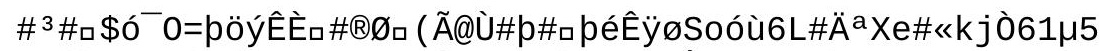

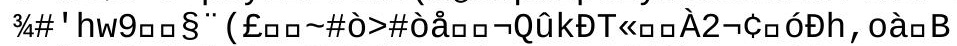

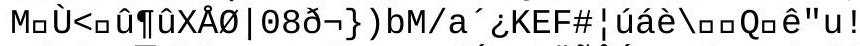

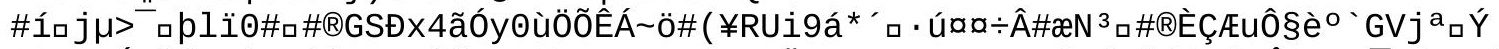

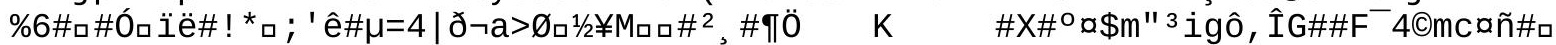

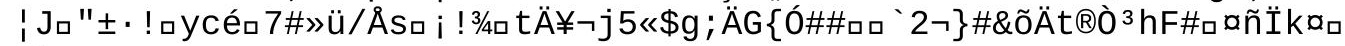
«ò 


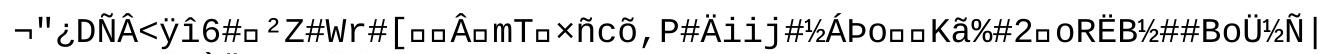

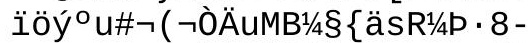

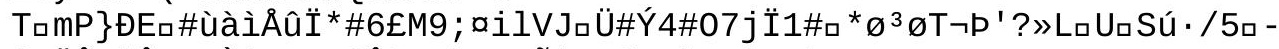

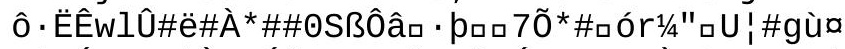

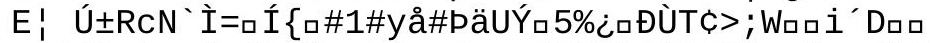

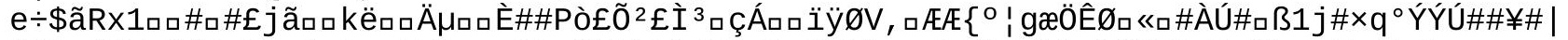

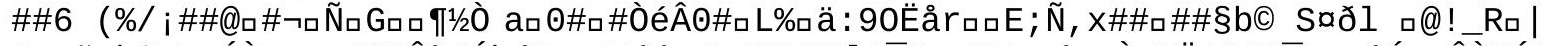

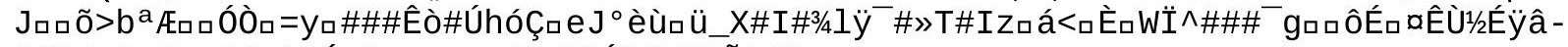

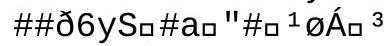
KXa\$\#É ${ }^{2} \# ¥ \dot{1} \hat{I} \tilde{A}: \# \S \sim$. -

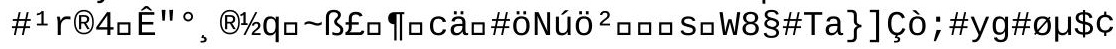




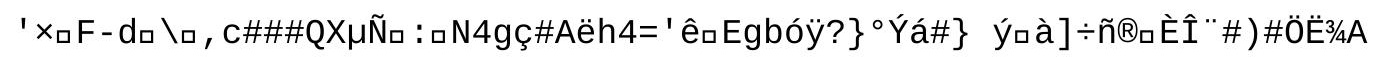




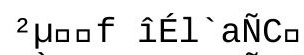

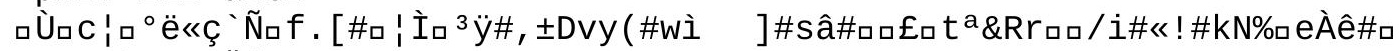

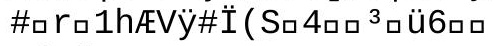

$\# \S ; \tilde{n} \#$

T\#möÕCQQ̇̀̇ F\#

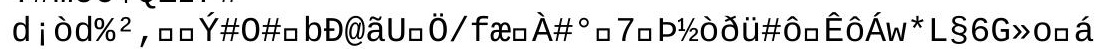

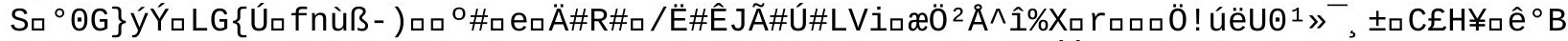

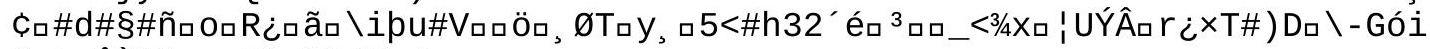

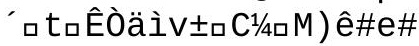




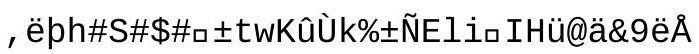




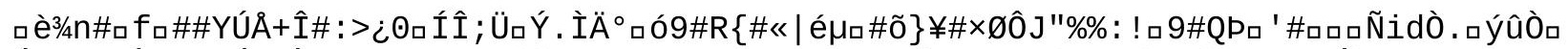

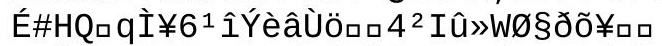

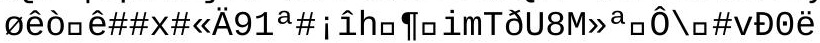

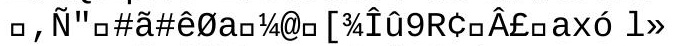

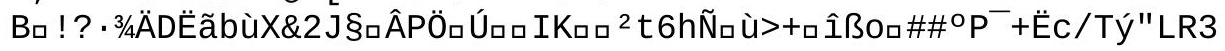


KıÕEØ×ÕÈóhF\#ロ $x_{\square} æ X W$

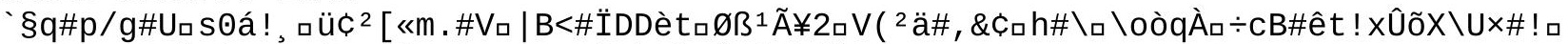

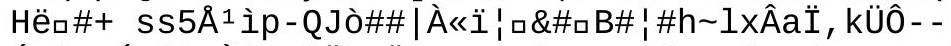

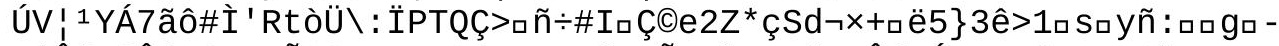

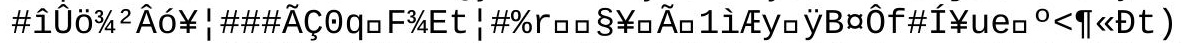

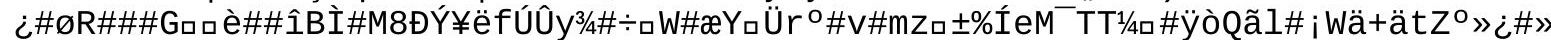

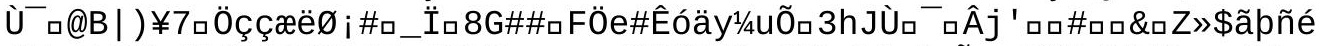

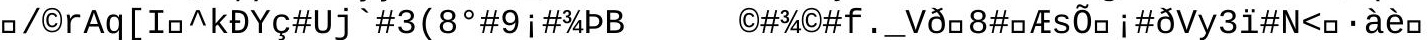
ë8bå3/4\} 9û\#' Ç\#ロ\#'

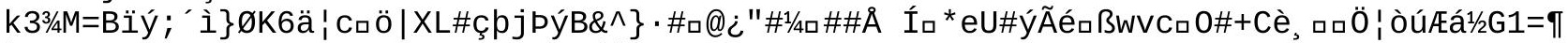

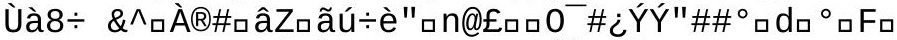

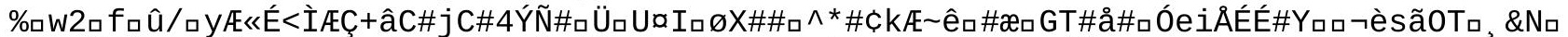

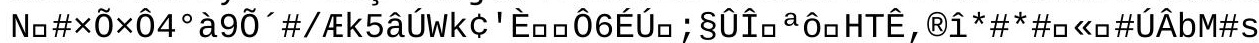

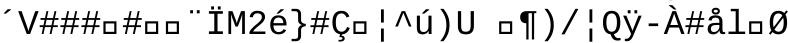

endstream

endobj

1110 obj

$<</$ Subtype/Type1

/FontDescriptor $112 \odot \mathrm{R}$

/LastChar 222

/Widths [250

333

$\odot$

0

$\odot$

500

500

500

0

500

$\odot$

$\odot$

500

500

0

250

$\odot$

$\odot$

$\odot$

$\odot$

$\odot$

666

614

552

666

614

552

666

718

333

447

0 




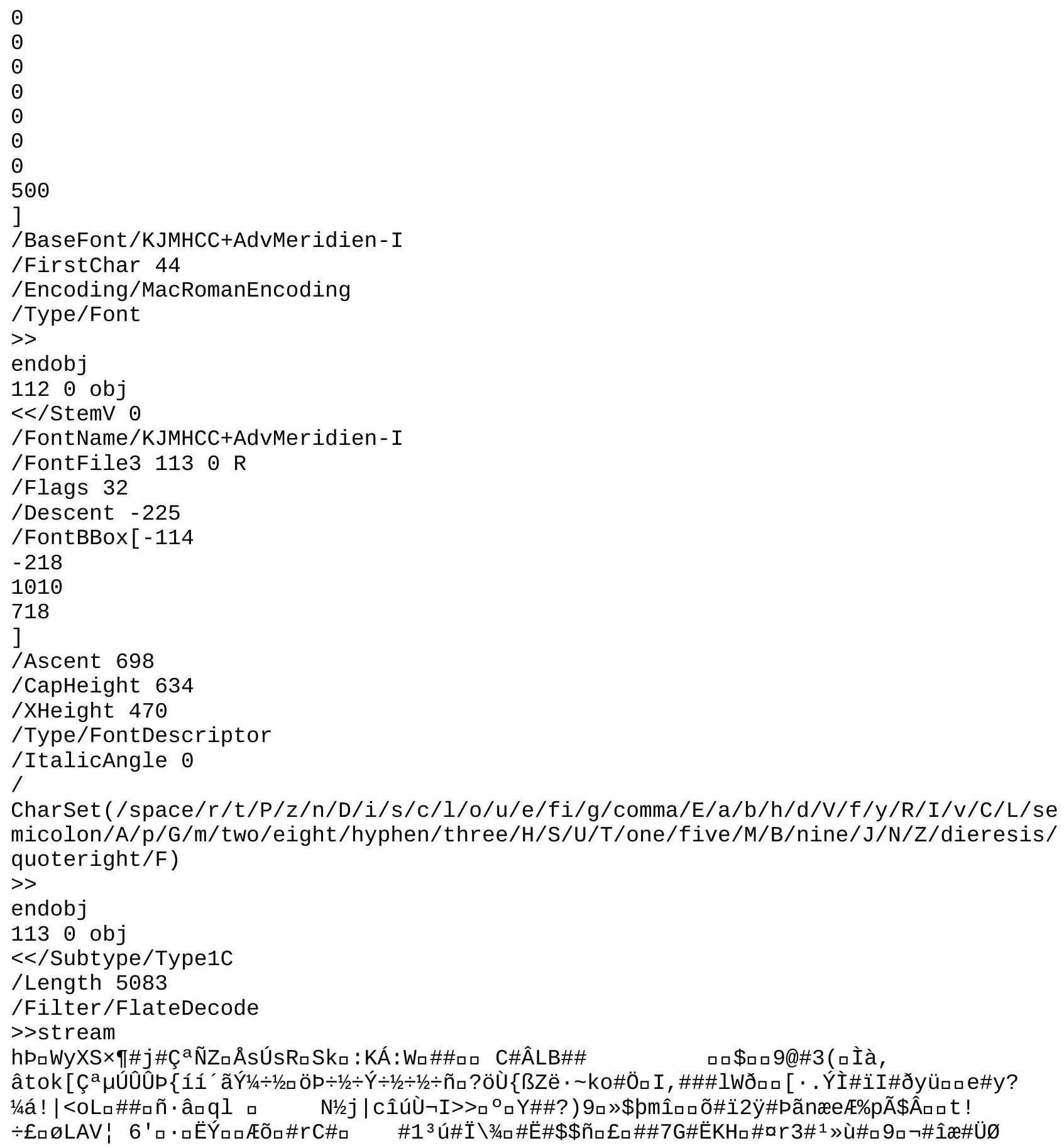




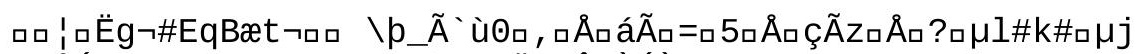

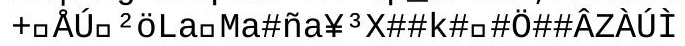

a9Y?ølò1ùıIロ '

'=ロ 3ùÖ॰À) · Ù1ì»ロ 


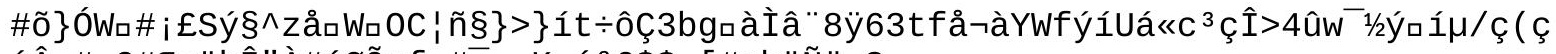

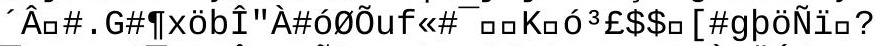

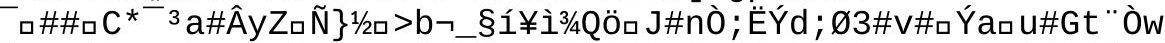

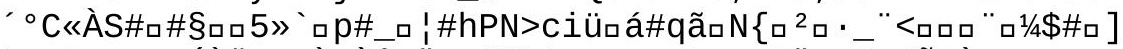

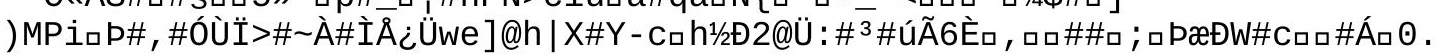




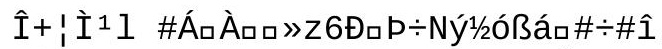

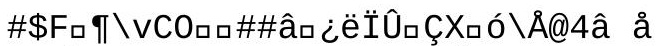

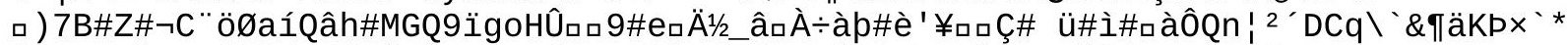

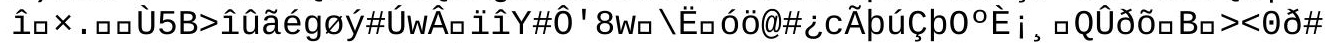

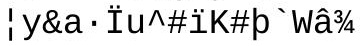


$2 \pm-1 / 4 \hat{U}$

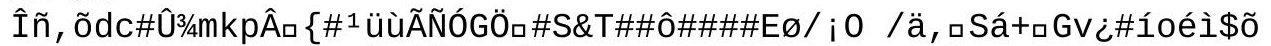

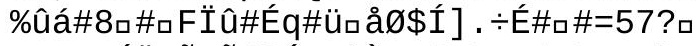

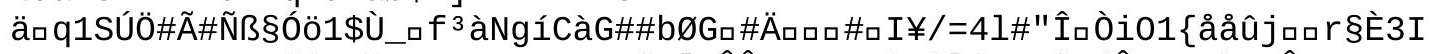

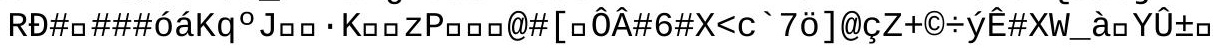




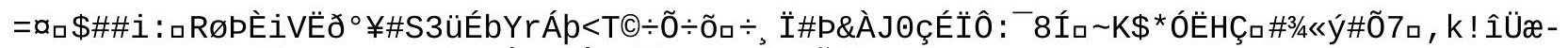

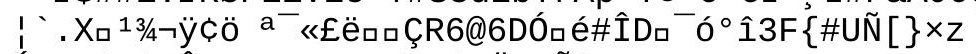

ÍCฉ c 'æ: 
口\#71¿\#à"à\#_\#\#ロåñ \#=ロ ${ }^{\circ}$ 


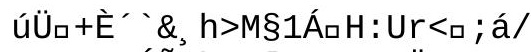
' $\mu$

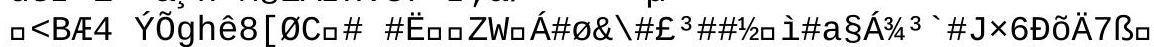

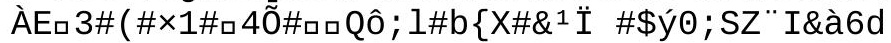




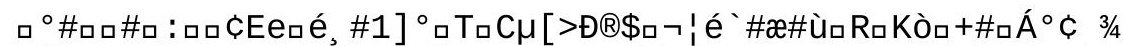

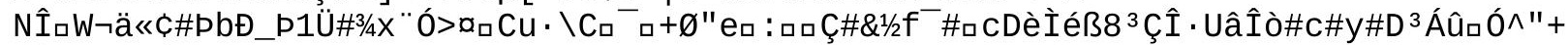
£ôü ${ }^{2} \mathrm{a}^{2} \mathrm{t}^{\prime}$

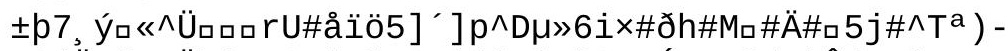

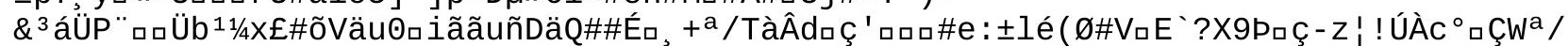

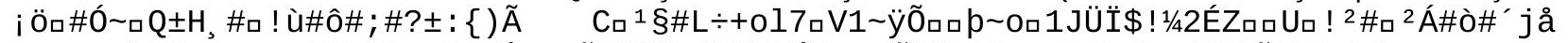

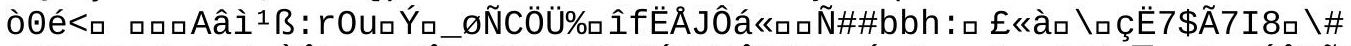

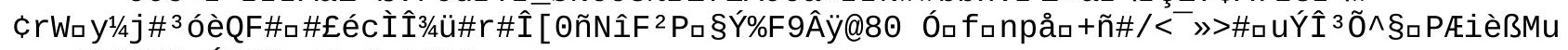
æ_ıäâ\#\#N1/4. ÚH\#KıdGı ( çù\#ðÇ

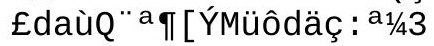





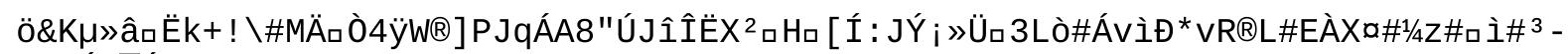
Í\#ロÓN ${ }^{-}$ǾXm 
\#\#IÓロ\#ロ\#a=ù2üÃeðû 


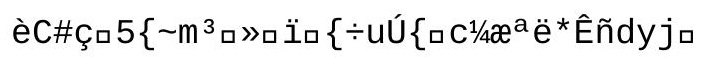





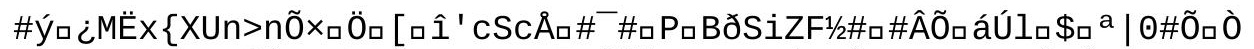

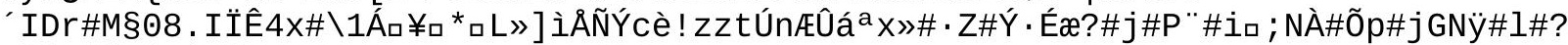

口] ú\# ¿lX (\#*\%[K

sõÁÂap $\left.\urcorner^{1 / 2} \tilde{A} \oplus N o ̂ y ́\right]$ ĖÃ巛

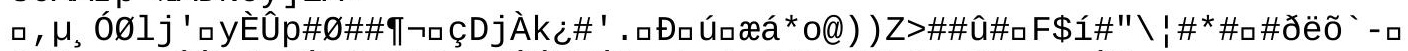

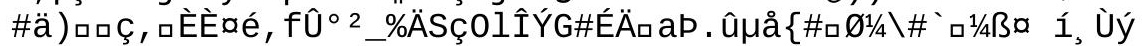

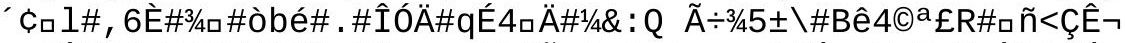

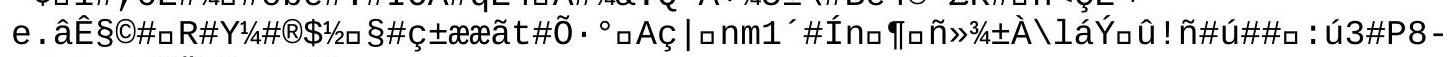

a\#口\# i $9 \# 3 / 40 \ddot{T} J_{\square}\left(\square \tilde{n} 1 / 4 V_{\square}\right.$

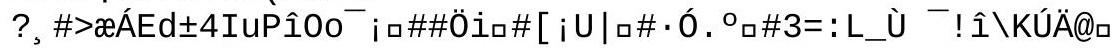

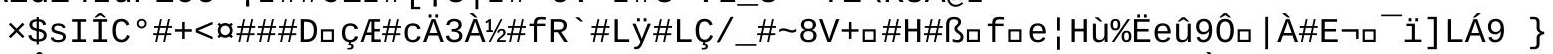

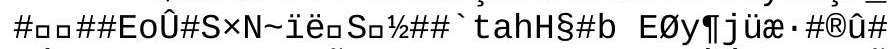

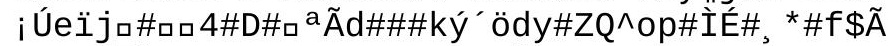

$æ ! N \S N C ̧\left(Z \square a U . \hat{1} B^{*} . \dot{U} / 1\right.$.

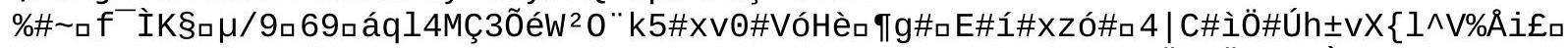

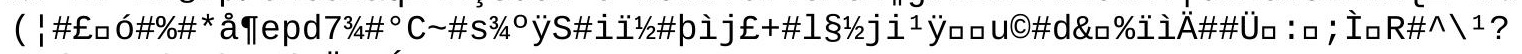

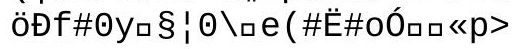


口 Kà\#R\# | d\#

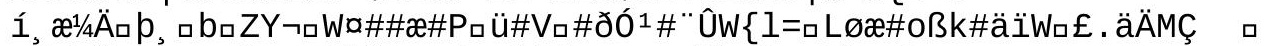

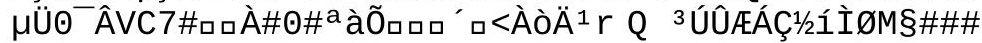




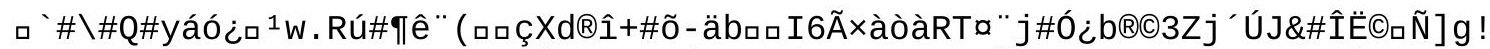

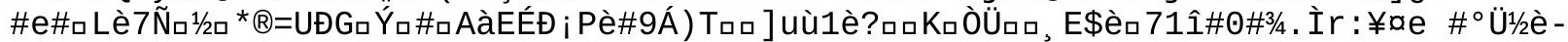
口'00\#MÖì\#ロ

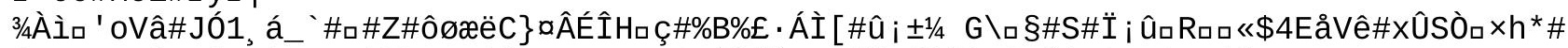

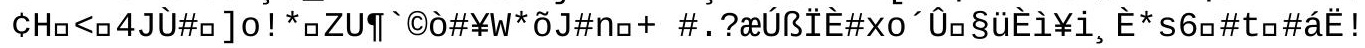




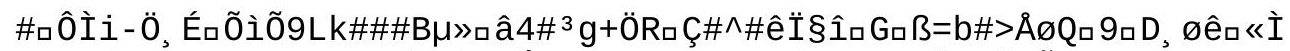

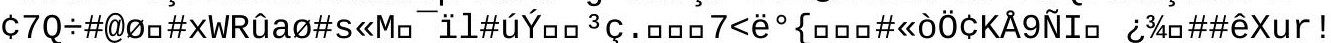

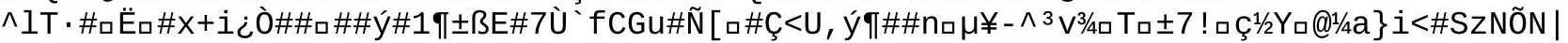

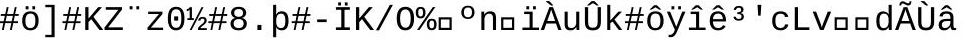




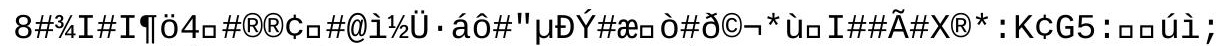

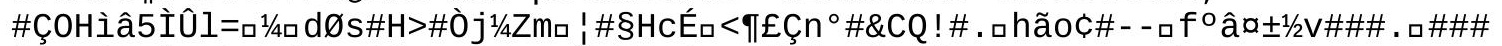

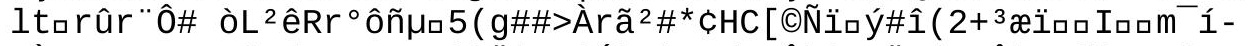

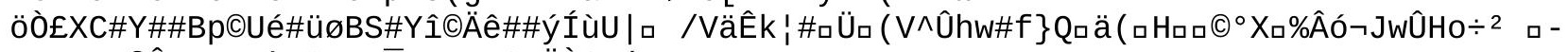

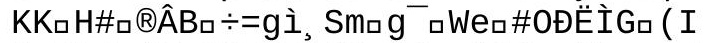

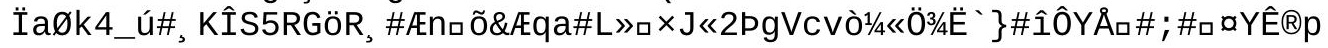




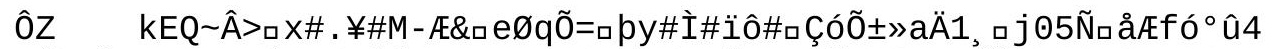

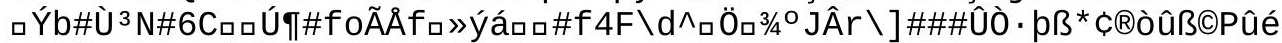

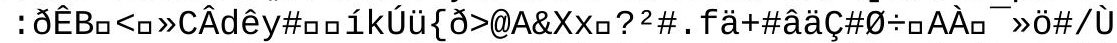

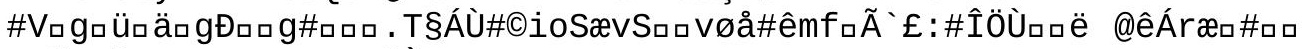

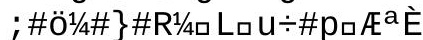

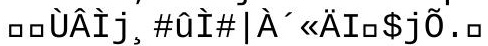

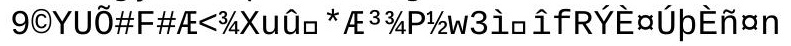




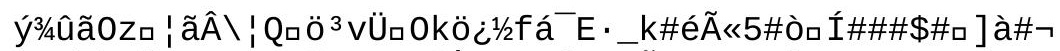

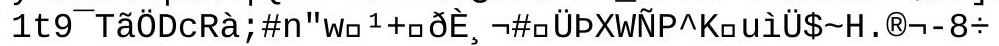




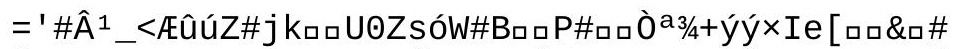

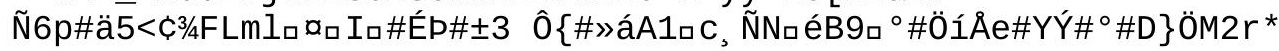




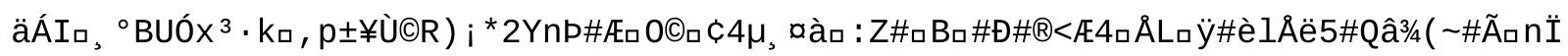




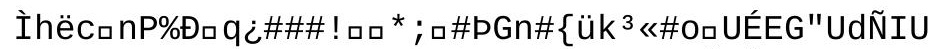

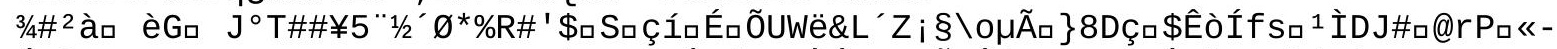

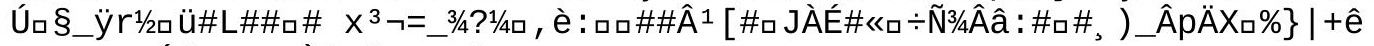

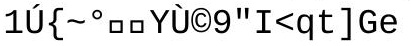


õ ${ }^{\circ} \div{ }^{*} \mathrm{~h} \# \mathrm{~g} \# \# ?^{1} 4 \cdot \mathrm{Ht} \# \cdot \mathrm{qg} \# 9$ aq $¥ \mathrm{i} \mathrm{T \mu}$

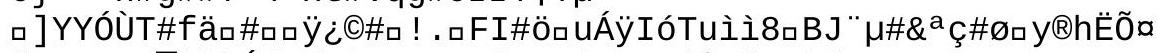

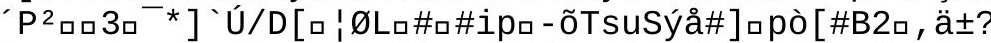

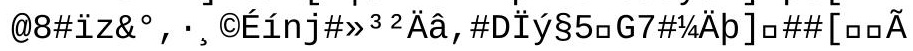

endstream

endobj

1140 obj

$<</$ Subtype/Type1

/FontDescriptor $115 \odot \mathrm{R}$

/LastChar 111

/Widths [979

]

/BaseFont/KJMHCD+AdvEls-ent4

/FirstChar 111

/Encoding/WinAnsiEncoding

/Type/Font

$>$

endobj

$115 \odot$ obj

$</$ StemV 0

/FontName/KJMHCD+AdvEls-ent4

/FontFile3 $116 \odot \mathrm{R}$

/Flags 32

/Descent -324

/FontBBox $[0$

$-343$

1166

1218

]

/Ascent 926

/CapHeight 1224

/XHeight 716

/Type/FontDescriptor

ItalicAngle 0

/CharSet(/o)

$>$

endobj

$116 \odot$ obj

$<</$ Subtype/Type1C

/Length 112

/Filter/FlateDecode

>stream

hpbd`ad `dd\#ööòõpvÑvL)sÍ)ÖMÍ+1\#

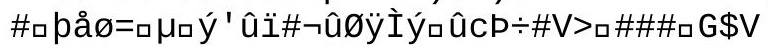

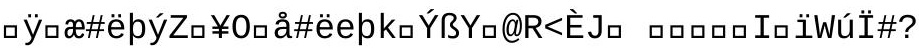

endstream

endobj

117 ○ obj

$<</$ Subtype/Type1

/FontDescriptor $118 \odot \mathrm{R}$

/LastChar 122

/Widths $[770$

1000

770

770

770

$\odot$

0

$\odot$

$\odot$

$\odot$

$\odot$

$\odot$ 


$$
\mid
$$




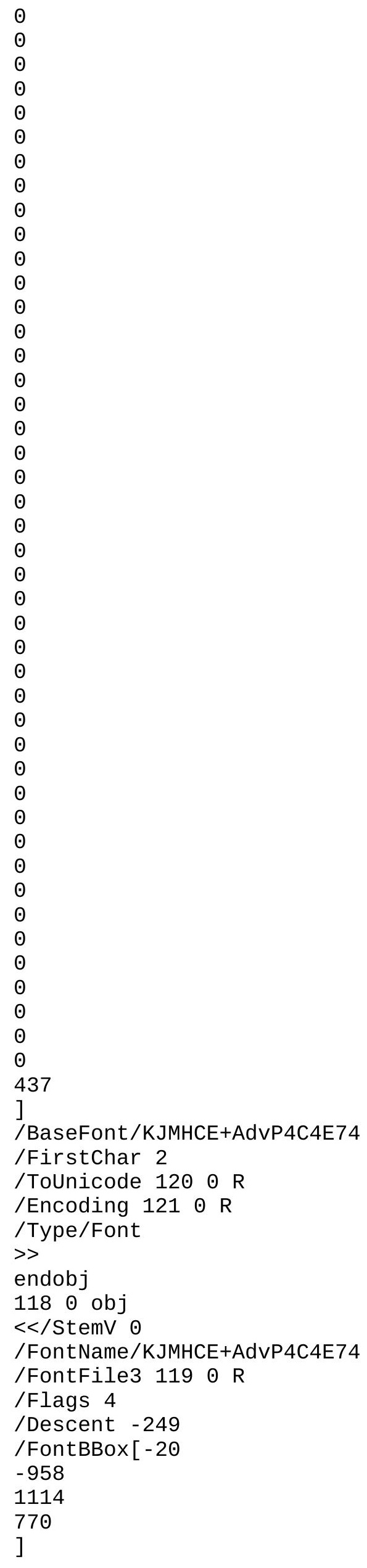


/Ascent 750

/CapHeight 717

/XHeight 705

/Type/FontDescriptor

/ItalicAngle $\odot$

/CharSet (/space/C2/C13/C6/C20/Co/z)

$>>$

endobj

$119 \odot$ obj

$<</$ Subtype/Type1C

/Length 701

/Filter/FlateDecode

>stream

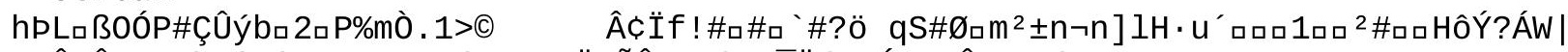

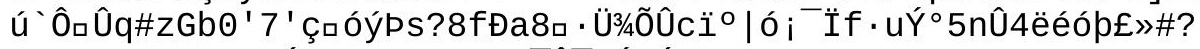

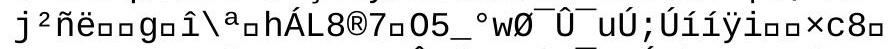

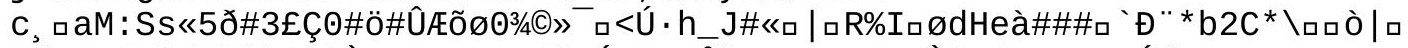

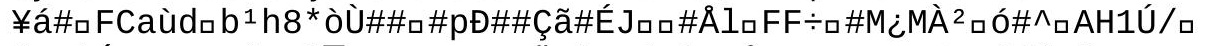

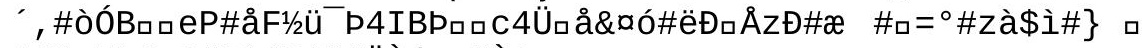

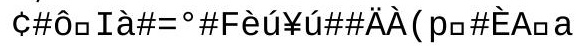




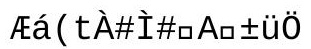




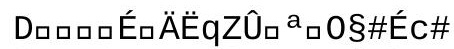

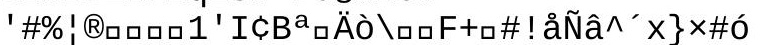

〜 _

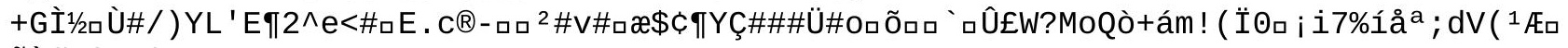
ñèØ\#G/EO 


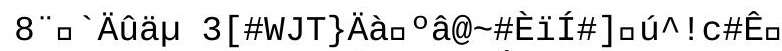

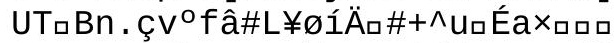

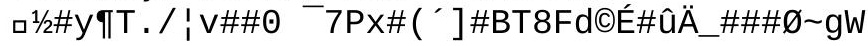

endstream

endobj

120 ○ obj

$<</$ Length 216

/Filter/FlateDecode

>stream

hPTPËロÃ $\odot$ 


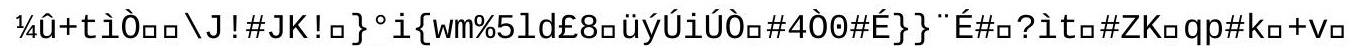

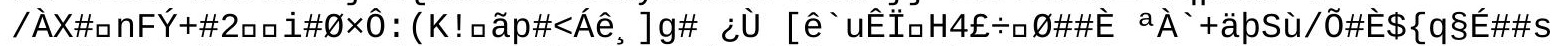

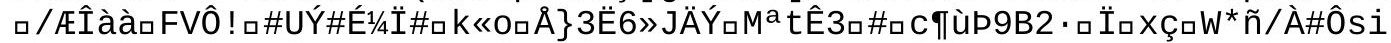




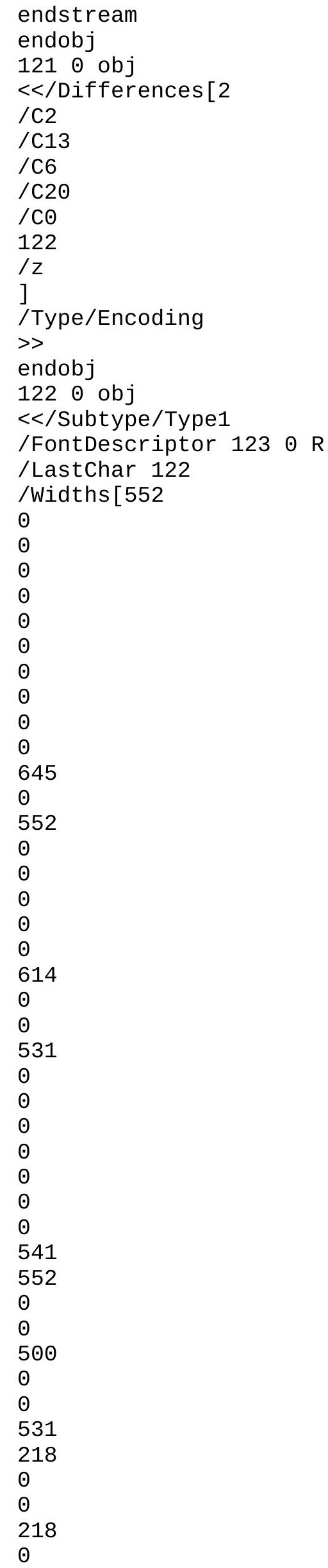


0

$\odot$

$\odot$

322

$\odot$

$\odot$

$\odot$

479

$\odot$

437

]

/BaseFont/KJMHDF+AdvCgsli

/FirstChar 67

/Encoding/WinAnsiEncoding

/Type/Font

$>>$

endobj

$123 \odot$ obj

$<</$ StemV $\odot$

/FontName/KJMHDF+AdvCgsli

/FontFile3 $124 \odot \mathrm{R}$

/Flags 32

/Descent - 224

/FontBBox [-208

$-218$

1000

739

]

/Ascent 731

/CapHeight 667

/XHeight 508

/Type/FontDescriptor

/ItalicAngle $\odot$

/CharSet (/space/V/e/t/C/l/i/n/P/a/h/o/Y/x/b/N/z)

$>>$

endobj

$124 \odot$ obj

$<</$ Subtype/Type1C

/Length 1318

/Filter/FlateDecode

>stream

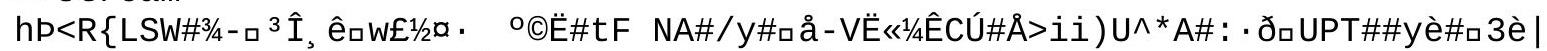

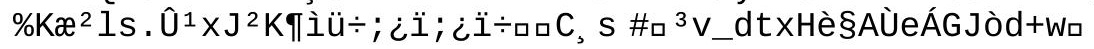




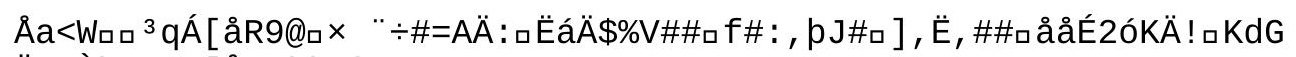
ÄQ¥Ùb_a ¿\# $[\AA$ riiyfñ

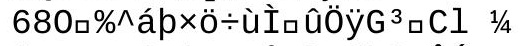

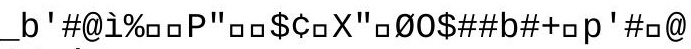
'\#ロ $r<9 \odot \#$ ' W\#WÅnà>ãpêๆÛÍäöĐÝË]æròùMİ 


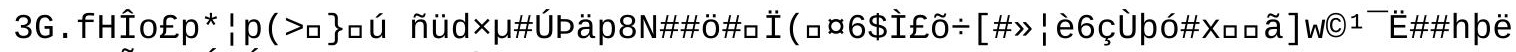

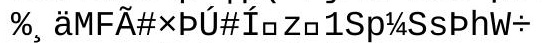

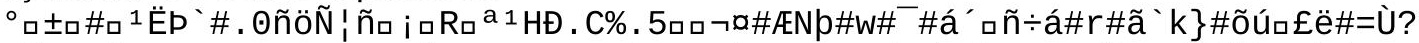

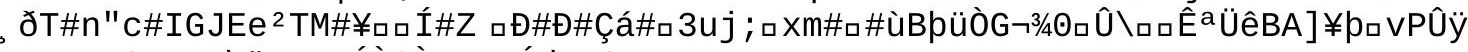

E\# i \#m\Ö\#x!_Íİ ${ }^{\circ}$ à

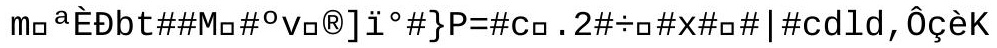




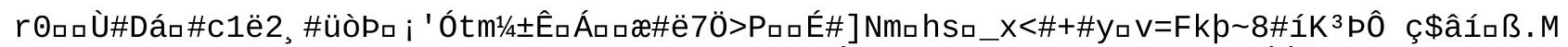

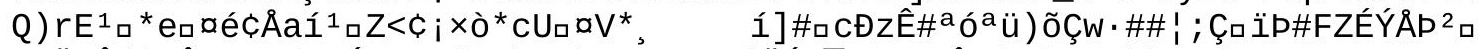

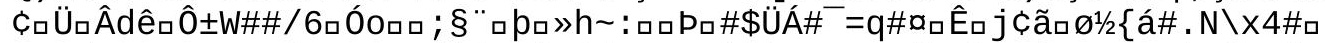

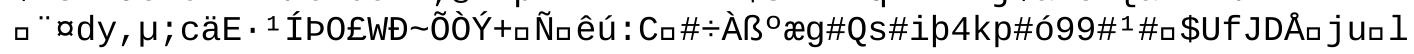




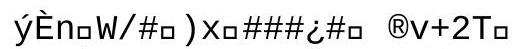

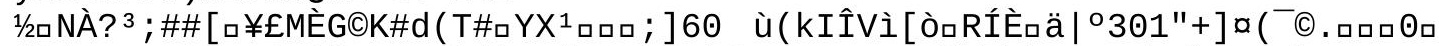

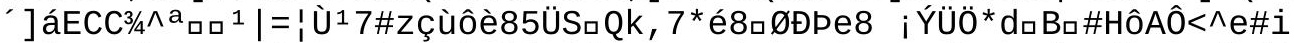

DఃÝ॰\#NÄkgCĐ»ロûø6r\#!; ;

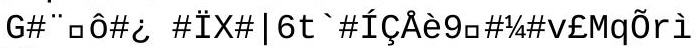

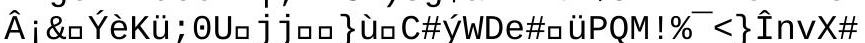

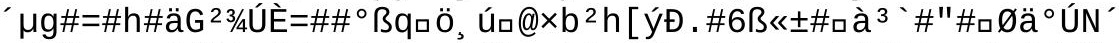

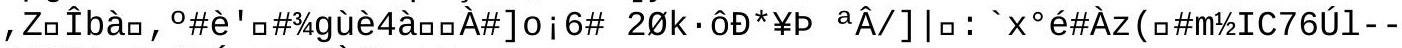

ö\&K\#úqáyðQÁzÆIp\#À\# ! zb5

endstream

endobj

$125 \odot$ obj

$<</ O P M \odot$

/HT 126 ○ R

/OP false

/op false

/Type/ExtGState

/SA false

/SM 0.02

$>$

endobj

$126 \odot$ obj

$<$ /Angle 45

/Frequency 75

/SpotFunction/Round

/Type/Halftone

/HalftoneType 1

$>$

endobj

$127 \odot$ obj

$<</$ Rect [92.183

481.946

171.553

489.43

]

/Subtype/Link

/A<</URI (mailto: n . bourges-abella@envt . fr )

/S/URI

$>$

/Border $[\odot$

0

$\odot$

]

/Type/Annot

$>$

endobj

$128 \odot$ obj

$<</$ Length 748

/Filter/FlateDecode

>stream

HロฉロÛnÚ@\#ロÏy

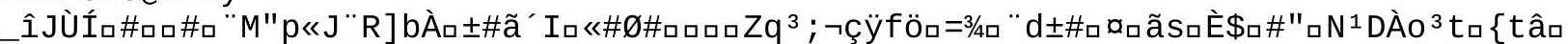

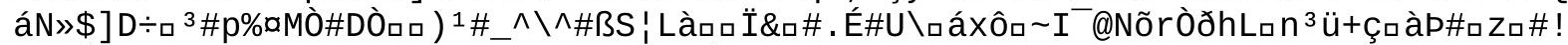

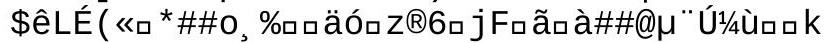




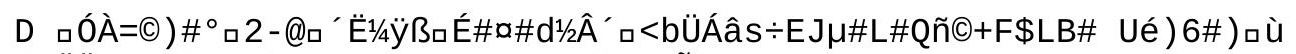

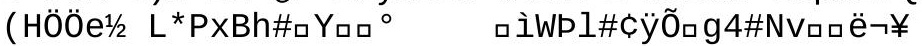




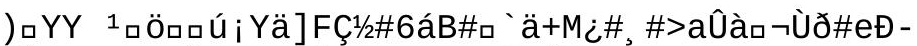

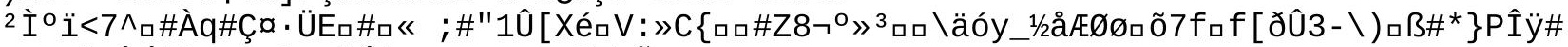

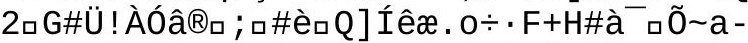

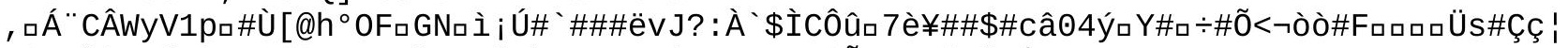

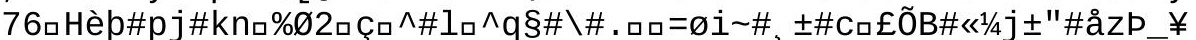

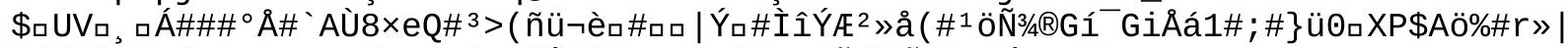

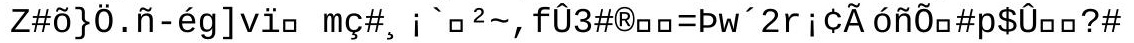


$\# 11^{\wedge 1 / 4}$

endstream

endobj

$129 \odot$ obj

$<$ /Length 824

/Filter/FlateDecode

>stream

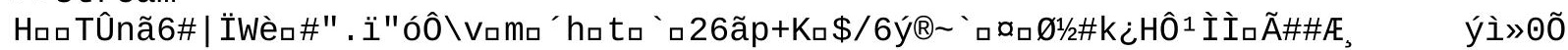

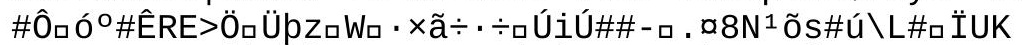

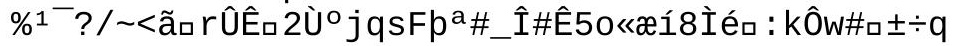


çù ${ }^{3} v T 3 C ̧ \ll \square S e ́$ 


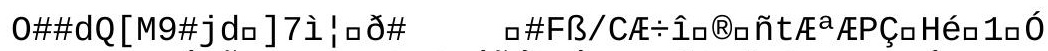

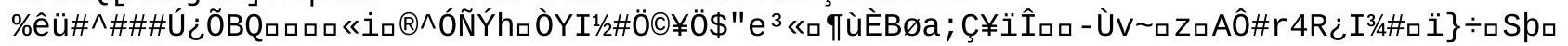

$\backslash P \# \square ; A N R_{\square} q$ 


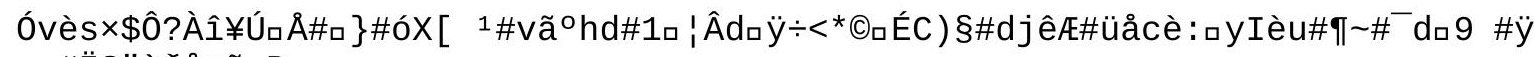
口. \#İS"èðånã»B 


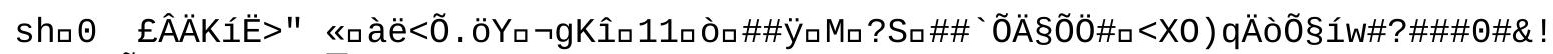

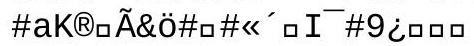




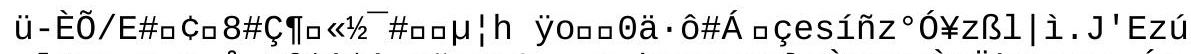

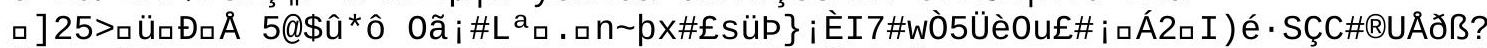

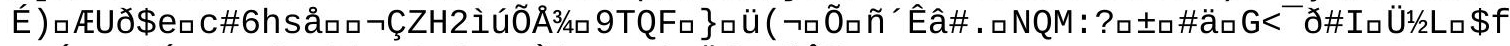

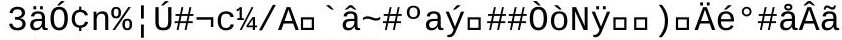

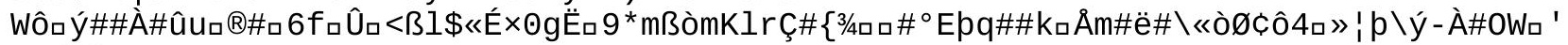
endstream

endobj

130 ○ obj

$<</$ Length 829

/Filter/FlateDecode

>sstream

HฉฉTM॰Ü6 


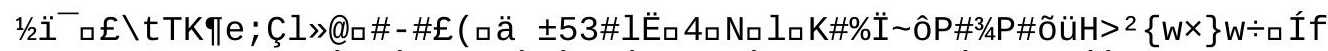

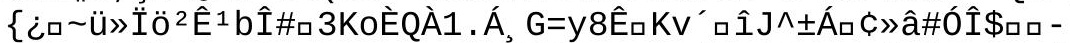

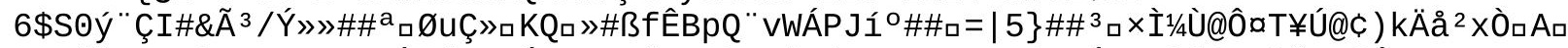

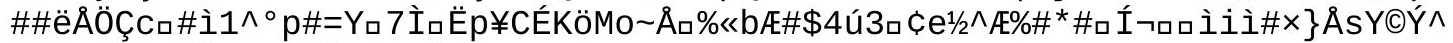

Ë] $\div \# \emptyset 1 / 47^{\circ} l$ ÿUa8\$ó\}oÝ@ . ' 8

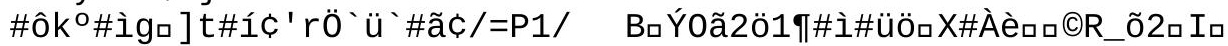

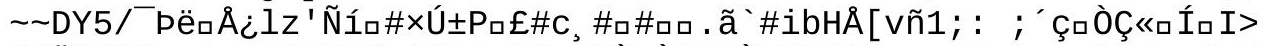

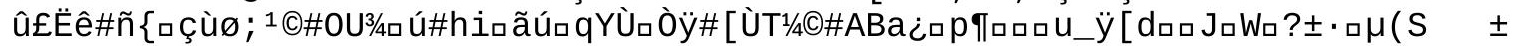

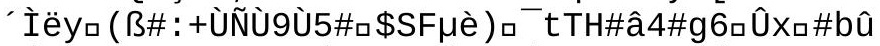

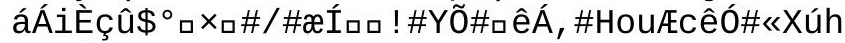

6 [\#Ý\#C 


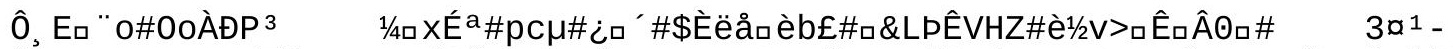

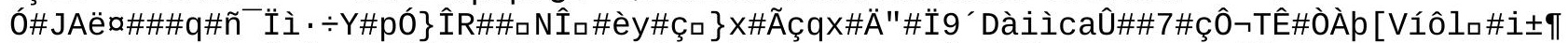

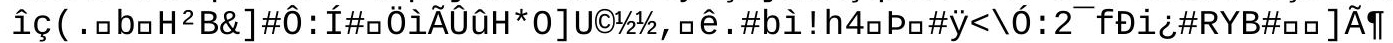




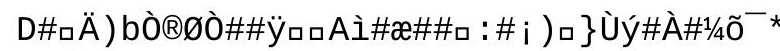

endstream

endobj

1310 obj

$<</$ Length 802

/Filter/FlateDecode

>sstream

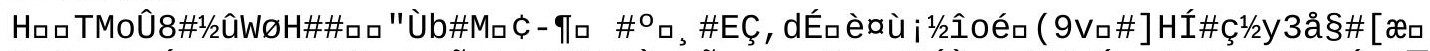

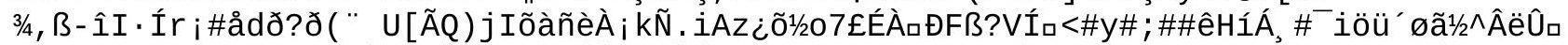

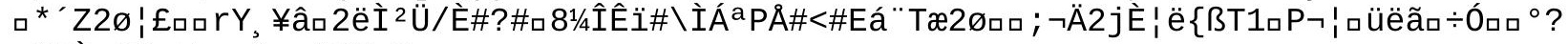

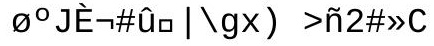




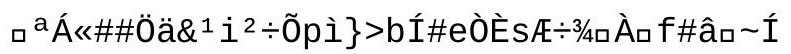




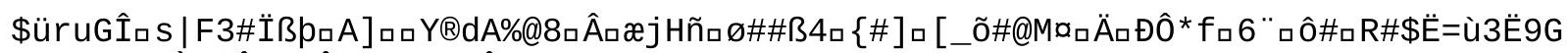

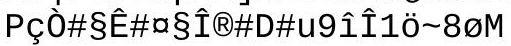




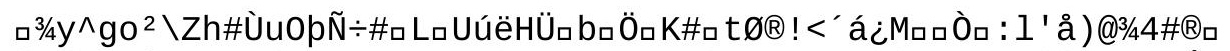

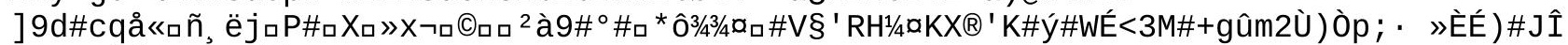
$=\square 13 / 4 \hat{\mathrm{I}}\left(\mathrm{V}_{\square} ¥ 3 / 4 ; \hat{C}\right.$

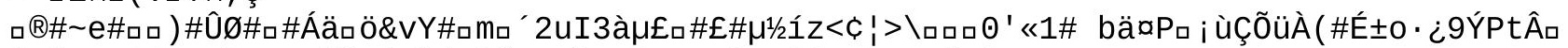

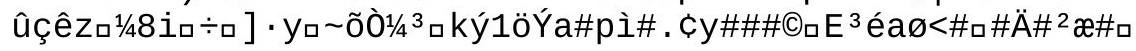




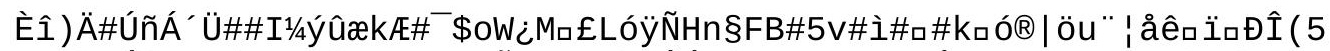

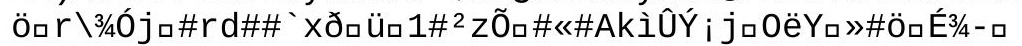
$\Theta \# \&\} \square f$ endstream

endobj

$132 \odot$ obj

$<</$ Length 715

/Filter/FlateDecode

>stream

H口aTKoÛO 


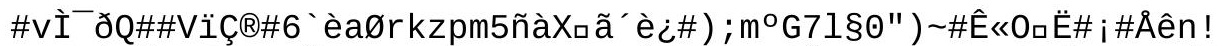

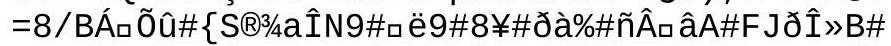

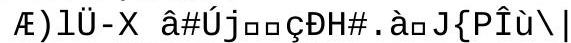

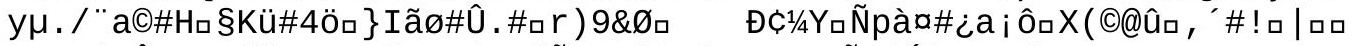

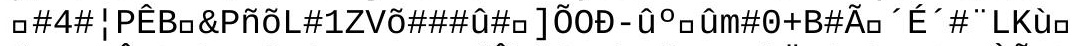

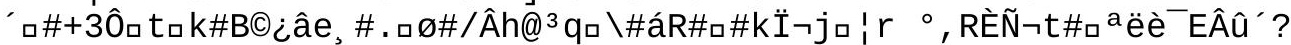

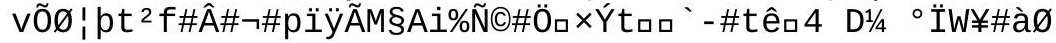

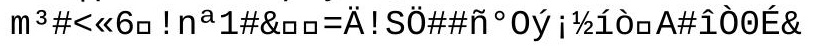




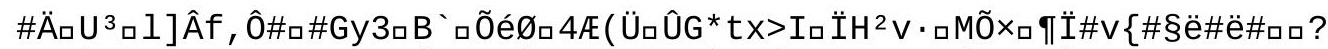

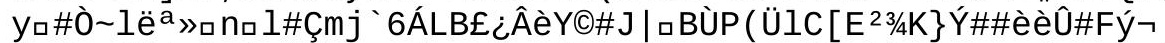

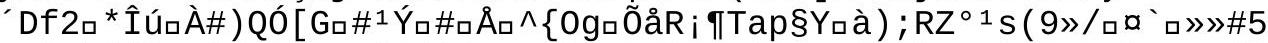

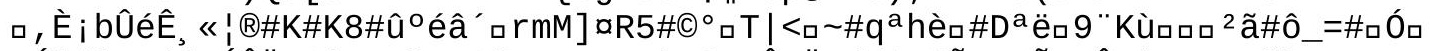

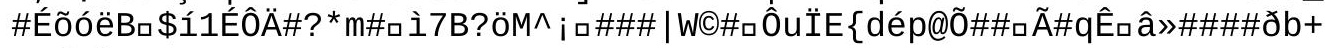

endstream

endobj

$133 \odot$ obj

$</$ Length 831

/Filter/FlateDecode

>stream

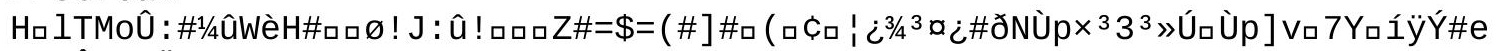
\%०\&Ô\#ロgöOy! ê! ìXïF

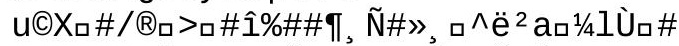

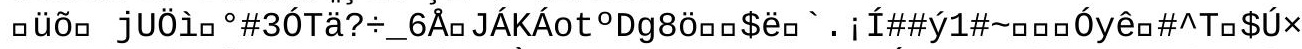

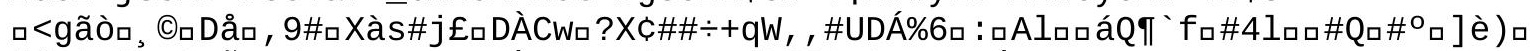

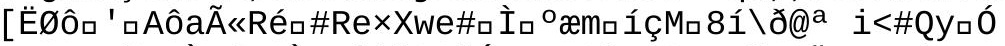

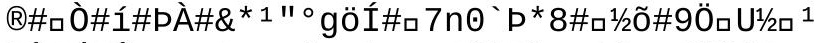

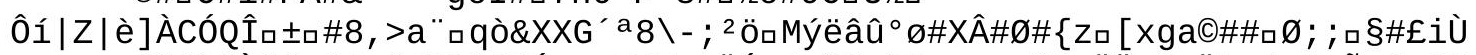

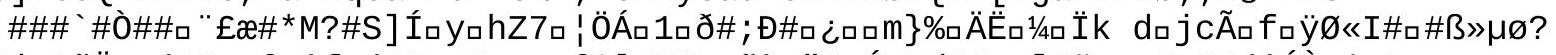

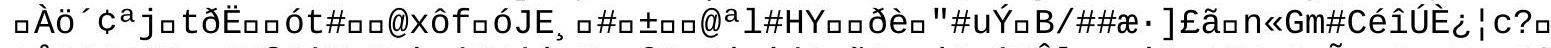

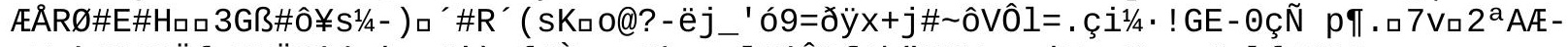

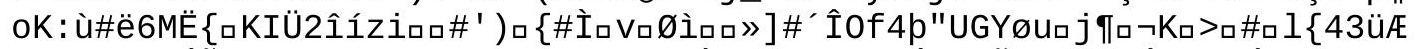

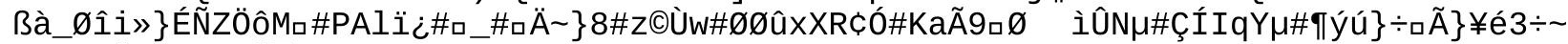

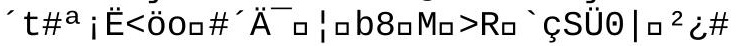


\#ô! (C) \#

endstream

endobj

$134 \odot$ obj

$<</$ Length 815

/Filter/FlateDecode

>stream

Hı tTÉaÛ $8 \# 1 / 2 \div$ WèH\#mı ,

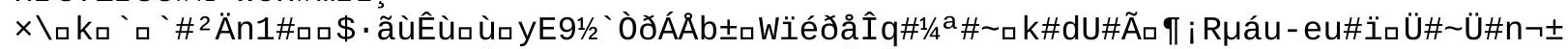

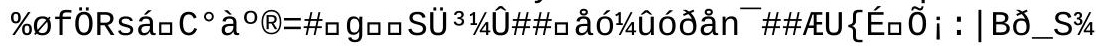




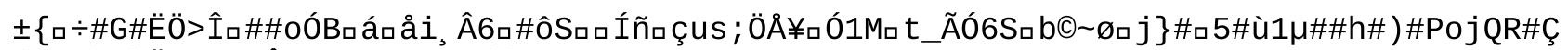
$\{j a s \backslash \Uparrow k E ̈ o ̀ q m a c U ̂ n=; R \# B O ́ a y ́ a / d$

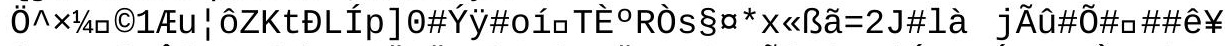

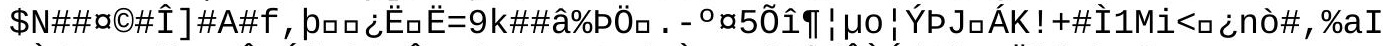

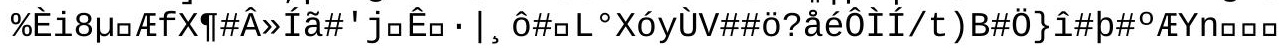




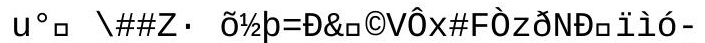

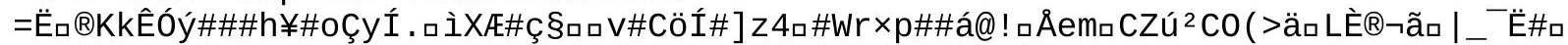

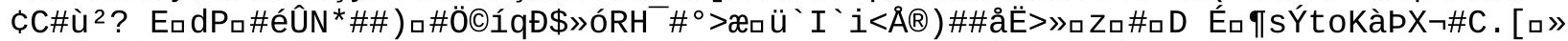

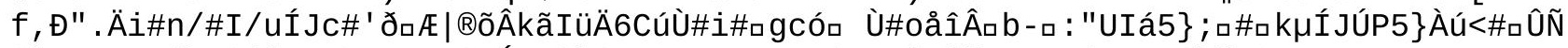

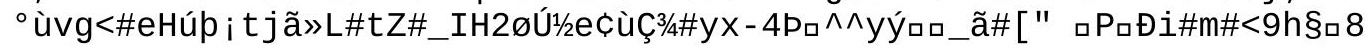


ûđalZ $\left.{ }^{\circ}, y ́\right] \varnothing S>_{\square}{ }^{-}$yiHÉ§| : ã\#Vý 'À\#ÝA /

endstream

endobj

$135 \odot$ obj

$<$ /Length 771

/Filter/FlateDecode

>sstream

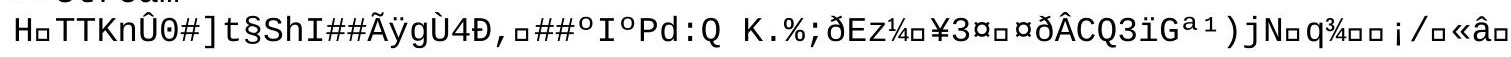


íòocUKm ( 'Ýaq£a, 0 ! =\$»öya ${ }^{1}$ Üô̂ि ¿ÝaXN, ôôa mô\$İKû@ôóS?

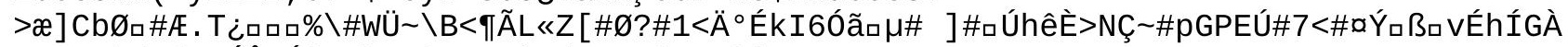

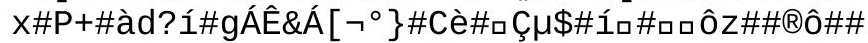




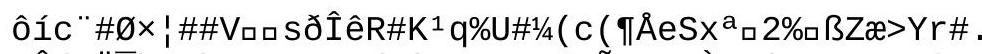

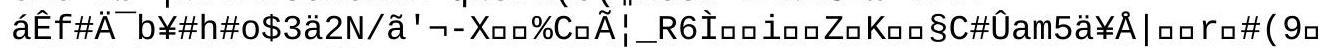

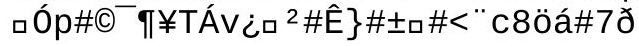




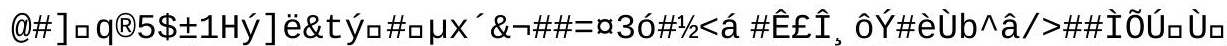

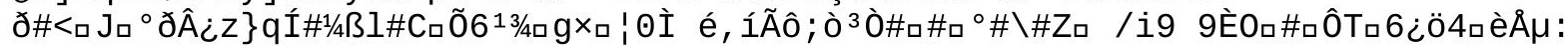

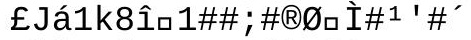

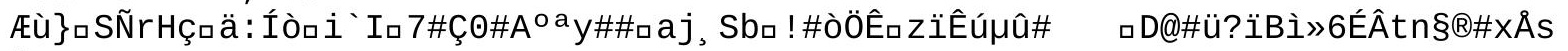
$\left[a_{\square} \pm\right.$

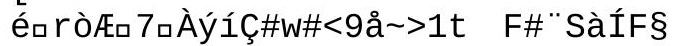




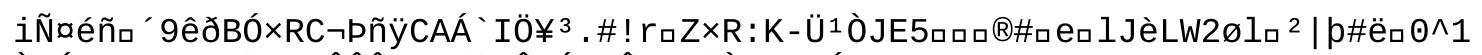

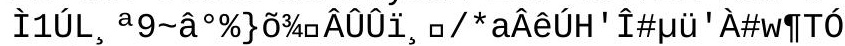

endstream

endobj

$1 \odot$ obj

$<<$

/Type/Page

/Resources $2 \odot \mathrm{R}$

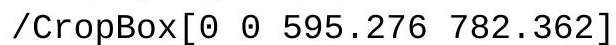

/MediaBox[0 $\left.\odot \begin{array}{lll}\odot & 055.276 & 782.362\end{array}\right]$

/Rotate 0

/Parent $82 \odot \mathrm{R}$

/Contents $3 \odot \mathrm{R}$

/Thumb $67 \odot \mathrm{R}$

$>>$

endobj

$2 \odot$ obj

$<</$ Font $<</ F 295 \odot \mathrm{R}$

/F4 $101 \odot \mathrm{R}$

/F5 $105 \odot \mathrm{R}$

/F6 $108 \odot \mathrm{R}$

/F9 $1170 \mathrm{R}$

$/ \mathrm{F} 10122 \odot \mathrm{R}$

/F11 $50 \odot \mathrm{R}$

$>>$

/ProcSet [/PDF

/Text

]

/ExtGState $<</ G S 2125 \odot \mathrm{R}$

$>>$

$>>$

endobj

$3 \odot$ obj

$<</$ Length 7993

/Filter/FlateDecode

>sstream

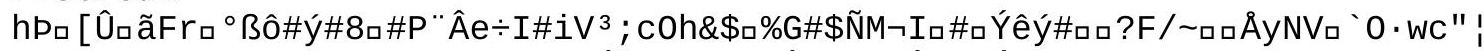

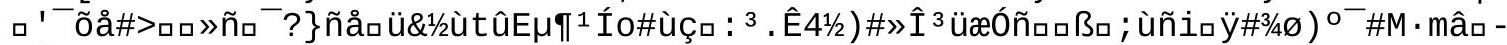
ìúEm॰_ù ${ }^{\circ} \mathrm{P}$ 


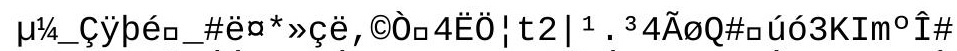

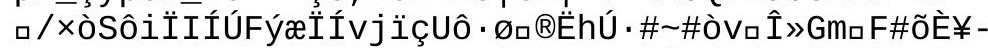

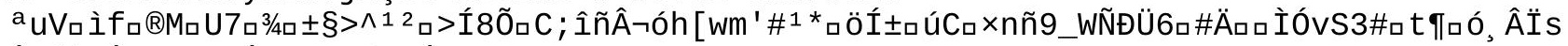

Û\#ñ $\tilde{n}^{*} \hat{E} \mu_{\square} 6 \varnothing_{\square} \hat{E} \# \# \# C ̧ c o ́ r<E ́$

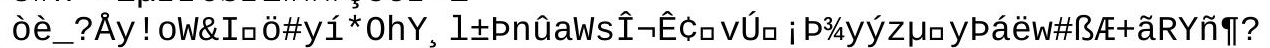

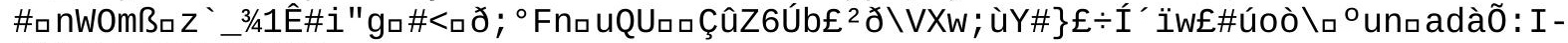

$\#$ Ãü\#7y)\#kb! Í / §Ø

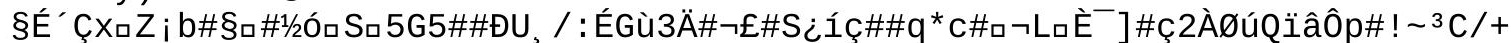

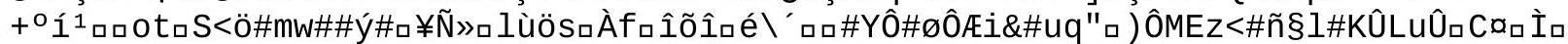

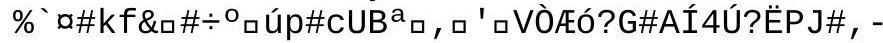

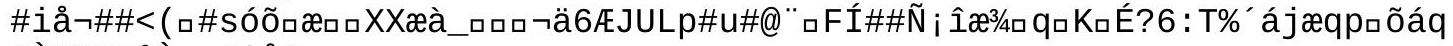

KÙâX3ÿåÀ= ú 'Å०ロ

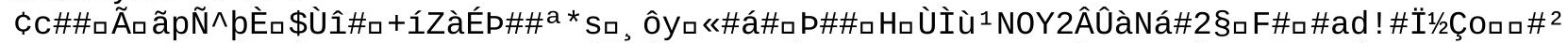

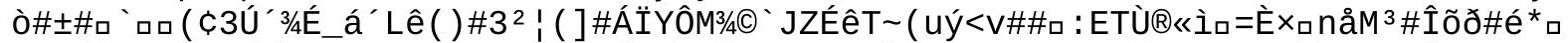

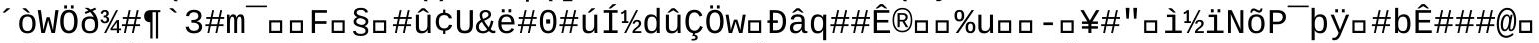

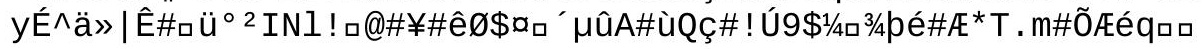




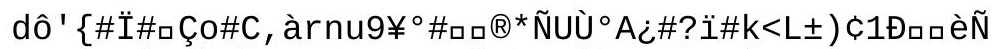

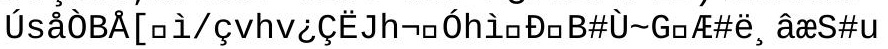




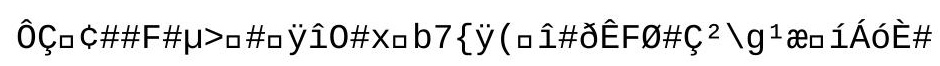


oäロ ${ }^{\circ} \mathrm{K}$ • S $\Theta \cdot \# a ̃ a u ̈ O ̀ \_P e 3 / 4 G \# R$ 


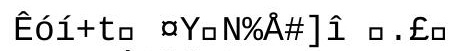

8\#M\#SÔ®ÜË. hW\#T' '

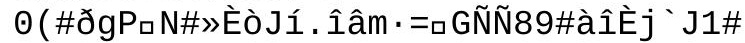


$æ^{\wedge} \wedge^{\prime}$ a $i ¥ \mathrm{Jp}\{\tilde{0} \circledast \# \mu] \square^{\prime}$

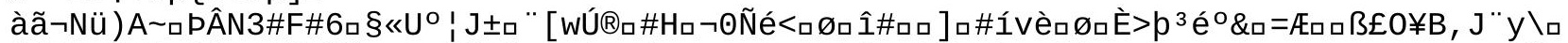

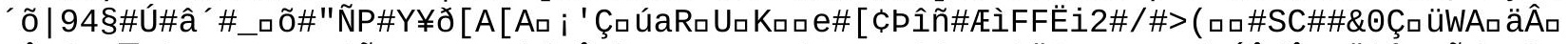

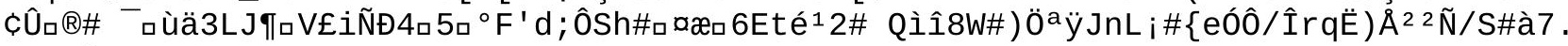
$! \square \notin \#^{\circ} \mathrm{X}$ 


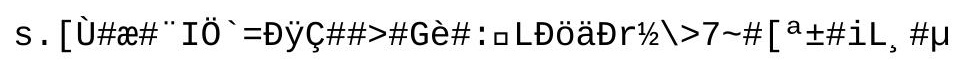




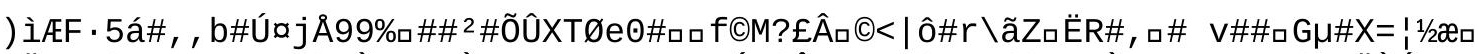

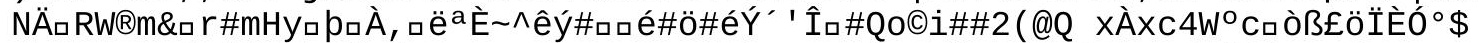

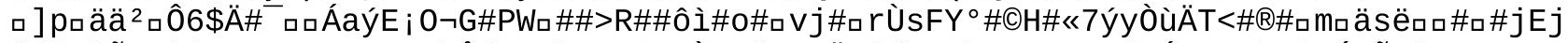

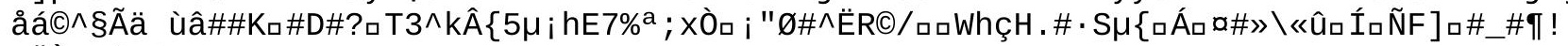
DÄÒ1/4\#fVO 


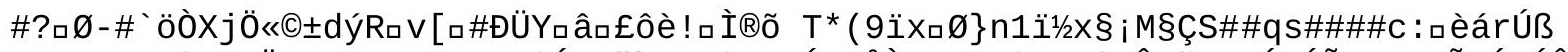

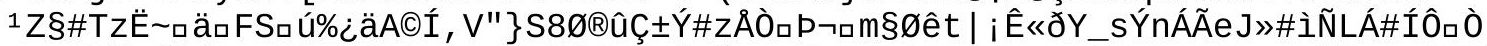

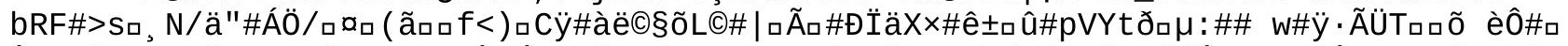

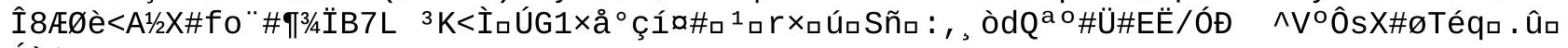
ÓùG

$\square Q ? A \backslash £ \tilde{Z Z \# ~}$

$=\#$ eûÂ $\left[\right.$ ClÕĖ\# $\cdot=\hat{I}^{\prime}$ ' Æ\#-

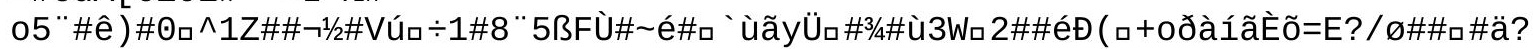

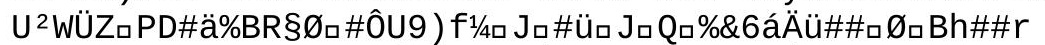

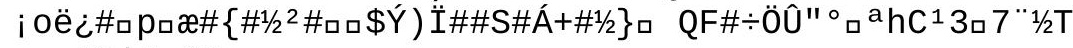

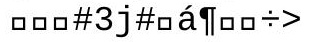

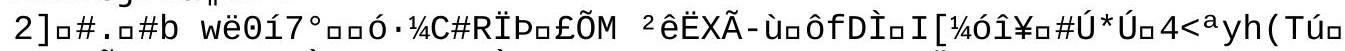

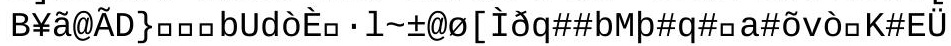

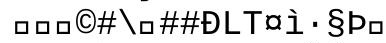

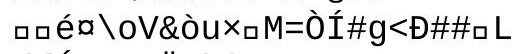

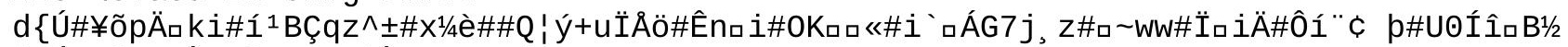

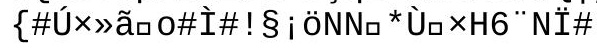


W`Am81ï\#ª̈a\#\#v\#ý ${ }^{2}$ mNSð@ü+áôî 


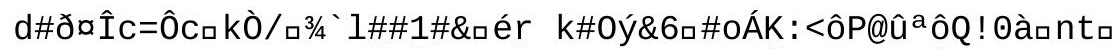

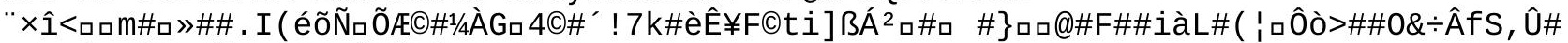

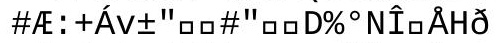

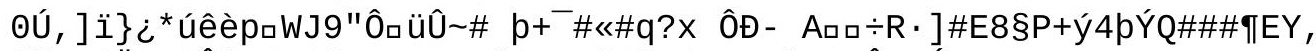

¿"\#口

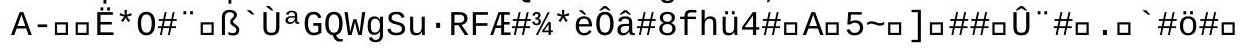




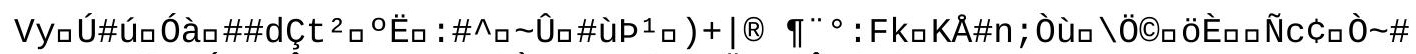

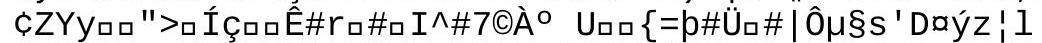

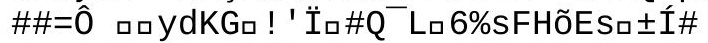

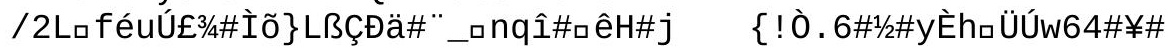




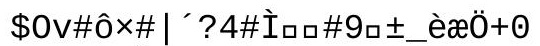

$3 \neg \#$ ฉ ÒX\#ロí@đ

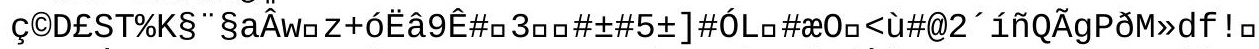

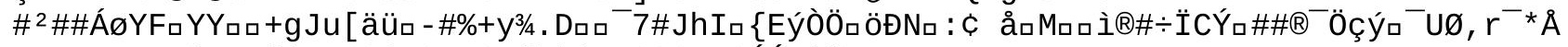

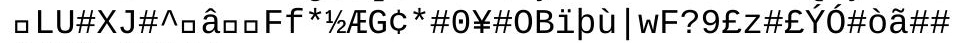

ê\#[ÙyÒR] đóa

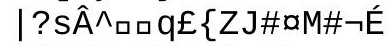

$1\left\{0 \ddot{y} \# \square B W^{*} \# \# \square M\right\} a_{\square}=$

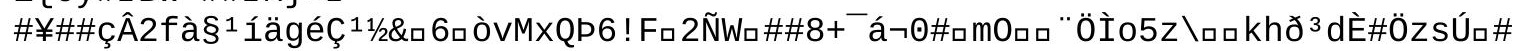
$\sim^{\wedge} \mathrm{dj}$ jaÂMÛ $1 / 2 z \#$ ! 


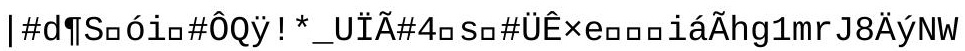




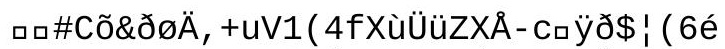

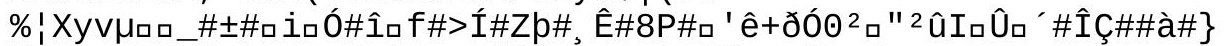

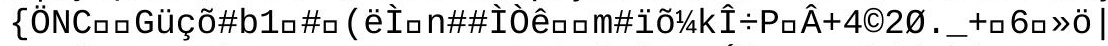

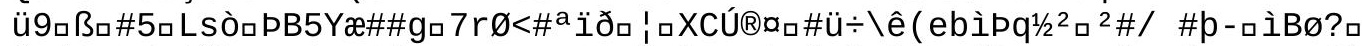

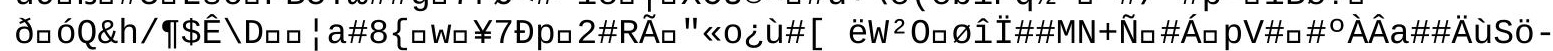

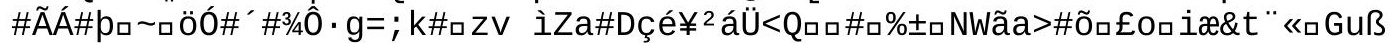




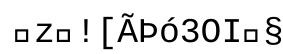

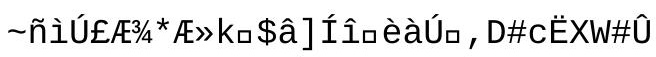




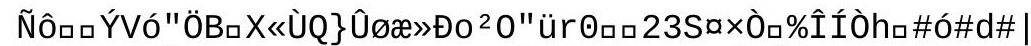

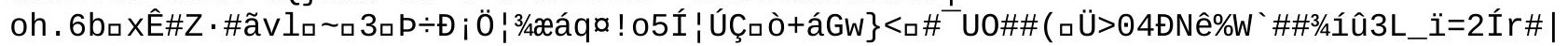
$\emptyset_{\square} \wedge$

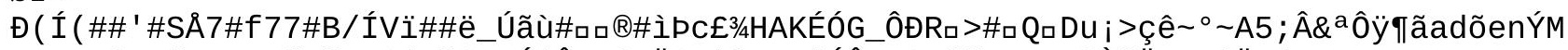

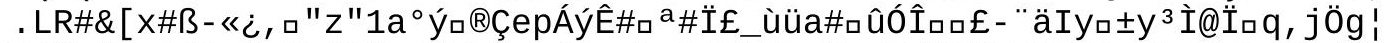

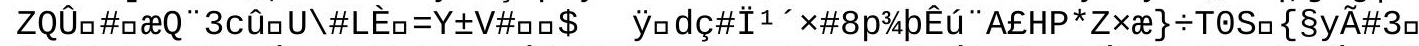

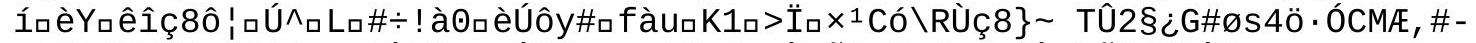

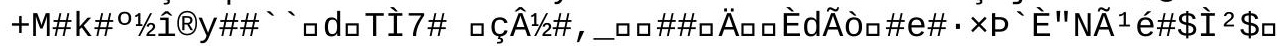

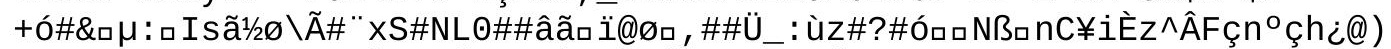

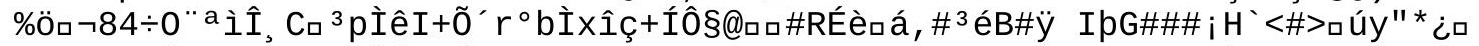

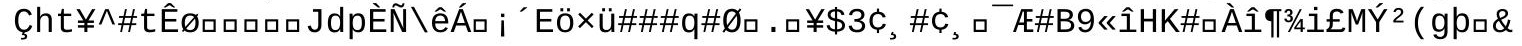




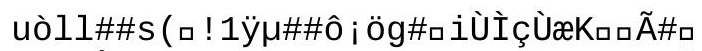

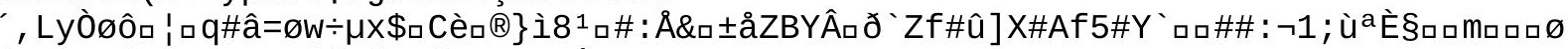

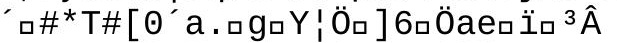


E\#wÉpっìıí\#Yょ[eËë 


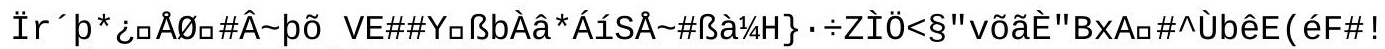

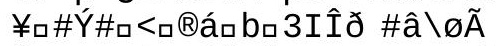

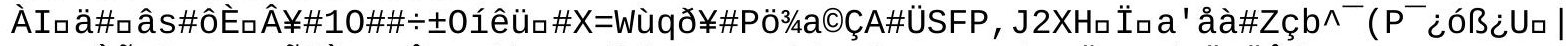

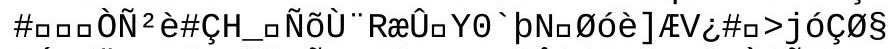

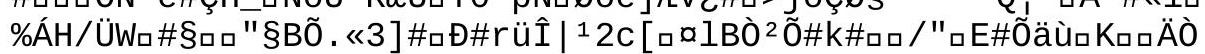

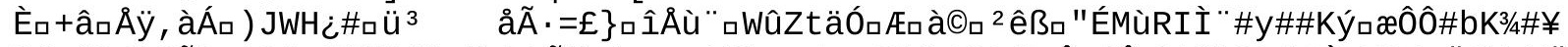

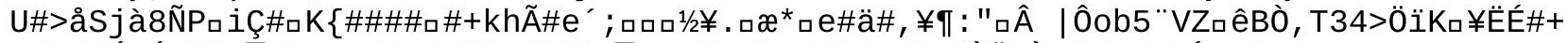

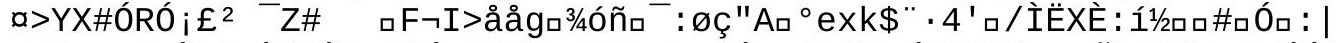

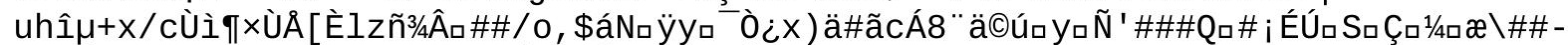

○1FÚĐ\#nE\#®ロXâ\# \#Lß\#ロ3/4XkロÁFï5Ã\#ロÄG`| Õ\#M\#» 


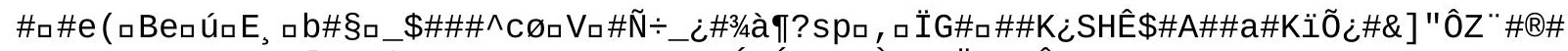

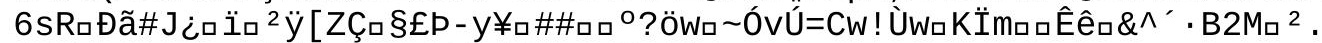

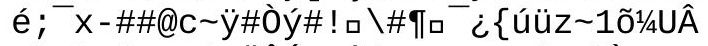

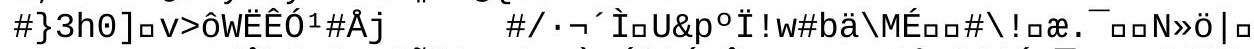

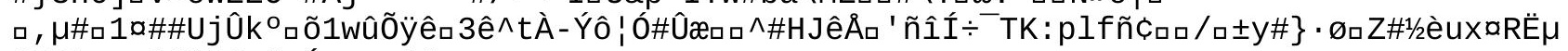

\#7\#суу ; ö\#ê]3¿êcú; ㅁ口 *\# 


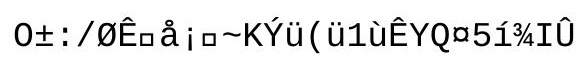




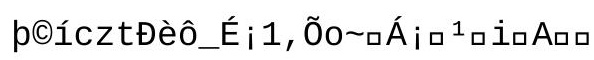




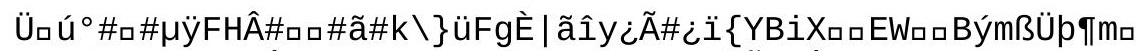

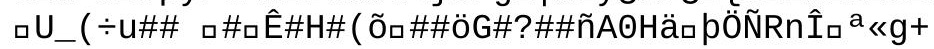




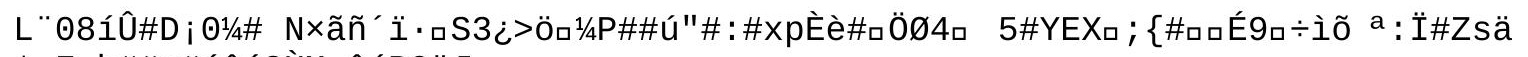
çFab\#\#ø\#éôó?Ùm ôéPO"Æ

ôô" "\#\#R\#巴

$c \llbracket 3^{*} \mathrm{f}^{3}$ ù $9 u ́ \partial$. f\#

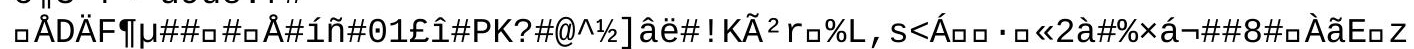

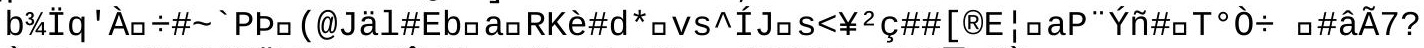

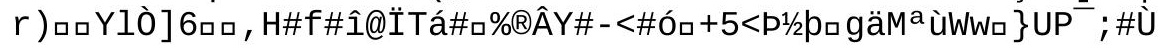


ëìzÙó ${ }^{0} \mathcal{C}_{\square} g \mid Z^{\mathrm{a}} Q R \pm Y \# a ̀ n q a$ 


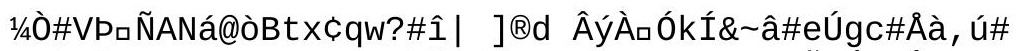

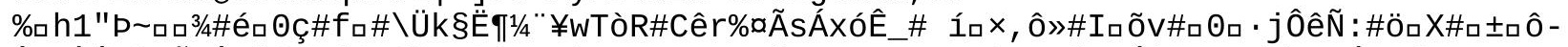

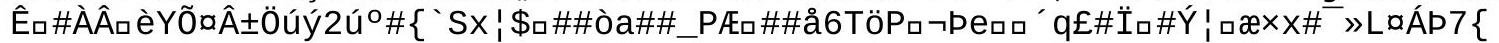




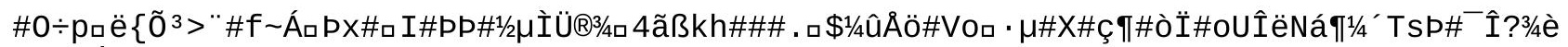
C口) $Y$

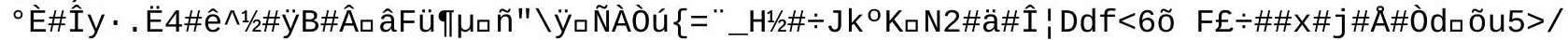
$6 ; ; \mathrm{s}^{\wedge} \mathrm{l}_{\mathrm{a}} \mathrm{k}$

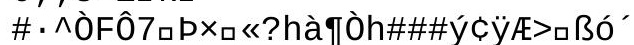

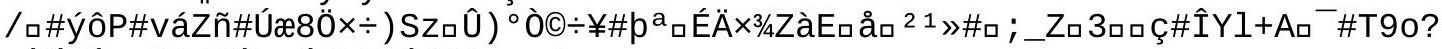

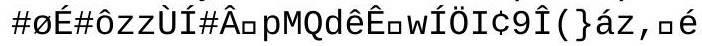




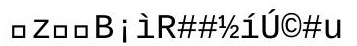

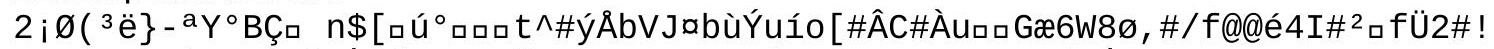

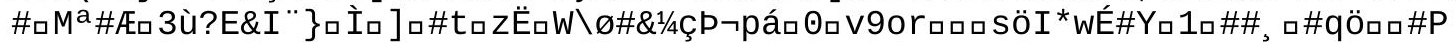




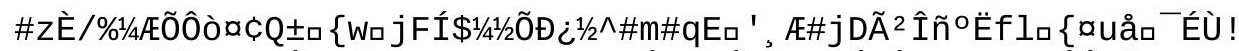

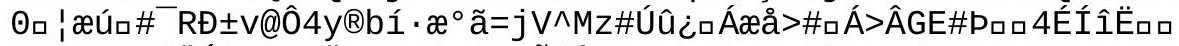

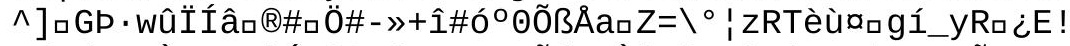

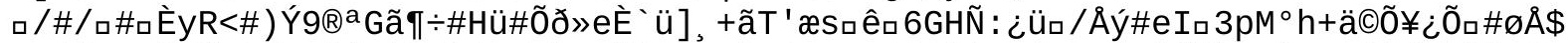

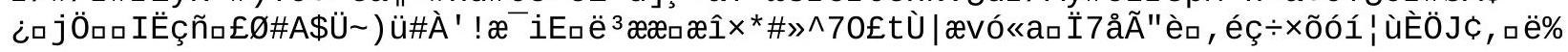




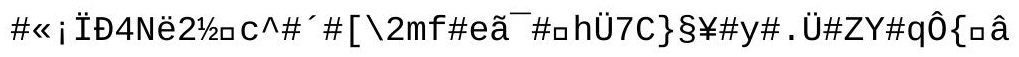




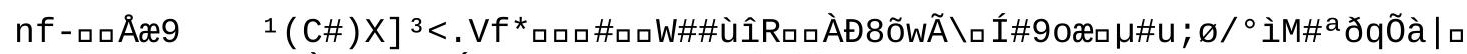

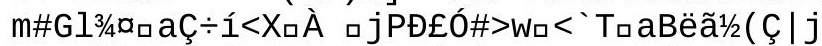

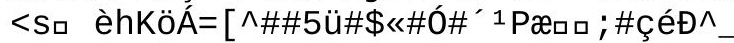




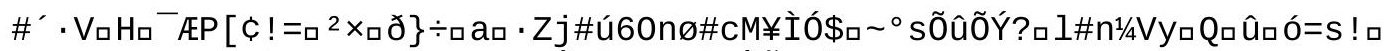

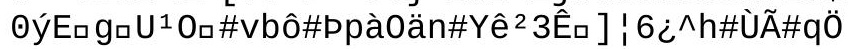


üYÈÂ\#Ü

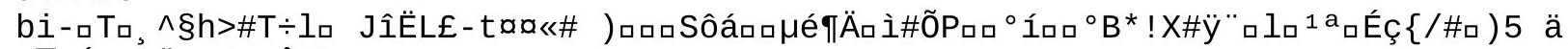
c- ${ }^{-}$İĐn) Ë, XúØoÛfò $\cdot y \#$ 


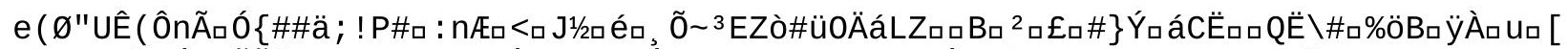

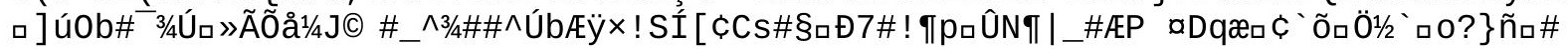


\#ĐÓîh

endstream

endobj

4 o obj

$<<$

/Type/Page

/Resources $5 \odot \mathrm{R}$

/CropBox[ $\left[\begin{array}{llll}0 & 0 & 595.276 & 782.362\end{array}\right]$

/MediaBox[ $\left[\begin{array}{llll}\odot & \odot & 595.276 & 782.362\end{array}\right]$

/Rotate 0

/Parent $82 \odot \mathrm{R}$

/Contents $6 \odot \mathrm{R}$

/Thumb $68 \odot \mathrm{R}$

$>$

endobj

$5 \odot$ obj

$<</$ Font $<</ F 1253 \odot \mathrm{R}$

/F2 $95 \odot \mathrm{R}$

/F4 $101 \odot \mathrm{R}$

/F5 $105 \odot \mathrm{R}$

/F6 108 ○ R

/F7 $1110 \mathrm{R}$

/F8 $1140 \mathrm{R}$

/F9 $1170 \mathrm{R}$

/F10 $122 \odot \mathrm{R}$

$/ F 1150 \odot R$

$>>$

/ProcSet [/PDF

/Text

]

/ExtGState $<</ G S 2125 \odot \mathrm{R}$

$>$

$>>$

endobj

6 $\odot$ obj

$<</$ Length 6844

/Filter/FlateDecode

>stream

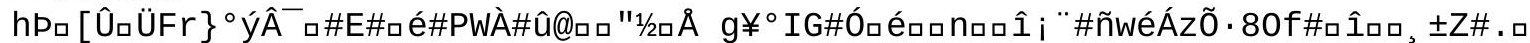

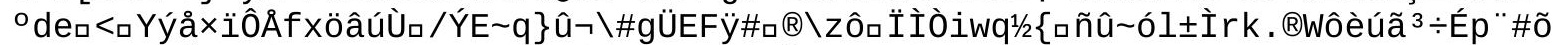

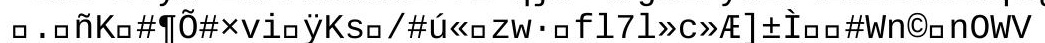

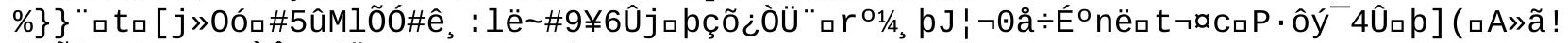

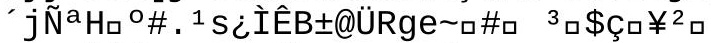

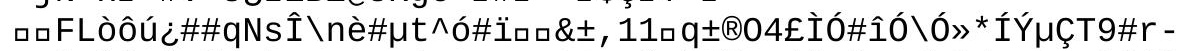

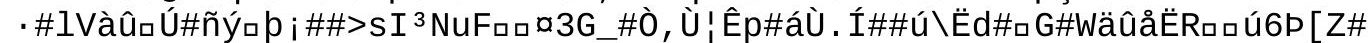

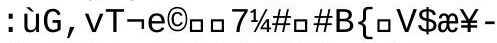

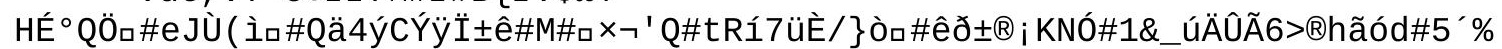

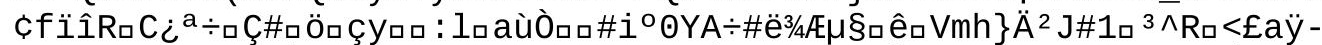

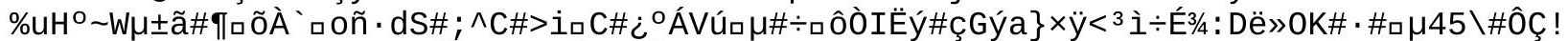

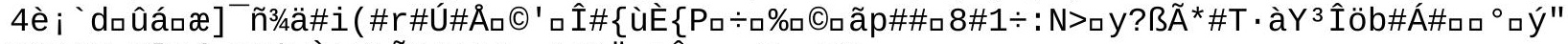

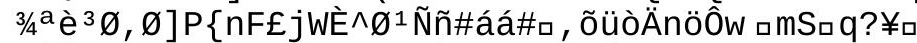

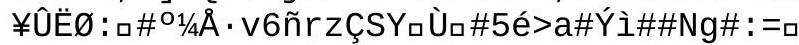




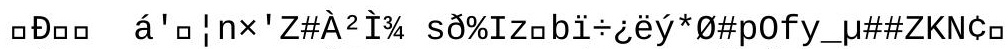

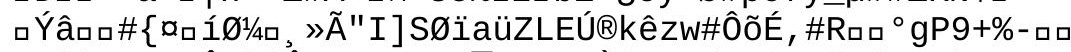

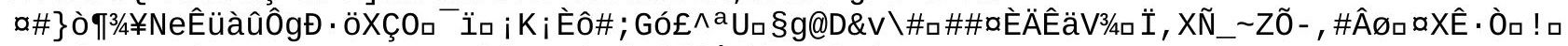

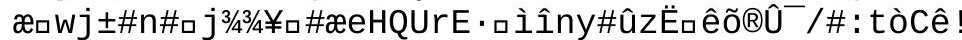

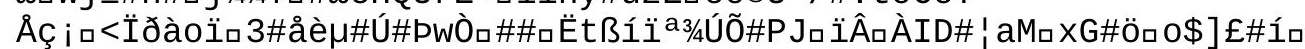

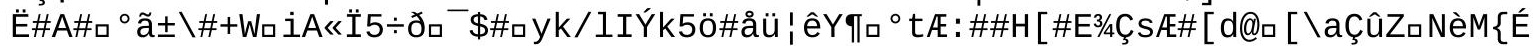

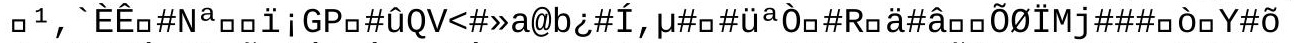

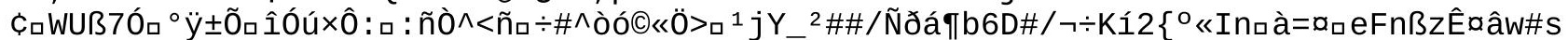

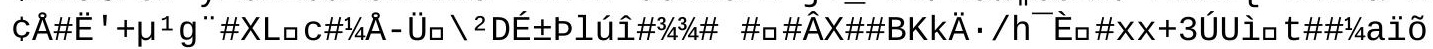

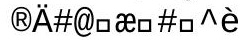




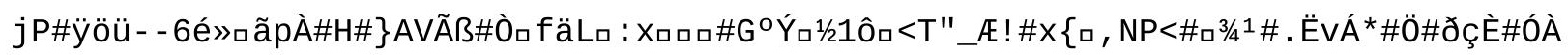




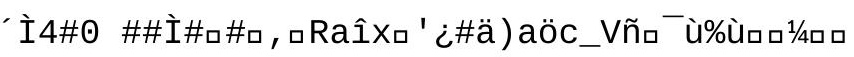




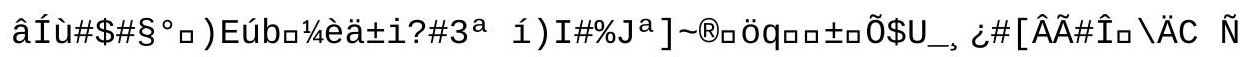




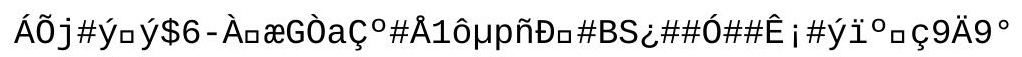




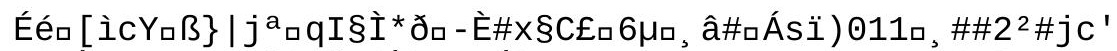

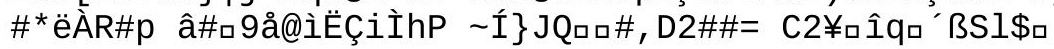




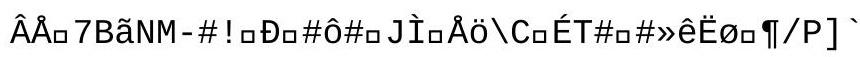


í( âN\#â5

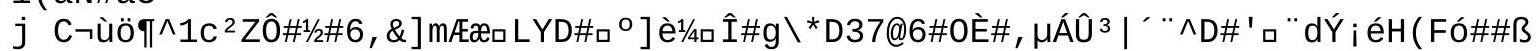




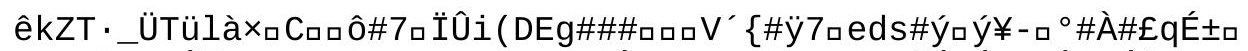

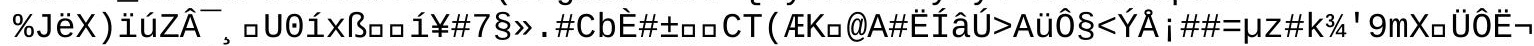

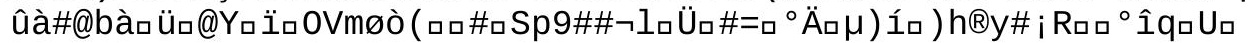




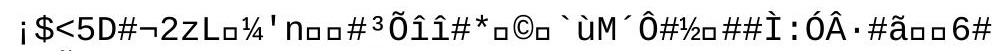

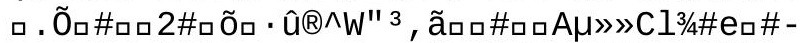

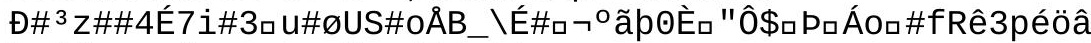

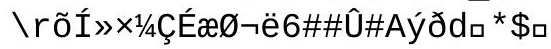




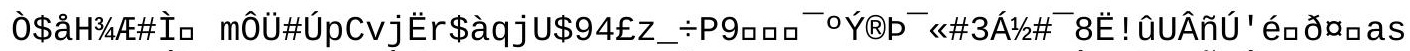

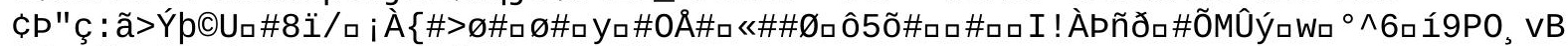

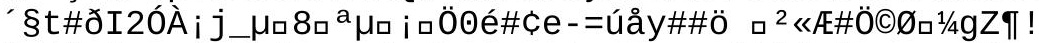

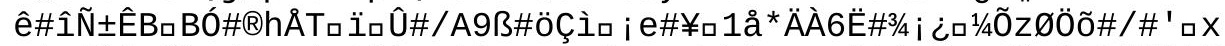

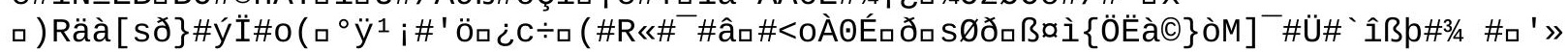


ㅁ

ÙQ

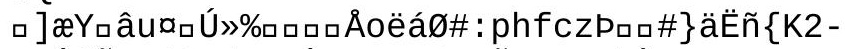

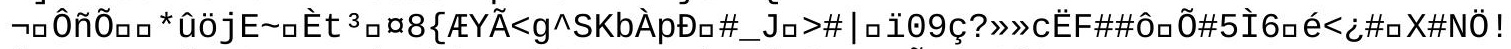

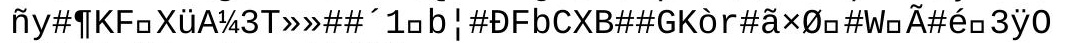

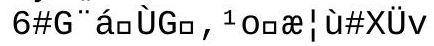




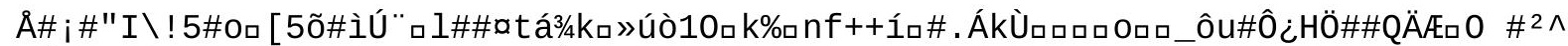

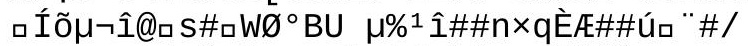

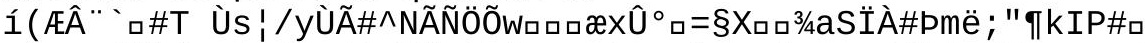

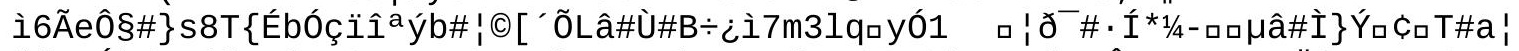

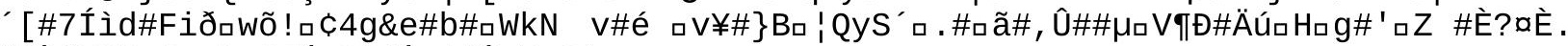

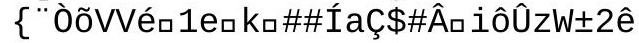




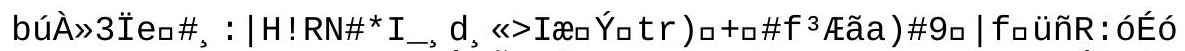

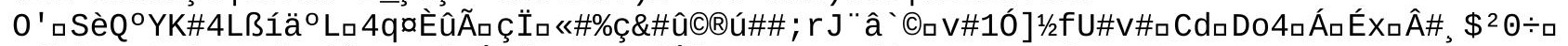

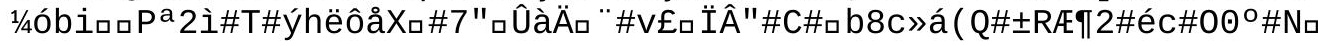


ó:\#åㅁL\#ñc1òj6! 


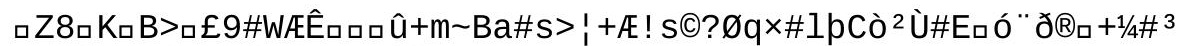

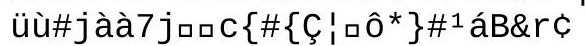


$A^{1} / 4 \square \square, f$

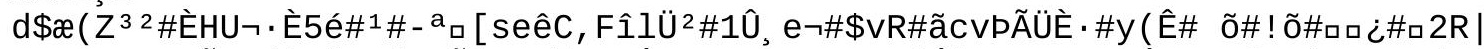

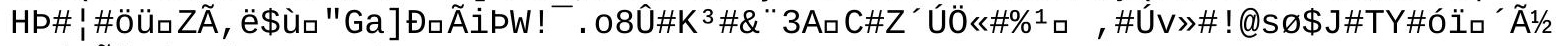

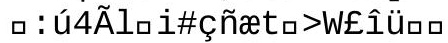


h]R 


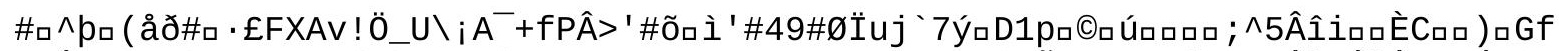

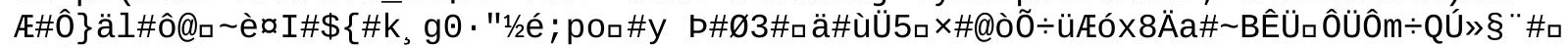
\#Ç [a\#ロrOúaI×S ${ }^{\circ}$

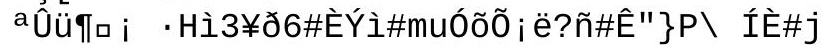


Óì f\#ÔEÉ1/4p\#Ï@\#H

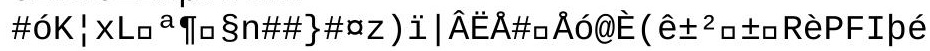




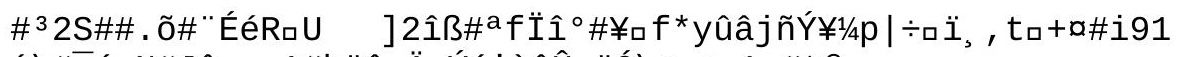

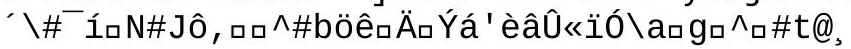




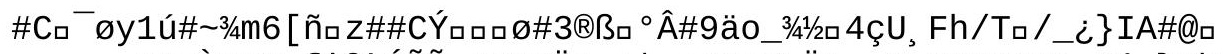

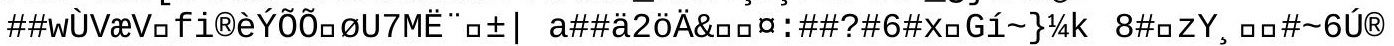

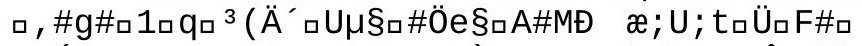

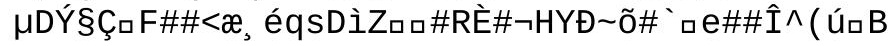




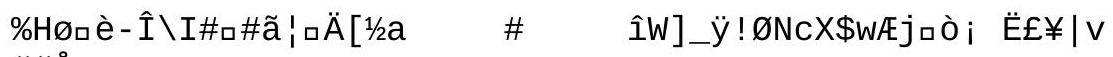

$\# \# \AA$

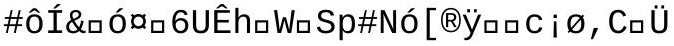




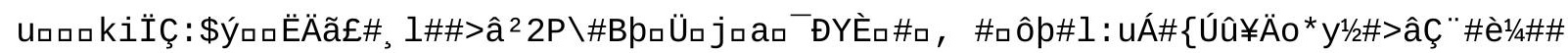
e

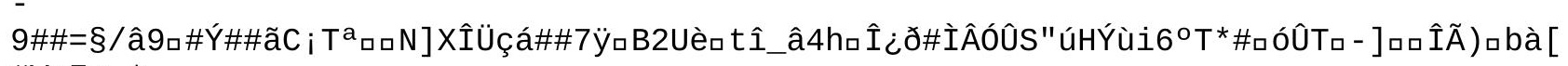
$\# \mathrm{VqFp}$ c

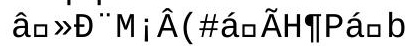
$i^{\prime}$ 
๑òpÿEqó

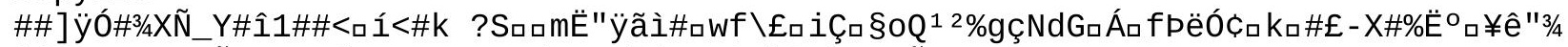

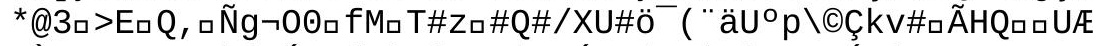

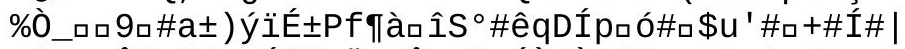

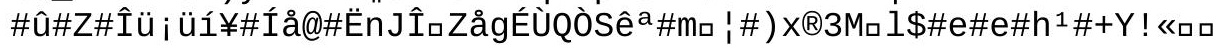




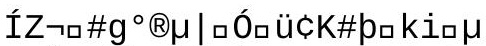

口KÛÎØ 


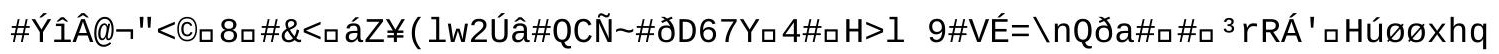




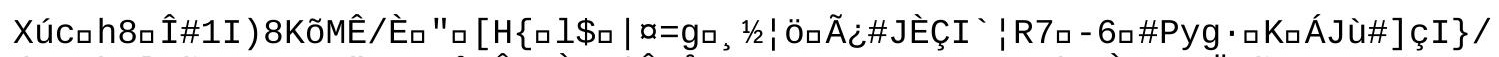

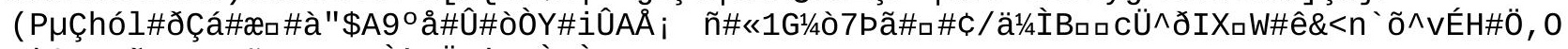

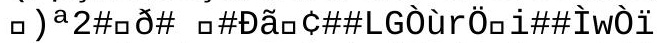

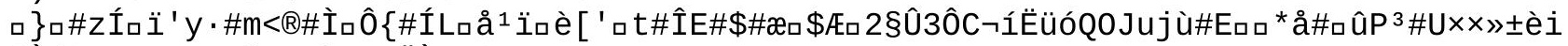

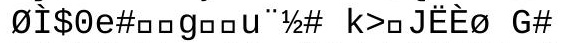

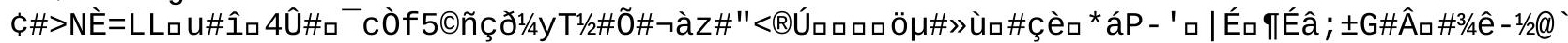

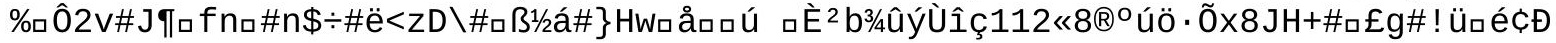




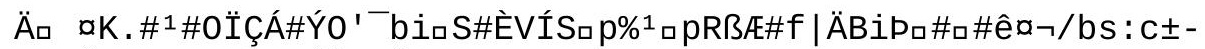

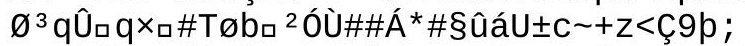

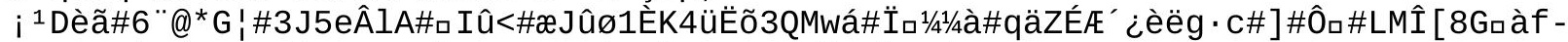

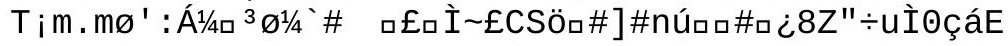




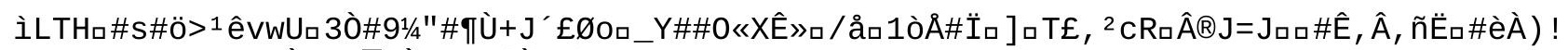

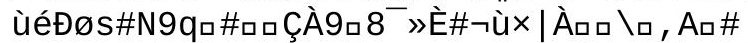

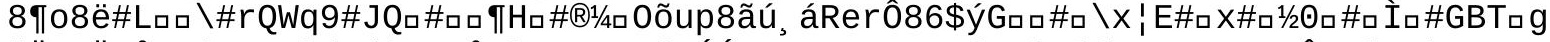

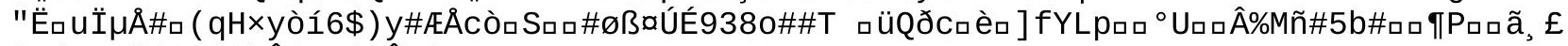
$\left(p\left(c T^{1} / 4 C{ }^{*} C^{2} \hat{a ̂}^{a} \backslash \hat{E} G \& \varnothing(\square \hat{E} \# \$\right.\right.$

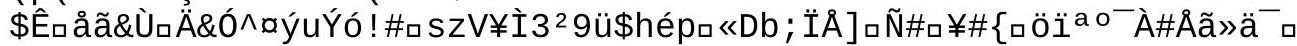
- 口GÝV1/20Xf\#"s6|\#Â+ 


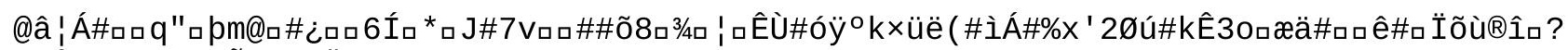

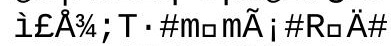

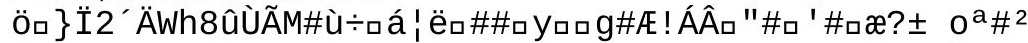




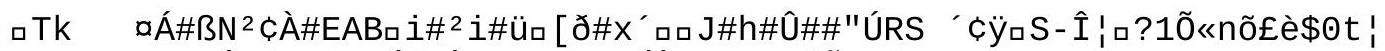

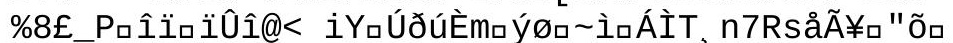

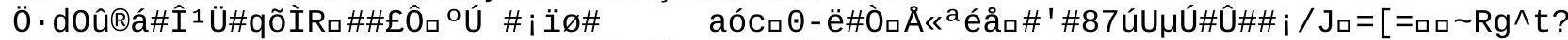

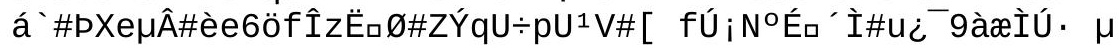




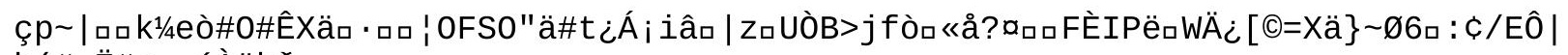
p (\#ロË\#<ロロáÀïkठ 


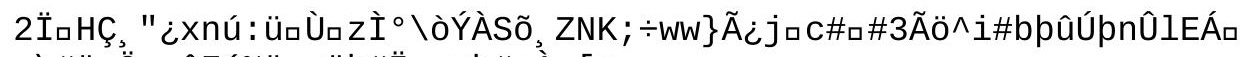

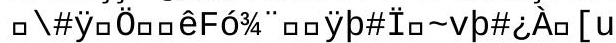

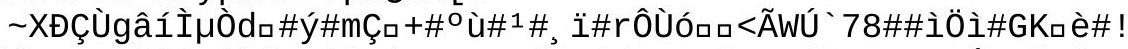

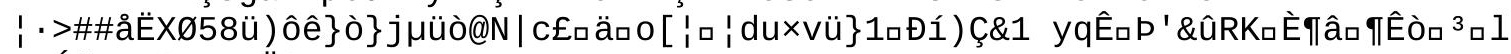

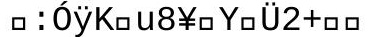

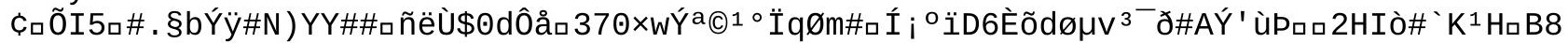

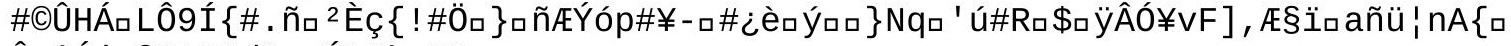

Îpî́j_@ÇLQ\#/1/4ロ eÝ1\#à_2? 


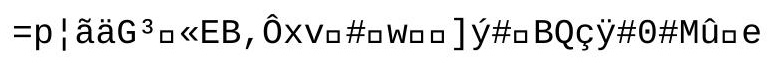

endstream

endobj

7 ○ obj

$<<$

/Type/Page

/Resources $8 \odot \mathrm{R}$

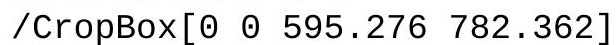

/MediaBox[ $\left[\begin{array}{llll}\odot & \odot & 595.276 & 782.362\end{array}\right]$

/Rotate 0

/Parent $82 \odot \mathrm{R}$

/Contents $10 \odot \mathrm{R}$

/Thumb $69 \odot \mathrm{R}$

$>>$

endobj

$8 \odot$ obj

$<</$ XObject $<</$ Im1 $9 \odot \mathrm{R}$

$>>$

/Font $<</ F 1253 \odot \mathrm{R}$

/F2 $95 \odot \mathrm{R}$

/F4 $101 \odot \mathrm{R}$

/F5 $105 \odot \mathrm{R}$

/F6 108 ○ $R$

/F7 $1110 \mathrm{R}$

/F8 $1140 \mathrm{R}$

/F9 $117 \odot \mathrm{R}$

$/ F 10122 \odot \mathrm{R}$

$>>$

/Procset [/PDF

/Text

/ImageB

]

/ExtGState $<</ G S 2125 \odot \mathrm{R}$

$>$

$>>$

endobj

$9 \odot$ obj

$<</$ Subtype/Image

/Length 9361

/Filter/FlateDecode

/BitsPerComponent 8

/ColorSpace/DeviceGray

/Width 280

/Height 474

/Type/XObject

>sstream

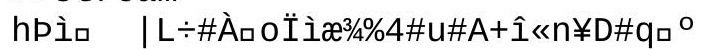

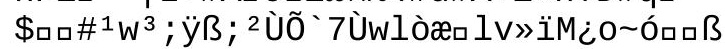

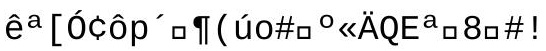




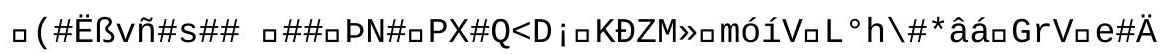




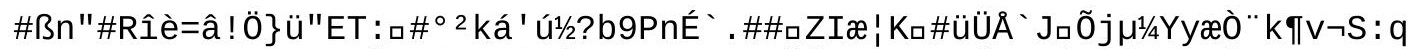

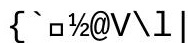

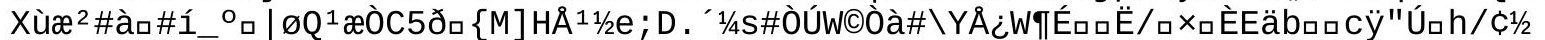

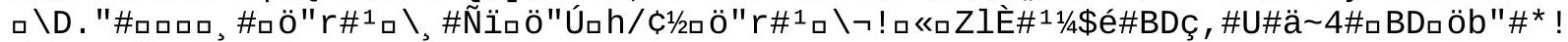
$\mathrm{d}^{-} \mathrm{B}$ "ös $\tilde{N}^{\wedge}$ a åa 


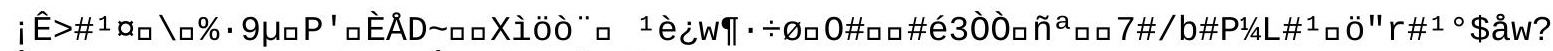

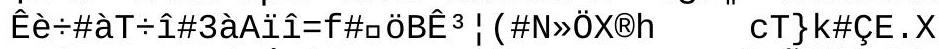

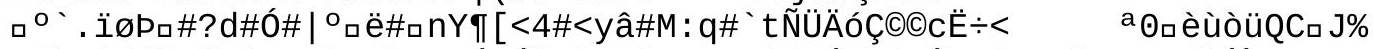

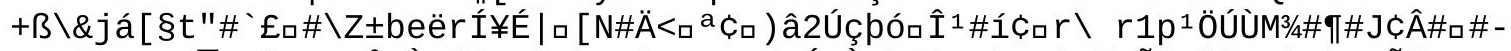

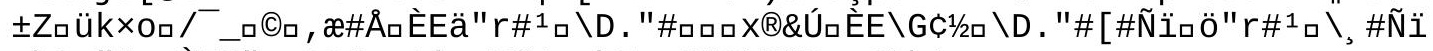

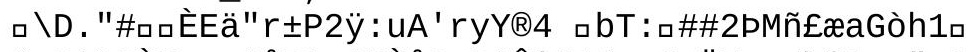

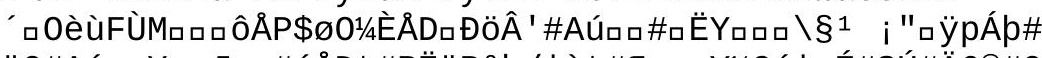

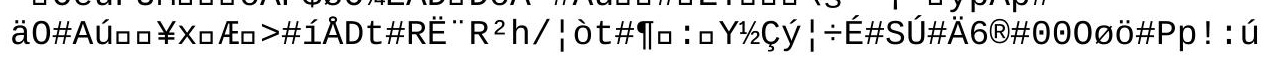

$» X j 2$ 


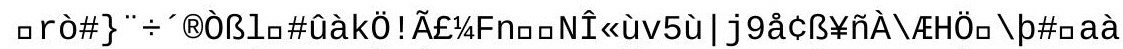

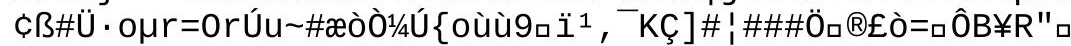




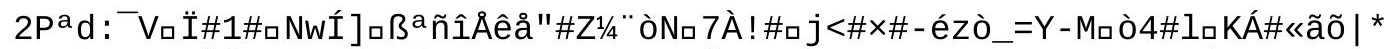

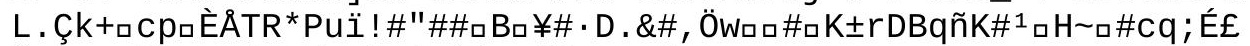

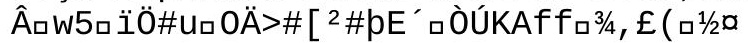




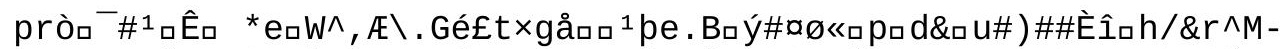

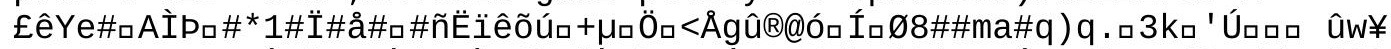

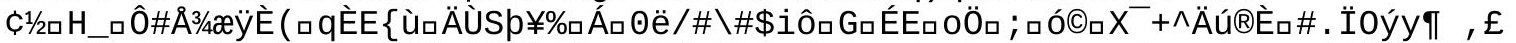

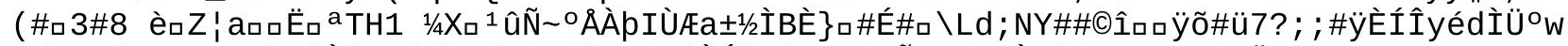

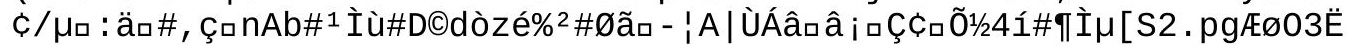




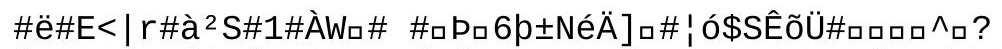

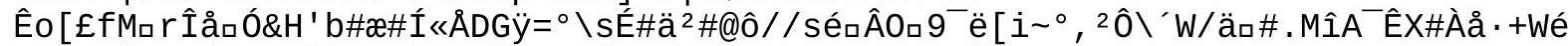

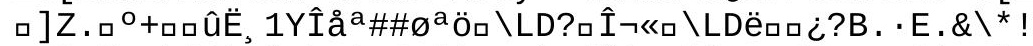

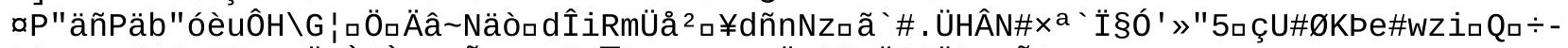

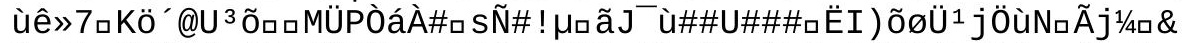

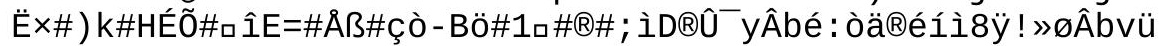

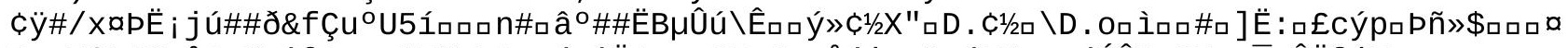

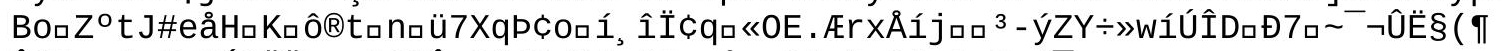

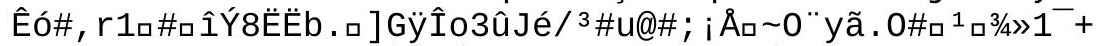

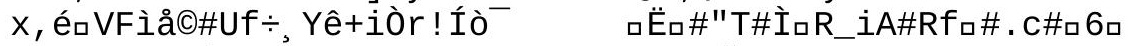

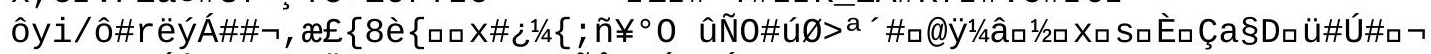

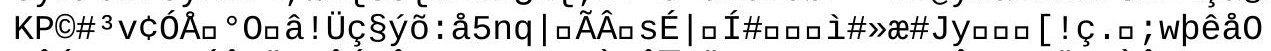

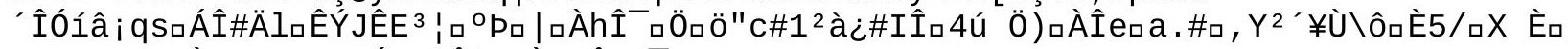

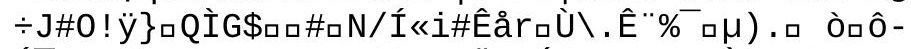

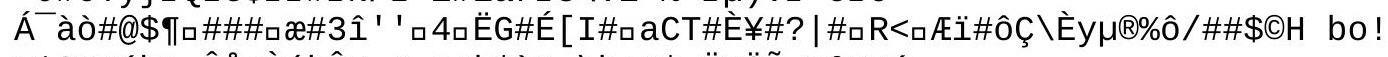

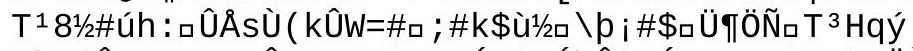

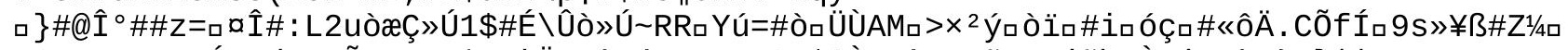

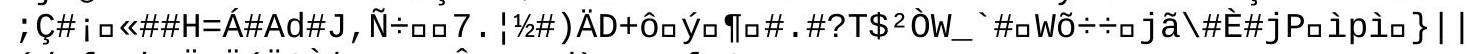

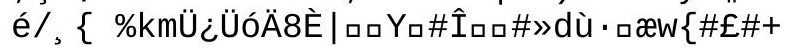




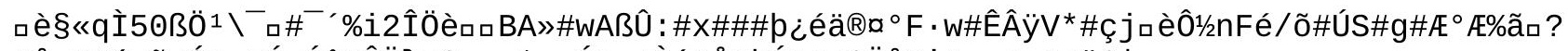

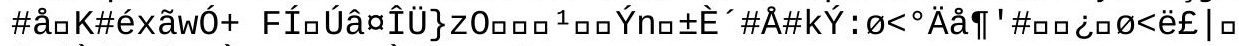

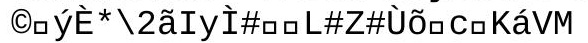




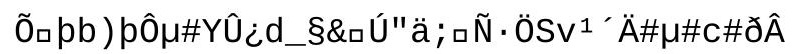
\#û 
Íøı ̊̊ $\mathrm{u}^{1} \mathrm{~h} ? \mathrm{O} \neg$

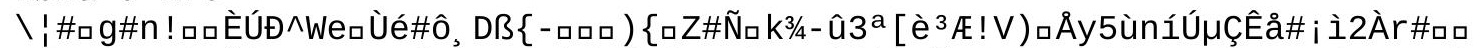

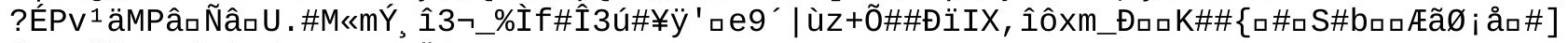

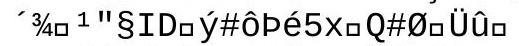

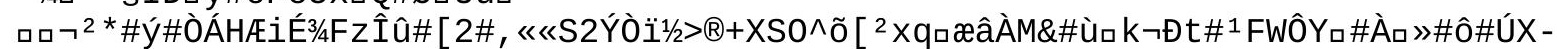

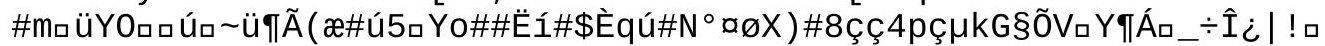

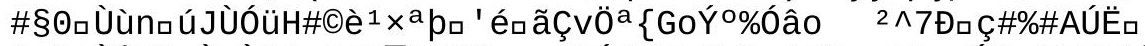

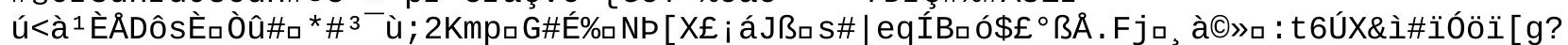

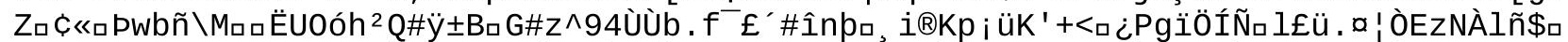

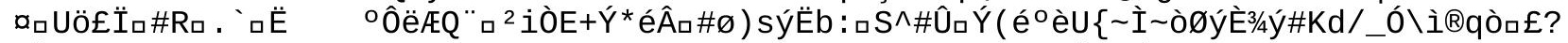

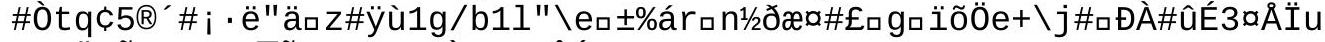

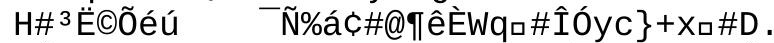

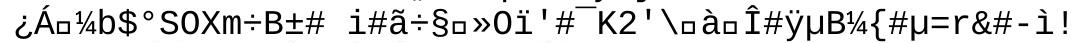

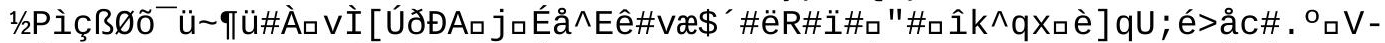

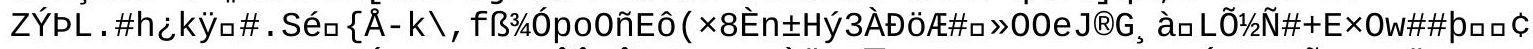

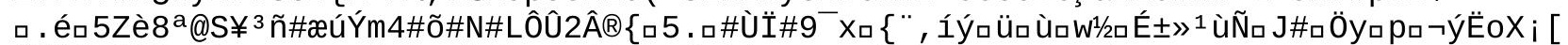

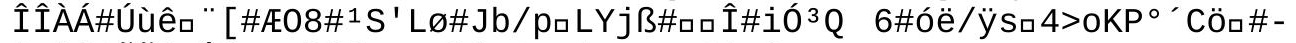

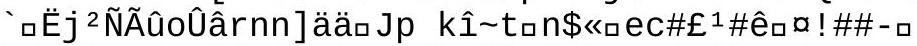

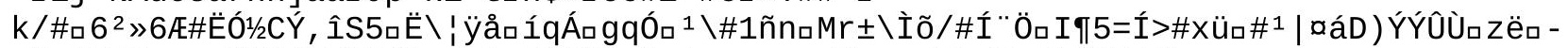

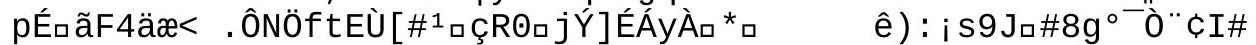

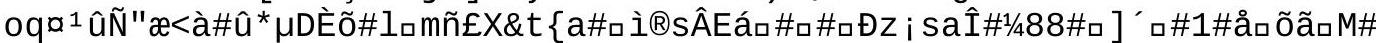

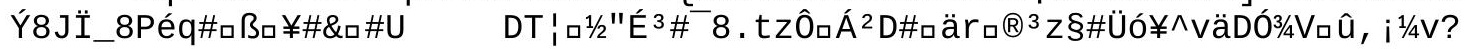

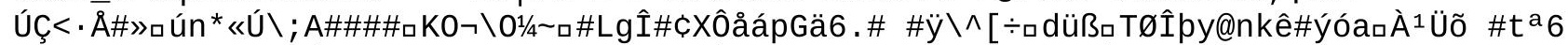

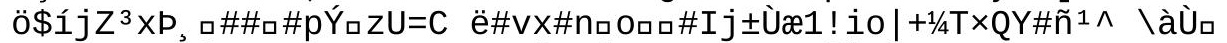

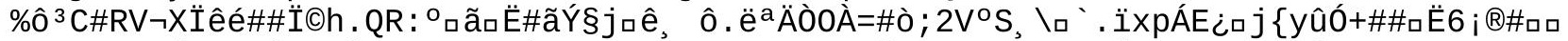

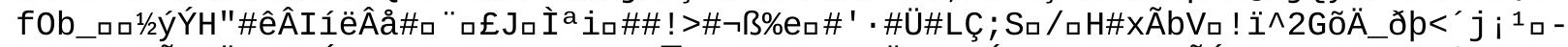

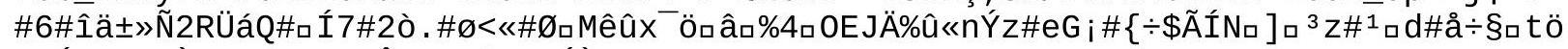

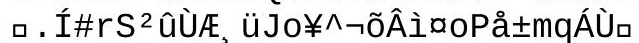




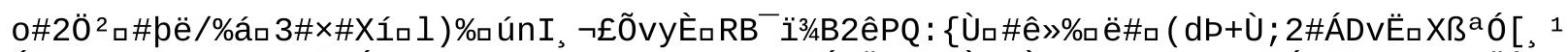

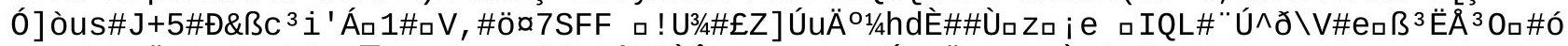

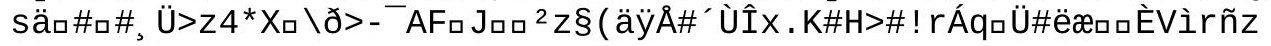




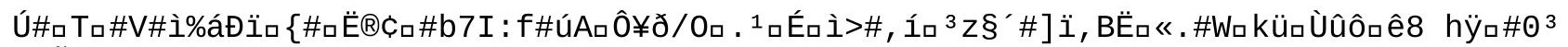

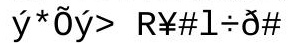




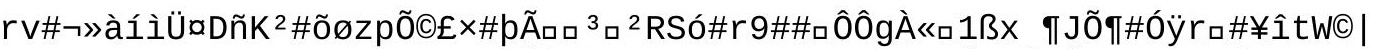

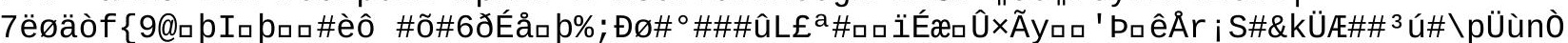

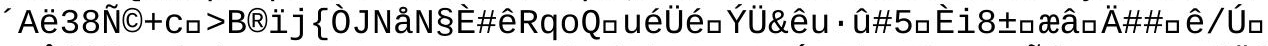

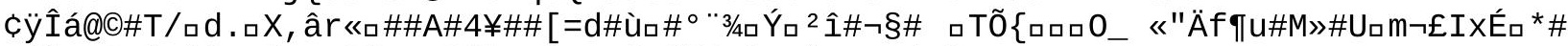

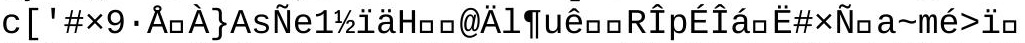

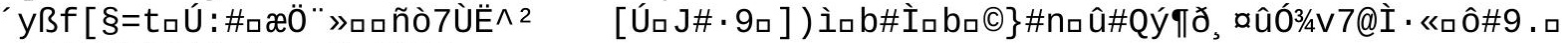
口 ] B\#5jDD०Á\#口åaczÃ : JÄOß口 


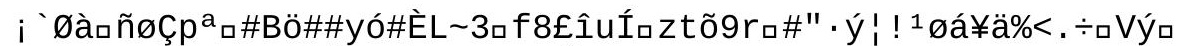

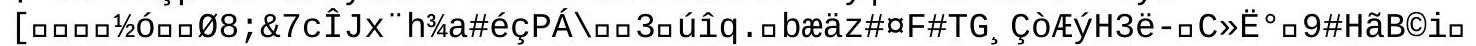

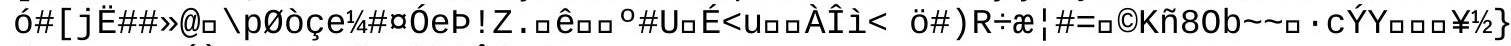
\{ r r\#R\#67ÁÈv\#YK\#oð“"ù̀ \ô ÛSýø

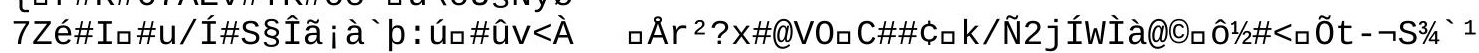




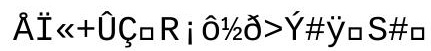




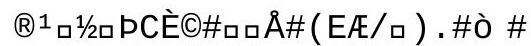

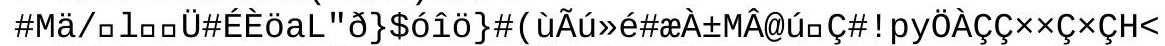

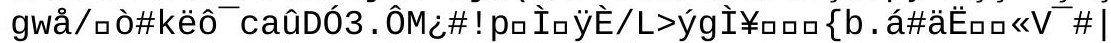

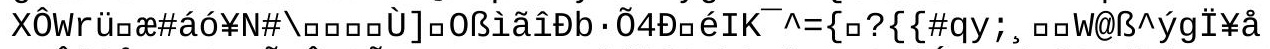

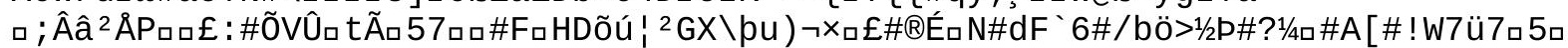

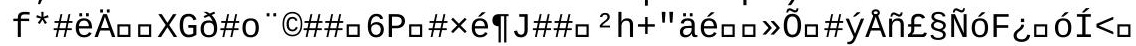

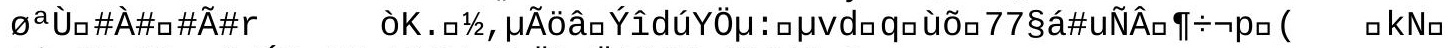

pån\#2gñ8gm\#/ÚQw“õaù\}\#Yı! IÏbıËjû\#\#s\#\#\#j\#æ\&

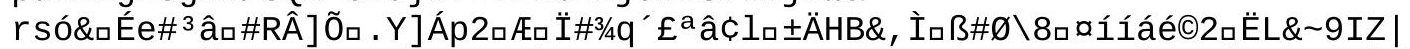

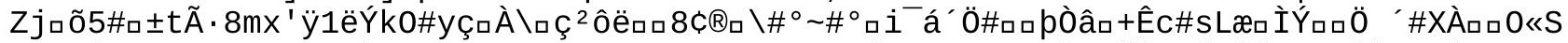

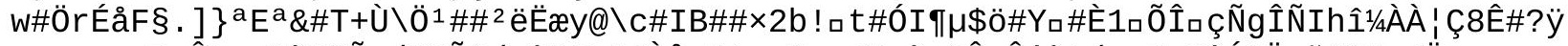

R\}ma $\operatorname{mag}_{\square}$

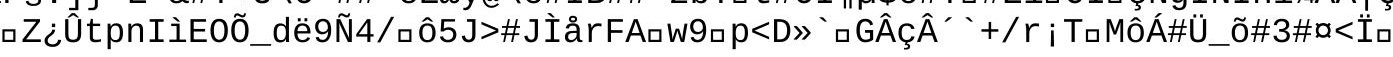

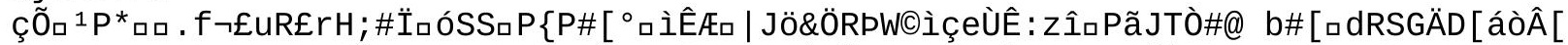

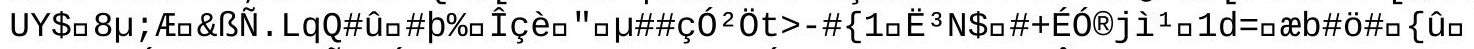

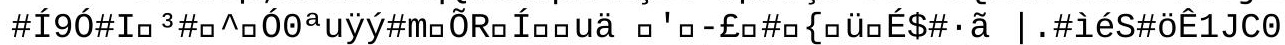

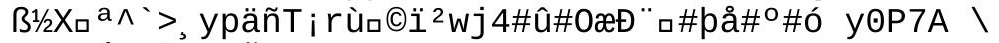

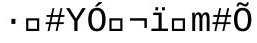




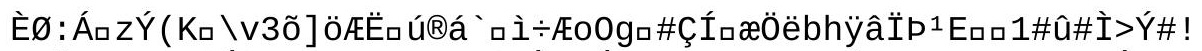

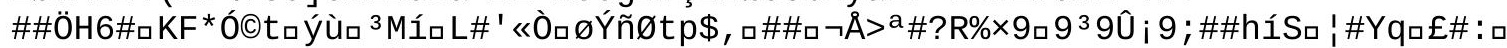

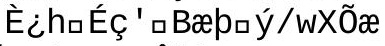

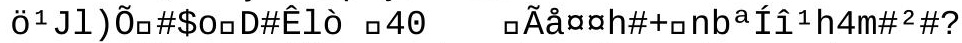

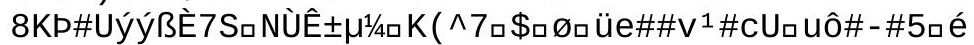


口/\#םäup\#Ç,\#lÄÄ×ä/\#Çם 


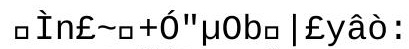

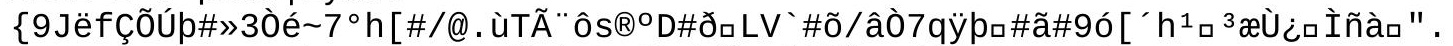

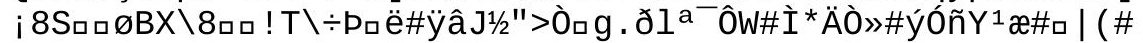


É ; êa Õ\#! ßæ|İঃL\#Ü|£̂̂ÉóÂcF 


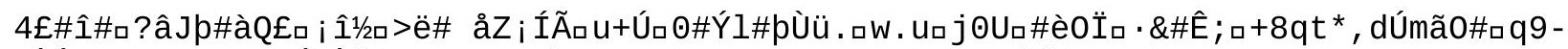

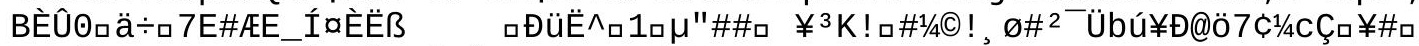

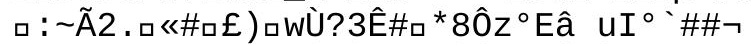




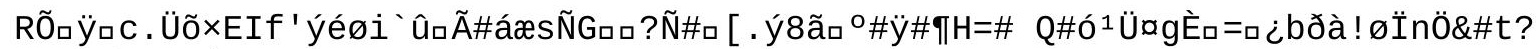

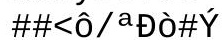

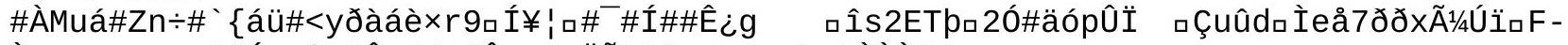

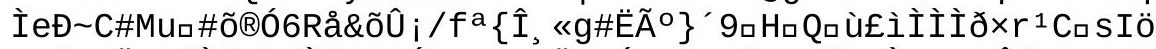

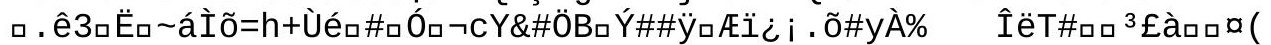

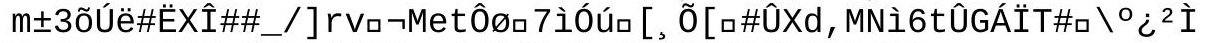

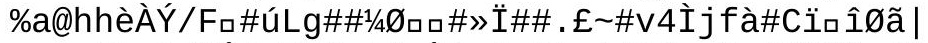

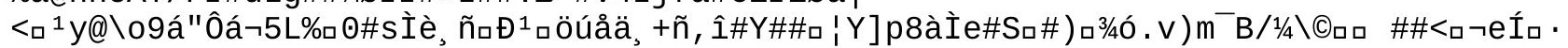

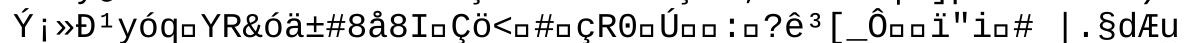

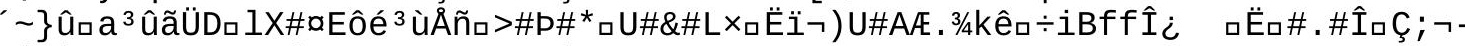

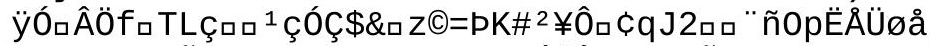

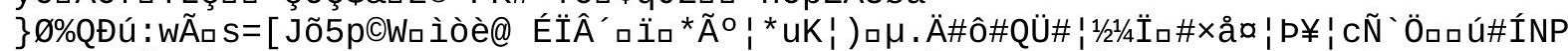

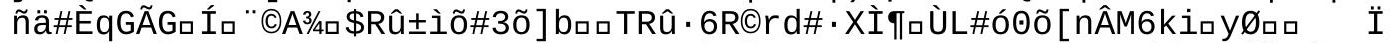

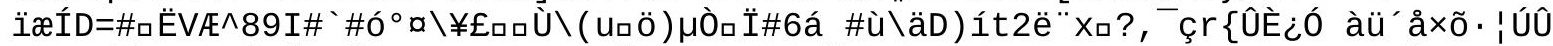

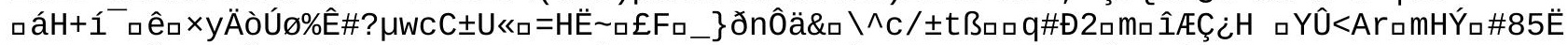

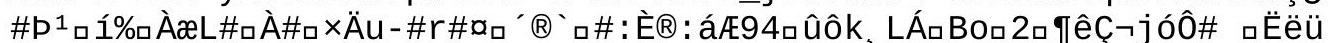

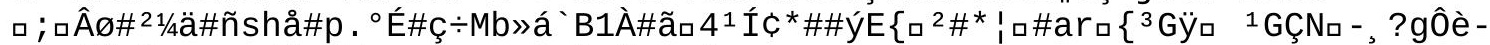

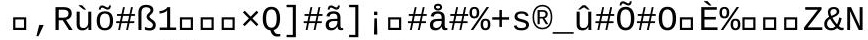

$\# \quad ¥ 1 \hat{0} \sim \& r \Delta A 8 \# \#^{\prime \prime}$ ]

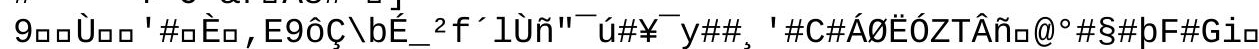

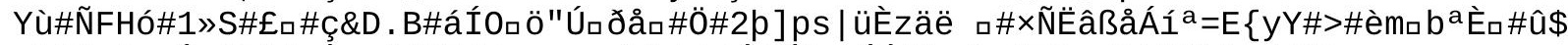

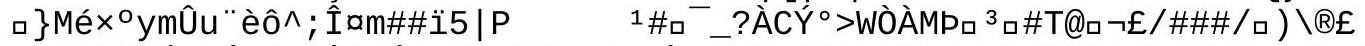

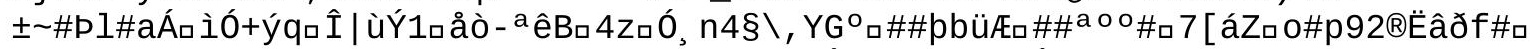

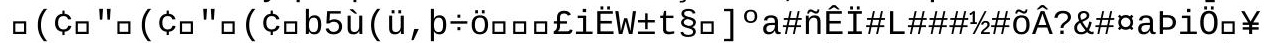

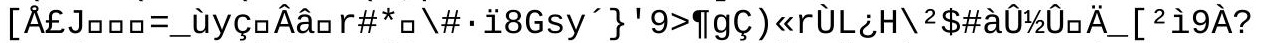

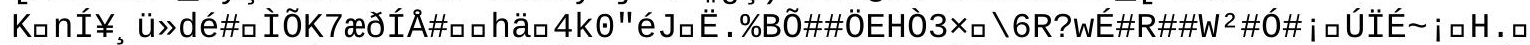

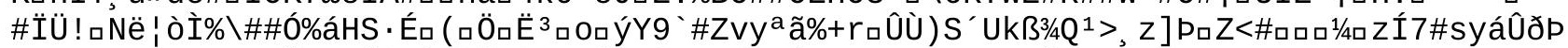

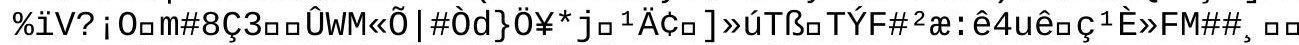

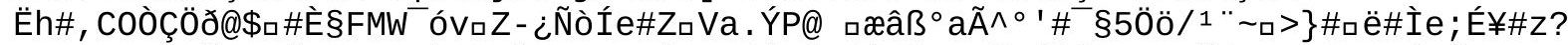

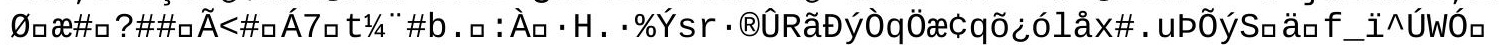

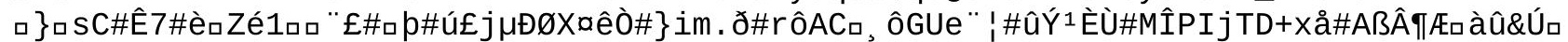

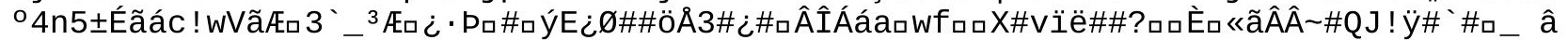

endstream

endobj

$10 \odot$ obj

$<</$ Length 5634

/Filter/FlateDecode

>stream

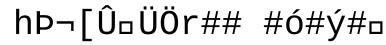




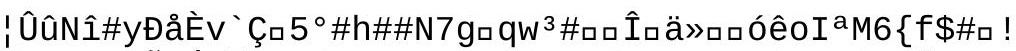

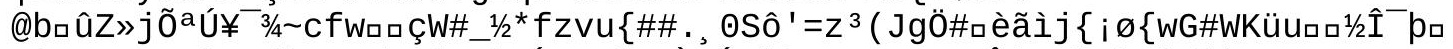

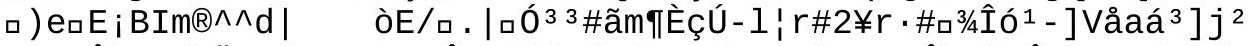

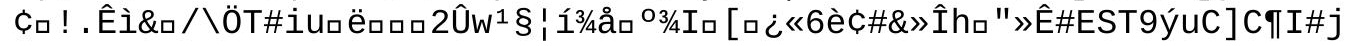




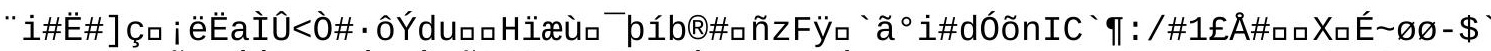

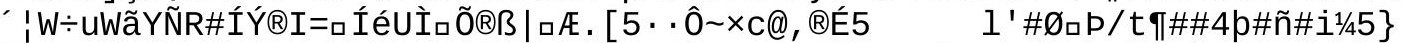

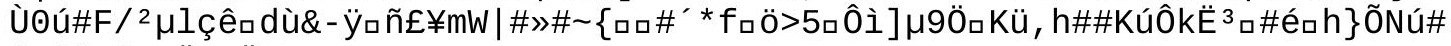
'ロôå6 '॰CÖSRË 


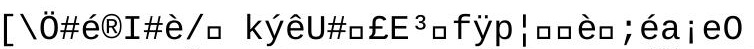

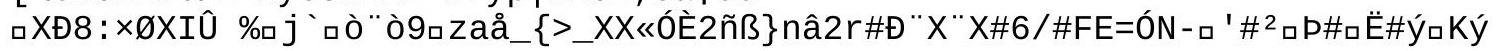




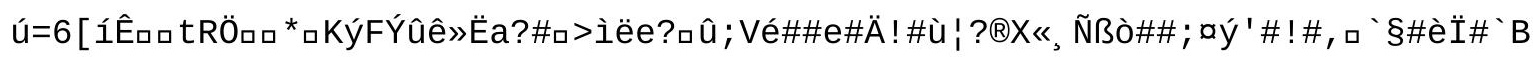




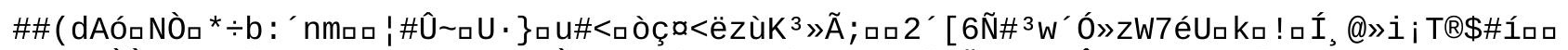

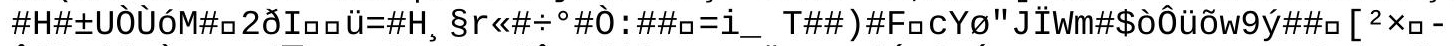

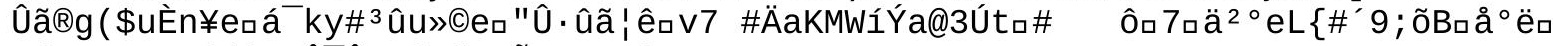

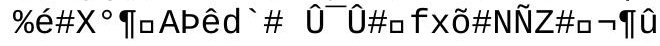




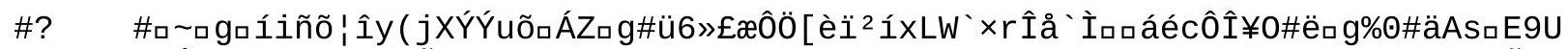

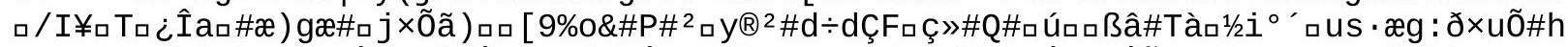

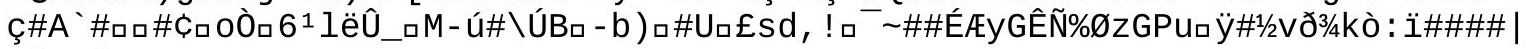

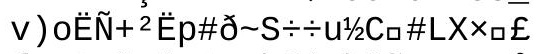

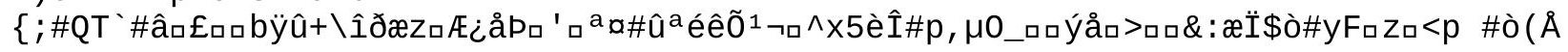




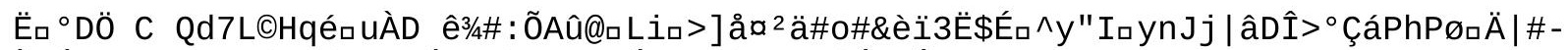

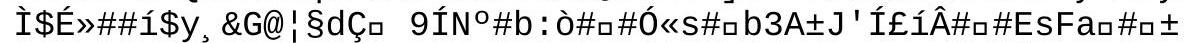

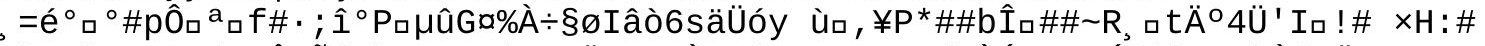

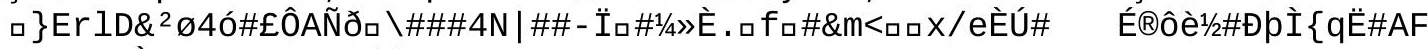

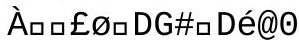

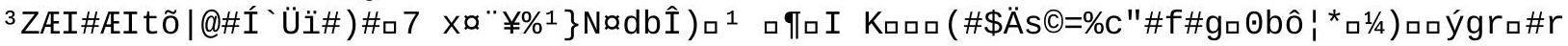




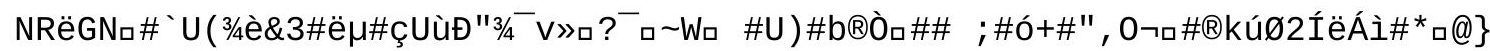

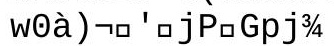




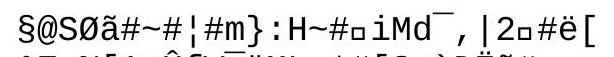

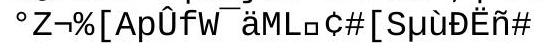

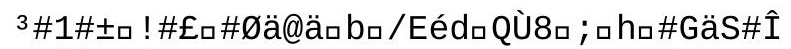

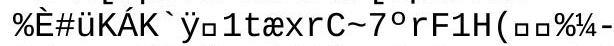

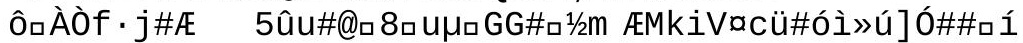

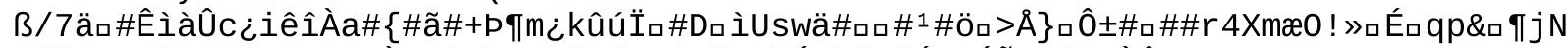

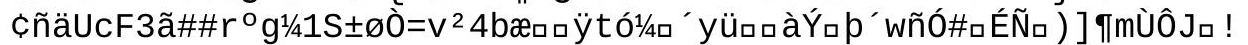

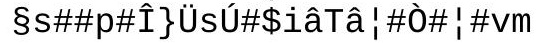




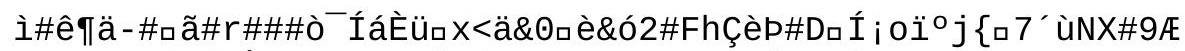

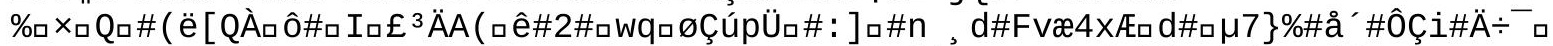

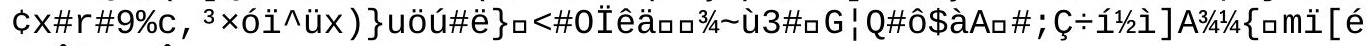

c?ôû K\#Ôq

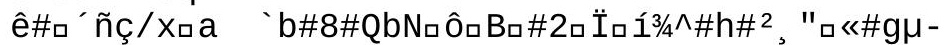

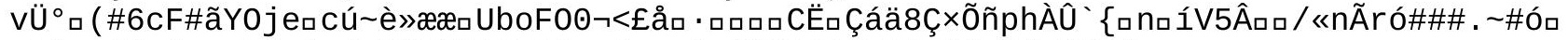

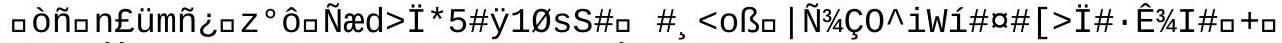

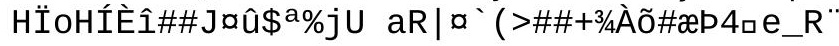




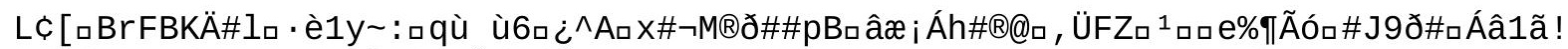

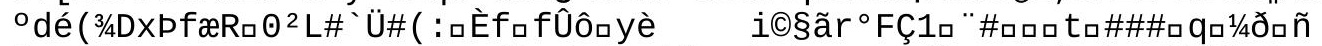

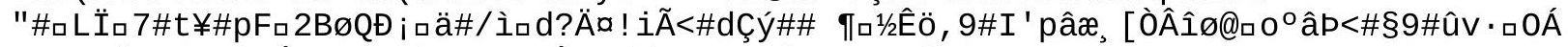

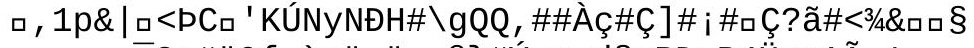

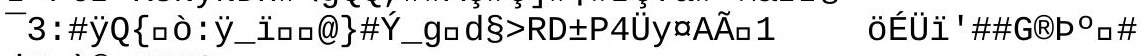

$\left.; \div 1{ }^{\circ} \mathrm{A} a j \# \ll\right)(-\# \# \#$

'dıÉáX 


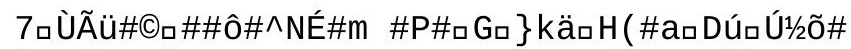

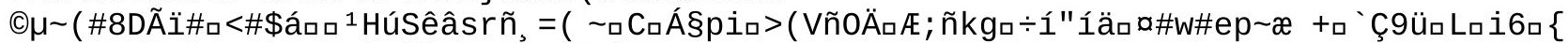

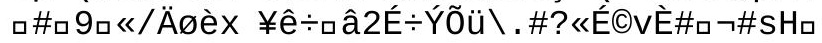




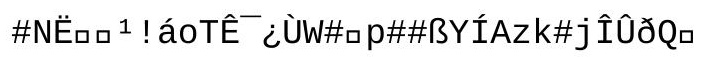




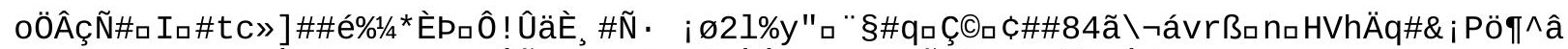

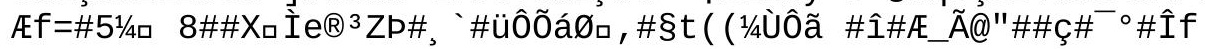


oÛ\#ñロ G G/4ロ İÀË\# 


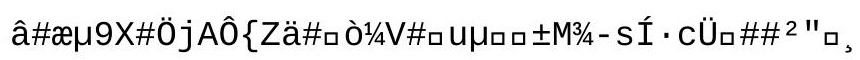




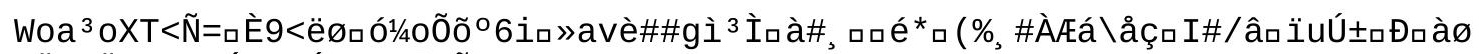

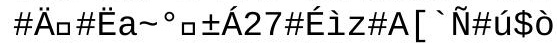

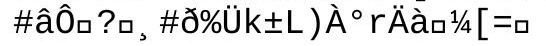




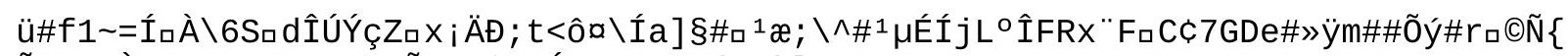

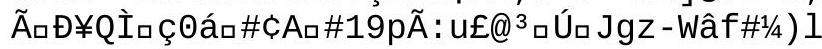




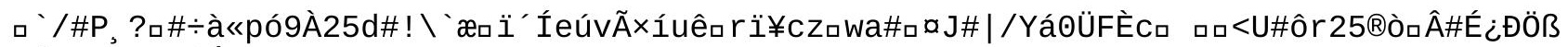
口Ë口 $\#^{3} \tilde{n} M,\left(I^{3}{ }^{3 / 4}\right.$

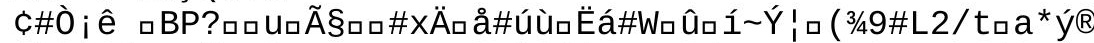

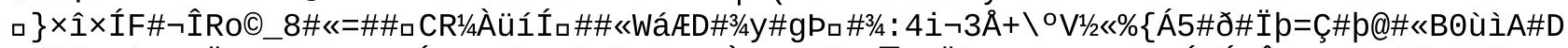

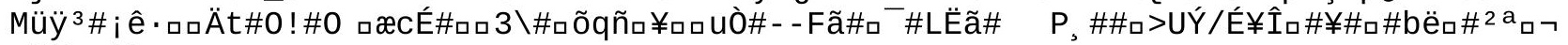
añFı_êL 


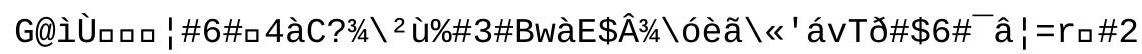
\#\#\#Ù\#\{ ${ }^{\circ} 0 \tilde{c}>_{\square}=\neg S q_{\square} f$ \#\#\#E. à 4

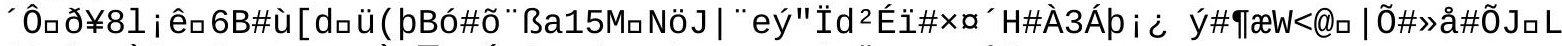

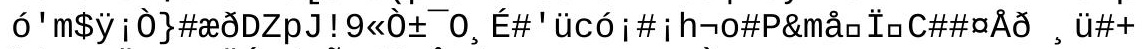

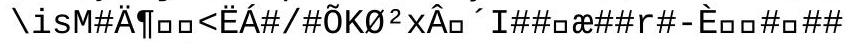

bAıábıฉ $3 / 4 \S$ · \# 


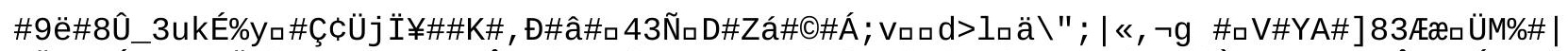

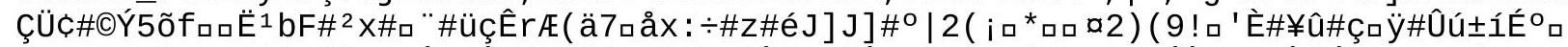

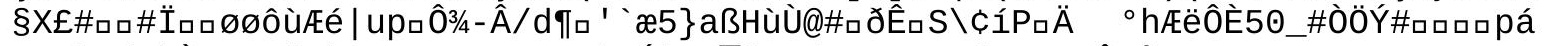

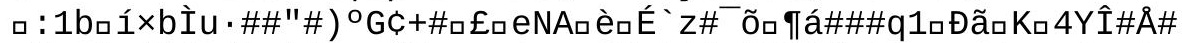

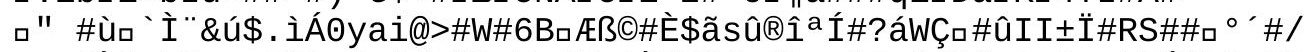

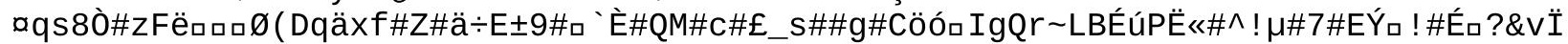

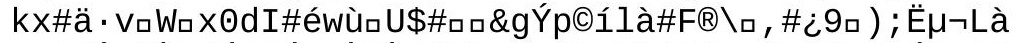

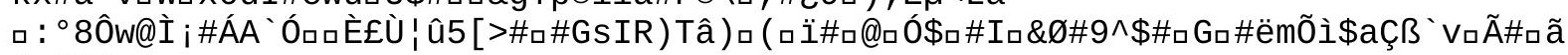
a\#ß\#u) 


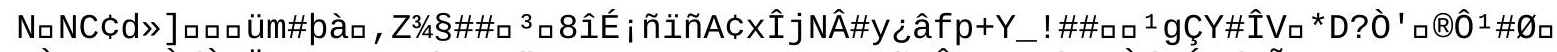

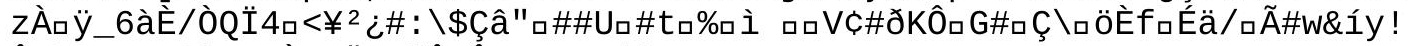

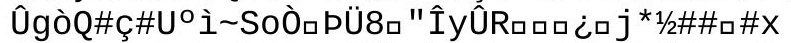

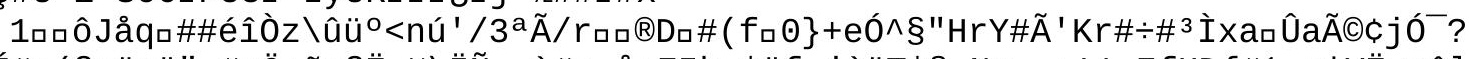

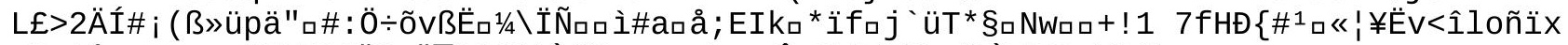
ם"ם`ÅX $\#$ \#ѐM!1 $1 \ddot{I} \# \times \ddot{I}^{-} \circ \mathrm{J}^{\prime}$ àÀ\#囚c

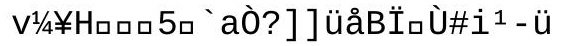




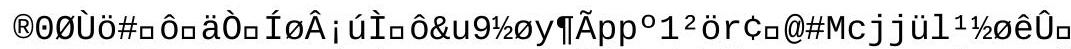

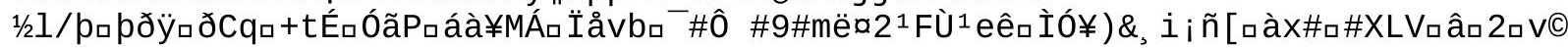




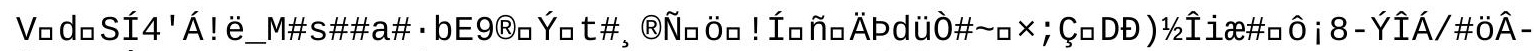

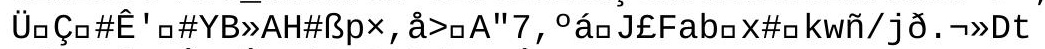

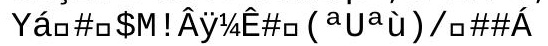




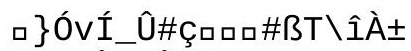

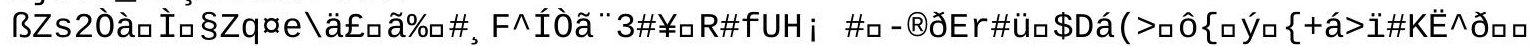
\#Vý: 7ÈhUÒロXÇ1 âá¿\# 


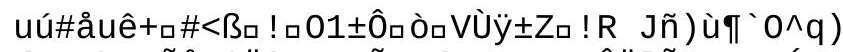

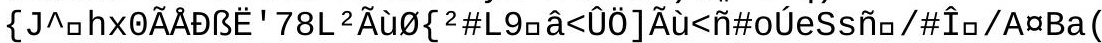

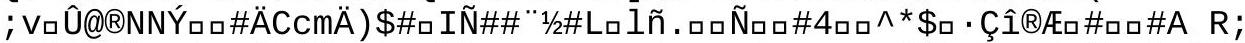

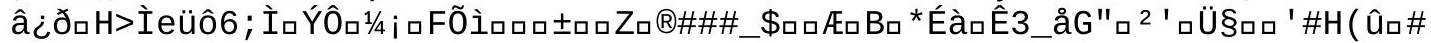


\#JA\#

endstream

endobj

$11 \odot$ obj

$<$

/Type/Page

/Resources $12 \odot \mathrm{R}$

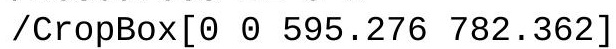

/MediaBox[ $\left[\begin{array}{llll}\odot & \odot & 595.276 & 782.362\end{array}\right]$

/Rotate 0

/Parent $82 \odot \mathrm{R}$

/Contents $13 \odot \mathrm{R}$

/Thumb $70 \odot \mathrm{R}$

$>$

endobj

$12 \odot$ obj

$<</$ Font $<</ F 1356 \odot \mathrm{R}$

$/ \mathrm{F} 1460 \odot \mathrm{R}$

/F15 $63 \odot \mathrm{R}$

/F4 $1010 \mathrm{R}$

/F5 $105 \odot \mathrm{R}$

/F9 $1170 \mathrm{R}$

/F10 $122 \odot \mathrm{R}$

$>$

/Procset [/PDF

/Text

]

/ExtGState $<</ G S 2125 \odot \mathrm{R}$

$>$

$>$

endobj

$13 \odot$ obj

$<</$ Length 4646

/Filter/FlateDecode

>sstream

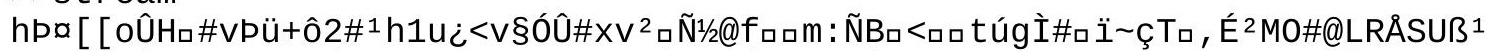

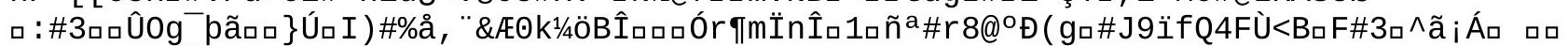
\#\#qèz ${ }^{*} F 6 R A ̈ b$ 


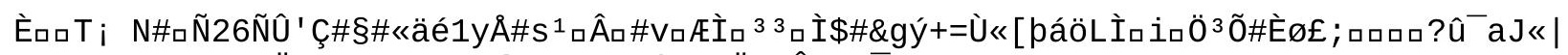

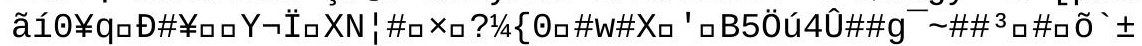




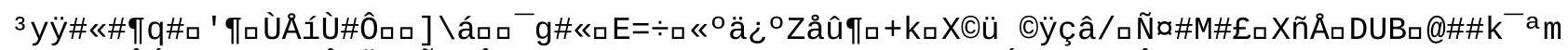

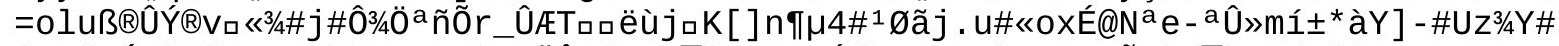

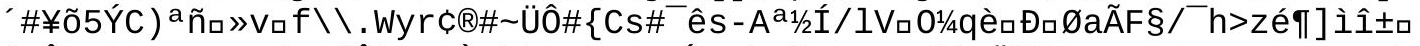

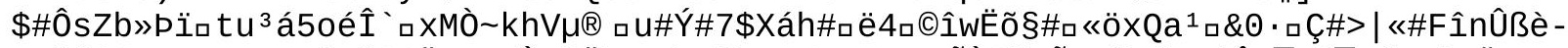

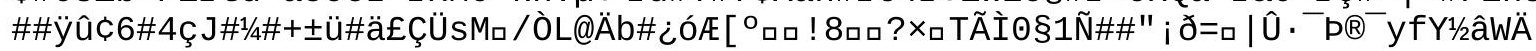




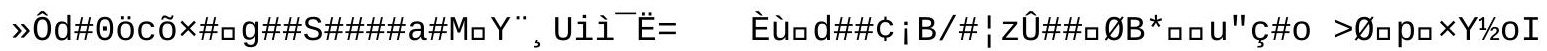
$\square \# \mid \ll / p \ddot{i}$ 


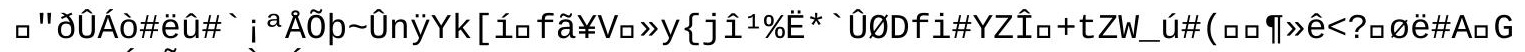

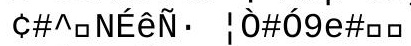

K\&ÁN\#价

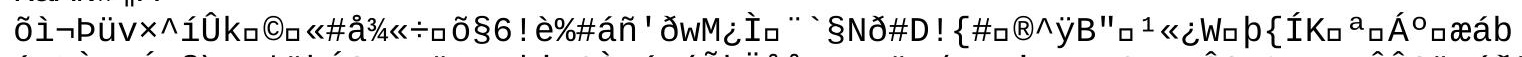

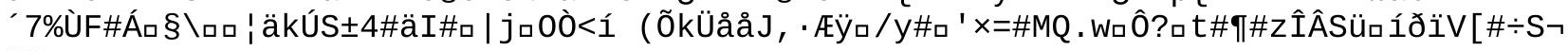
AT

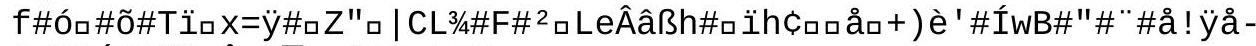

\#\#3/4TÁéùi $1 / 2 Z 0 \hat{a} J^{-}$ฉp $\{\ddot{a ̈ ß}, \backslash \# \#$ 


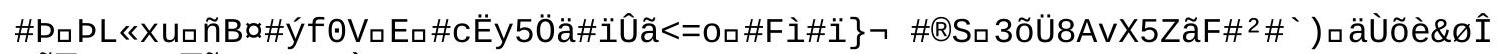

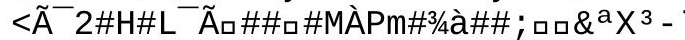

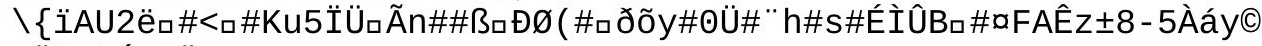

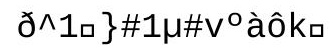
口 İ̂िøbÁ\#!Ö

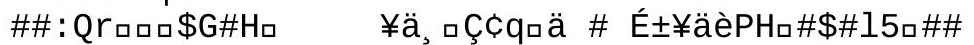

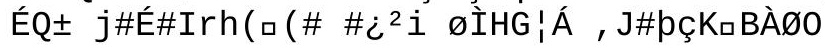




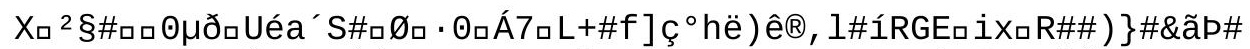

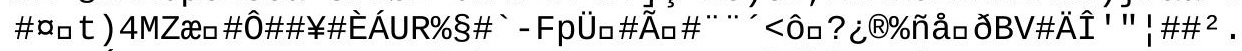

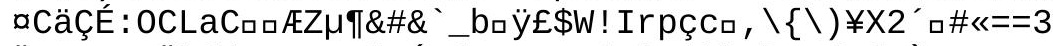

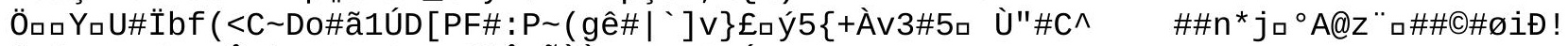

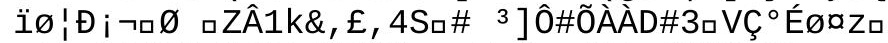

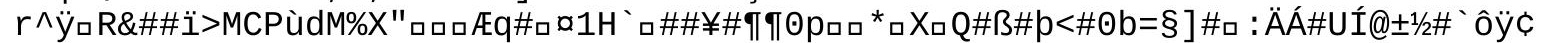

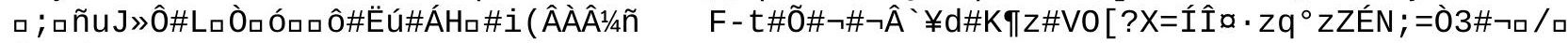
\#ard 


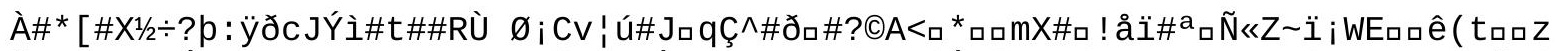

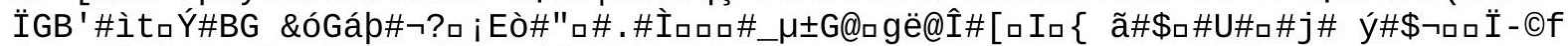

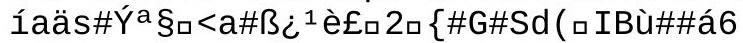


å\#ロ8Âa ( 8J5\&\#"Eýi ${ }^{3}{ }_{1}$ \#ó "\#HDÚ 


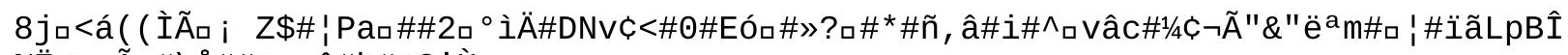
$\% \ddot{E}<$ «

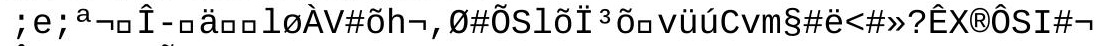

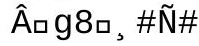




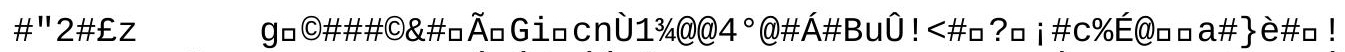

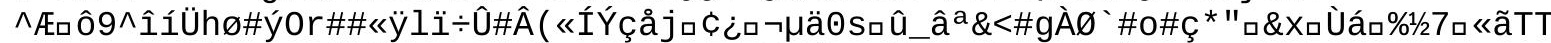

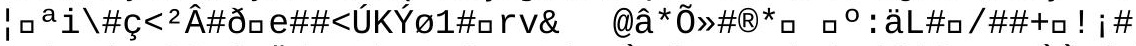

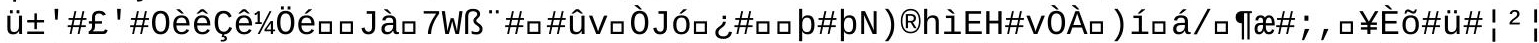

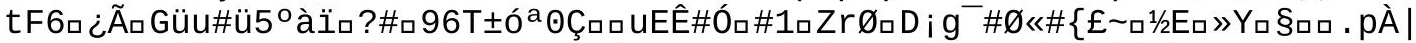

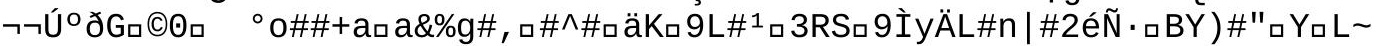




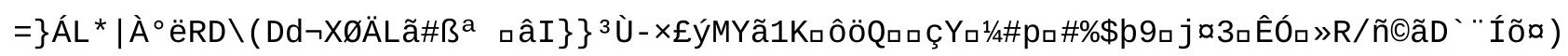




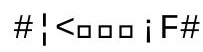




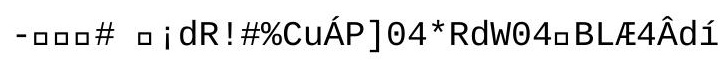




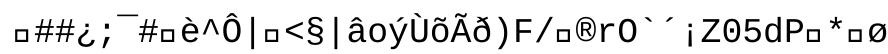


§\#ם pàRa 'Ú¿¿ÓÃL\# 


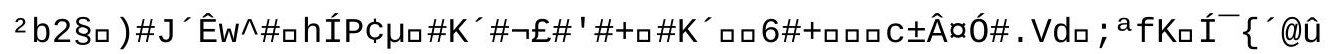




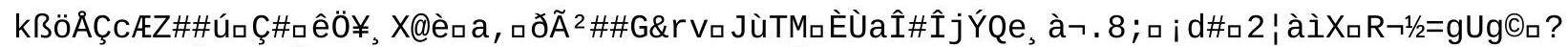

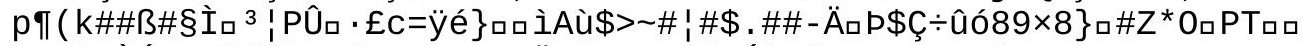

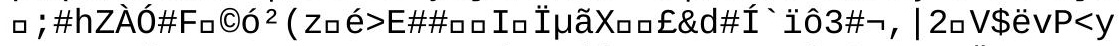

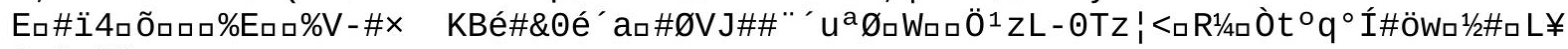
(m"ם®â\#, \#v:\#ロ 


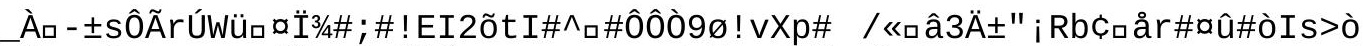
口

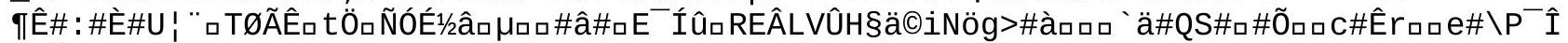

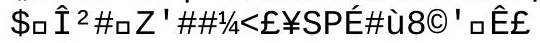

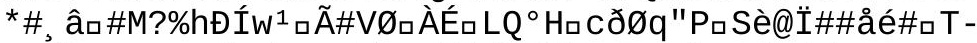

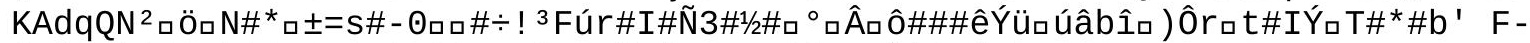

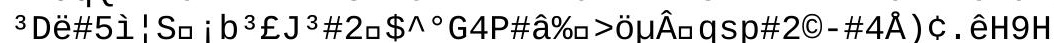

ê $L \# a \#^{2} W \mid \sim 1 \# ; i \sim$

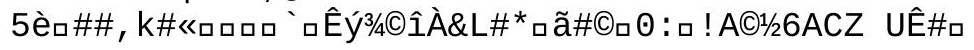




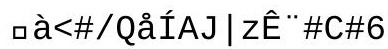

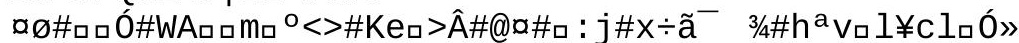

à! $\mathrm{vJ} \# ; 0: \operatorname{Rex}^{2} \mathrm{C} z \mathrm{~V} a \mathrm{I} \pm \mathrm{Li} \mathrm{i}^{*}$. i \#\#5

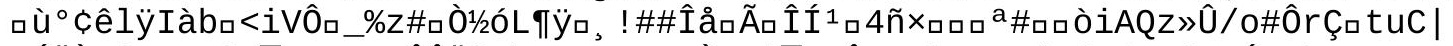

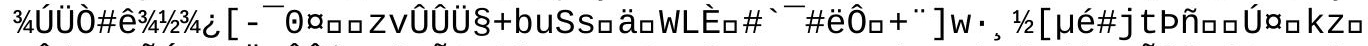

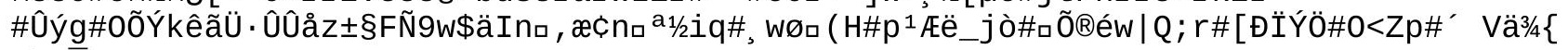
口Pm 


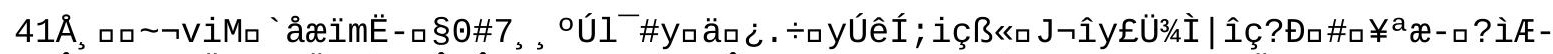

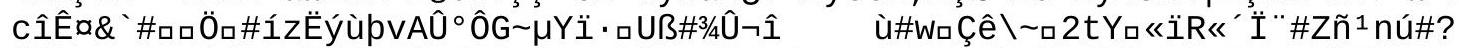

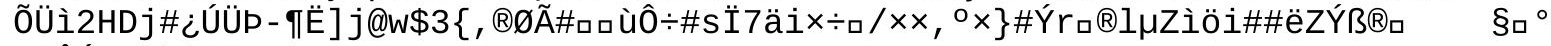

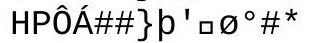




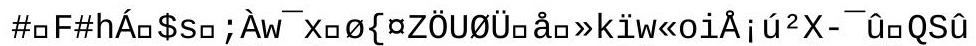

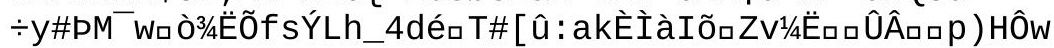

$\left\{\mathrm{SÝ} 1 \mathrm{~K} \% \pm \mathrm{i}{ }^{`} \#\right.$ 


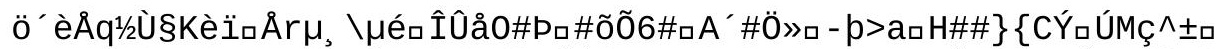

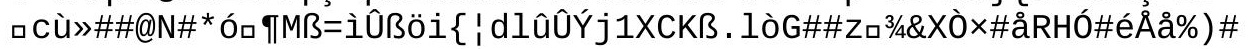




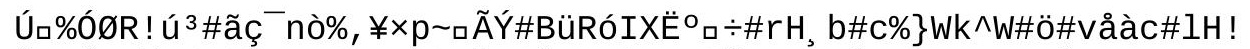

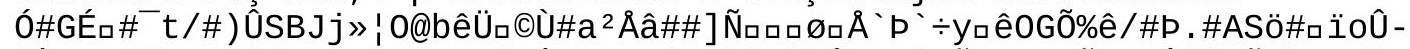

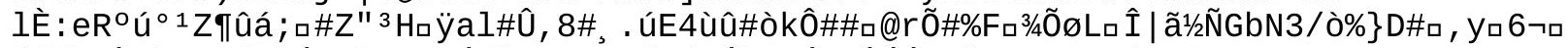

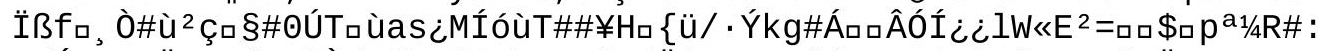

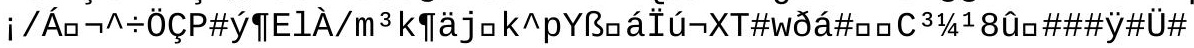

endstream

endobj

$14 \odot \mathrm{obj}$

$<<$

/Type/Page

/Resources $15 \odot \mathrm{R}$

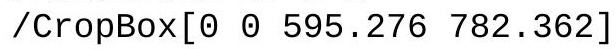

/MediaBox[ $\left[\begin{array}{llll}0 & \odot & 595.276 & 782.362\end{array}\right]$

/Rotate 0

/Parent $82 \odot \mathrm{R}$

/Contents $21 \odot \mathrm{R}$

/Thumb $71 \odot \mathrm{R}$

$>$

endobj

$15 \odot$ obj

$</$ Font $<</ F 1253 \odot \mathrm{R}$

$/ \mathrm{F} 1356 \odot \mathrm{R}$

/F14 $600 \mathrm{R}$

/F15 $63 \odot \mathrm{R}$

/F16 $160 \mathrm{R}$

/F4 $101 \odot \mathrm{R}$

/F5 $105 \odot \mathrm{R}$

/F6 108 ○ R

/F7 $1110 \mathrm{R}$

/F8 $1140 \mathrm{R}$

/F9 $1170 \mathrm{R}$

$/ \mathrm{F} 10122 \odot \mathrm{R}$

$>>$

/Procset [/PDF

/Text

]

/ExtGState $<</ G S 2125 \odot \mathrm{R}$

$>>$

$>>$

endobj

$16 \odot$ obj

$<</$ Subtype/Type1

/FontDescriptor $17 \odot \mathrm{R}$

/LastChar 49

/Widths [833

]

/BaseFont/KJMJNL+AdVMPi-One

/Firstchar 49

/ToUnicode $19 \odot \mathrm{R}$

/Encoding $20 \odot \mathrm{R}$

/Type/Font

$>$

endobj

$17 \odot$ obj

$</$ StemV $\odot$

/FontName/KJMJNL+AdVMPi-One

/FontFile3 $18 \odot \mathrm{R}$

/Flags 6

/Descent - 205

/FontBBox [-20

$-208$

1000

770 
]

/Ascent 696

/CapHeight 712

/XHeight 442

/Type/FontDescriptor

/ ItalicAngle 0

/CharSet (/space/one)

$>$

endobj

$18 \odot$ obj

$<</$ Subtype/Type1C

/Length 108

/Filter/FlateDecode

>stream

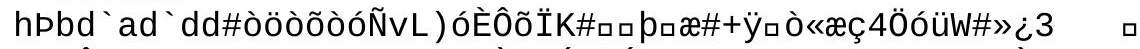

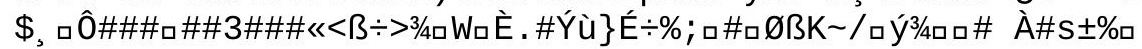

endstream

endobj

$19 \odot$ obj

$</$ Length 215

/Filter/FlateDecode

>stream

hPTP1nÃ@ 
Üõ

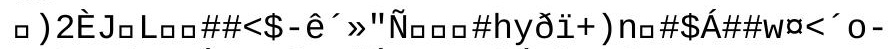

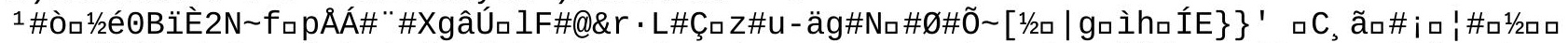

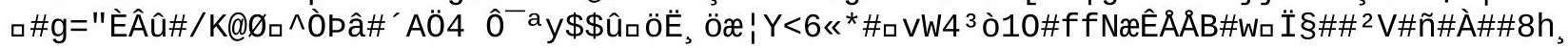

endstream

endobj

$20 \odot$ obj

$<</$ Differences $[49$

/one

]

/Type/Encoding

$>$

endobj

$21 \odot$ obj

$<</$ Length 6603

/Filter/FlateDecode

>stream

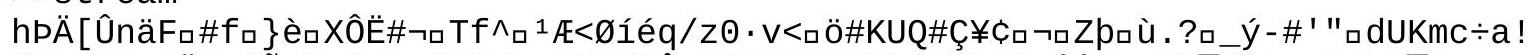

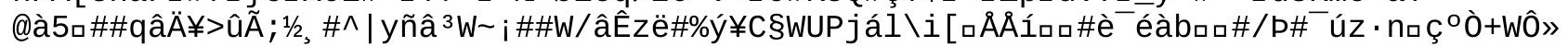
$\# u^{\circ}$

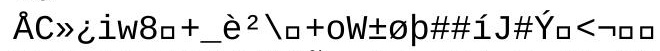

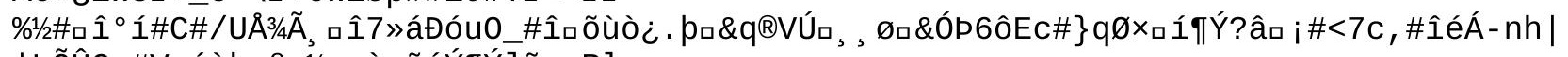

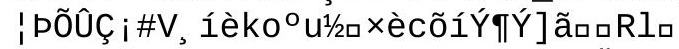

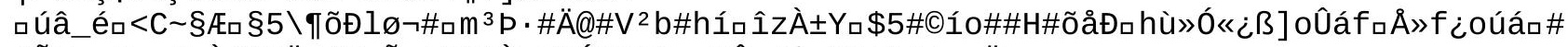

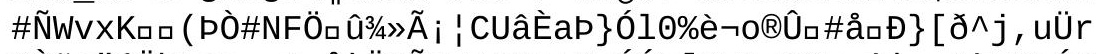

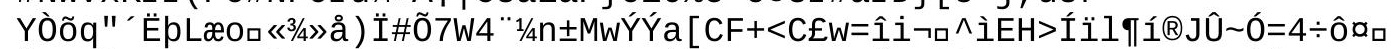

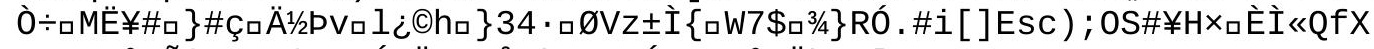

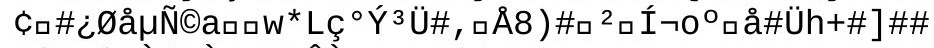

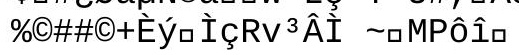




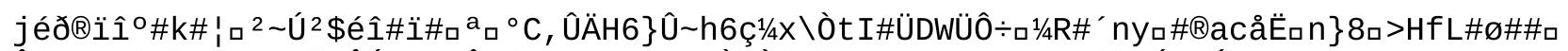

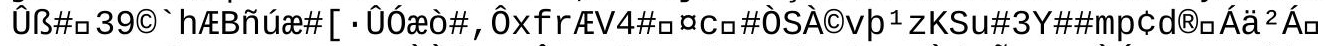

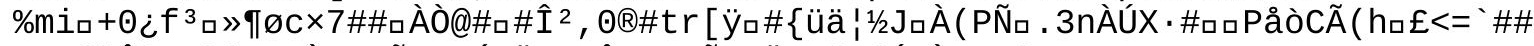

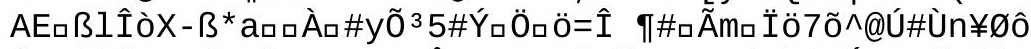

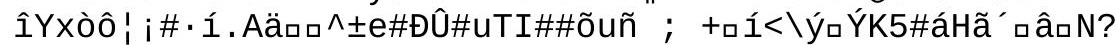

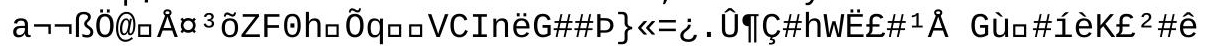

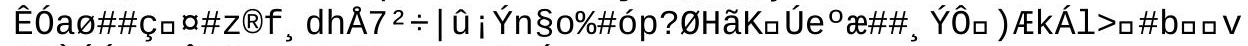

äGĖÓÁl\#ロÔ^å; ;æT»Îë»ロV=_ânó

Ta [\#IÖロ ¡UZロÓ

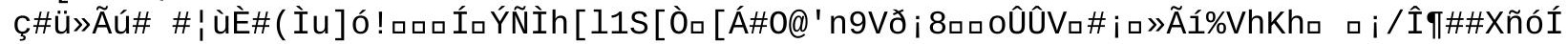




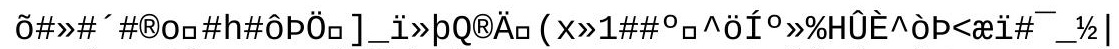

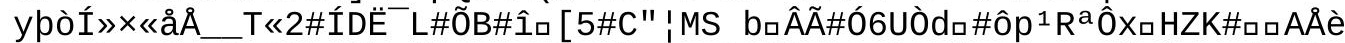

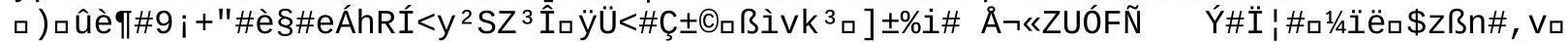

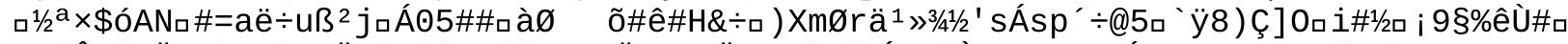

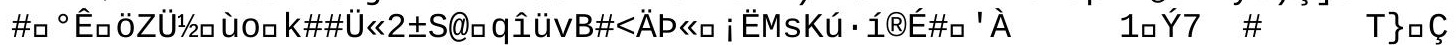

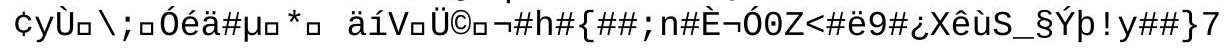




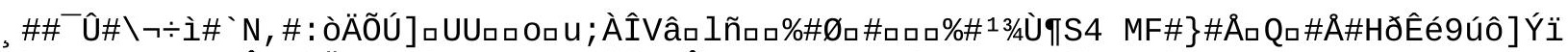

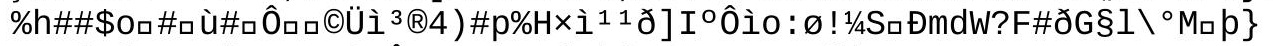

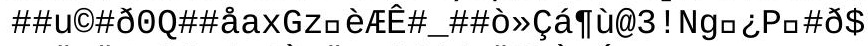

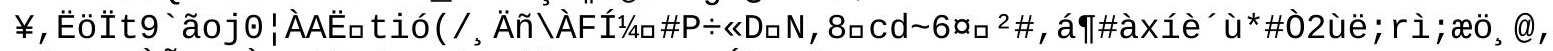

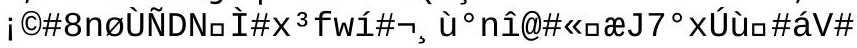




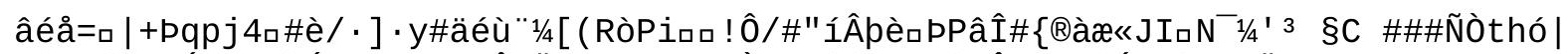

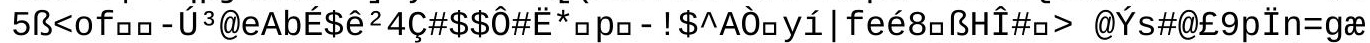

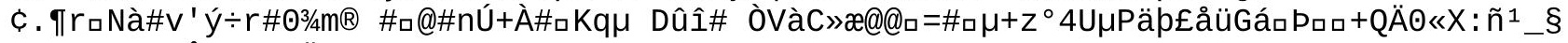

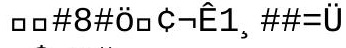

$\square$ \$ X\#ם

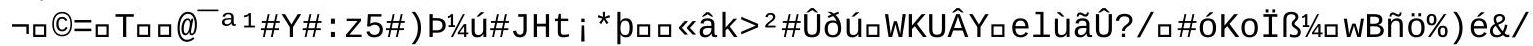

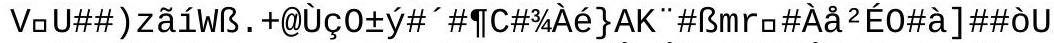

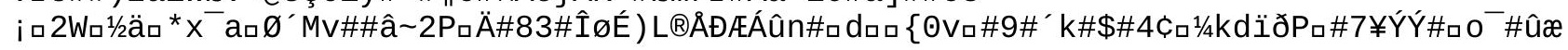




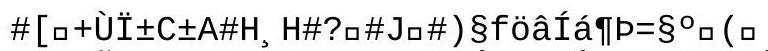

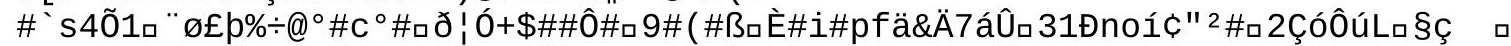

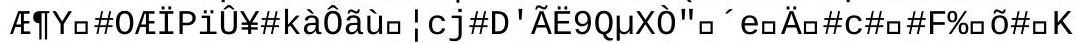

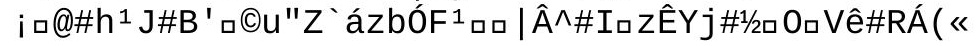




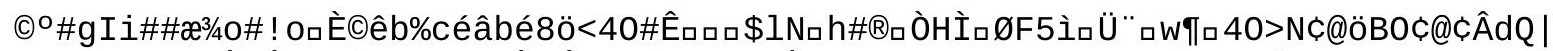

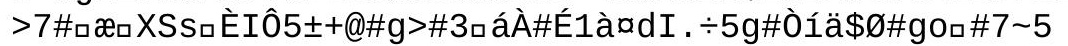
口уð口 " $\mathrm{v}^{\mathrm{A}} \mathrm{A} \%$ 


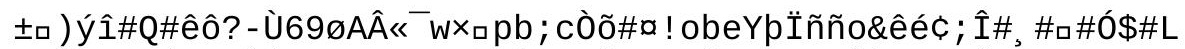

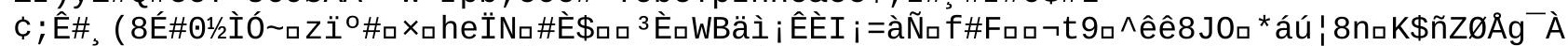

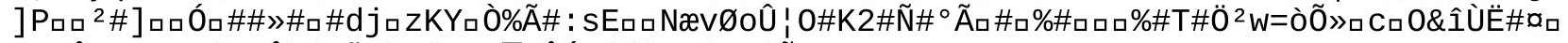

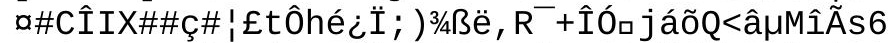

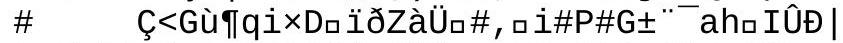

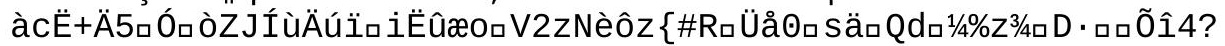

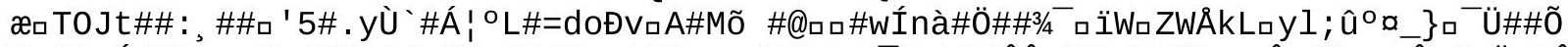

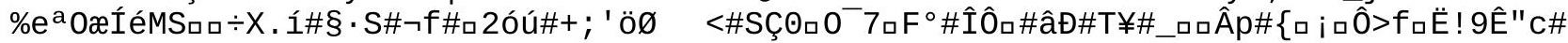

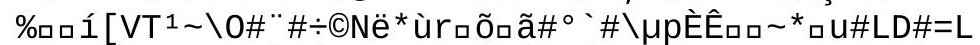

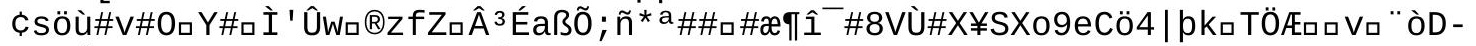

\&: fóäıX\#y' i\#\#ロU5Nı\#£âı\#Ú! 


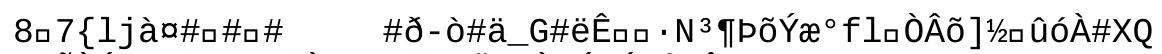

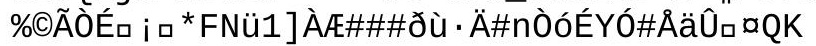




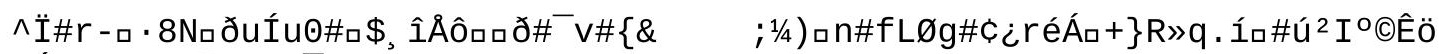
'Úı Ô\#1ロ\#N]\#\#0ø ÝQ?

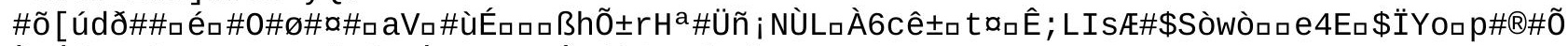

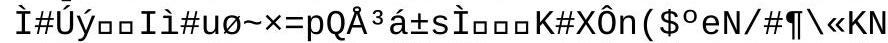

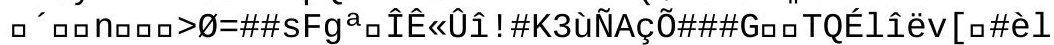

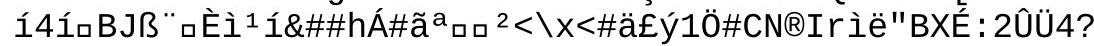

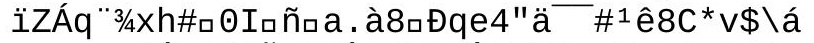

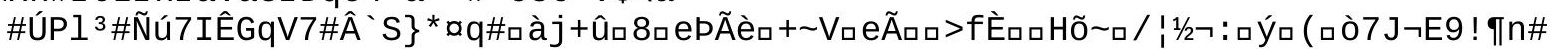

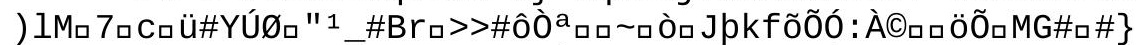

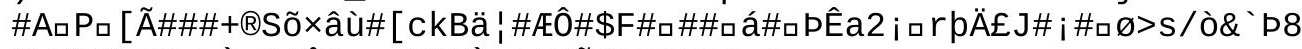

âFø6"S\#Æøhòw9ãÊ\#=ャp£ú\%Àa ' h\%õ\#)\#\#0\%\#ロ\# 


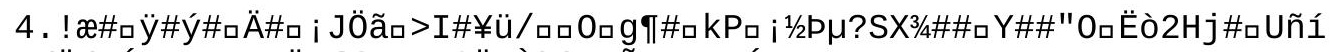

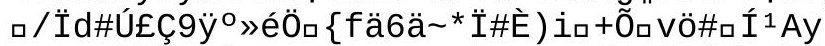

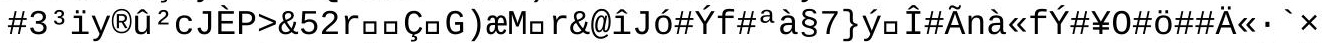

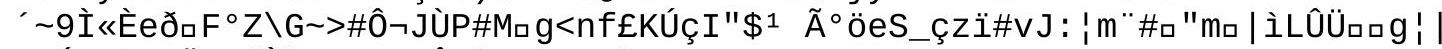

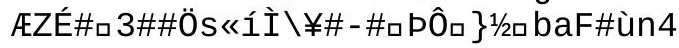




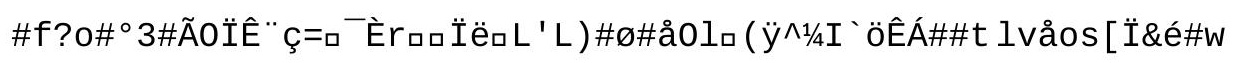




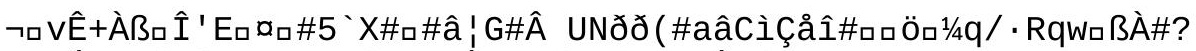

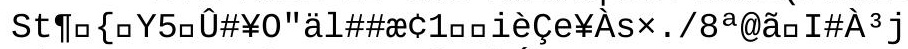

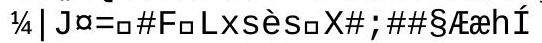




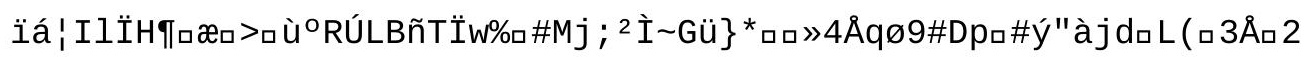




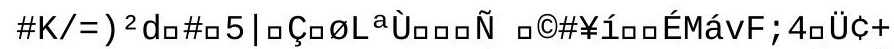

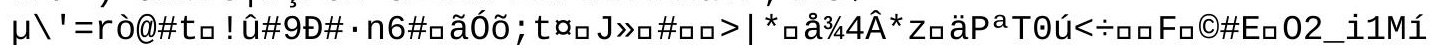

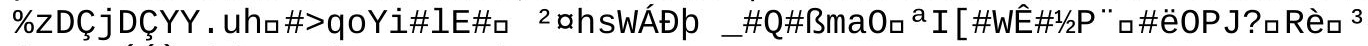

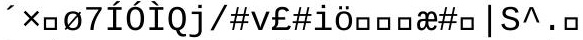

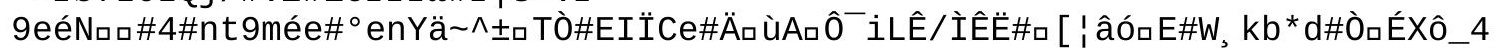

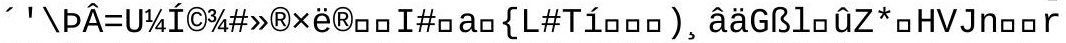

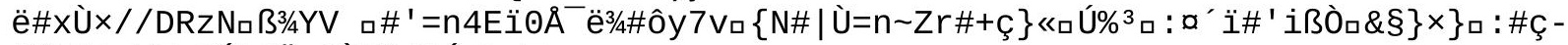

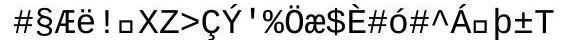

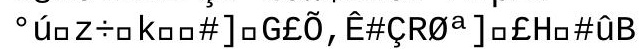




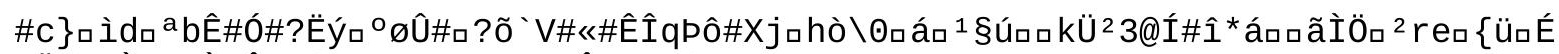

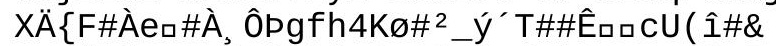

๑.MÚgð\#ZTyóQ] 
Øெ\#а̊

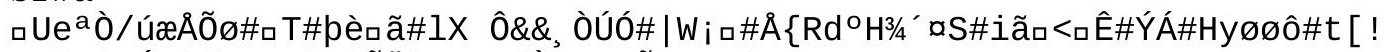

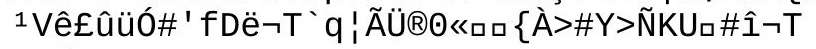

g? $\{$ eeP

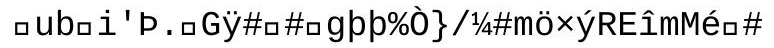




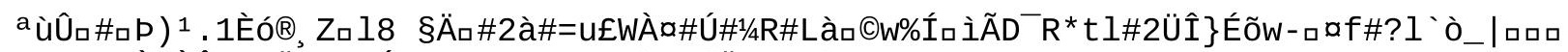

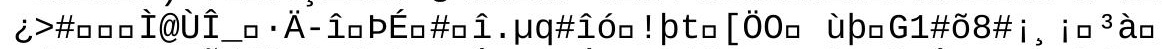

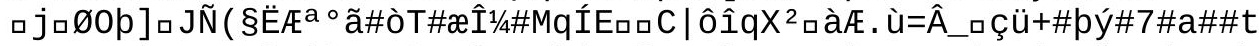

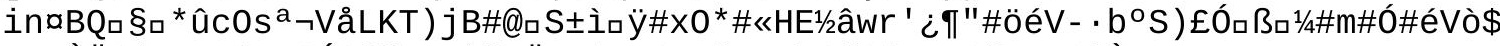

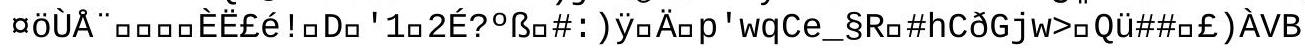




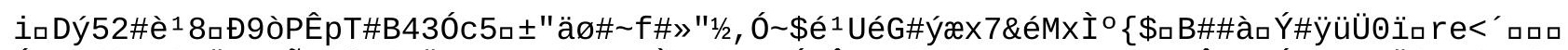

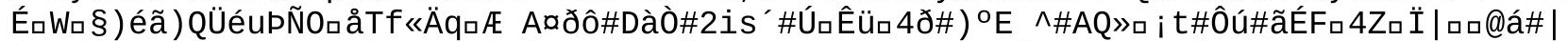

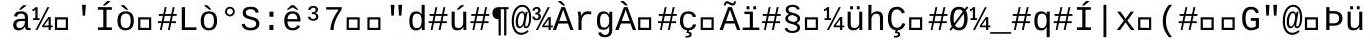

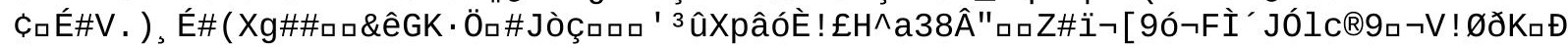


1/4>U«ÒVÿ $\{1 / 2 \#$ a

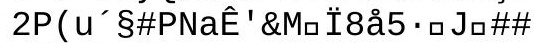

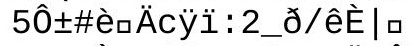

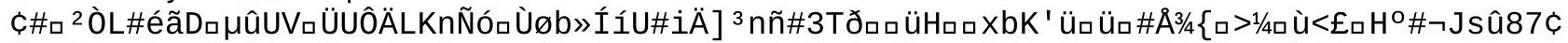

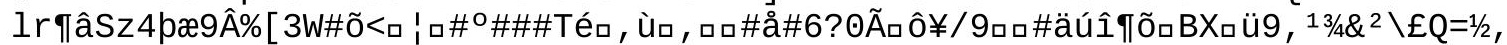

$\% \div$ ¿ËOérqã $)<\left[w^{1} / 2<\right.$ èaN\%« 
ÉÚ(

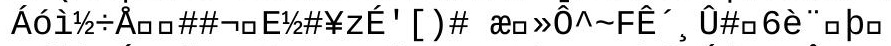

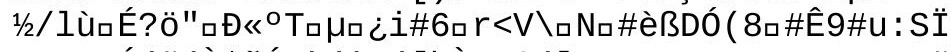

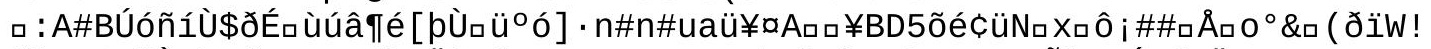

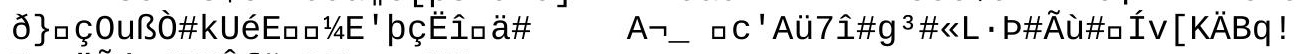

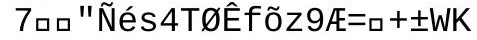

\#§\#UJ ${ }^{1}$ múQåôํำ ' u\#\#! \#Jáÿÿÿ@\#ロ , o\#

endstream

endobj

$22 \odot$ obj

$<<$

/Type/Page

/Resources $23 \odot \mathrm{R}$

/CropBox $\left[\begin{array}{llll}\odot & \odot & 595.276 & 782.362\end{array}\right]$

/MediaBox[[ $\left[\begin{array}{llll}0 & \odot & 595.276 & 782.362\end{array}\right]$

/Rotate $\odot$

/Parent $82 \odot \mathrm{R}$

/Contents $24 \odot \mathrm{R}$

/Thumb $72 \odot \mathrm{R}$

$>>$

endobj

$23 \odot$ obj

$<</$ Font $<</ F 1356 \odot \mathrm{R}$

/F15 $63 \odot \mathrm{R}$

/F4 $1010 \mathrm{R}$

/F5 $105 \odot \mathrm{R}$

/F6 $108 \odot \mathrm{R}$

/F8 $1140 \mathrm{R}$

/F9 $1170 \mathrm{R}$

/F10 $122 \odot \mathrm{R}$

$>>$

/ProcSet [/PDF

/Text

]

/ExtGState<//GS2 $125 \odot \mathrm{R}$

$>>$

$>>$

endobj

$24 \odot$ obj

$<</$ Length 5374

/Filter/FlateDecode

>stream

hD1/4[Ùn\#Éø\#føyðG 


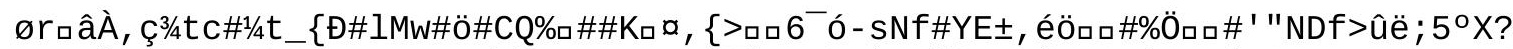

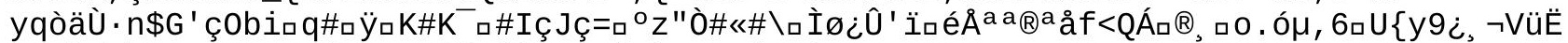

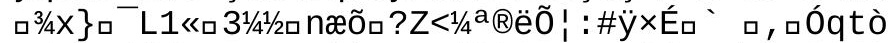

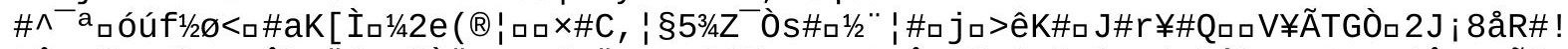

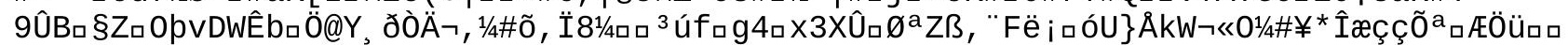

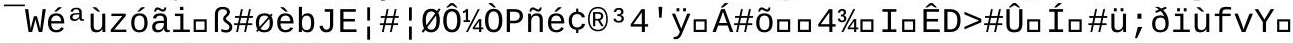

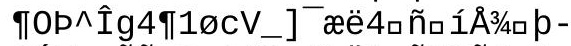

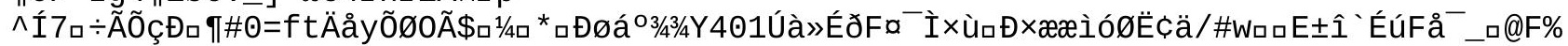
\#3/4 


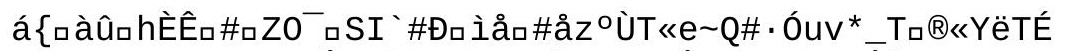

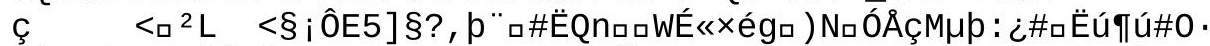

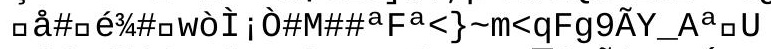

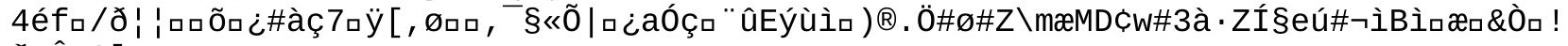

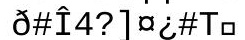

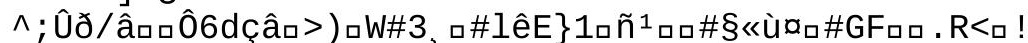

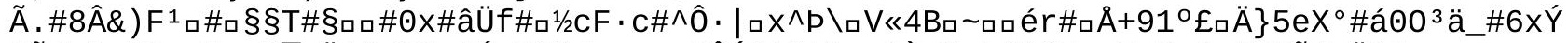

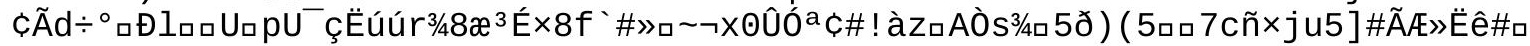

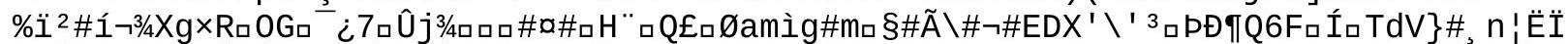

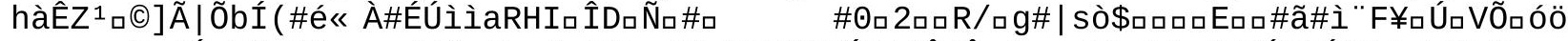

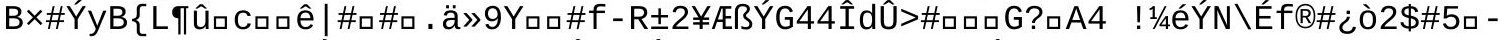

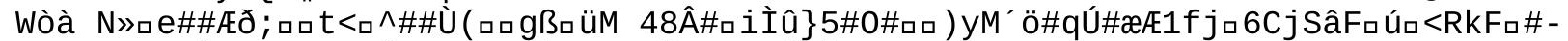

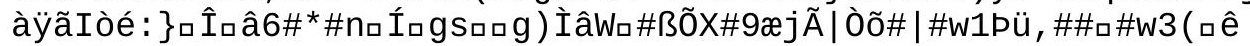




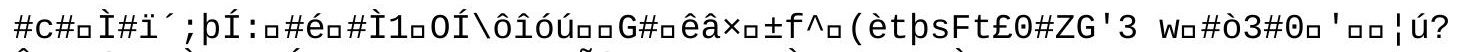

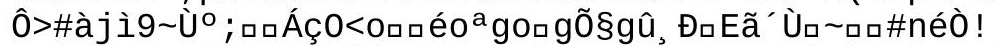

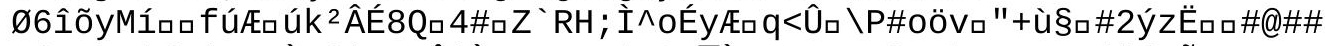

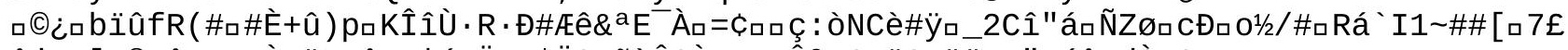

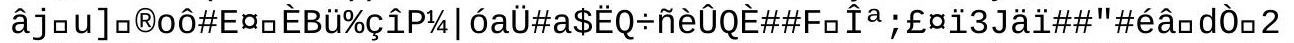

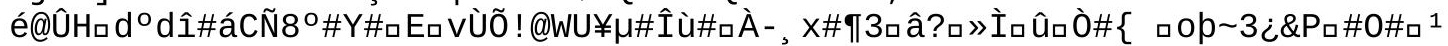

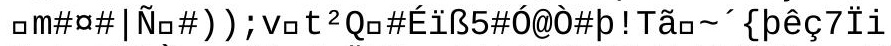
WH\%\#F .

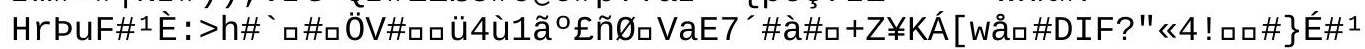

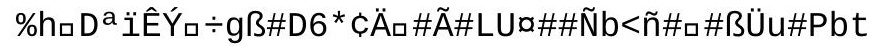




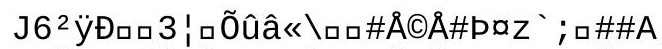

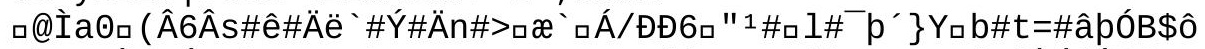

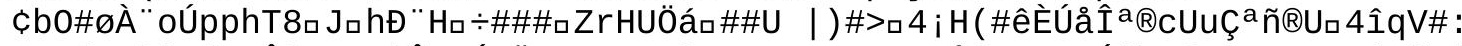

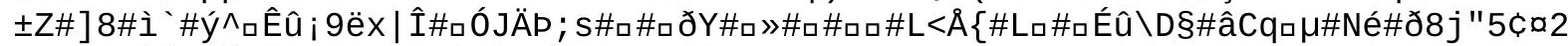

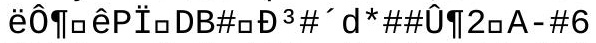

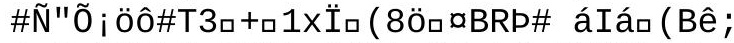

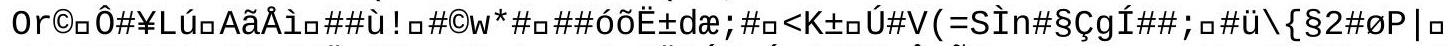

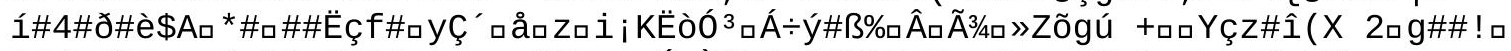

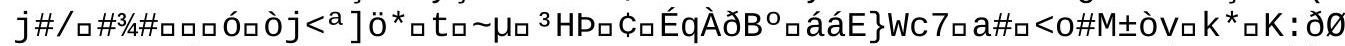

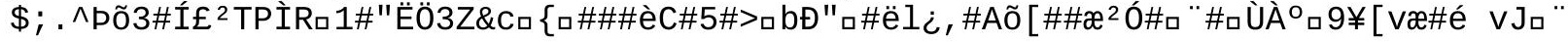




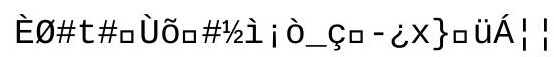


Ê?Ùøロ âwp\#Bi i »

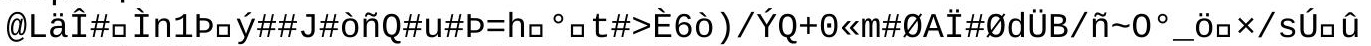

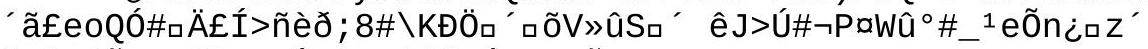

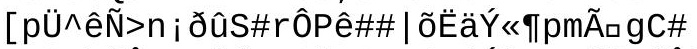

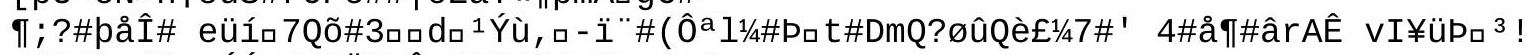

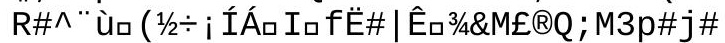

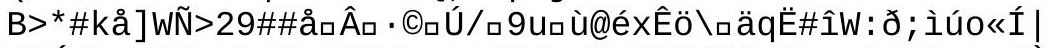

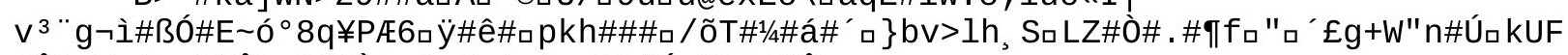

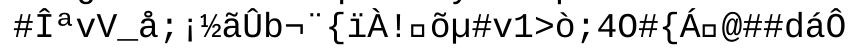




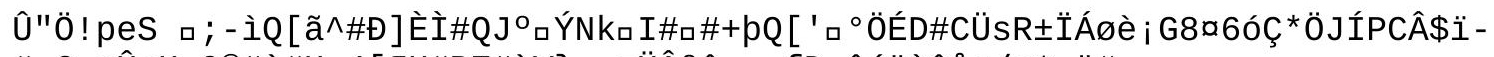

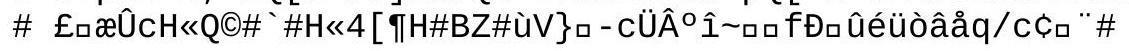




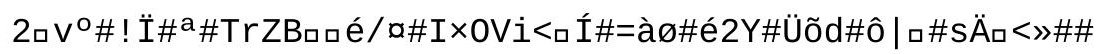




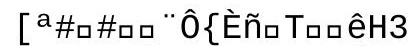

\#æ0\#“\#@\}y/aïê i \#Q\#3/4Lå»\#Ù ¿\#\#h\#3ロÛqu SaıİÜ`hjh\#\#å äÜa\#

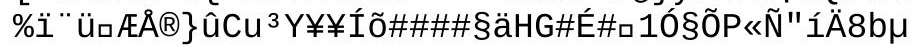




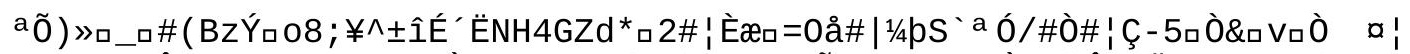

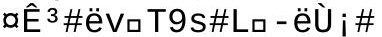

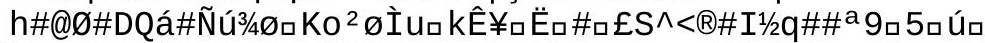

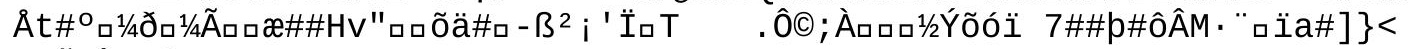
$1 / 4 \# 0 ̃$ À巛\#ठ

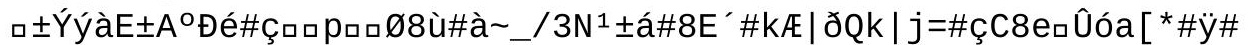

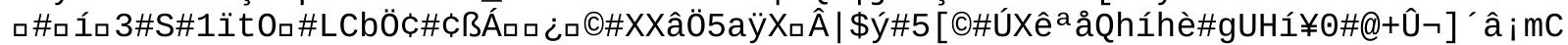

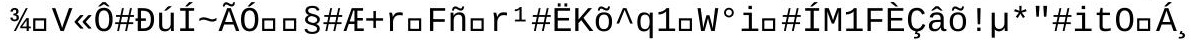

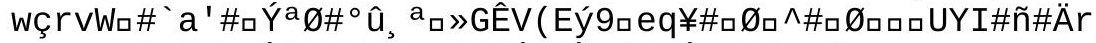

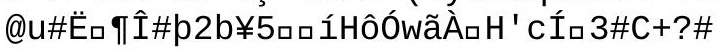




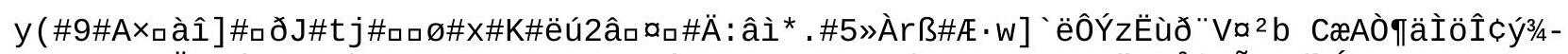

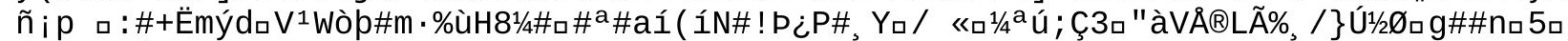
fWE $\tilde{A}$

ÜåİËÊÆÉÉ ; k4

口EÅGÑ \#Û

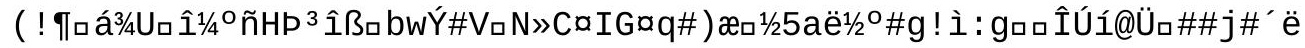




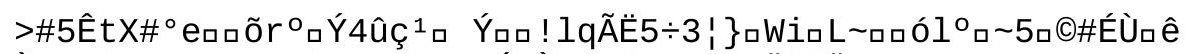

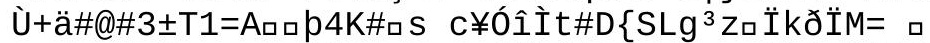

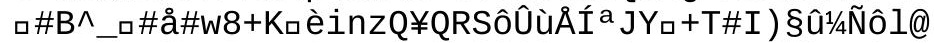


$\mathrm{mu \# \# H \{ b}$ 


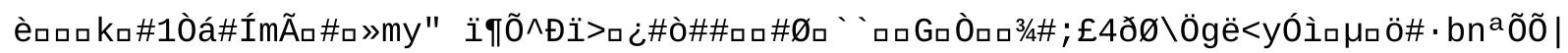
9M4\#\#J\# 


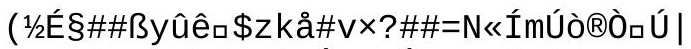

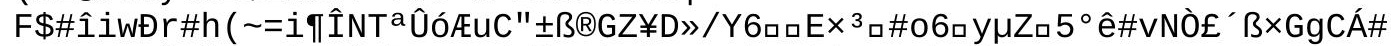

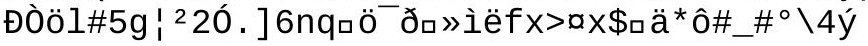




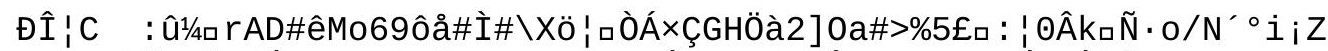

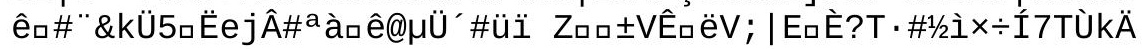




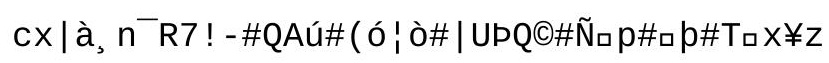


$<$ è f fề"

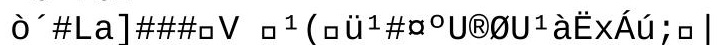

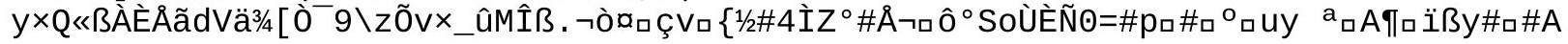
$\mathrm{SK}_{\square} \mathrm{X}$ 


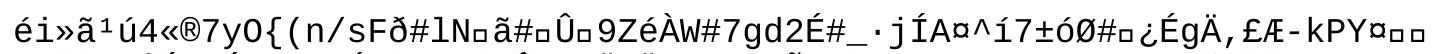

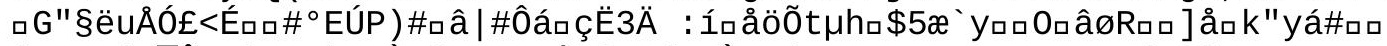

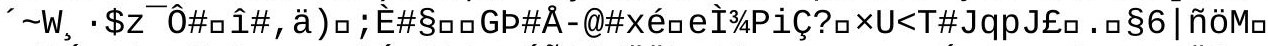

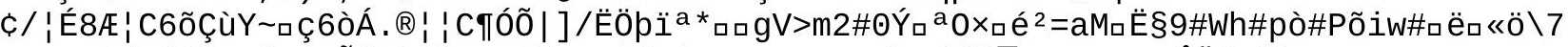

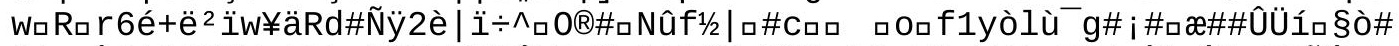

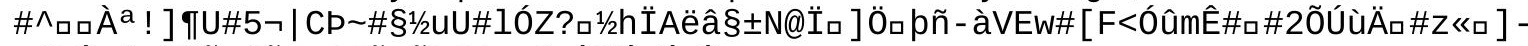

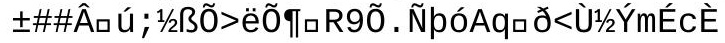




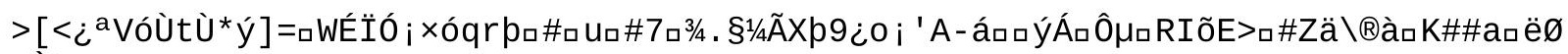
çúk\&âarax 
$\left.I C \hat{I}^{1} \mathrm{èE}\right\}^{3}{ }^{\circ} \mathrm{H} \mid \mathrm{I} \pm a b \quad>\mathrm{I}^{3 / 4}$ 


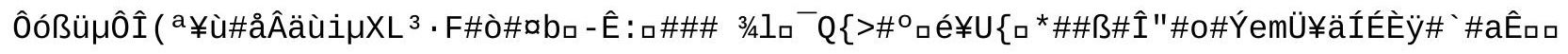
endstream

endobj

$25 \odot$ obj

$<<$

/Type/Page

/Resources $26 \odot \mathrm{R}$

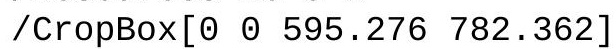

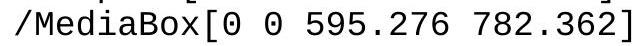

/Rotate 0

/Parent $82 \odot \mathrm{R}$

/Contents $29 \odot \mathrm{R}$

/Thumb $73 \odot \mathrm{R}$

$>$

endobj

$26 \odot \mathrm{obj}$

$<</$ XObject $<</$ Im2 $27 \odot \mathrm{R}$

/Im3 $28 \odot \mathrm{R}$

$>$

/Font $<</ F 4101 \odot \mathrm{R}$

/F5 $105 \odot \mathrm{R}$

/F6 108 ○ R

/F9 $1170 \mathrm{R}$

$/ \mathrm{F} 10122 \odot \mathrm{R}$

$>$

/ProcSet [/PDF

/Text

/Imagec

]

/ExtGState $<</ G S 2125 \odot \mathrm{R}$

$>>$

$>>$

endobj

$27 \odot$ obj

$<</$ Subtype/Image

/Length 97800

/Filter/FlateDecode

/BitsPerComponent 8

/ColorSpace/DeviceRGB

/Width 520

/Height 475

/Type/XObject

>sstream

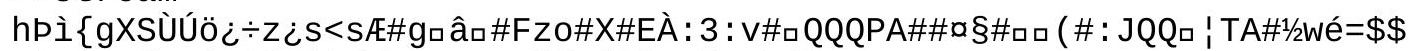

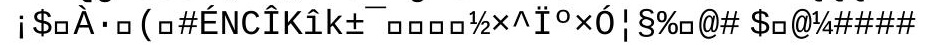

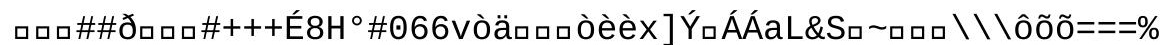

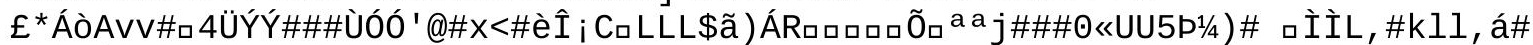
\#\#)lÝj $T \backslash \backslash n b r f \div \hat{1} \_0 \square 8 ; 4 D a ̂ \cdot \square \square \square$

// ó§O\#? \2a\#,\#XZ^uvv)))ฉロú

|TQQËÊÊE\#,7\$\#

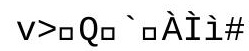




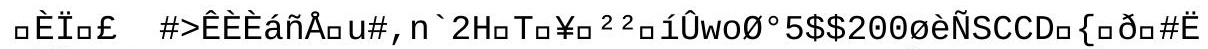

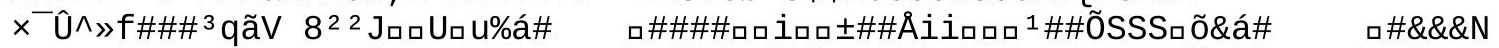

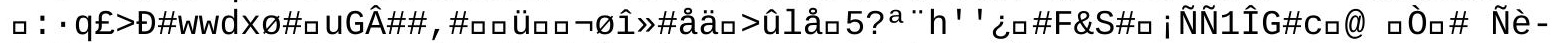

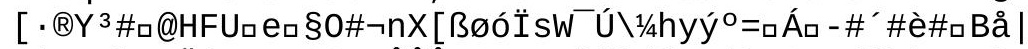

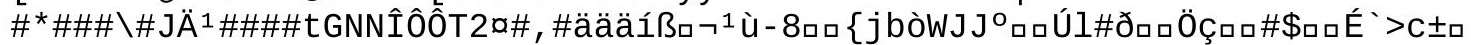

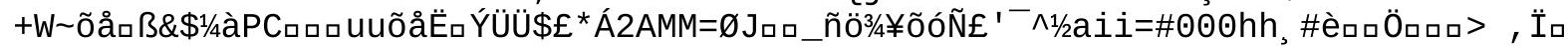

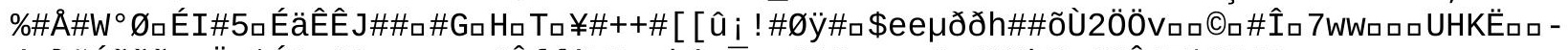

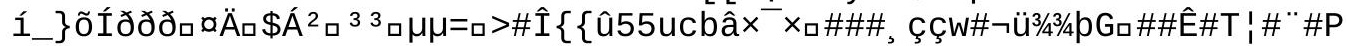

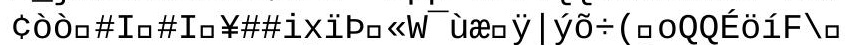

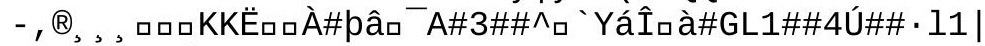

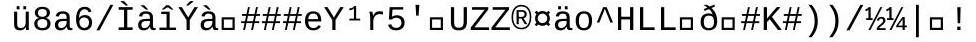




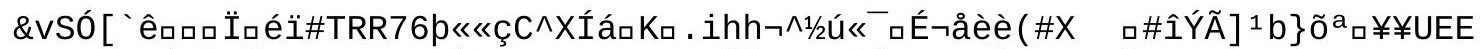

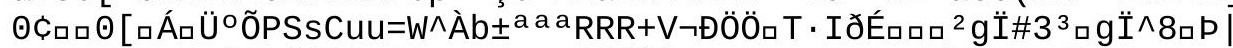

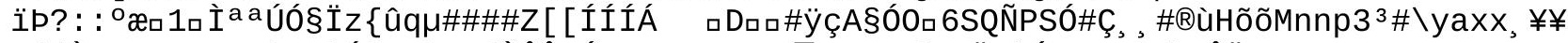

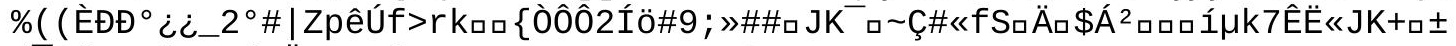

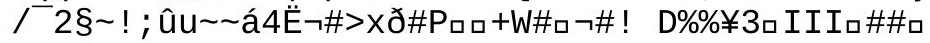




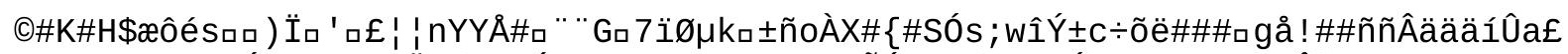

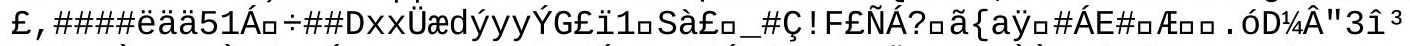

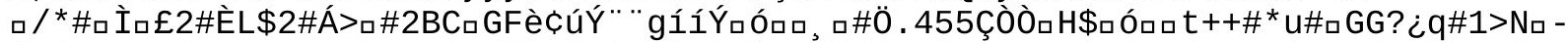

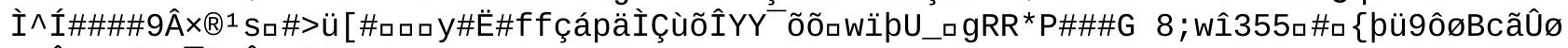

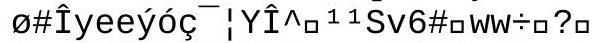

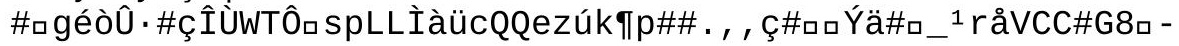




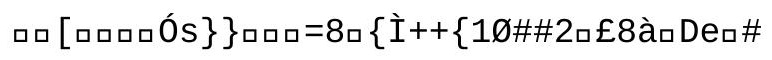




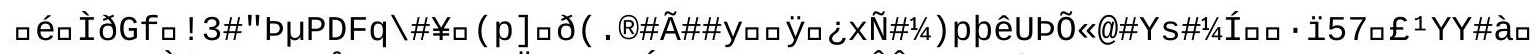

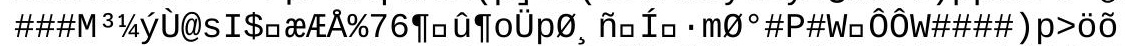

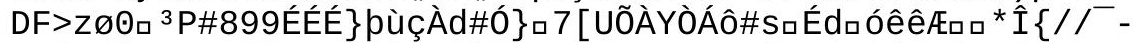

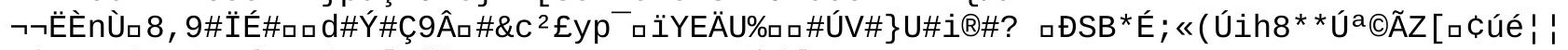

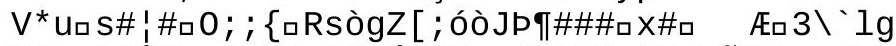

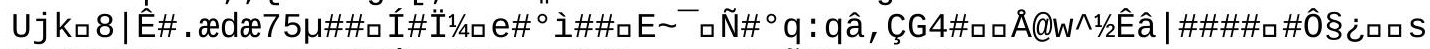

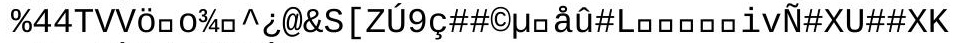

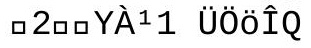


3/4íììn / 


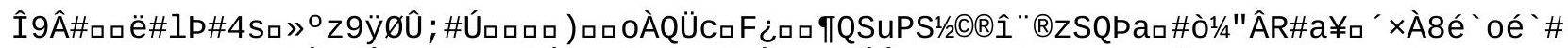

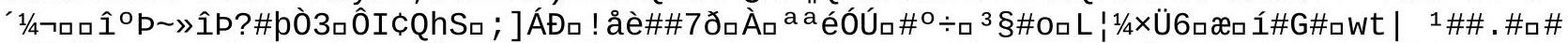

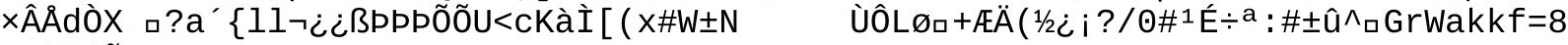

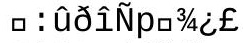

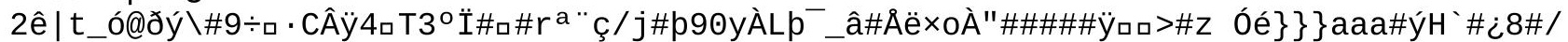

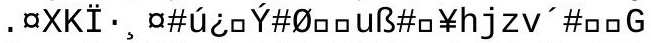

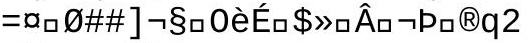

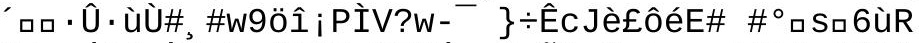

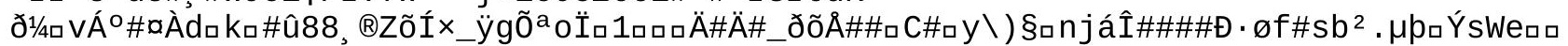

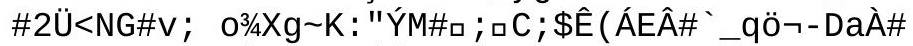

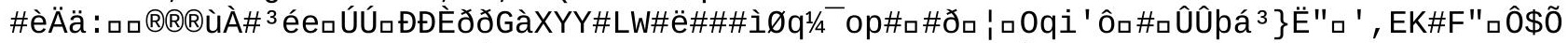

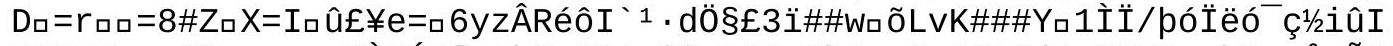

口

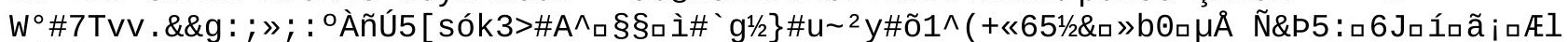

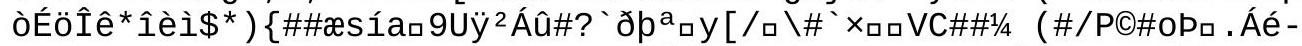

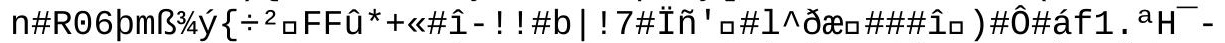

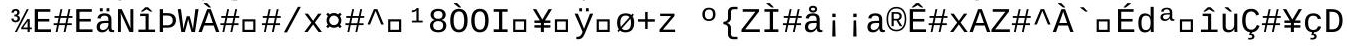


qםU ${ }^{3}{ }^{3}$ S\#èêæÍロ?pøã

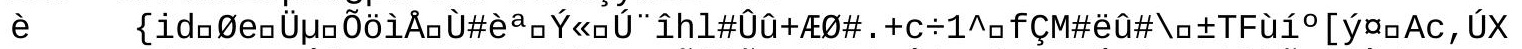

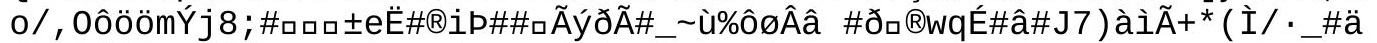

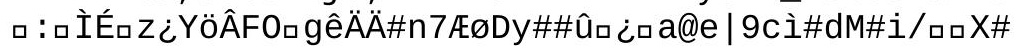




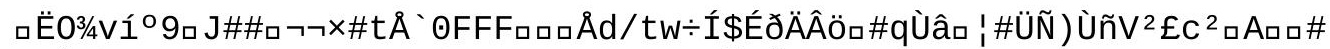

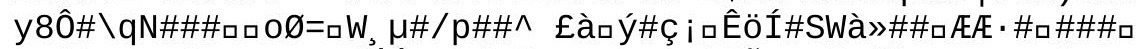

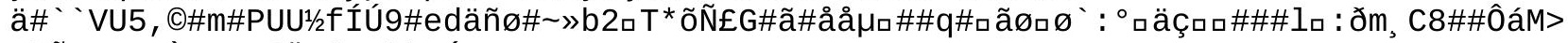

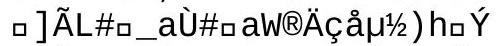

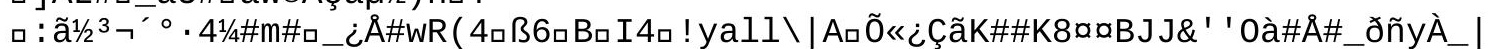

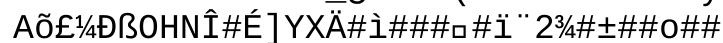




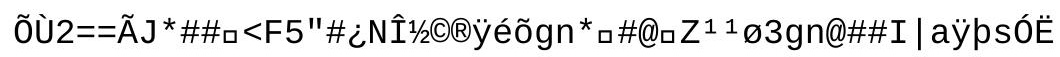




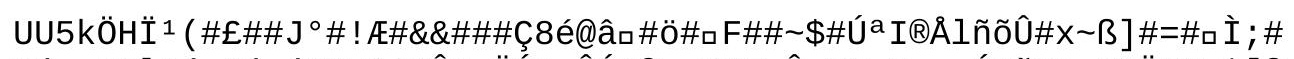

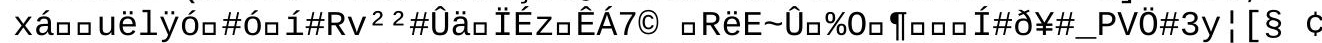

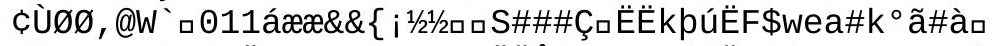

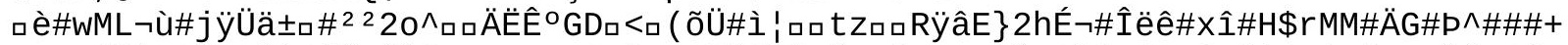

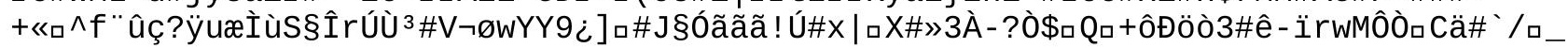




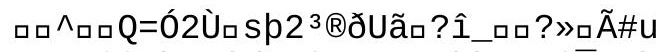

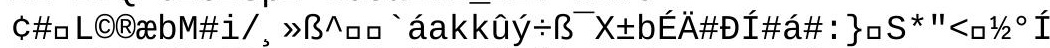

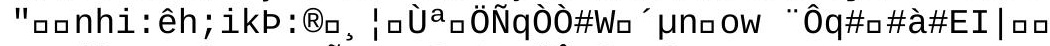

4ๆİM\#\#k\#3ÇRàà\#/\#ðäı

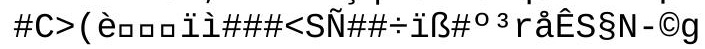


áÄ॰ùá\#Àq] ]\}"tqa 


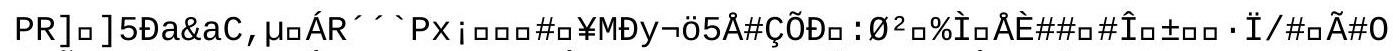

\#\#\#1ั̀\#-

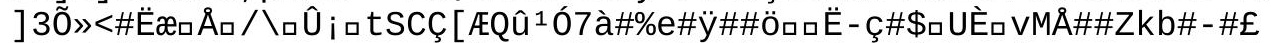

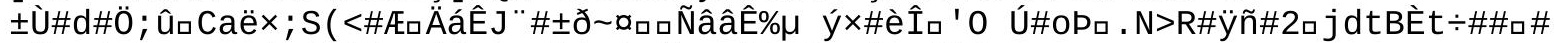

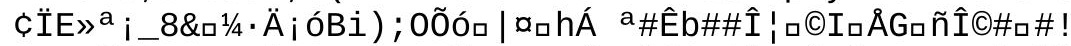

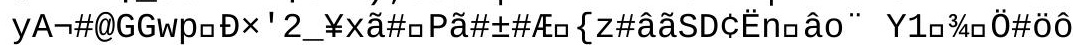




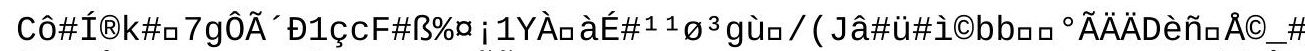

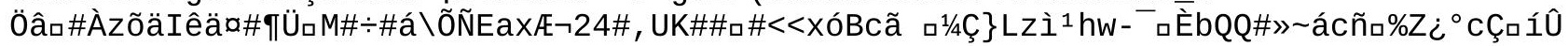


口\#\#/\# 


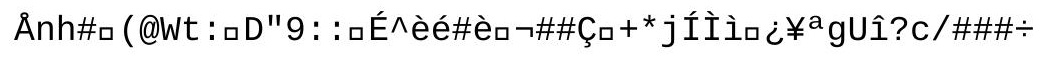




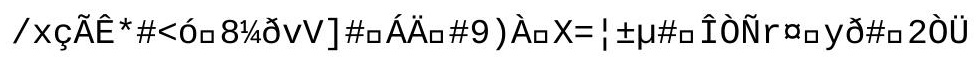




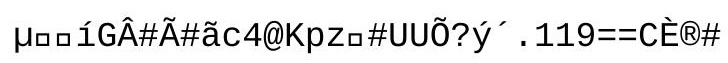




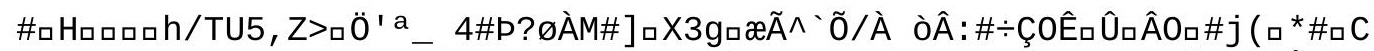

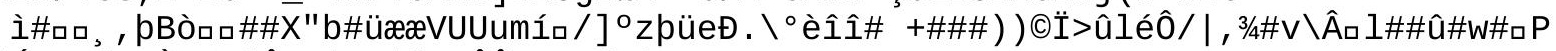

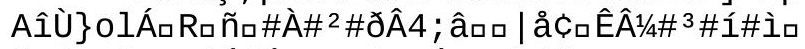

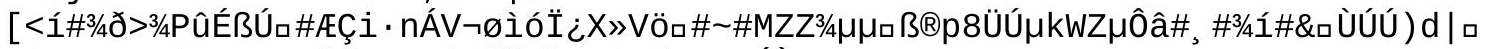

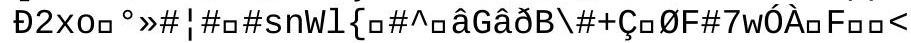




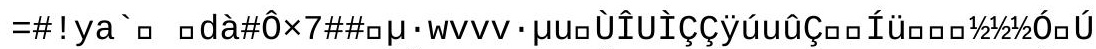

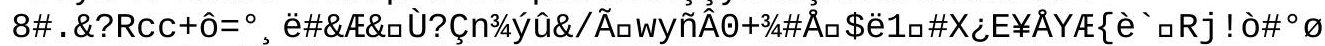

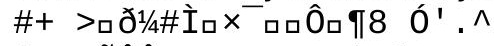

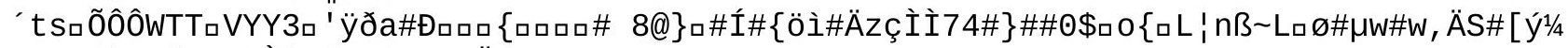

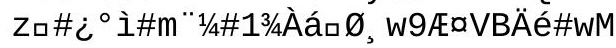


${ }^{\circ}$ \#at (

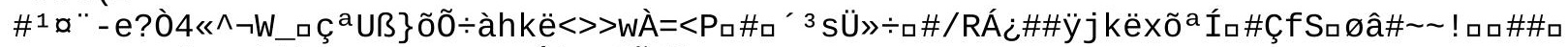

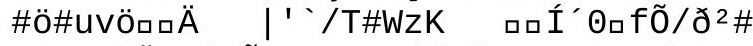

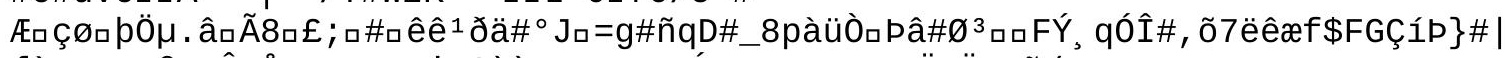

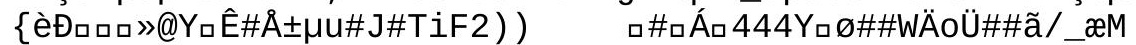

○ם]Đ3Ûg “-

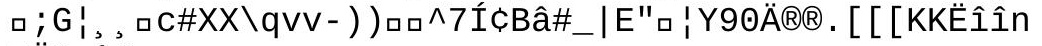

$E \ddot{A}^{1} \mu{ }^{\prime} \#$ 


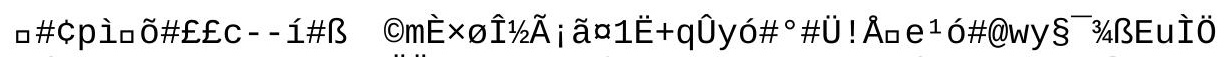

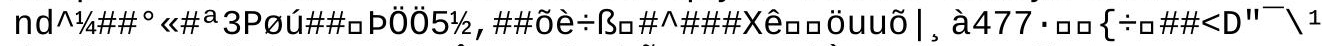

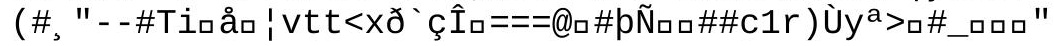




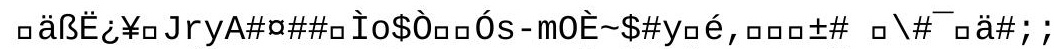

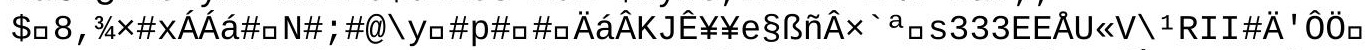

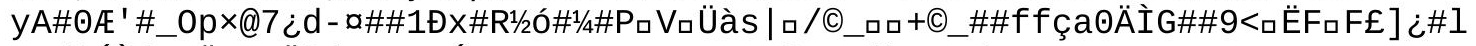

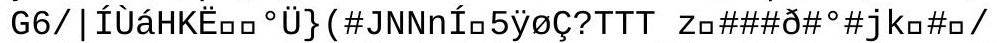

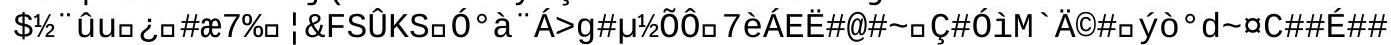

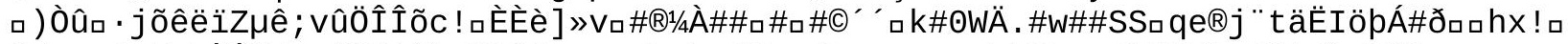

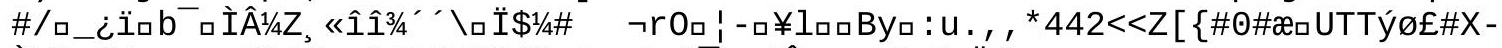

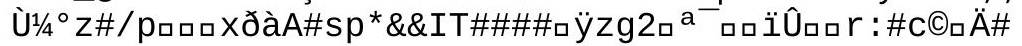

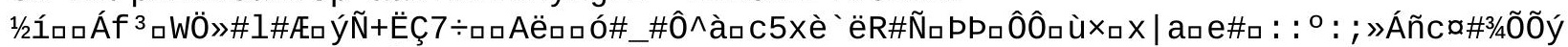

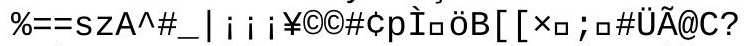

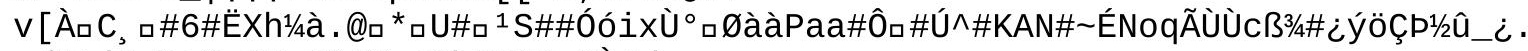

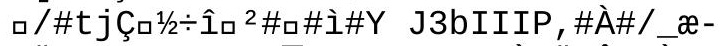

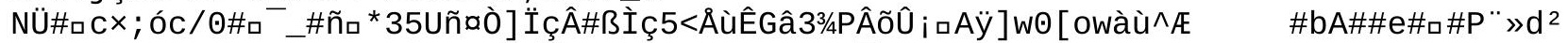

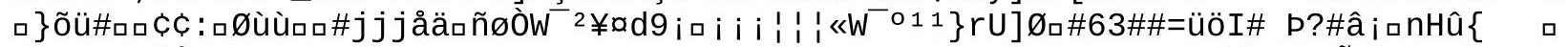

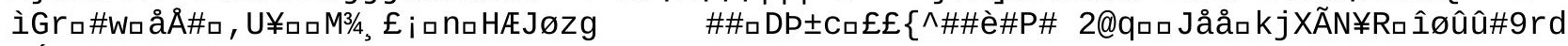

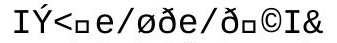

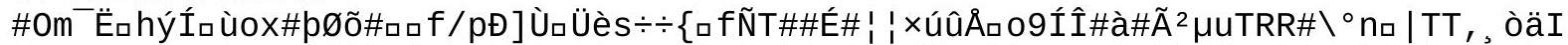

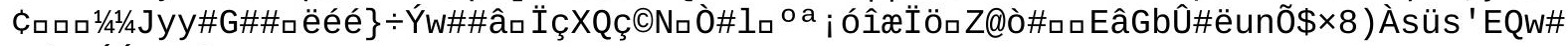
๑/ \#ÁÁan ${ }^{a}$

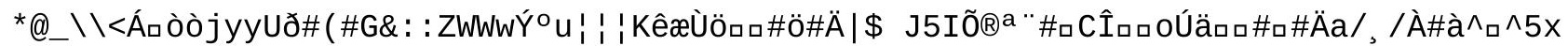

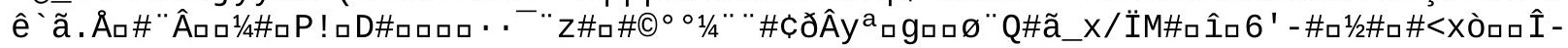

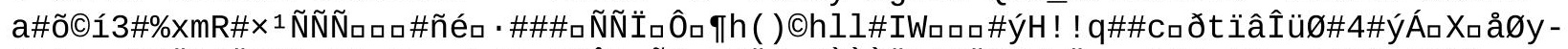

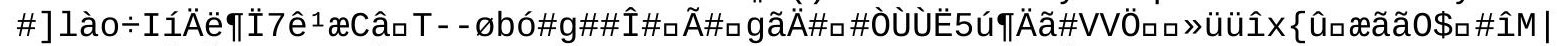

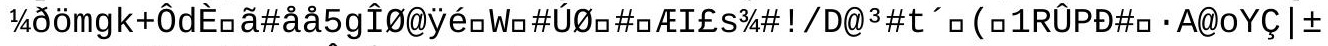
i i áòr " ë1/4ðy ${ }^{a} T e ̂\left(\times E \hat{r} a ̊ \# 3^{3} \# p, h\right.$ 


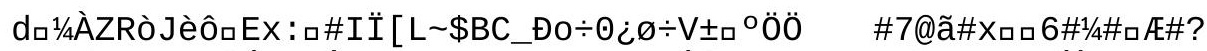

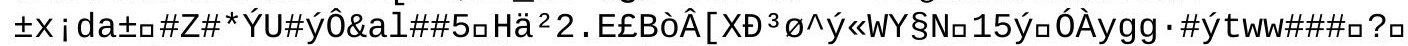

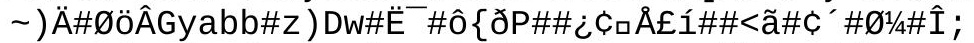

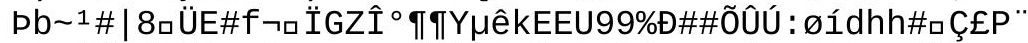

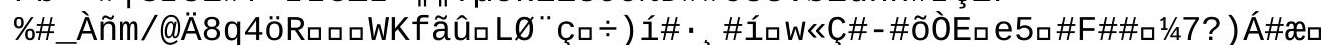

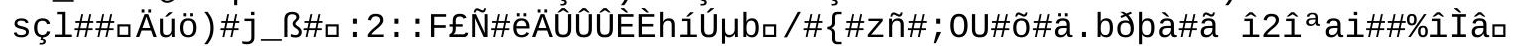

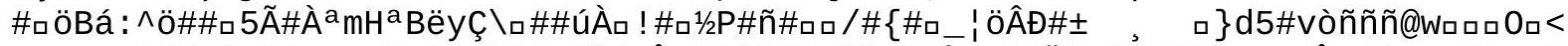

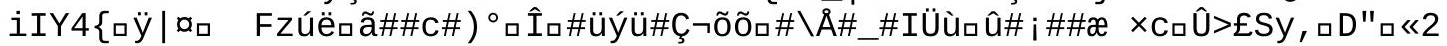

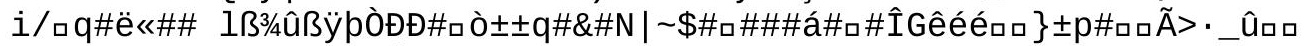

'WG_ú\#ÄÇ/ $/ \times \#$ =

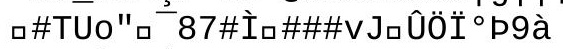

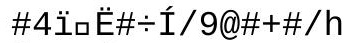

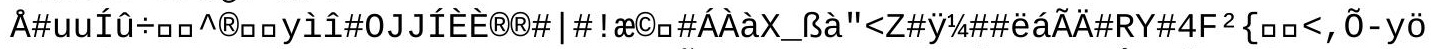

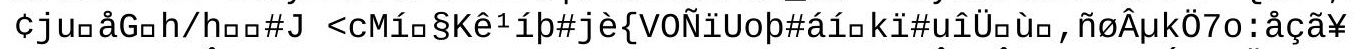

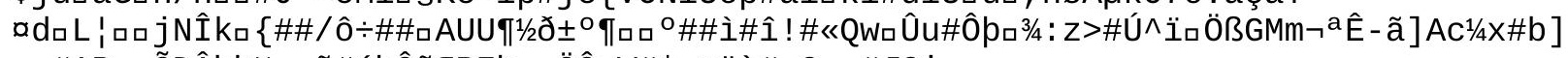

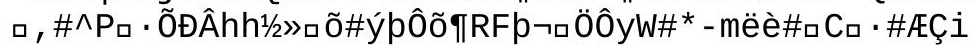

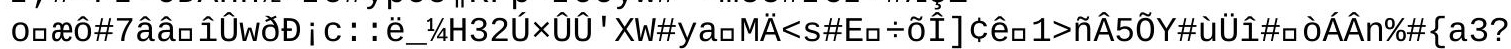

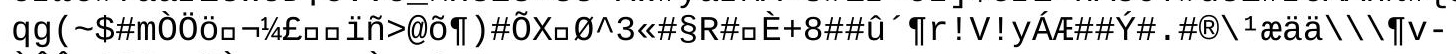

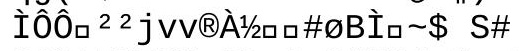

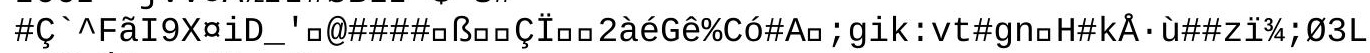

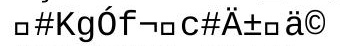

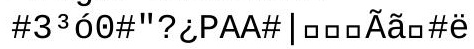

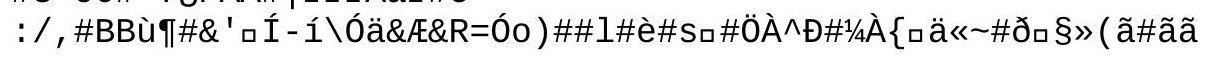




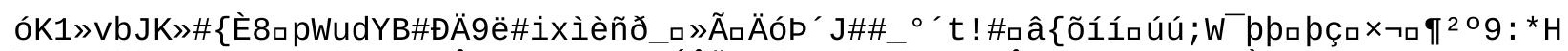

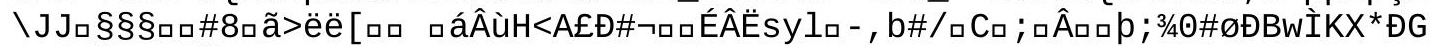
口3/4i3Ếp>\#âÃ

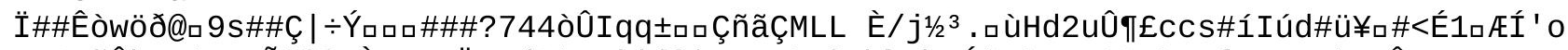

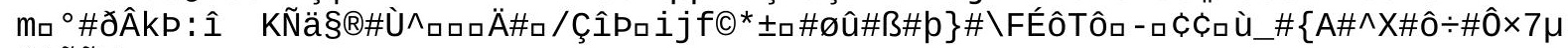

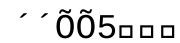
ÖIzz: : 


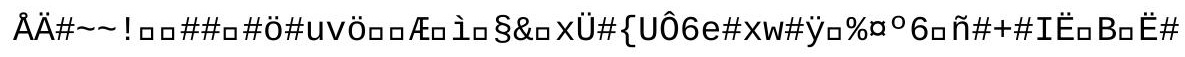




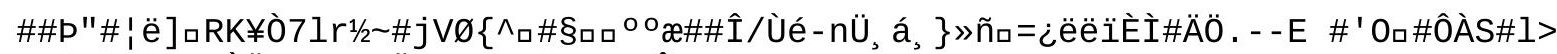

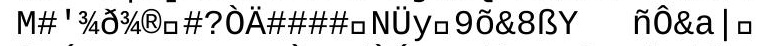

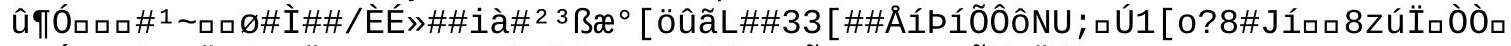

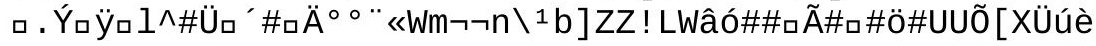




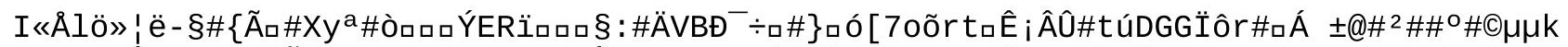

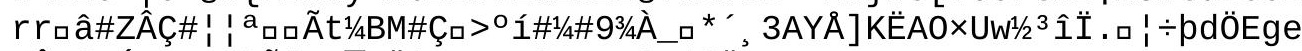

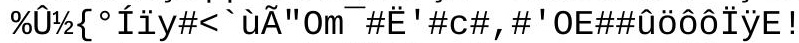

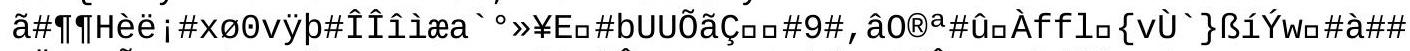

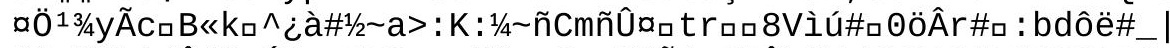

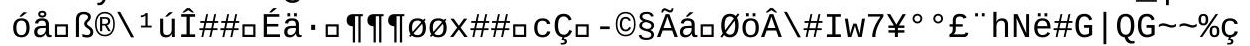

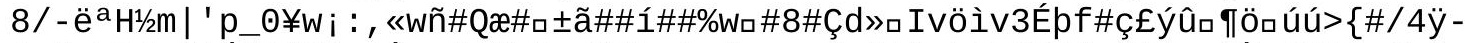

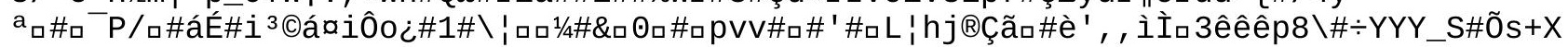

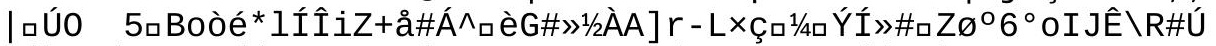

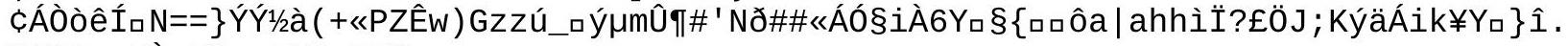

\%1́3/4òr - RÒıRàa, LC\#yM\#ã -

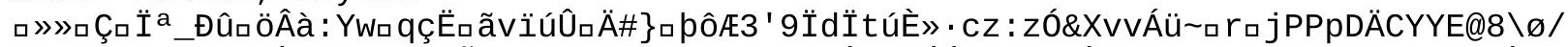

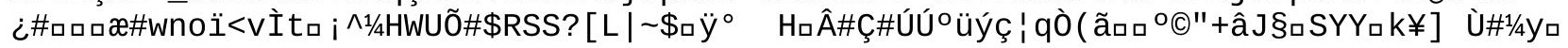




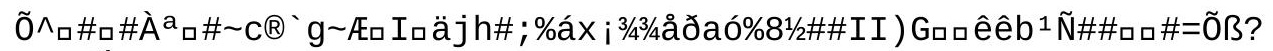

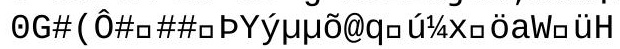




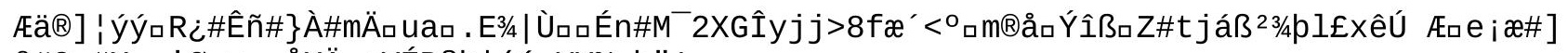

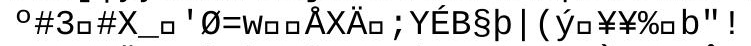

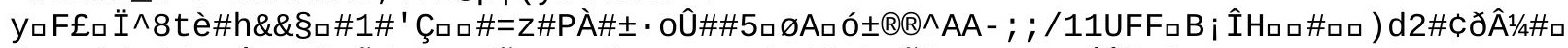

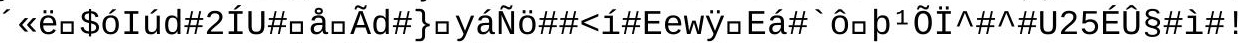

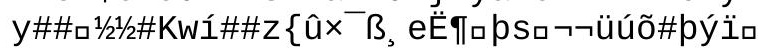

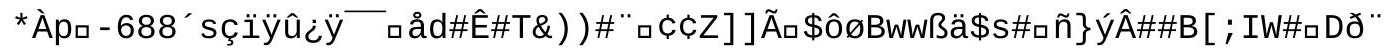




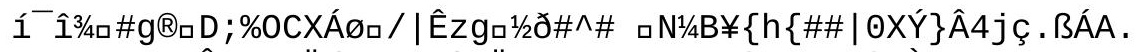

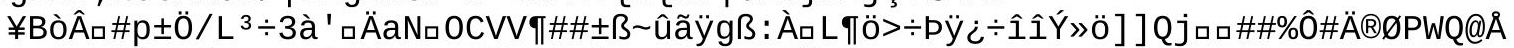

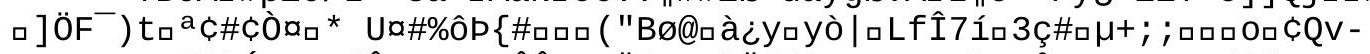

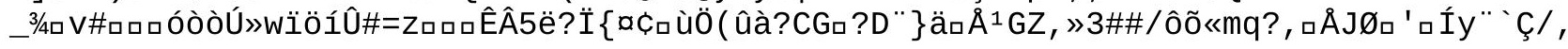


d2\#ìíñ̃I@@\#ロם)

* * úäÉ\#\#ロ ${ }^{\circ}$ DPÑ̃̈Ñ

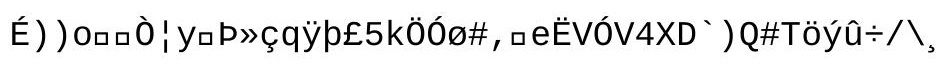


$\sim{ }^{\mathrm{a}} 4 \wedge^{\circ} \boldsymbol{\eta}$

ฉñ 


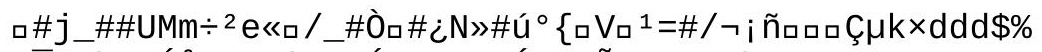

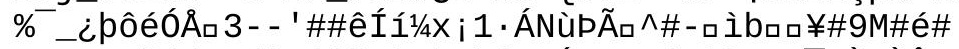

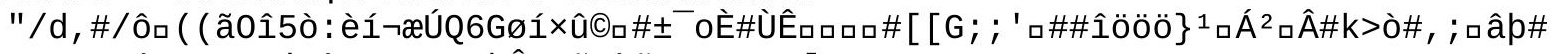

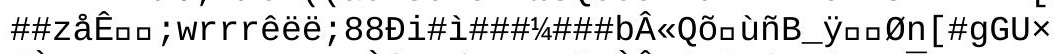

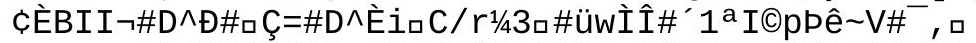

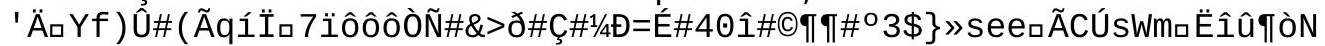

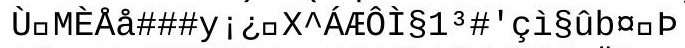

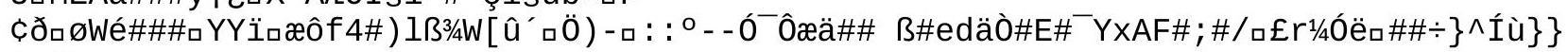
\$口\#

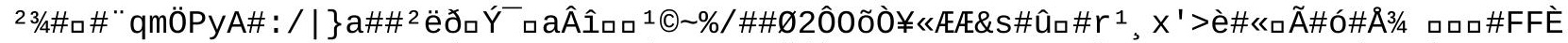

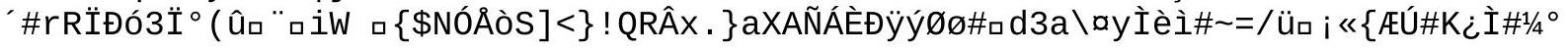

,

/ðャ¥4Y 


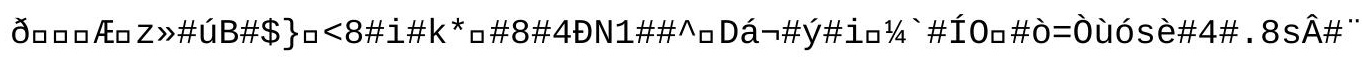




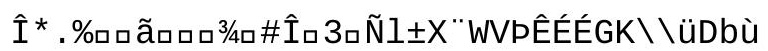




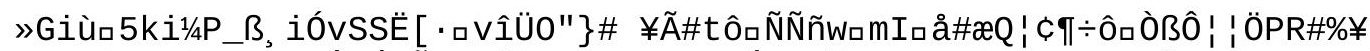

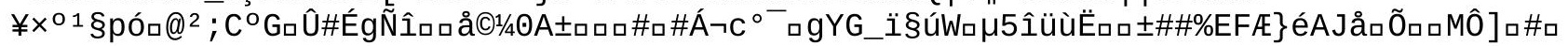




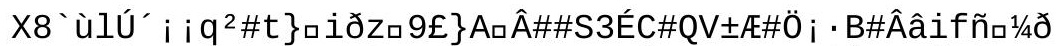

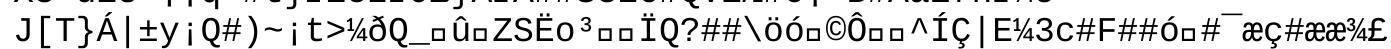

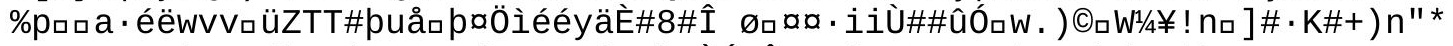

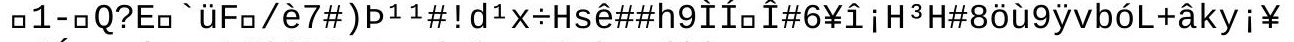

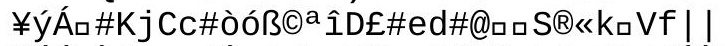

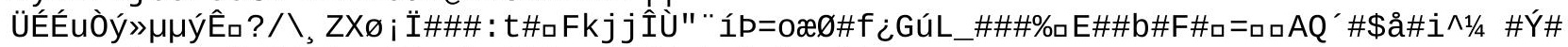

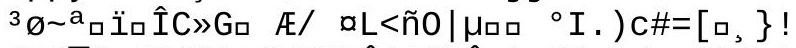

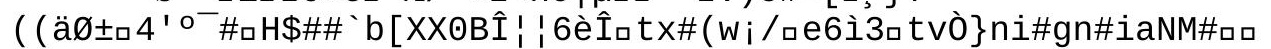

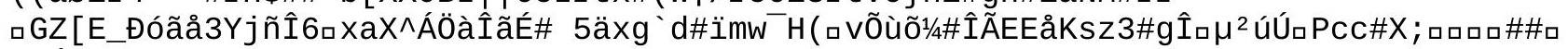

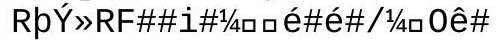


7ロ ○\#Æロ Ý

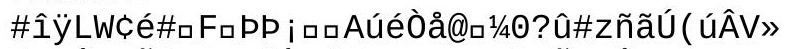

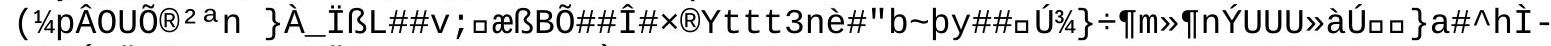

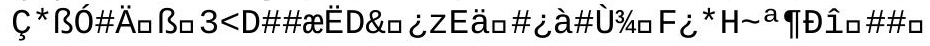




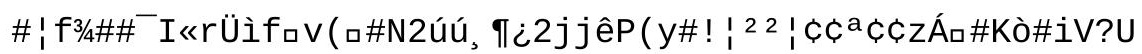




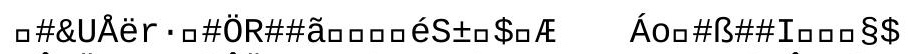

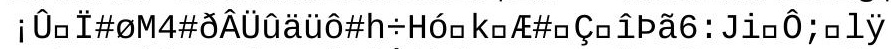

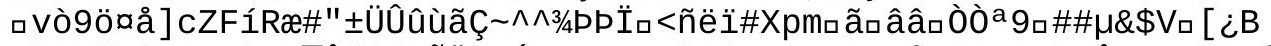

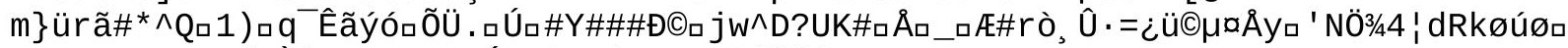

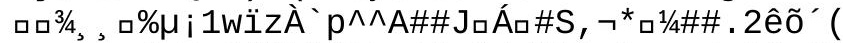

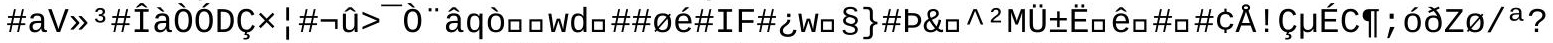

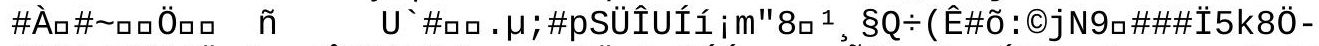

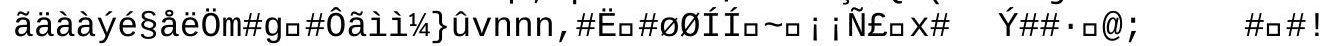




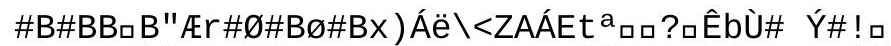

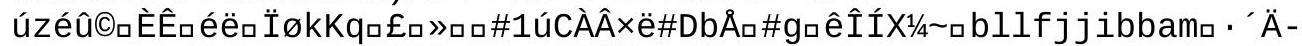

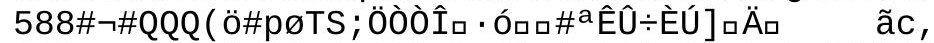

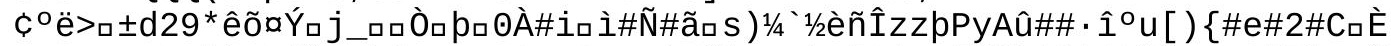

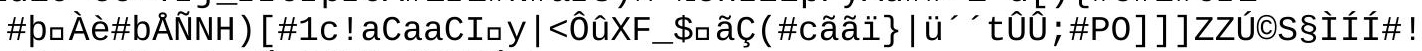
gWWOWW/ý2mm\#a * $\AA_{\square} \mathrm{xÝ} \div \mathrm{J} \# \#^{3}$ q $\{$ URVÚT

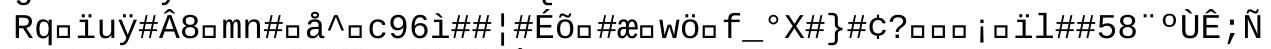

'-. / $\#$ \#bCCóÄ_\#IIC口a@ET'。À

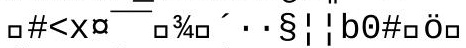

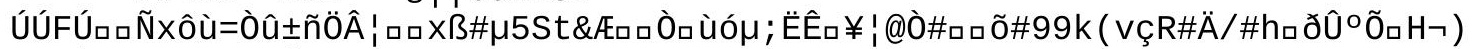
$¥ m D 0 ̂ D, k$ a $"$ $\oslash X F \tilde{A} ¥ \# t D \tilde{n} \#$

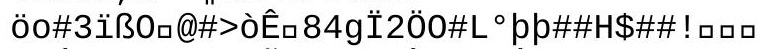

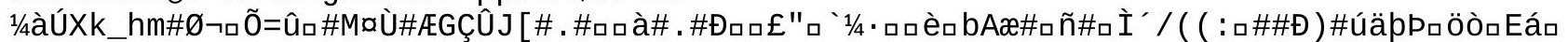

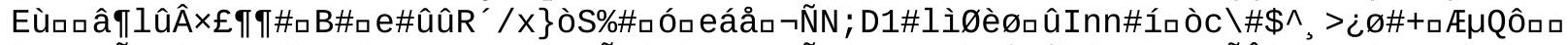

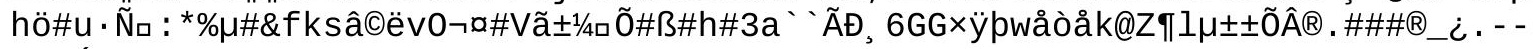
mmmÍ\#9óòø\#

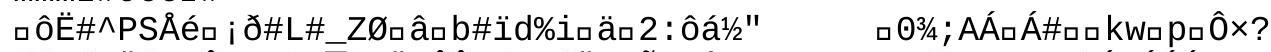

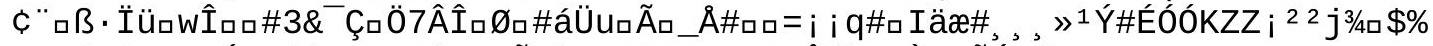

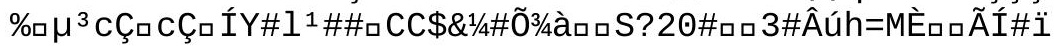




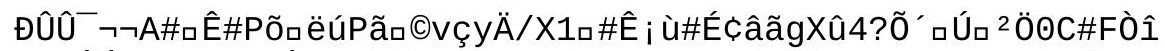

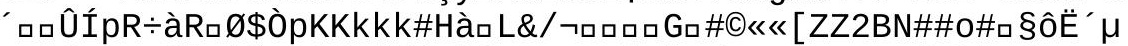

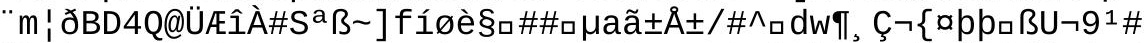

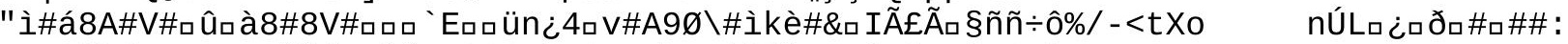

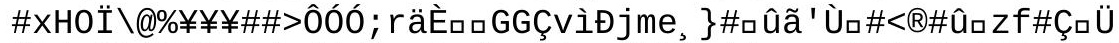

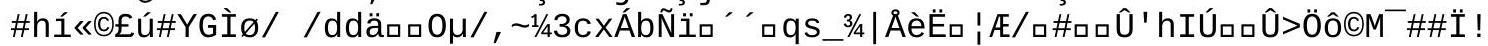

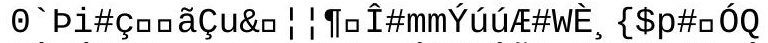

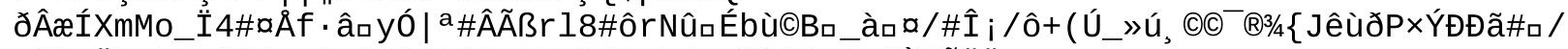

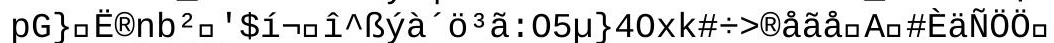

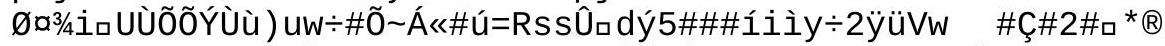

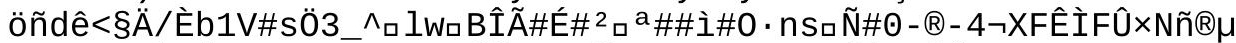

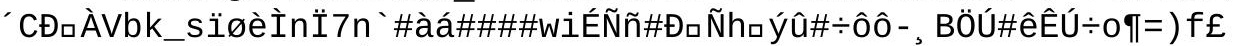

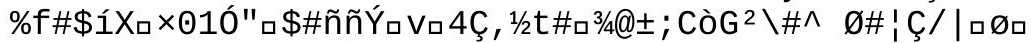

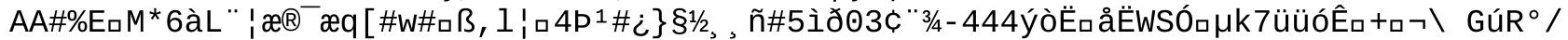




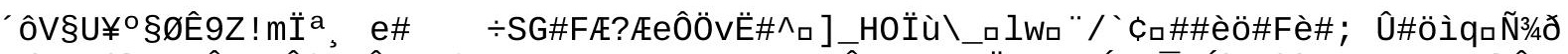

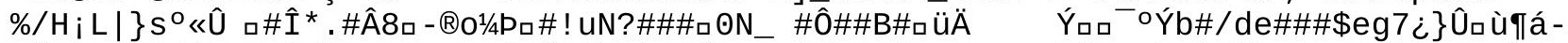

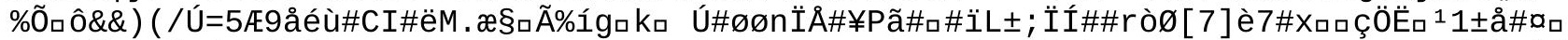

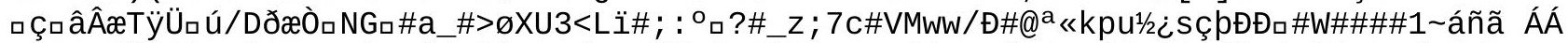

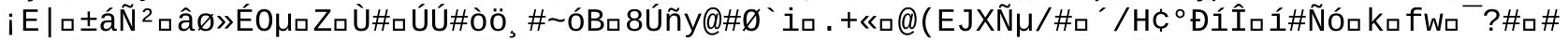

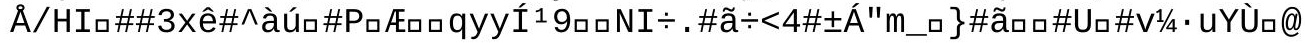




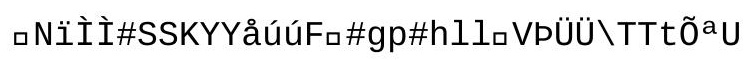




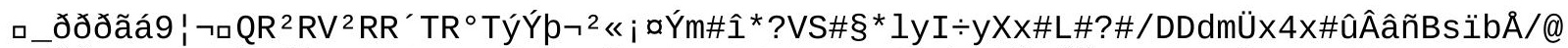

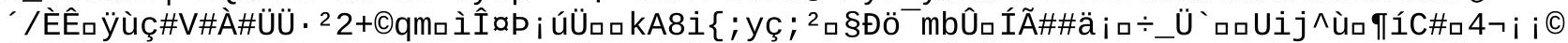

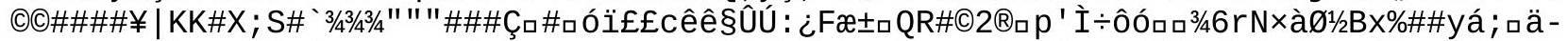

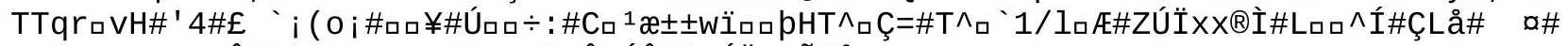

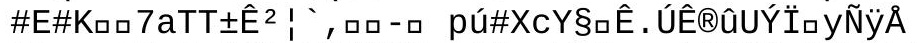

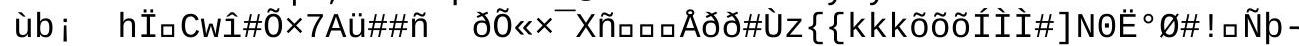

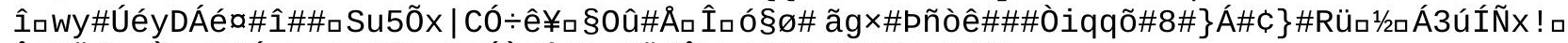

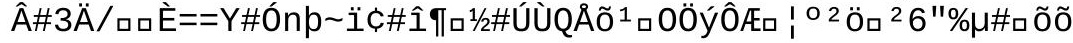




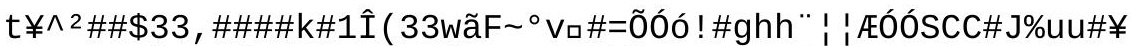

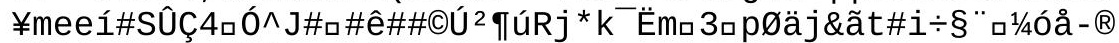

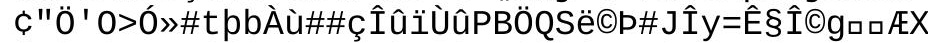




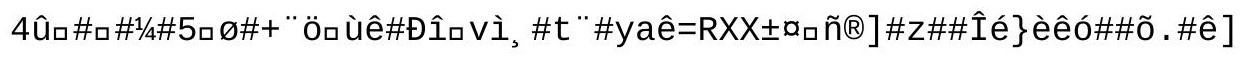




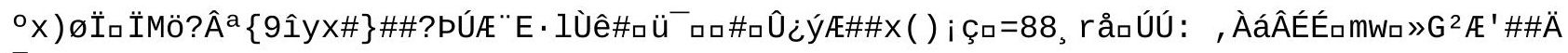
$Z$

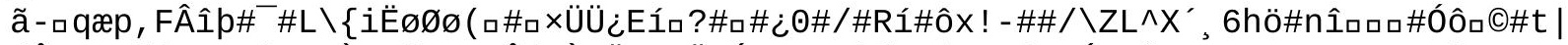

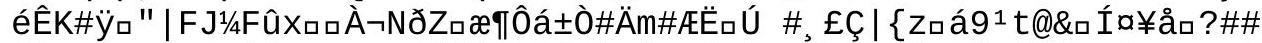
$\square \# \#$ a $)<<\square D C \times \# C$

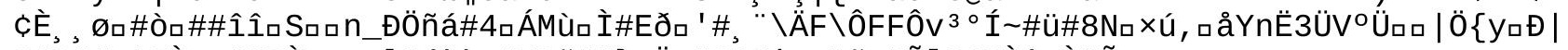

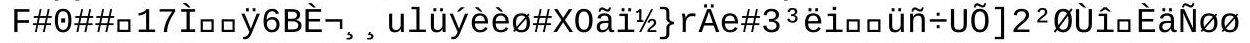

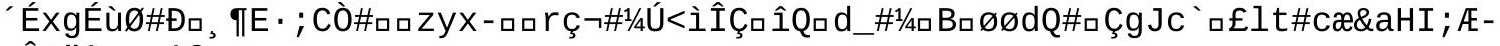

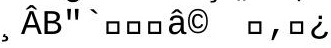






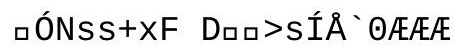




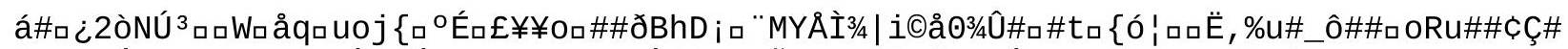

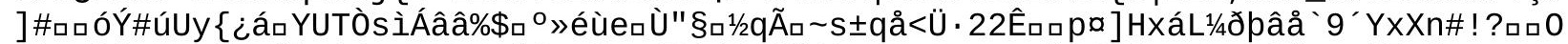

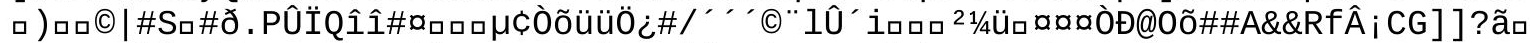

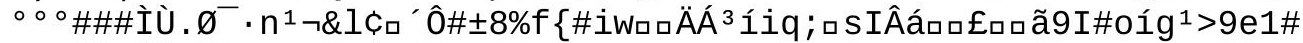

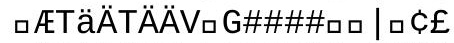

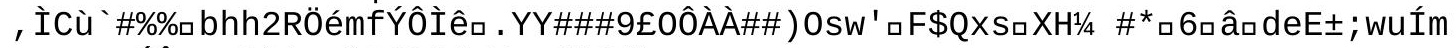

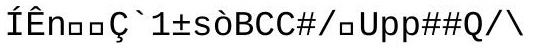




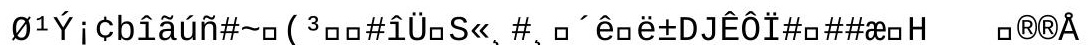

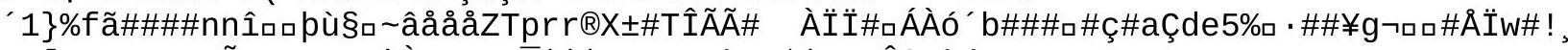

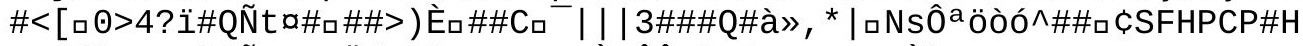

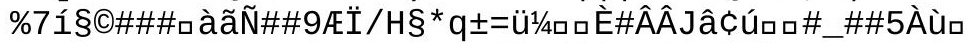

!.

aW+\# i ÄÅtlü, QÖ̈[

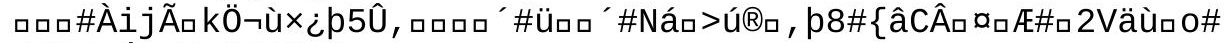

If\#\#vÁ«ロFo\#\#\#\# \# 
\#\#DÄ\#ロñ\#\#Ù $\tilde{a} 1 / 2 \check{I} \ddot{I} /=u \# q \# \%$, **a

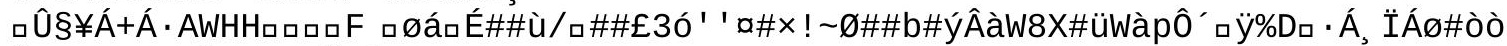
Ò\#ロロ\#\#ロ Àロ9E\#/Å»ñ\# 
v\#\#ロi_\# 


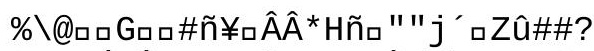

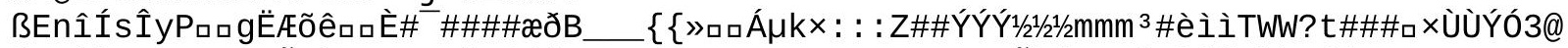

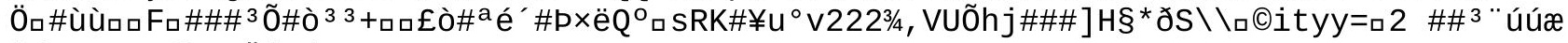
i/\#VzZZ\#íkccË]çdor 


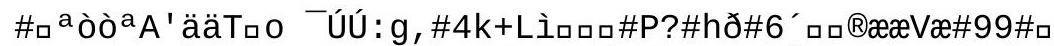

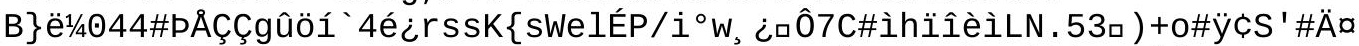

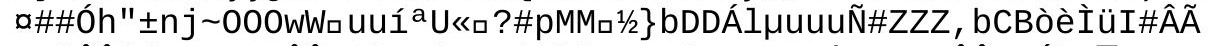

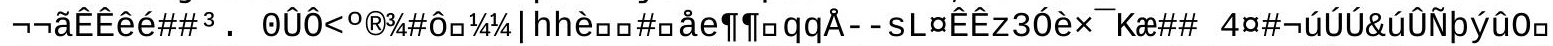

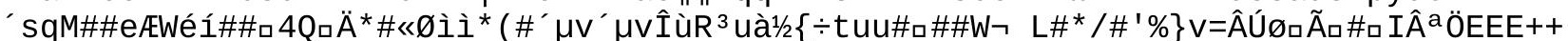

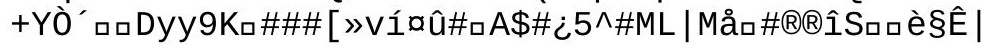




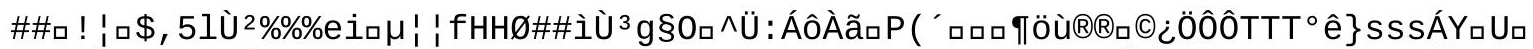

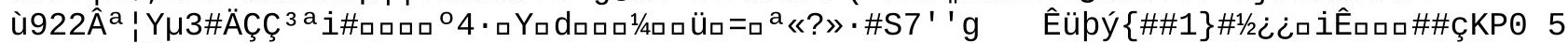

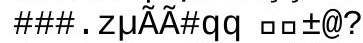

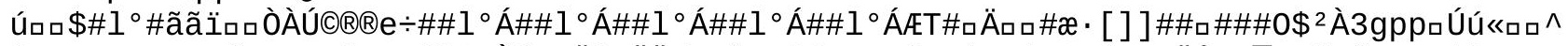

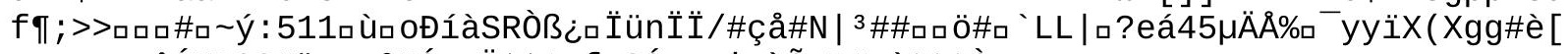

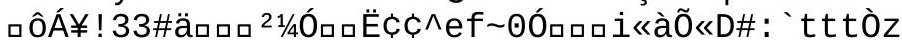


, ÆÂÂAि॰ $3:$ : : व 


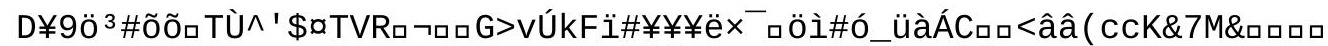

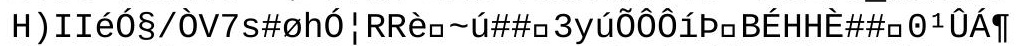

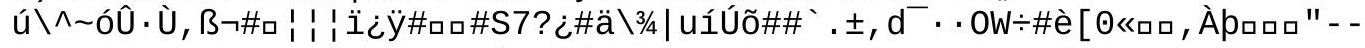

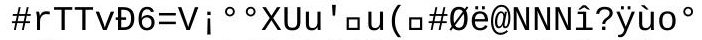

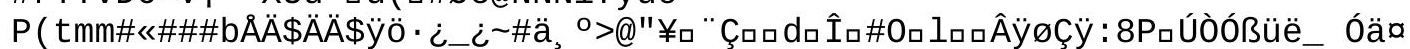

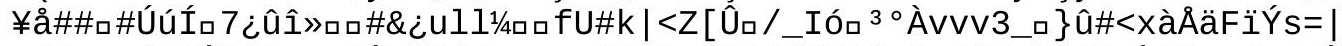

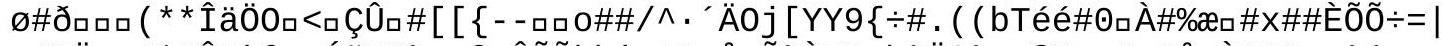

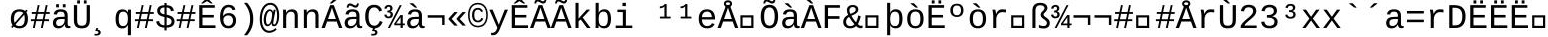
\&' 'ËËË̈̃áp???\&¿/\#॰ [ף]\#' ' 


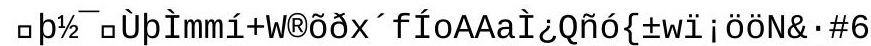

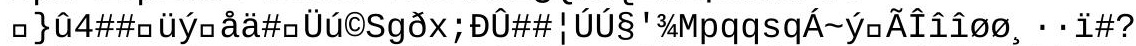

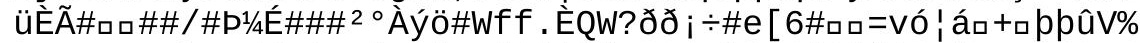

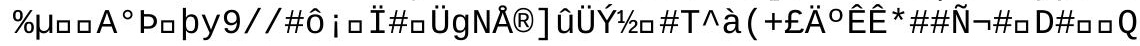




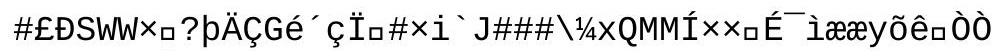

$\left\{\left\{c ̧ y ́ u ̂ 5 \_U ̈ z G G \times \# ?\right.\right.$

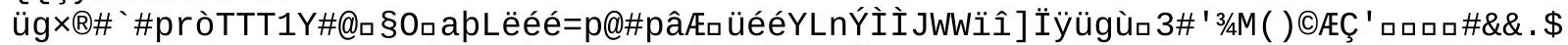

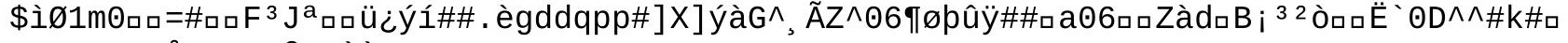
q 7+口r«Iån\#yAn@aa“\#

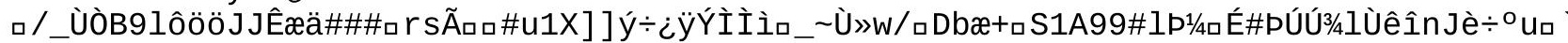

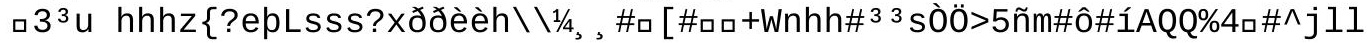




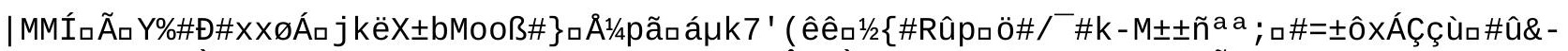

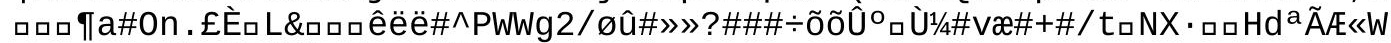

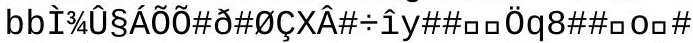

' $\hat{I} 0 \ddot{0} \times 7$ @+ /

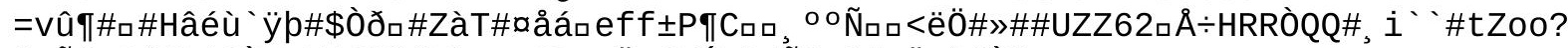

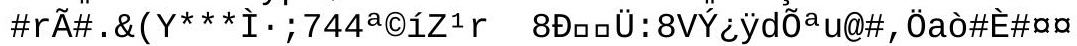

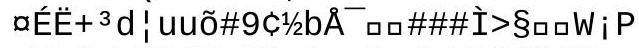

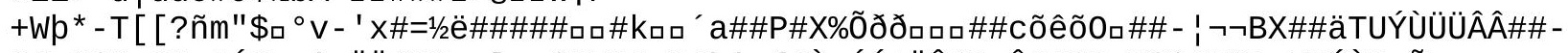

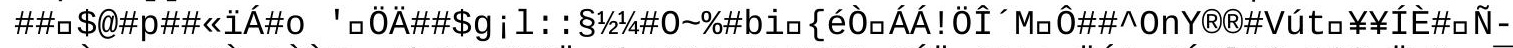

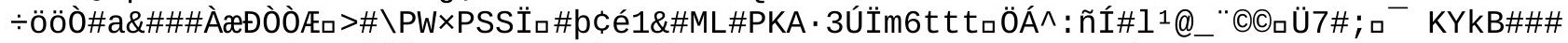

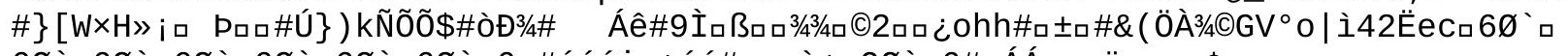

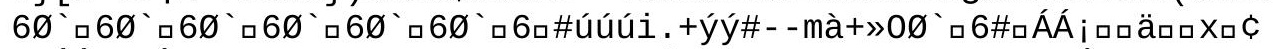

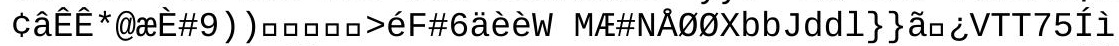

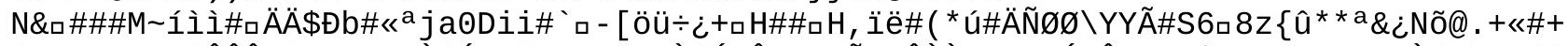

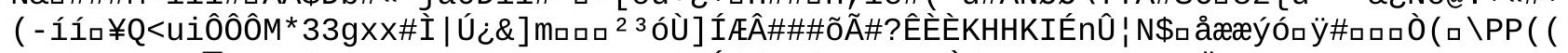

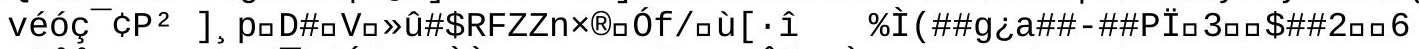

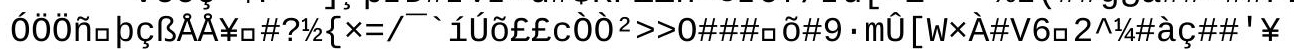

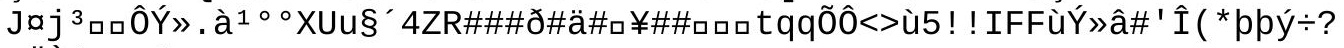

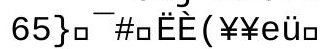

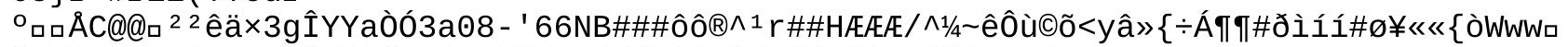
ãçoXZâiúa££Ëëx) '

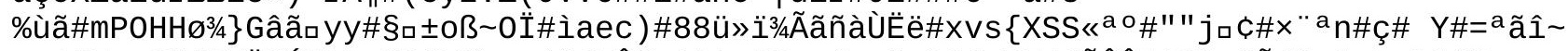

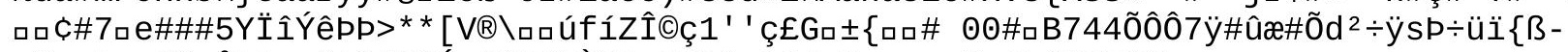

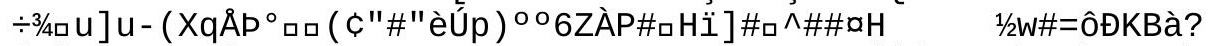

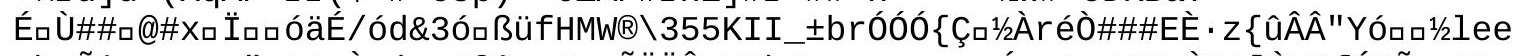

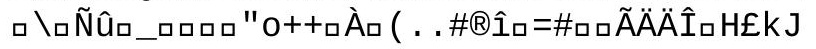

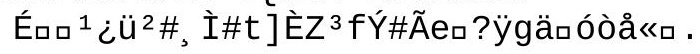






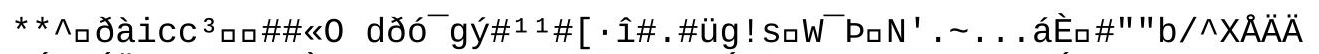

mcÚ\#\#9?ם

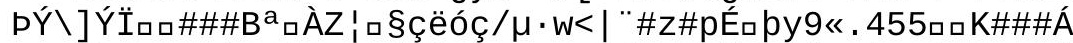

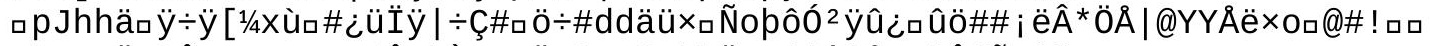

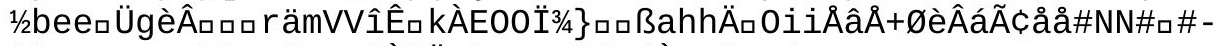

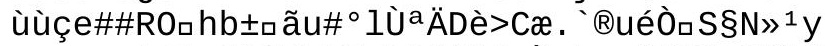

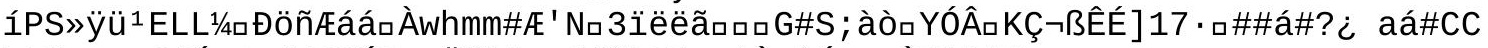

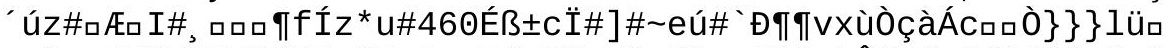

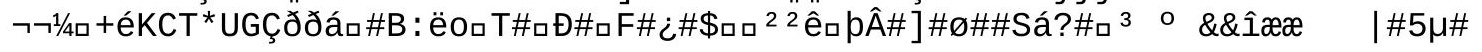




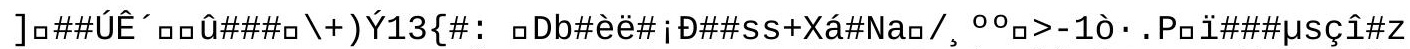

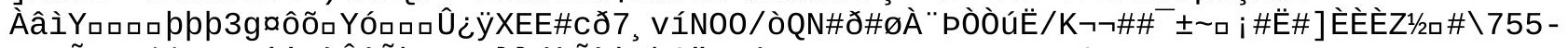

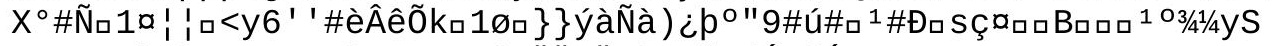

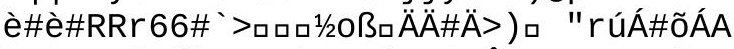

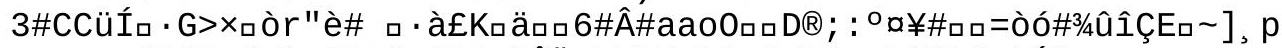

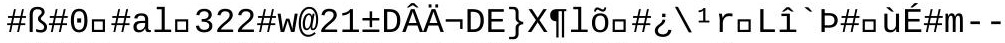

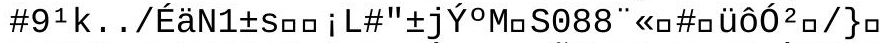

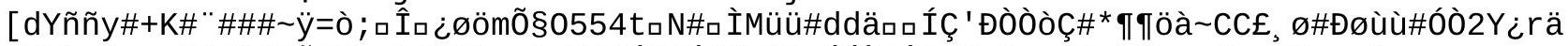

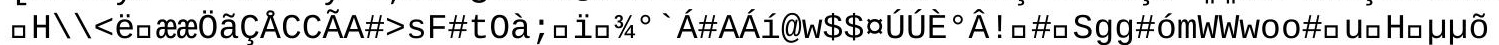

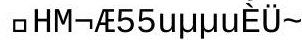




\section{,}


oh \#\#!SÀäíûÈcçÄÀ 


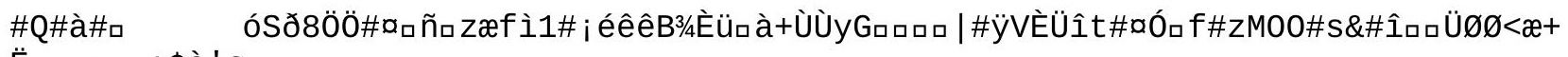

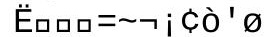

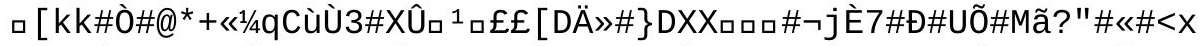

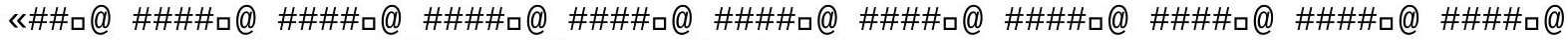

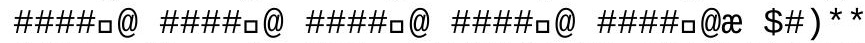

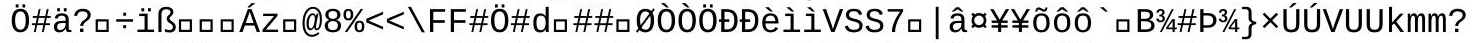

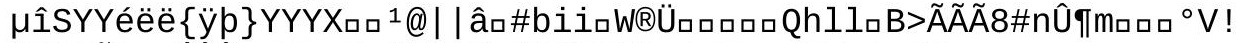

ß\#QQÑ Çם 


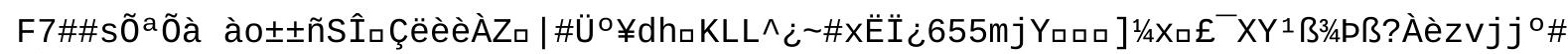

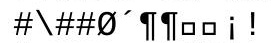

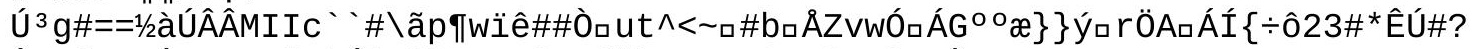

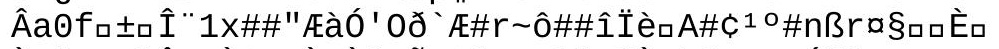

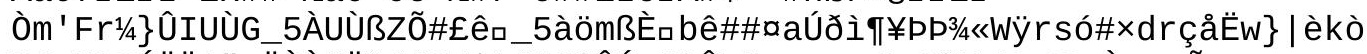

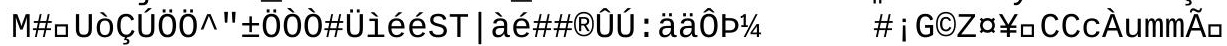

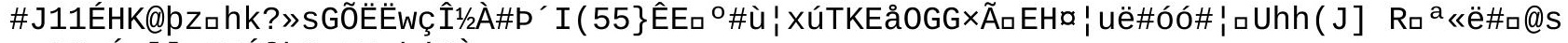
yy\#\#xÁu] ] cEEÍSk\#ロJEqkj\#Àrq 


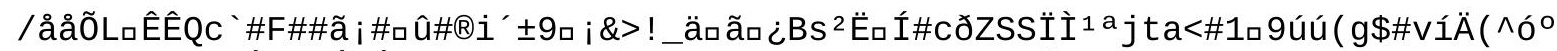

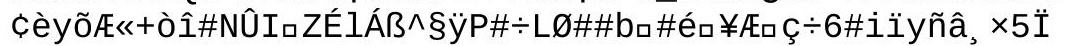




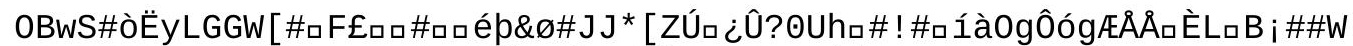

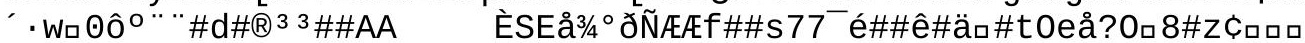

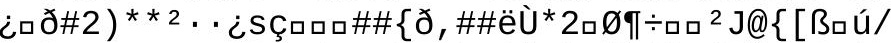

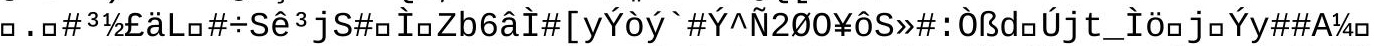

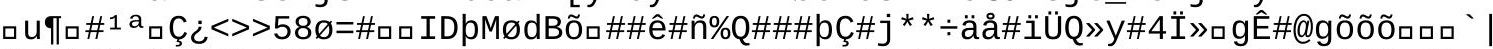

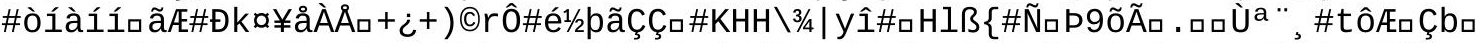

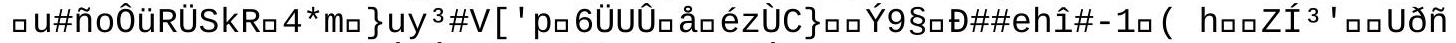

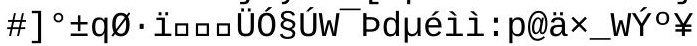
口\#pCC 'àx ùv -

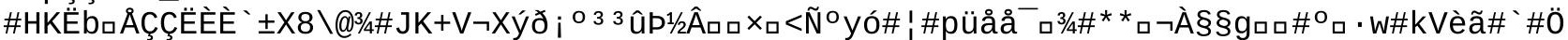

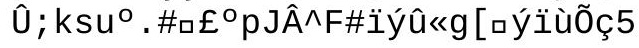




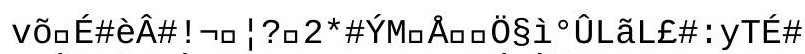

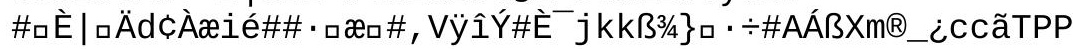




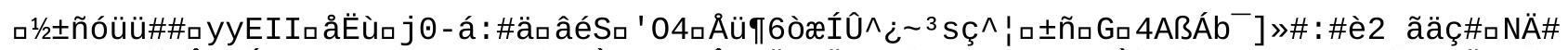

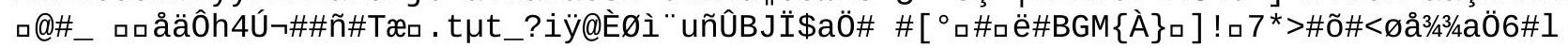




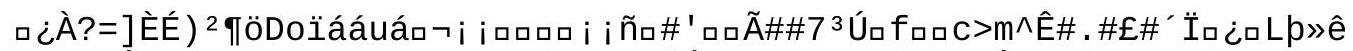

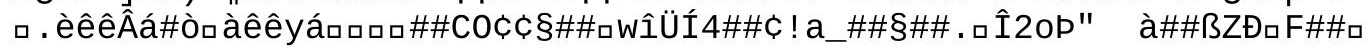






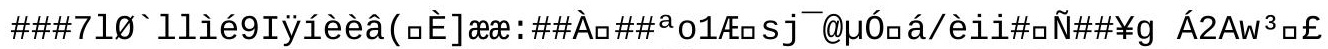

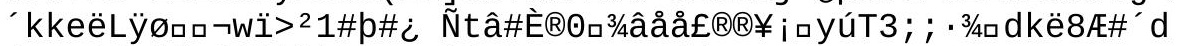
ó\#\#\#óó\# ' Ô\#[[ [ ] ) ) Đ#\#\#À' ÇÜÜ\#\#\#ロO\#//_ 


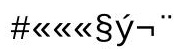

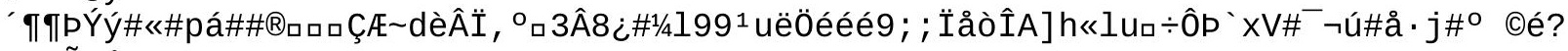
$\wedge \# \#$ ÃC' 


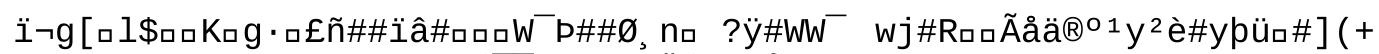

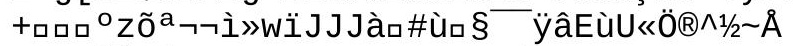

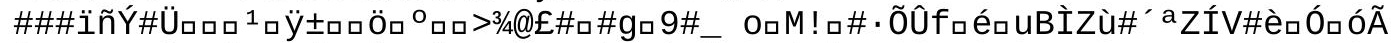
ôๆ\#[ $\left.\square^{2} 0\right) \square A i \# \# \#$ ¿øÏXGÂOg\#) 33ÿùsGôöÀSPUý\#İs

$\mathrm{J} a$ Ë\#\#ロRR\#CıUb

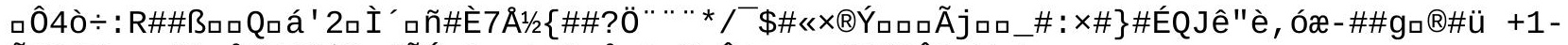

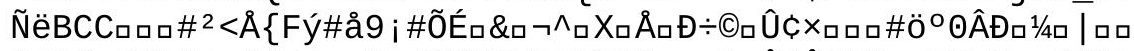

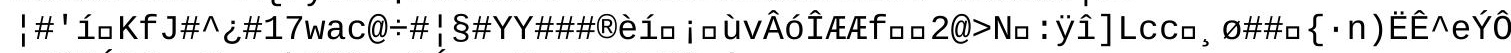

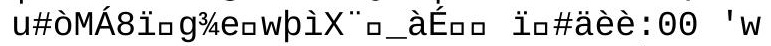

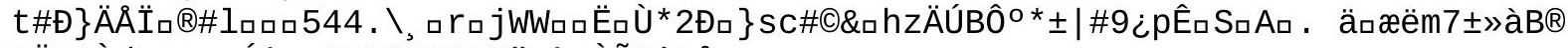
\%ÏDAÀd > ! ! ฉÁôu॰SRS3DDN\#9" zèıÈÑE ' äåo

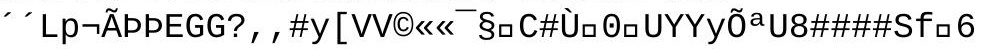




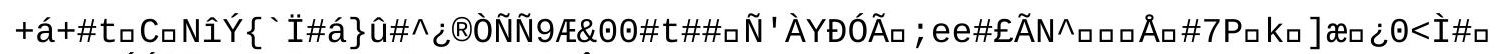

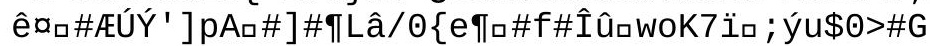




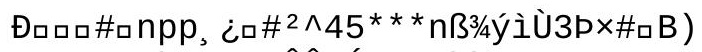

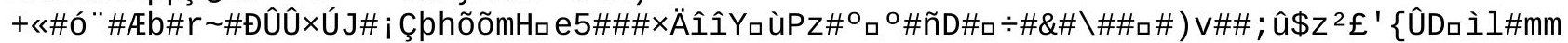
$>$ FE\#\# 
PyðÃh

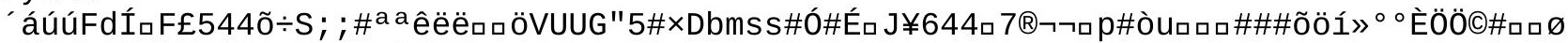

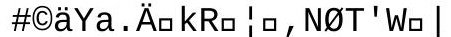

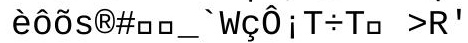




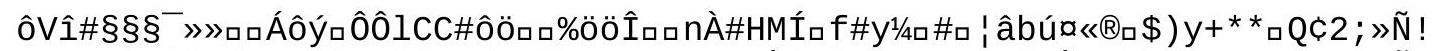

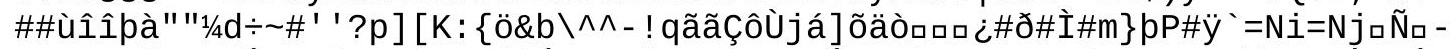

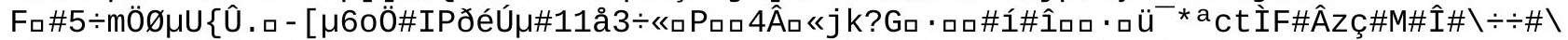

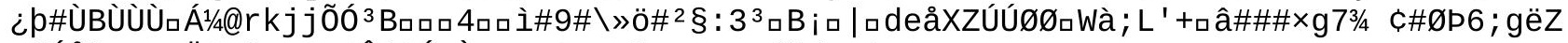

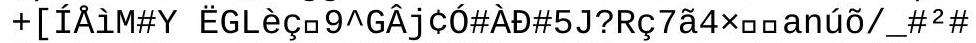

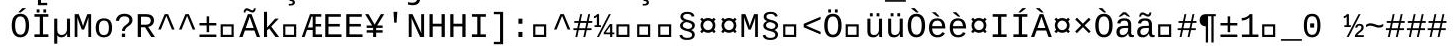

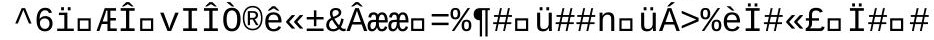

\#ßÛoñ

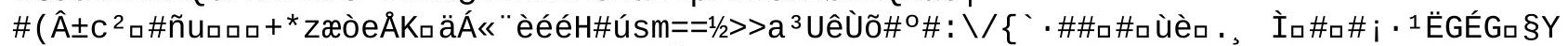

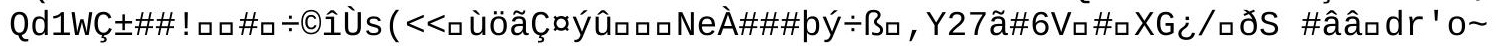




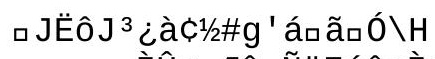

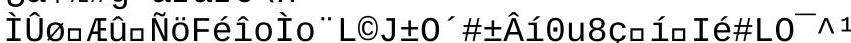

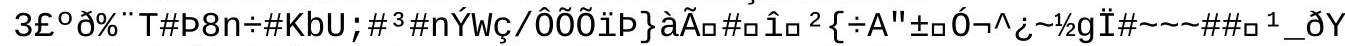

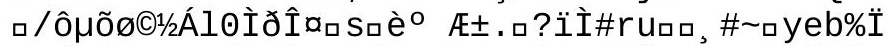

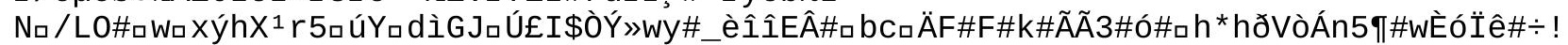

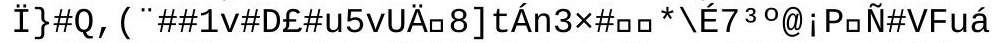

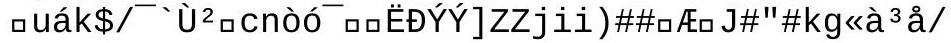


RcıPa6áı\#ıhı

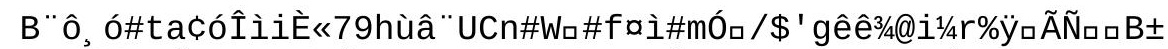

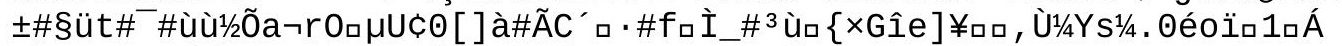

ah $\quad \mathrm{mZ}-\ddot{y} n \& u ;$ a 


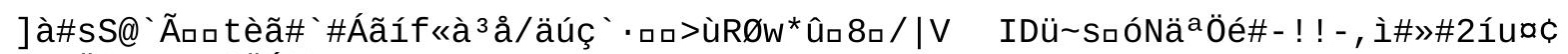
crOÏ@口Æÿû¿ßËÉ) $\backslash{ }^{\circ} \$ \square a$

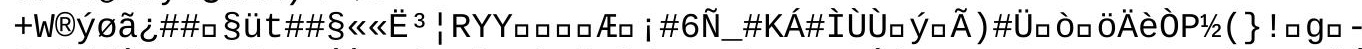

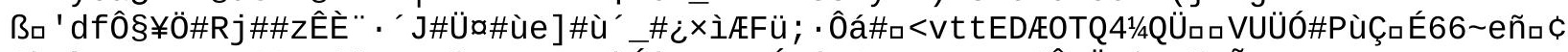

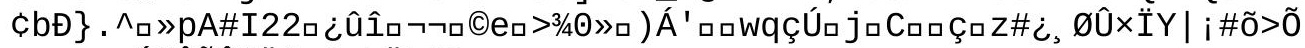

É\#Ễ̃̂िก̃Ûuि0_kÏL\#?

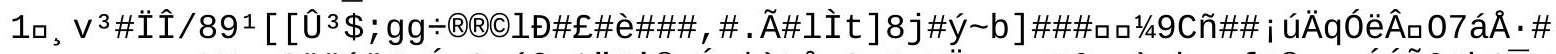

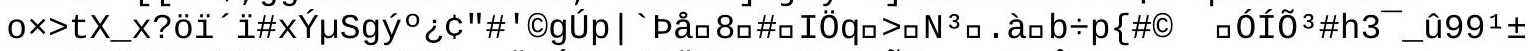

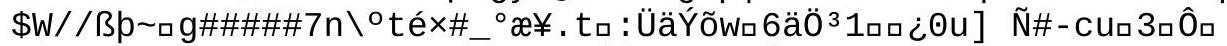

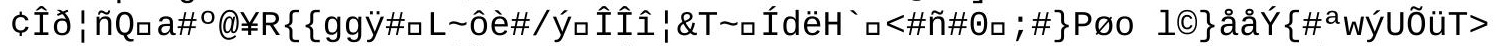

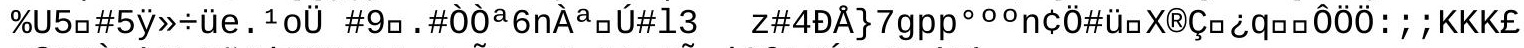

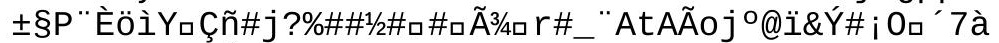

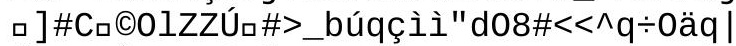

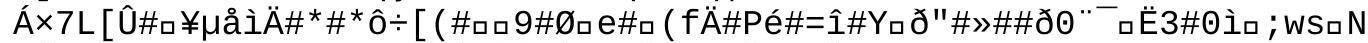

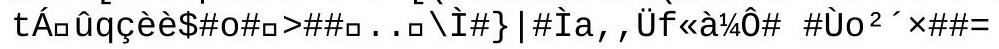




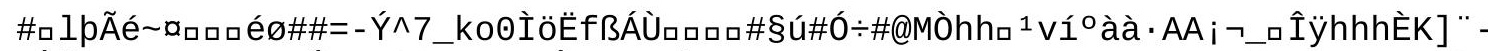

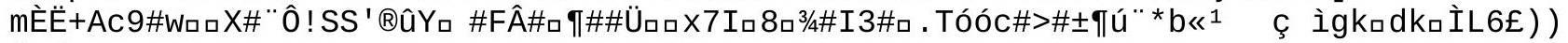

$" 2 d$

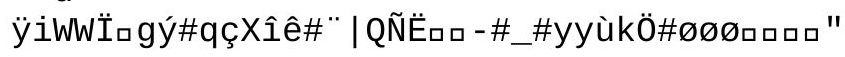




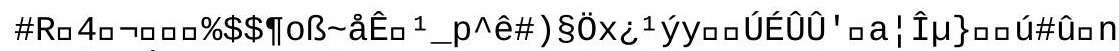

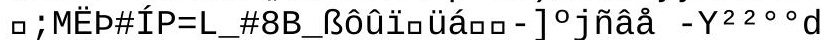

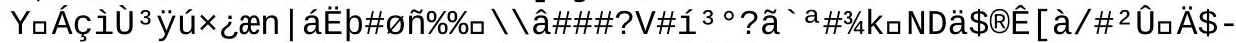

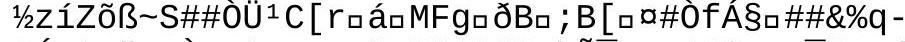

9ÍĐù\#" $\pm v I^{3} / 4,{ }^{1} \#$ \#\#<

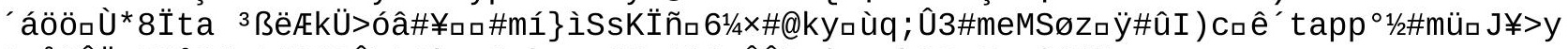

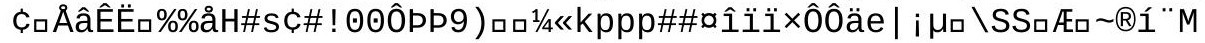




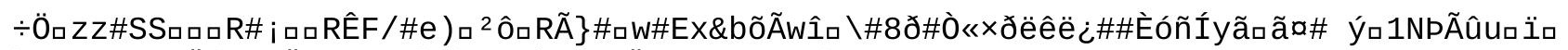

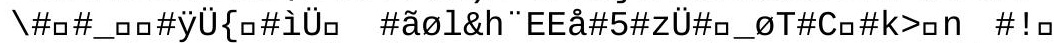

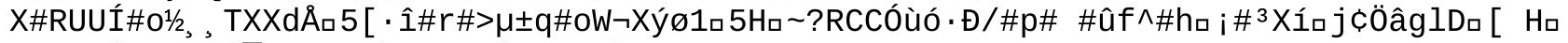

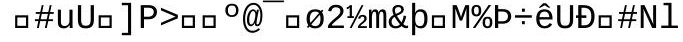




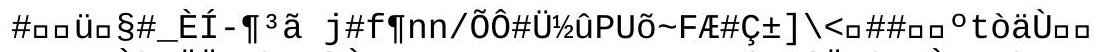

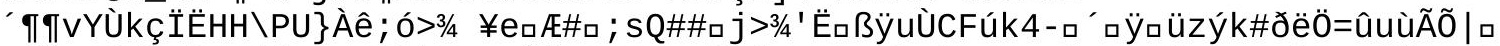

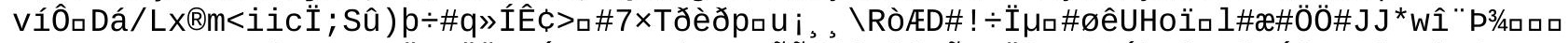

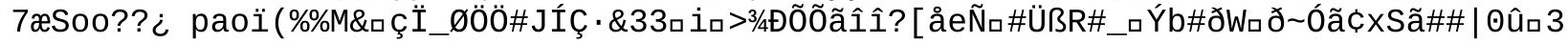


Ú1^S]ĐÓâa . 


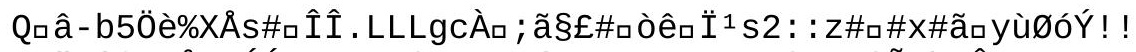

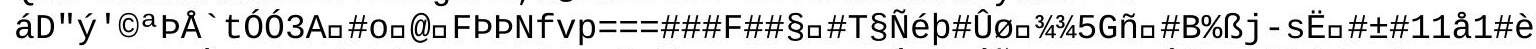

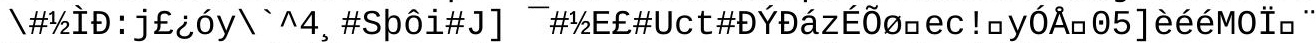

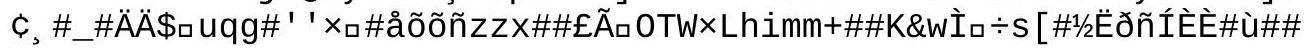




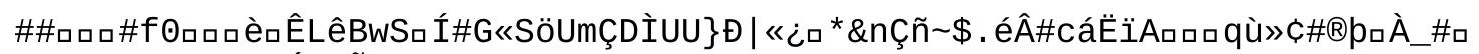

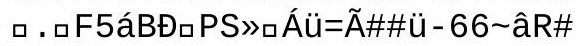


( ( $73^{3} \#>\# \#$ À̀̀ $*$. ü 


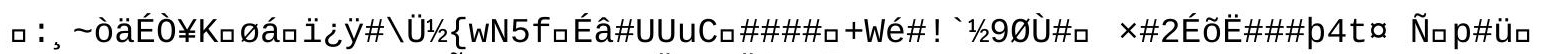

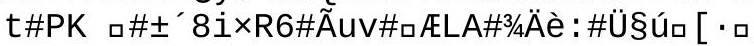





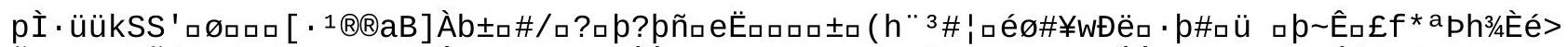

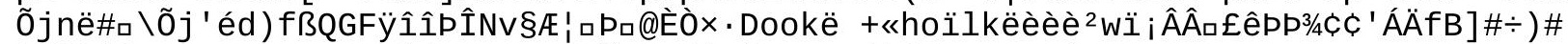

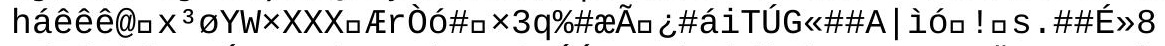

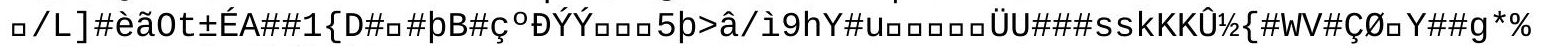
\%G\#7\&Ò\#ĐYÀロËßßÿìù ${ }^{3} \mathrm{C}$ 


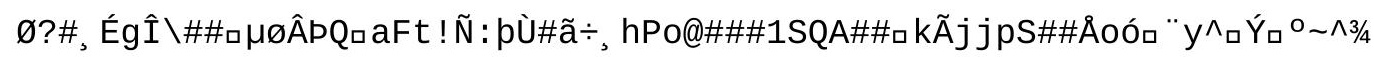




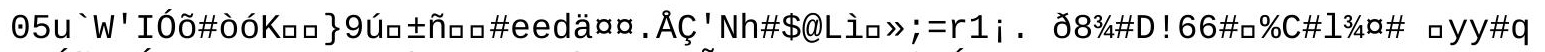

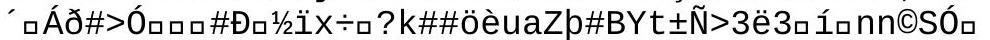

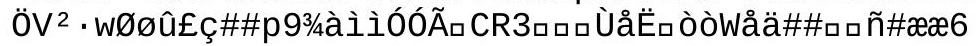

7ロ\# ฉ/ĐKฉฉOMFF\#Ó 


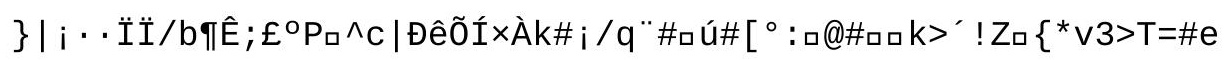




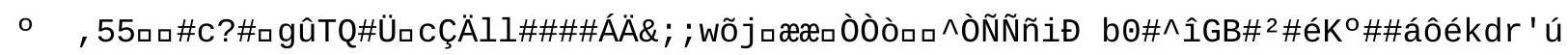




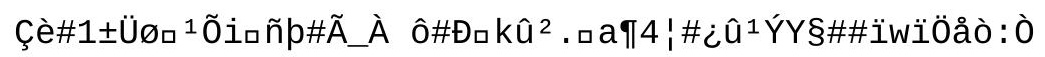




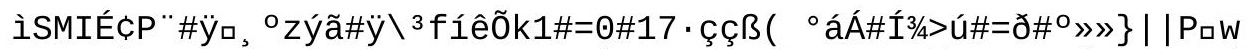

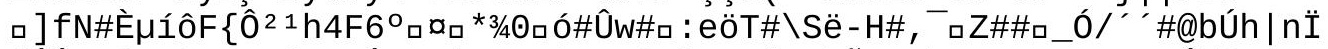

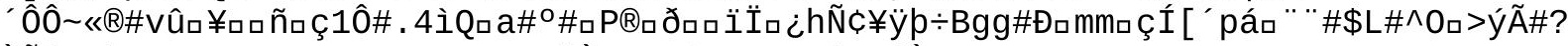

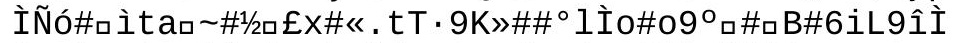

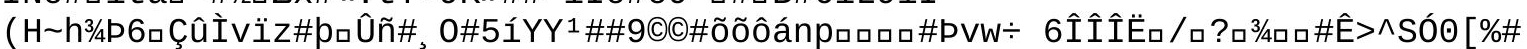

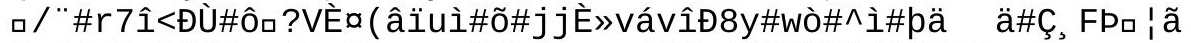

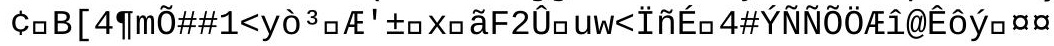




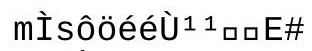

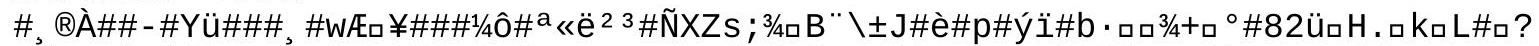

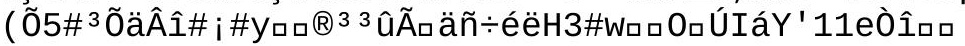

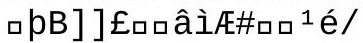




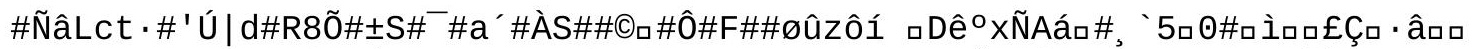

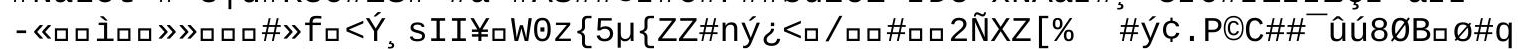
$\ddot{i q} q \tilde{N}: \square p$

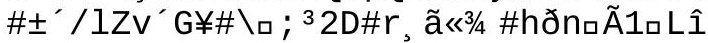




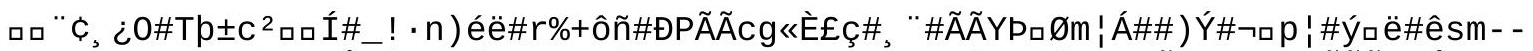

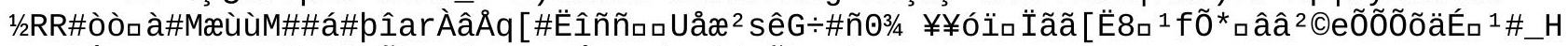

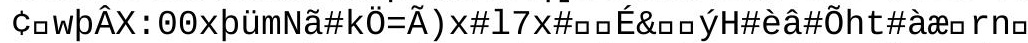

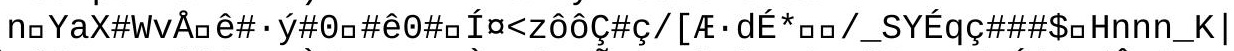

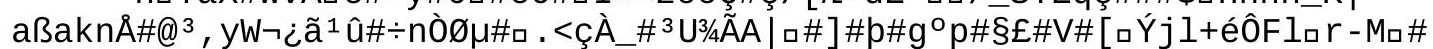

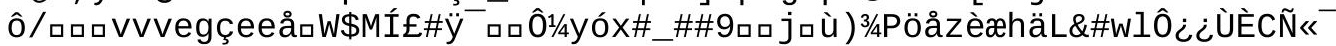

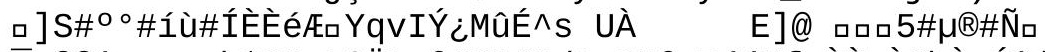

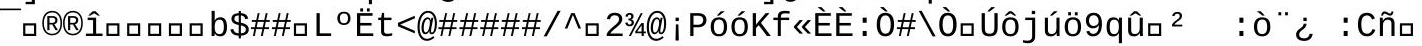

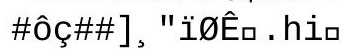





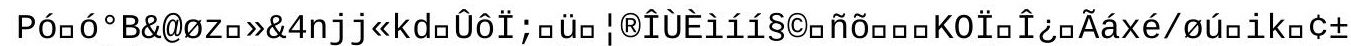

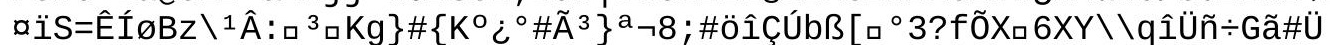

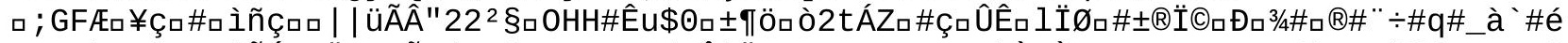

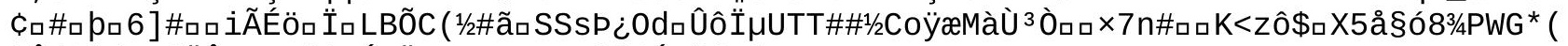

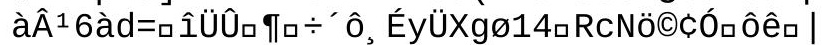

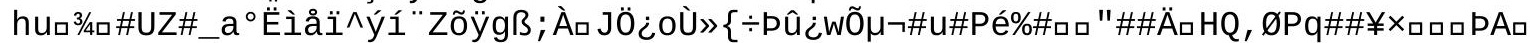

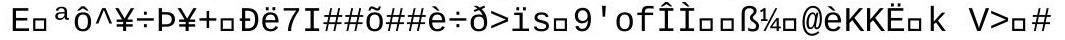




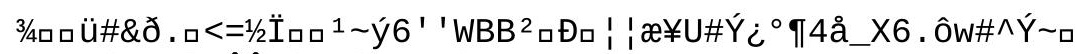

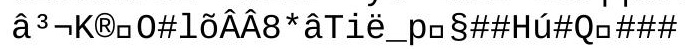




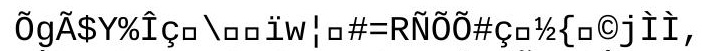

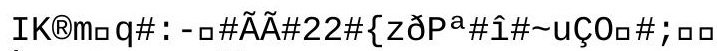

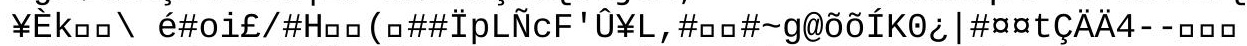

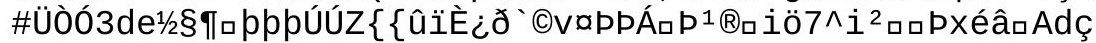




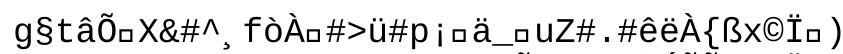

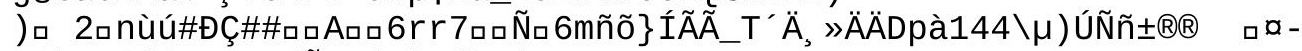
Mà̀ $q^{a}$ ýýıPü\#\#ýÃ\# : áFì\#ókıû\# 


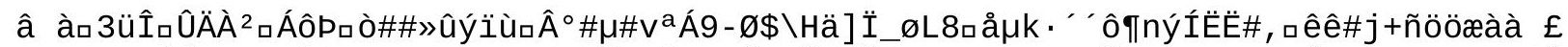

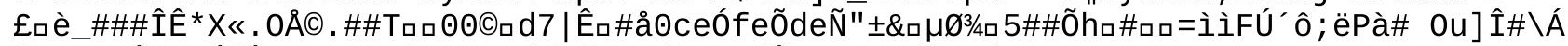

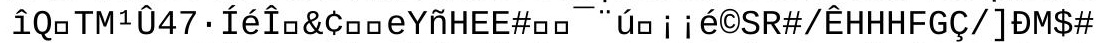

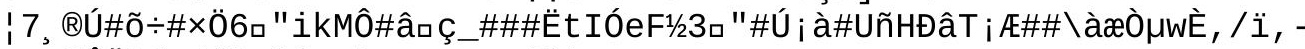

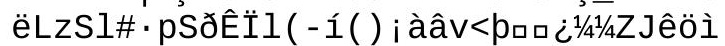

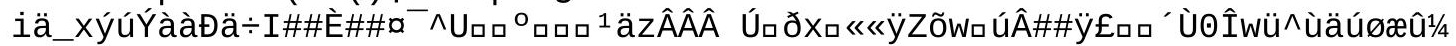

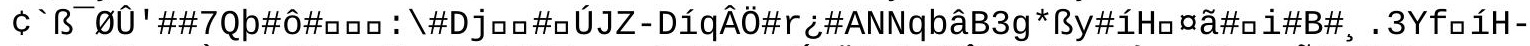

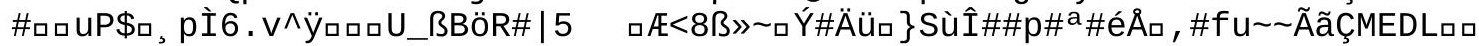

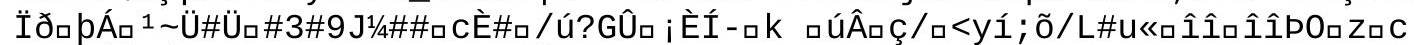
û3/4ü\#ÊÊKKÉWם\#\#7Q ýÇas ×oBÇâfû ) ¿3uù ÎPãT\#§qa\#rpq, ap\#-

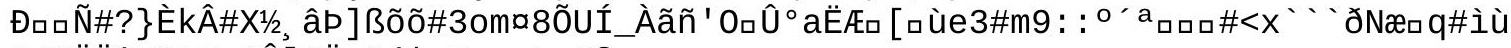

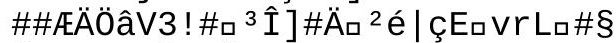

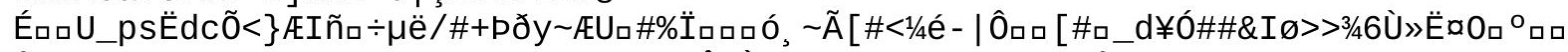
Å:

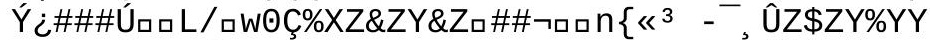

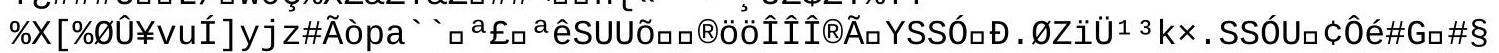

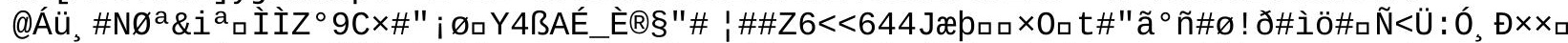

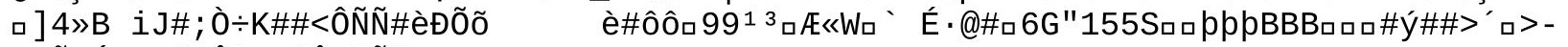
, LÕ $\sim E \#>=ß Q U \hat{u} \# \# @ \hat{A} \backsim \tilde{n} \tilde{N} \tilde{n} 4 \square$ 


\section{Cfa\#;ZTÔ}

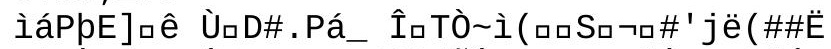

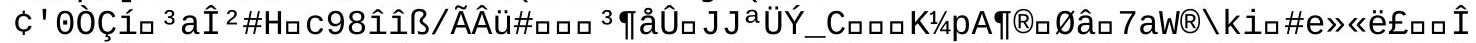

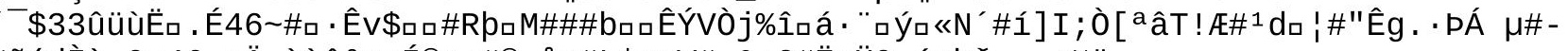

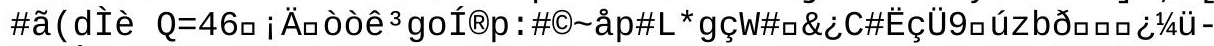

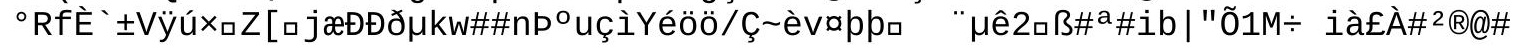




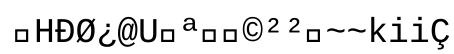


. +ë( Îk\#P\#6dÃ

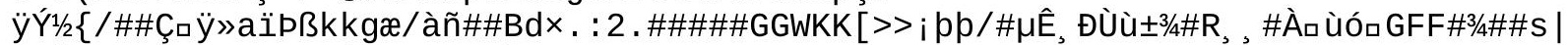
ñöÒV

$\ddot{y} \square \hat{I} r p_{\square}, a z \# \# \ddot{y}$ 
$\ddot{0}=\square \mid g \#$

$\# \mid C_{\square} X J \backslash \# Z g q n \tilde{n} \# /$ 


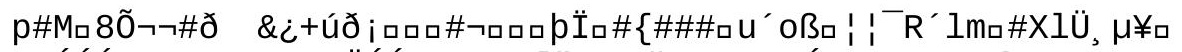

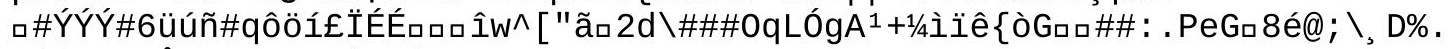

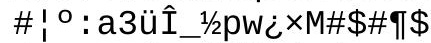

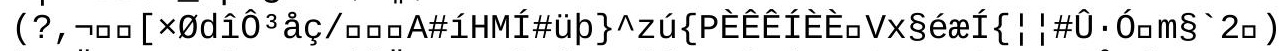

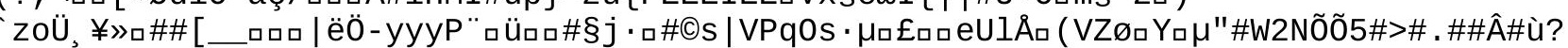

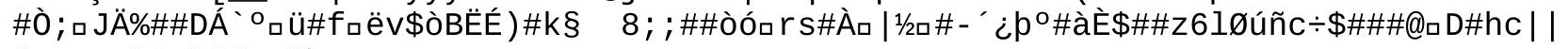
1/4ロ ??口JJJqqñb_a 


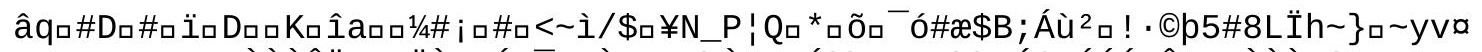

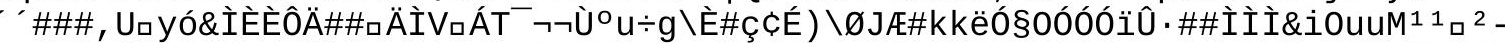

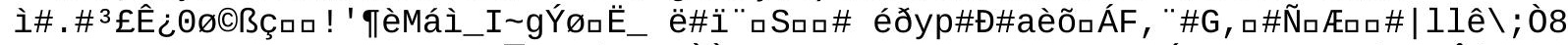

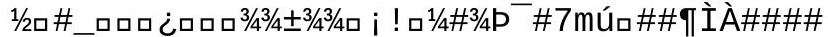
\#\#ロÍø7ロ9sFEEeá\#ロロÛ\$ \$®口S

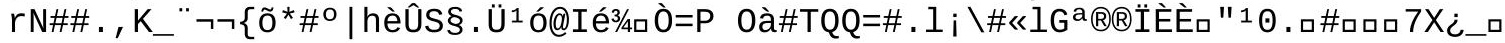




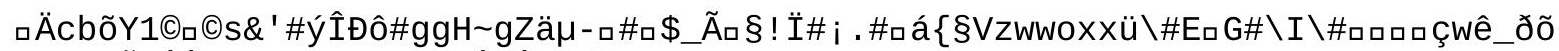

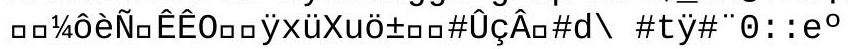


$\# \#$ !

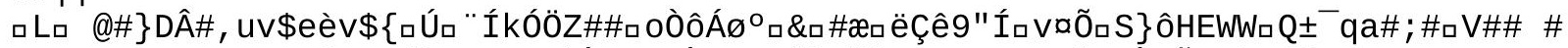

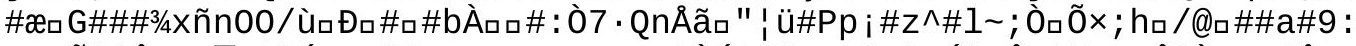

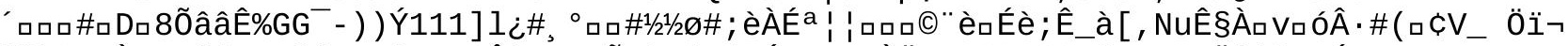

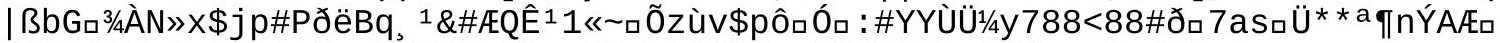

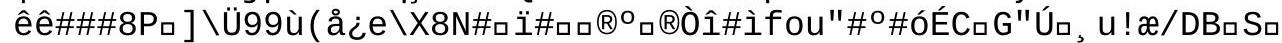

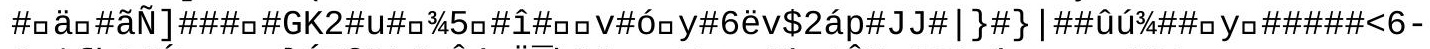

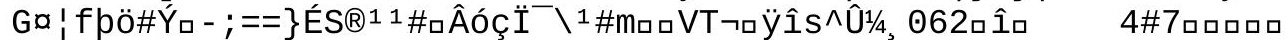

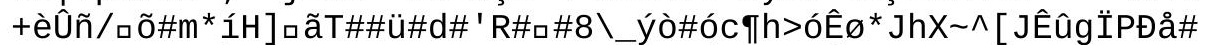

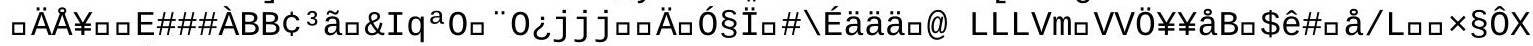

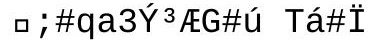

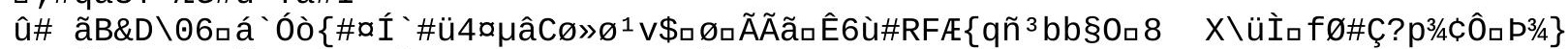

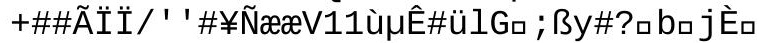

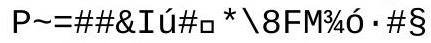

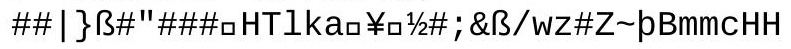




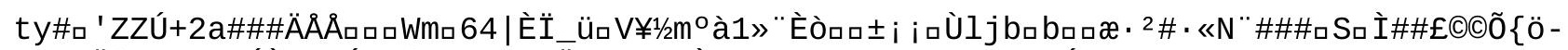

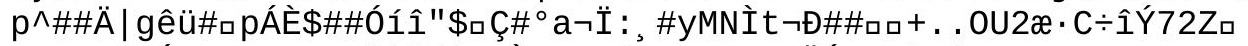

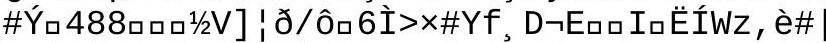

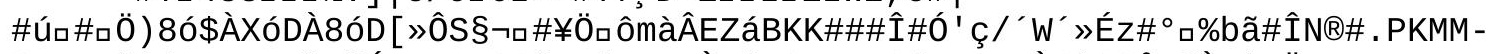

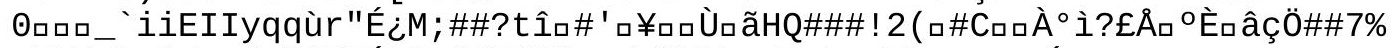

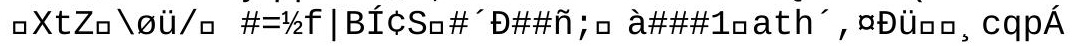




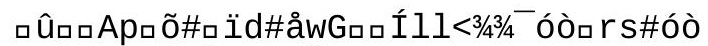

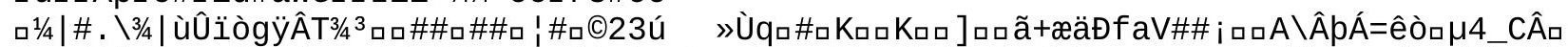

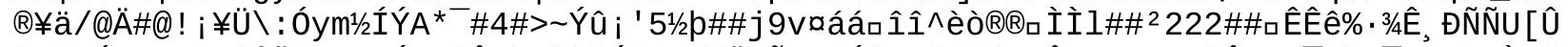

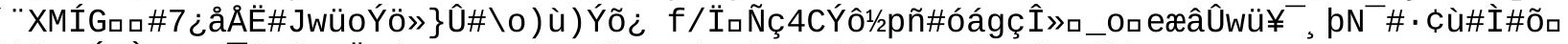

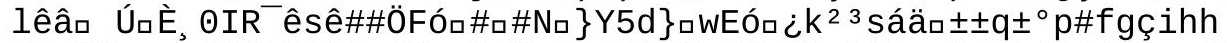

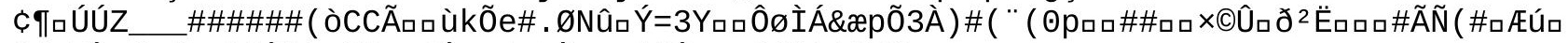

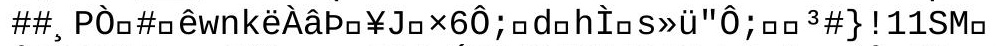

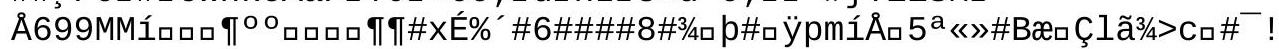

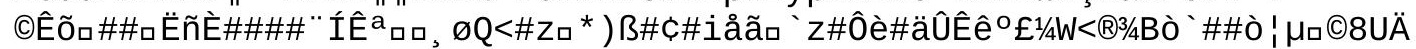
ÇC\#\#1/4¥ 


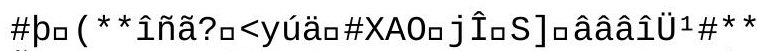

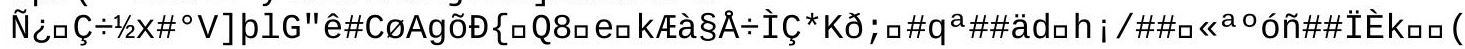




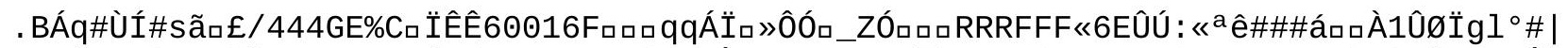

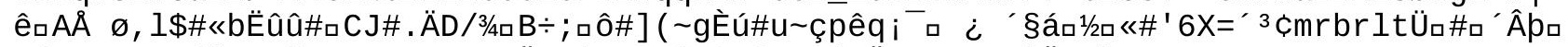

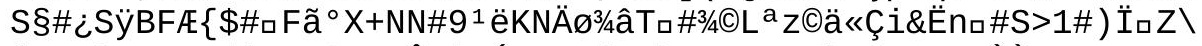

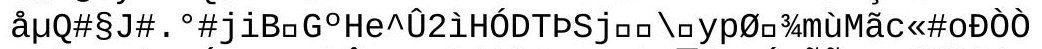

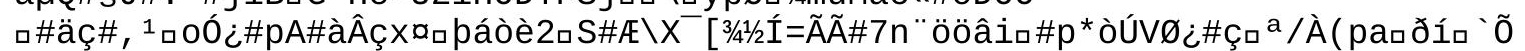

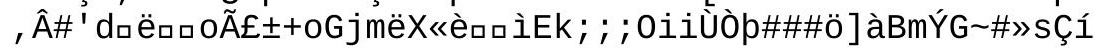




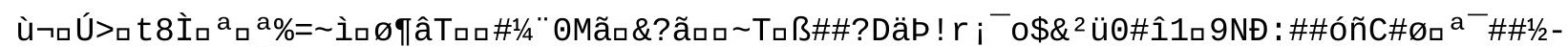
T\#ロòó\#q8+\#\#\#ÄÖÖvGם\#-+«XòÄ>sæİjê\#- - \#P\#1 Éúaà1ÛquêOu] !

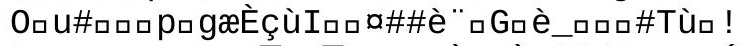

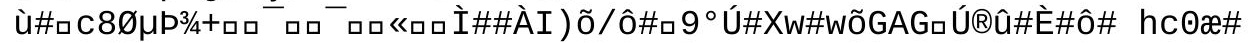




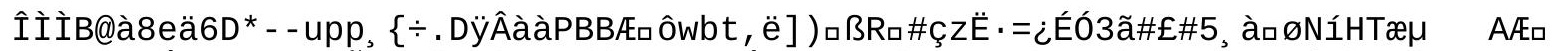

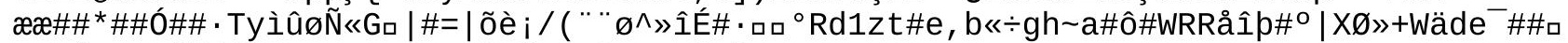

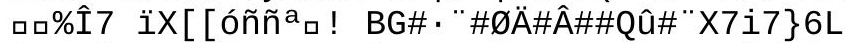

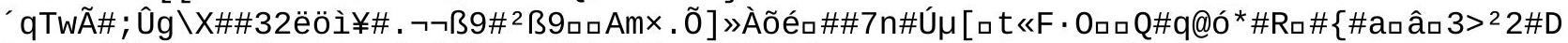

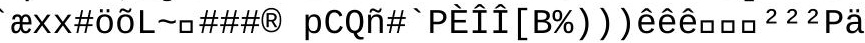

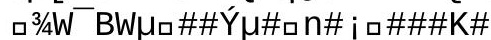




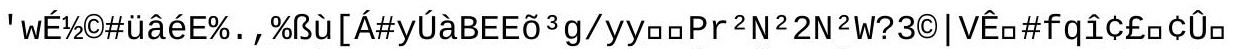

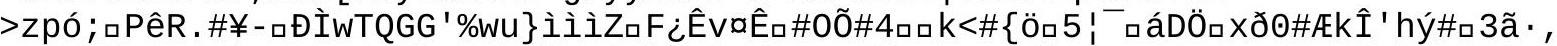

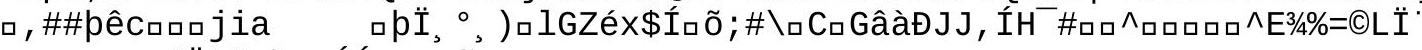
, äáÄòñYh\#XÚÉ $1 / 24^{1} \#$ đ\# 


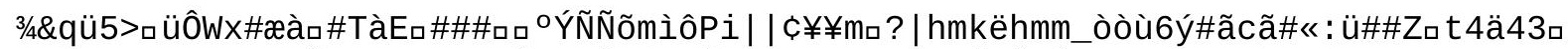

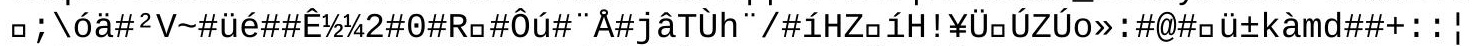

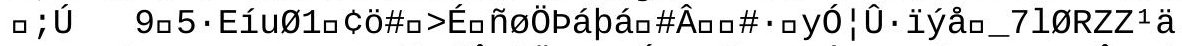

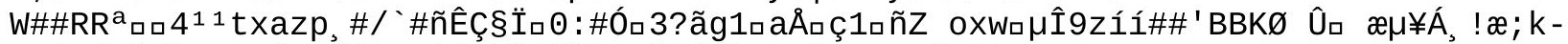

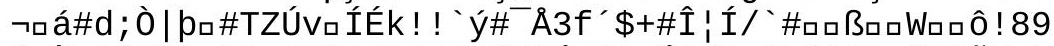

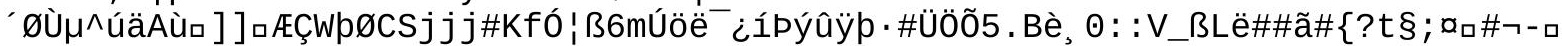

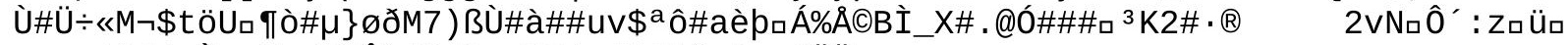

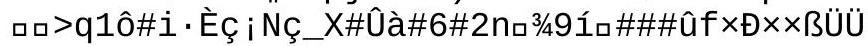

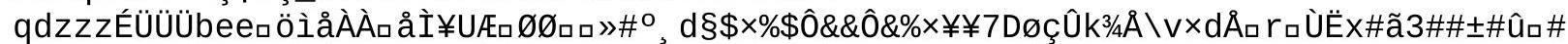

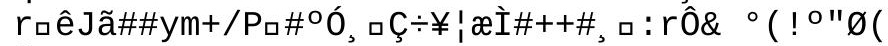

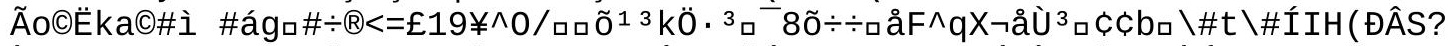

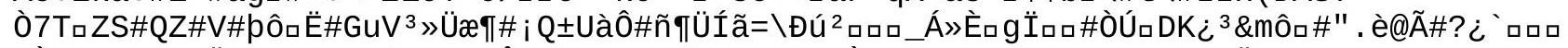

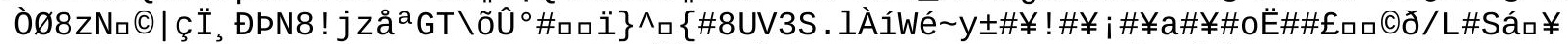

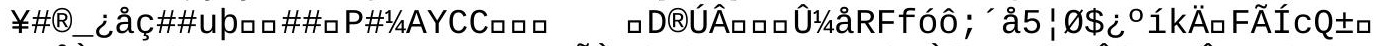

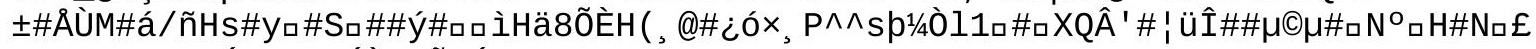

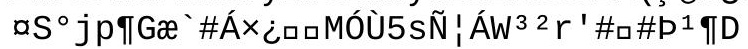




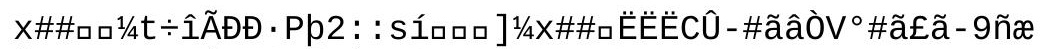
$>\div \ddot{\mathrm{uMy}}{ }^{\circ} \odot \odot \# \# \mathrm{M} / 2$

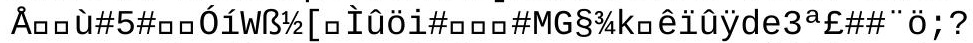

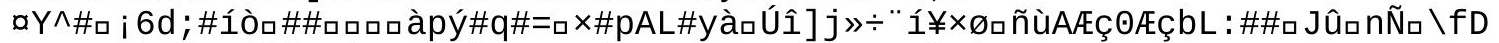

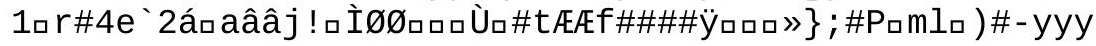

ロロロ«ף*ãâ

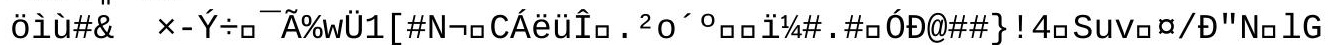

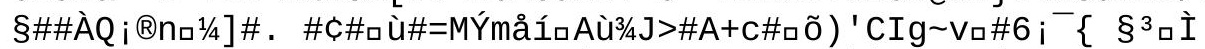

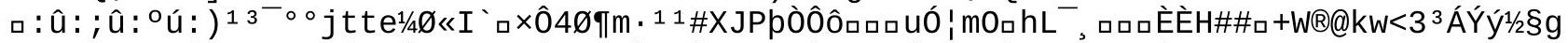

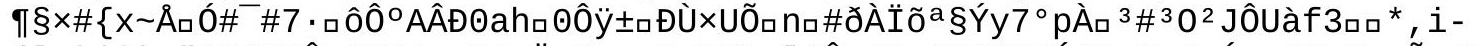

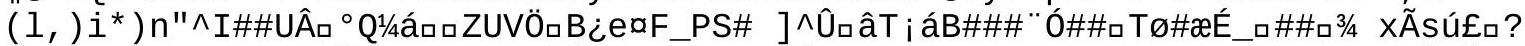

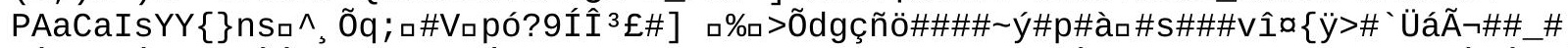

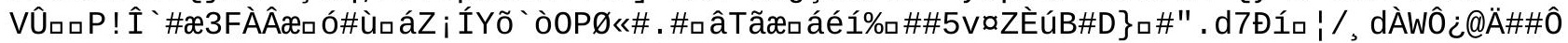




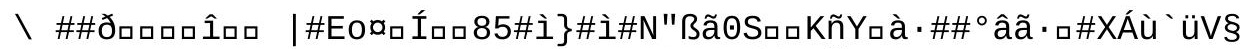

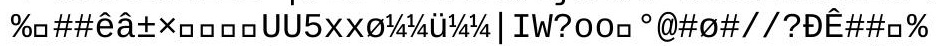

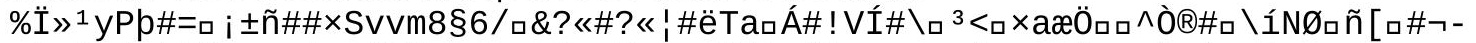

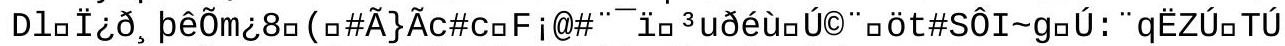

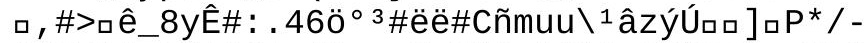

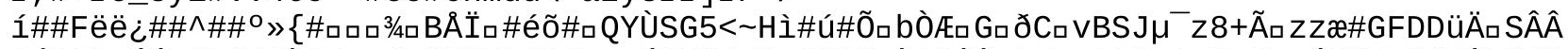

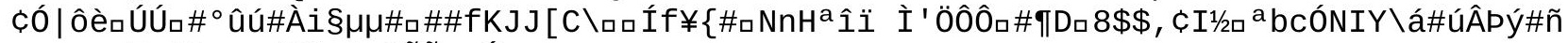
$5 \square \mathrm{J}^{\circ} \gg \mathrm{Ç}_{\square} / \# \# » \mathrm{H}_{\square} \mathrm{QN} \tilde{O} \neg, u ́$

口

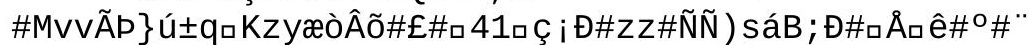

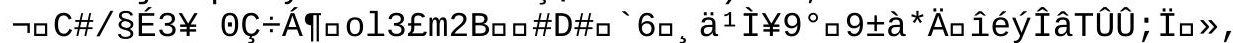

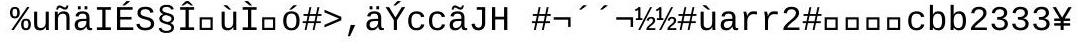




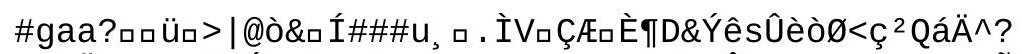

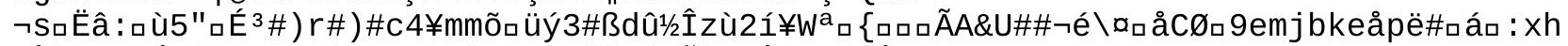

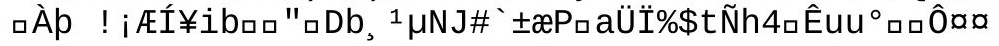


๑\#ёE口 


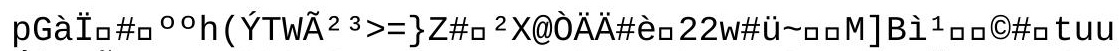

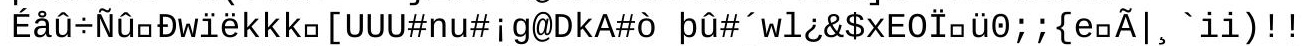

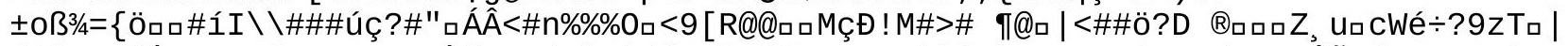

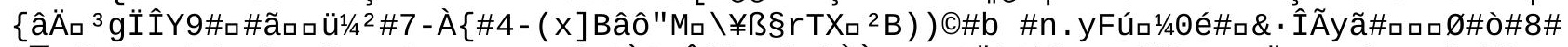

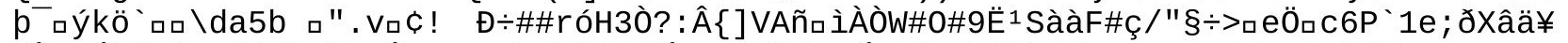

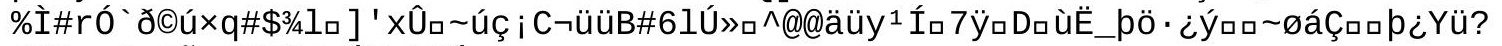

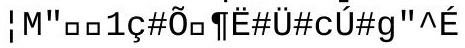




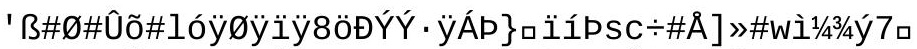

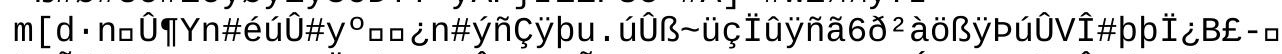

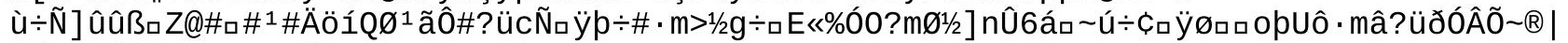
âÎW6làúç? ^ $Z^{\circ} \hat{E}$ 




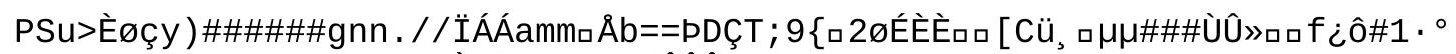

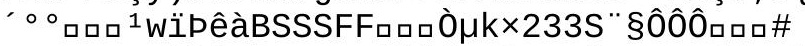

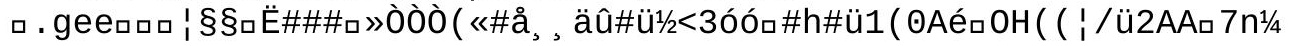




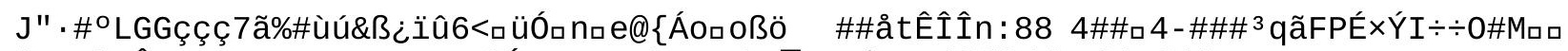

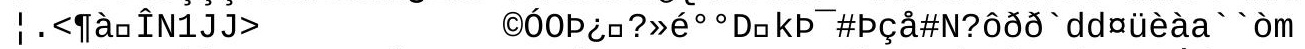

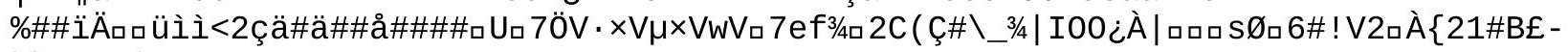

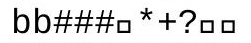

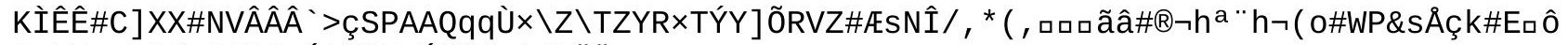
SsIImaañôkäååQロÁi\#\#ๆnÝ\#\#\#_^^püÜ 


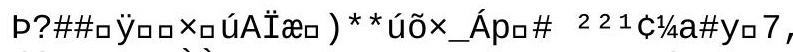

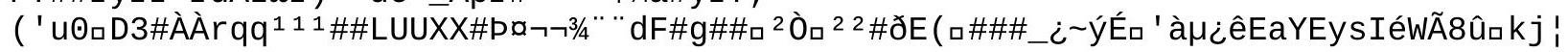




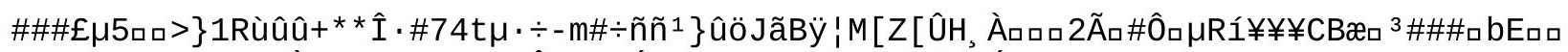

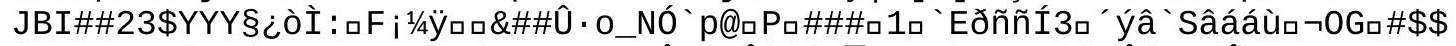

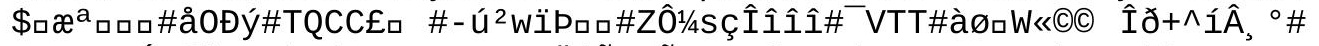

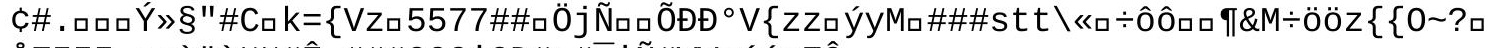

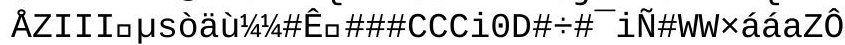




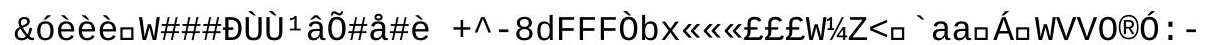

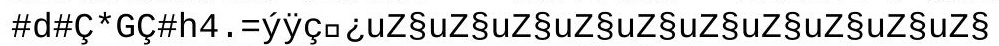

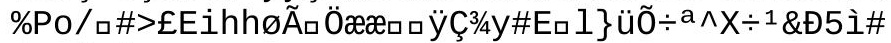

( Jロ (QÔ5 व००E 


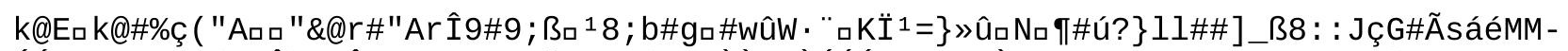

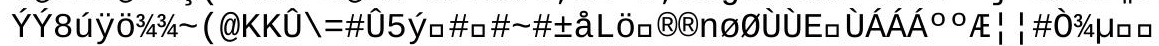

\#囚ロ\#\#mú, “\#\#]TZ\#\#ロZ`x-

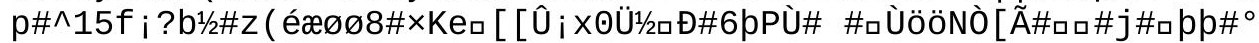




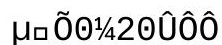




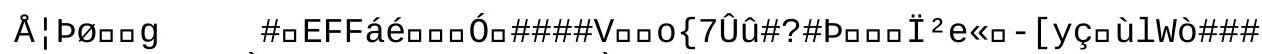

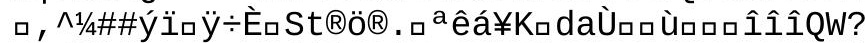

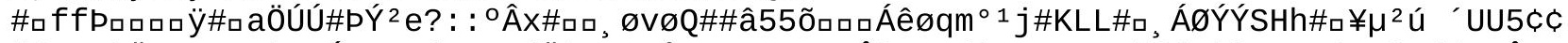

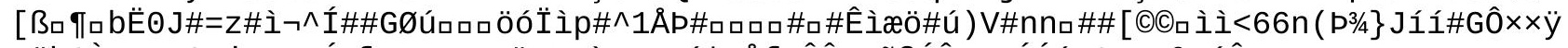

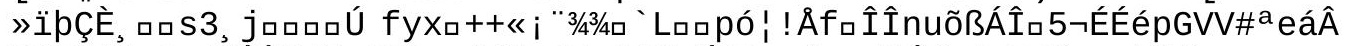

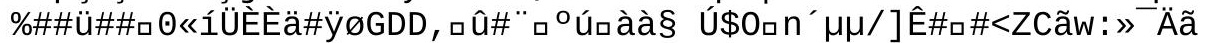




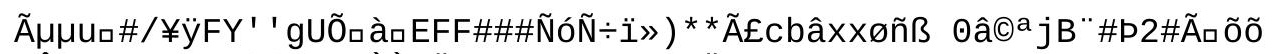

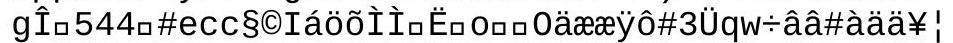

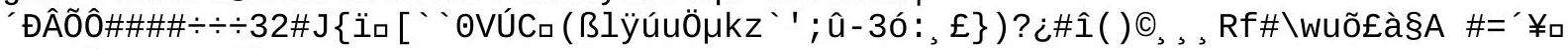

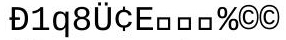

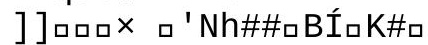




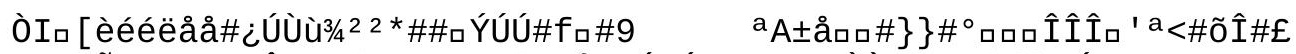

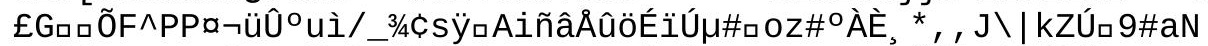

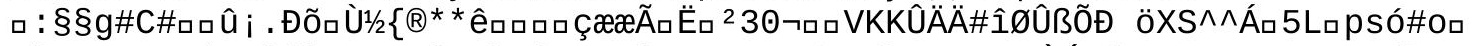

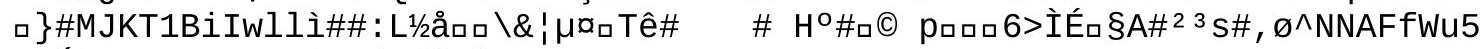

口PÁOVVV\#·ロ $\mathrm{a} \% \frac{1}{4} 8 \mathrm{qqq} \# \varnothing i R$

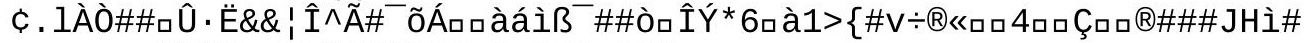

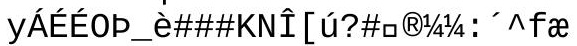

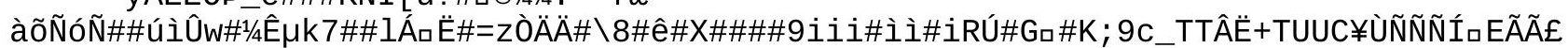

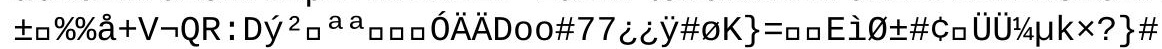

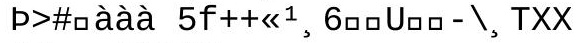

\#\#nn॰òòJ *\# 


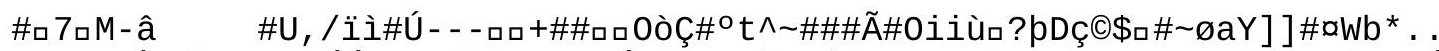

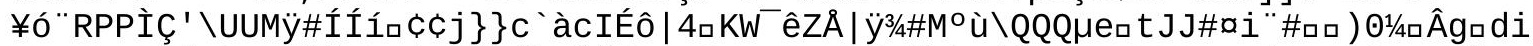

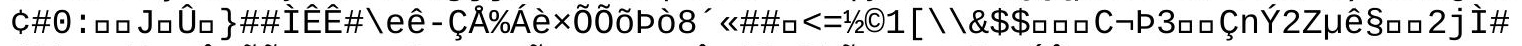

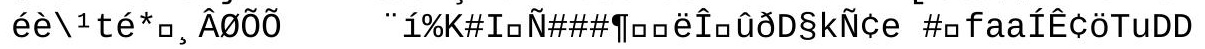




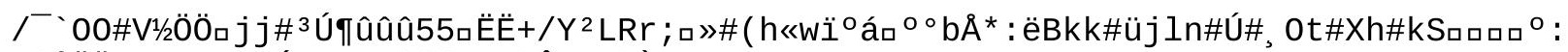

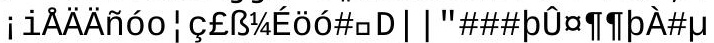

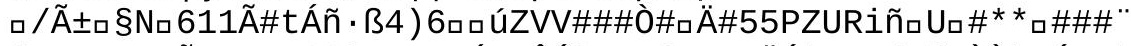

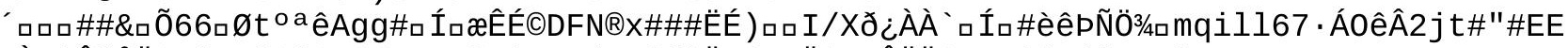

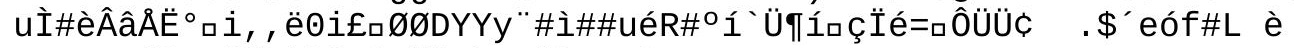
\#GzzÆðhAAQmí+\#\#ロt.ロ ${ }^{2}{ }^{2}$ ฉロÇם 


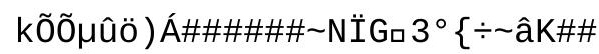

Ánqôôôñò

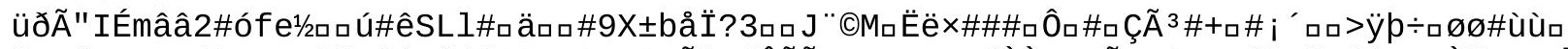

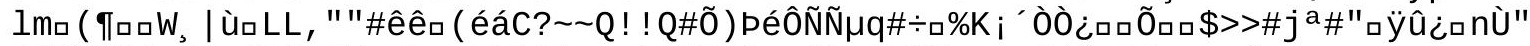

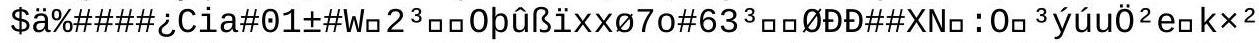

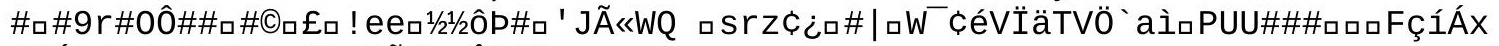
C*ÁE\#\#S'\&páiA ãÇÇ 'Ã\#ロâ̂tóò

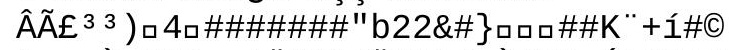

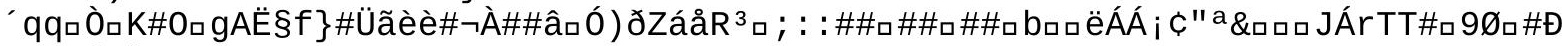

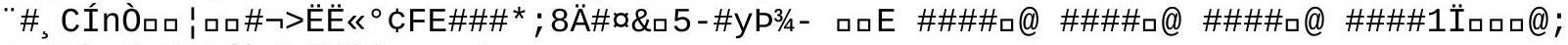

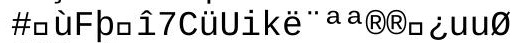

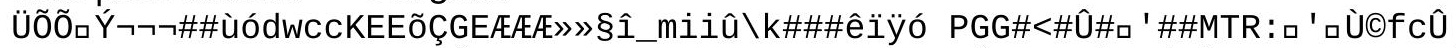

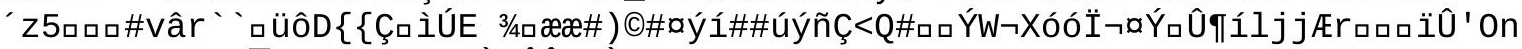

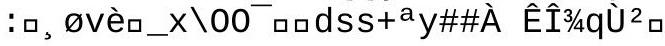



«àúé§uñ̃̃ )p_ \\zñâå 



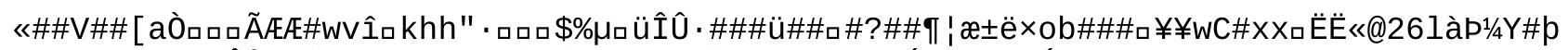

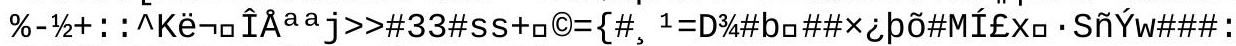

íöõõkëÖ]åån\#\#Ùìi<iio K22®Âööãagp×c' 7uèĐ\#33Kð口

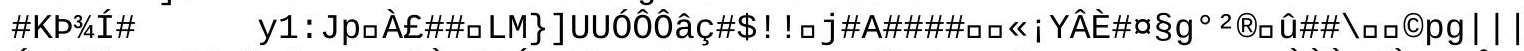

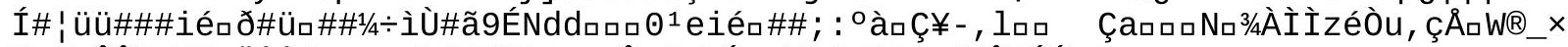

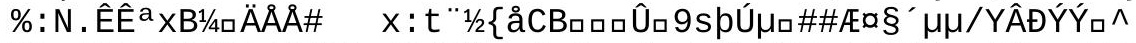

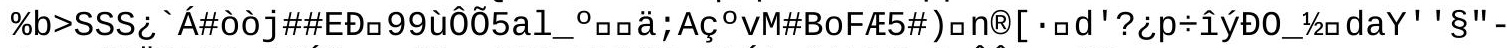

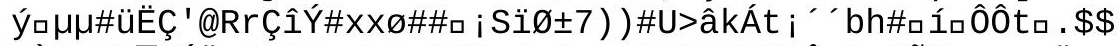

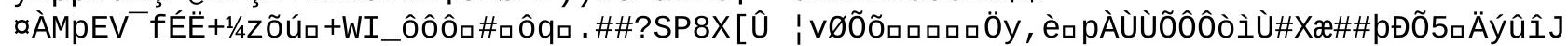

Jja\#IâââA\# \# ${ }^{3}$ vín\# 



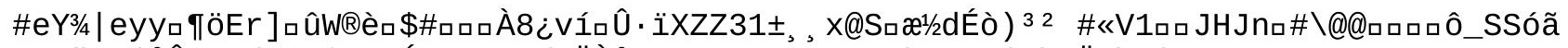

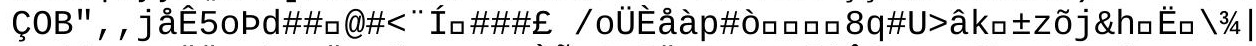

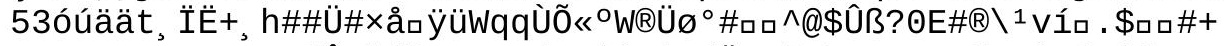

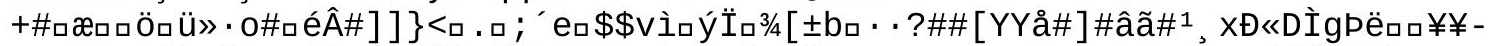

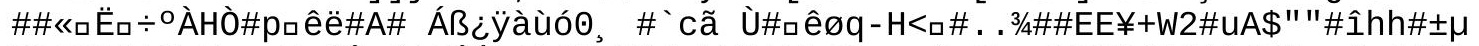

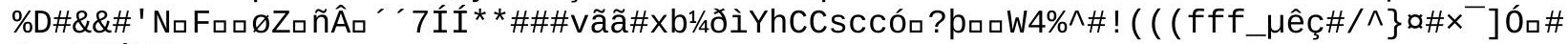
'yEEÕó§/ 


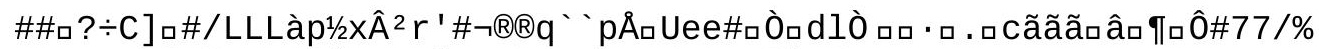

\#ャýýý\#\#[‘ðÇ\#\#_]Ý;

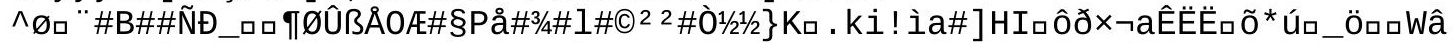

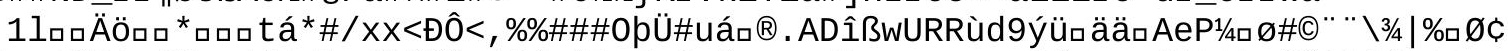

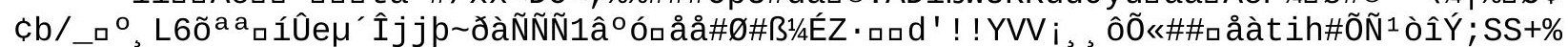

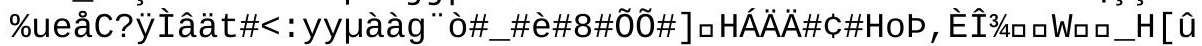

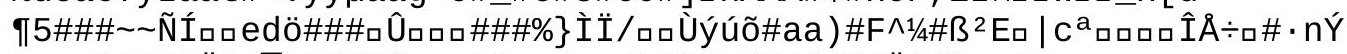

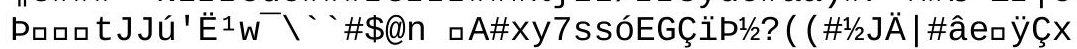

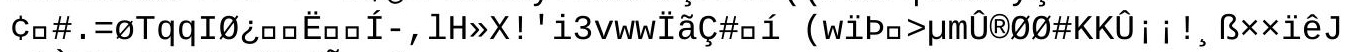

ฉ\}òĐĐtt1/4\#\#ÖJ@@\#0̃<ฉ2

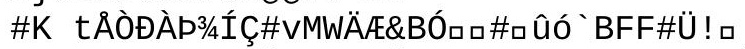

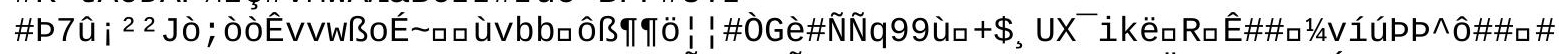

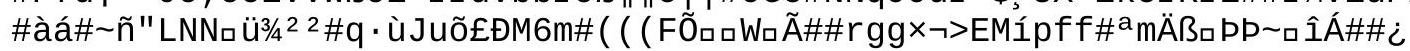

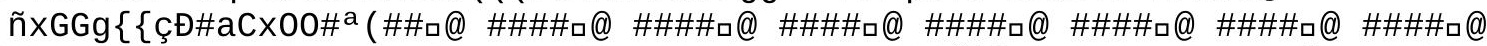

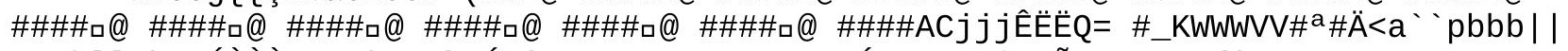

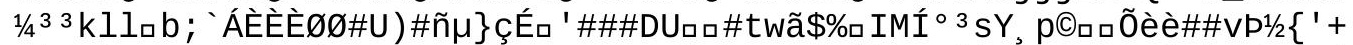

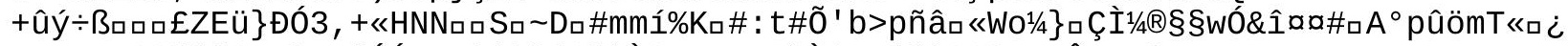

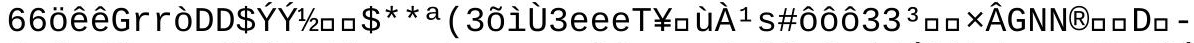

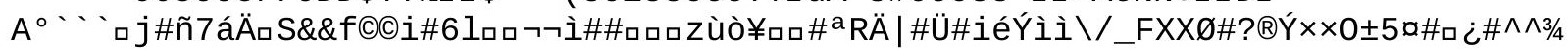

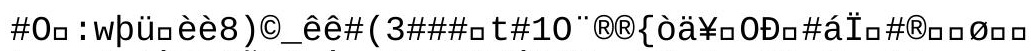

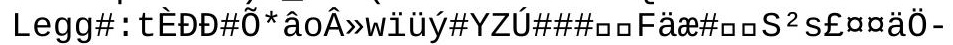

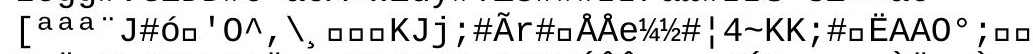

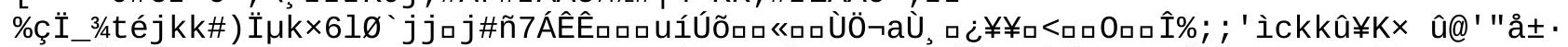

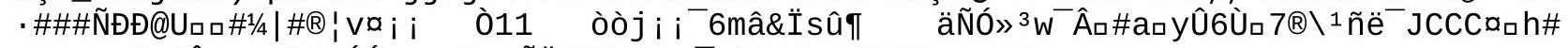

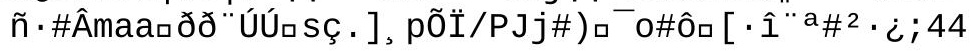




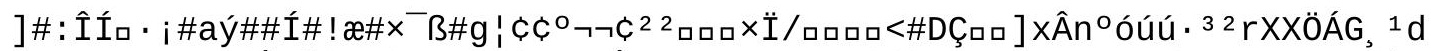

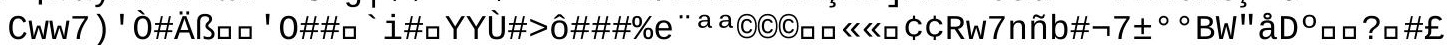

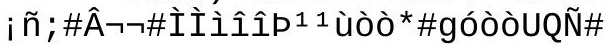




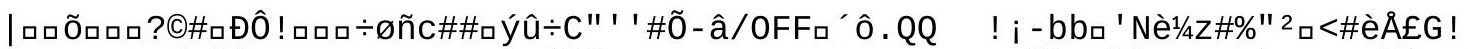

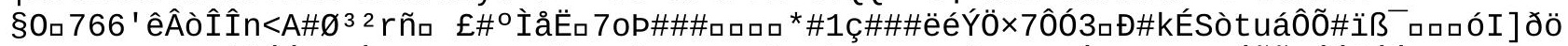

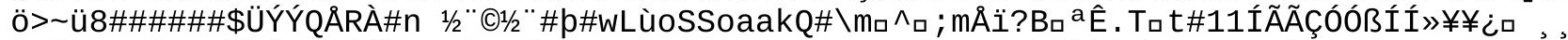

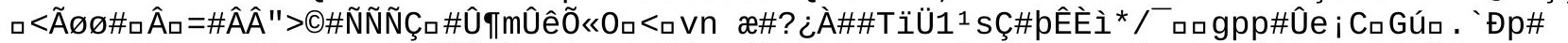

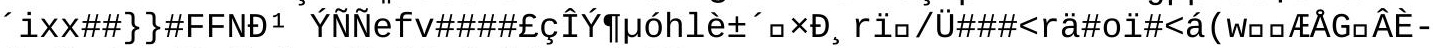

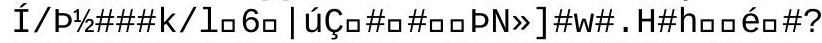

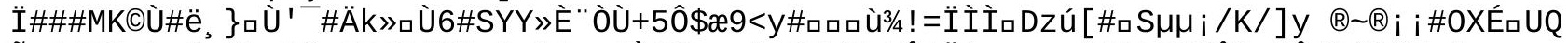

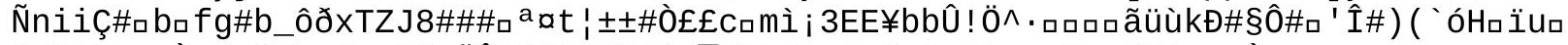

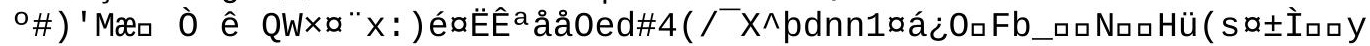

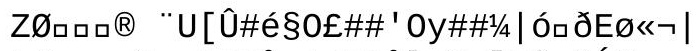

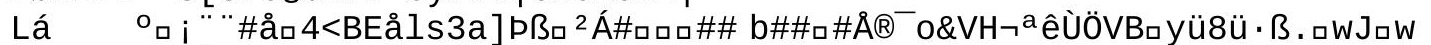

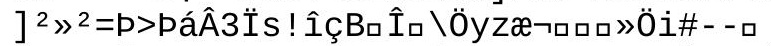

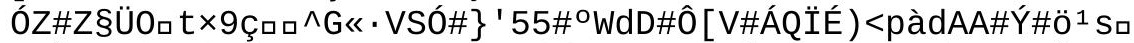

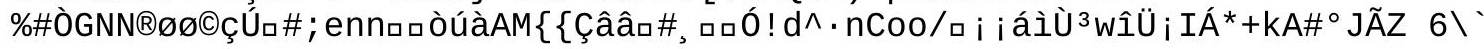


Æ¥òònæ|

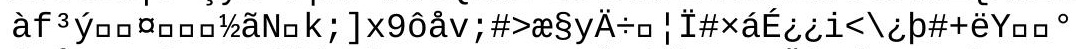

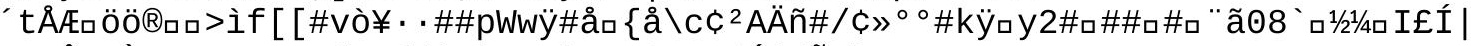
æäIÂ ${ }^{1} 60 ̀ G V V o ̈ 70>8 \times f a a\left\{u ́ o ̂ \# e ̀ ; 7 n e ̀ c<y^{0}\right.$. . oúúááÑĐ\} \#AWC -

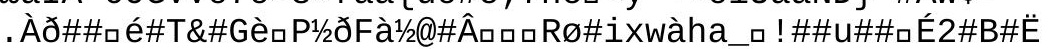




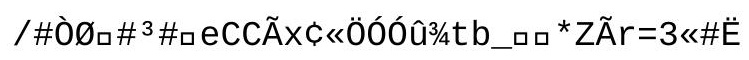




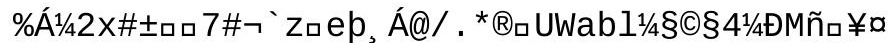

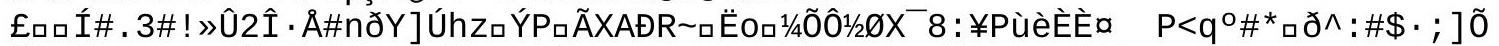

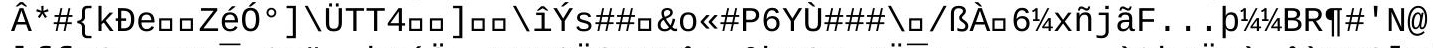

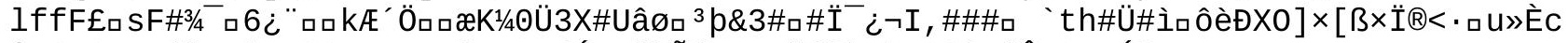

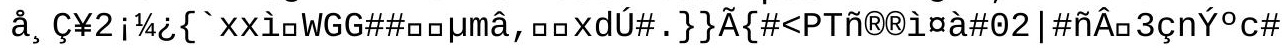




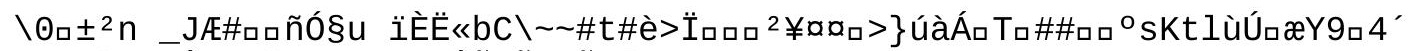

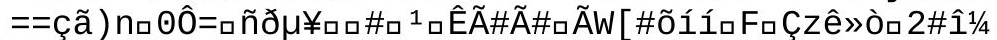

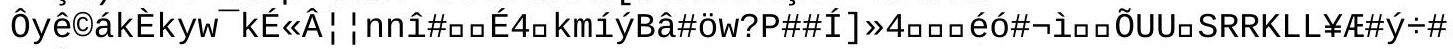

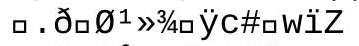

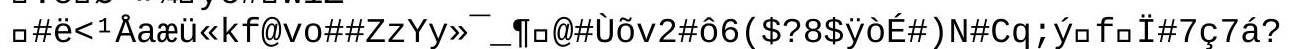

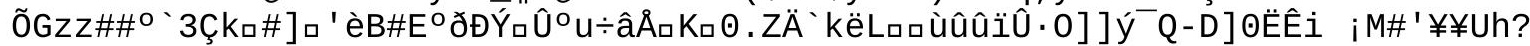

*3/4 $\{$ W\# $]$ â $\div\{$ ÀM6CฉÇo\#å 


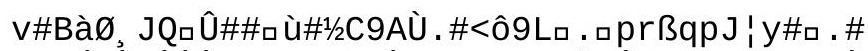

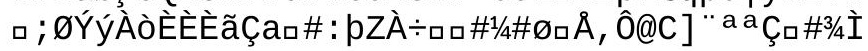




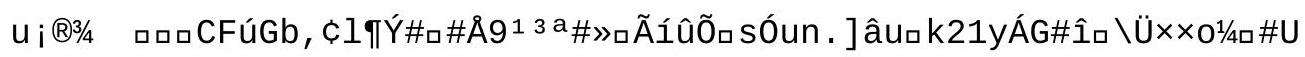




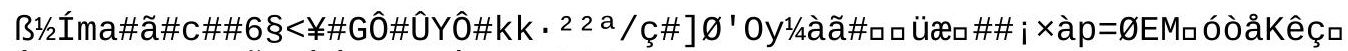

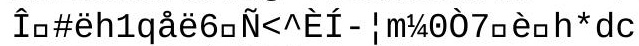

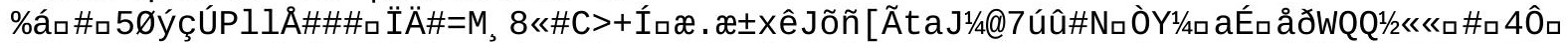

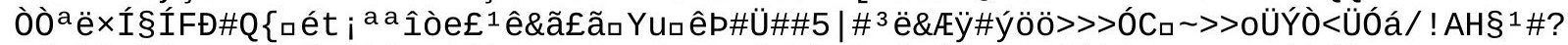

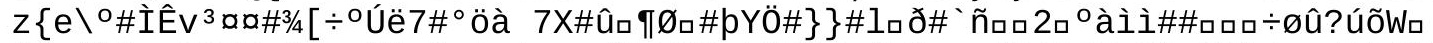

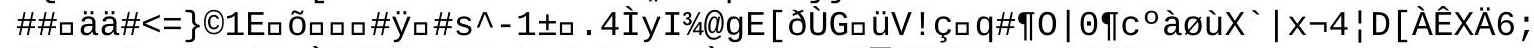

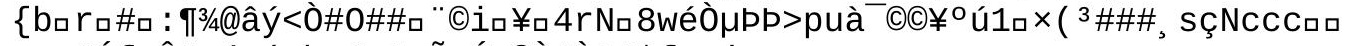

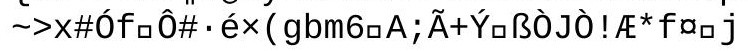




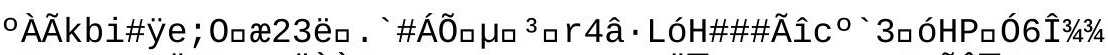

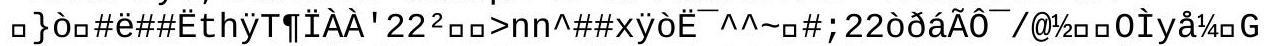

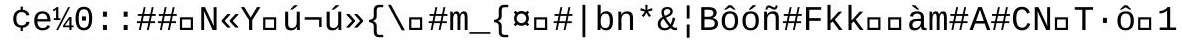

Ëöñ $[\{\# / \mid$

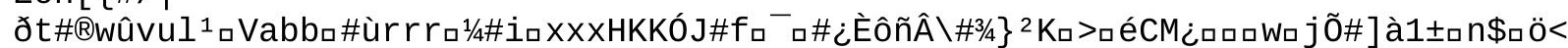

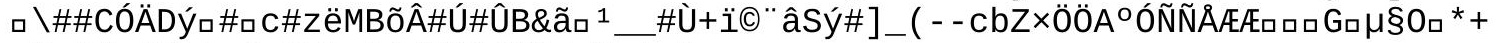

+ÿeÖ\#ロÓH4^W\#aUFFa\& ${ }^{3}$ dõÙõ

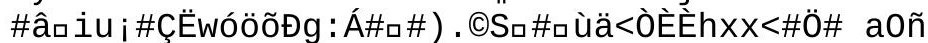

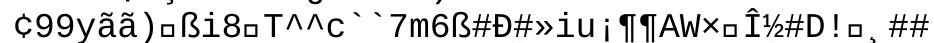

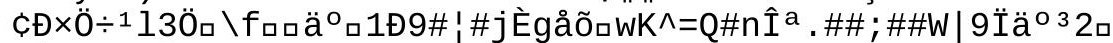

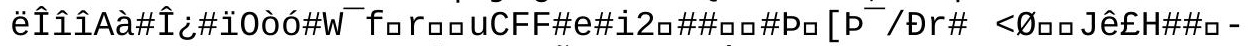

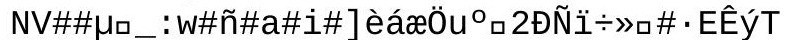

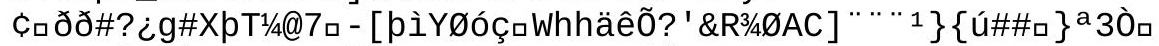

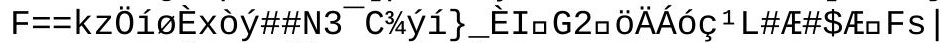

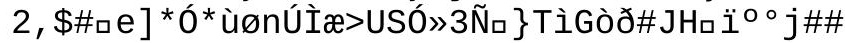


V\#Ï\#\#ロX»ロýÍロlê 


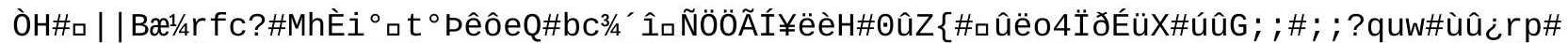

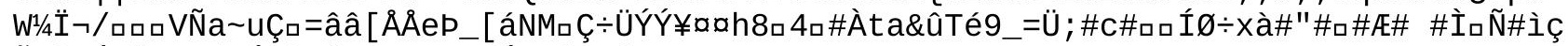

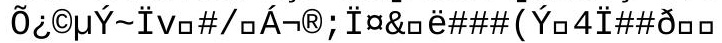


õß口 ¿hùò5 



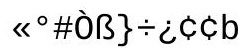

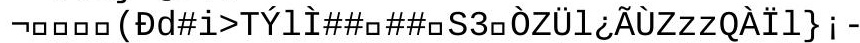

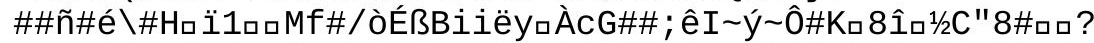

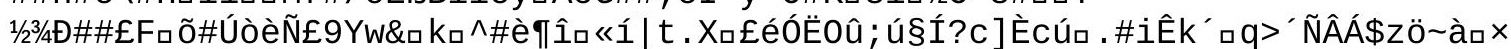

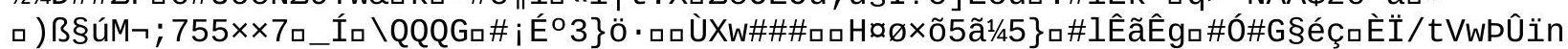

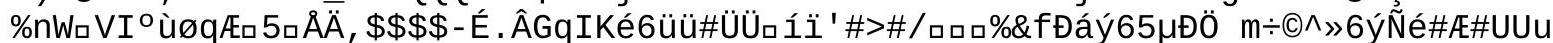

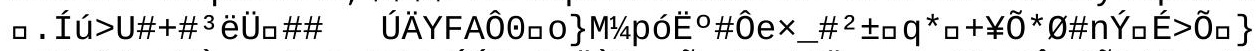

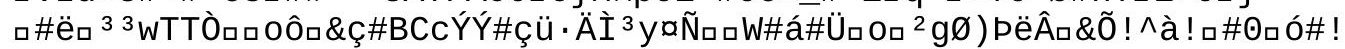

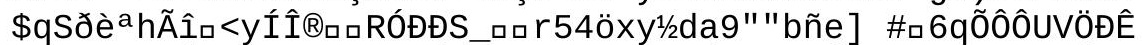

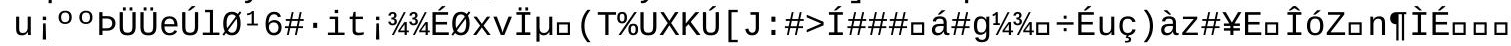

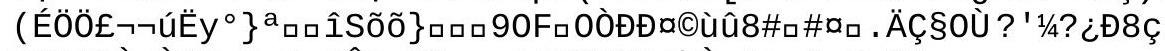

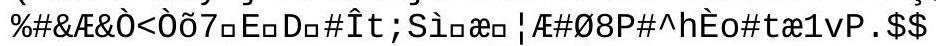

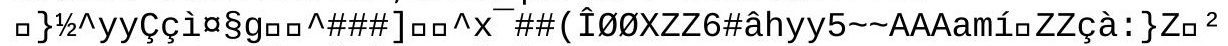

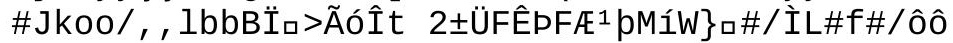




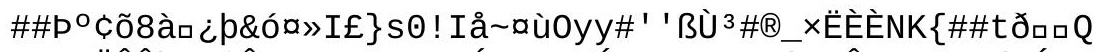

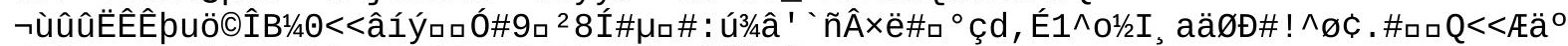

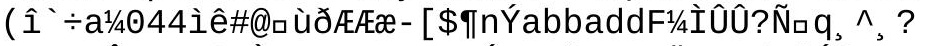

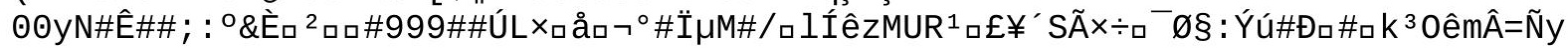




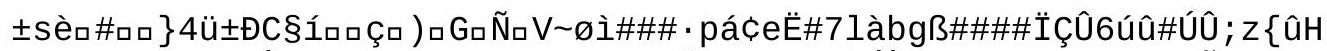

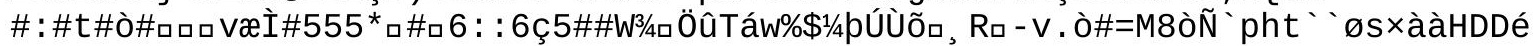




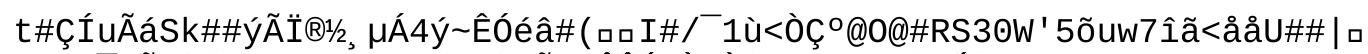

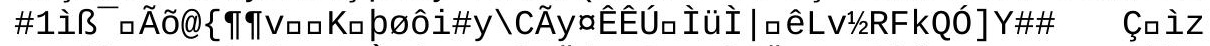

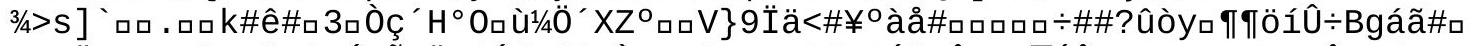

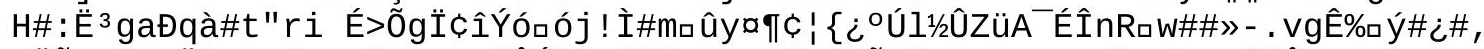

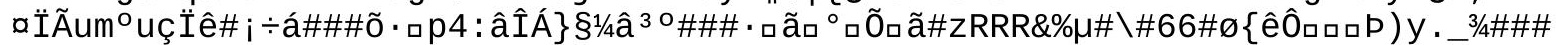
$\div 0_{\square}<\operatorname{chh}$ qu $\ddot{E} \hat{A} \hat{A}$

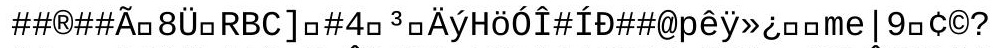

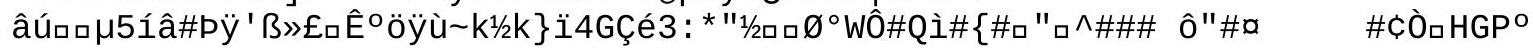

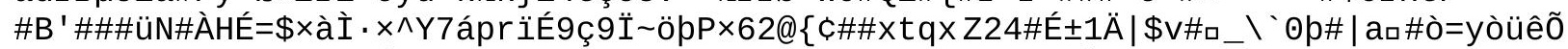

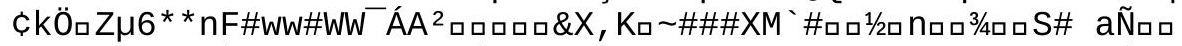

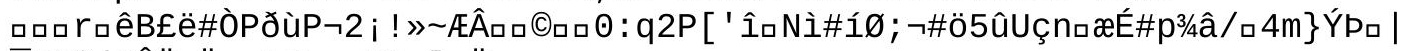

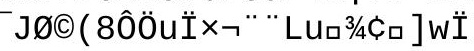




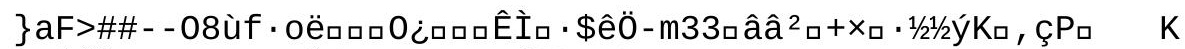

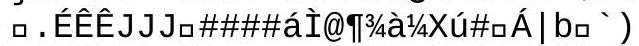

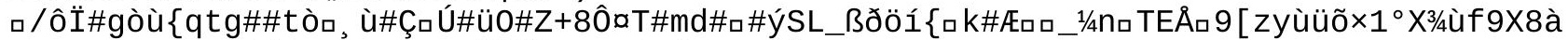

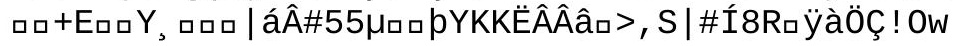




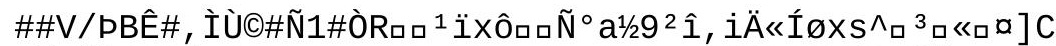

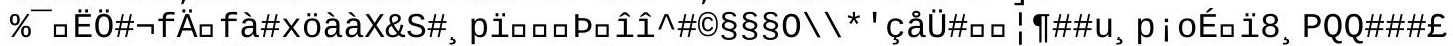

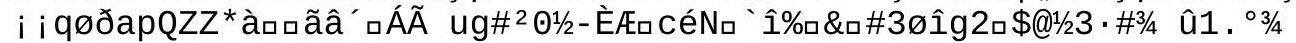

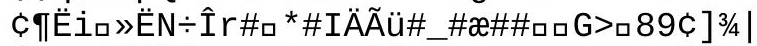

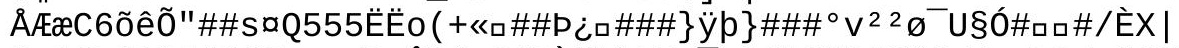

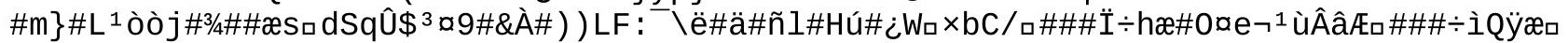

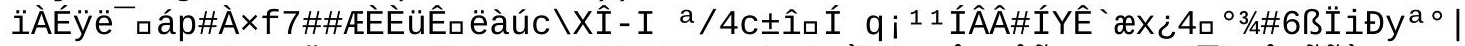

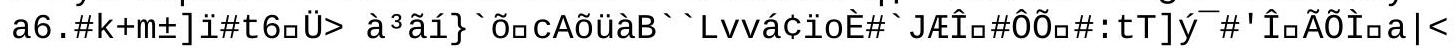

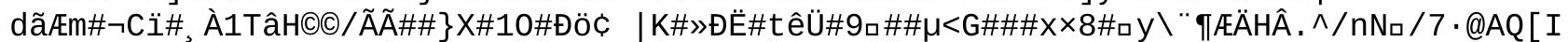

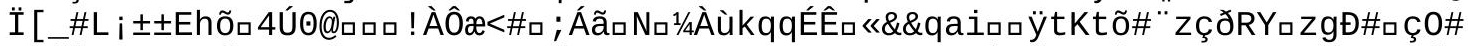

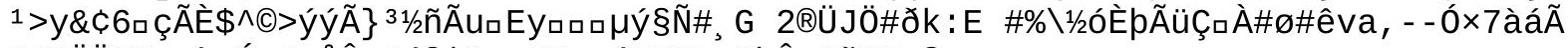

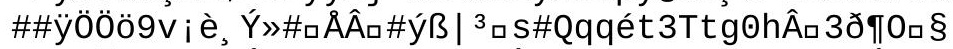

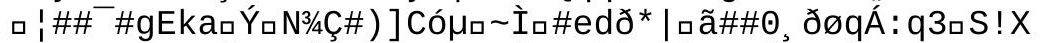

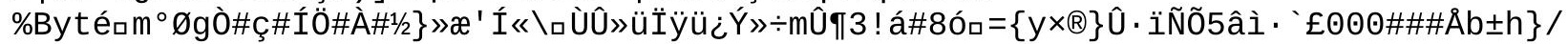




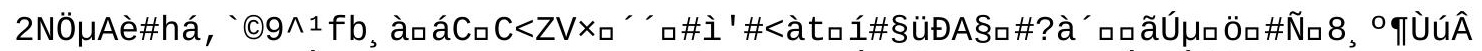

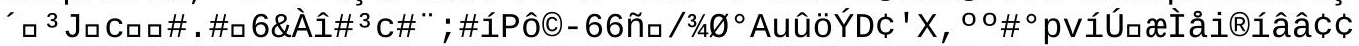

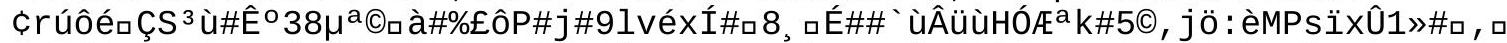

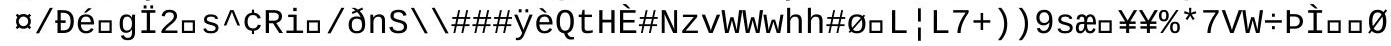




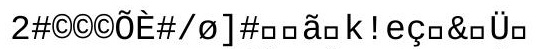

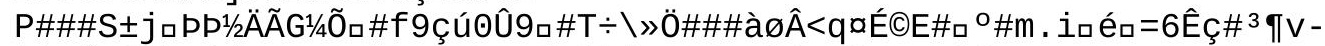

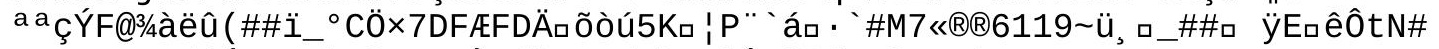

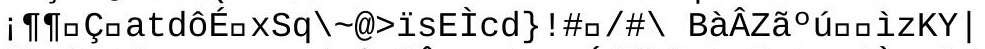

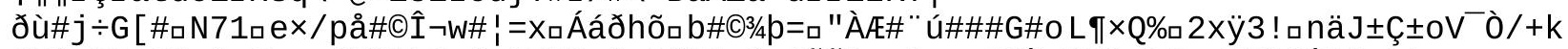

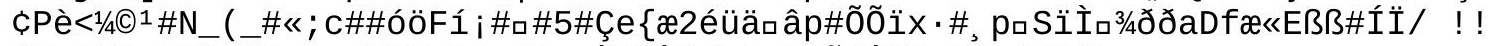

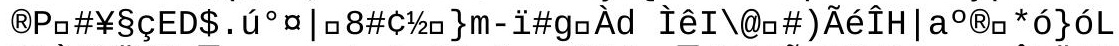

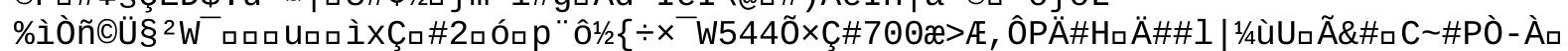

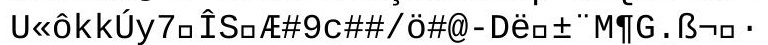

\#Gú $\S \emptyset_{\square}+W o ß 3 / 4[Y Y S \wedge p \# x E E$

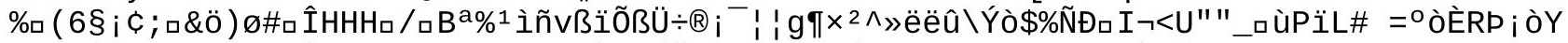

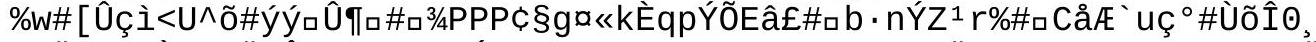

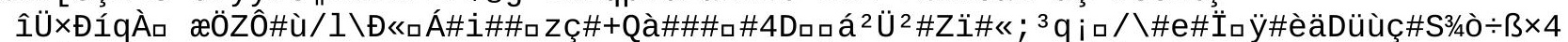

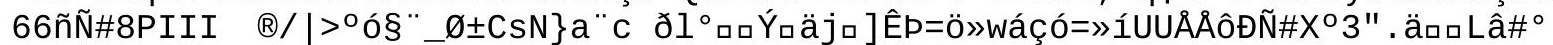

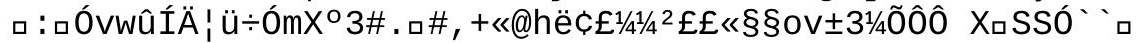




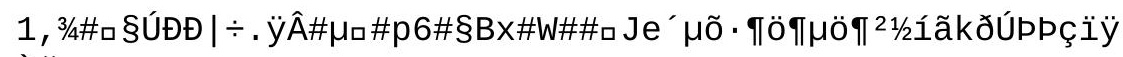

à\# 


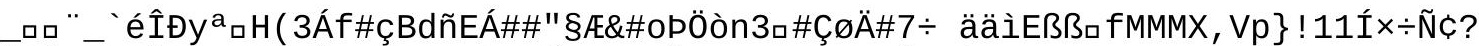

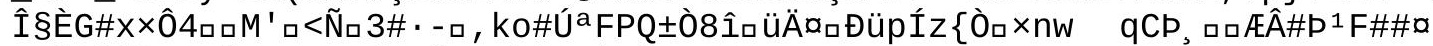

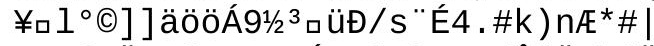

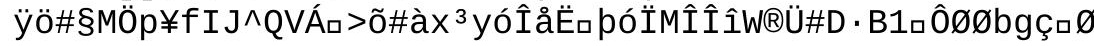




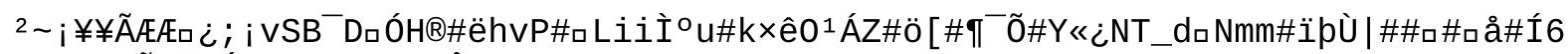

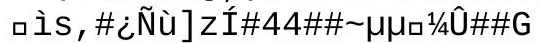

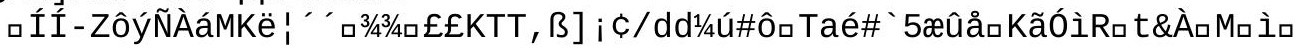




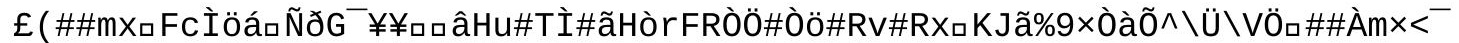
'Rpa ${ }^{1} \#=28$ ü $^{*}$ ¿XEåî \úÂG"

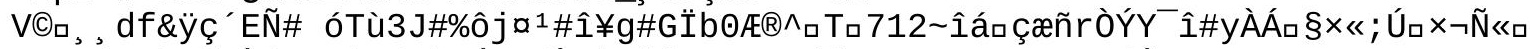
口\#а i âHpùHð, Àúör . â\#/· \} pÝH=/Ė8ùH|ÓququHsýú\#\#\#0 $i \hat{A} \gg X_{\square}$

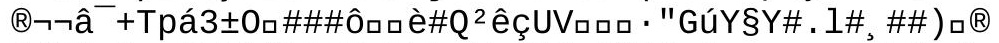

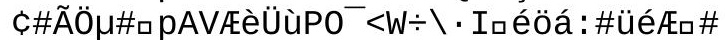

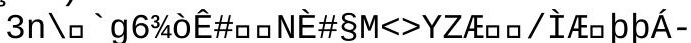

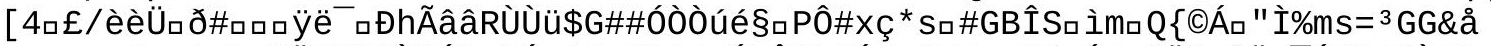

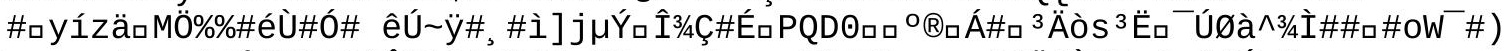

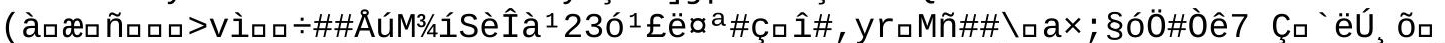

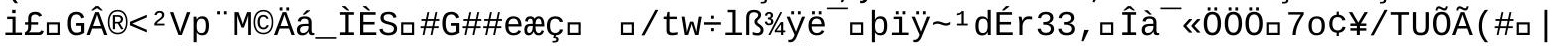

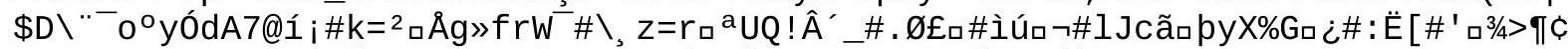

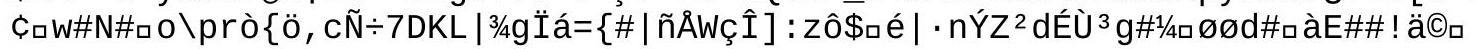




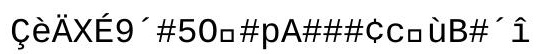




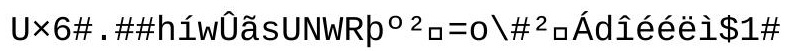


AB1॰0̂ôôF\$"6ロÔ\#ÚÚ: : \#?øo§Li

$\# \#$ \# $>$ NabáÂ $\mu \mathrm{ÈM \#}$

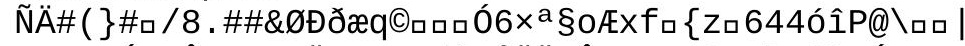

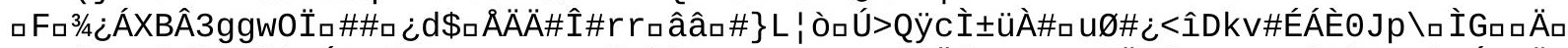

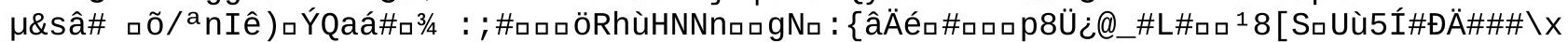

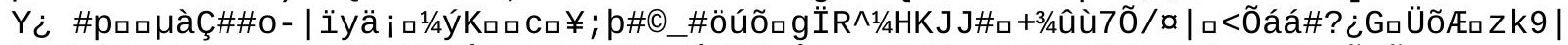

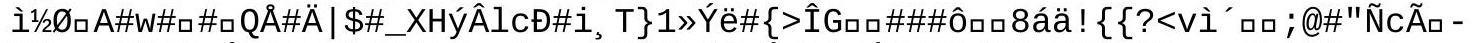

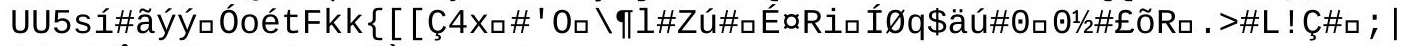

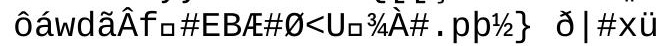




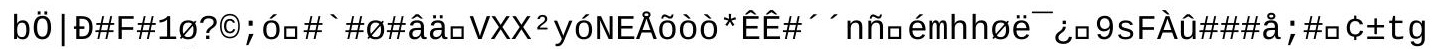

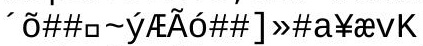

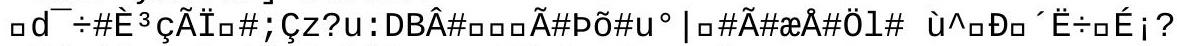

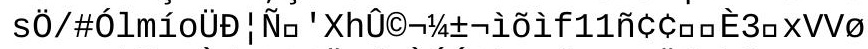

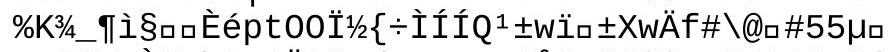

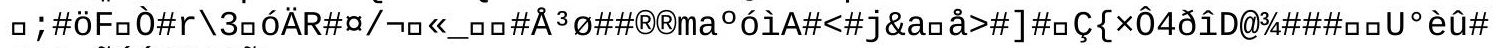

c] ${ }^{1}$ rõúúfúíêõk

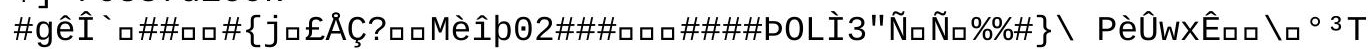

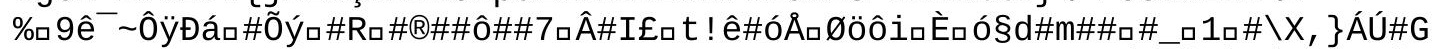

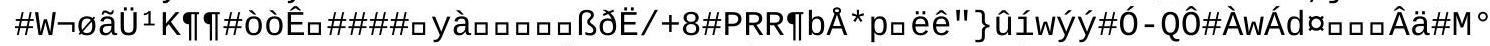

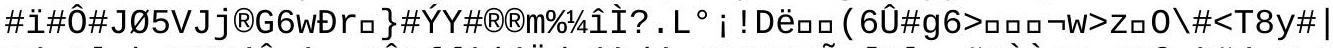

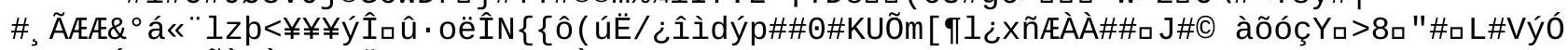

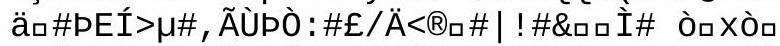

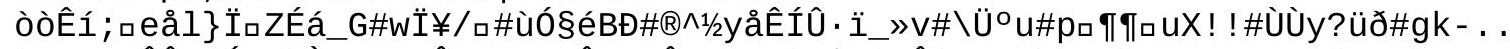

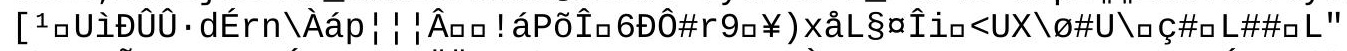

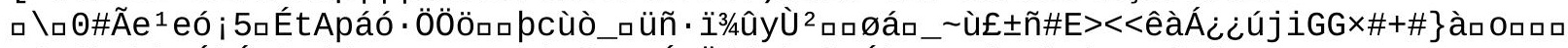

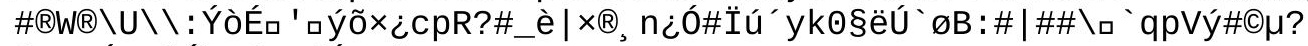

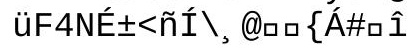




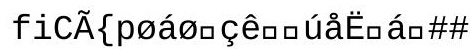




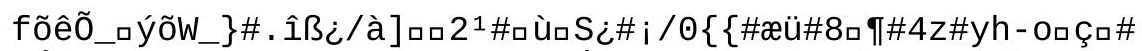

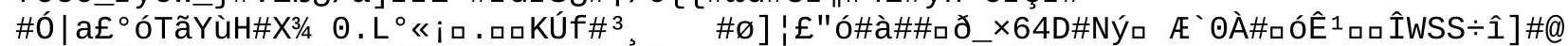

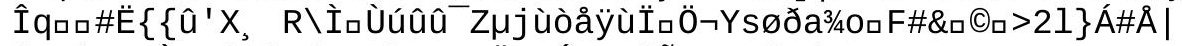

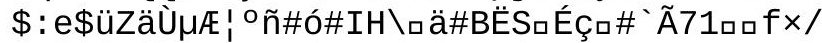




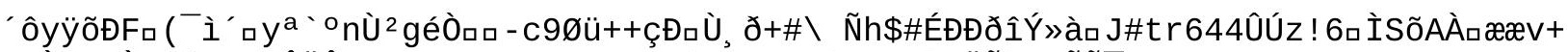

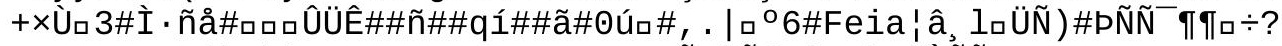

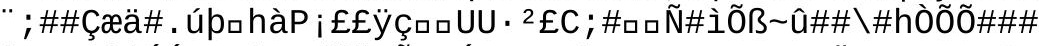

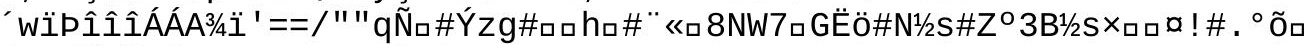

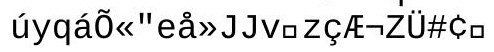




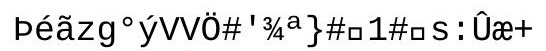

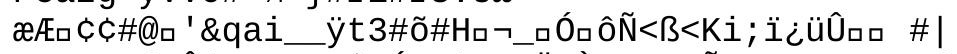

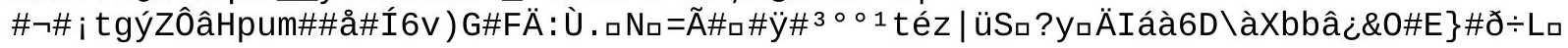




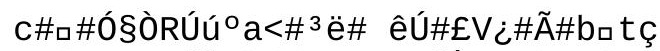

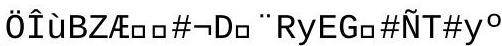

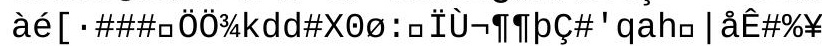

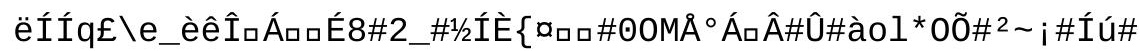




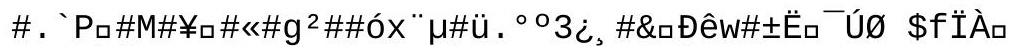

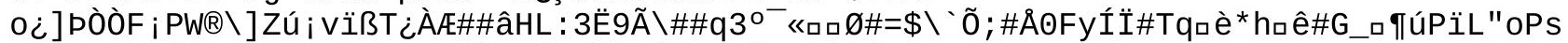

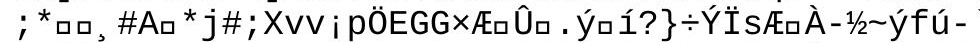

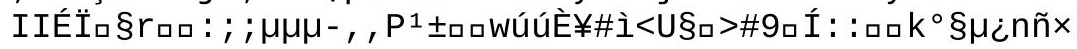




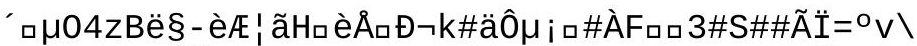

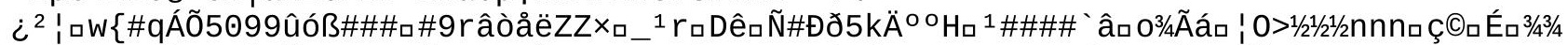

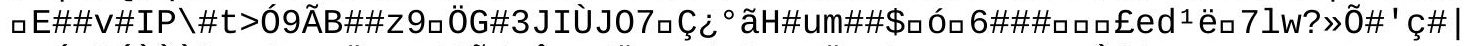

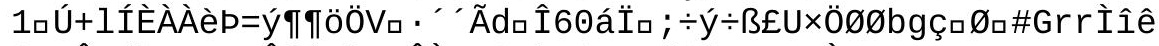

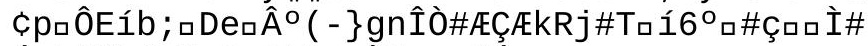

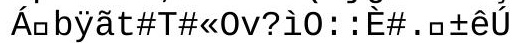

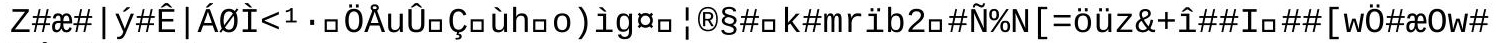

ËÍ - ú\#ıàc\# 



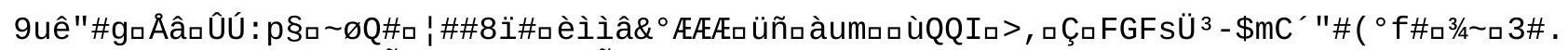

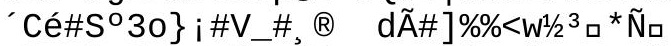

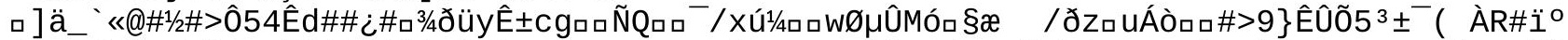

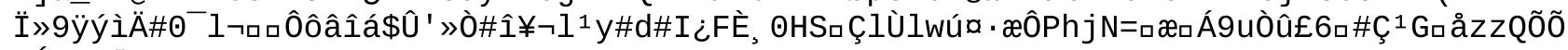
aÉpÇÇ [

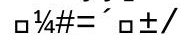




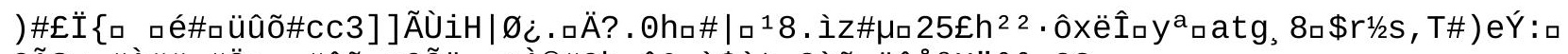

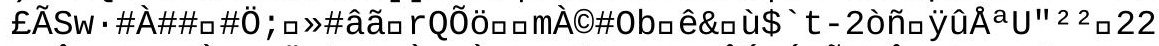

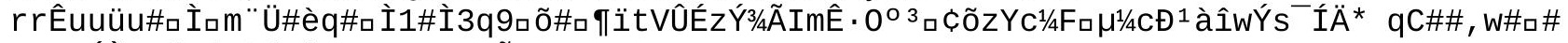

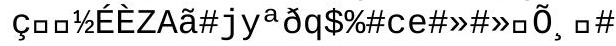




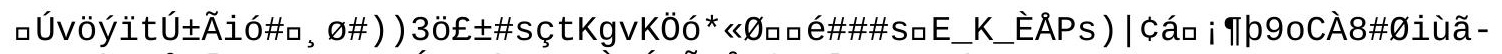

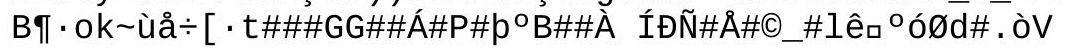




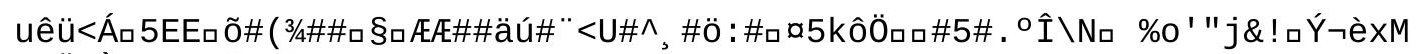

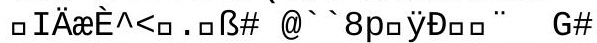

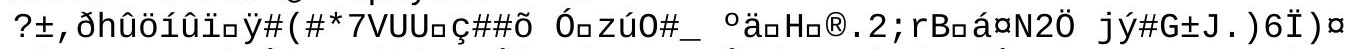

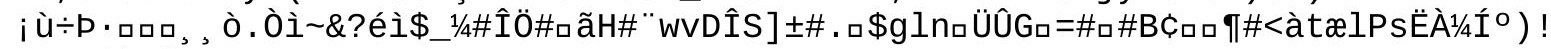
i\%Jロ | àıûे+ 

q_Ù¥\#v@Xe 'っ-®oâÊiý3ç 


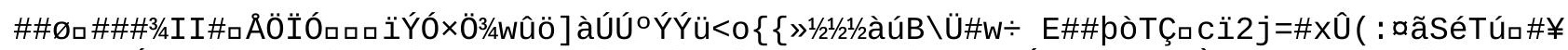

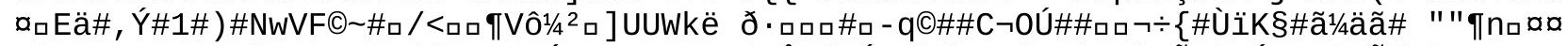

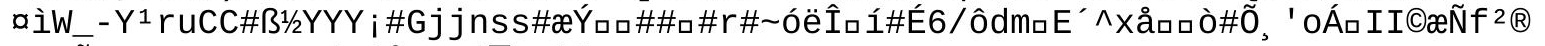

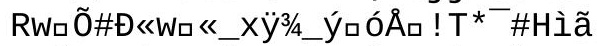

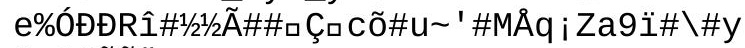

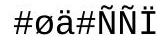

^/úpıh\#\# RRr 22

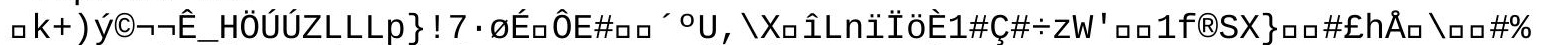

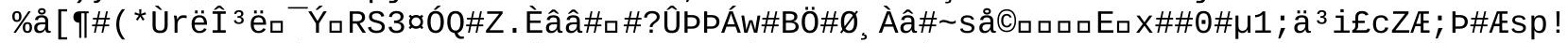

\#\#\#`ón\# ${ }^{3}$ Òn \#GaÂaA

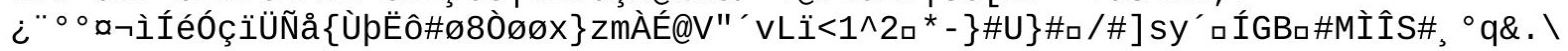
¿\#1 q£\#a. 


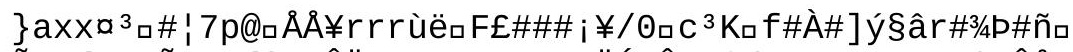

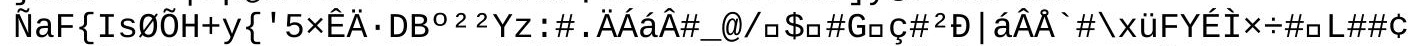

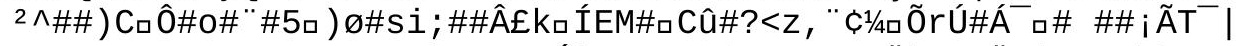

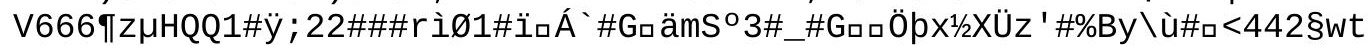




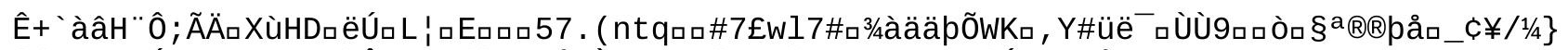

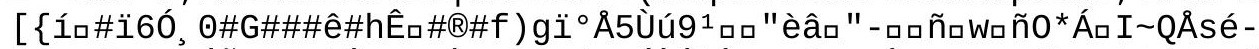

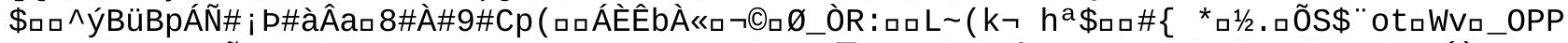

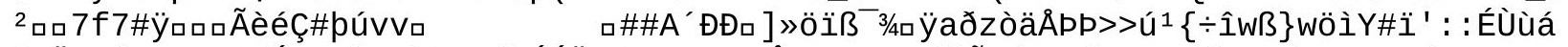

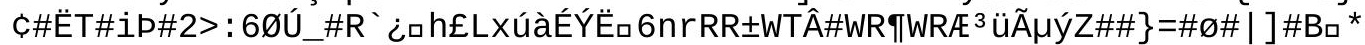

*\#

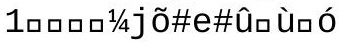




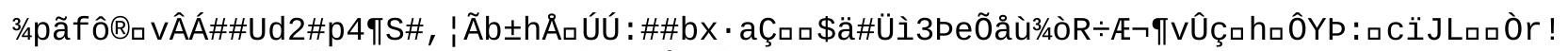

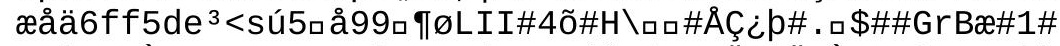

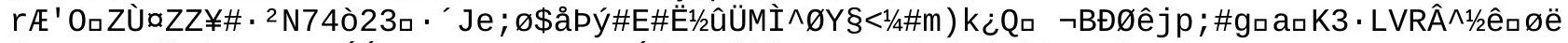

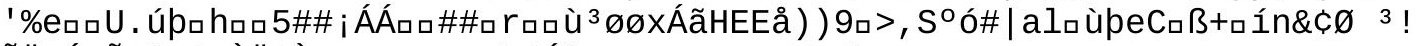

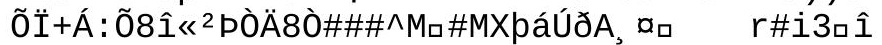




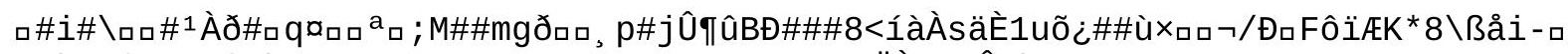
$7 \wedge \mathrm{k} \# \mathrm{t} * \circ, ! 1 / 4 \S \uparrow \mathrm{kFK} 7 \cdot \# \pm \mathrm{u} \# \mathrm{âa} \#$

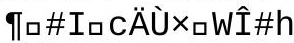

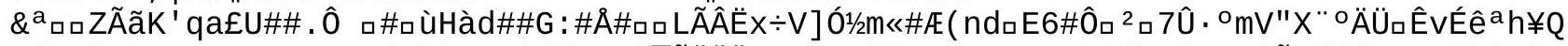

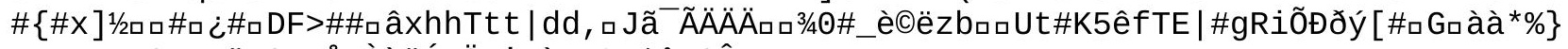
10UロUї\#Ç\#xÅロÒàëÚ\#Öם ' >è\#\#tç ${ }^{1}$ âHQÛT

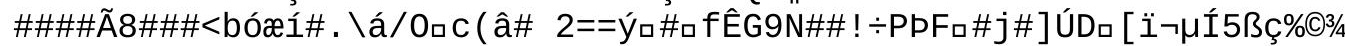

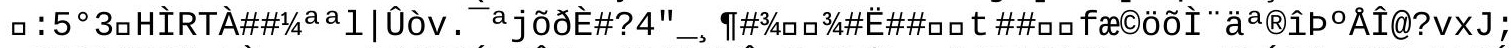

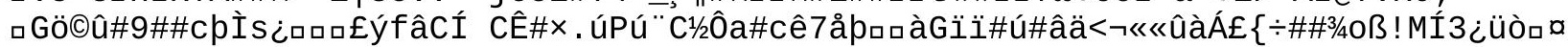

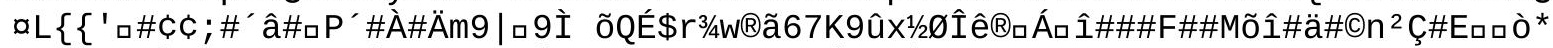

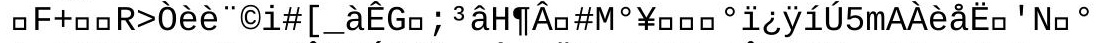

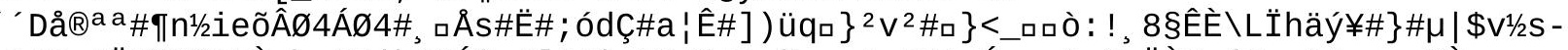

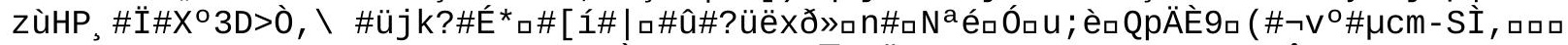

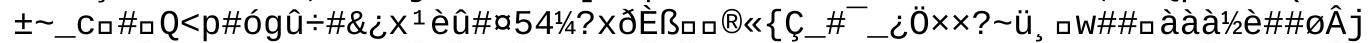

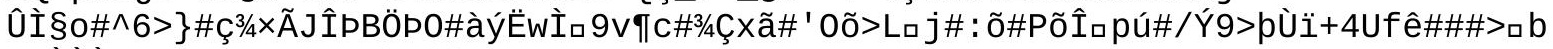

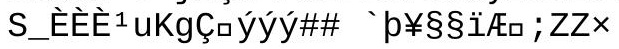

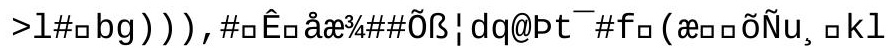




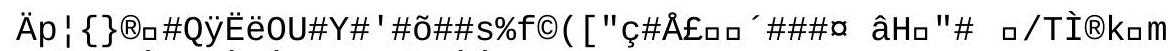

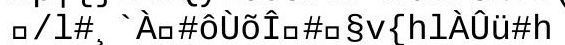

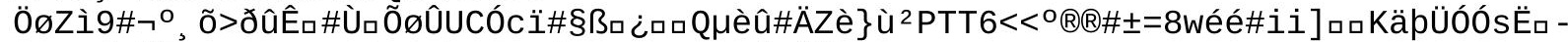

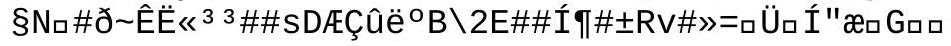




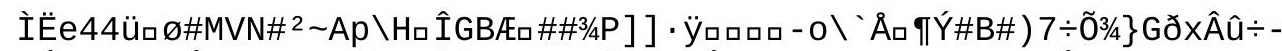

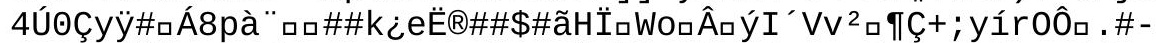

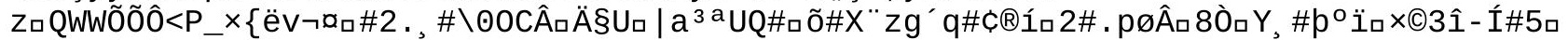
MoòêrÝ ${ }^{2} Y ́$ T\#-eì-\#\#m. ô̂\#

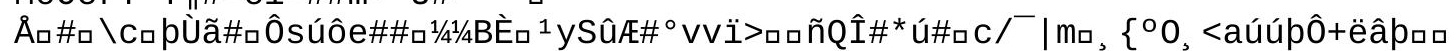

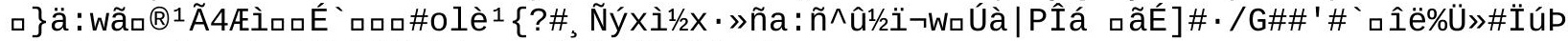

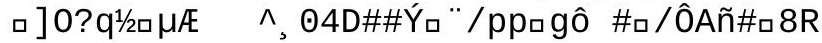




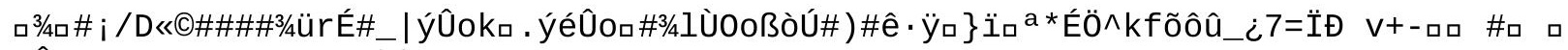

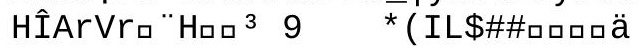




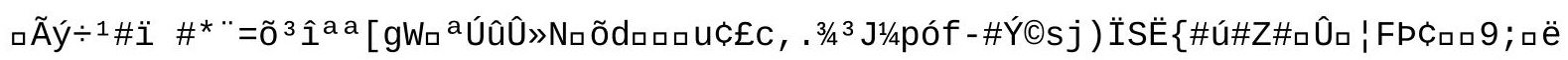




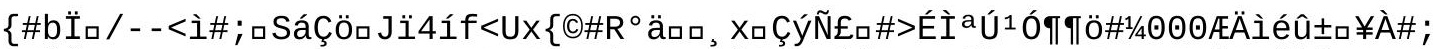

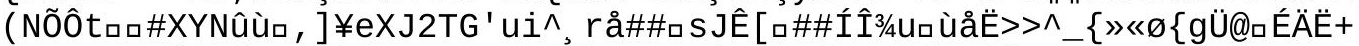

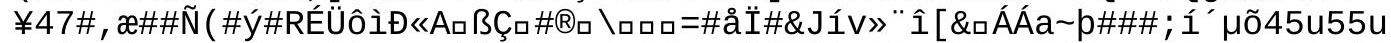

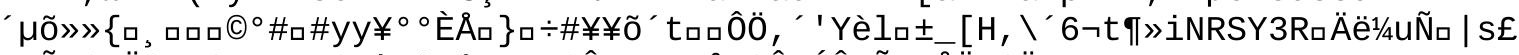

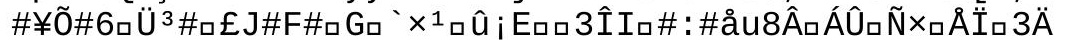

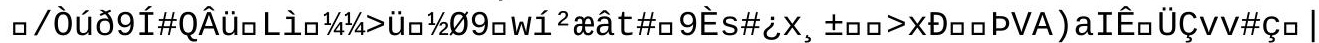

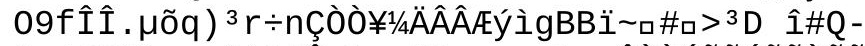

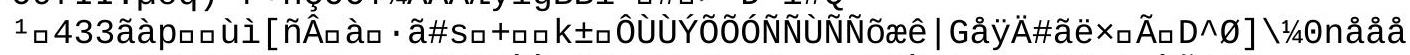

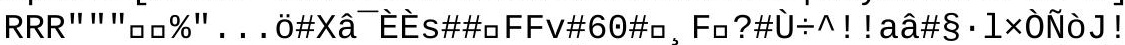

â1²\#ãã\#0̃õa·mãvp LLLúP»Đm++ i்

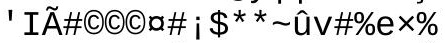

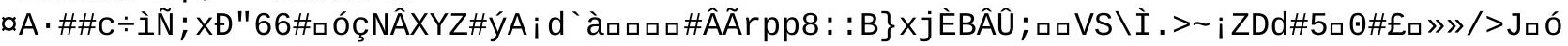
çcèèTøù 


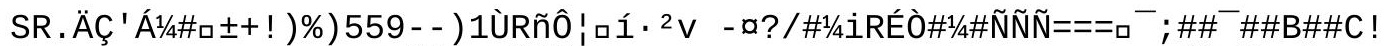

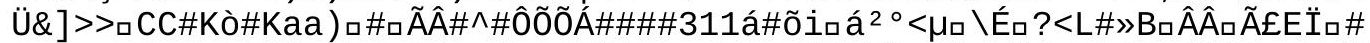

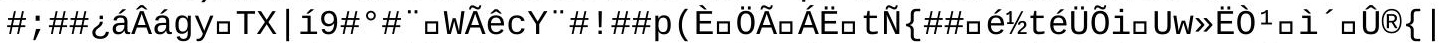

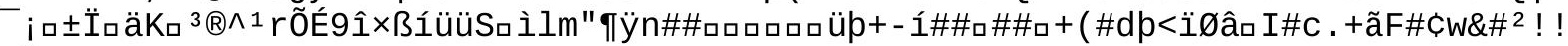
qİ॰

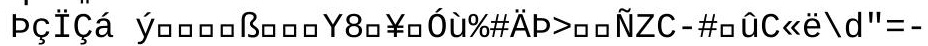

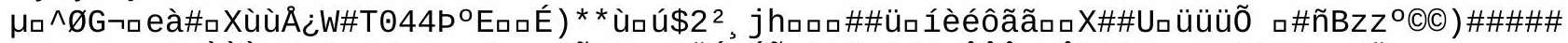

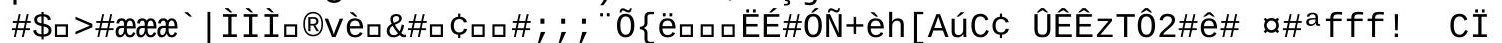

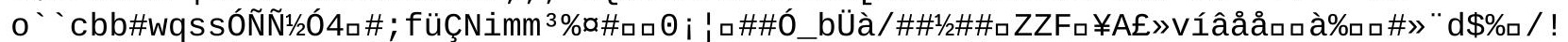

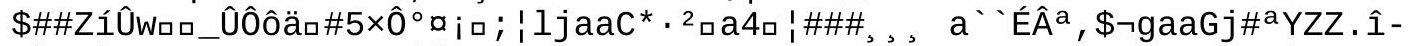

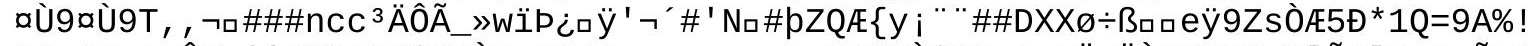

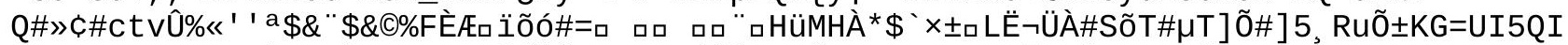

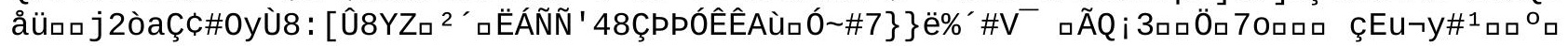
$\square$ p 


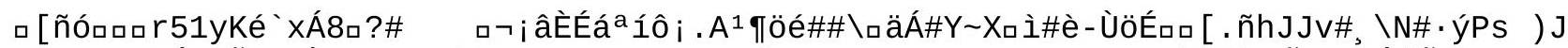

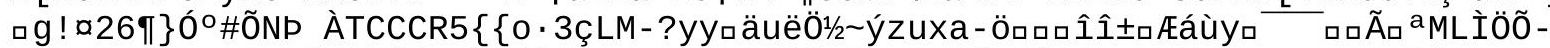

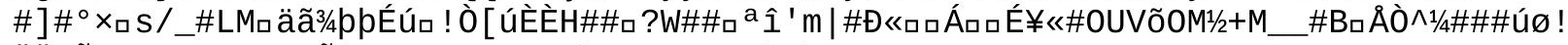

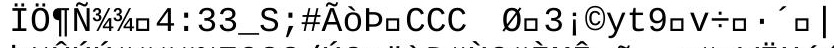

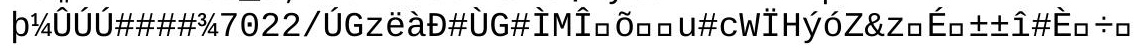




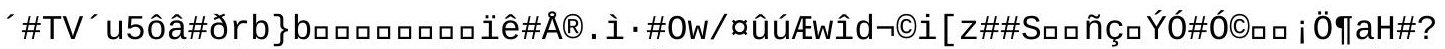

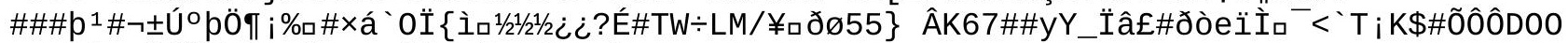

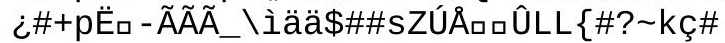


TTTÔWÑ,

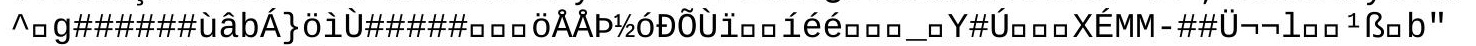

Eロロ $/ 4 \# \# \# \# Y ́ \tilde{n} \div \# E £ \tilde{n} \# \#$ ロ 


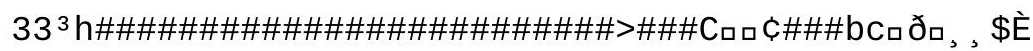
ㅁ 


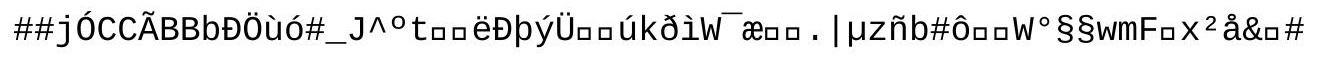



åä<ÈÁÁ\} ú 'ëa 6

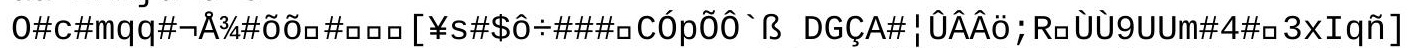

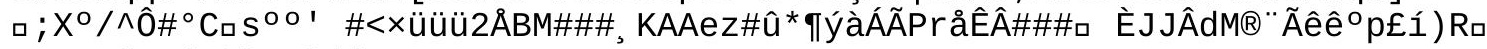

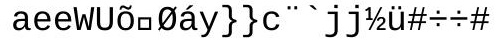

\#\#ロ»\#\#N

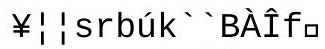
$\square \mathrm{C}^{\prime} \cdots$,

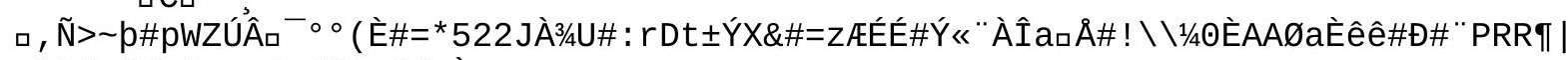

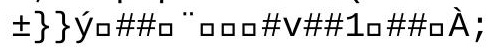




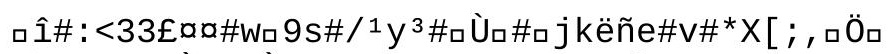

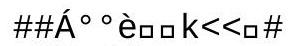

$n \backslash » v a ̃ \oplus ! A E \# \# G A ̀ p^{a} \sim \tilde{n} \tilde{n} \# \#>\# m \tilde{n} \tilde{n}$

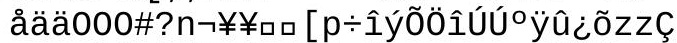

$\varnothing !$

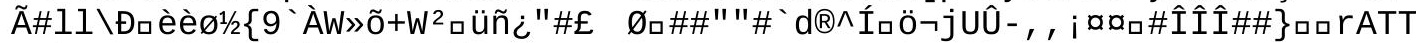

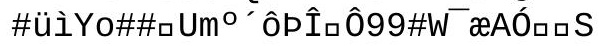

Êææ ' 3 3-UT<ùıXÁĐĐÜ/Ææ4 


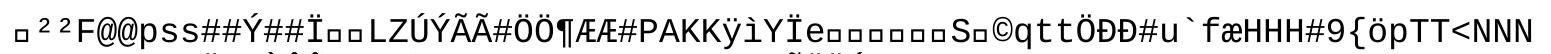

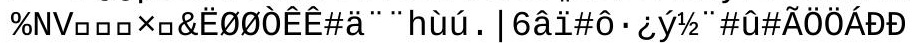




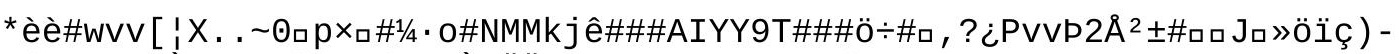

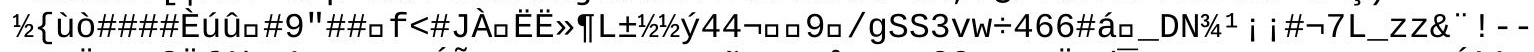

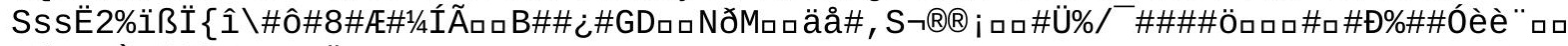

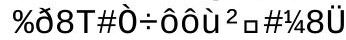


ÁÂKJJ ; zT 


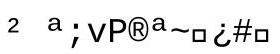




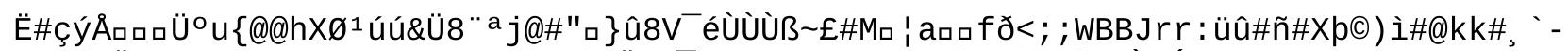

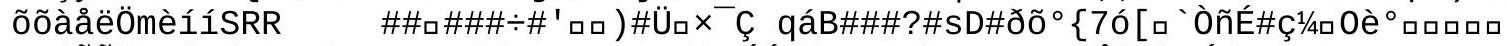

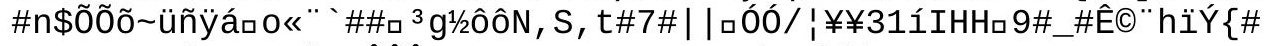

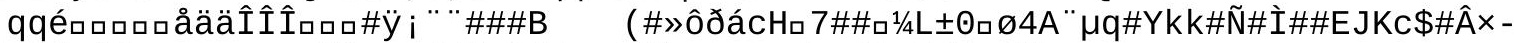

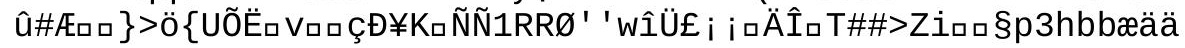


\#yûÖロ\#lロg\#ú ÆSVV!

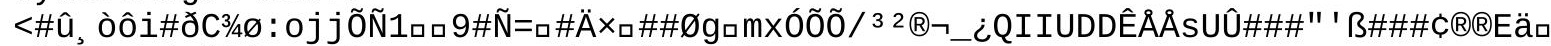

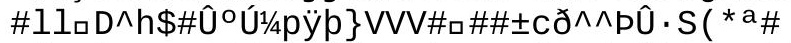

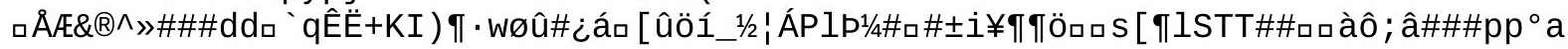




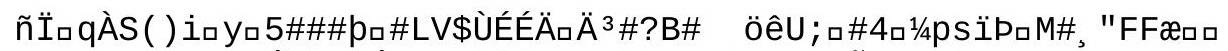

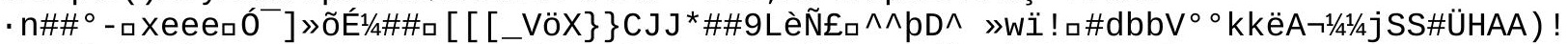

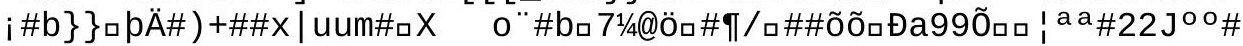

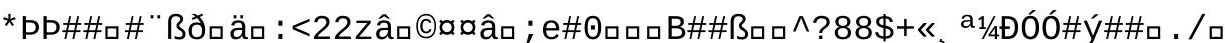

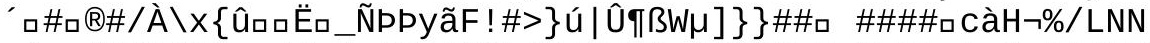

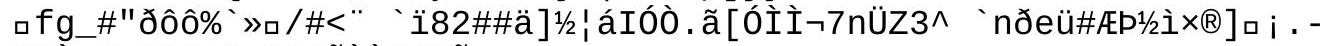

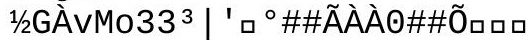

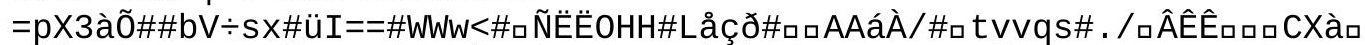

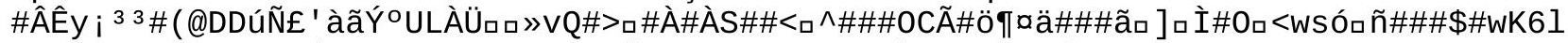

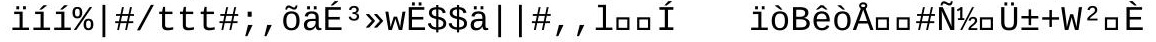




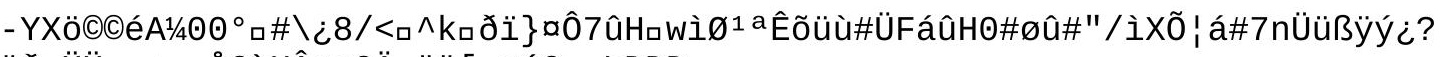

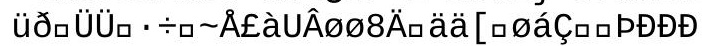




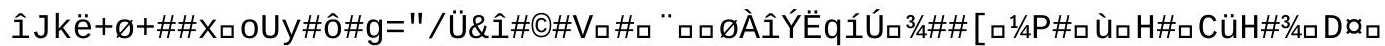

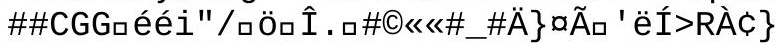

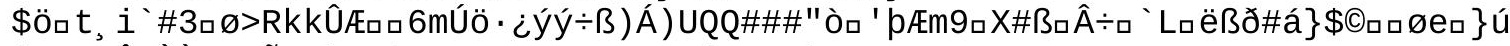

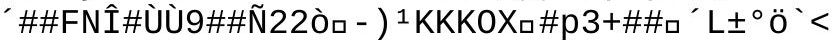

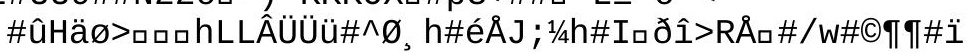


3/4 \#\# $\mathrm{V}^{\circ} \cdot$ 


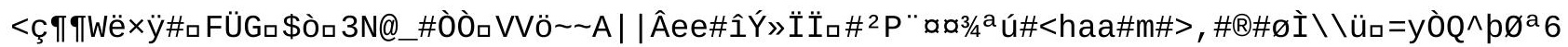
$D<44$ 
$j+I^{-} \#$

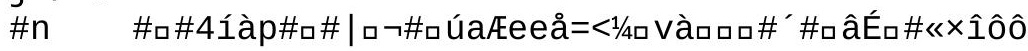




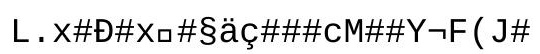




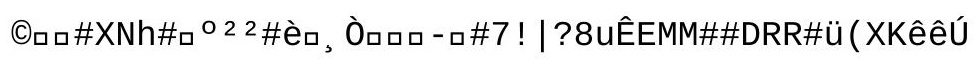





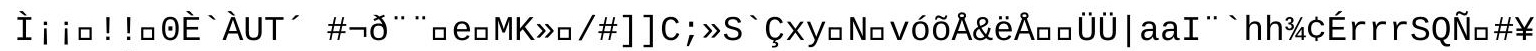
a\#q»\#Ё\#ѐ\#ѐ\&3ó

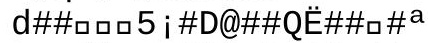

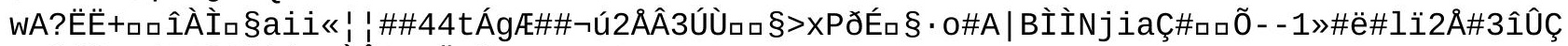

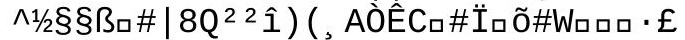

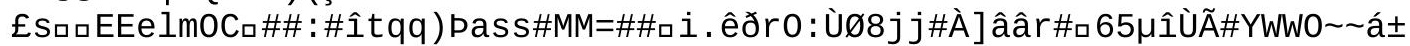

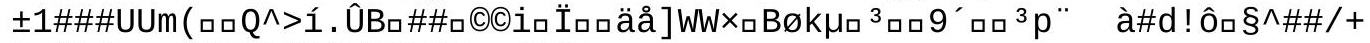

$\{x \tilde{n} b \#$. . 3/4\#Mëë\#â\#1/2\#HOÏ̈̈̈ - \#Ø1̂A

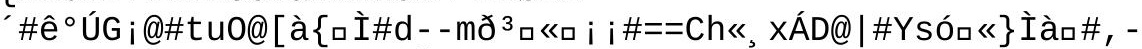

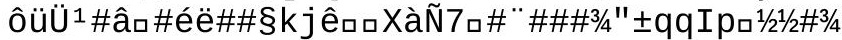




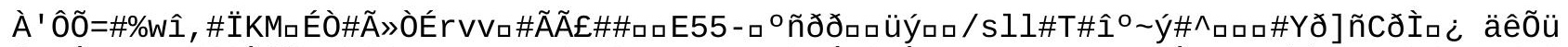

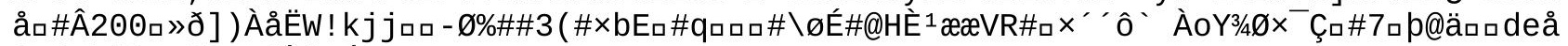

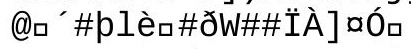

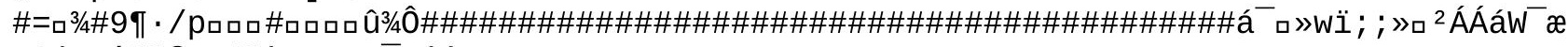

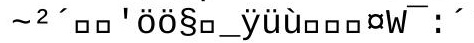




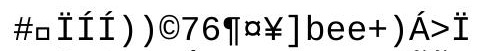

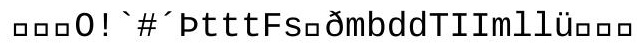

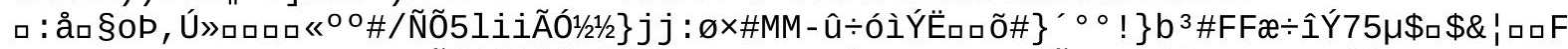

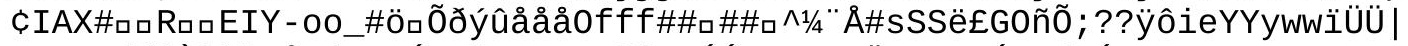

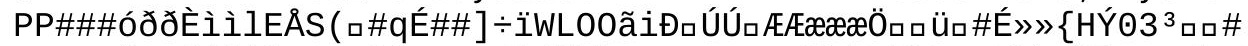

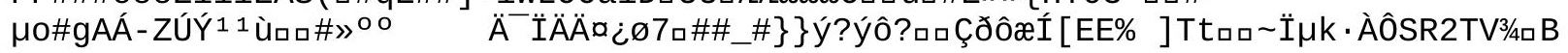

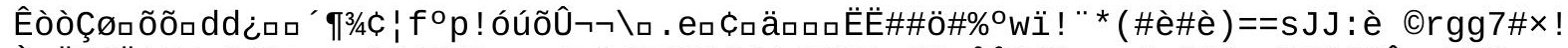

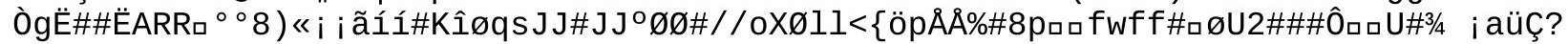

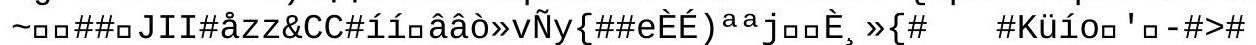

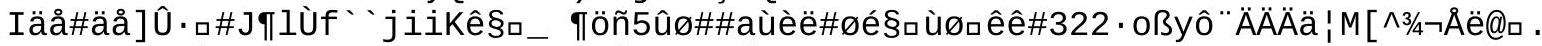

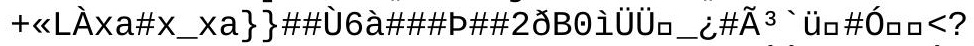

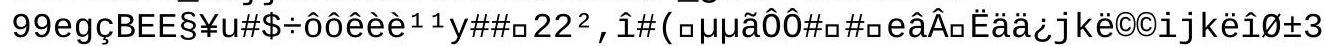

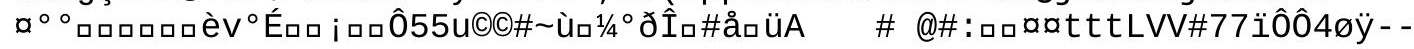

$X 9+\ddot{q} \ddot{\mathrm{e}} \times 0 \# \# \# ;$ i

kÛÄÄ

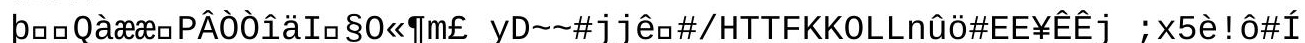

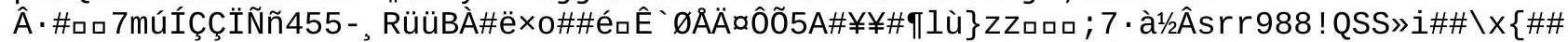

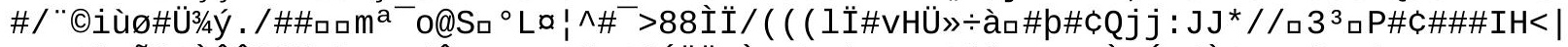

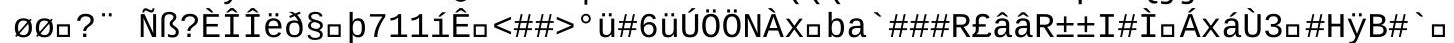

ãéêê, $\wedge \wedge \sim \# h_{\square} \mid \# \# i ß ß @ . . P_{i}$ !

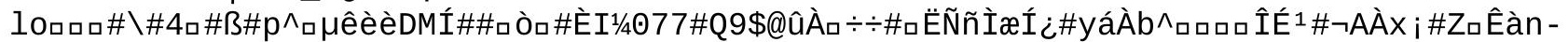

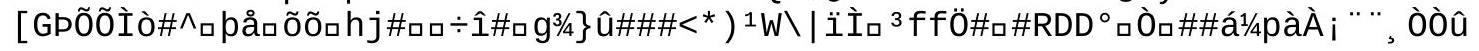





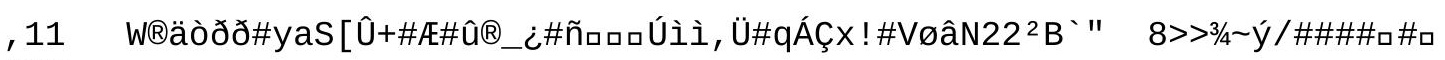
\#KĐ 


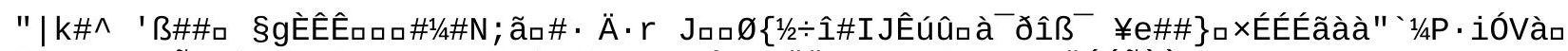

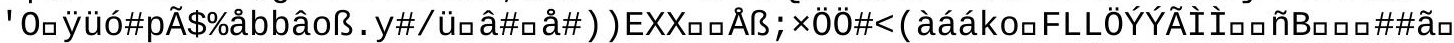

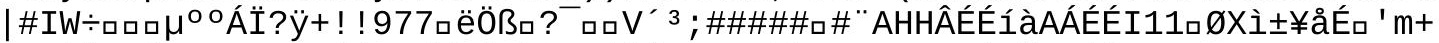

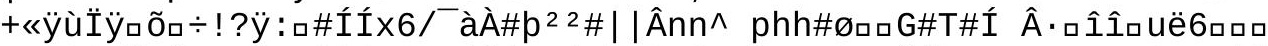

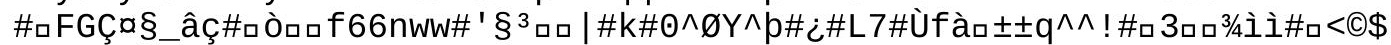

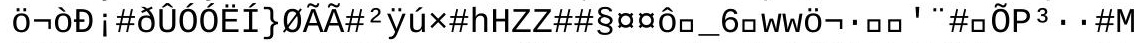

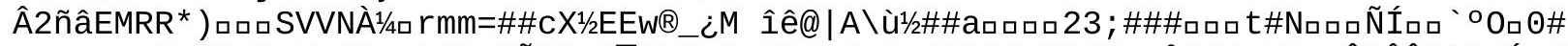

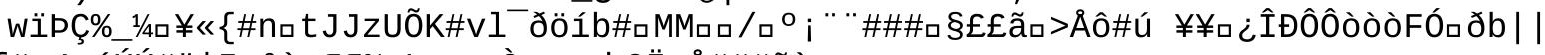

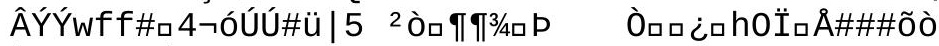

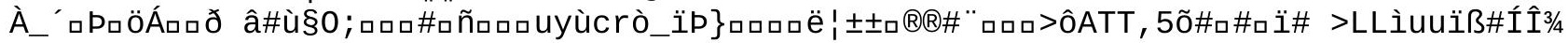

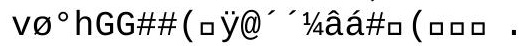

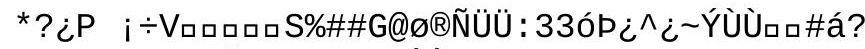

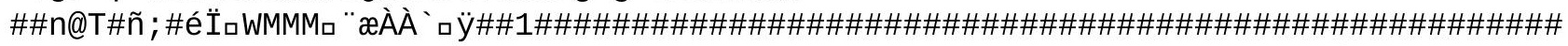

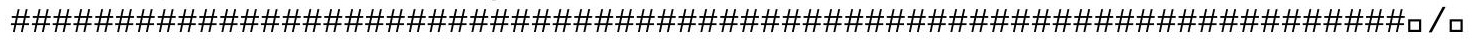

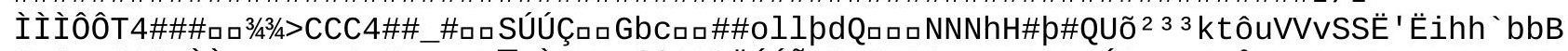

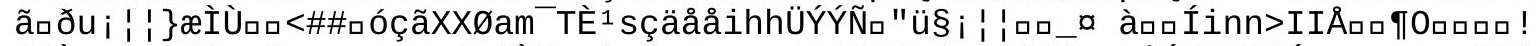

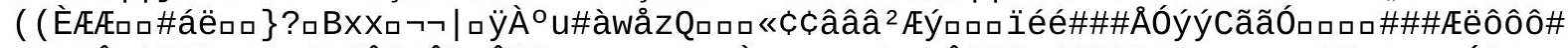

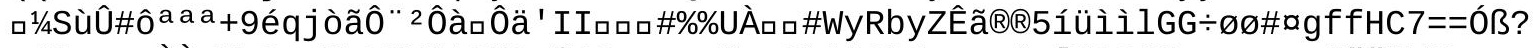

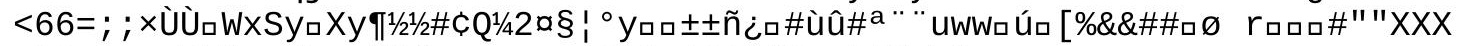

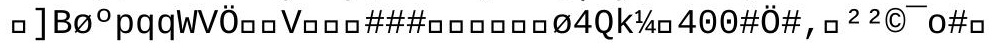


arr\#ø9\# 


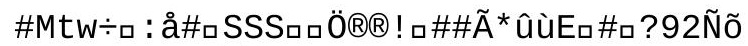

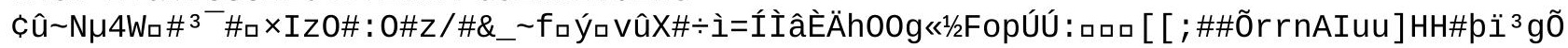

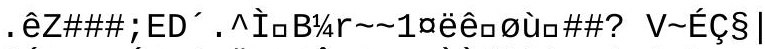

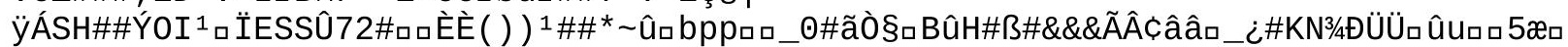
ôôıS§| D7\#

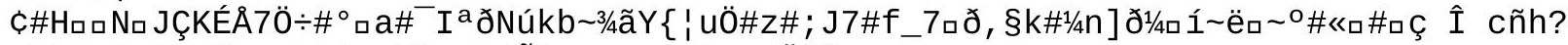

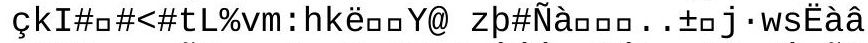

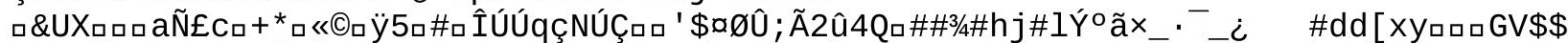

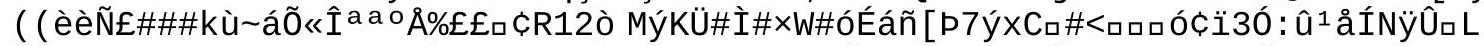

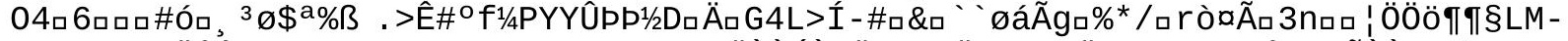

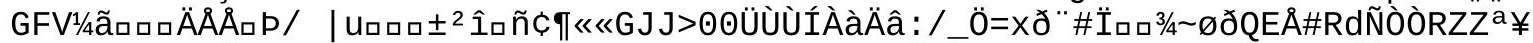

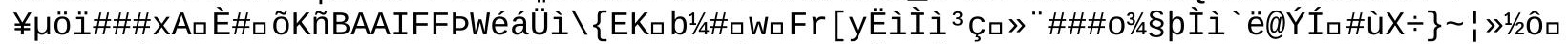

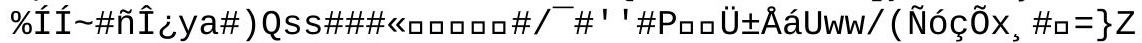

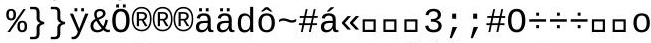

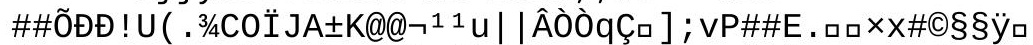

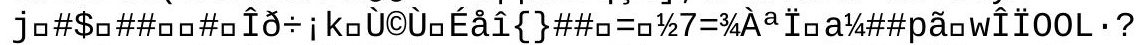

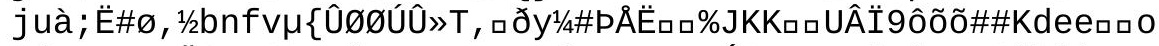

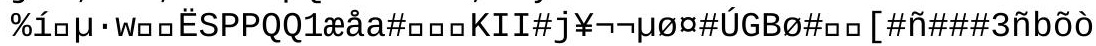



ÑöÖ\#Uðó\# . / 


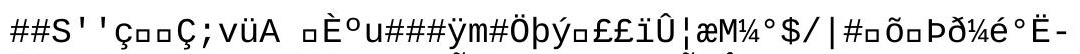

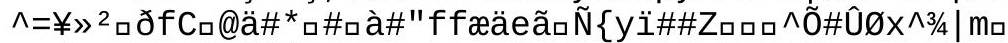

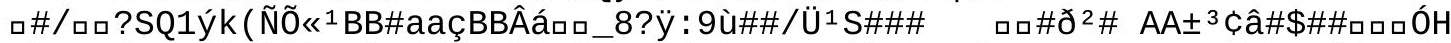

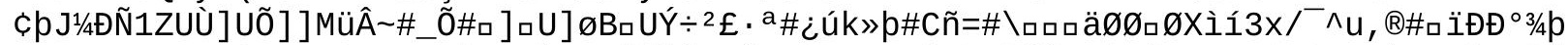

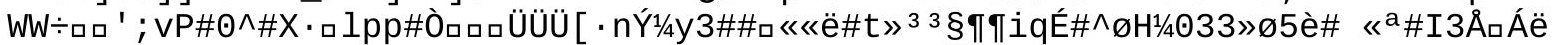

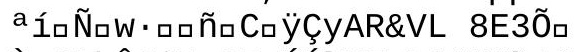

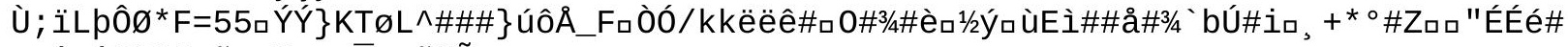

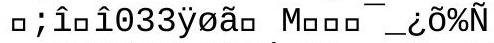

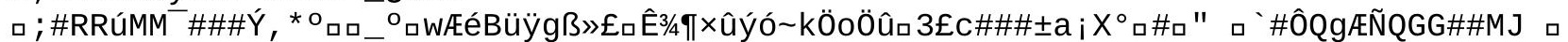

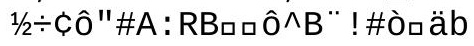


IHn

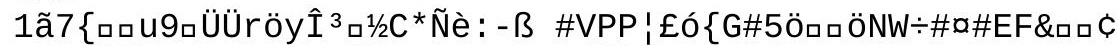

i /\#\#Å@- 6 6ø48>>\#Ê"\#Đ2 2

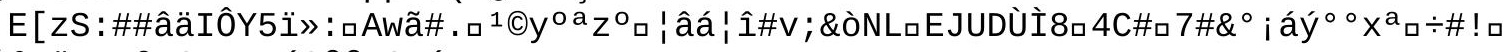
$\wedge{ }^{3} \ddot{a ̈}^{1} \AA^{2}{ }^{2} \# \ddot{y}<g g^{a} \# 8=$ ¿víQ@@\#8ÆáÆ 


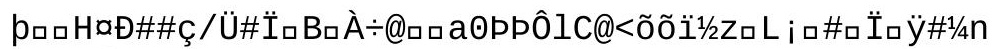

UQí?\{ÖロרาB

$-9 Đ ß ?$

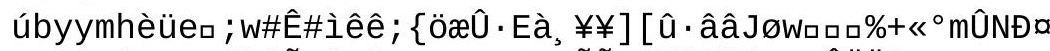

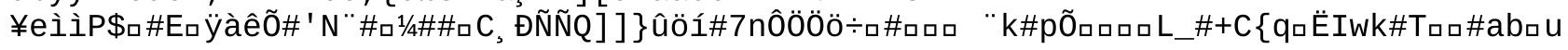




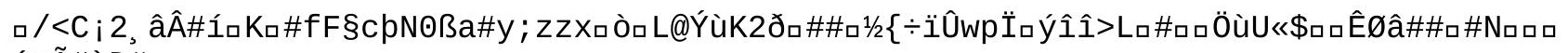
aÃ\#àD\# 
qé\$ãã\#uuÍxü 


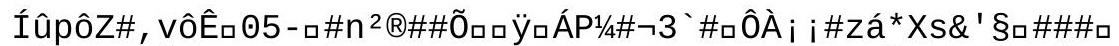

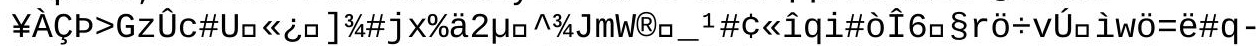

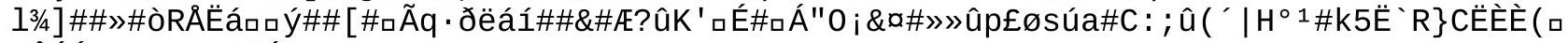
VÎ́ó́3¿Zロ\#\#Ö०ôÁàMAı\#>\#\#O\#1/4G"\#\# 
口ÎIL $\left[\div \mid>\square^{1} \#\right.$ 
Æ1\#S\#L 


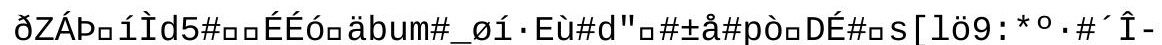

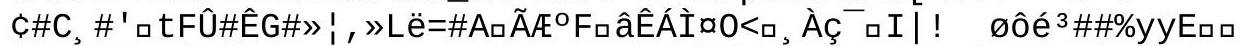

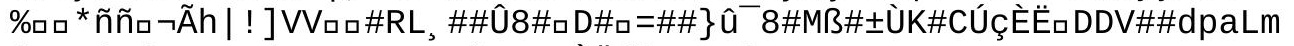

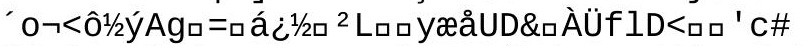

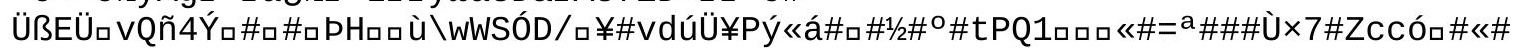
\%\#”\#/ßi

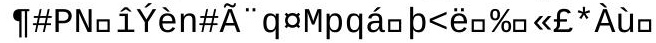




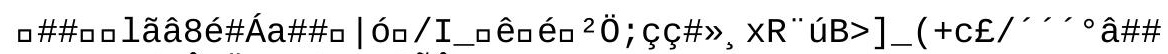
口; vì< $\left.{ }_{\square} d_{\square} \hat{U} \div A ̈ \# \# i ̀, \# \#\right]$ ÂÔ\#vGíy-\#ú!

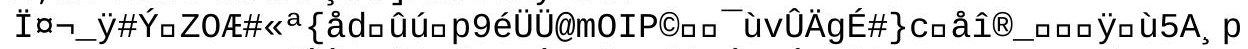

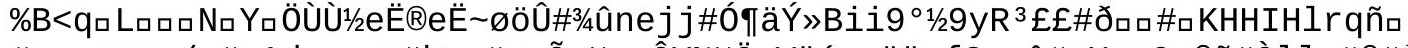

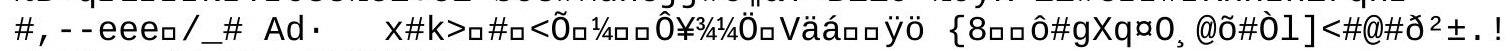
ÇSZ[»\#S(Y-DĐ| aOAEAV1t\#> 


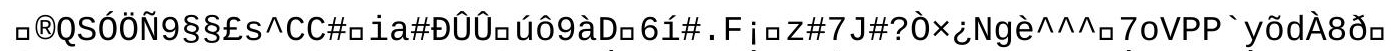

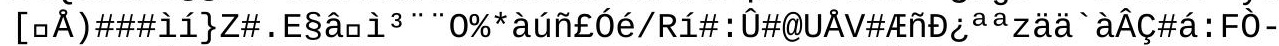

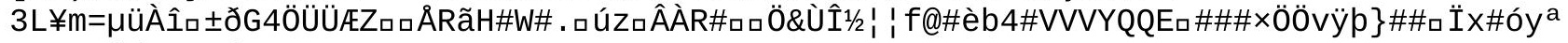
Ç${ }^{1}$ ä\#\% $\mathrm{d} \# \# C ̧$, é 


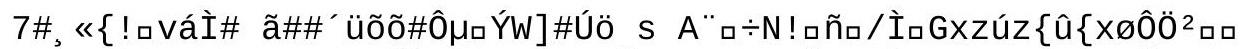

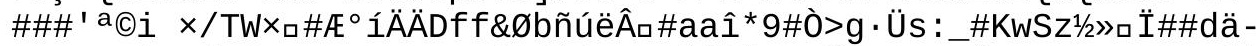

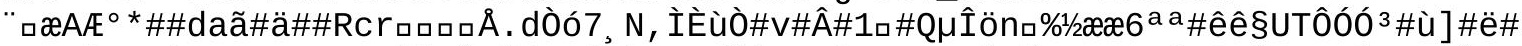

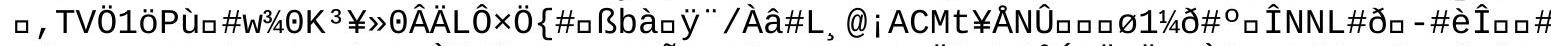

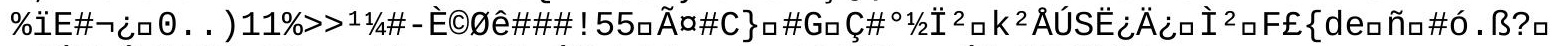

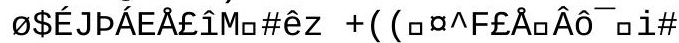
口םQR ${ }^{\circ} \ddot{U} U_{-}$口ÉÏロY\#\#Ü\#\#ロ

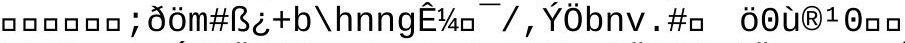

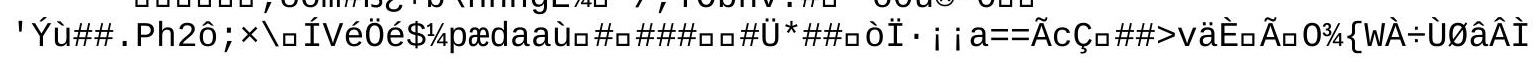


!66Md,\#\#ロĐÛ; àI \ฉamÉiöÖñ3Ýø\#\#8n\#! 


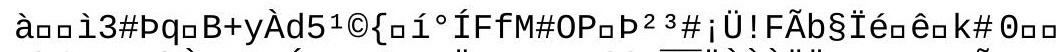

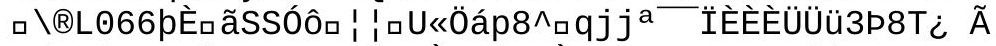

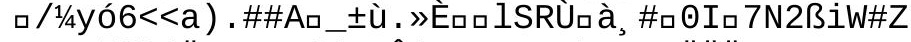

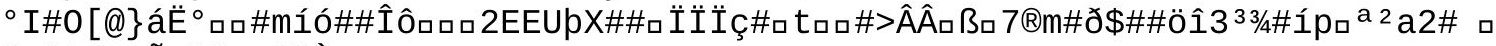

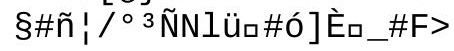




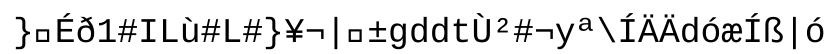




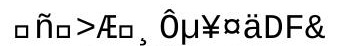

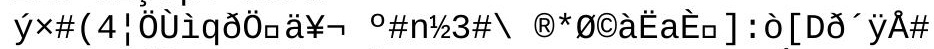

UY\#[ๆі̀ @@\#; 55Ï\#\#ロòq6WWW

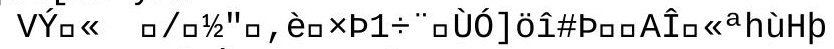

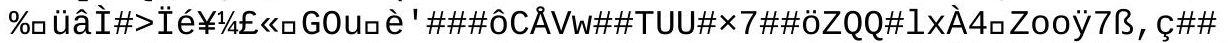

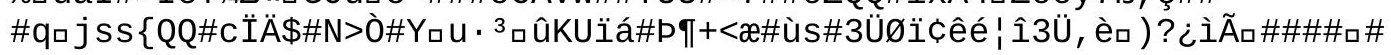





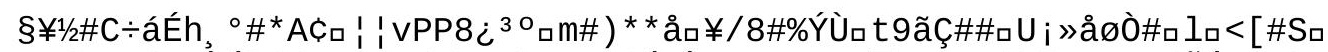

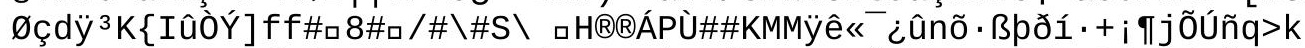

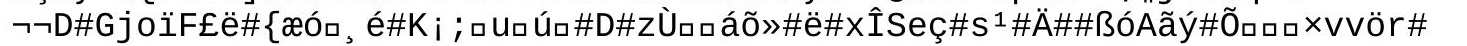




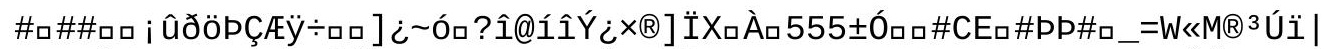

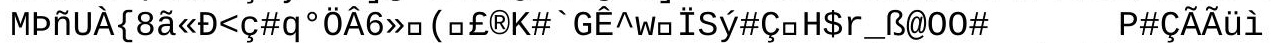

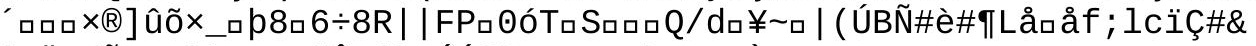

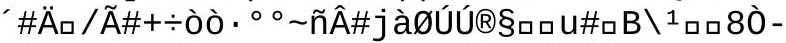

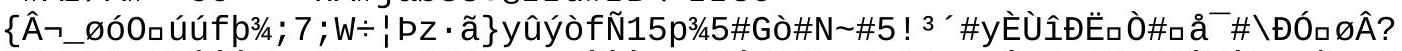

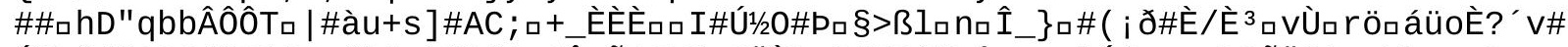

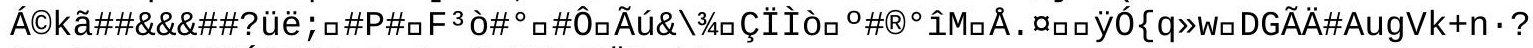
âbwĐ\#@ロĐß\#\#Ó66ÆR×Öロ/ロc\# ðM@sHÜRyóò

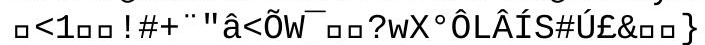

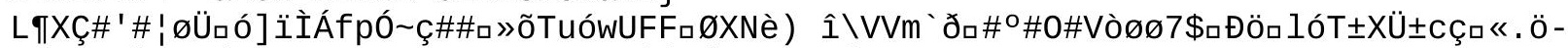

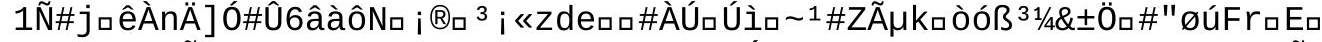

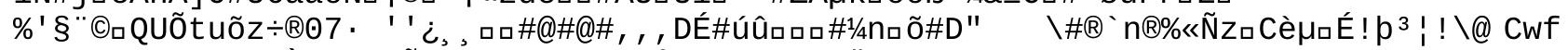

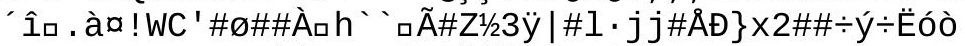

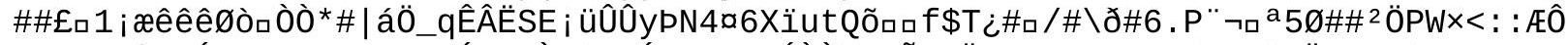

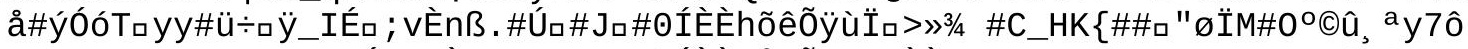
áÂß口 [66òıê

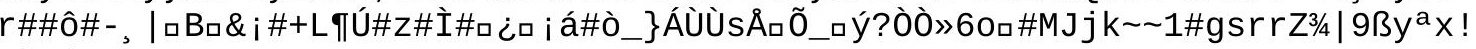




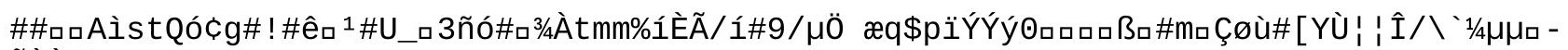
ÃÈÈ ( \} 



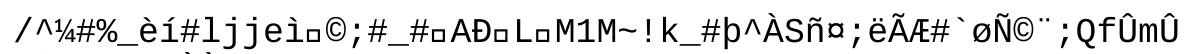
Z) $<$ ZDCã\#ÀÈ8 


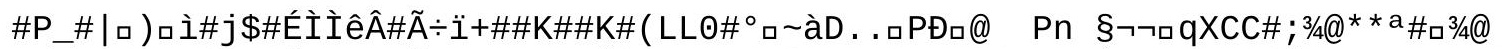

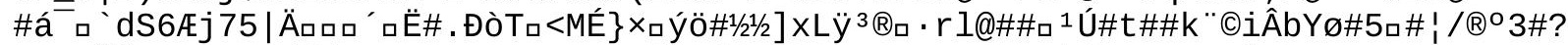

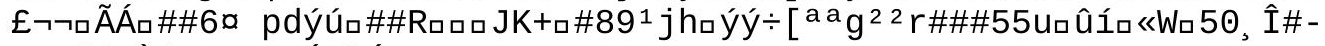

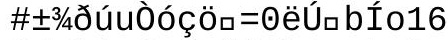

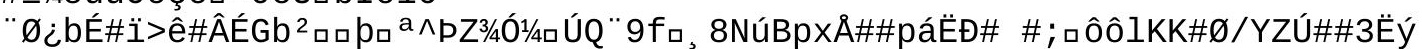

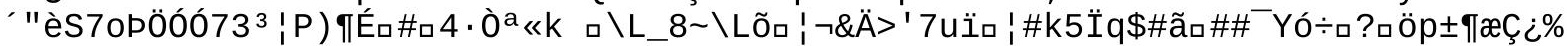

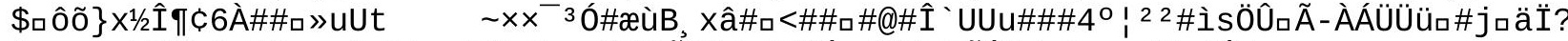

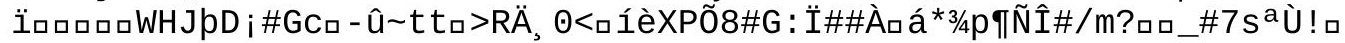
f§!ฉÔ6Ekô\#םãéibYY7ロ/8/ .\#« 


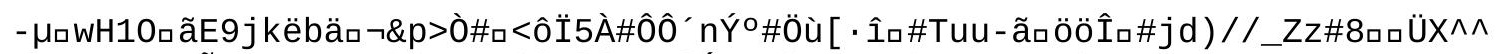

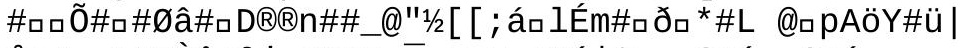

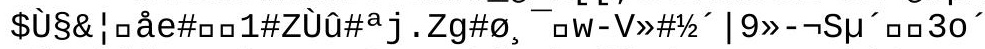

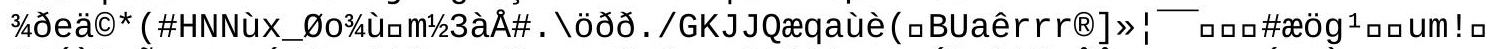

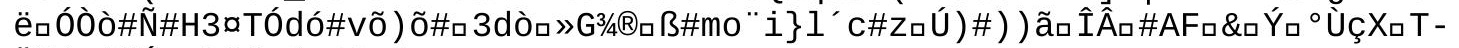

Ä§ýoý\#ÝquP\#\#ロéajja\#

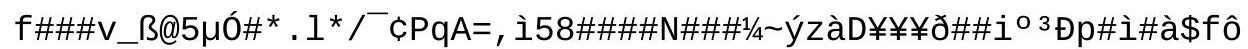

C»»^*19\#uíoìgý/aúB\#üú\#]1⁄2@m- '>1̂\#<ÿú7õ/dí\#

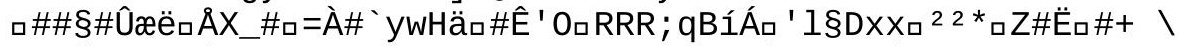

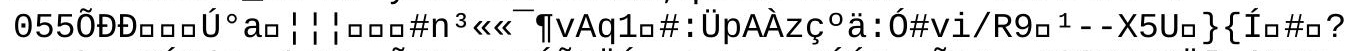

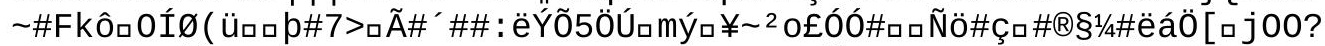

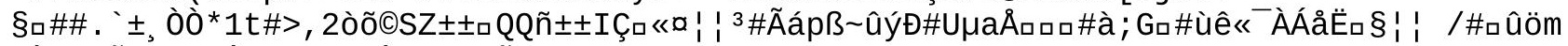

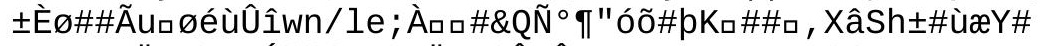

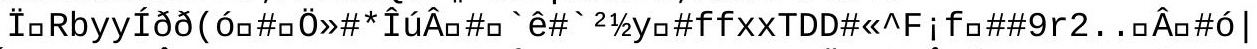

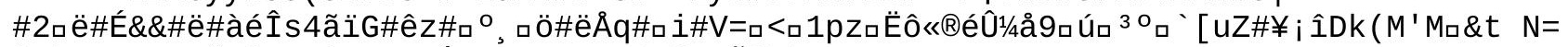

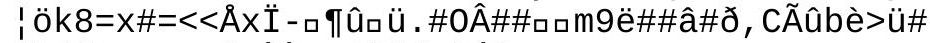

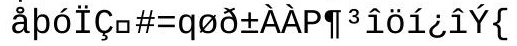




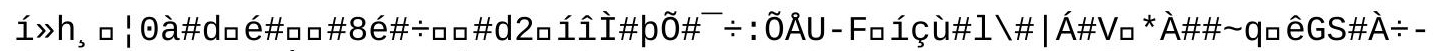

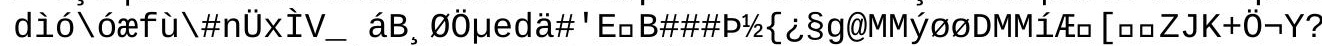

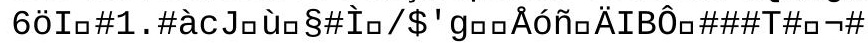




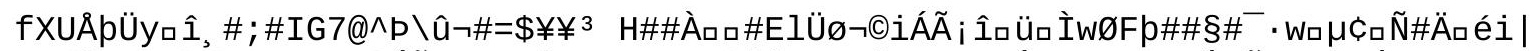

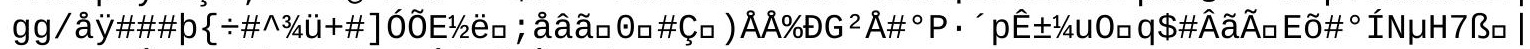

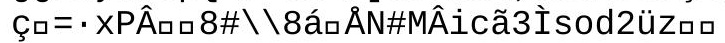




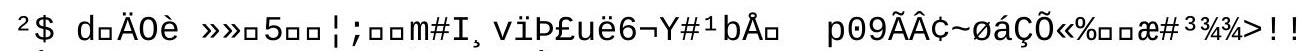

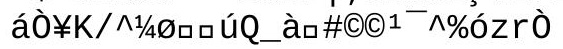


Sıé, y60̃2Ýlıma

Yà5äc] Ûè\#̈̈\#n[zúÛ5k\$Ö®ýiåÊuooú $\S €$

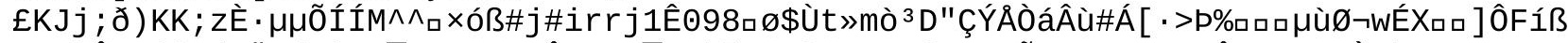

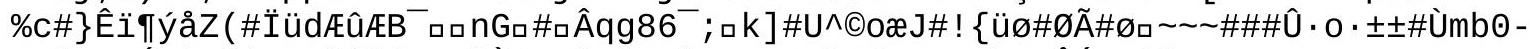

\#\#åa=tÝYèy ${ }^{a}$ `E-\#\}

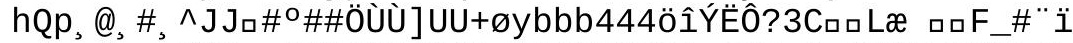

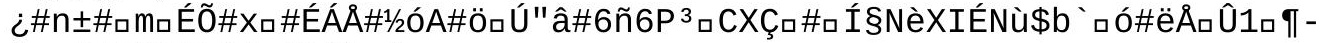

\#, 口\#ÓÆ\#22Ò $\left\{\left\{\square S^{\circ} A ̈ \backslash W \#\right.\right.$ 


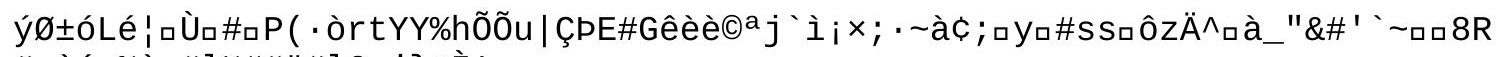

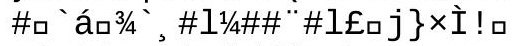

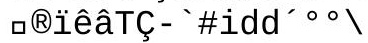




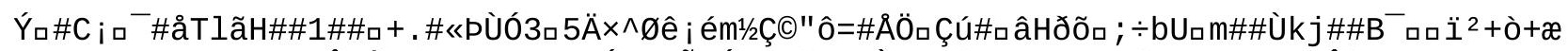

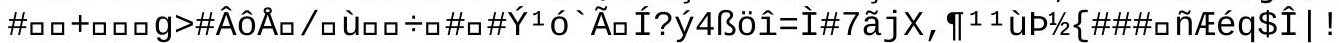

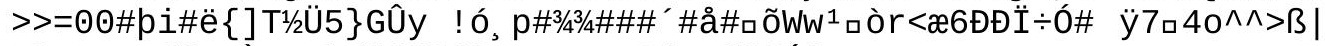

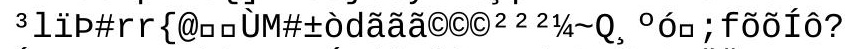

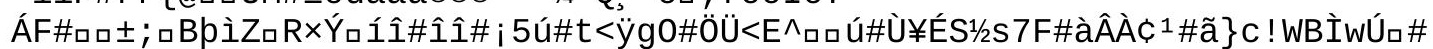

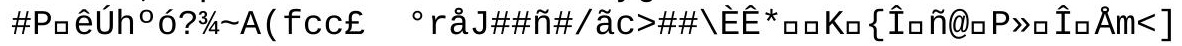




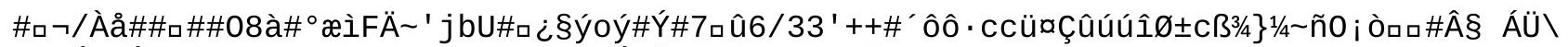

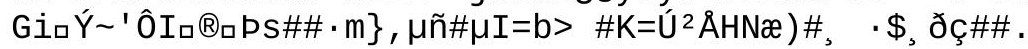




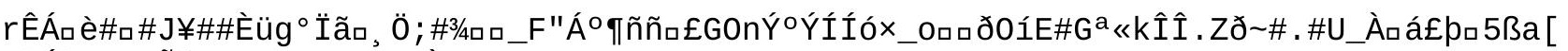

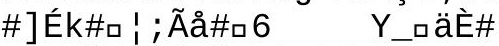

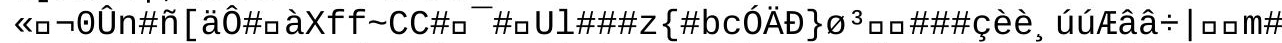

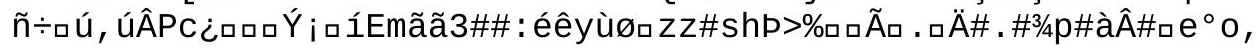




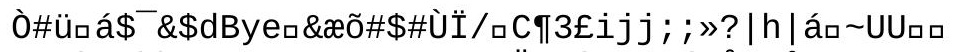

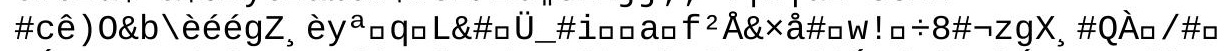

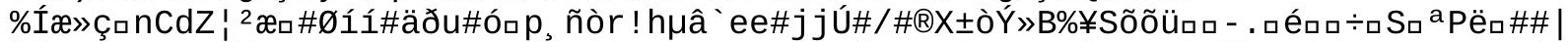

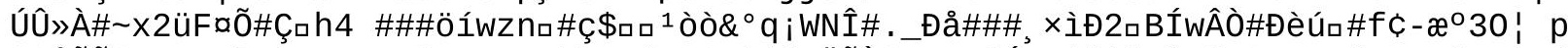

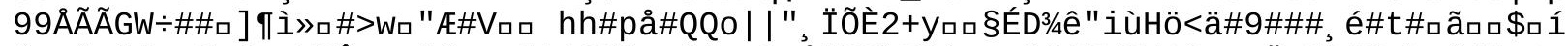

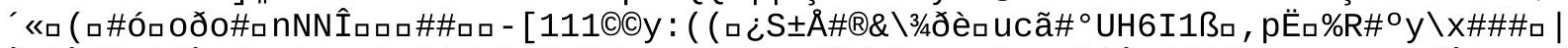

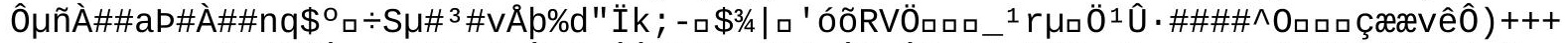

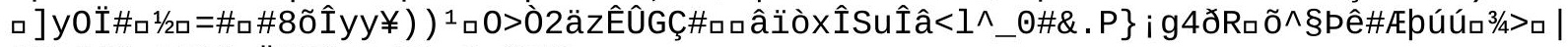

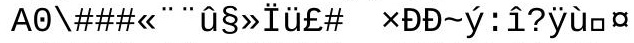

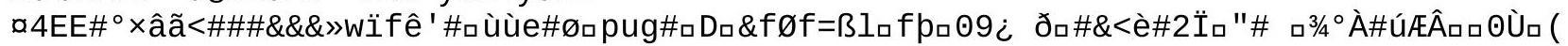
(á 5TU5`土ìø\#UW\#S \

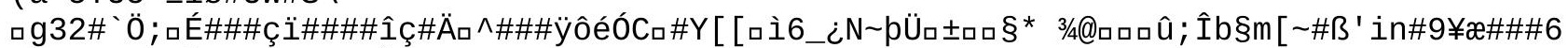

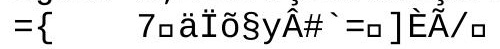




$$
\square \square
$$




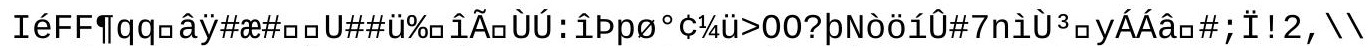

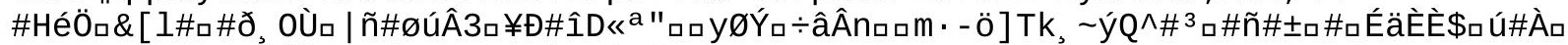

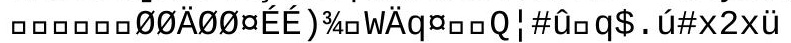




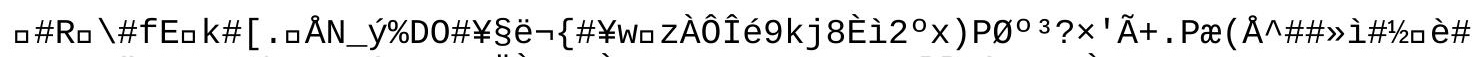

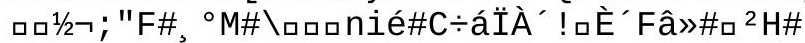

$x t] 3^{\prime 3 / 4} 1 / 480 ̀$ ì 


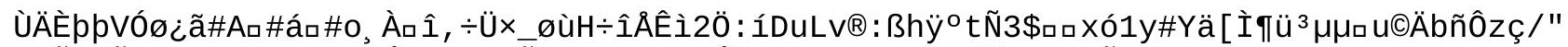

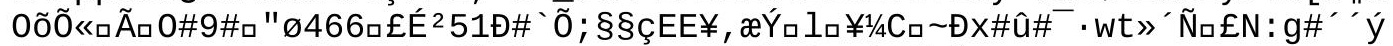

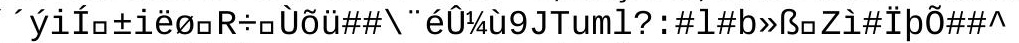

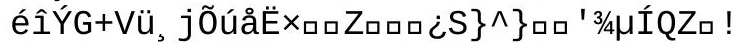

ø 


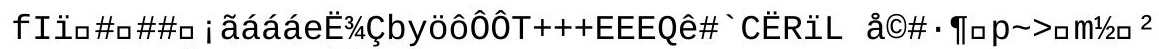

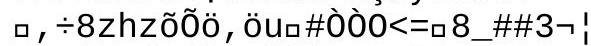

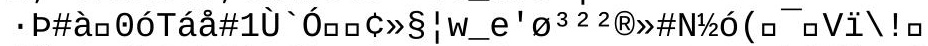

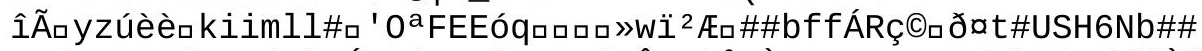

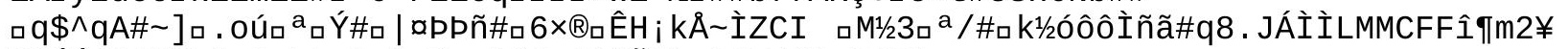

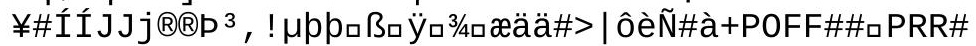

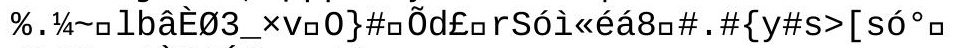

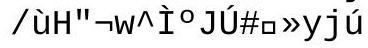




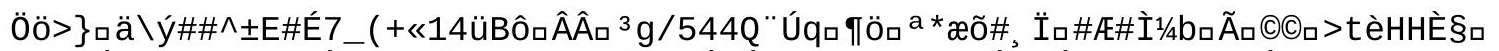

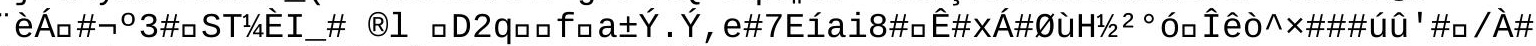

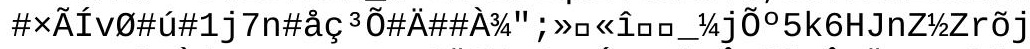

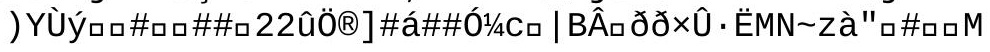




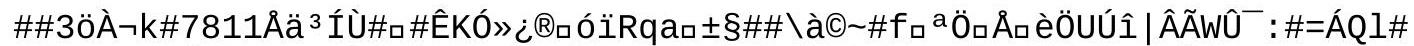

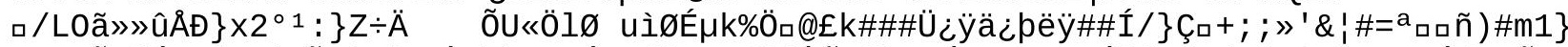
¡ $\mu \mu \_$Ãe H! papñĖ\#án ${ }^{2}$ ÈbंOZCOÏÀÔ\#3¥qêÚ 


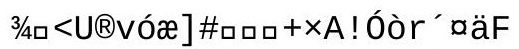

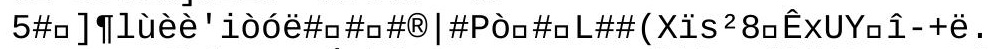

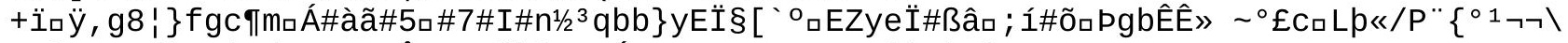

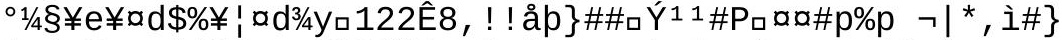

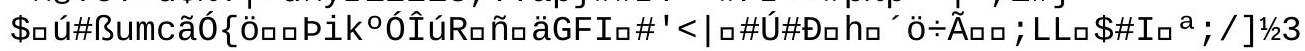




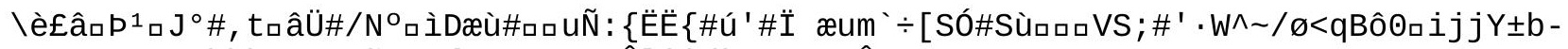

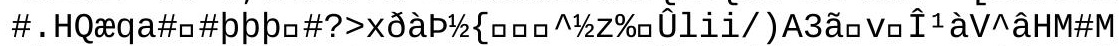

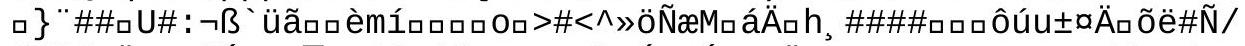

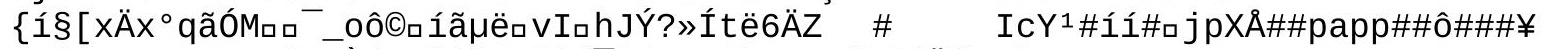

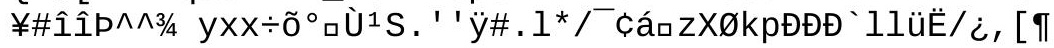




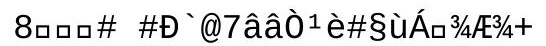

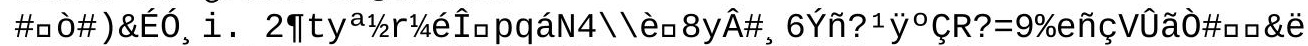

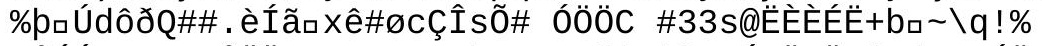

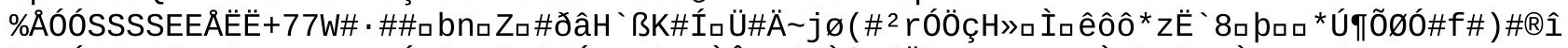

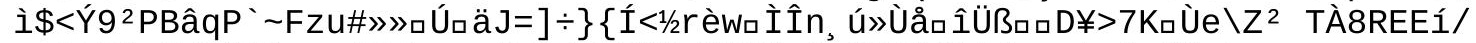

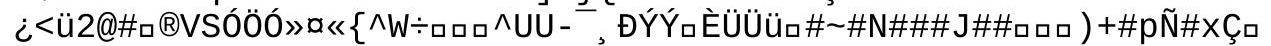

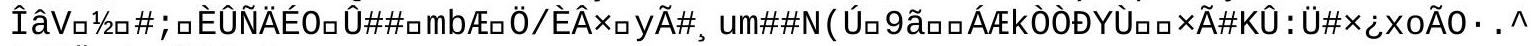

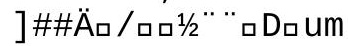

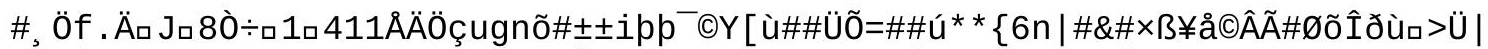

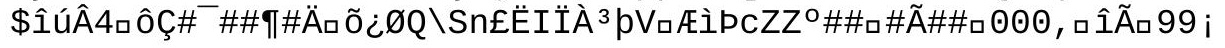




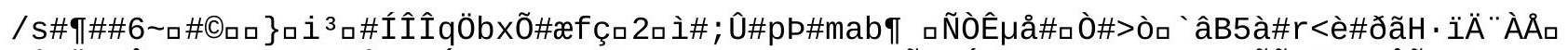

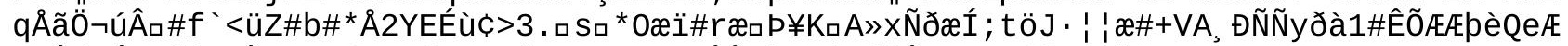

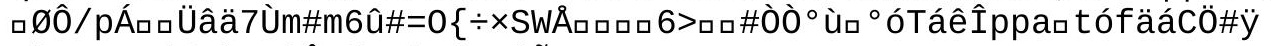

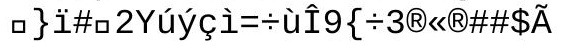




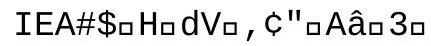

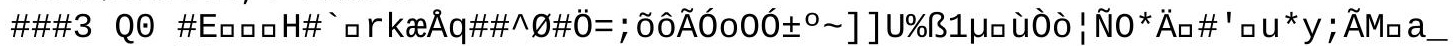

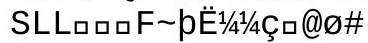


àgWãááËÉÉCònaG, - müüロ\$\%e_3/4ü\&\#ロ! ablc

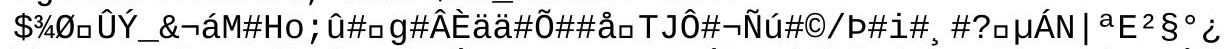

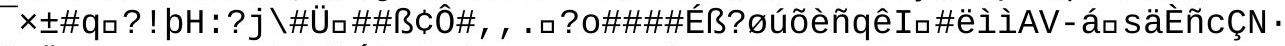

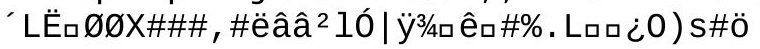




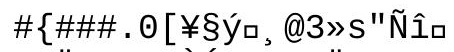

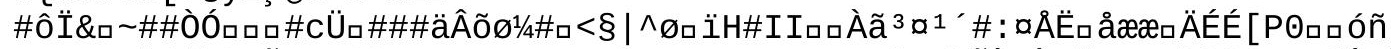

ljjpøÃ\#\#\& ${ }^{\circ} \mathrm{C} \#$ \#

E口æW ,

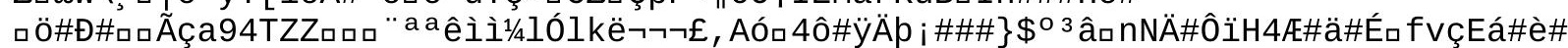

\%sáBEEmGÇt\# $\cdot \mid \mathrm{sõ}^{1}=\circledR$

û\#PZC\#ð1\#[0̂Gôr

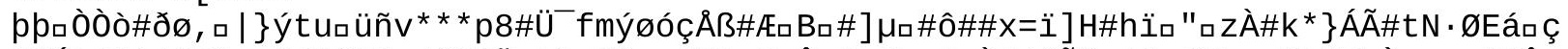

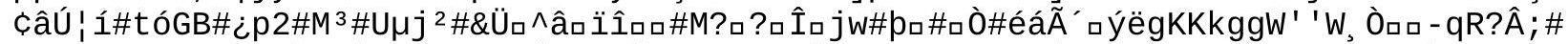

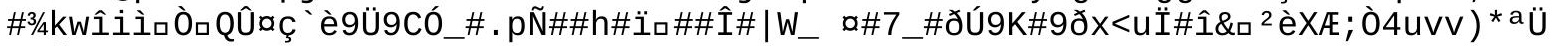

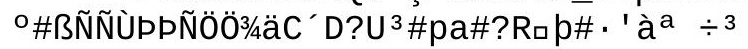

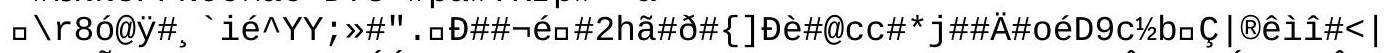

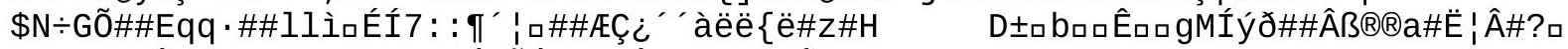

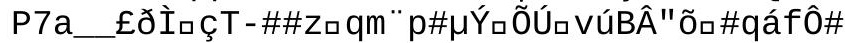




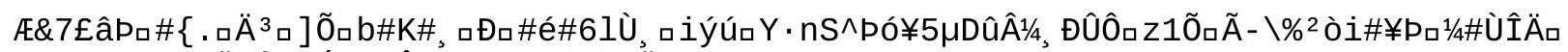

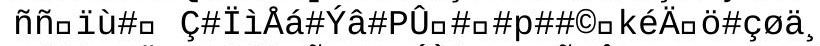

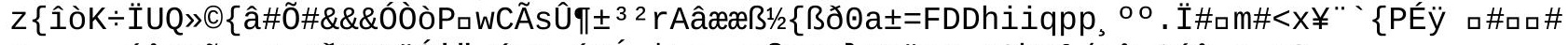

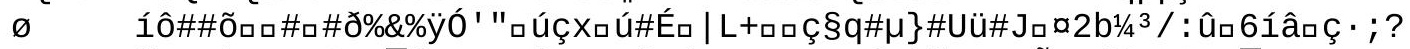

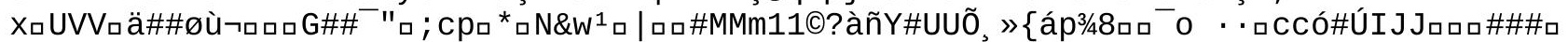

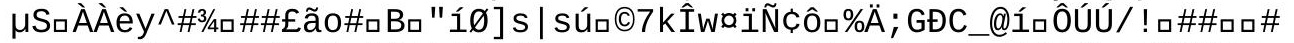

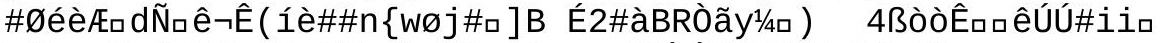

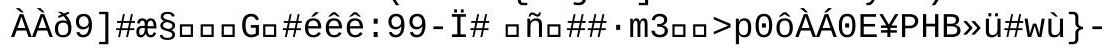




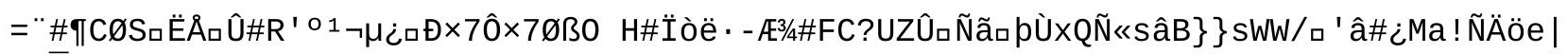
2. - Æg. $\ddot{y} \#$ 


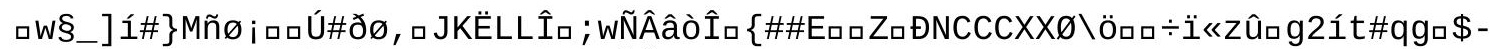

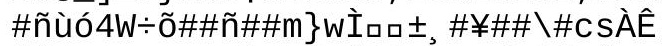

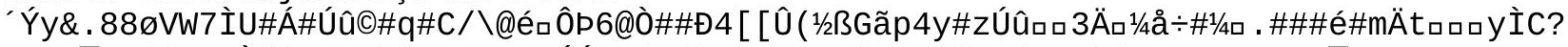

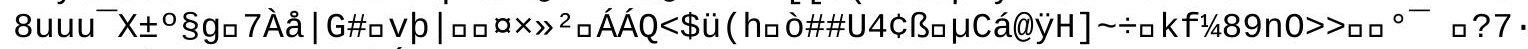
'×Y\#! ᄀ $\div$ t\#ÒÁ

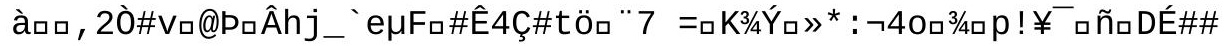

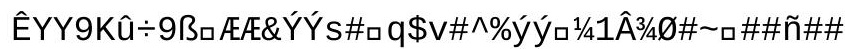

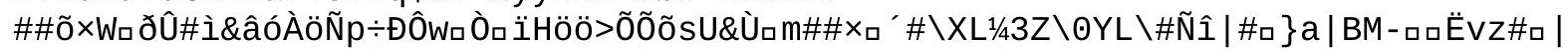

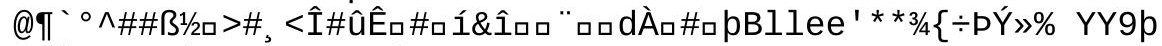

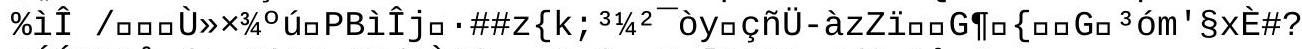

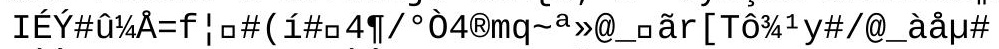

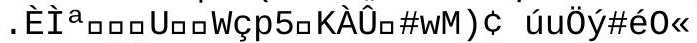

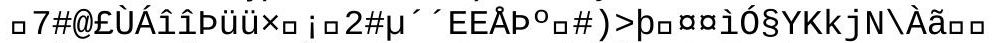

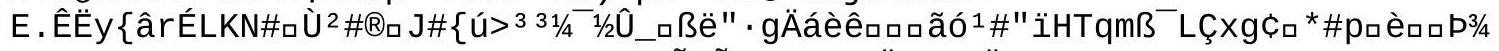

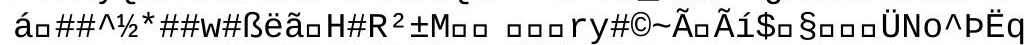

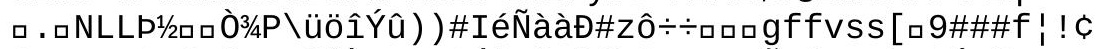

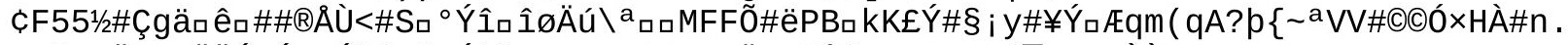

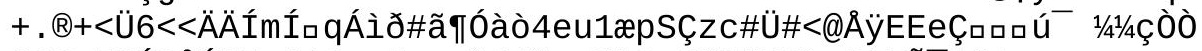

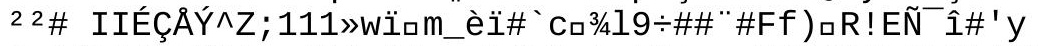

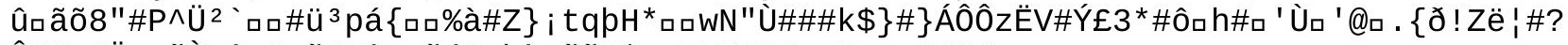

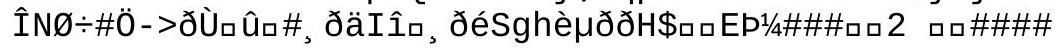

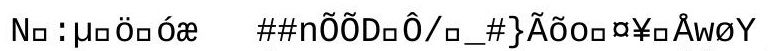

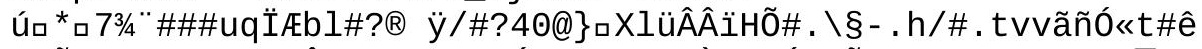

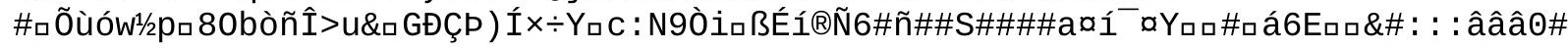




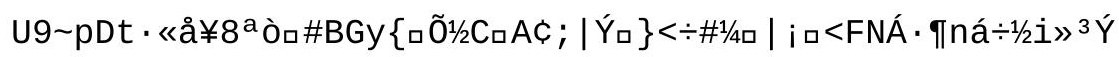




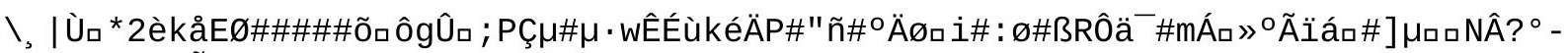

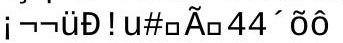




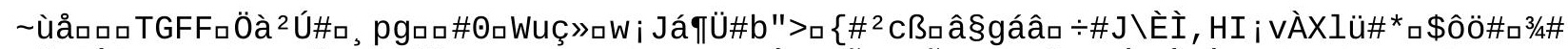

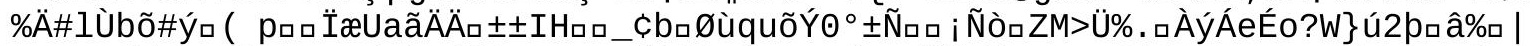

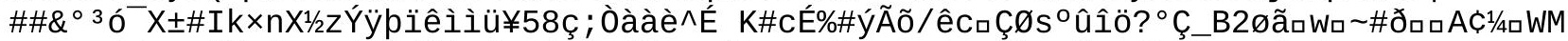

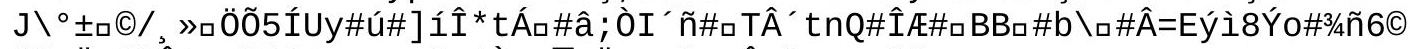

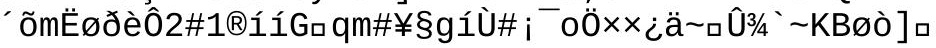




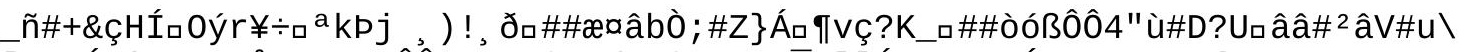

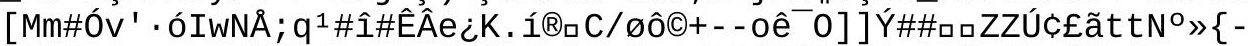

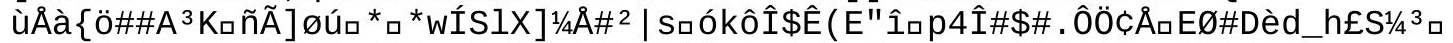

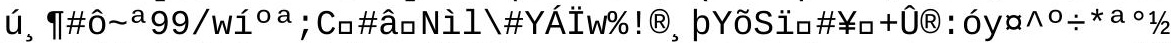

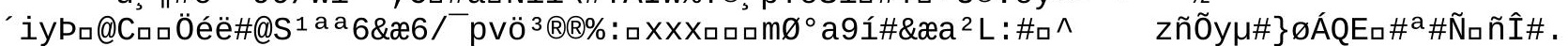

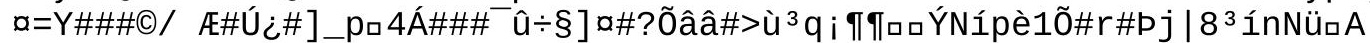




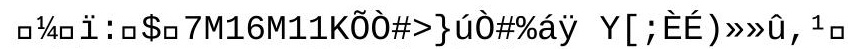

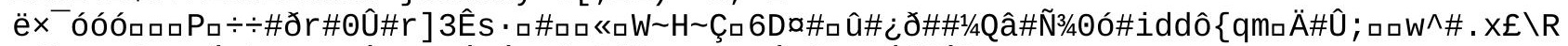

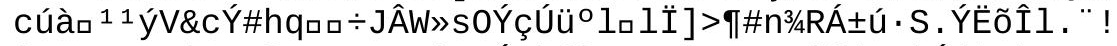

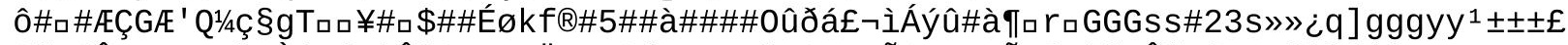

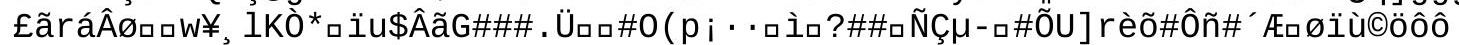

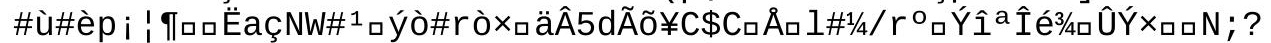

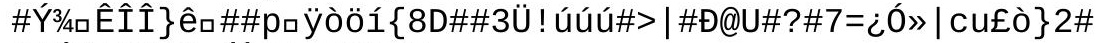

jûÚú ' ) â\#" pÁÀOQv?®००¥

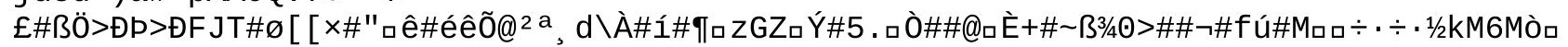
\#Õם 


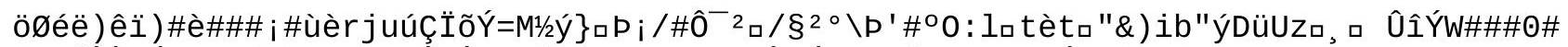

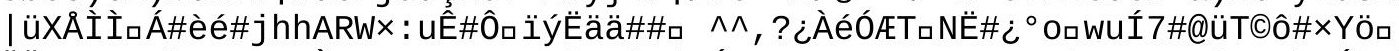

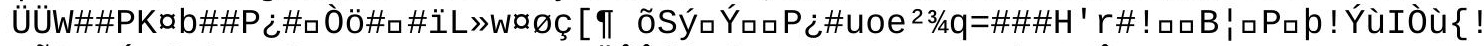

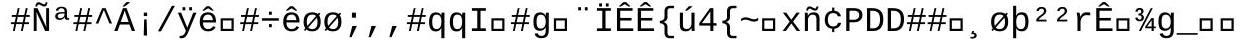




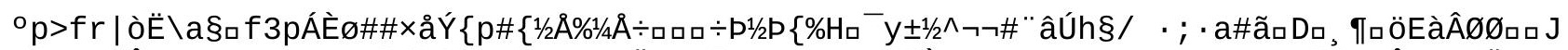

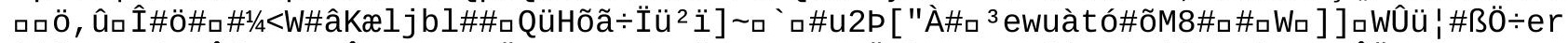

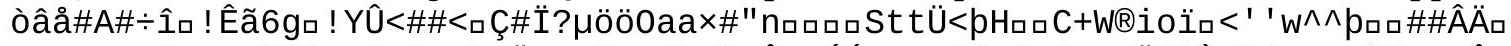

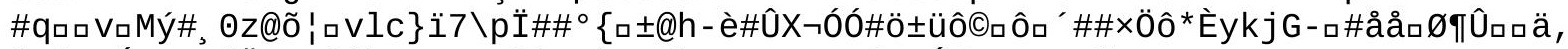

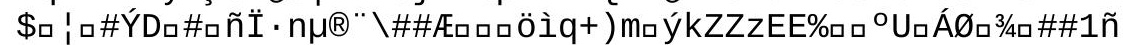

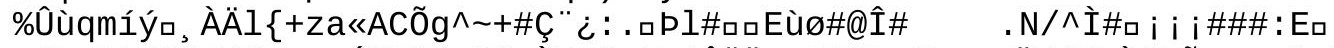

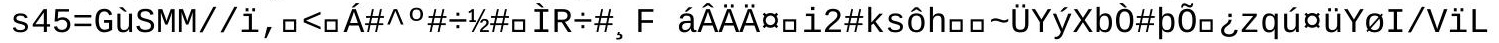




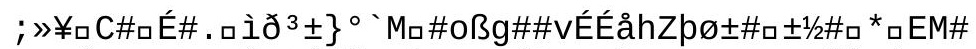

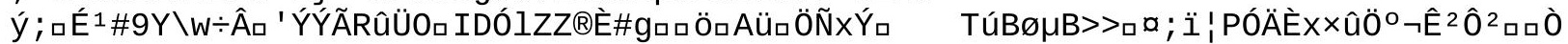




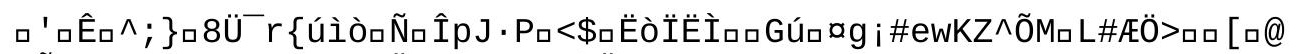

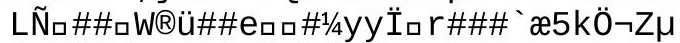

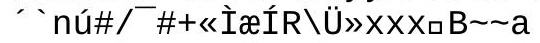


ö\#? ¿ロ\#V

. ] a j1/4\#ÛÔXı

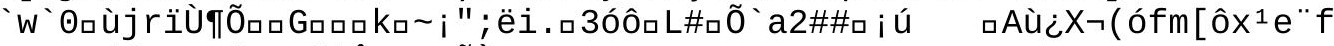

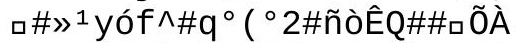

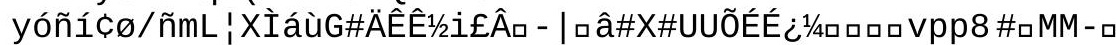

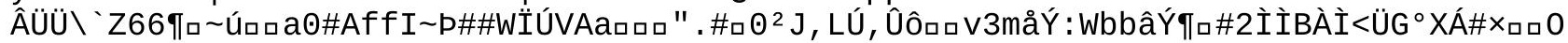

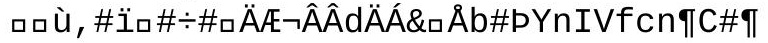




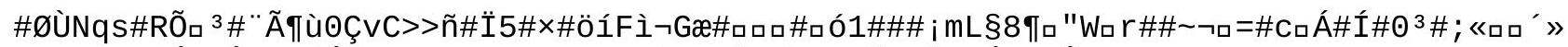

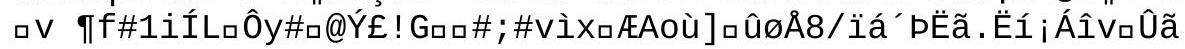




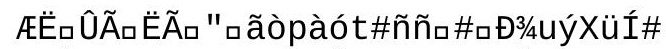

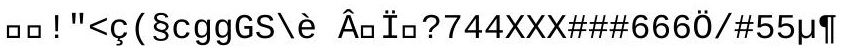

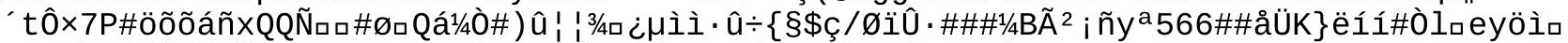

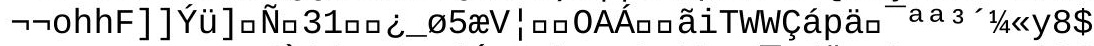

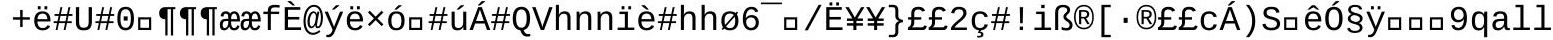




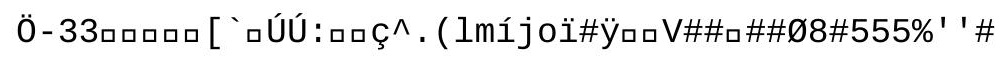

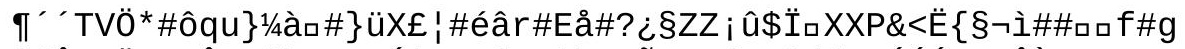

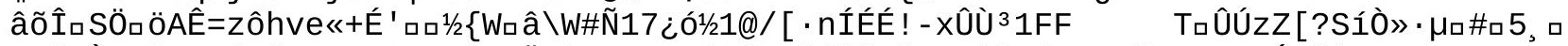

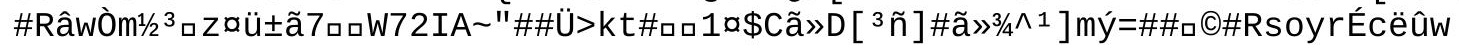




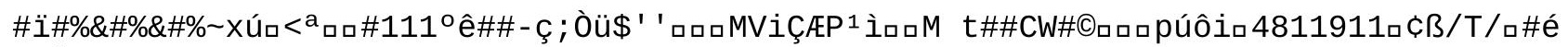
ééÅÆÆRı LN A\#Æ\#CC\#í\#ýç HooïÆa\#ÿ\#\$<-paFĐwmmíìwa

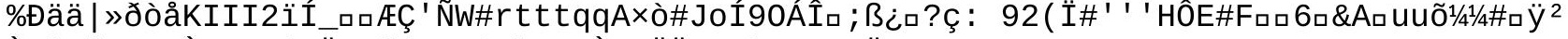

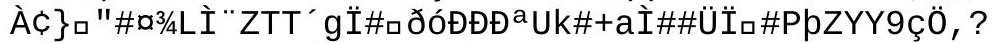

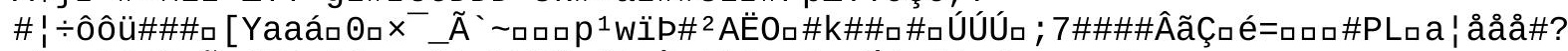

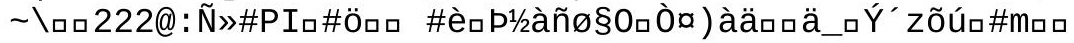

$\mathrm{S}$ 
$\mathrm{b} \# \square \# I 0 \tilde{0} \mu|| ; V^{-}$bMLL $¥ ¥ ¥ \square \pm$ 


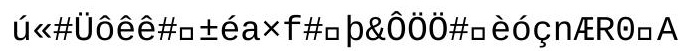


$\mathrm{b \# \square \# Ä} \mathrm{\# 1 \_ A}$ 
$\mathrm{b \# \square \# Ä} \mathrm{\# 1 \_ A}$ 
ú»QWWOFF6ùó

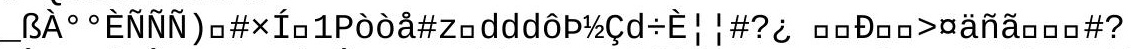

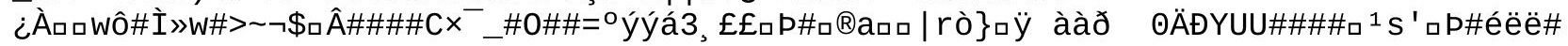





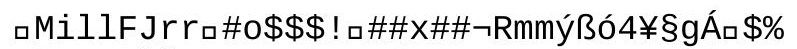

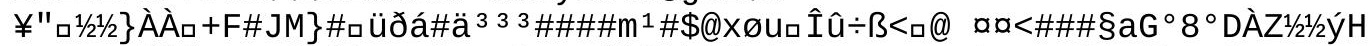

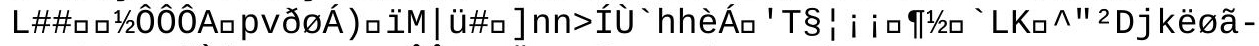

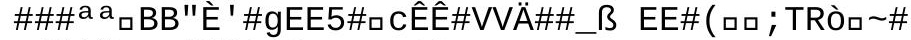

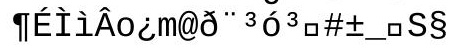




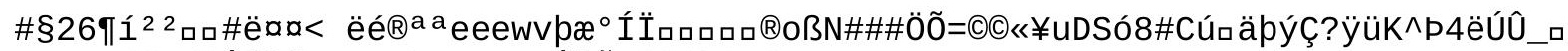

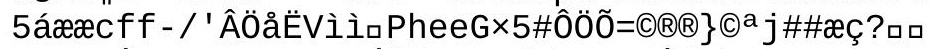

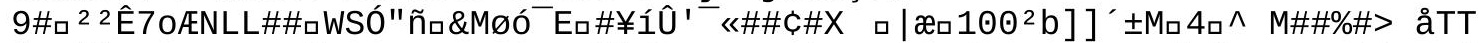

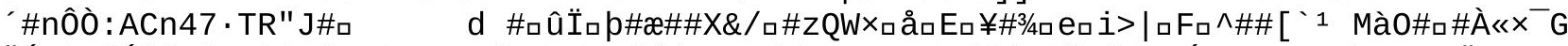

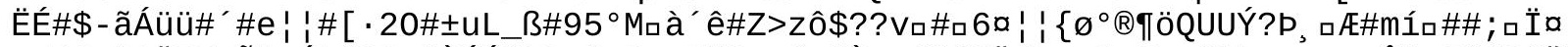

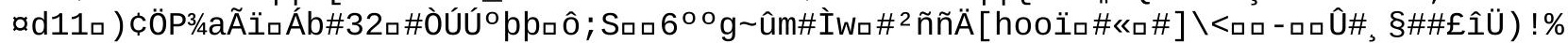

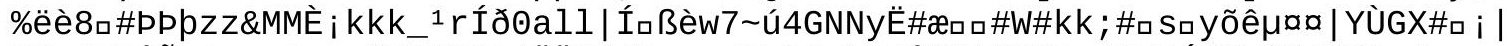

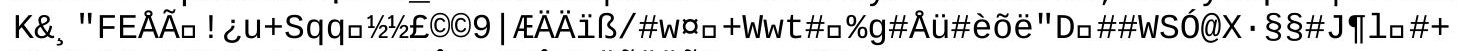

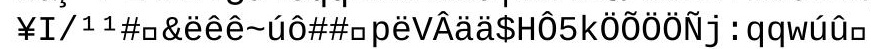




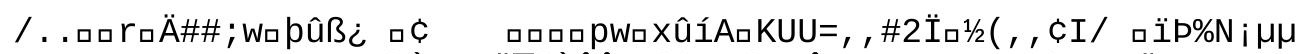

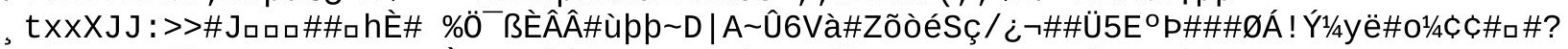

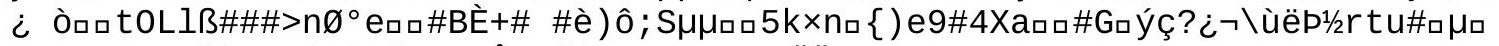

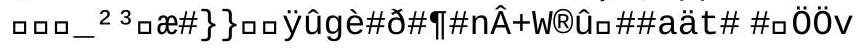




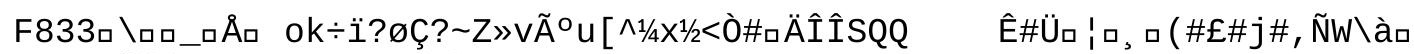
Cý\#ロìå"@gø\#,äan_èíí $[ \pm b-$

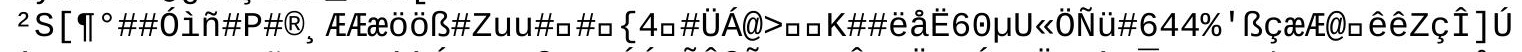

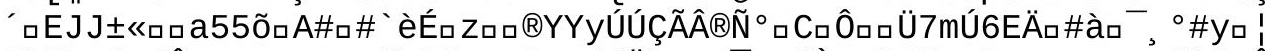

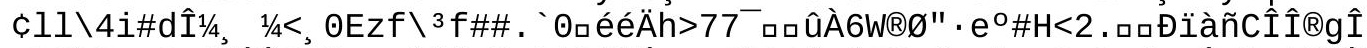

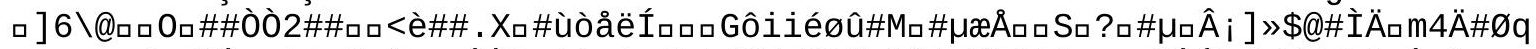

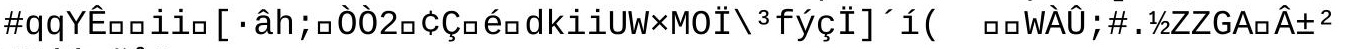

'1/1/4rpüE\#Öâêãaå\# 
1\#ðロ ÿRDD 


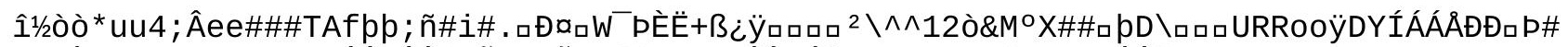

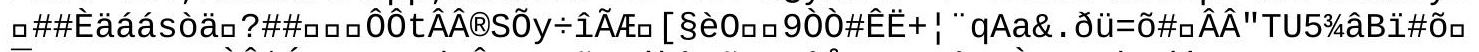

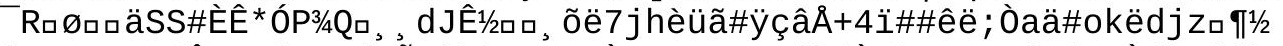

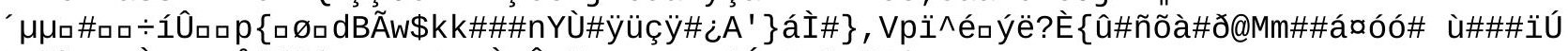

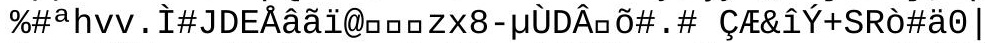

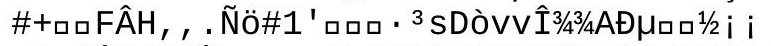

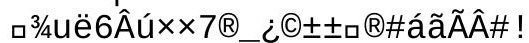

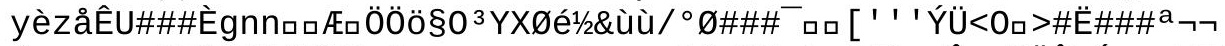

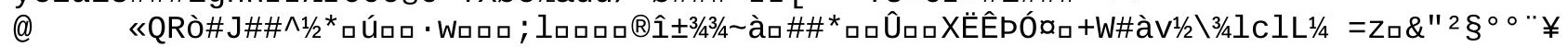

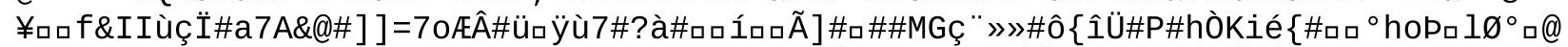

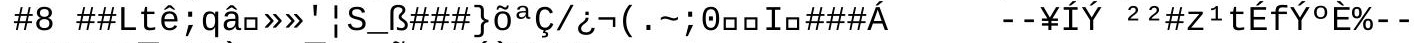

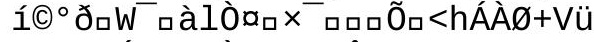

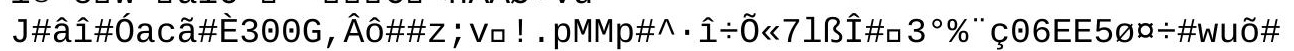





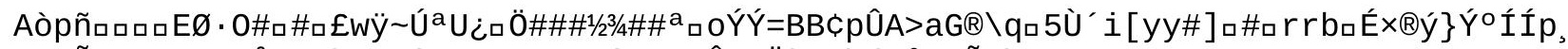

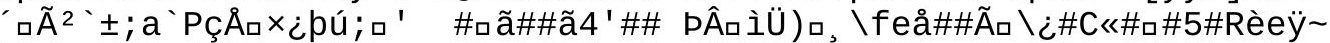

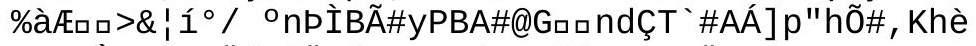

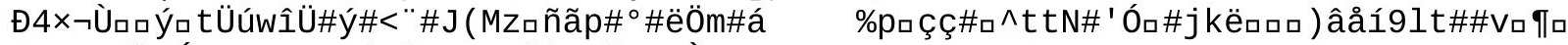

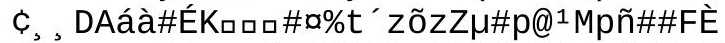

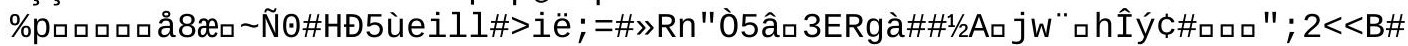




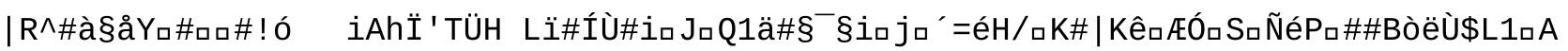


$\mathrm{b \# \square \# Ä} \mathrm{\# 1 \_ A}$ 
$\mathrm{b \# \square \# Ä} \mathrm{\# 1 \_ A}$ 
$\mathrm{b \# \square \# Ä} \mathrm{\# 1 \_ A}$ 


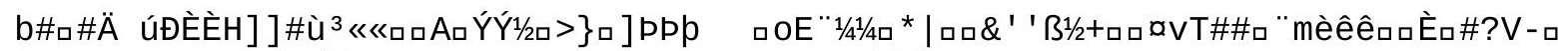

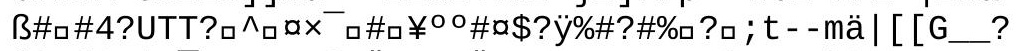

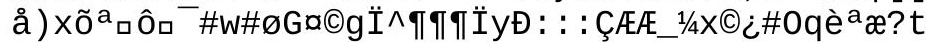




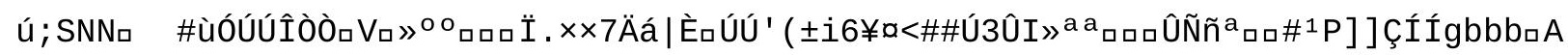




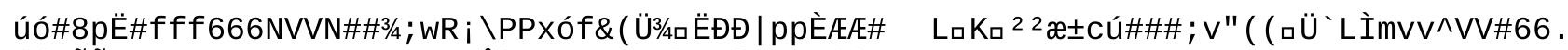

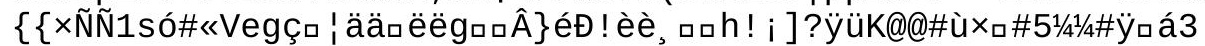




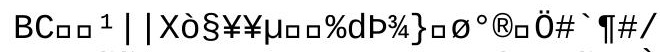

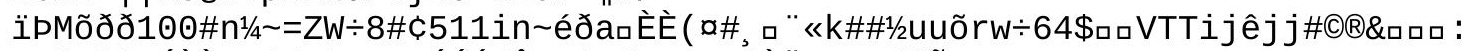

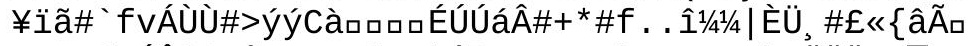

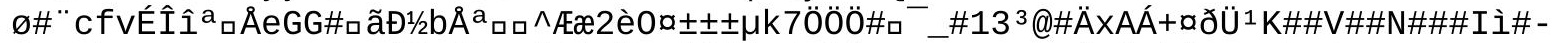

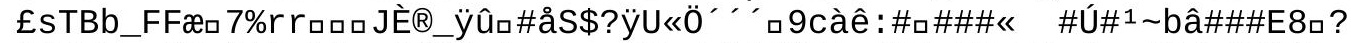

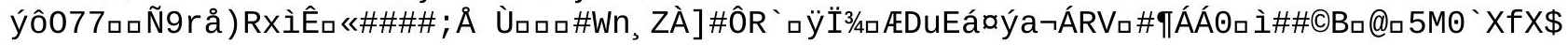





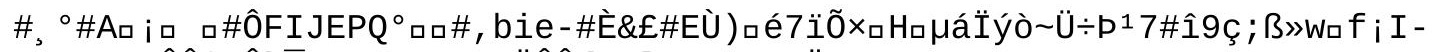

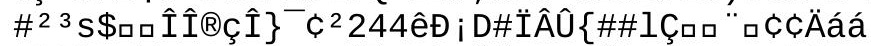

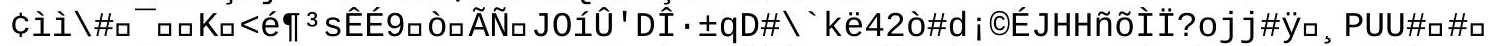

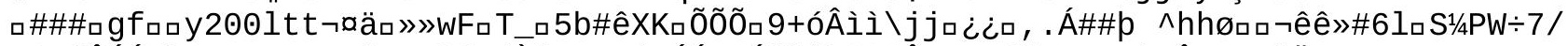

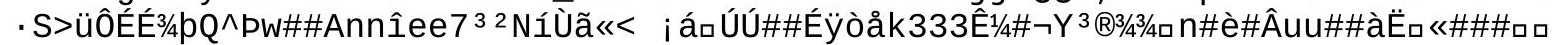

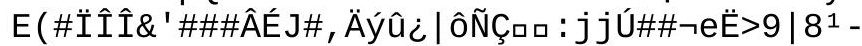

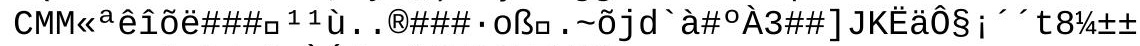
$n \#>l d \pm 0 \hat{0}$ ÒA $\#==1 / 20 F u ̀ 455 ¥ \circledast 3 / 4$

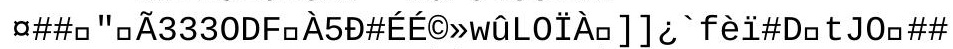

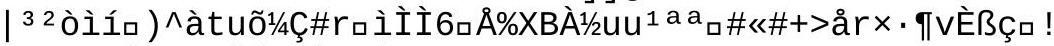

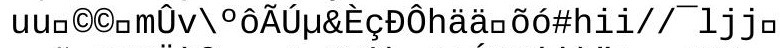

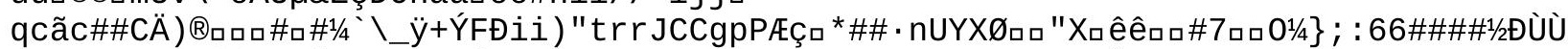

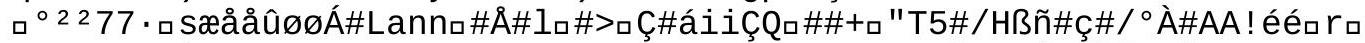

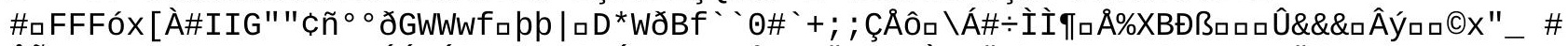

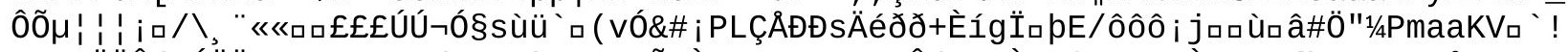

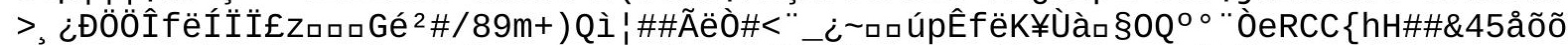

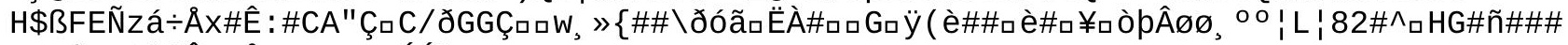

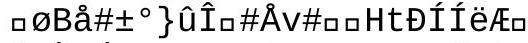

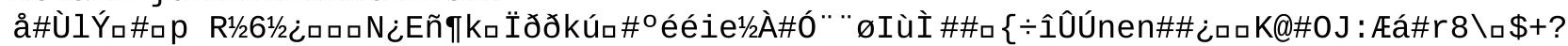

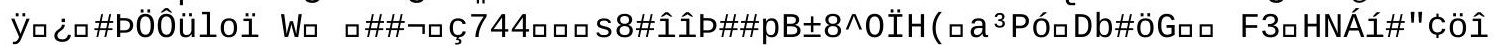

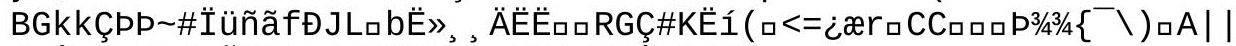

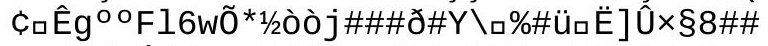

8sæ<R=ú\#7 - - 6"\#á ${ }^{\circ}$ ロ\#\}â\#Æ\#\#Fýý\#tßGロ\#ロ«5\#\#\#7 



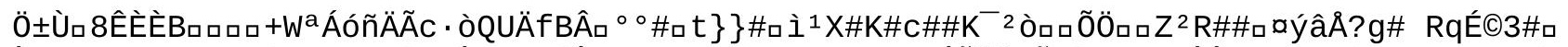

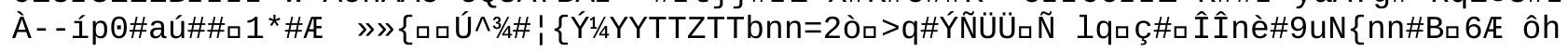




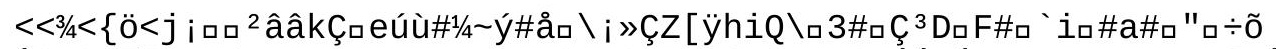

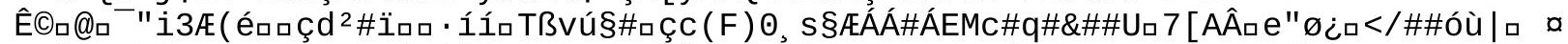

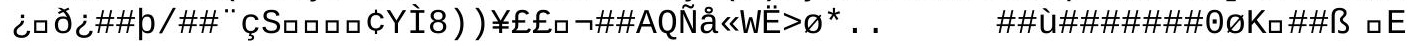

endstream

endobj

$28 \odot$ obj

$<</$ Subtype/Image

/Length 60997

/Filter/FlateDecode

/BitsPerComponent 8

/ColorSpace/DeviceRGB

/Width 520

/Height 351

/Type/Xobject

>sstream

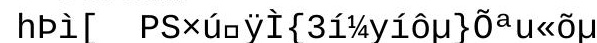

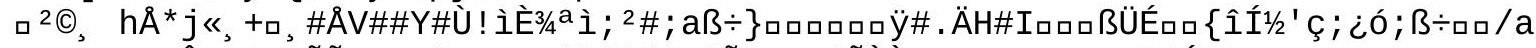

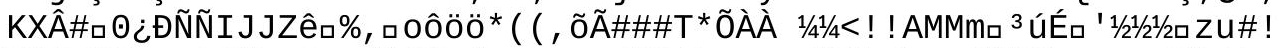

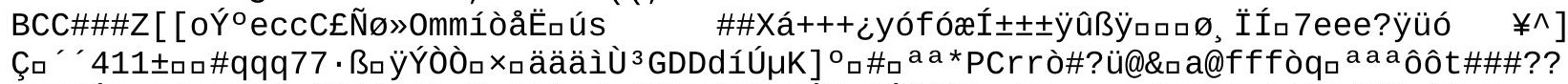

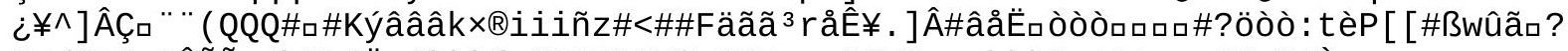

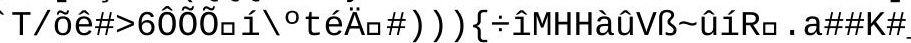
\%^XÂG\#ĐÚOCO) : 1 : 1 1 11 1 1 YYYÁÁÁ - - -\#

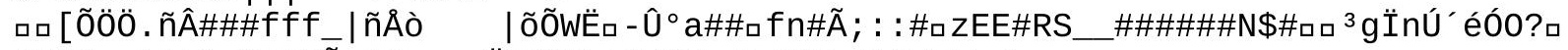

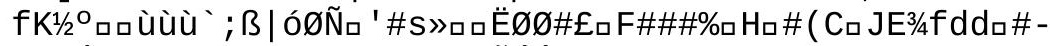

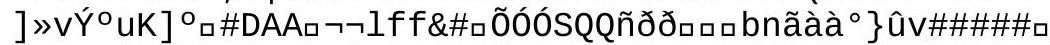

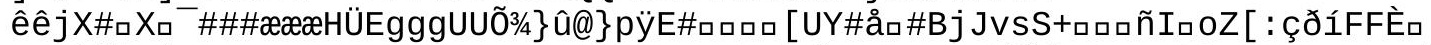

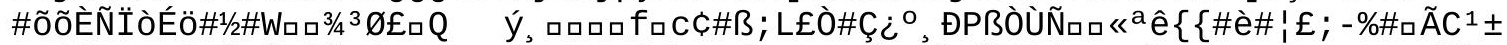

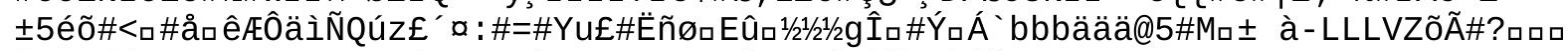

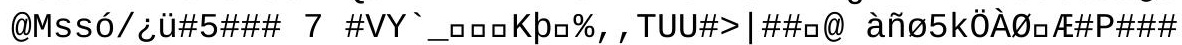

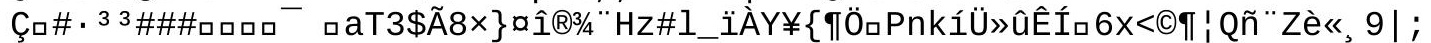

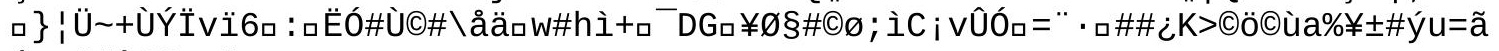

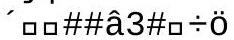

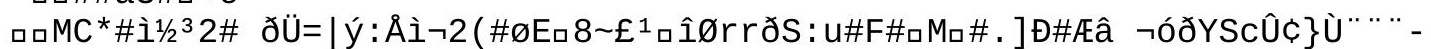

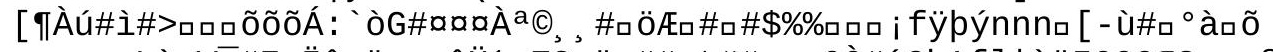

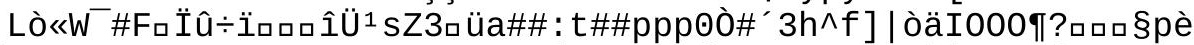

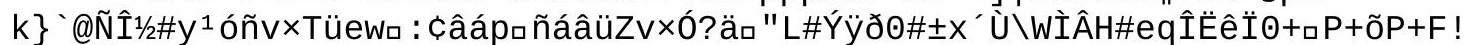

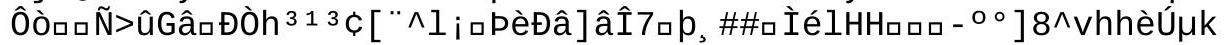




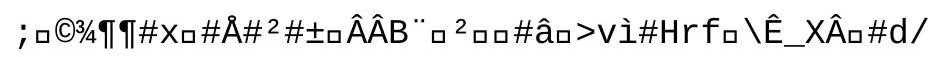




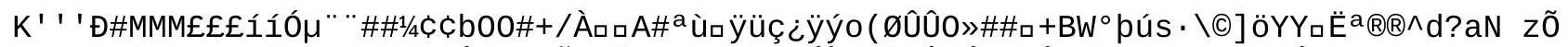

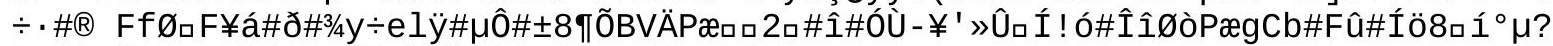
p<Ë)\#Û3L\#£ lã $\left.{ }^{a} 1 / 4 \neg f ` \# A ̃ e ́ l ` ` ̃ ̃ v\right) \# 4{ }^{\circ} \mathrm{Chp} \cdot \mathrm{a}^{a}$

$\square=\varnothing \#$

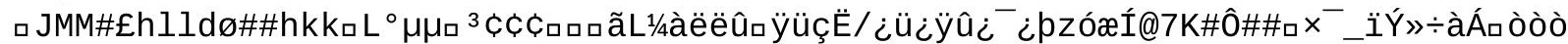

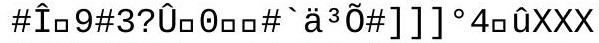

' c"

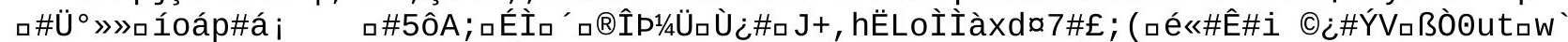

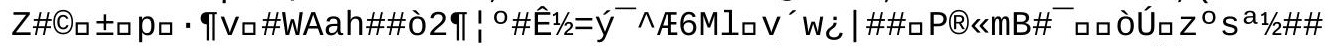

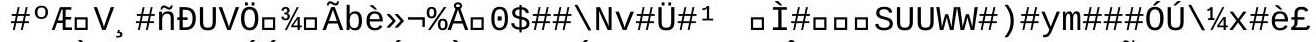

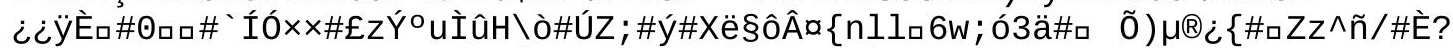

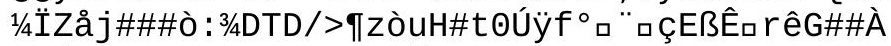

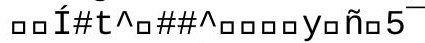

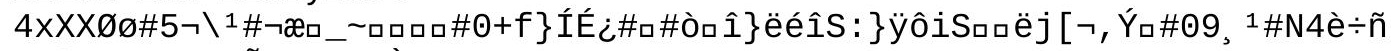
áû] êêúöï ${ }^{3} \# \#$ Õçr 


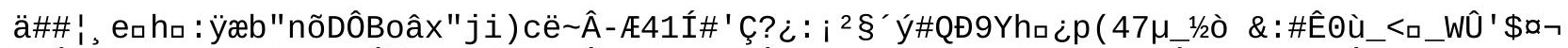

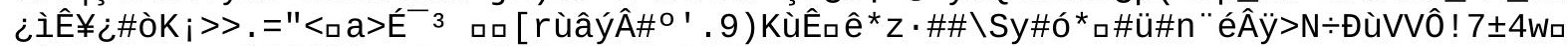

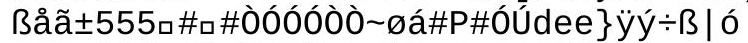

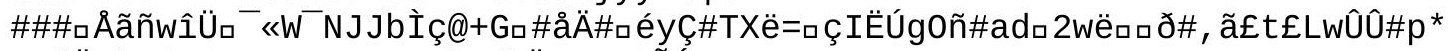

$+\# \hat{A ̂ A ̈} a ̀ I G \# £ T U \emptyset$ MJ (\#\#\}Ë\#\#FqGN̂́,

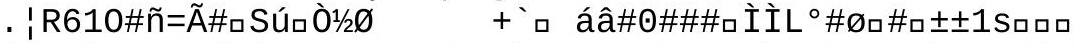

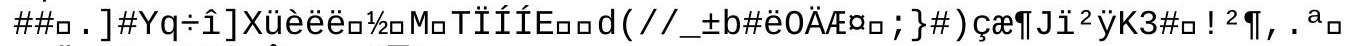

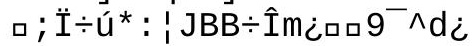

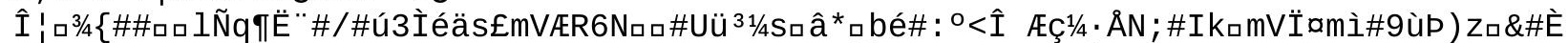

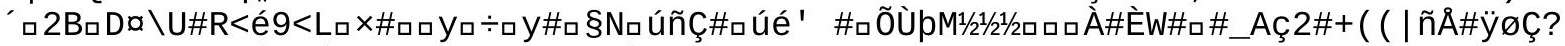

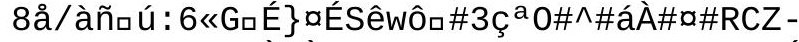

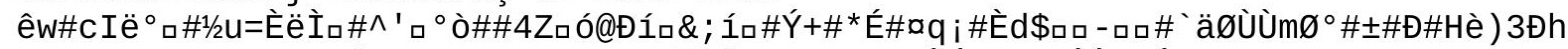

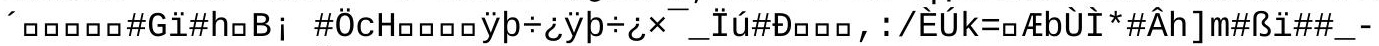

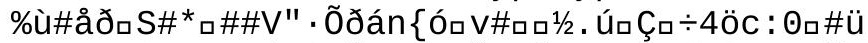

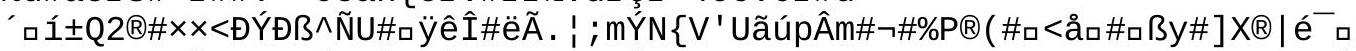

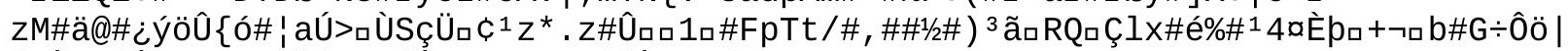

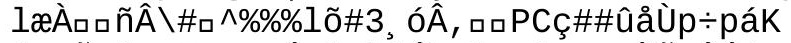

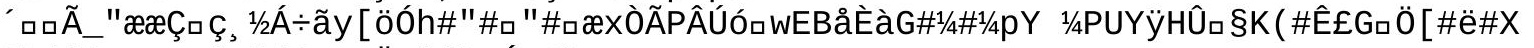

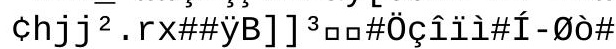


àò ${ }^{\circ}$ ç̧ç\} 


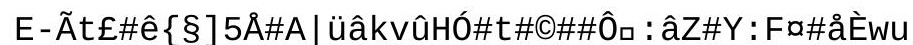

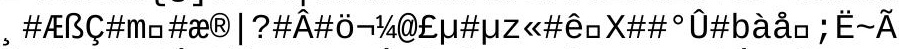

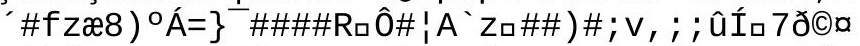

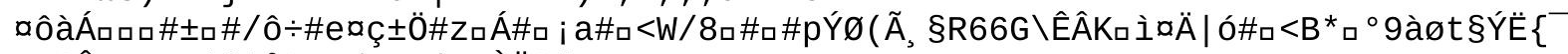

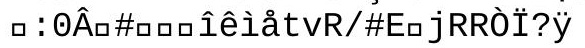

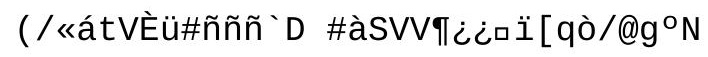




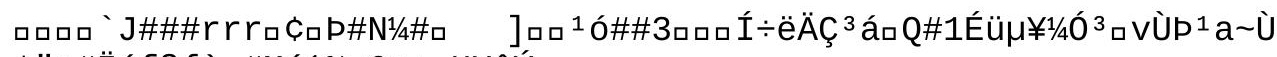

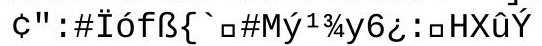




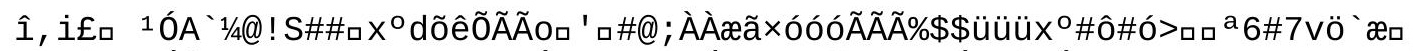

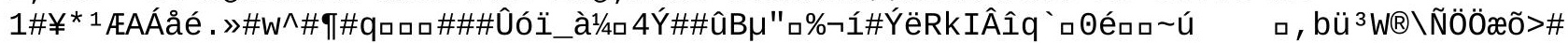




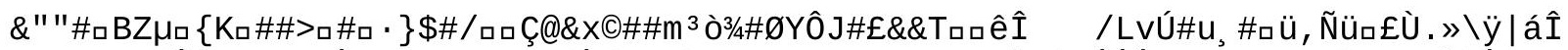

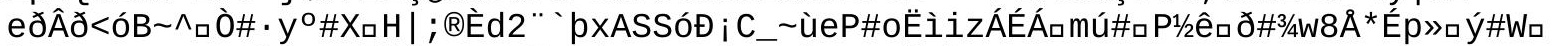

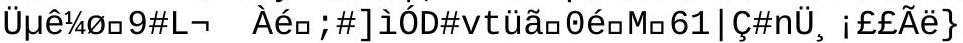

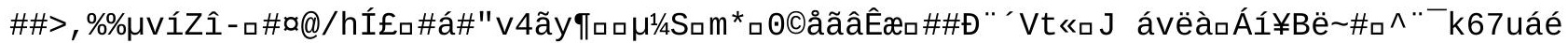

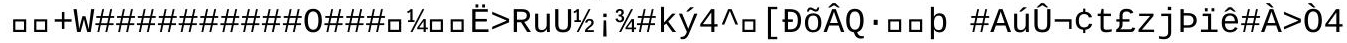




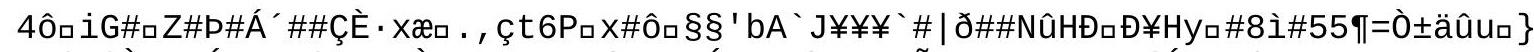

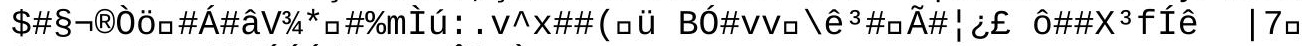

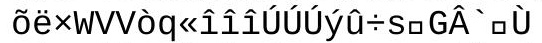

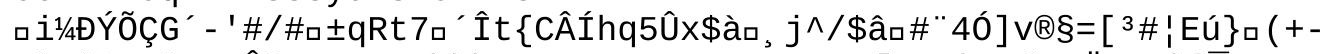

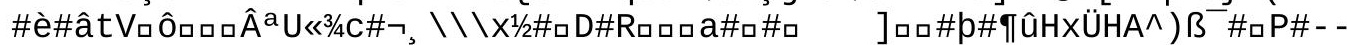

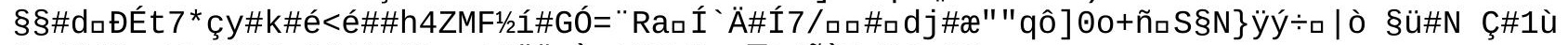

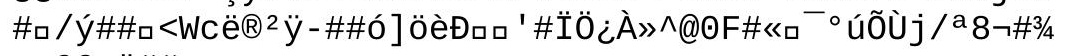

口- ○Cฉü\#\#

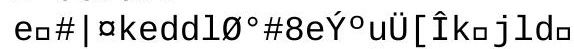

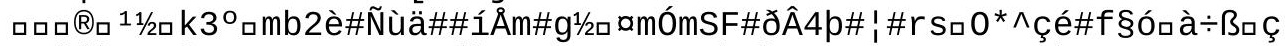

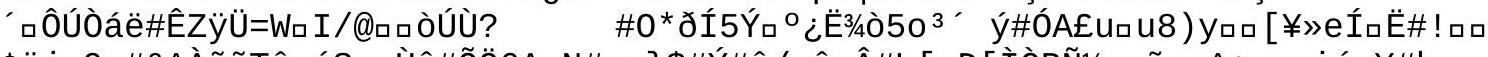

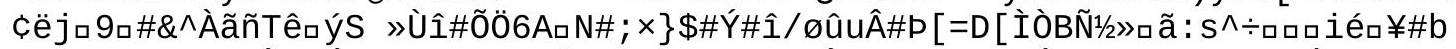

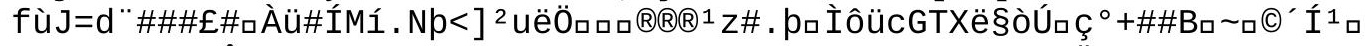

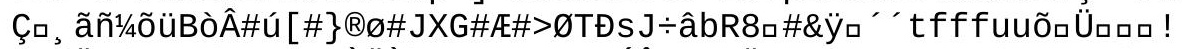

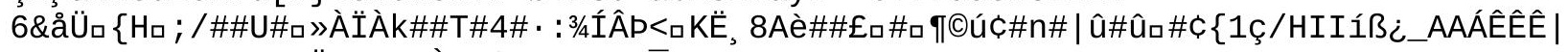

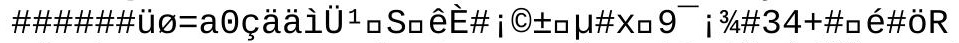

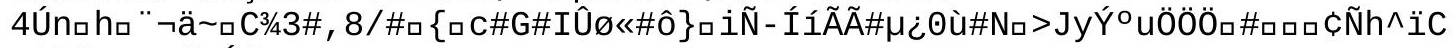

\#2 3 3 \#\#\#W@\ÉfH 


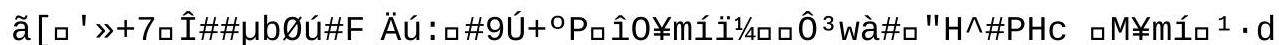

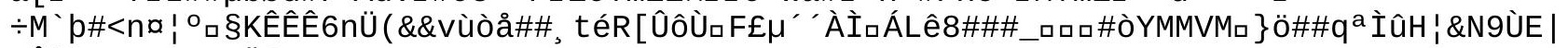
WẦ ûrë\#_amVıÖ@T

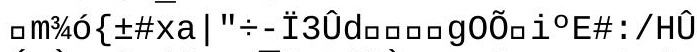

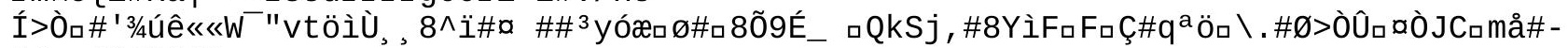
ä\#ロロ\#(£äñ\#\# 


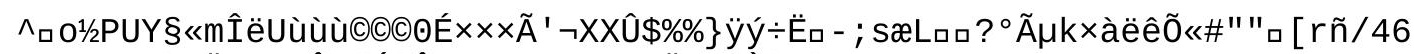

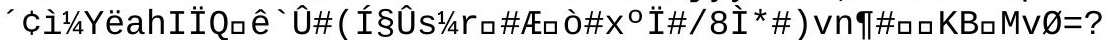

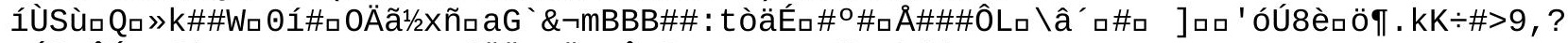

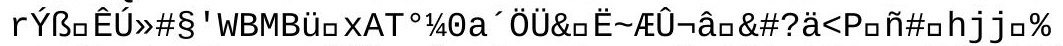

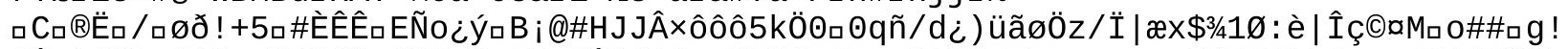

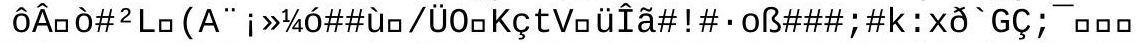

Vãééihh $(++; 66$ !

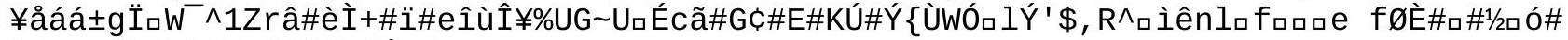
$\# x^{-} \_$¿dà,$]^{\circ} X, \# \# \#^{\circ} \backslash$ òún\#=

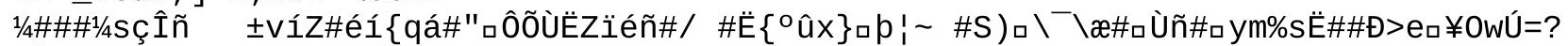

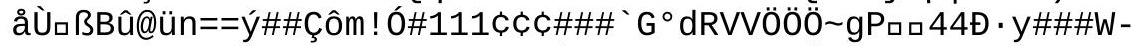

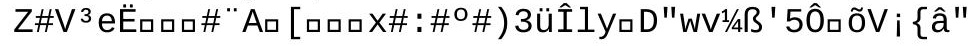




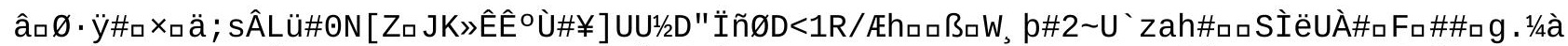

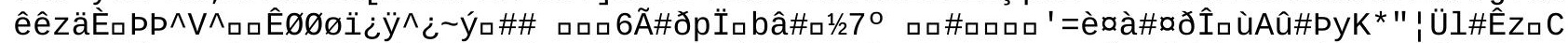

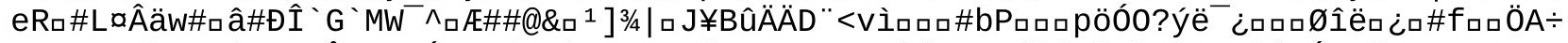

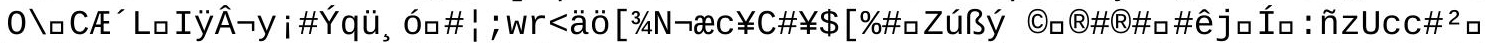

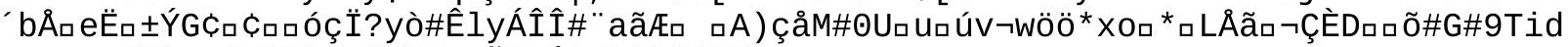

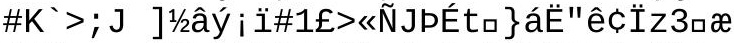

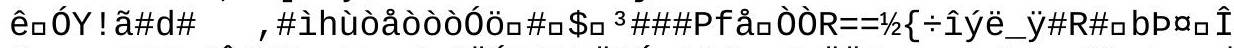

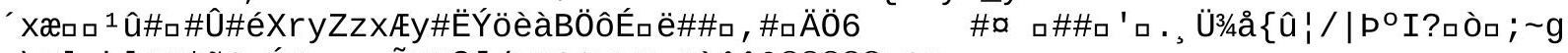

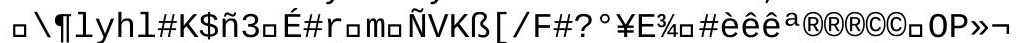

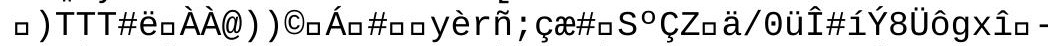

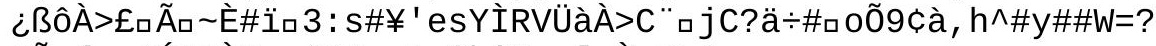

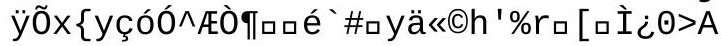




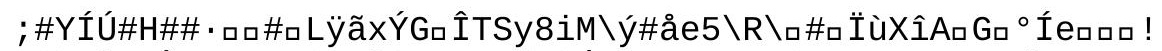

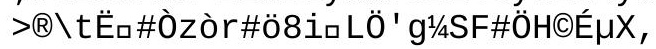

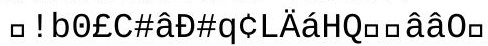




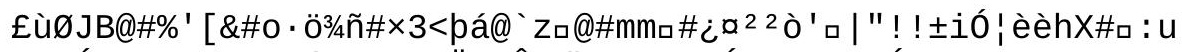

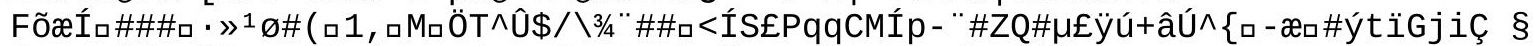

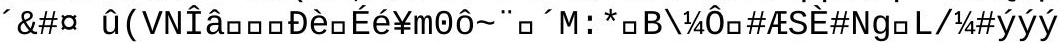

»wï\#

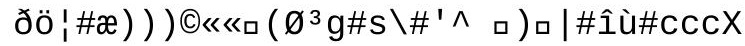




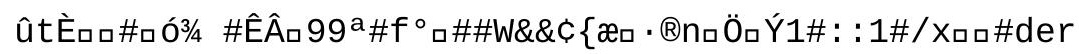


๑ON囚ß介Í́ロQ 


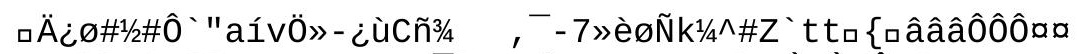

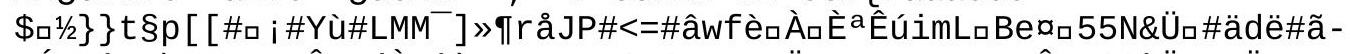

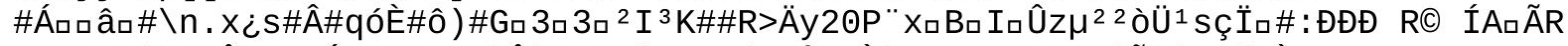

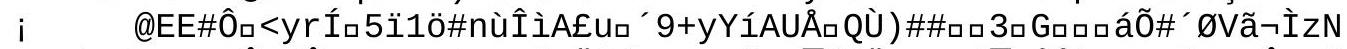

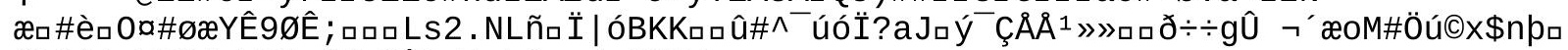

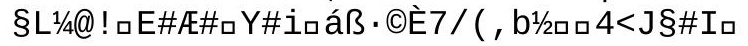




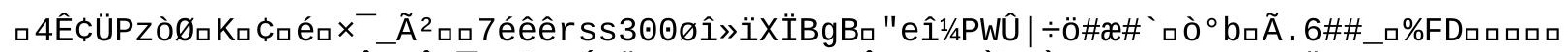

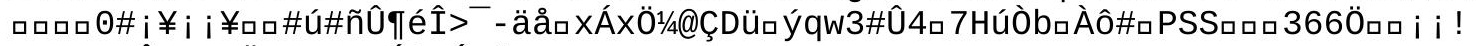

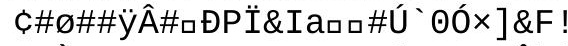

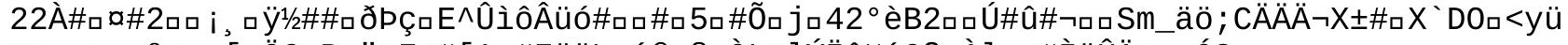

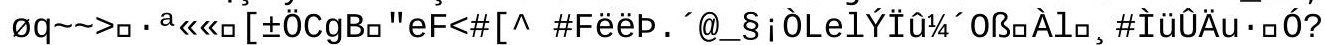

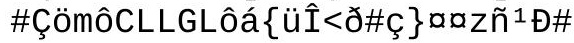




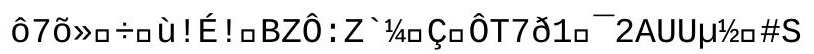



| úôé0?ýÄI/p\#ða'ì [^, pV\#*âõ’ $6 \mathrm{t}^{1 / 2}$ ó

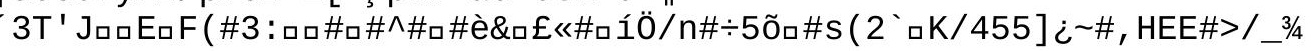




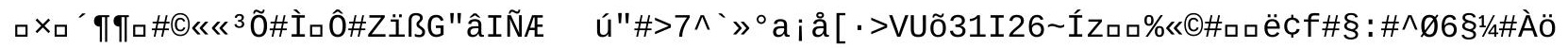




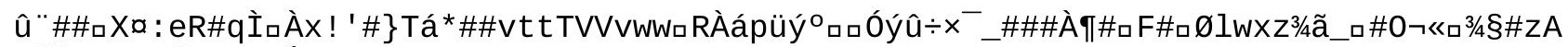

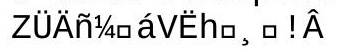




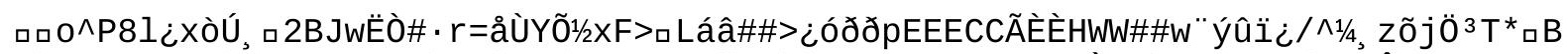

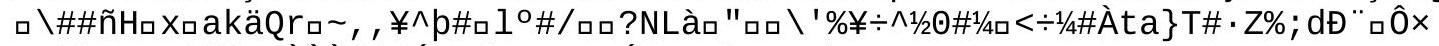

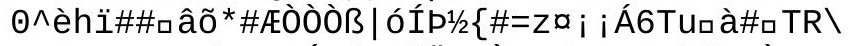

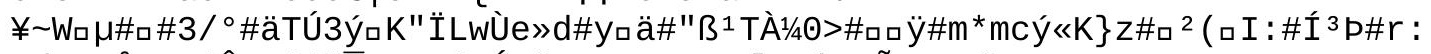

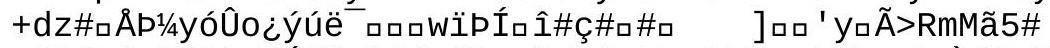

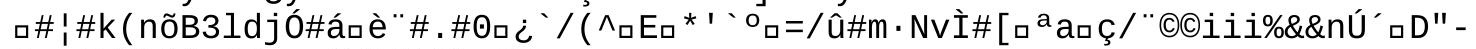

]»口\$3/4óLÀÅ¿@\$口: ; $\{$ XëéûHLqa $¥$

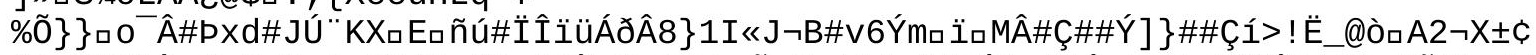

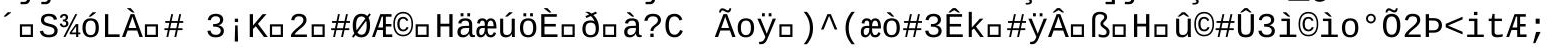




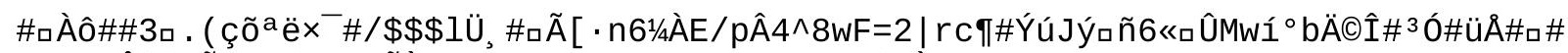

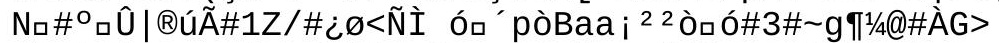

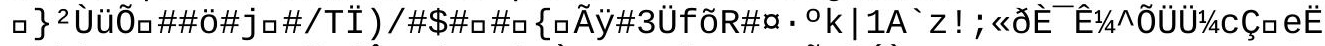

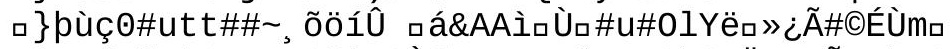

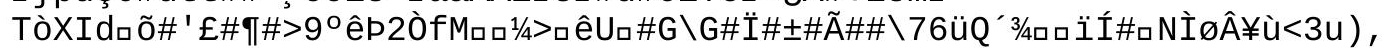

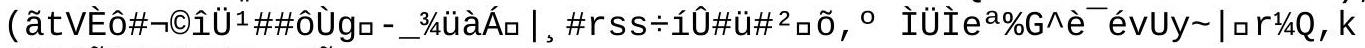
$\div \#$ ê\# ${ }^{1} \tilde{a} \# " i{ }^{3} / 4 Z \square \emptyset \tilde{N}, c ̧$ 


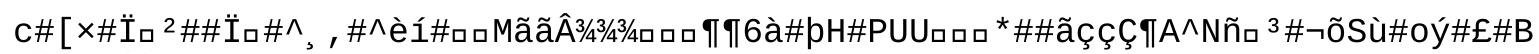

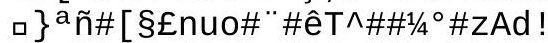

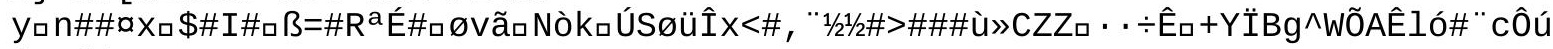
'ZçãîZqu ¿+D \#Ø 


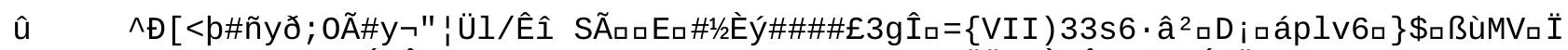

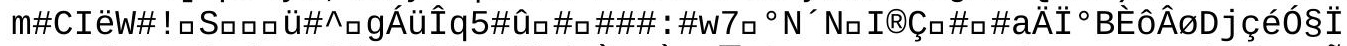

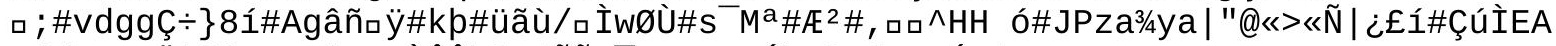

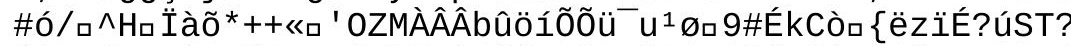

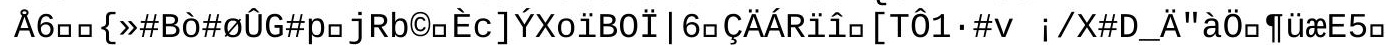

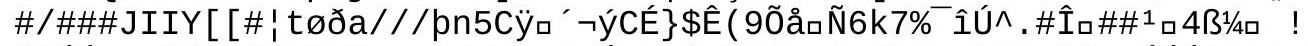

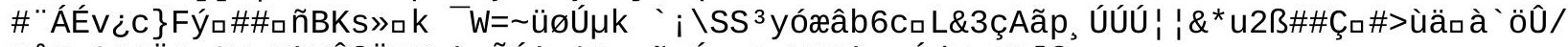

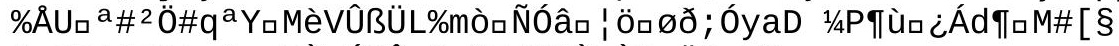

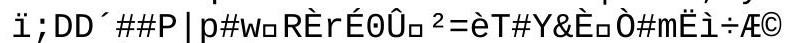

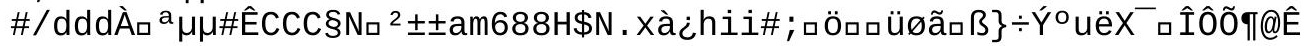




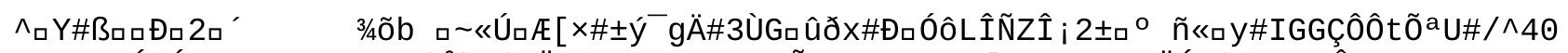

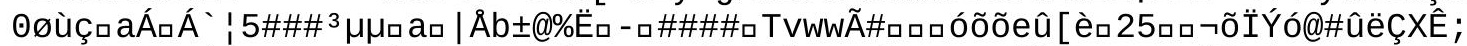

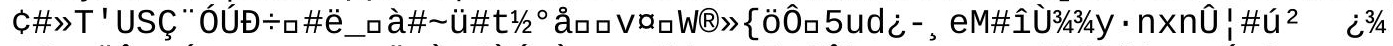

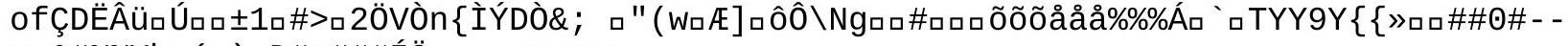

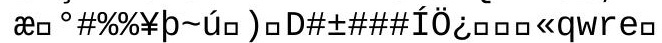

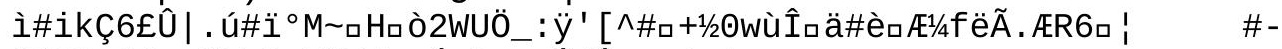

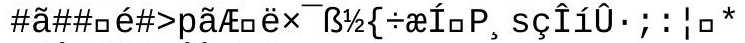

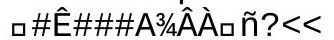




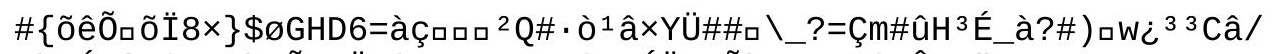

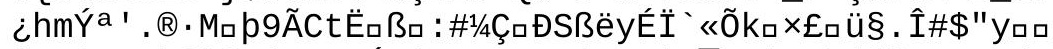

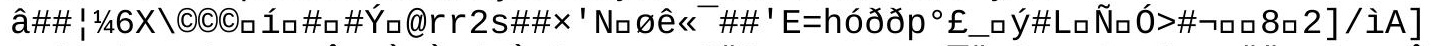

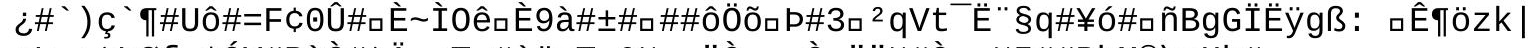

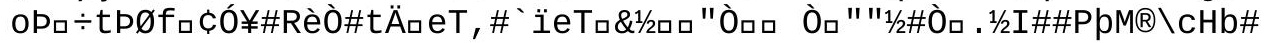




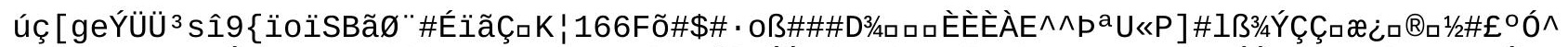

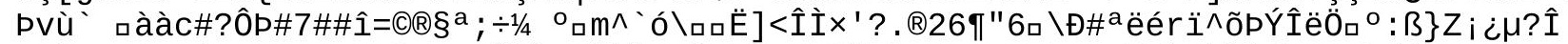

Æaà\#x8, . Ç’\#goèRıR=óÉ\#/Æh6r 


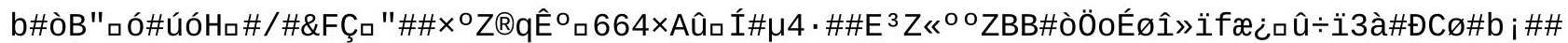

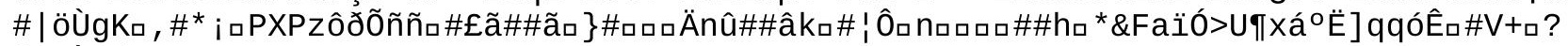

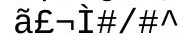




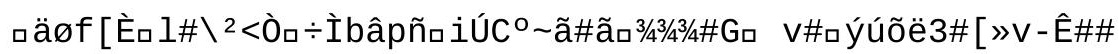




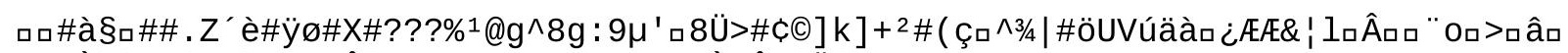

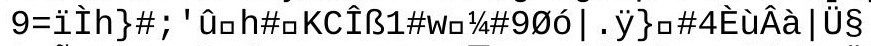

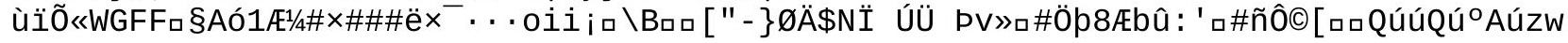




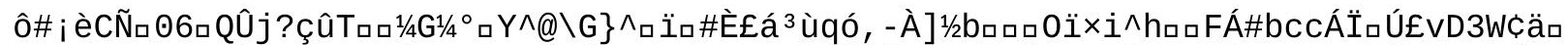

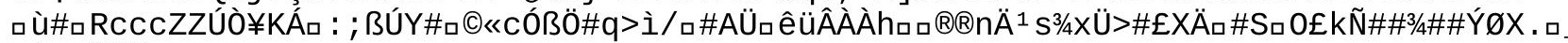
\#åp>0̃Ñ $¿ m$

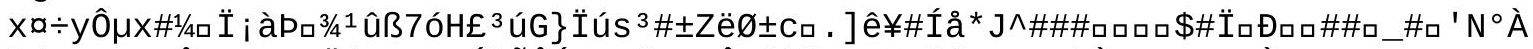

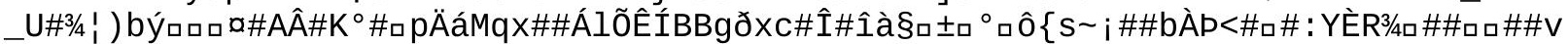

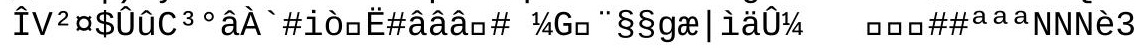




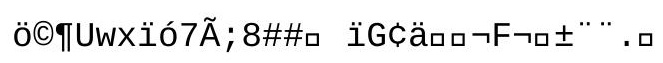




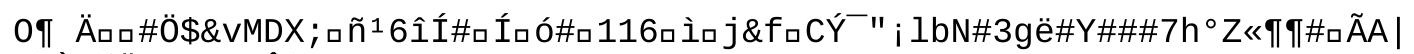

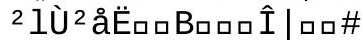

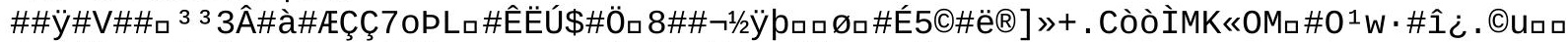

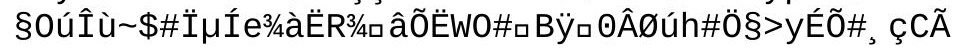




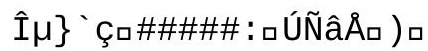


$P())$ i\#\#\#\#ロ!\#X\#İ\#1

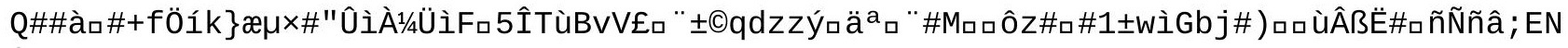
a 7,\%\#£ロ\#ם $\div$ 


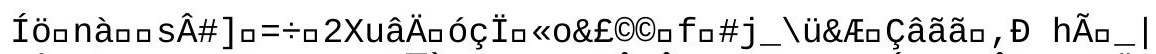

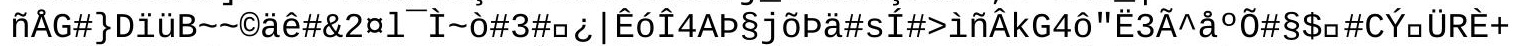

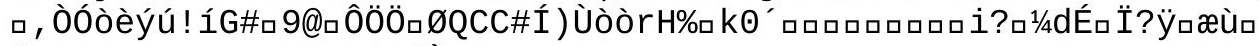

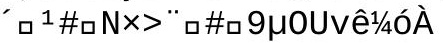


$\wedge \#$ ^X]UWìø\#ÍvFDa1/2Sajbb

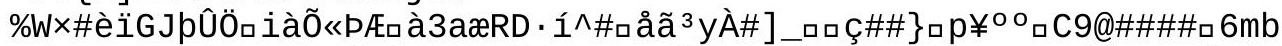

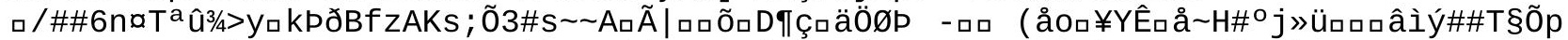

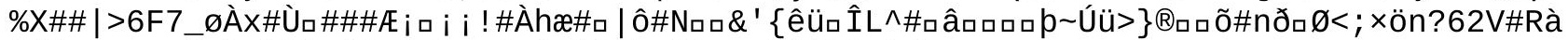

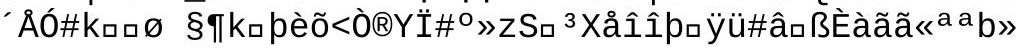




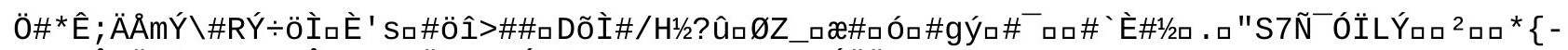

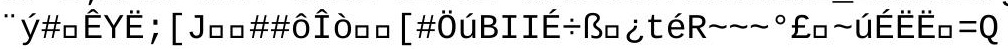




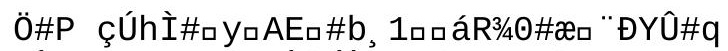

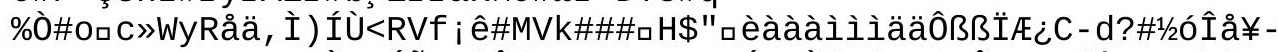

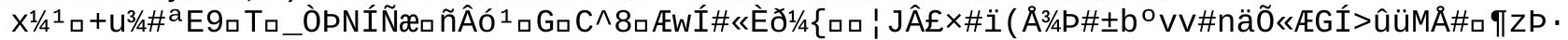

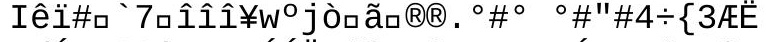

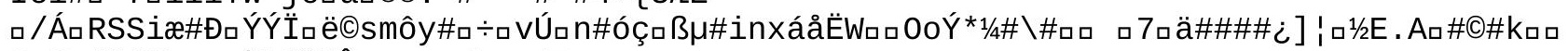

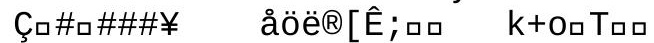

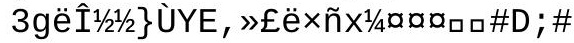




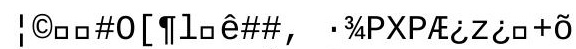

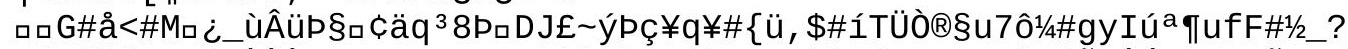

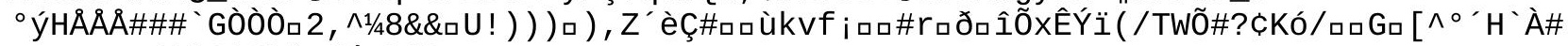

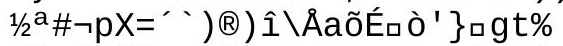

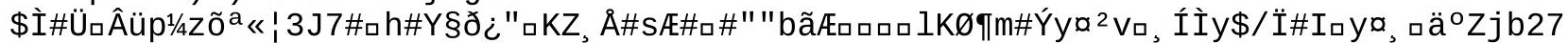

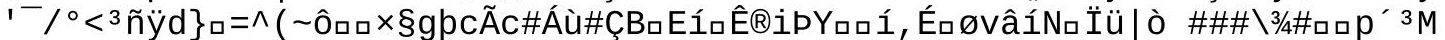

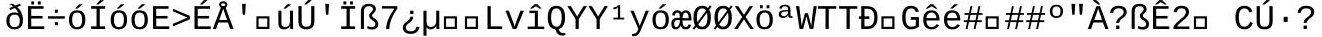

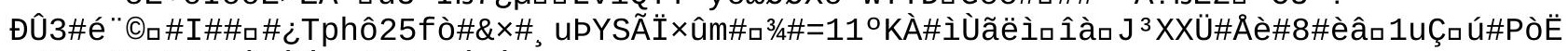

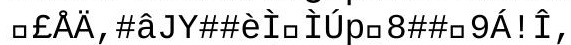

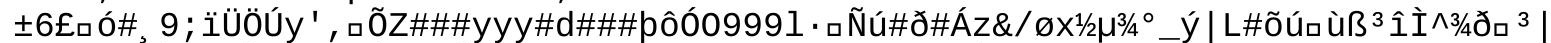

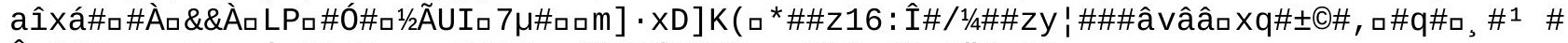

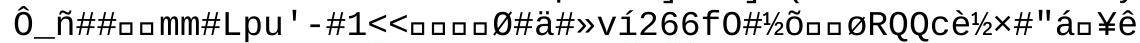

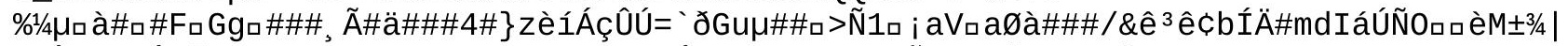

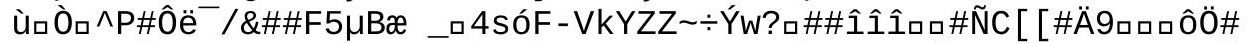

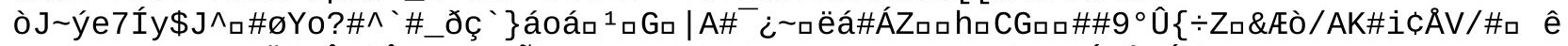

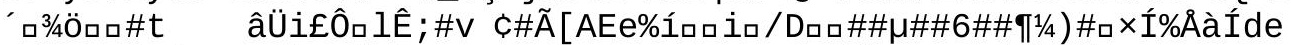

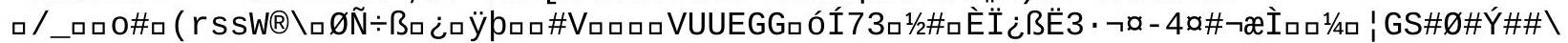

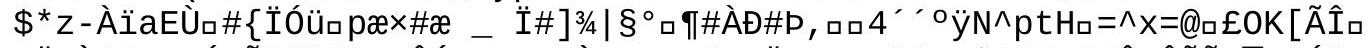

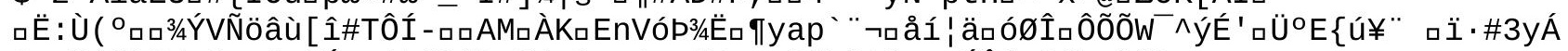
Ç; $\square$ Có\#l®/øÿ=ç\#æt

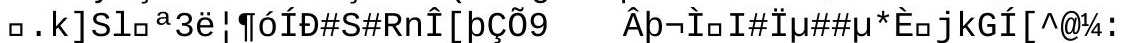

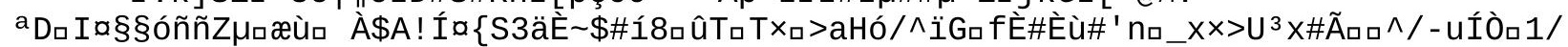

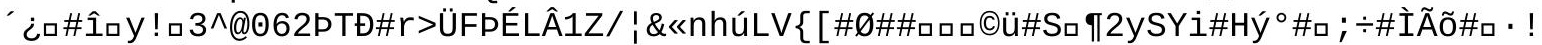

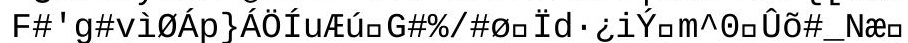

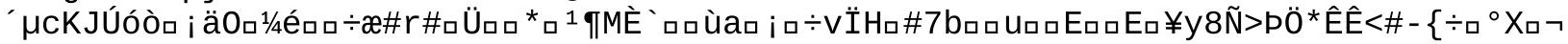




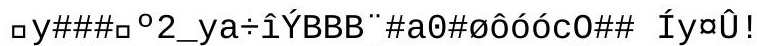

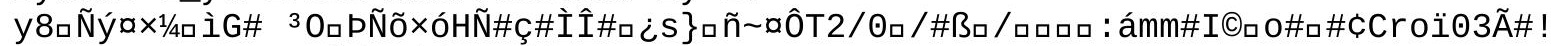

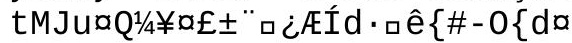




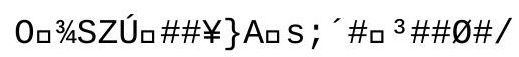





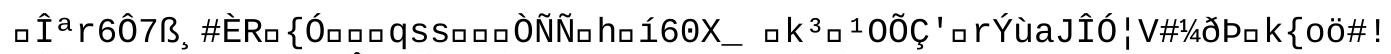

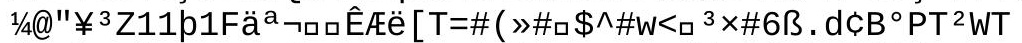

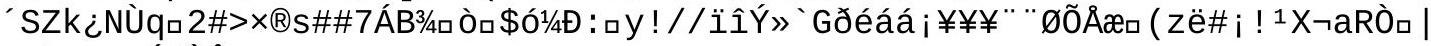

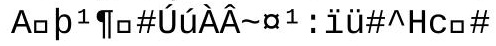




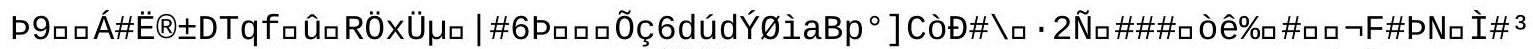

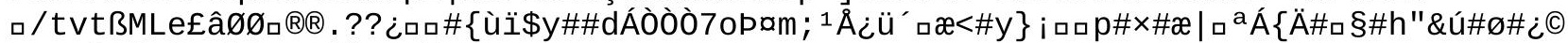
$B \# M M\} \square \ddot{I} \square$ êzZk $\left.{ }^{\circ} r=3 U ̈ 01 / 4 \neg \#\right]$ 


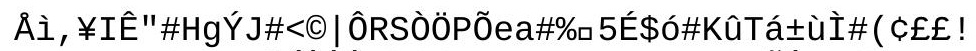
SPVV\#\#\#d£úóçİ̈́óòÀnh®/\#pLä\#øG9ロDïüBiIõîmÇip\#ロ\# 
$c c \#$ 


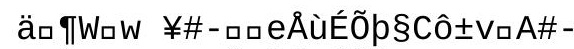

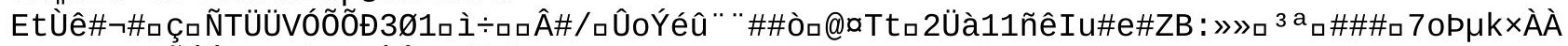

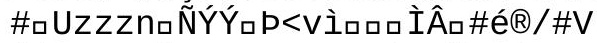

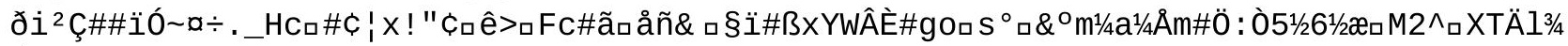
/g: :у⿺ ר 


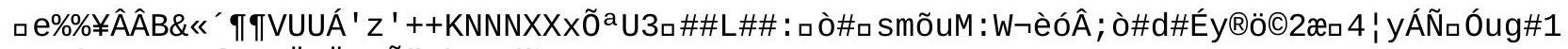

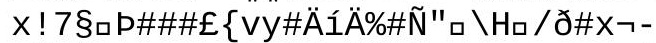

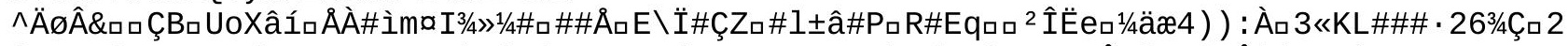

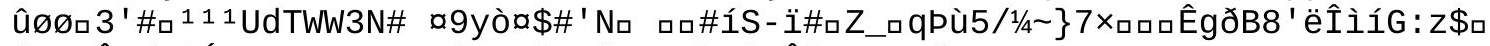

Ÿロ

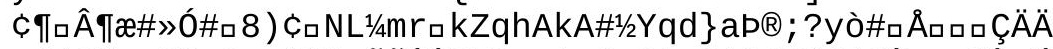

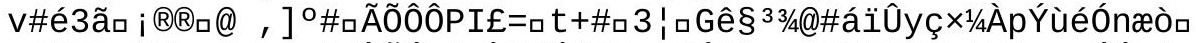

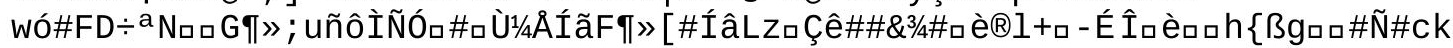

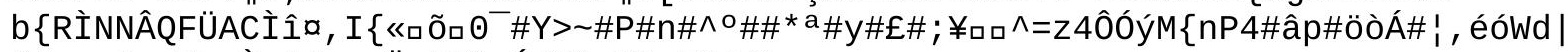

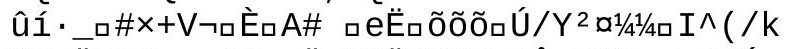

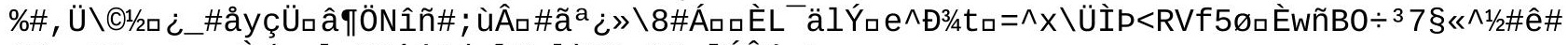

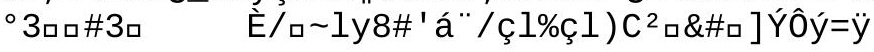




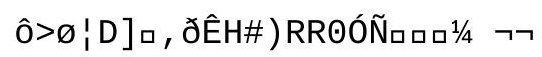


F\#V\#æロロ\#8|WWW\#U 


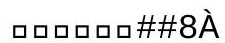


/ロロæA.S®; $\# \# \$ \square$ 


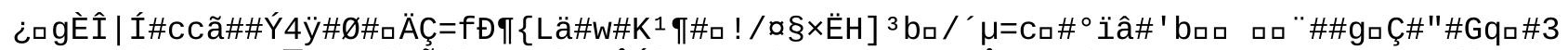

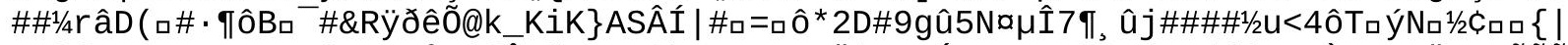

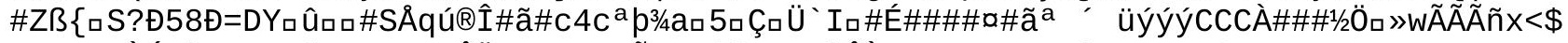

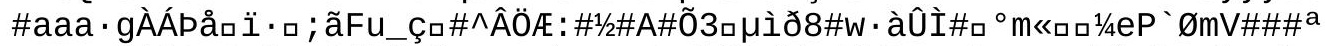

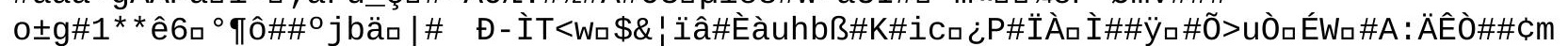

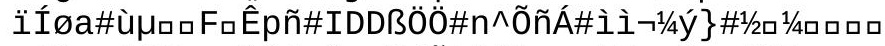

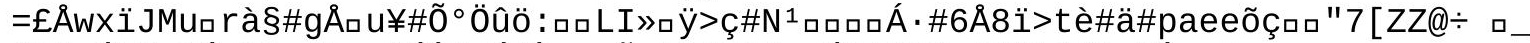

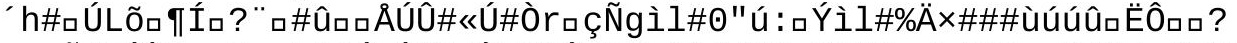

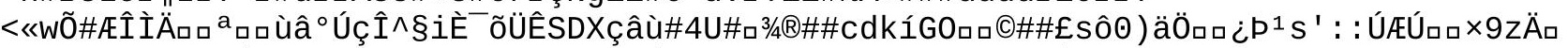

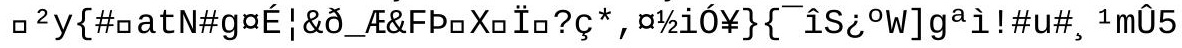




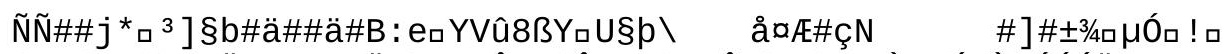

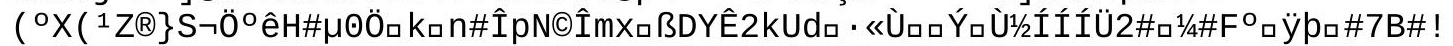

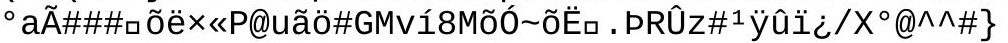




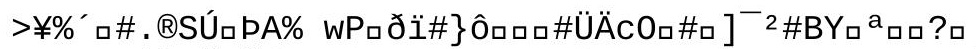

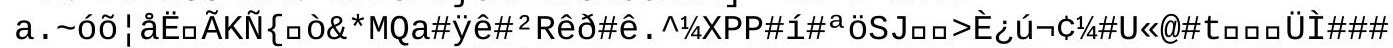

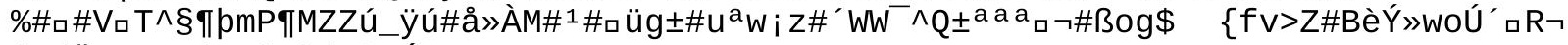

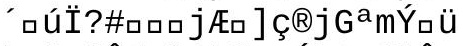

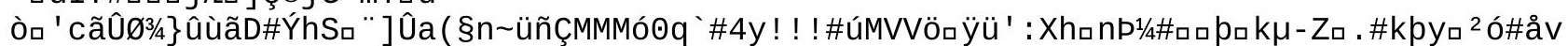

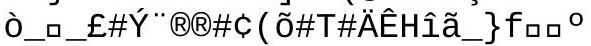

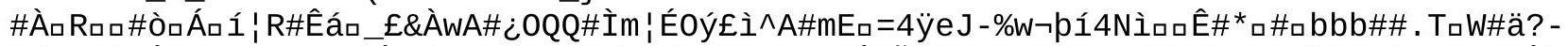

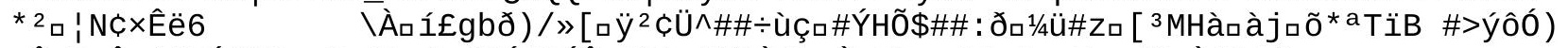

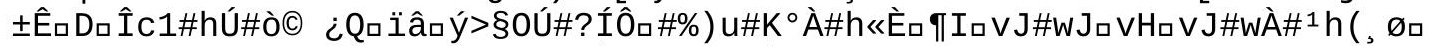

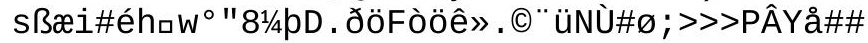




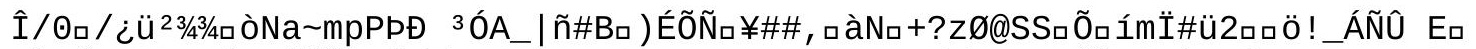

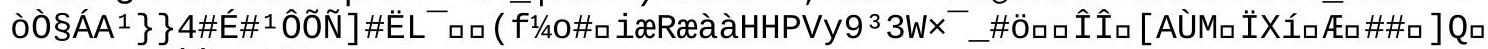
\#jÈò¥Kkkk? 


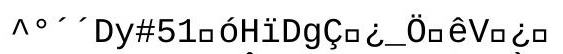

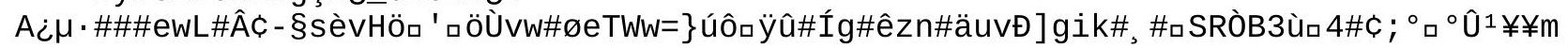




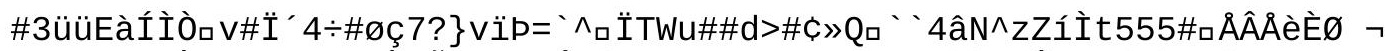

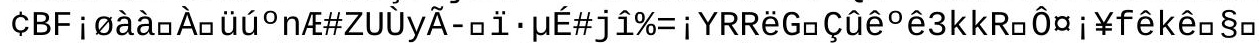

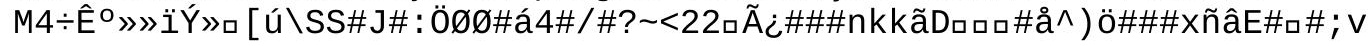




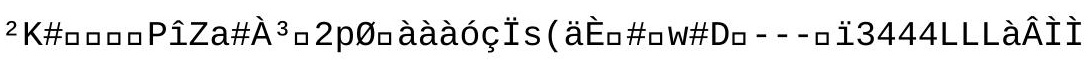




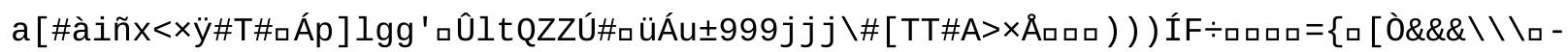
[\#aàääD5ロ

9ロø\#\#\#а̃PÂøø8ço

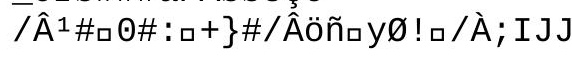

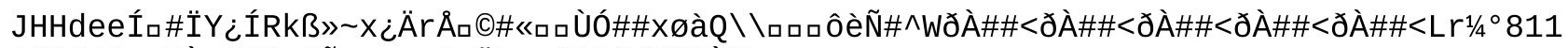
ñüùóááađ\%À¿\#\#øn\#Õ+ロ

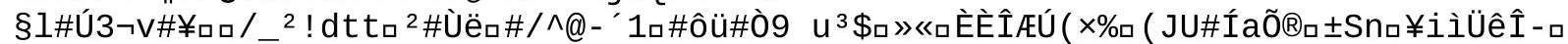

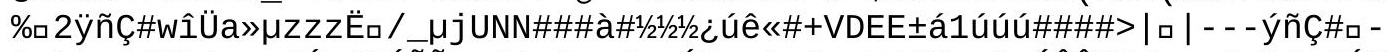

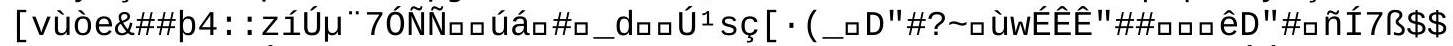

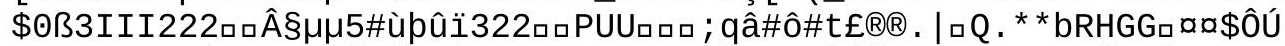

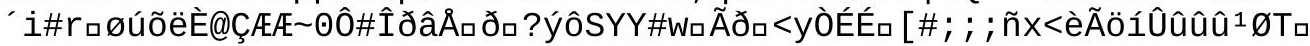




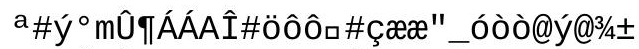

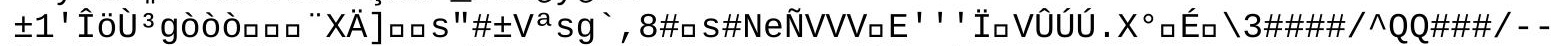

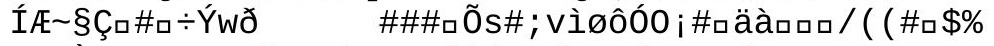

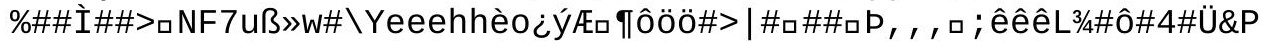




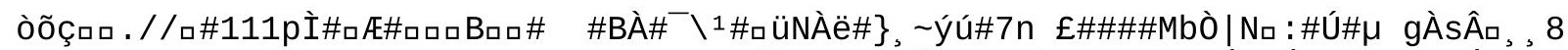

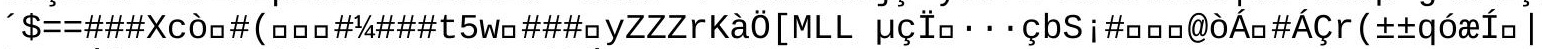

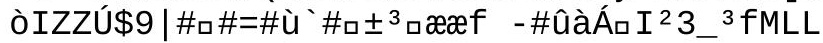




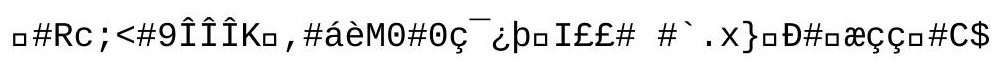




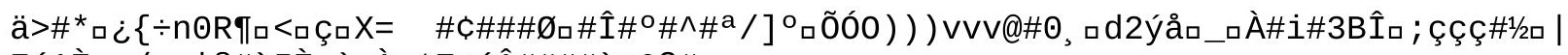

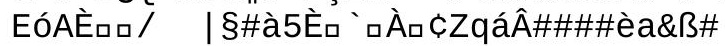




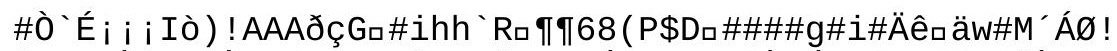

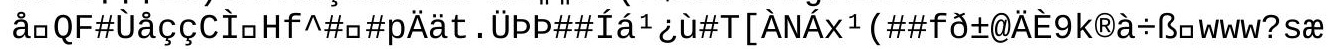




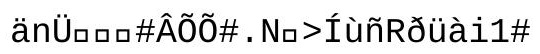


âÀı\# a ArU

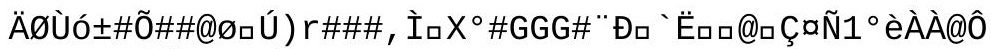

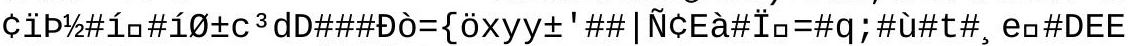

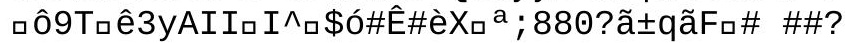




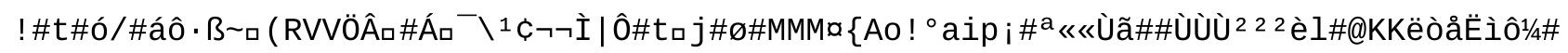




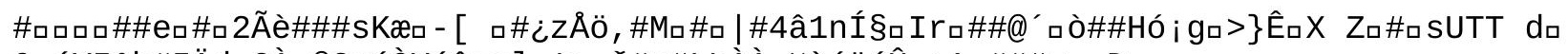

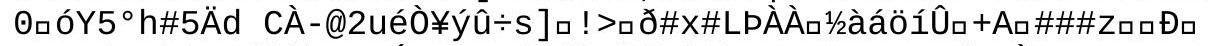

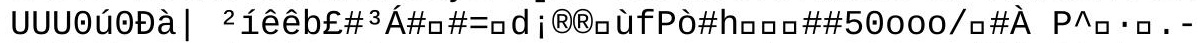

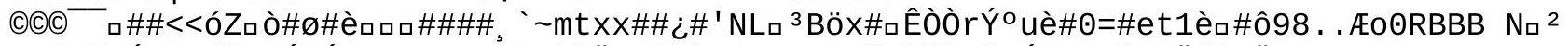

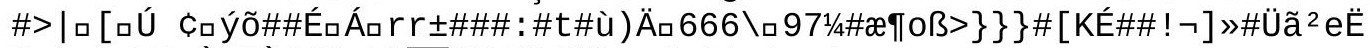

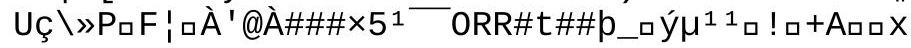


phlð\#ロÓH\#ÃãEGGOロ ${ }^{3} B$

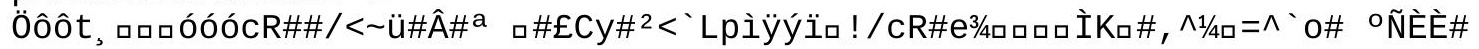




\section{Æ\#\#\#Ö ${ }^{3} \mathrm{Äp@F@}$}

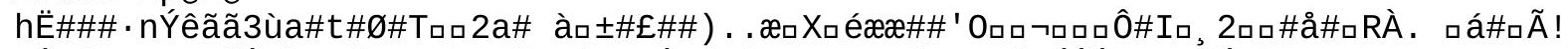

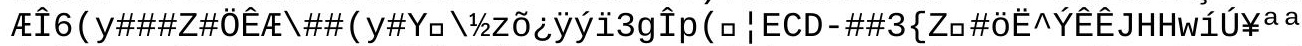

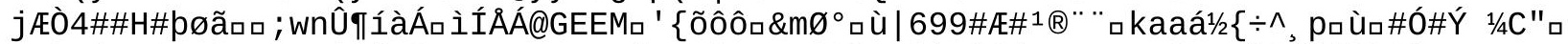

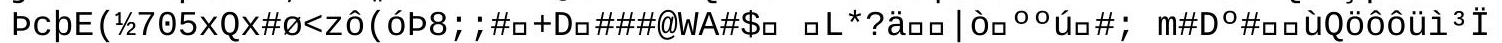

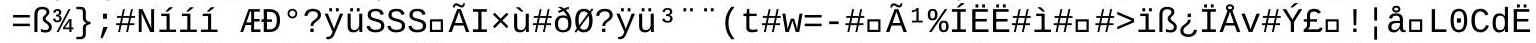

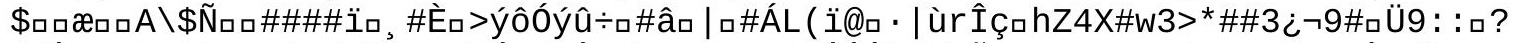

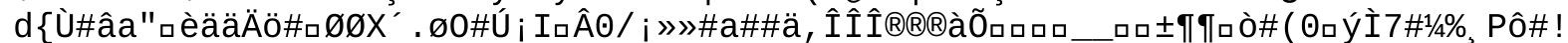

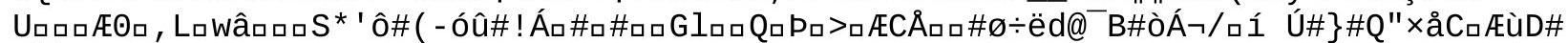

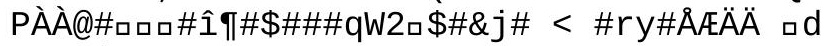

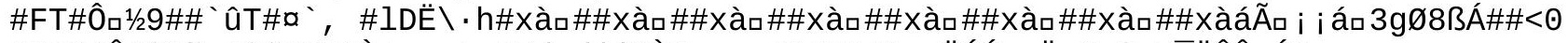

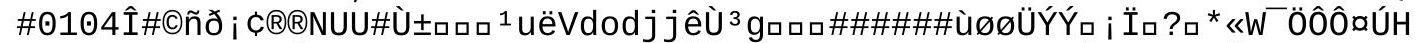

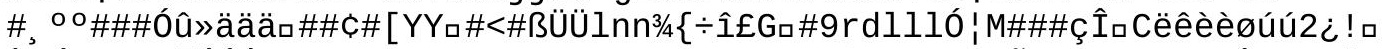

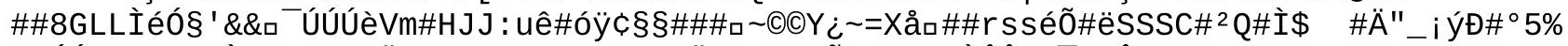

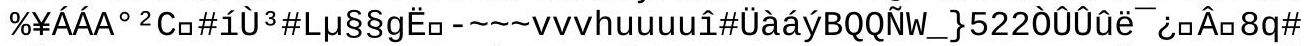

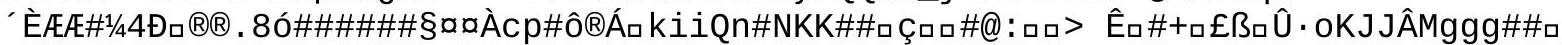

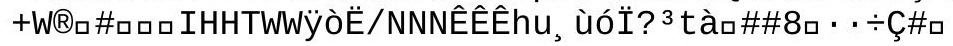




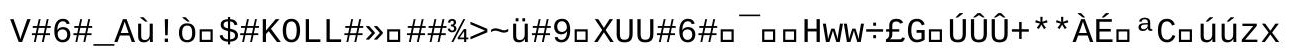


口\#Ç_ZZ

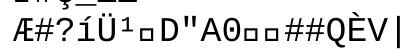




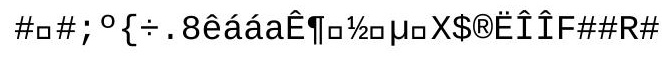
वप्

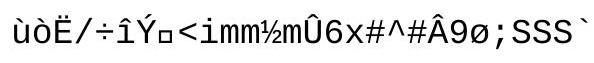




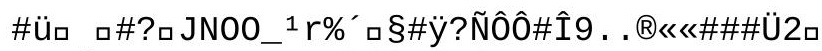

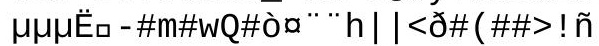




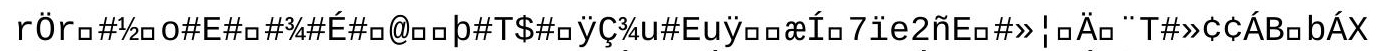

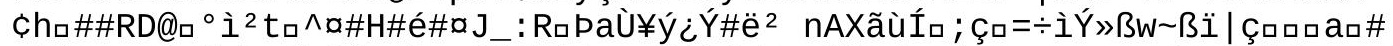

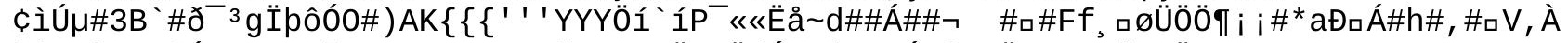

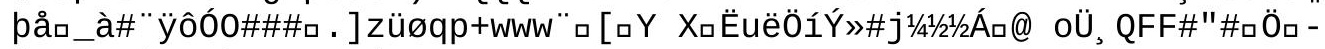

[\#L\#N\#a\#Frì»口nXÆ口i

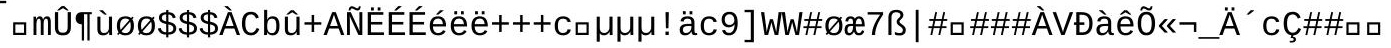




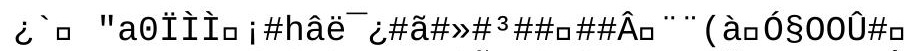

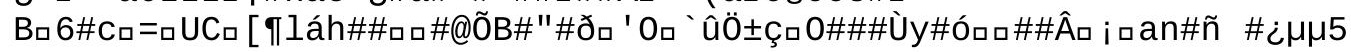




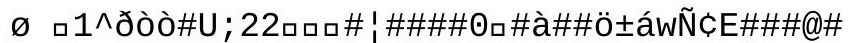

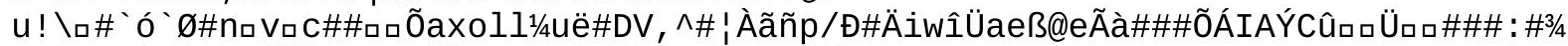

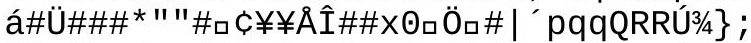

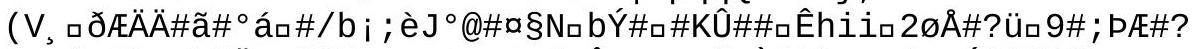

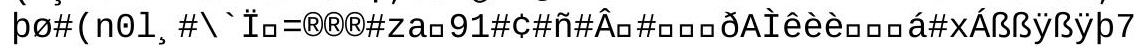




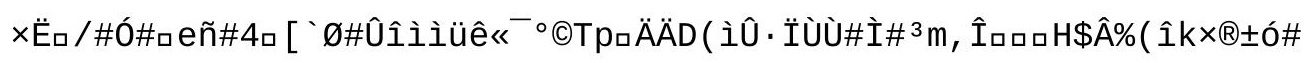




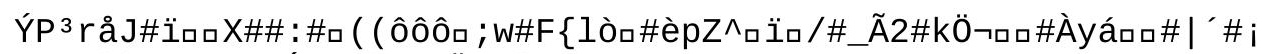
(\#\#6))

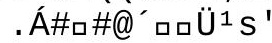




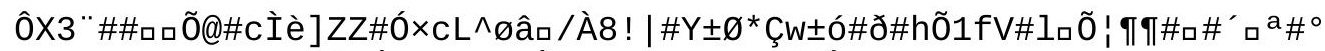
. Ä\#\#S\#ロ\#Æם ${ }^{3} \div \#$ À

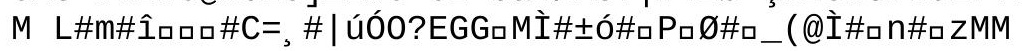


¿Өø_, paZ^\#)\#\#\#ôôéÓqם 


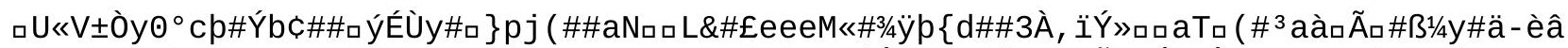

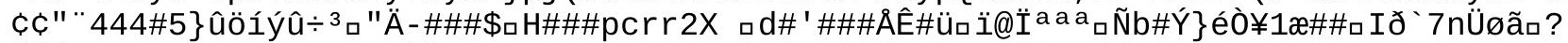

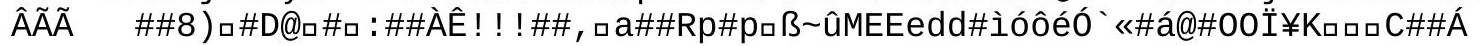




\section{Ü\{ùòeঃ\#p\#Ü\#\#ÙËËkÑ̂́CE}

\#êC\#\#ロàñxh\#0̂ôôrrr@@ロロ\#w@ם\#Z\#Ü\#zPTTd\#?

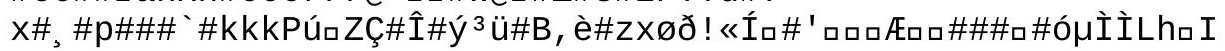




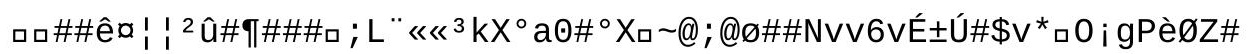




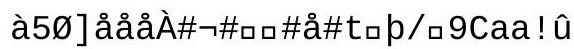

Ray

Æャá4ø\$ロÙ\#\#Á\% 


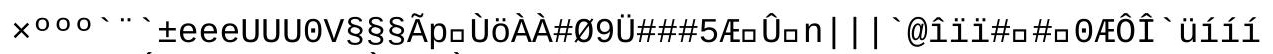

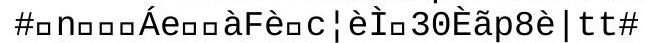

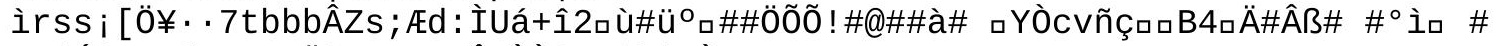

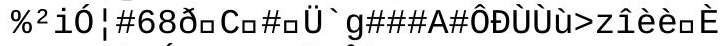

1/2an¿\#h4Ú·, \#\#l\#Åì

с $\mu \mu \mu_{\square} \mathrm{CE}^{*} \# \# \# \# \# \# \# \# \# \# \# \# \# \# \# \# \# \# \# \# \# \# \# \# \# \# \# \# \# \# \# \# \# \# \# \# \# \# \# \# \# \# \# \# \# \# \# \# \# \# \# \# \# \# \# \# \# \# \# \# \# \# \# \# \#$

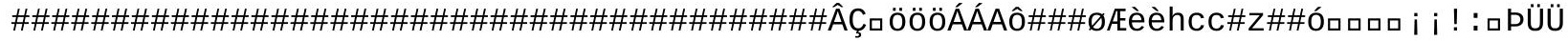




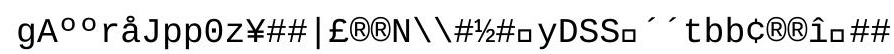






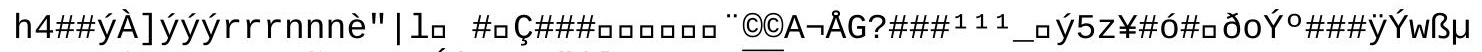

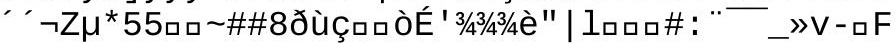

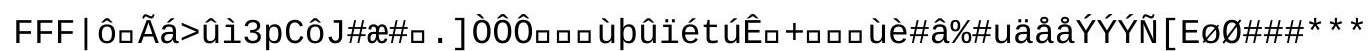

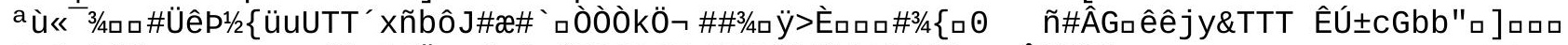

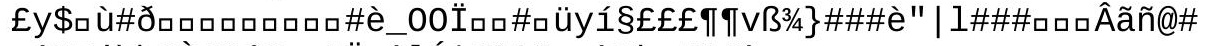

|

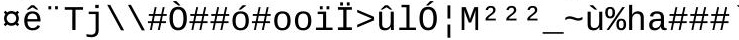

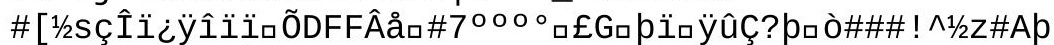

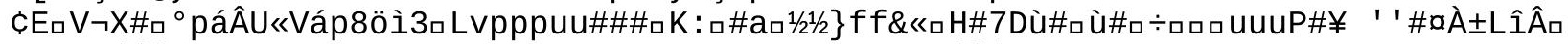

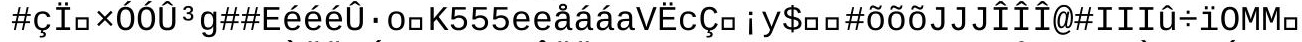

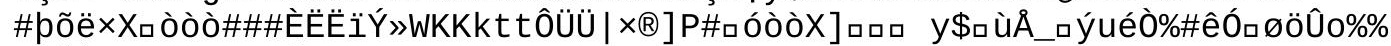

\%ÛÛÀS์raP8rä\#Ä3 16op 


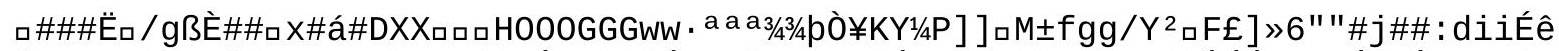

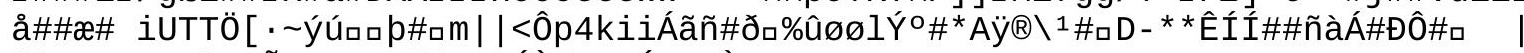

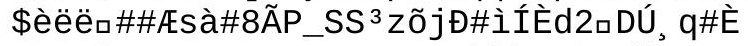

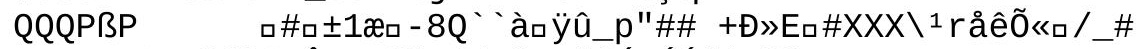

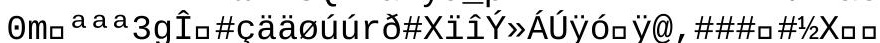

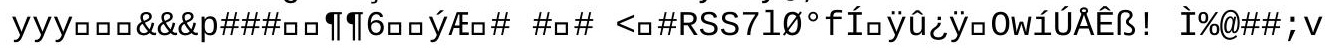


ฉÃ

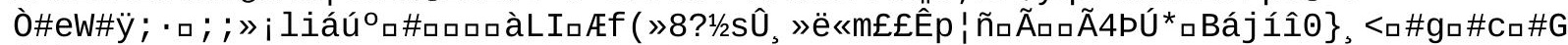
UbıSZE 


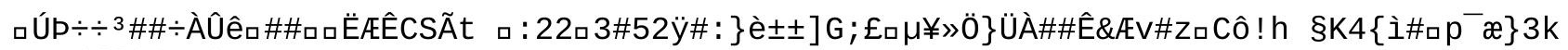
$+\mathscr{A} \$ \mathrm{~d}\left\{\left[\S \bigcirc_{\square}=1\right.\right.$

åú:

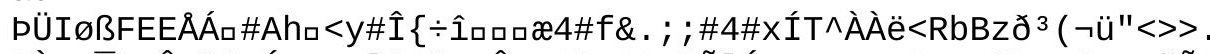

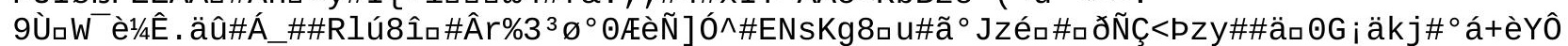

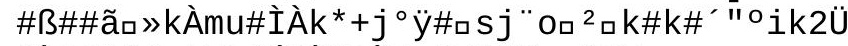

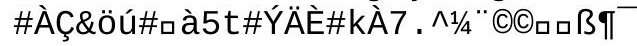


DÈÈ ( ( (\#\#\#00̈̈\#/^^ロロÃ\%p

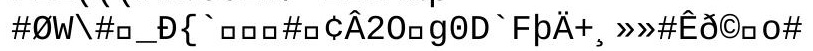




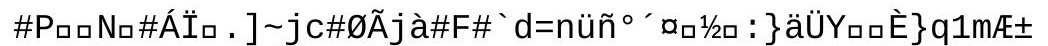

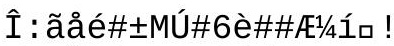

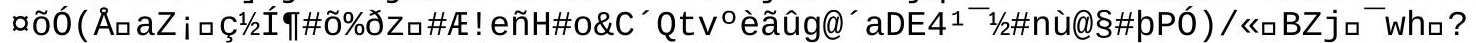

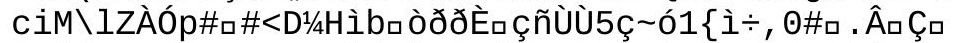

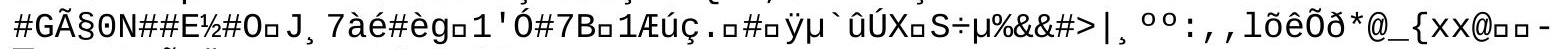

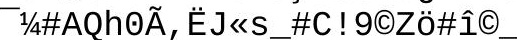

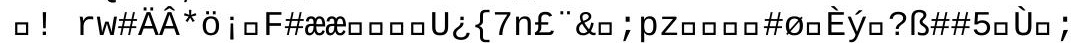

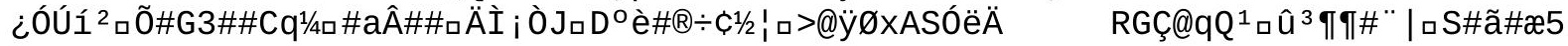


• ò\#W«ØロÔÖ口q\#\#/èì 
Оั๑) )บे๑3/40̃0 
口qp 


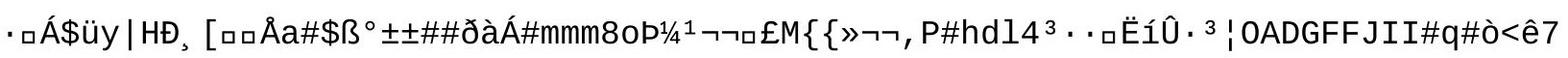




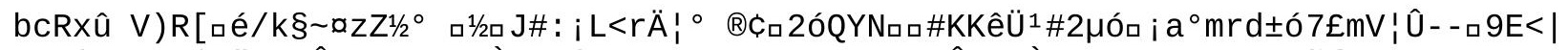

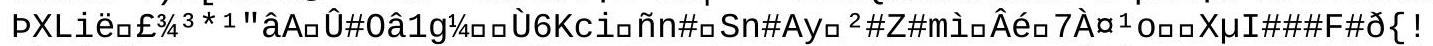

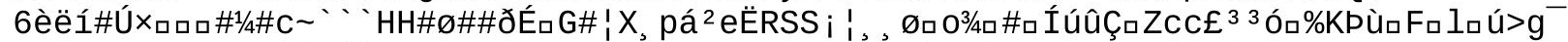

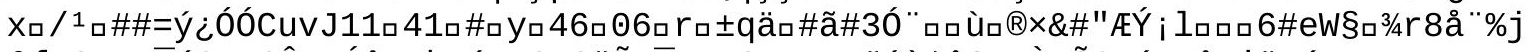

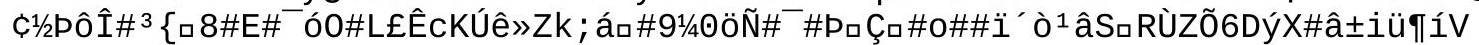

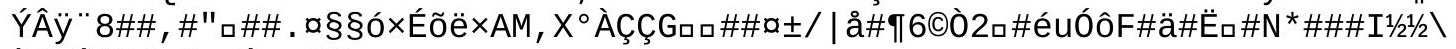

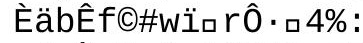

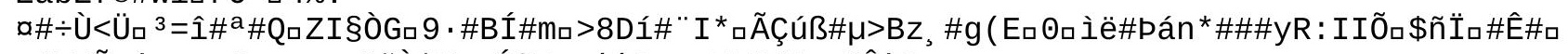

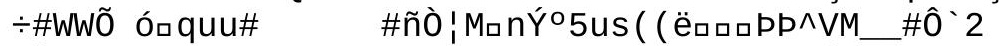

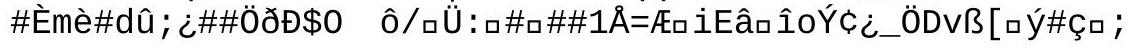




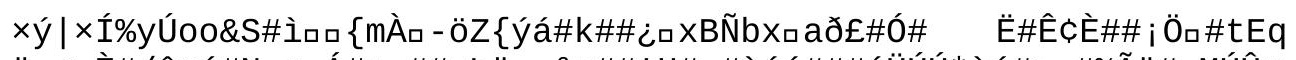

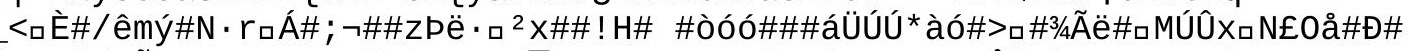

\óBq\#' / tõ

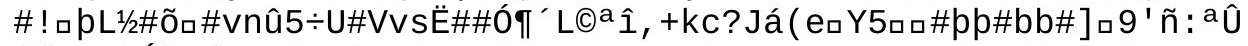

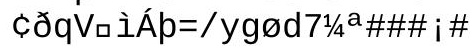




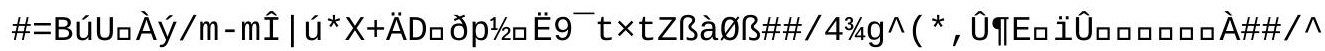




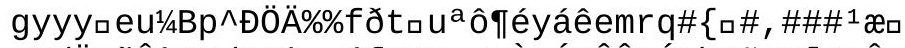

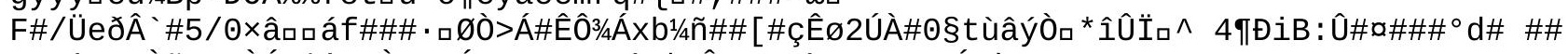

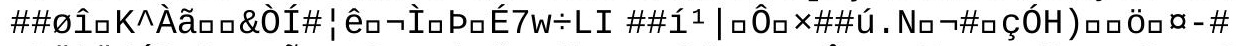

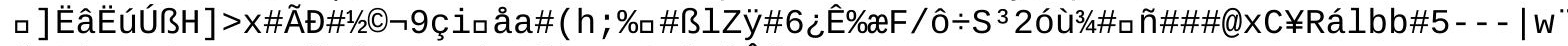

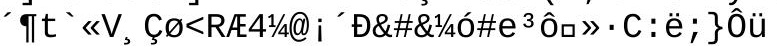




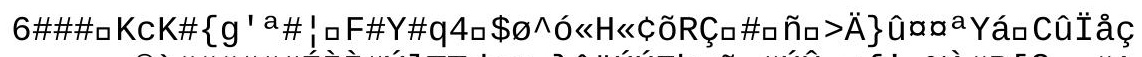

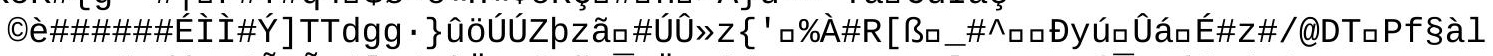

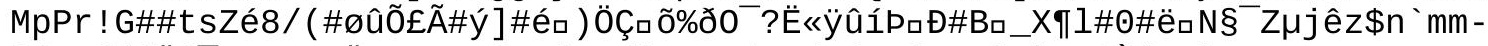

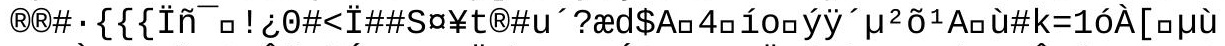

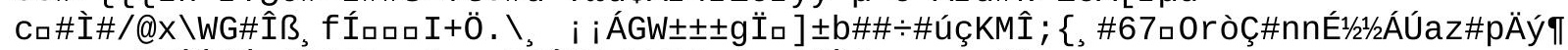

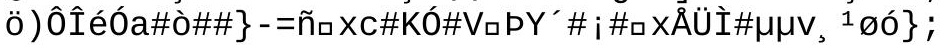


ãìs i À\#ß ûmJJ

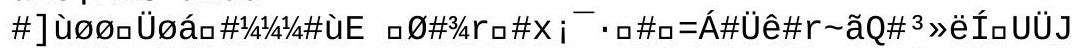

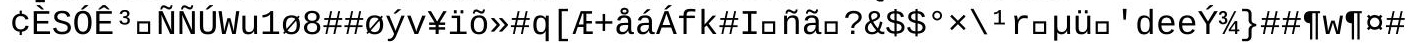




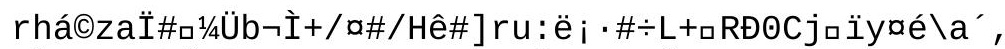

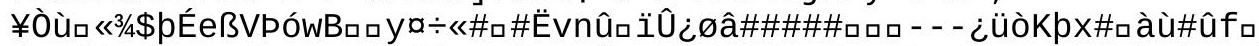

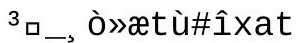

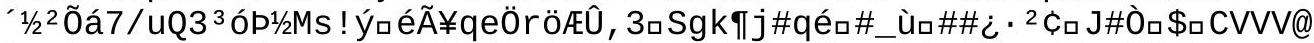

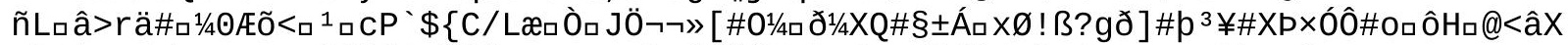

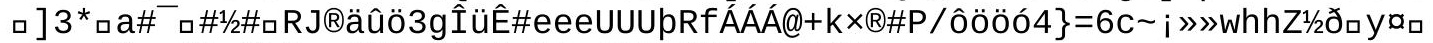

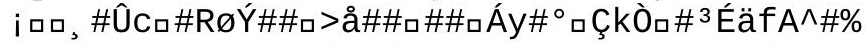

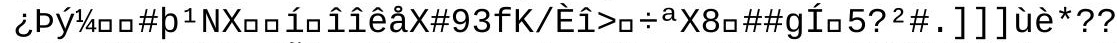

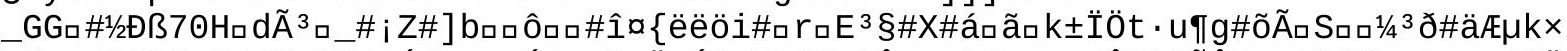

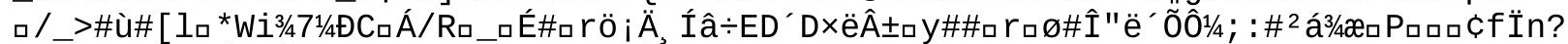

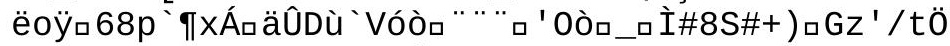

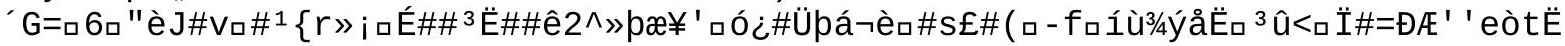




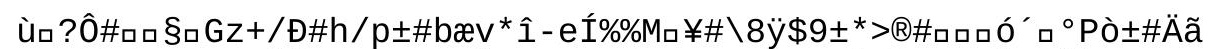

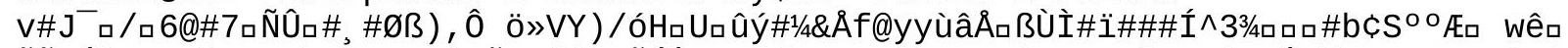

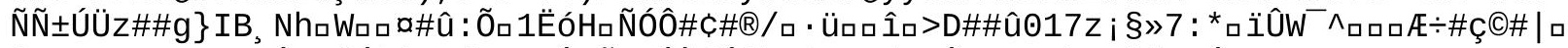

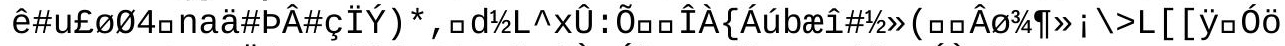

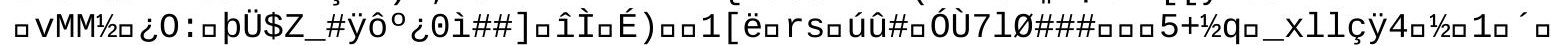
İyañáa òä

${ }^{3}=6$; Û, sC

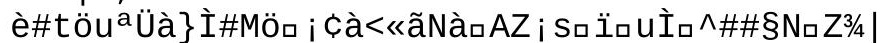

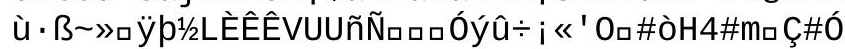




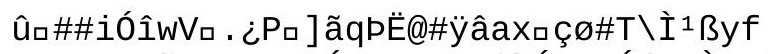

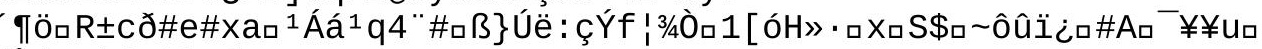
[ÒéC\#kí\#!ìiwf̧ 
1/400̂OËòÈ@ÜJ' ; êX\#?

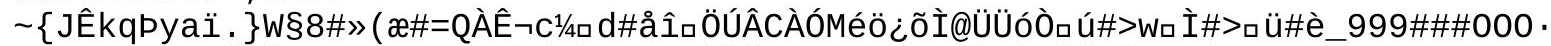

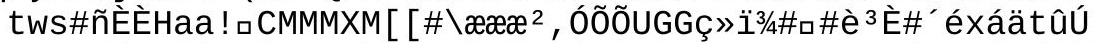

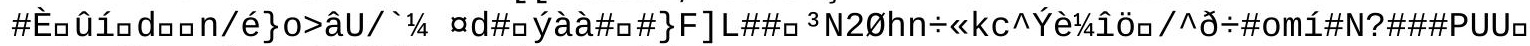

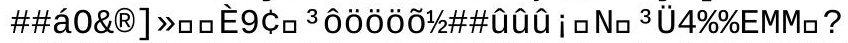

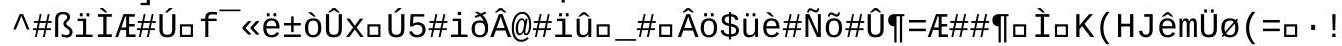

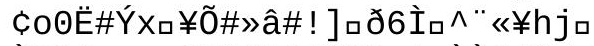

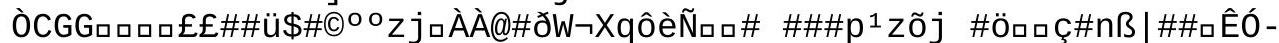

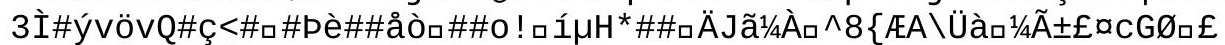

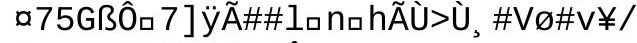

Gıæm õì@øäâr îûa 


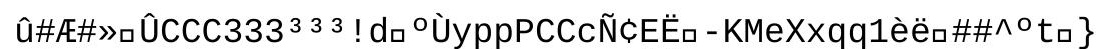

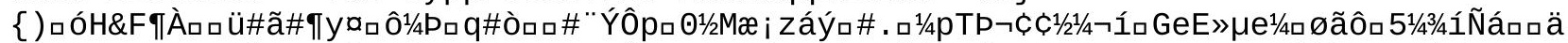

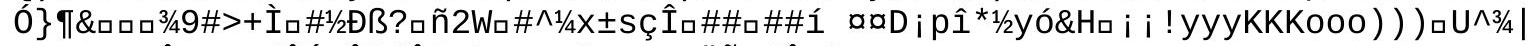

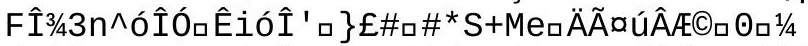

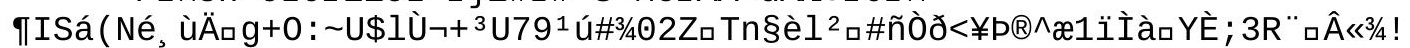

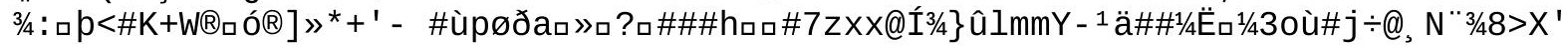




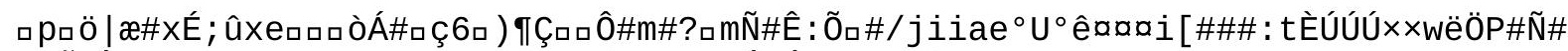

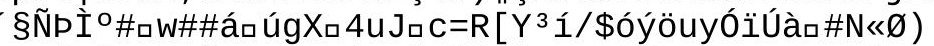

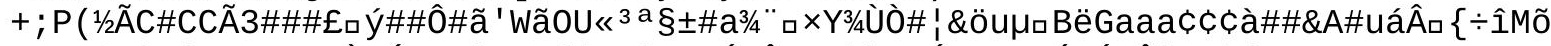

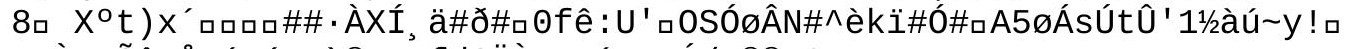

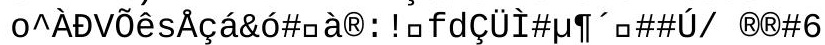




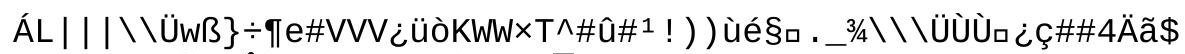

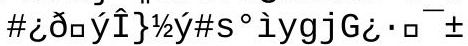




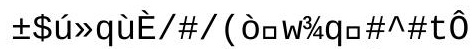

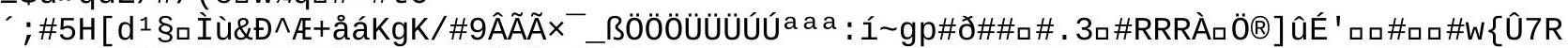

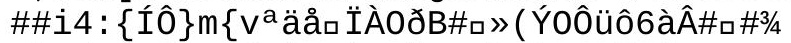

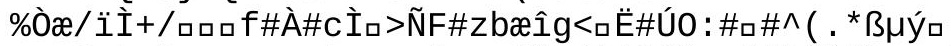

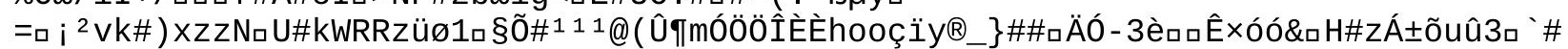

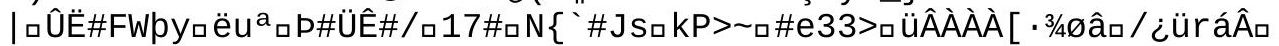

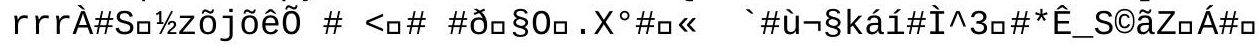




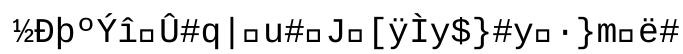




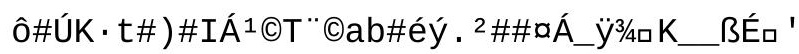





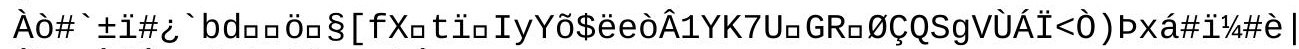

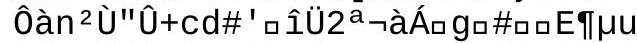

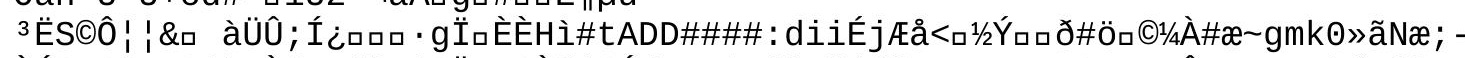

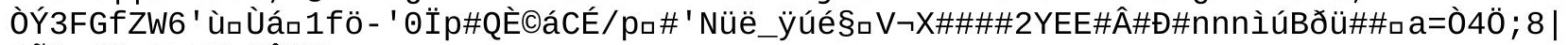

IÕåW\#\#c\#bTWæðÛã\% 


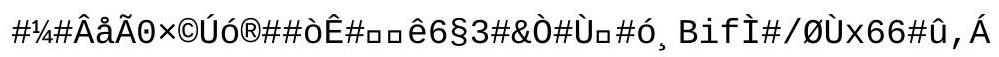




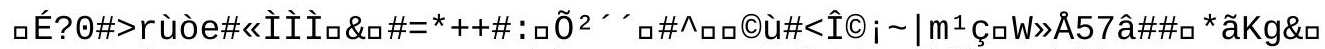

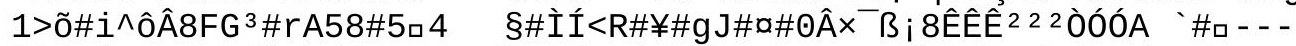

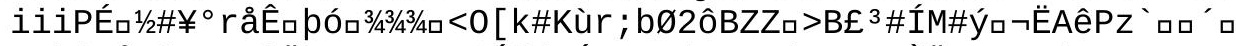

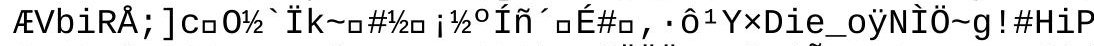

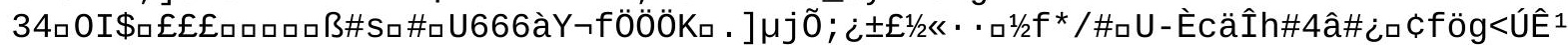

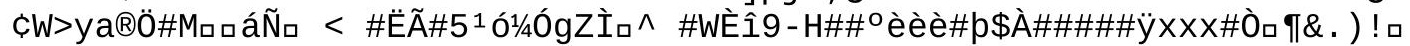

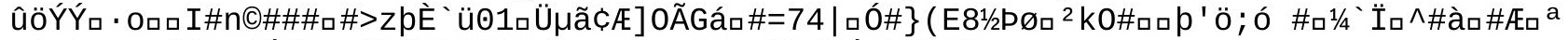

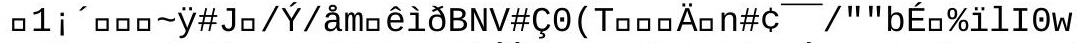

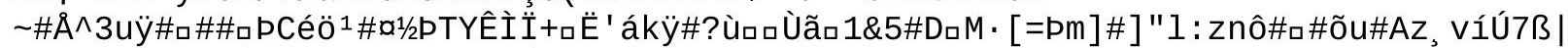

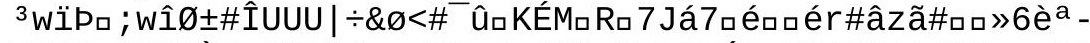

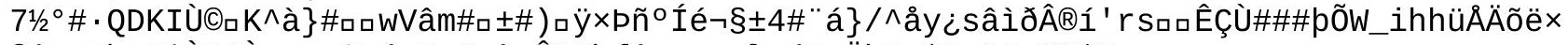

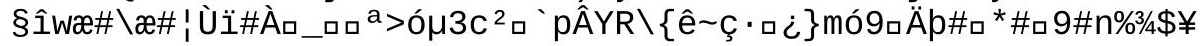




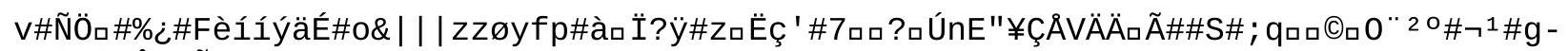

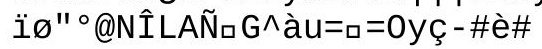




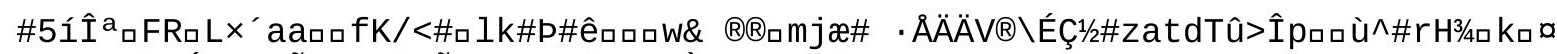

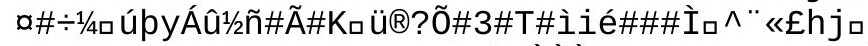

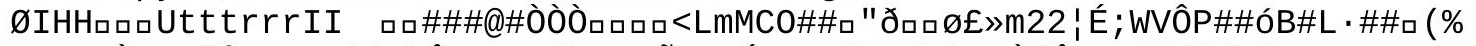

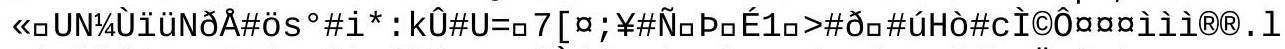

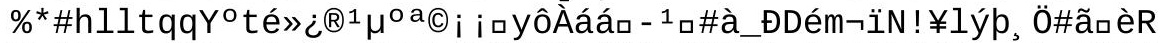

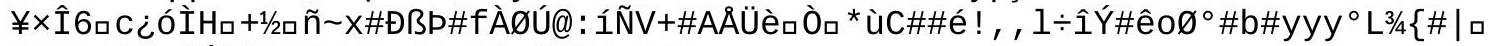

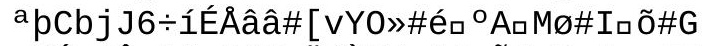

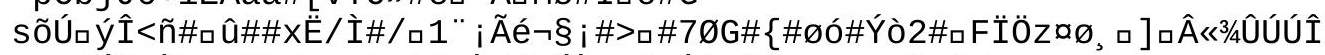

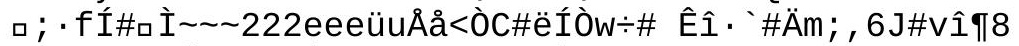

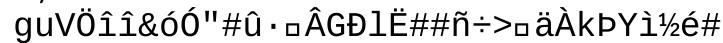




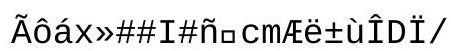




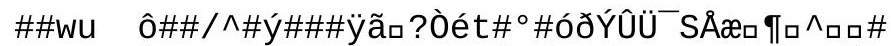

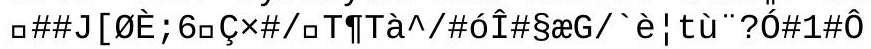

$£$ £ö 


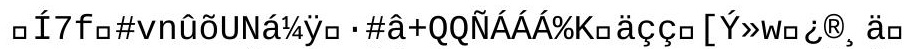

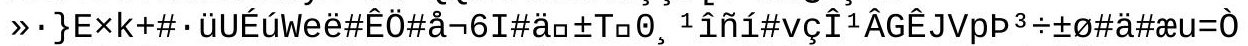

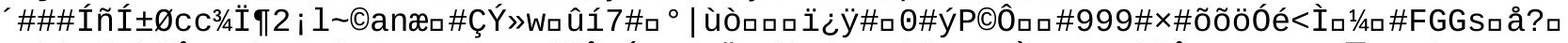

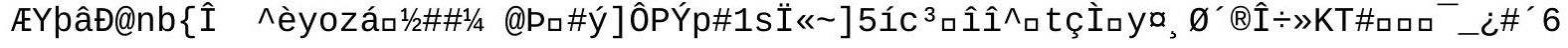




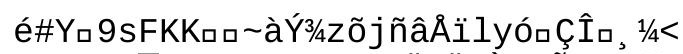

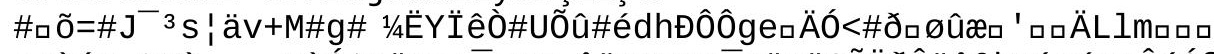

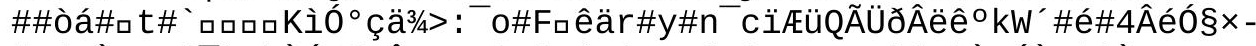

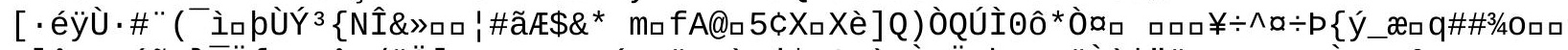

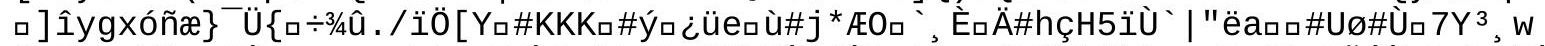

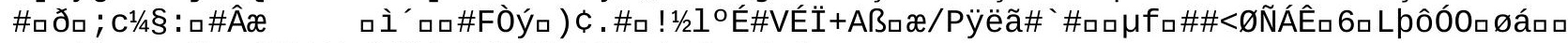

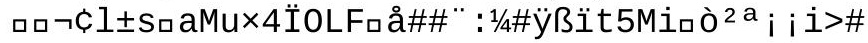




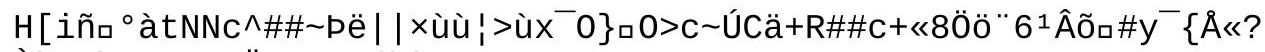

İkX\#\ロMaWZ1 ÄNFGG $f h) H$

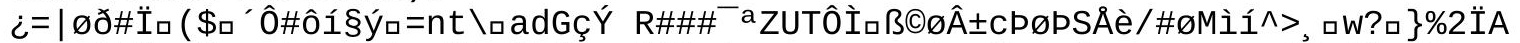

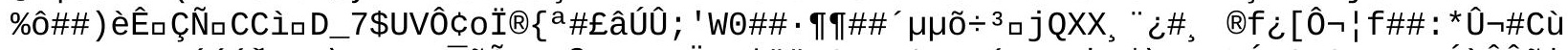

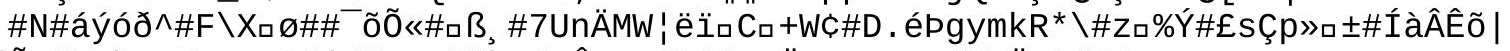

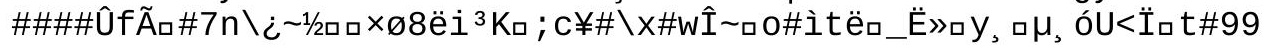


yø

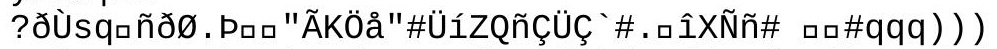

$++$

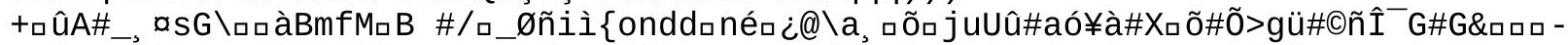

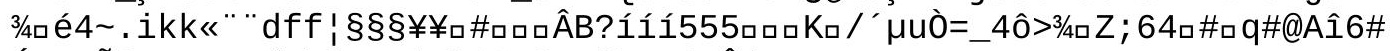

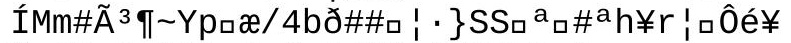

Úx\{\&mßnìêôä êa"C\#úgâT@JK®vP

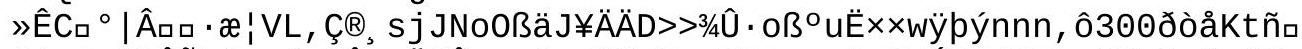

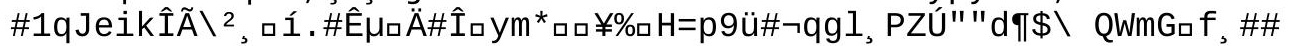

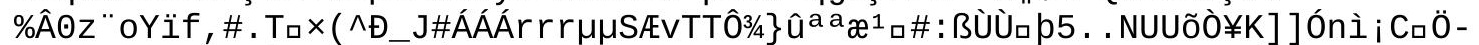

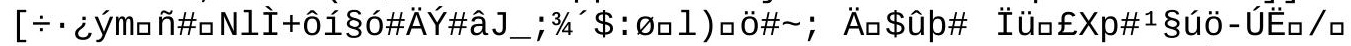

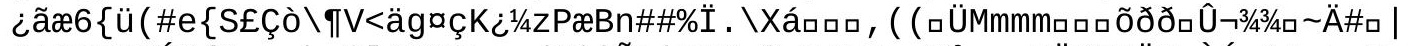

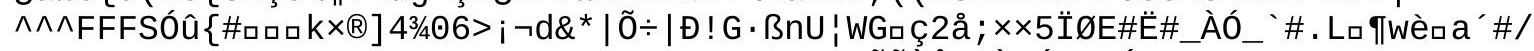

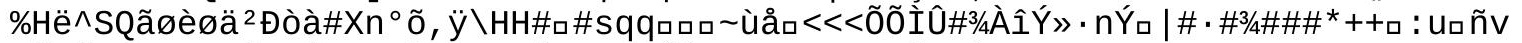

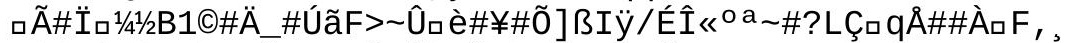

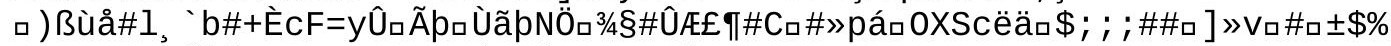

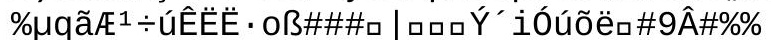

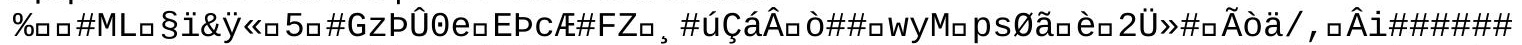

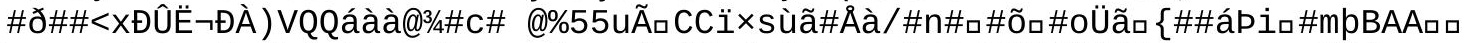

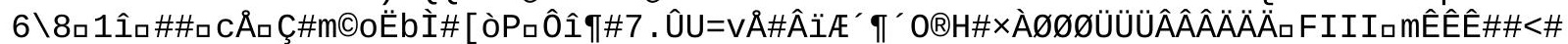

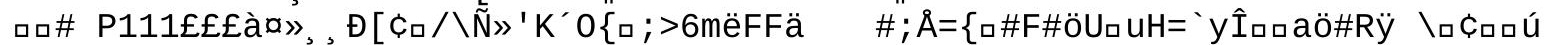

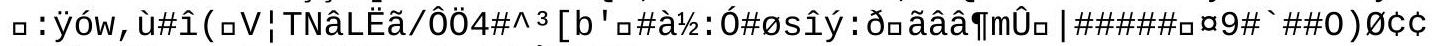

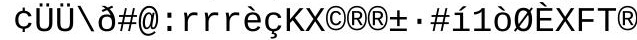


口B\# Æ\#aÍ1/2`\#B188Ë 


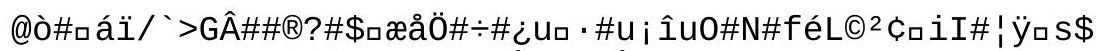

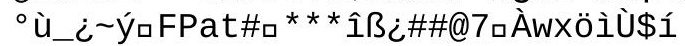




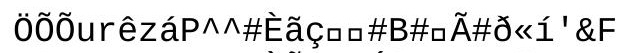

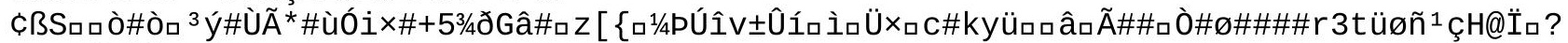

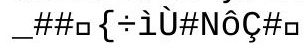




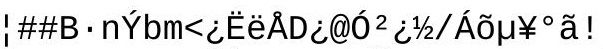

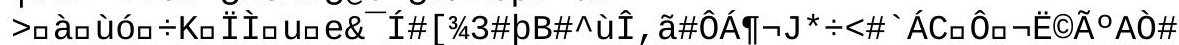

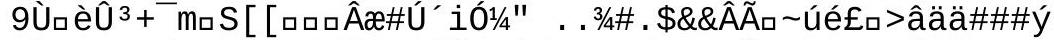

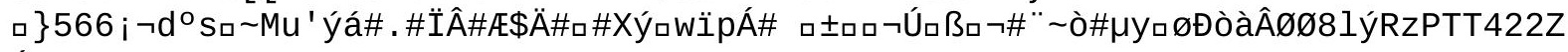
'\#\#. . व

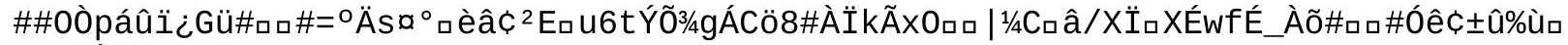

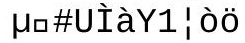




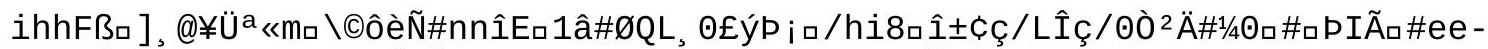

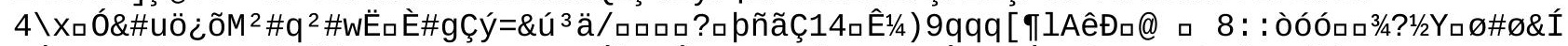

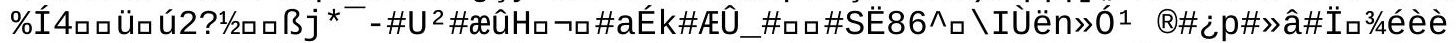

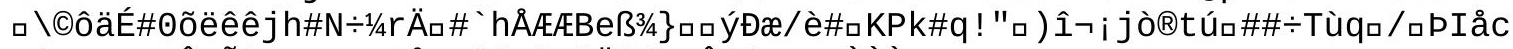

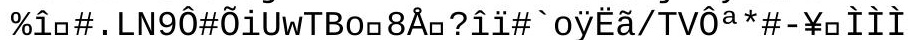

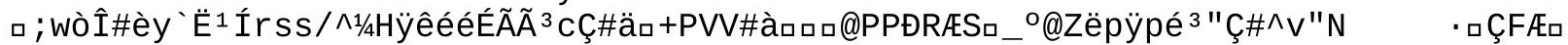

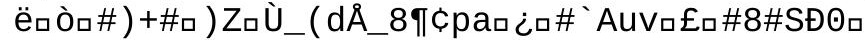

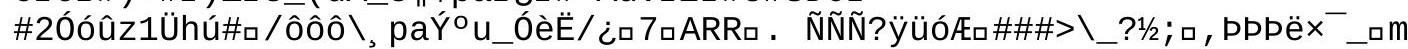

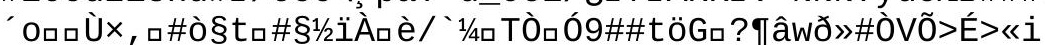

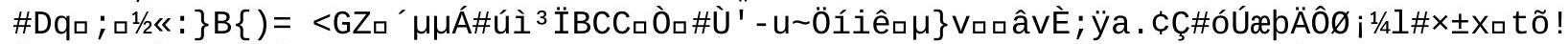

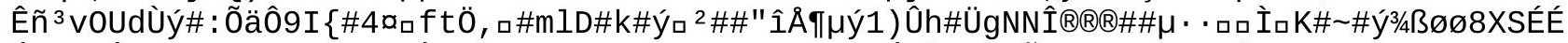

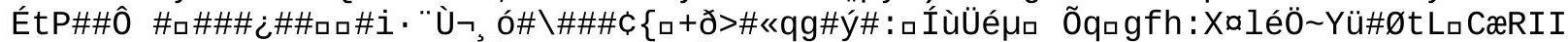
Xpaaa\#\#\#ùùùð9/?£\$ם

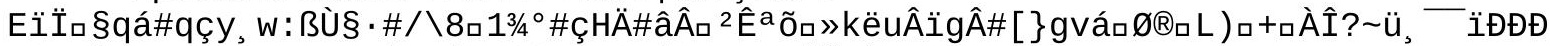

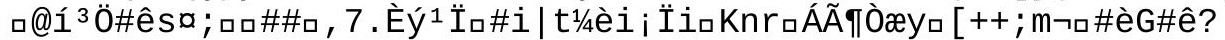

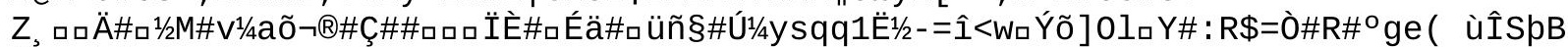

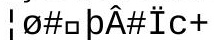

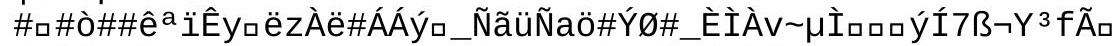

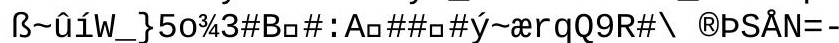

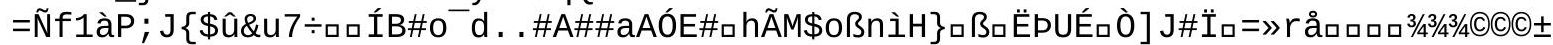

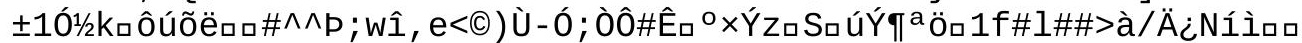

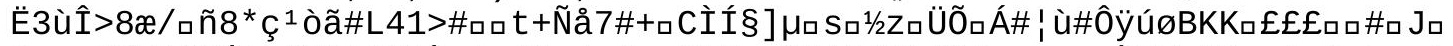

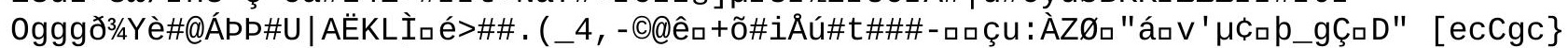
\#R\#fWøÏ॰ænS॰§Û amÛ 


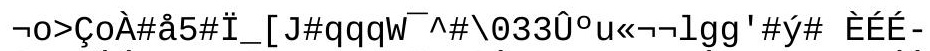

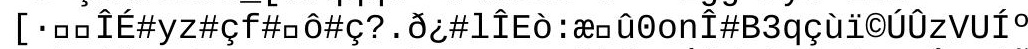

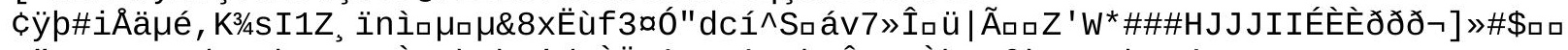

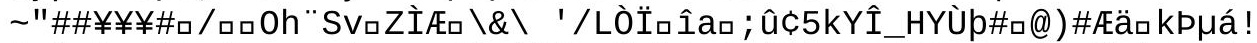

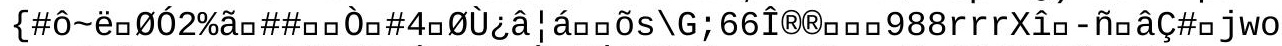

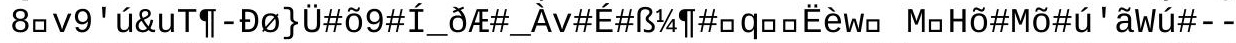

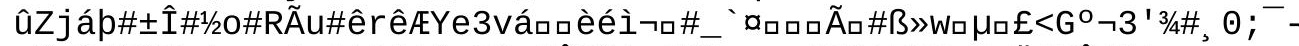

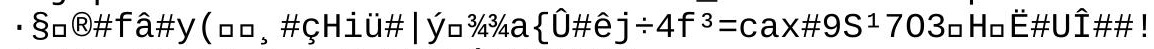

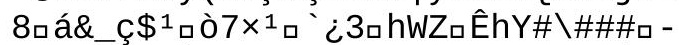

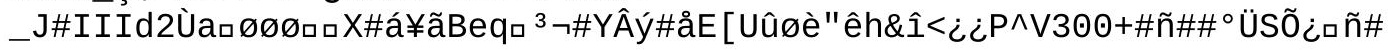

Ë\}\$\#üóú89 


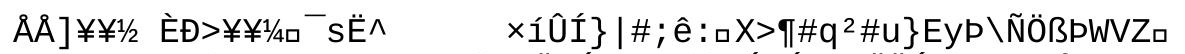

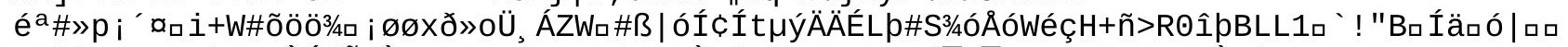

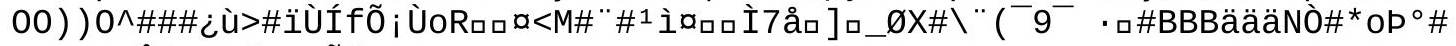

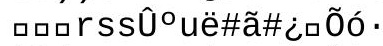

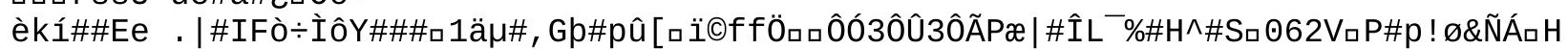

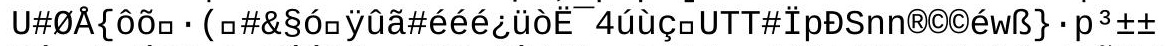

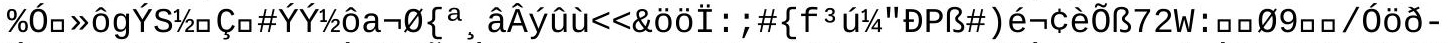

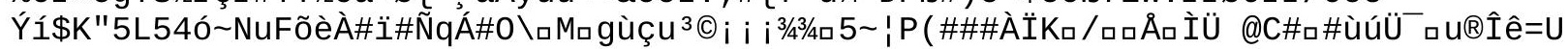




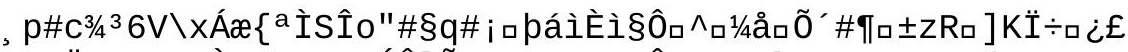

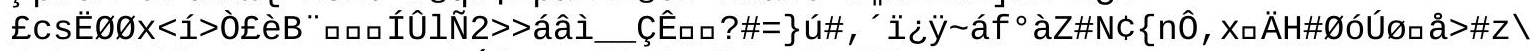

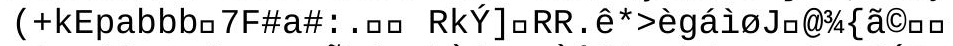

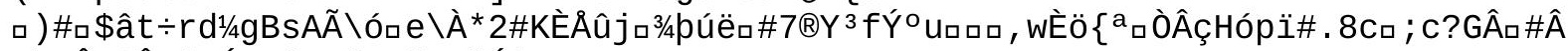
口 \^Û<SÎ̃o"\#Én, @WFí\#mÏPaßÓ ${ }^{1} \mathrm{~g}$

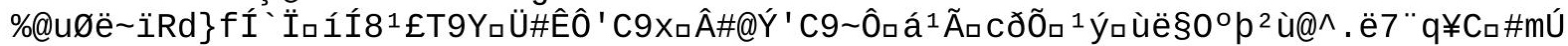

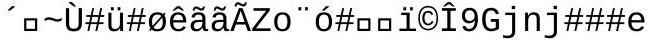

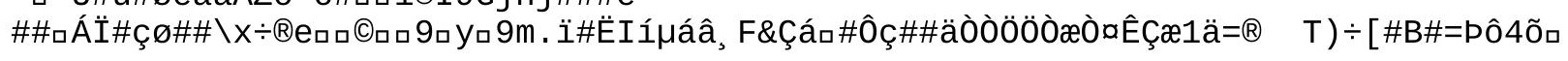




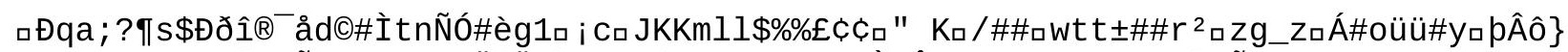

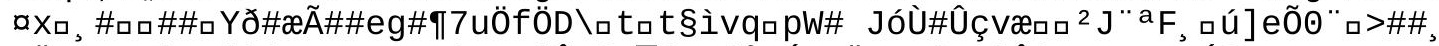

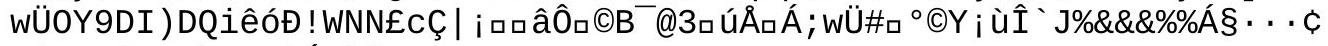

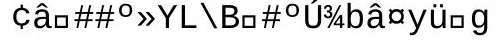


$\div$ T\#\#ã\#Xó\#ðö\#BOæ/Ta · Í\#6Û ${ }^{3} C ̧ I U 5$ 


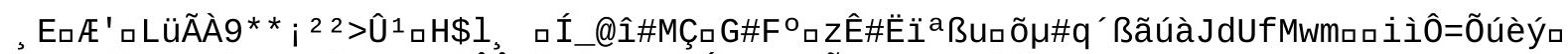

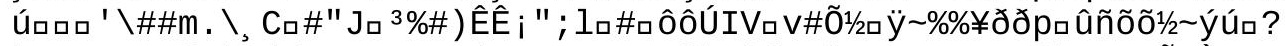

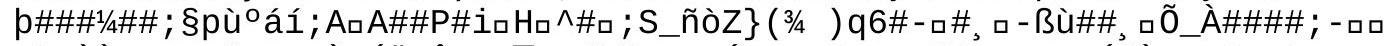

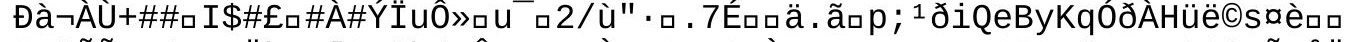

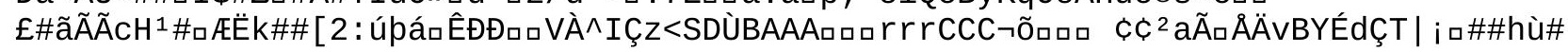




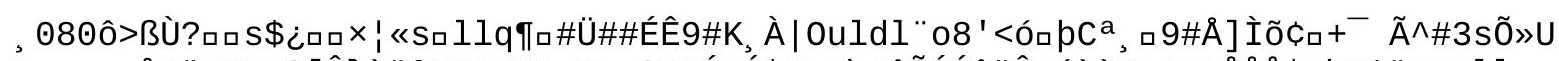

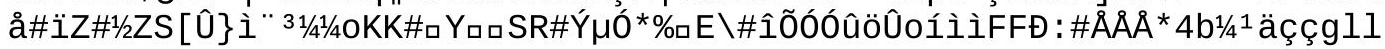




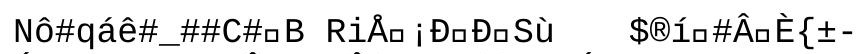

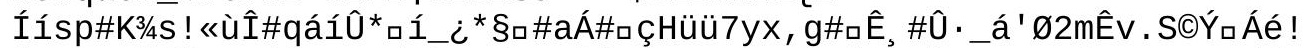

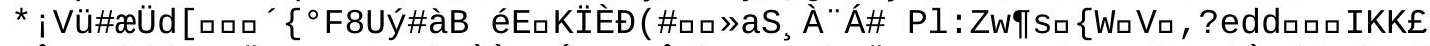

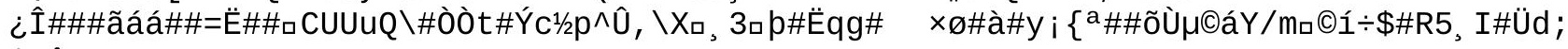

Cॄâ!

$\ddot{I} ;$; $\div$ Âa\#ÅX=IòzÛZÔ8\%\#Ã£a $\cdot n j$ ë

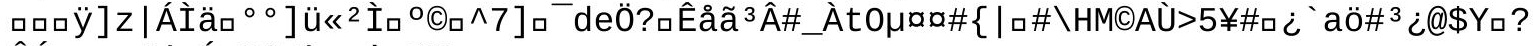

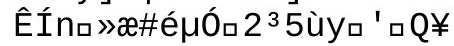

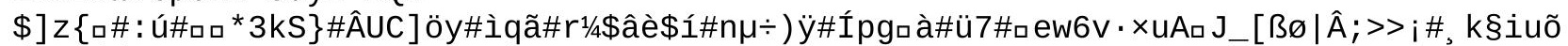

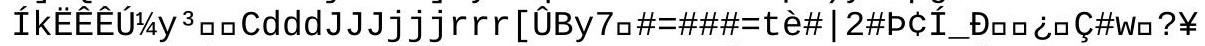

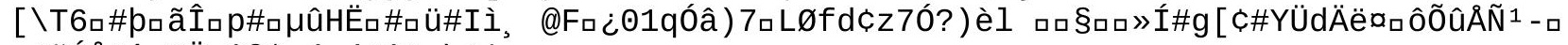

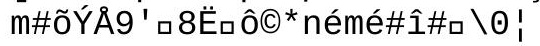




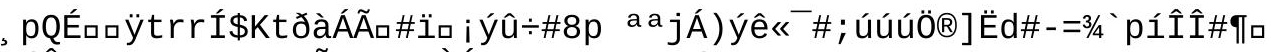

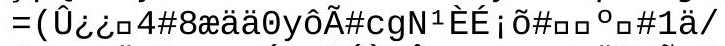

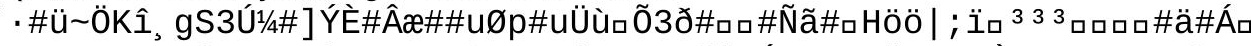

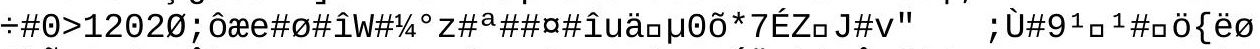

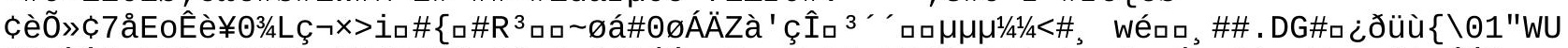

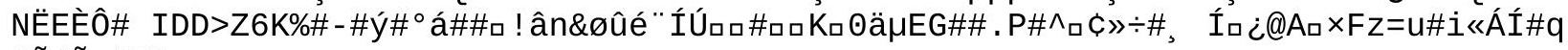

\#Ãý̂̃a `Mõ 


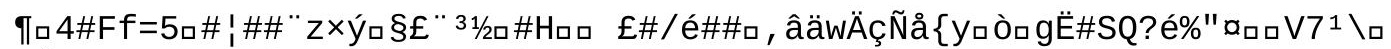
\$Ýtww\#.\#@\#\#0]\#\#_õú $\mu k e ̂ e ̂ e ̂ ß\} \div \# x \# * * *$, , ${ }^{\circ}$ ¿\#\#§ä ?

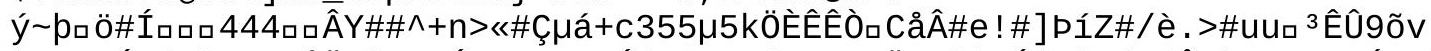

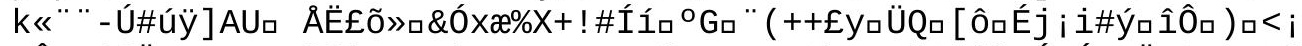

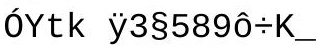

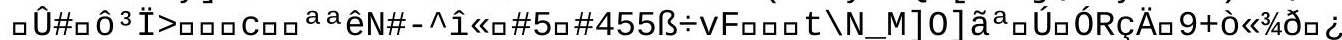

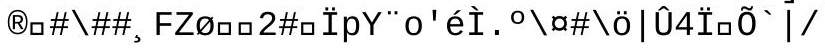




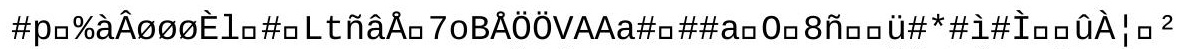

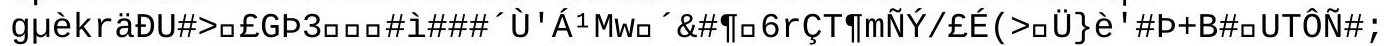




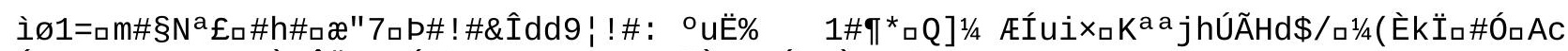

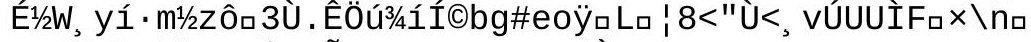

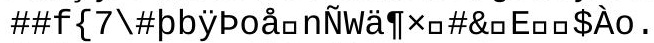

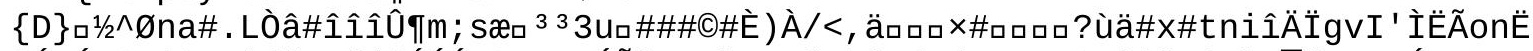

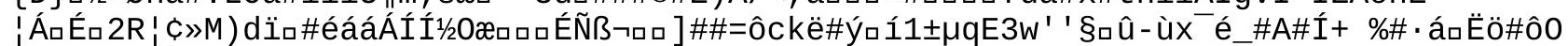

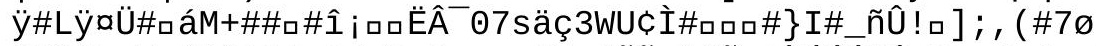

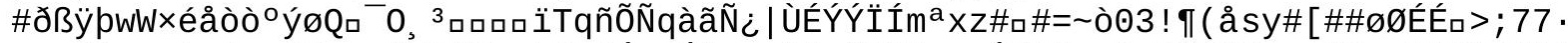

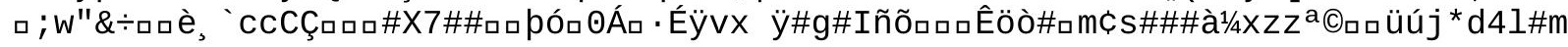


Hǚ\#\#j a

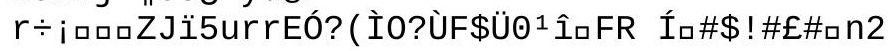





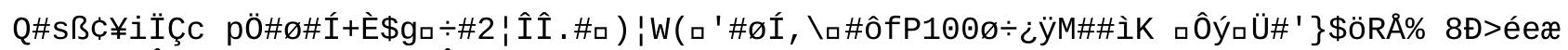

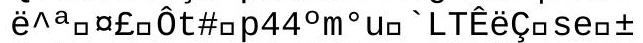

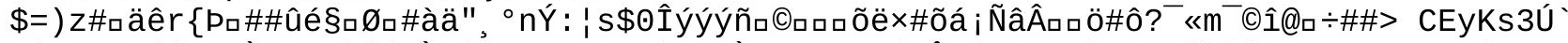

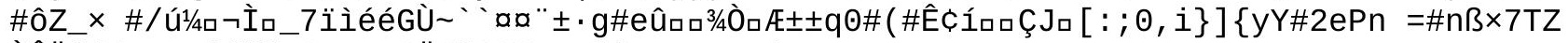

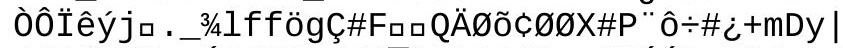

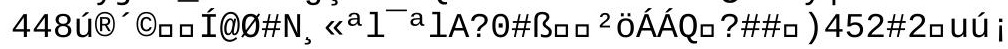




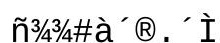




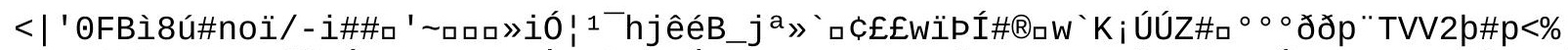

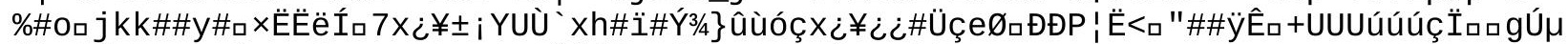

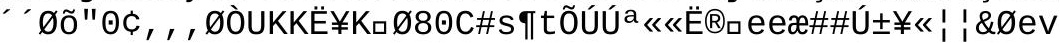




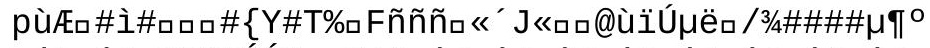

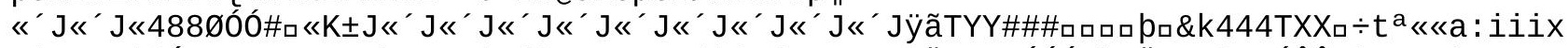

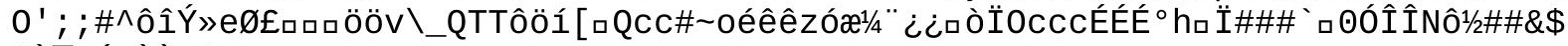

\$À æÝ ëÒÒRôr 6 : : : 


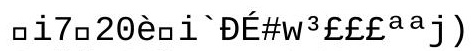

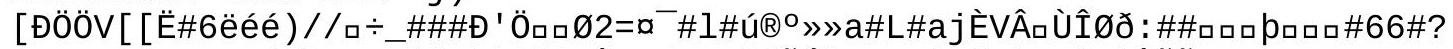

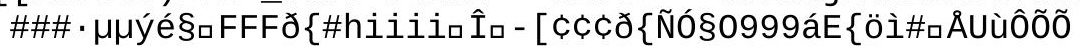

:99á-

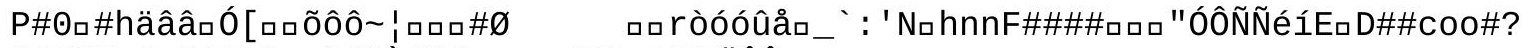

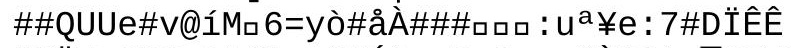

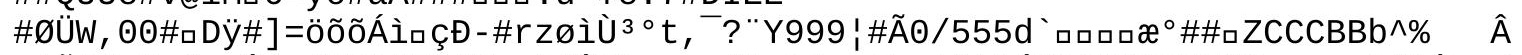

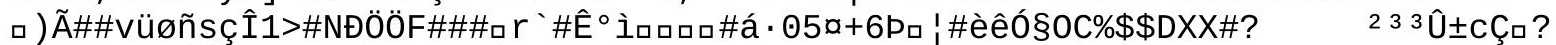

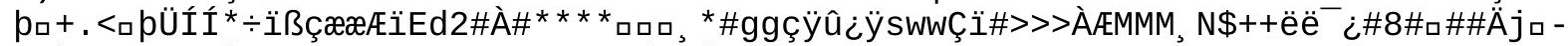

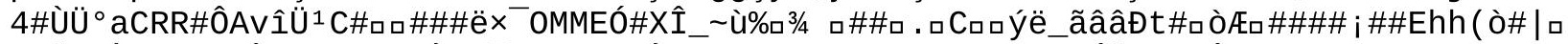

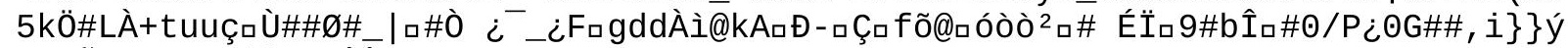

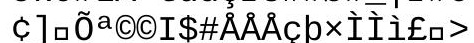

\#\#Æ : ○\&\HIIùpûïaâÀ\#êêêh 


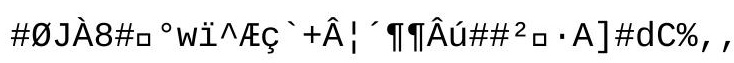




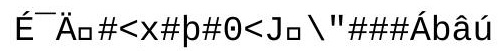

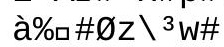

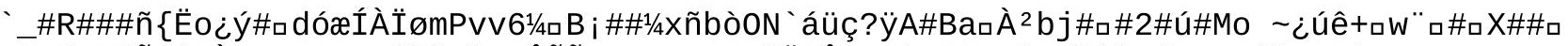

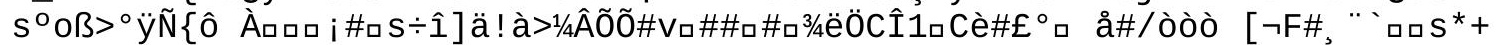

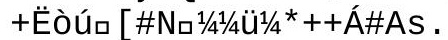

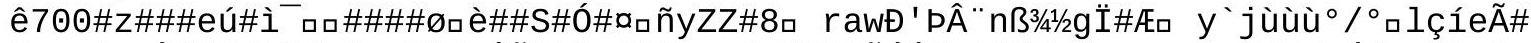

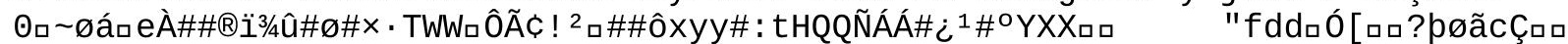
c\#fÆ5ロ \pm 
$(. \square: \mathrm{u}$

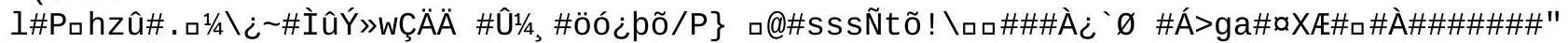

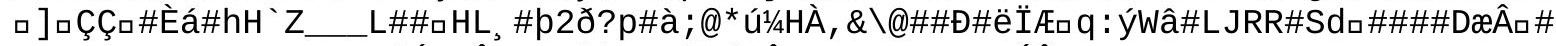

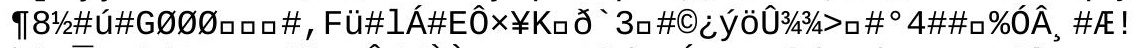

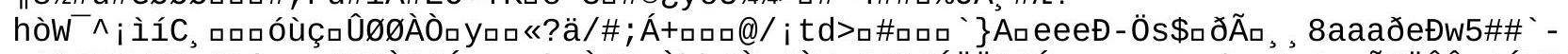

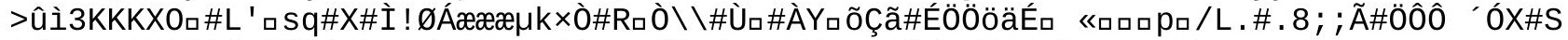

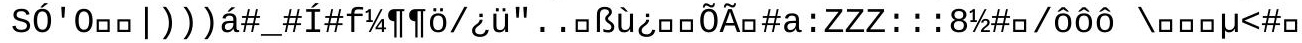




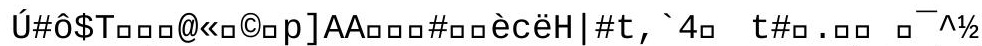
$<\# " \# z \# \mathbf{I} \# \# \# \# \#||<1 / 4] @ @ 44 \# \# \# \#$ a

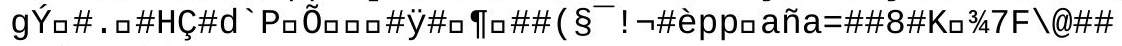
$\# \#$, . . ० ० \#V 


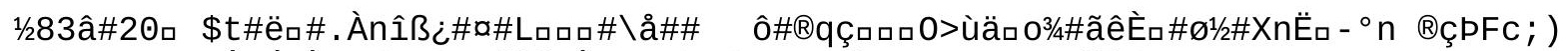

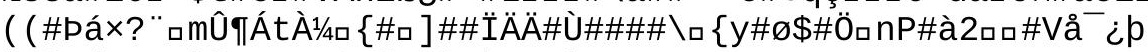

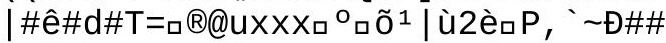

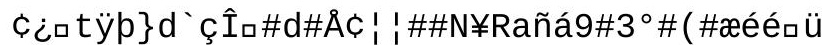

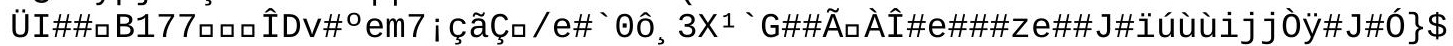

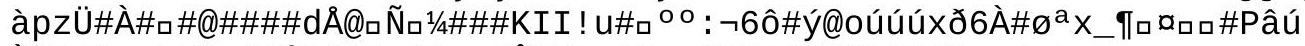

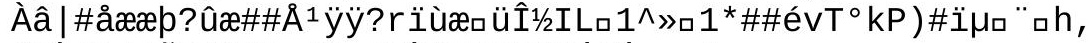

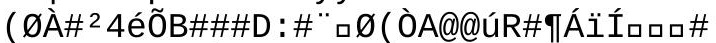

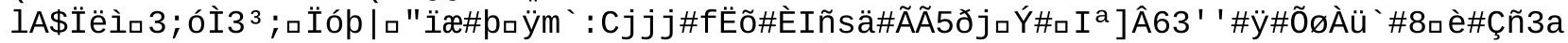

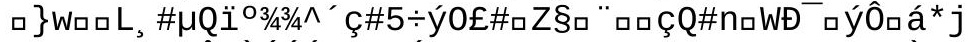

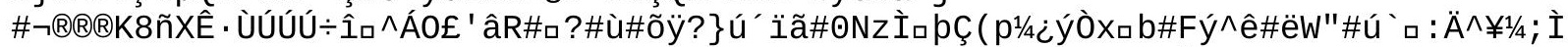

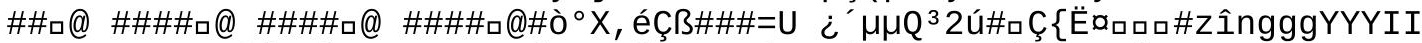

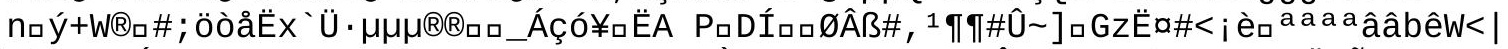

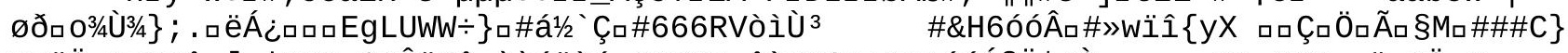

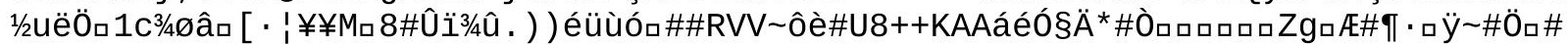


$\hat{\mathrm{I}} \mathrm{O}^{-} \mathrm{H}$ 


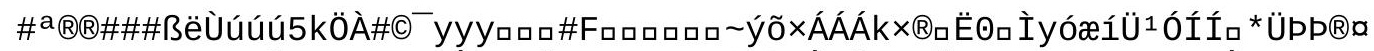

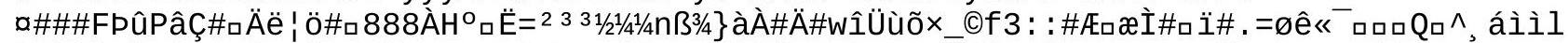

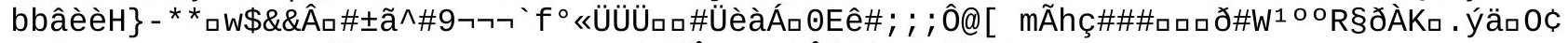

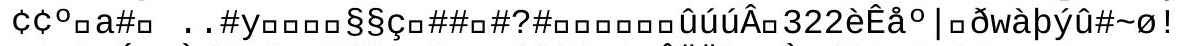

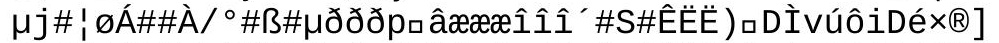

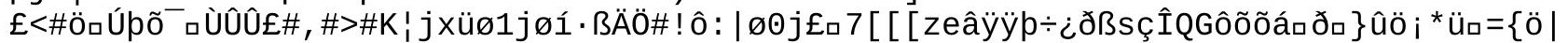

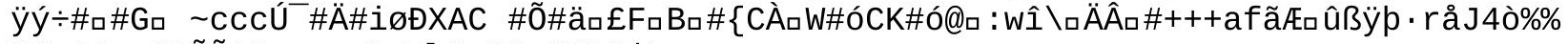

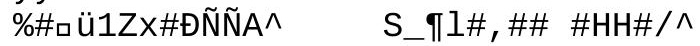




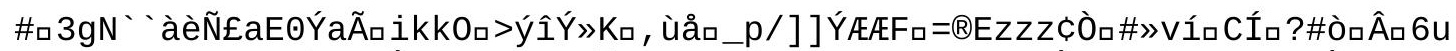

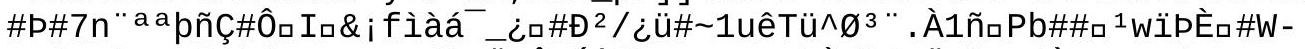

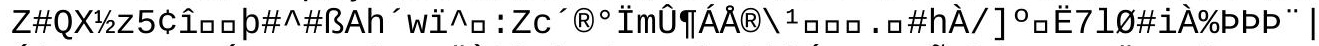

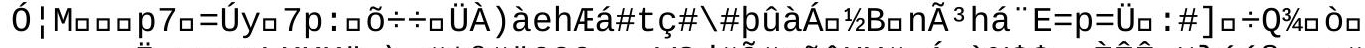

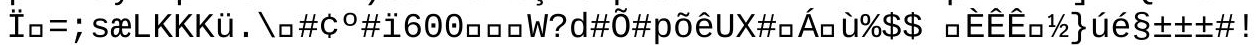

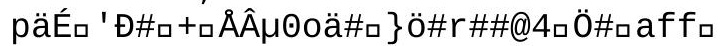

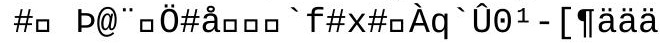




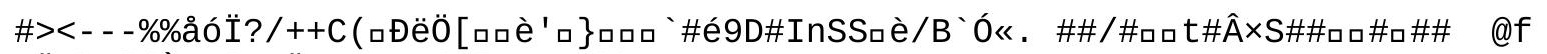

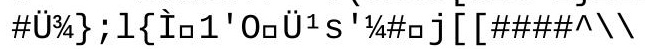




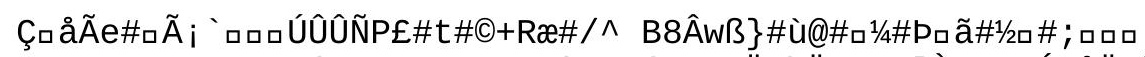

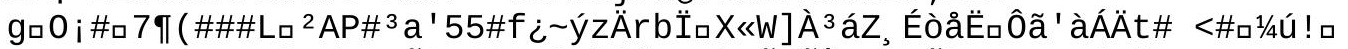

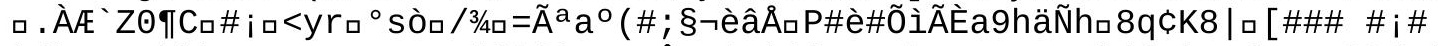

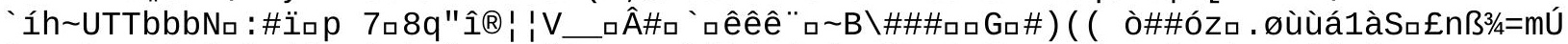

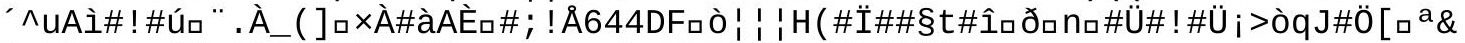

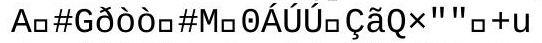
$\# ; \boxminus p / Z^{\prime} \# \mathrm{~m} » \emptyset 3 ¿ I \# \# \mathrm{Z}$ !

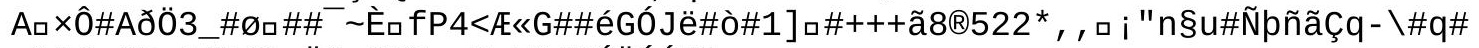

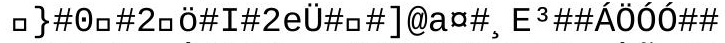

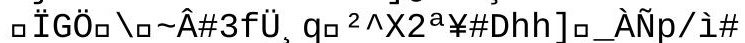

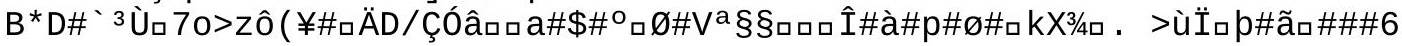




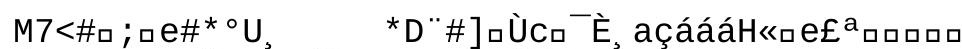

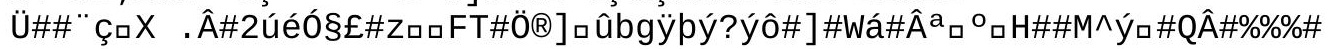




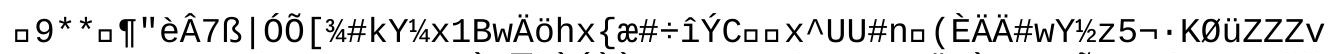

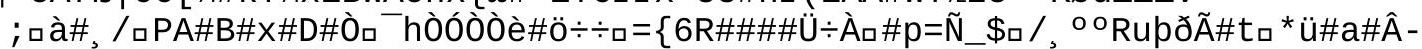

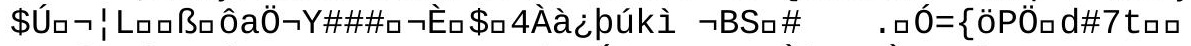

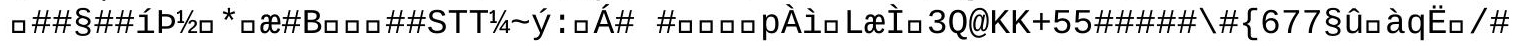





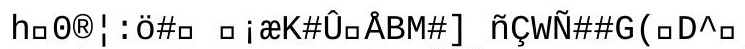

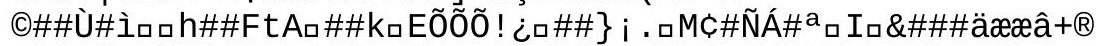

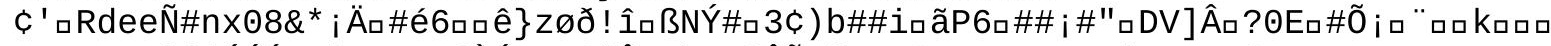

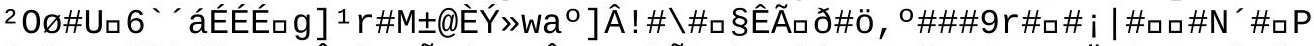

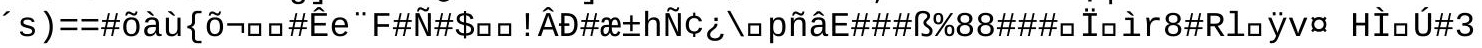


6 ¿Ѐ̀\#

$\$ \gg \# \#$ 


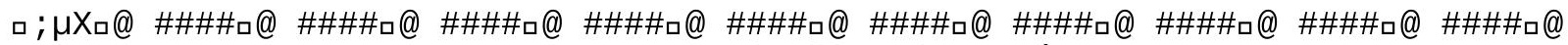

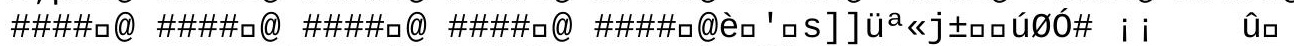

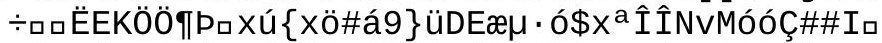

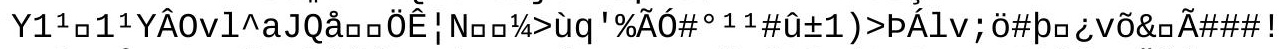

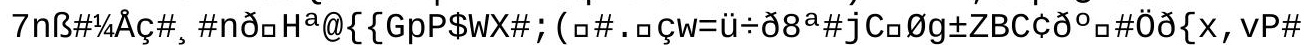

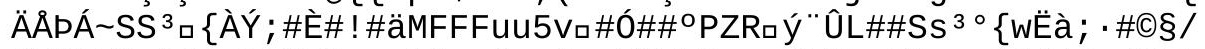

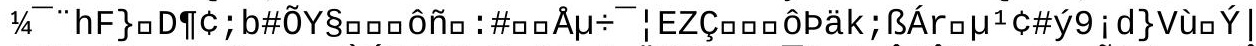

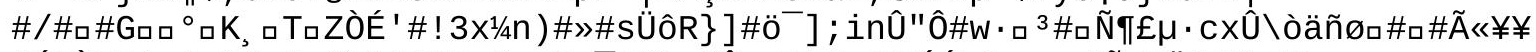

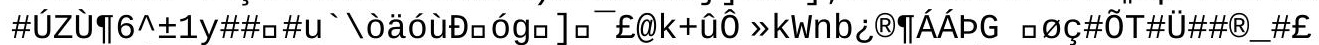

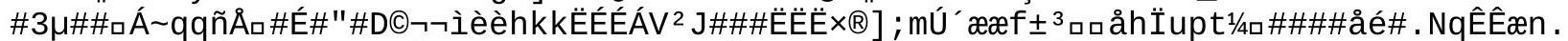

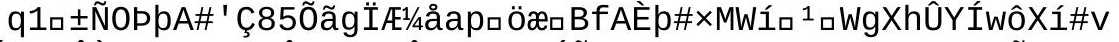

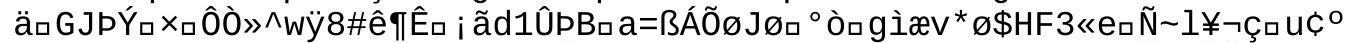

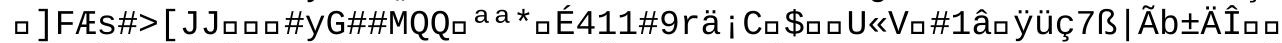

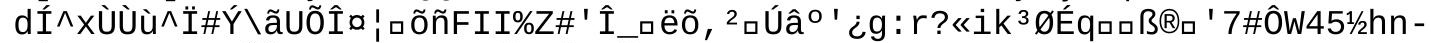

oåๆqPLüB $3 / 4$ × $\hat{\mathrm{I} A ̊ Y V M K c u s Q B \wedge ð p a ̀ E ̈ \#>0 ̈ o ́ \#-~}$

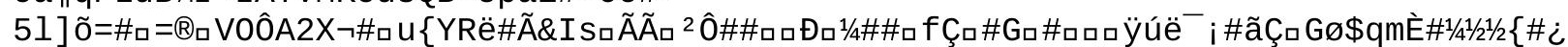

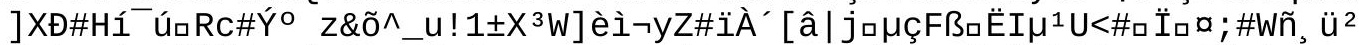

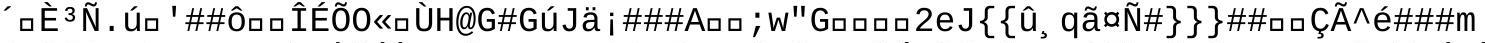

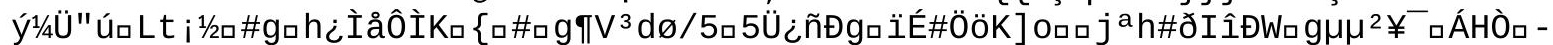

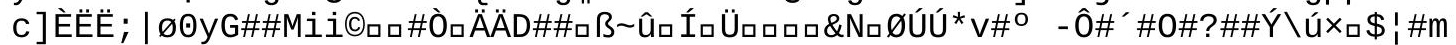

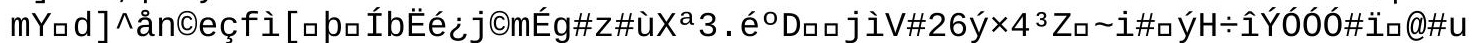

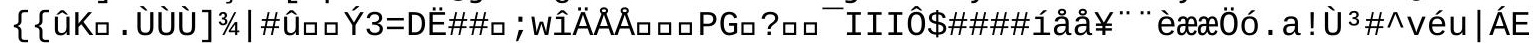

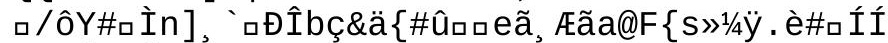

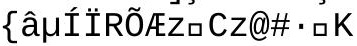




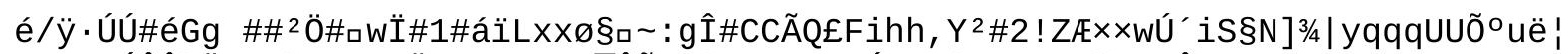

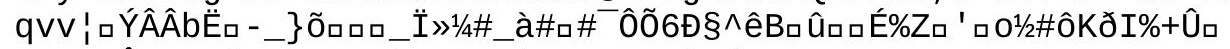

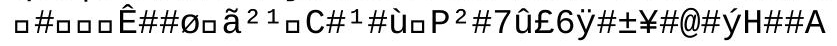




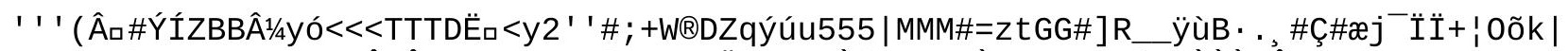

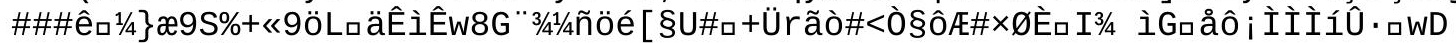

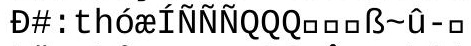

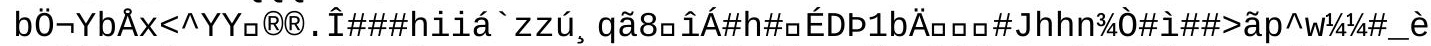

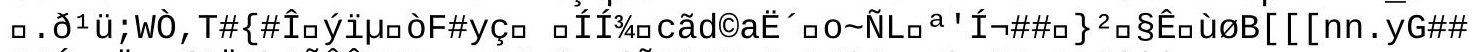

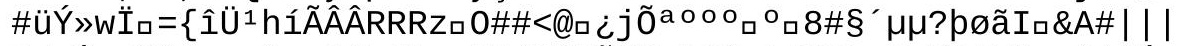

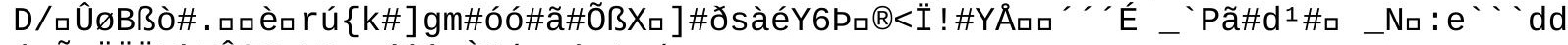

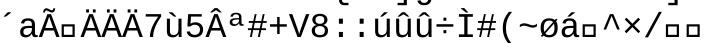

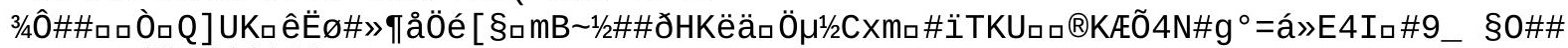
๑ . áX1DÁÃ ÃÃÍÍÝÝ]QQ1++Kᄀ 


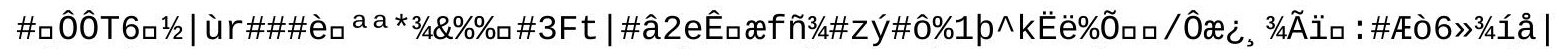

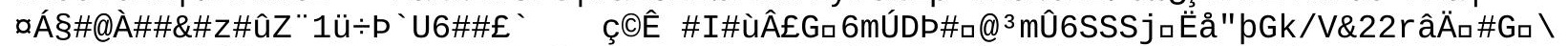

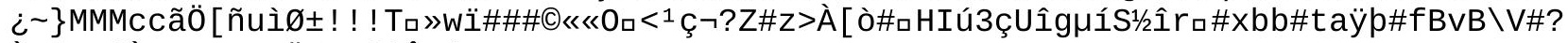
İ॰|VSûÀü¥mu-70Ü ${ }^{2}$ e $\tilde{I}^{\prime} \hat{I} \ll \$ 1 p \odot$ 


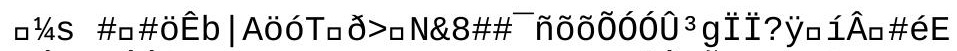

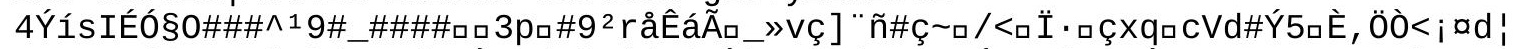

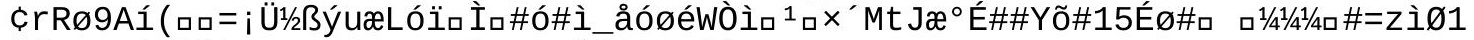

'í⿴囗< $\left.\left.\left.\left.\right|^{*}\right\}\right\}\right\} \# \#$ \} 


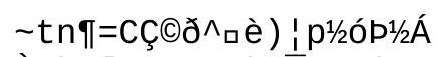

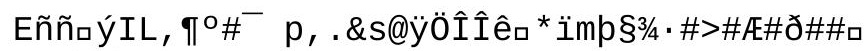

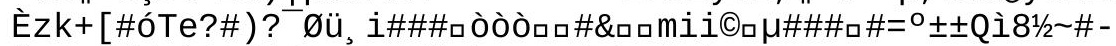

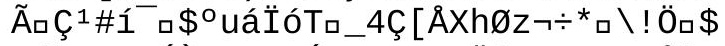

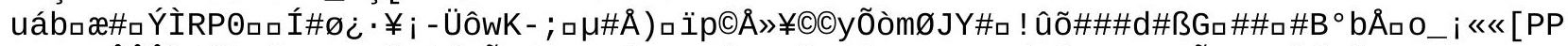

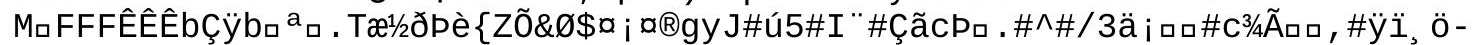

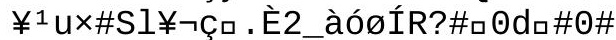




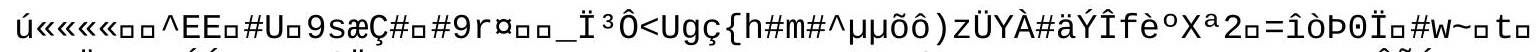

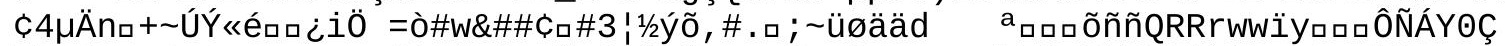

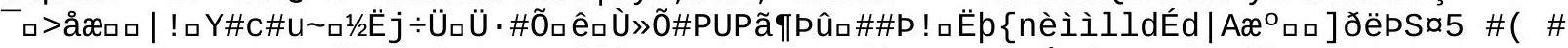

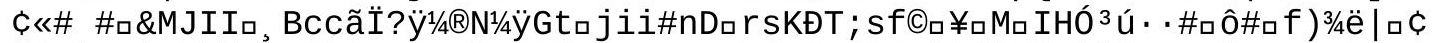

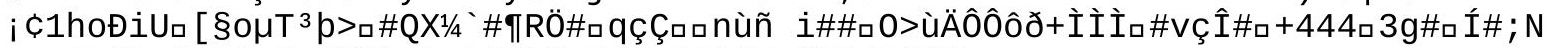

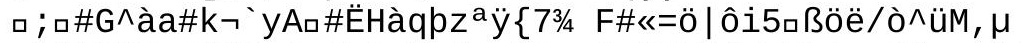

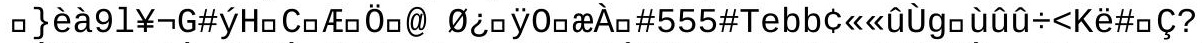

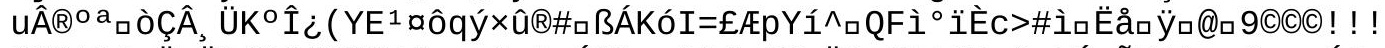

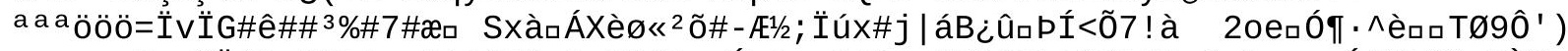

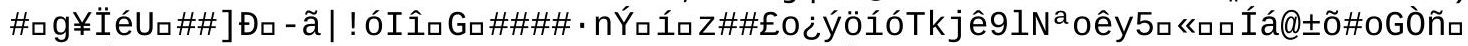

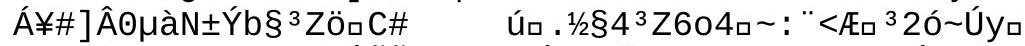

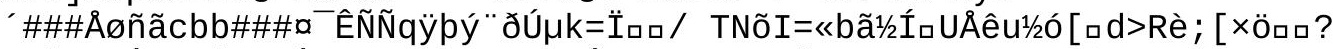

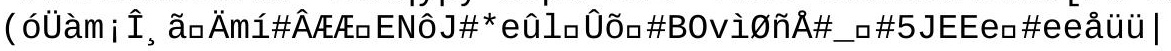





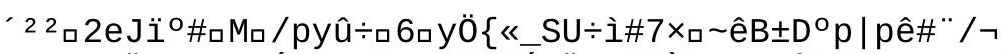

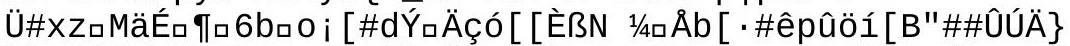

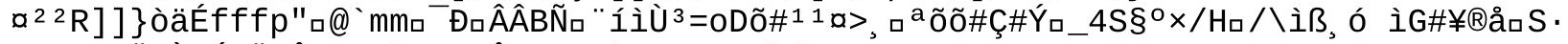

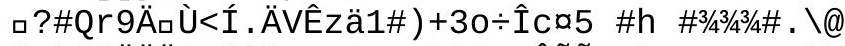

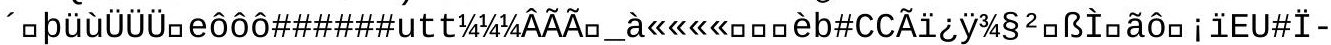

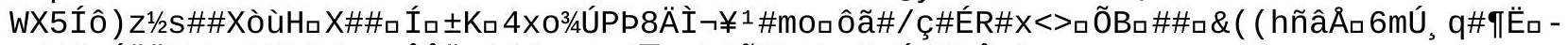

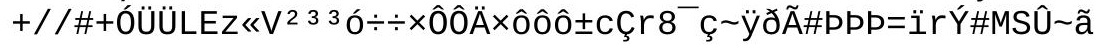




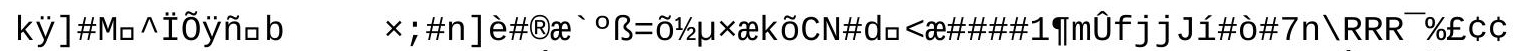

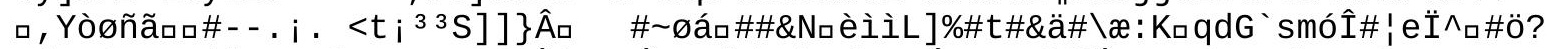

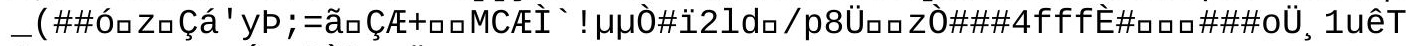
ü= $\mathrm{A} 2 \# \#<\# \# A ́ \sim 0] A ̀ p^{3} g \ddot{I}$

口.]

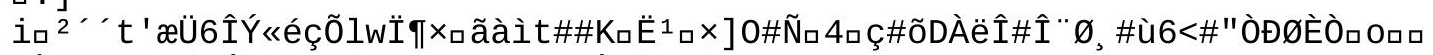
õÈcıjvfpÏ»ÍHS@Đ\#\#ロ]»vihh CGİ\#\#\#P3 


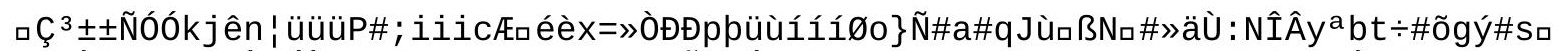

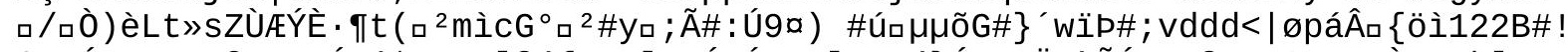

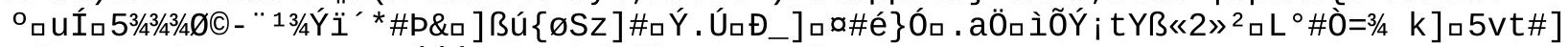

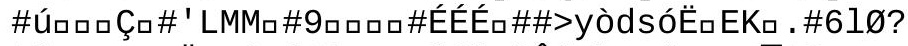

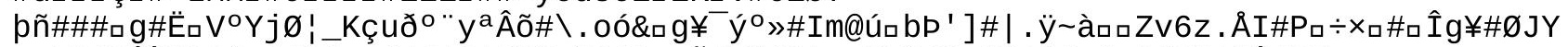

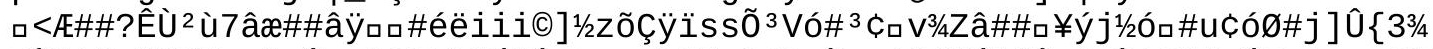

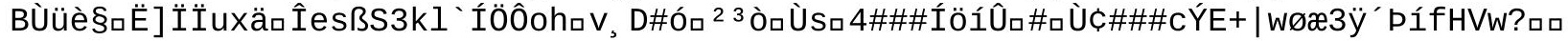




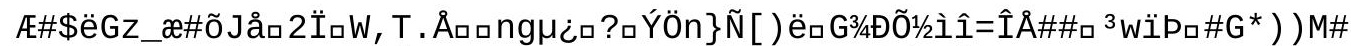

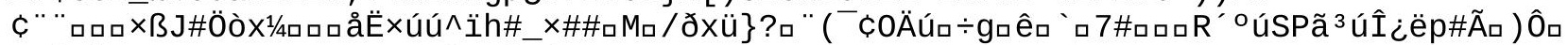

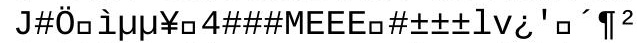

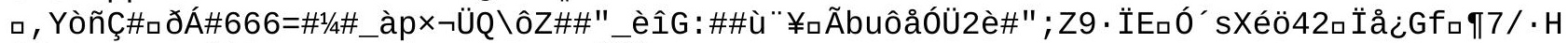

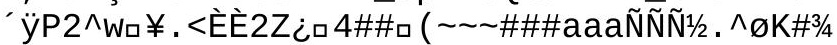




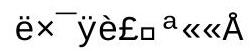

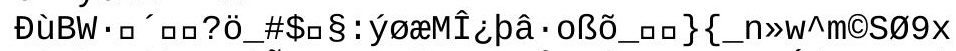

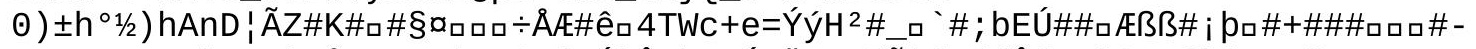

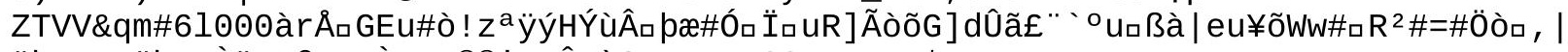

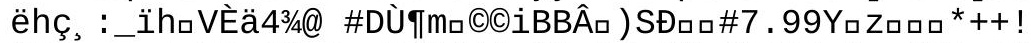

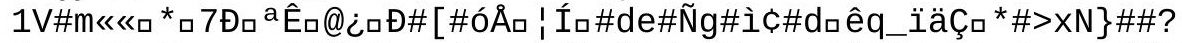

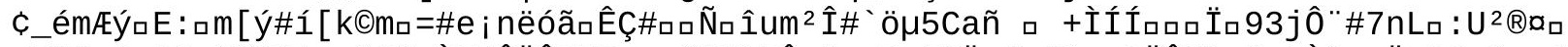

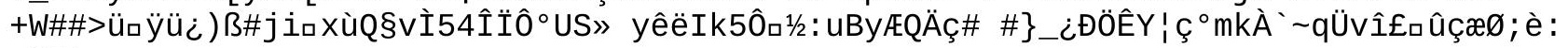

ç\%\#t

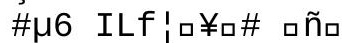

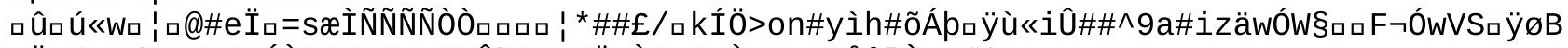

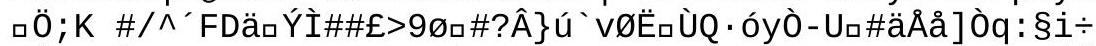

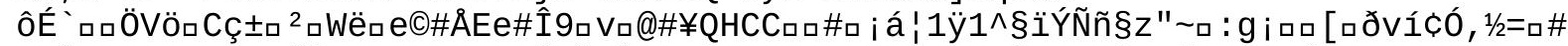

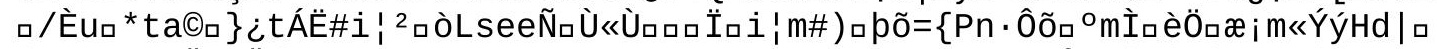

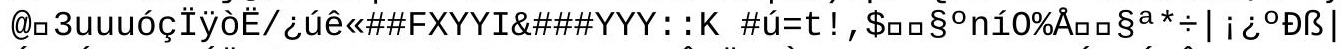

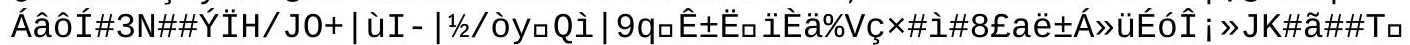

口:

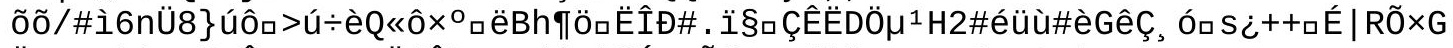

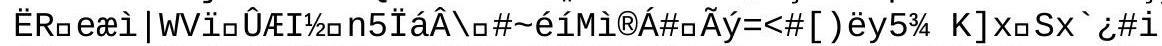

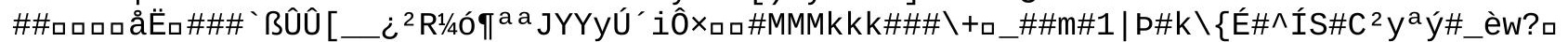

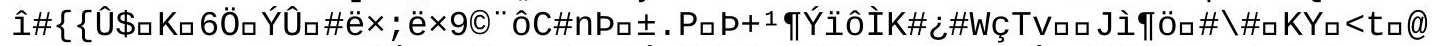

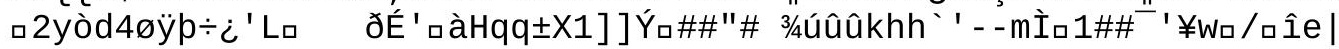

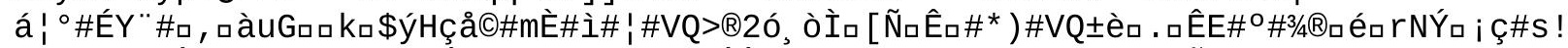

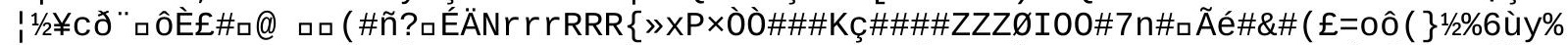

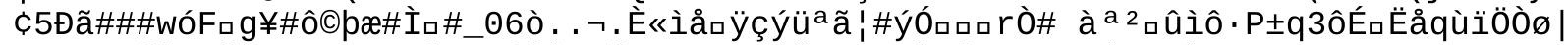

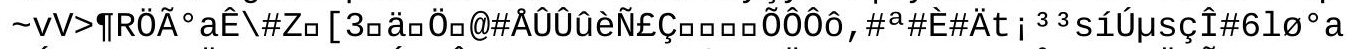

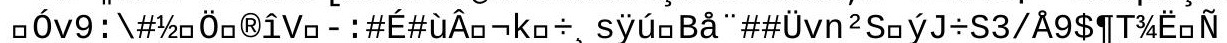

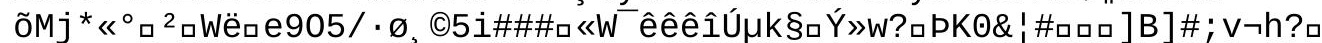

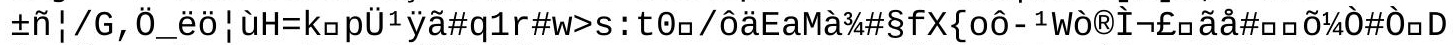

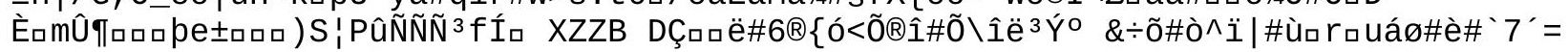




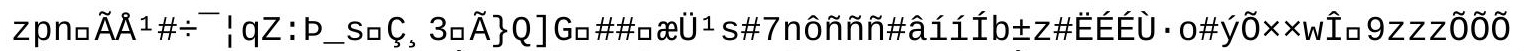

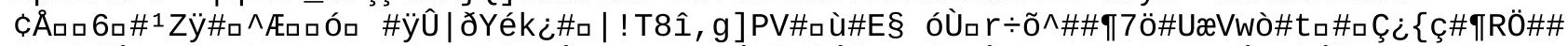

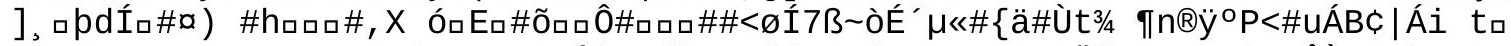

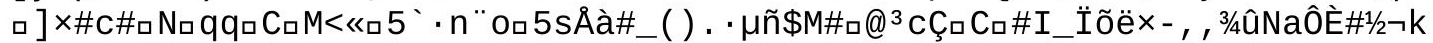

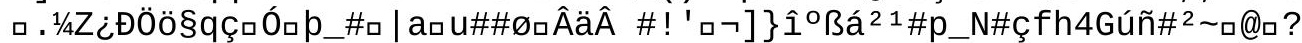

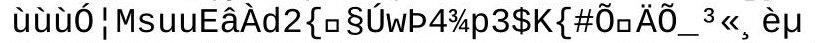

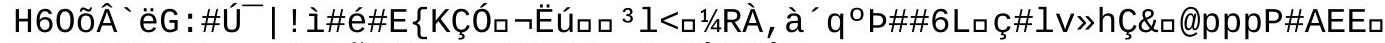

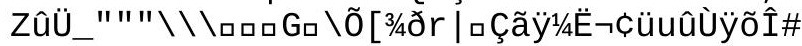

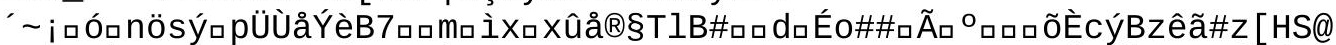

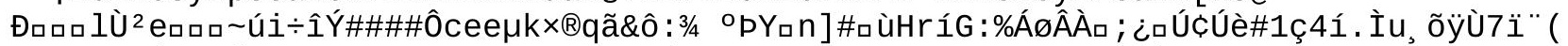

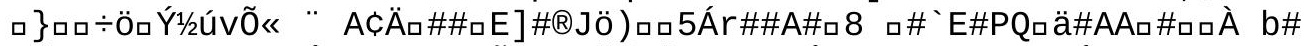

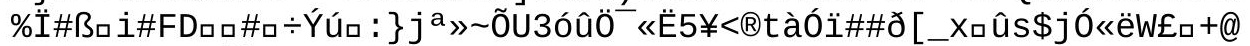

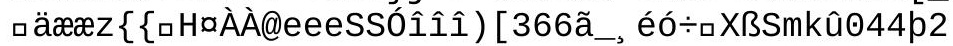




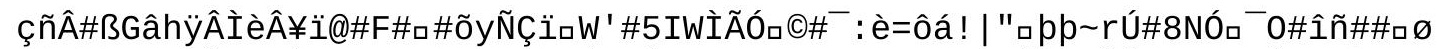

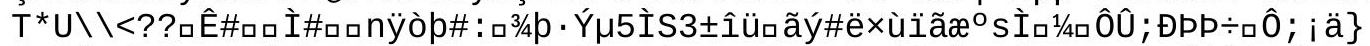

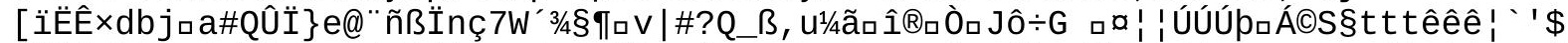

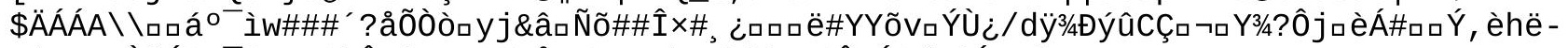

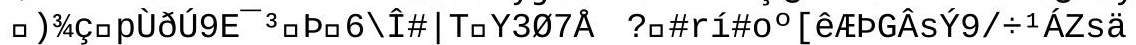

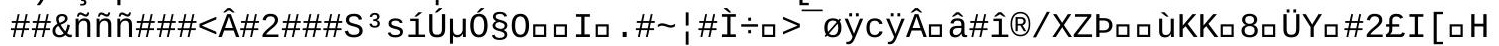

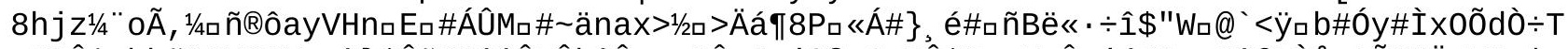
口 ë\#Ô

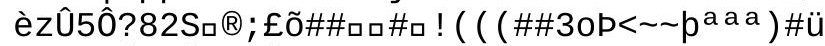

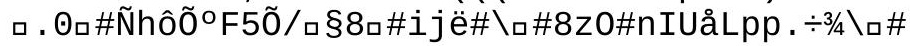




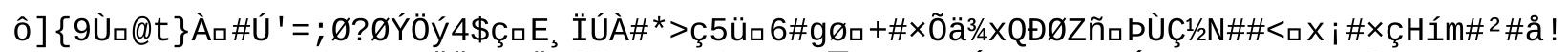

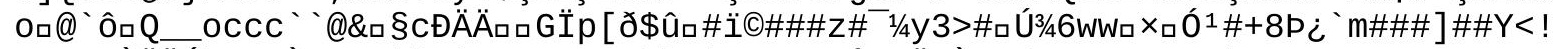

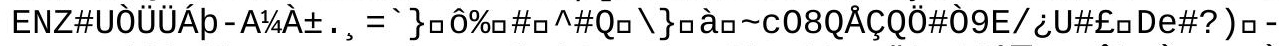

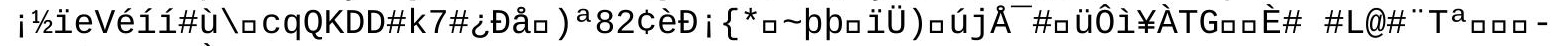
mrr2;U: : : È 


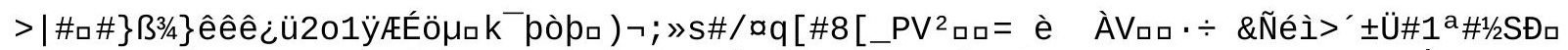

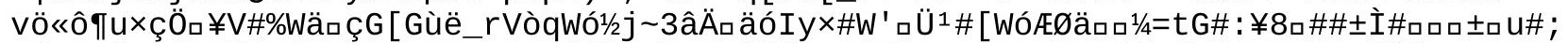

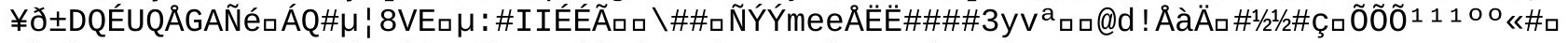

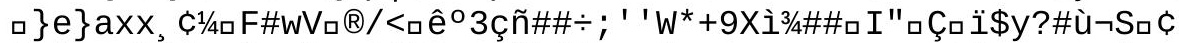

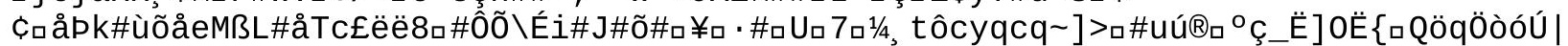

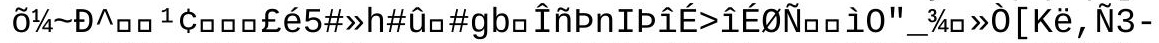

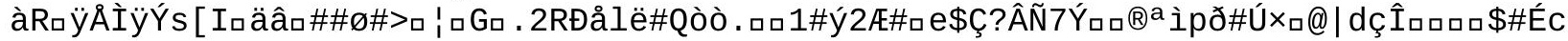


$00 \ddot{I} \|^{-}=F \# \ddot{Y} \ddot{I} \tilde{A} \tilde{A} \# \square B W W \times_{\square} \# \#(\#$

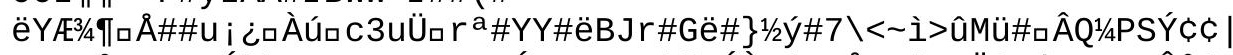

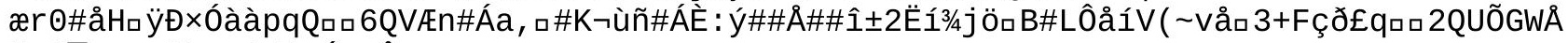

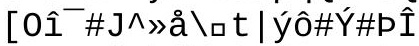

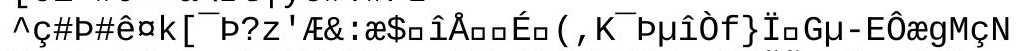

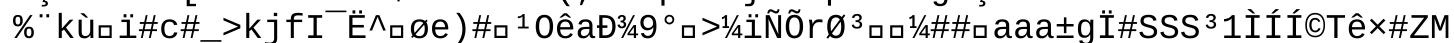

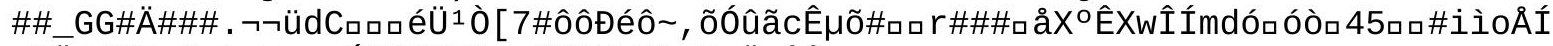

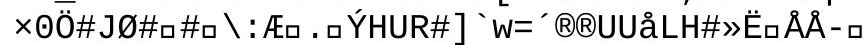

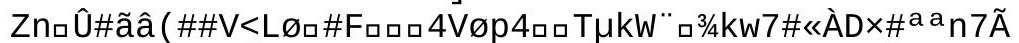

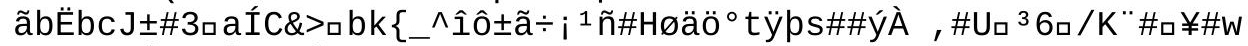

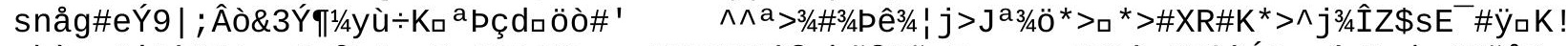

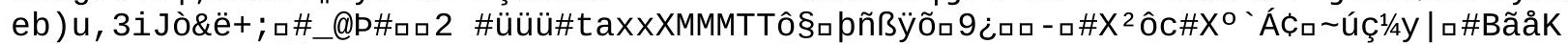

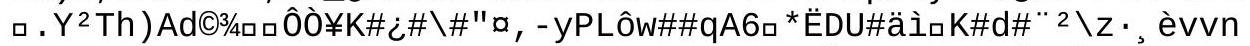

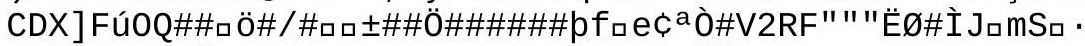

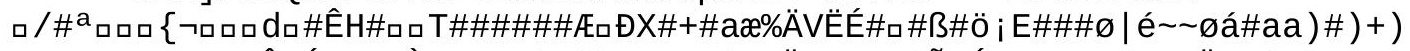

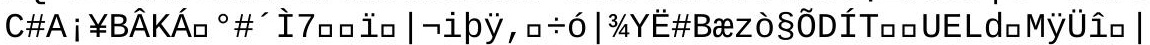

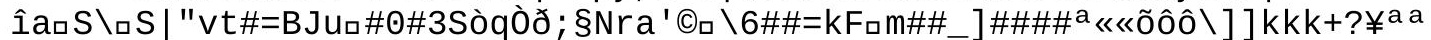

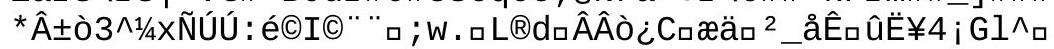

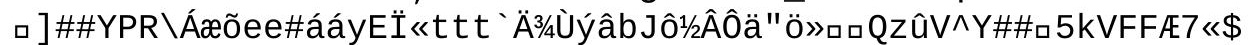

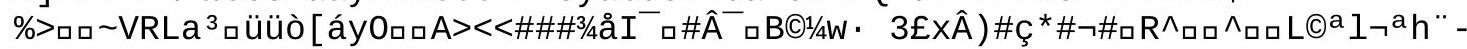

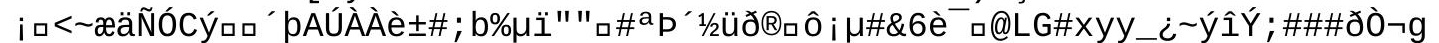

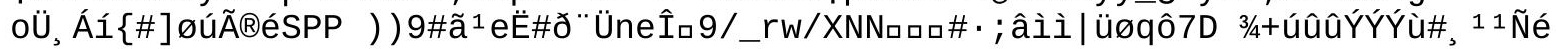

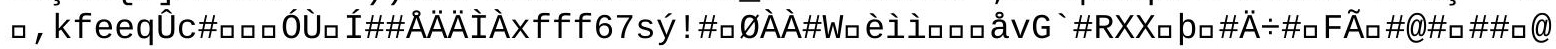

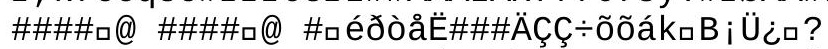

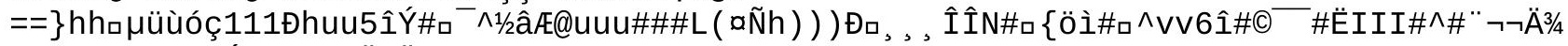

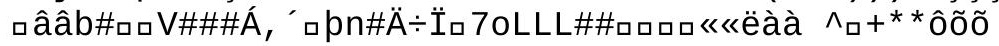




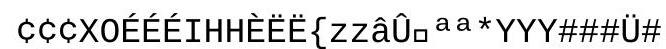

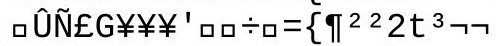


-

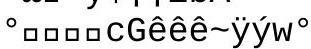




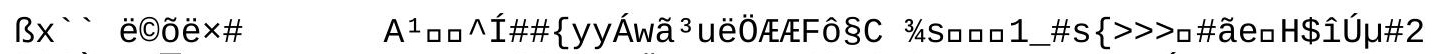

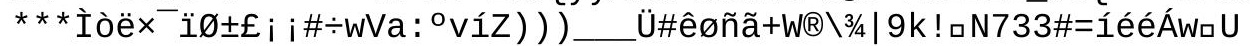




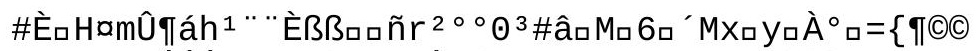

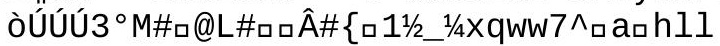




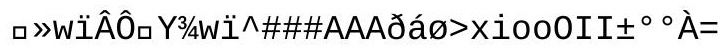




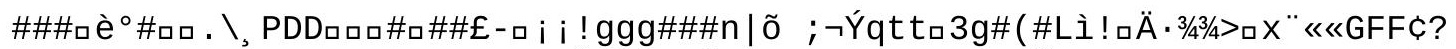

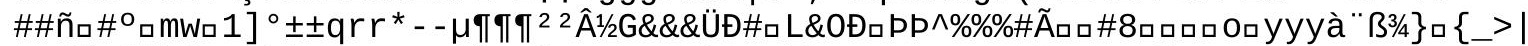

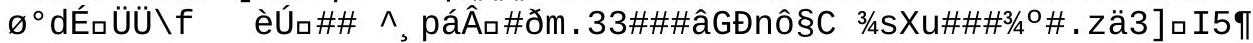


Íââ $2\left\{\div\right.$ nÜ $\left\{4 \square^{\circ}{ }^{\circ}\right.$ ÂXWÀ2222ø.\# 




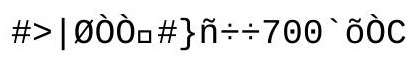




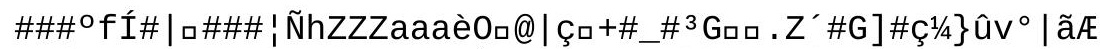

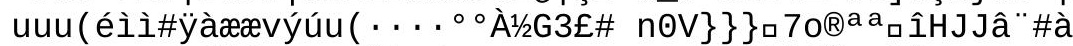

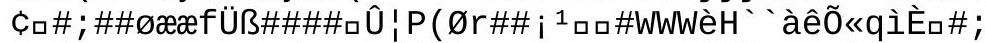

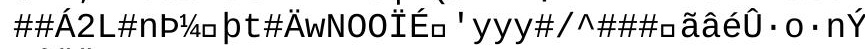

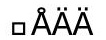

$\mathrm{z}\{\{\quad \# \mathrm{BSS} \# \mathrm{XQ} \% \%$

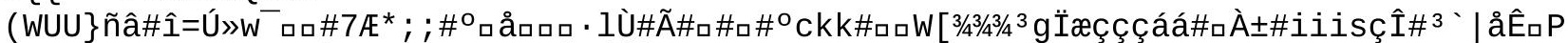

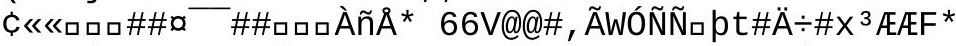

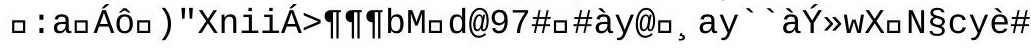


$\# \mathrm{t} \# ß F a \# 0 \mathrm{e}_{\mathrm{a}} \mathrm{a} \# \mathrm{q}^{\prime}$ 


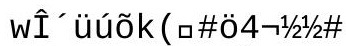

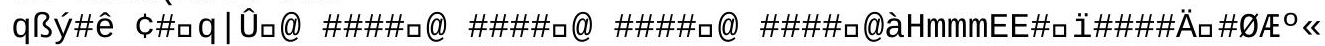




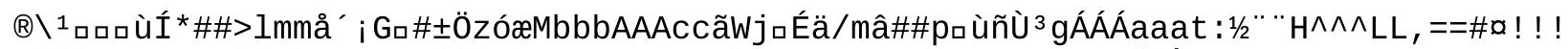

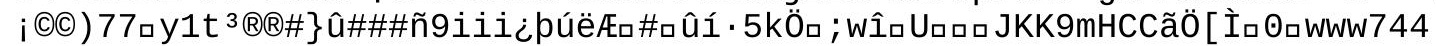






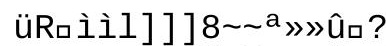

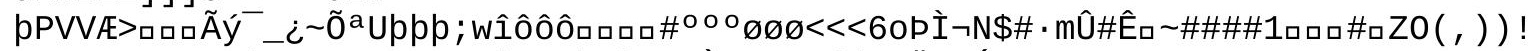

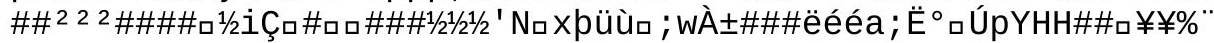




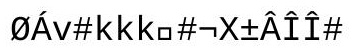


Â\#öööàÀnqu 'V/Ïロup\#óüq ' O\#=zÔĖÈ\#DdppĐİİ॰@\#

\#61PSSÃ>Ba\#C6

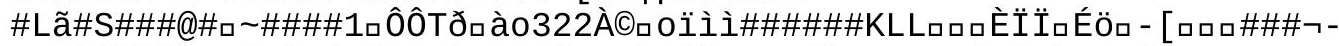

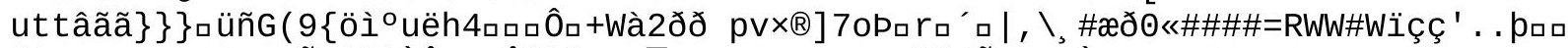

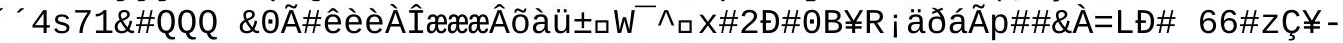

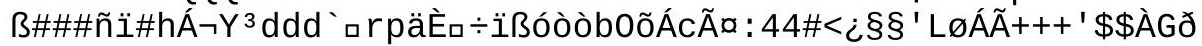

'

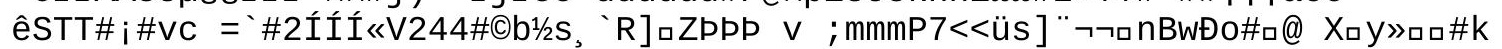

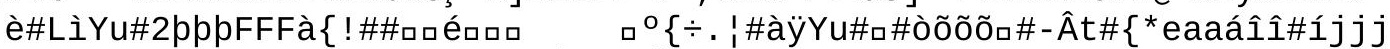

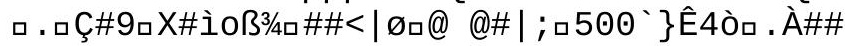




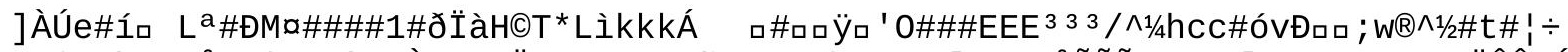

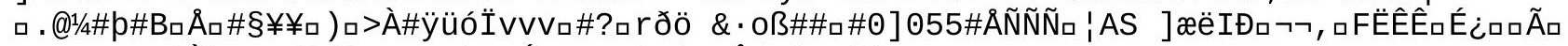

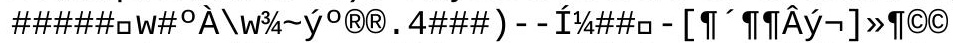

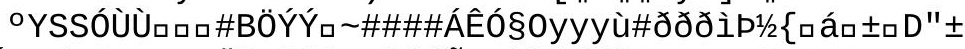

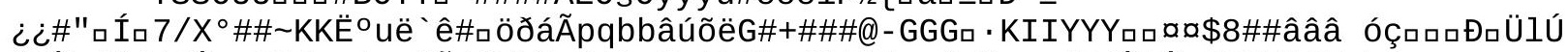

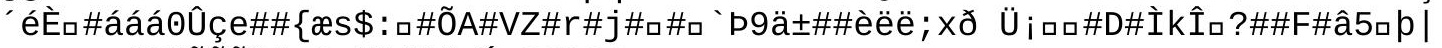

ฉ

!\#巴хpp\#» 


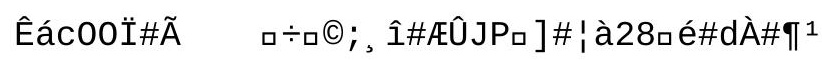


«5ÂpøP\#ロ`\#òĐ\#ャc3E\#\#ロ9 (NII

f\#B\#Ö\#XÛ\#@i é

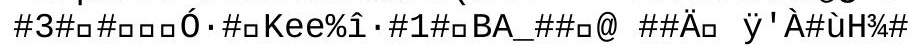

endstream

endobj

$29 \odot$ obj

$<</$ Length 2276

/Filter/FlateDecode

$>$ stream

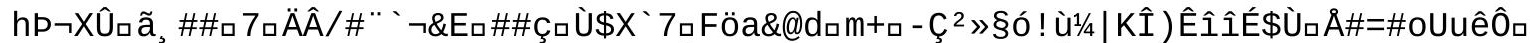

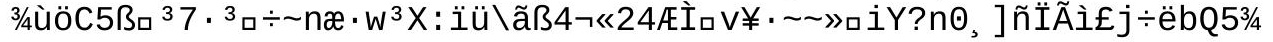




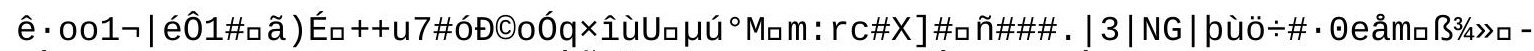

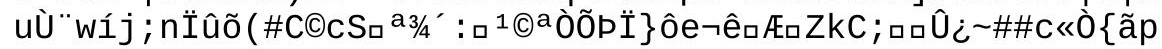




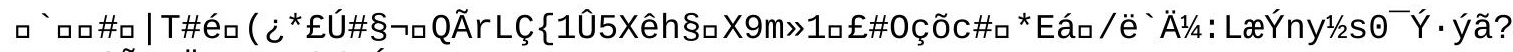
\#ñsuiõá8Üwë\$·\#|/ィÓaã

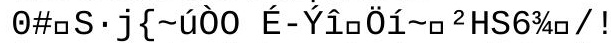

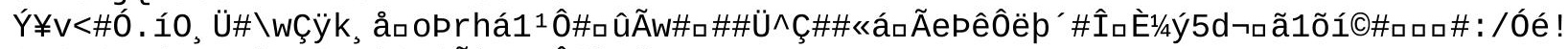
\% 6\#๑\%\#j TqĐàı1Q\#\#\$Q\#ì̃̃òKmmôøùĐf

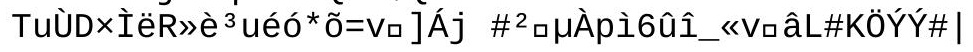




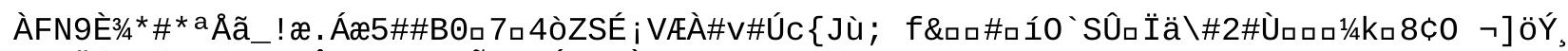

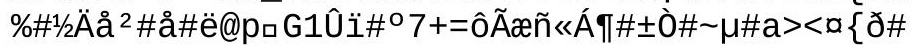




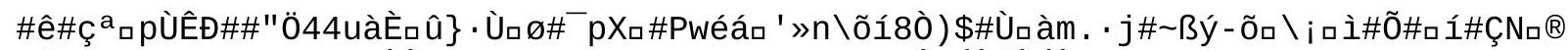

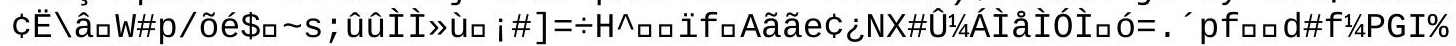

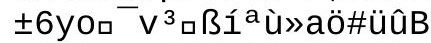

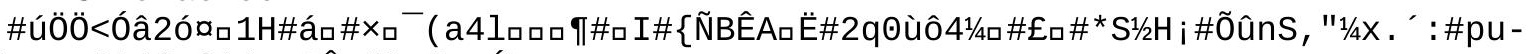

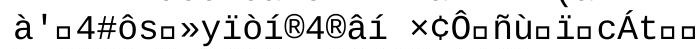

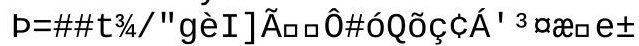

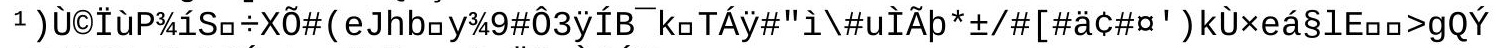

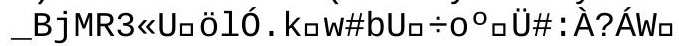


ß口 ${ }^{\circ} \mathrm{K} \AA$ ( $\left.₫ Y\right\} 6$

Ö\#uqbaXn§¥uq 


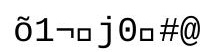




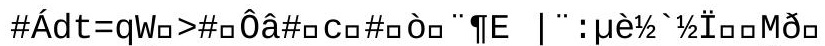

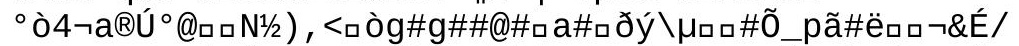

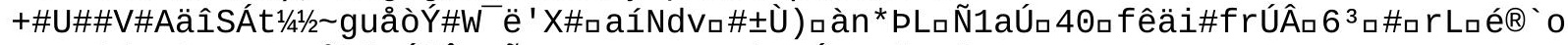

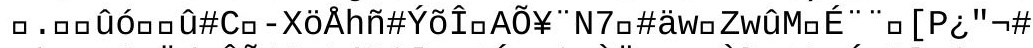

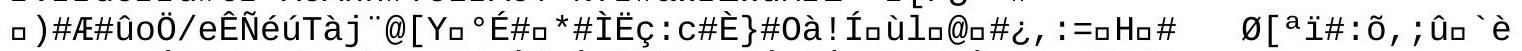

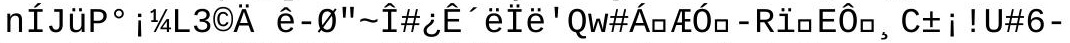

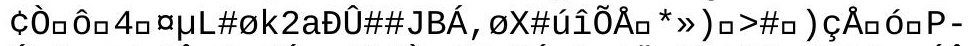

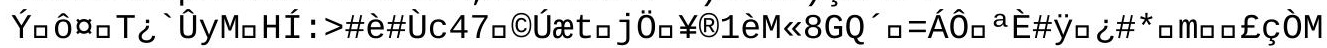




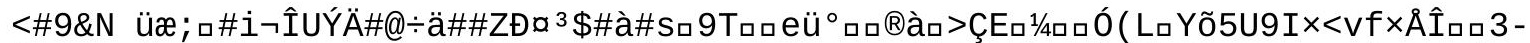

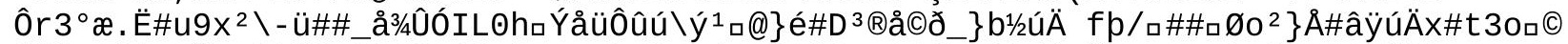

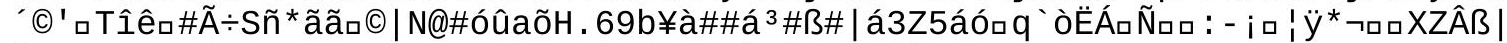

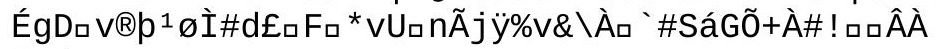

$3{ }^{1}$ 


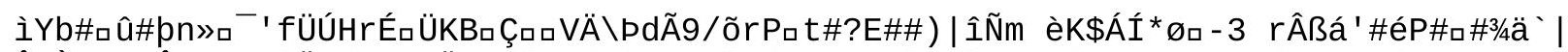

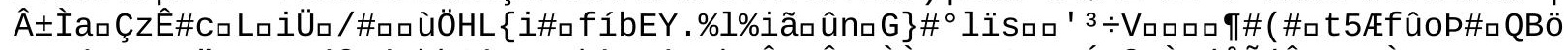

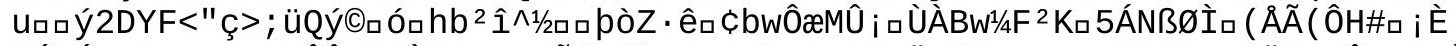

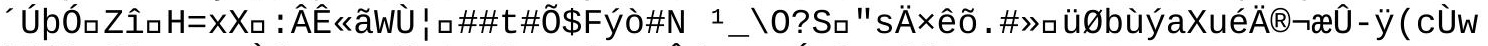

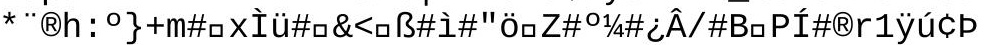




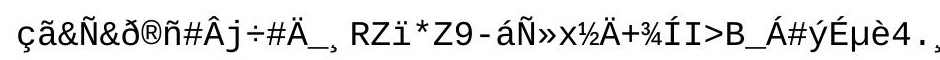




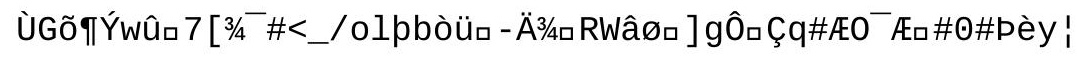

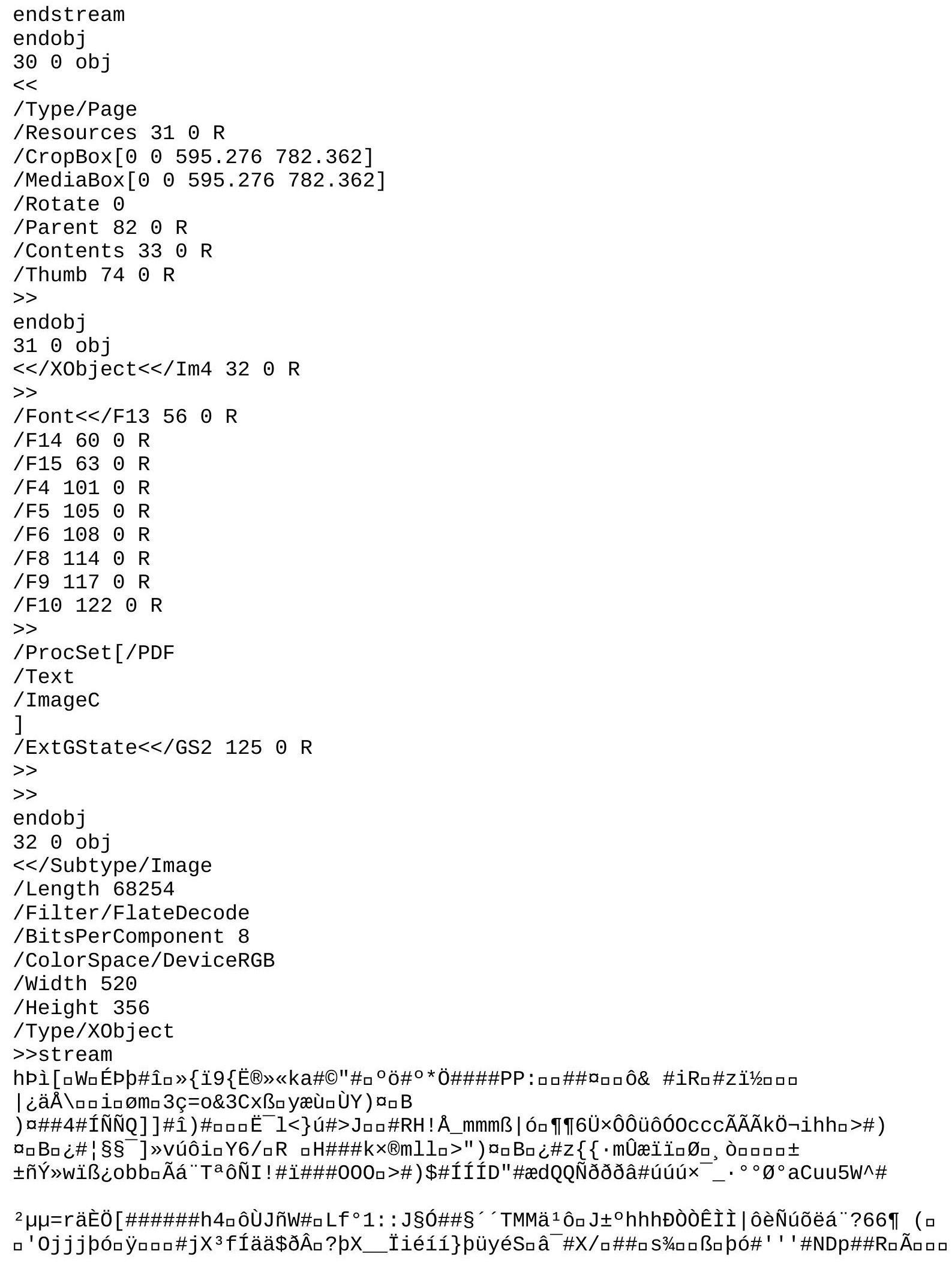


口Éq>U)V\#WîÜ\#!P\\ 


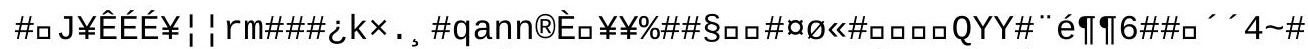

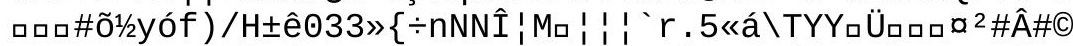

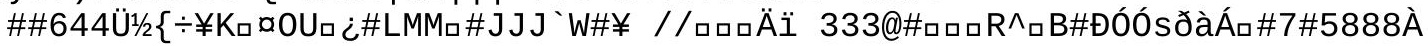




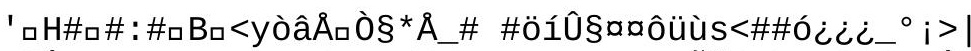

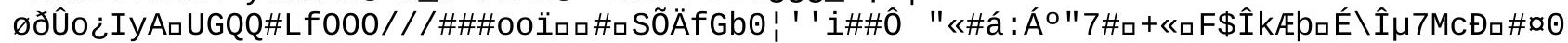
i 


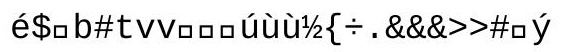




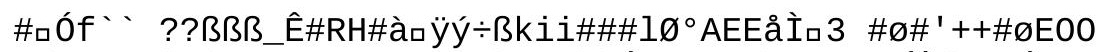

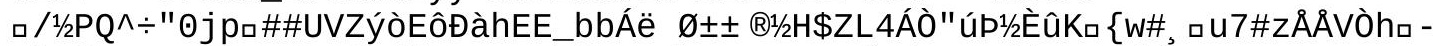

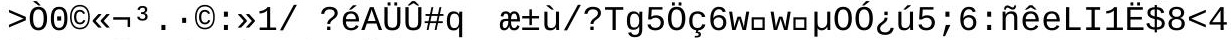

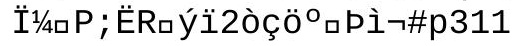
$/ \mathrm{C}^{\circ}$ 


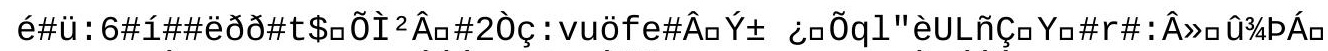

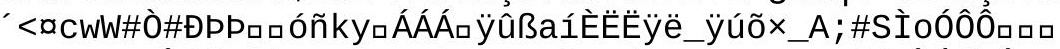

r\#Ùó@Têû

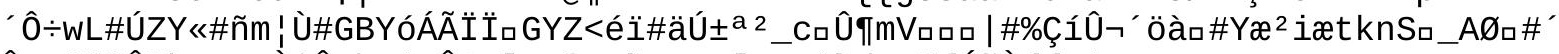

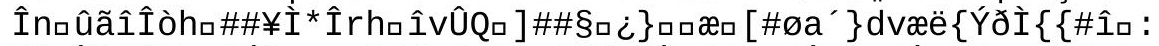

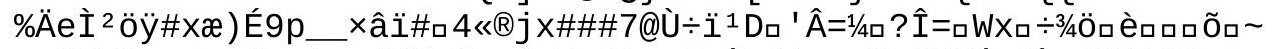

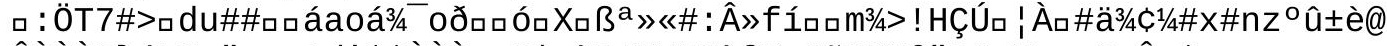

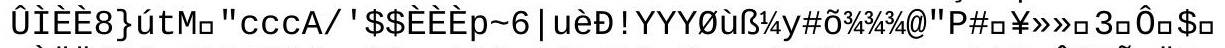

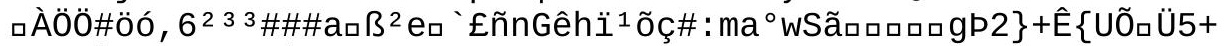

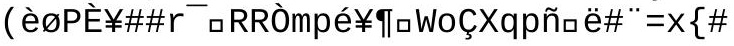




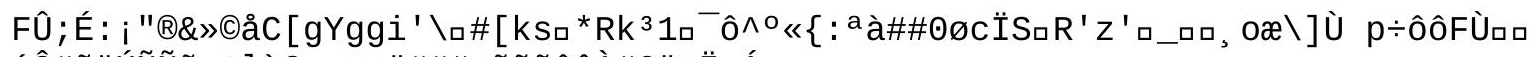

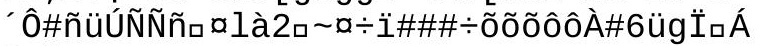

âül ㅁ YVD\#ᄀ/

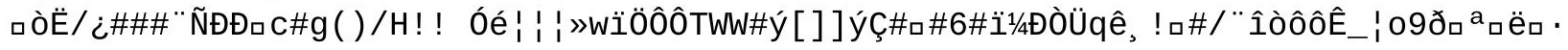


| 'É\{\&aûvČ<\#ú1 ìò@\#ðロ5ロi+h\#í\#ßMRgaÚaffaT:ihrakl"s

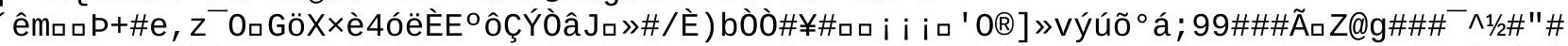

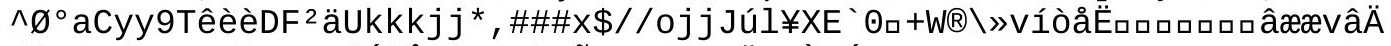

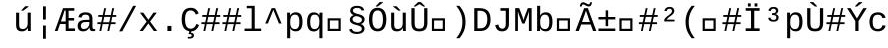

\#ý\# ! I\$òâ 


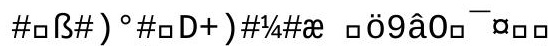

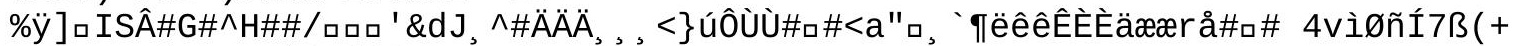

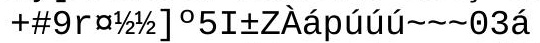

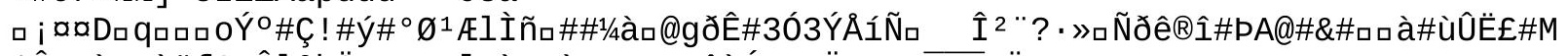

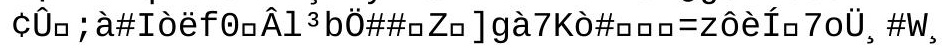
_Ü 


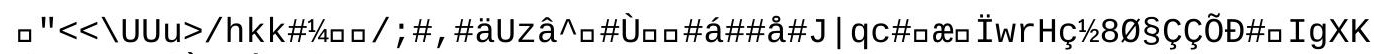
$\{$ ' 6 ; ò\#. ' 'Ù-3Å=\#囚y ù-

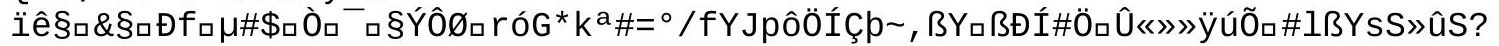

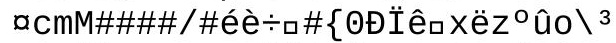

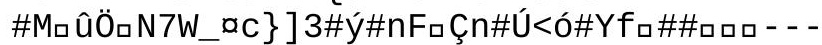

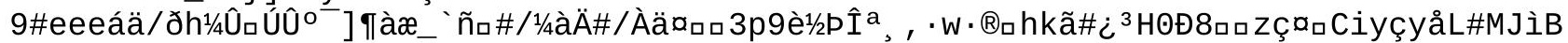

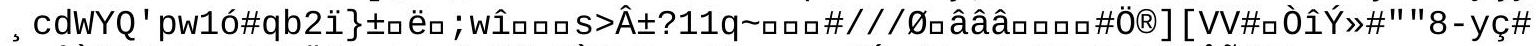

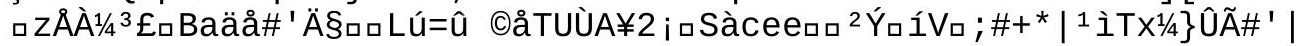

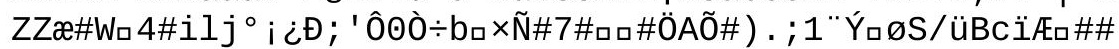




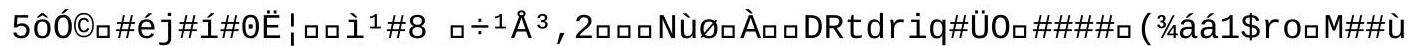
\%Ïロ\#\#\#ロ1/4\#ロûPĖê89ÉêX\Ä2DNLLFE\$ロ\#X!

\#ó; \# 


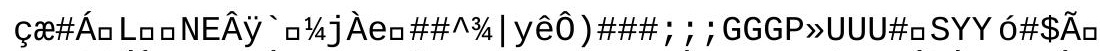

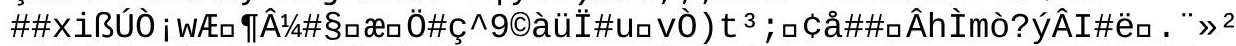

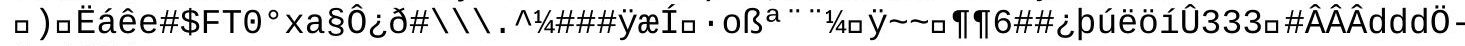
[wýúõáaVロVGG\#, \# 


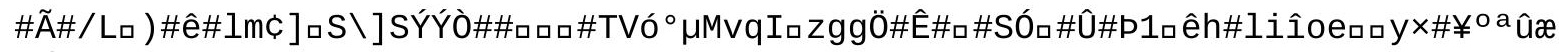
$\square /$ uuQuuCt

$£^{-} q^{\circ}$ ßó ß\#u075\#æ \#pôË $x_{\square} C ß 9 \# t_{\square}$

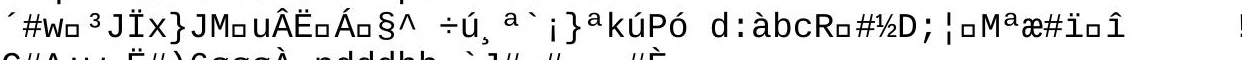

Ç\#A i Wa ̈̈\#) 6øøøÀ ndddhh, 'J\#ロ\#ロ

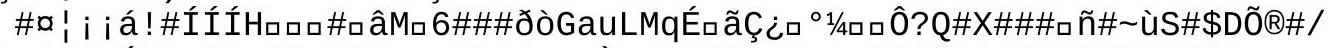

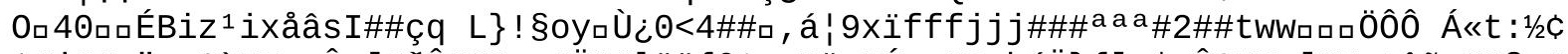

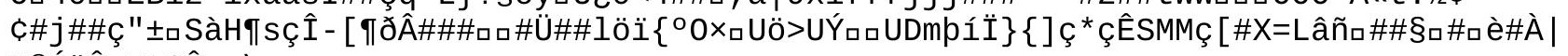
H®ÍÖÊqUÆ9Ê i_l

NrLa*\#Öם

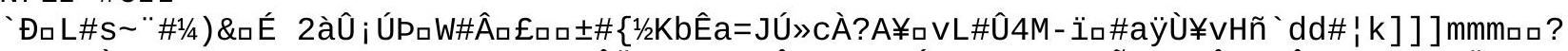

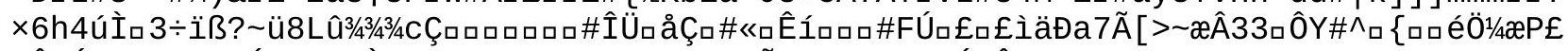

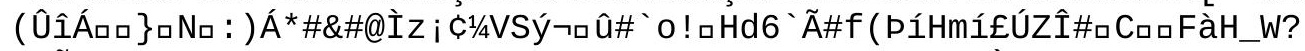

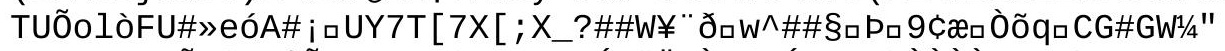

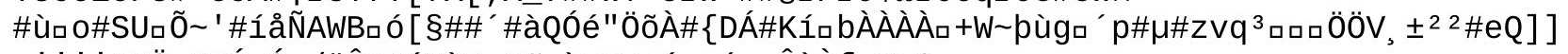

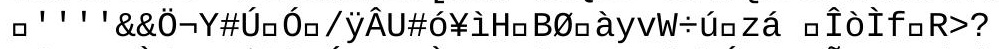

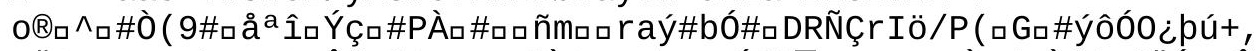

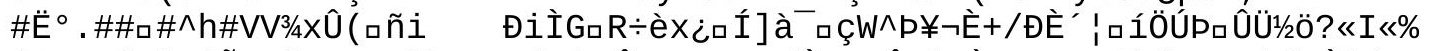

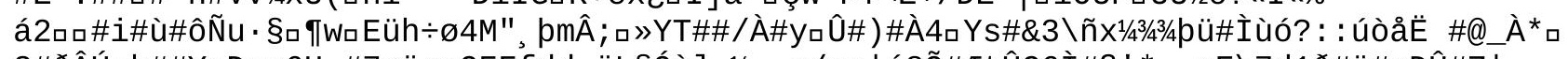

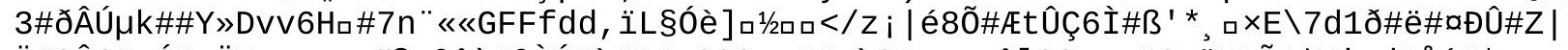
İ\#9ÊE\#ロÓH口ÄY ØßZ

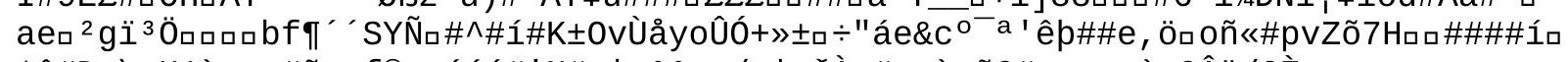

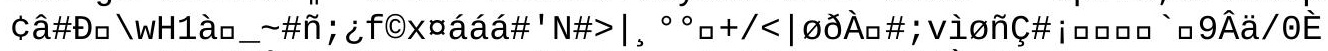

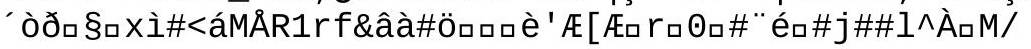




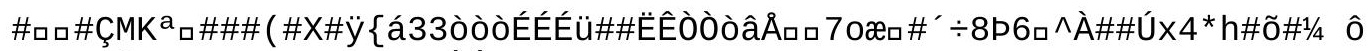

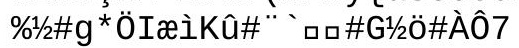




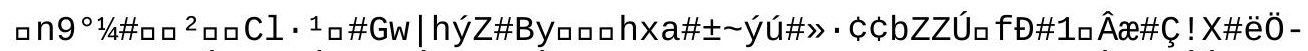

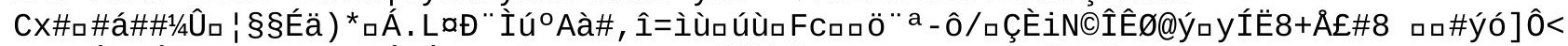

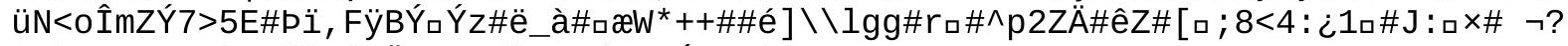

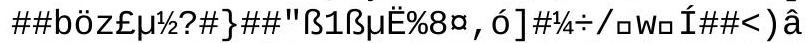

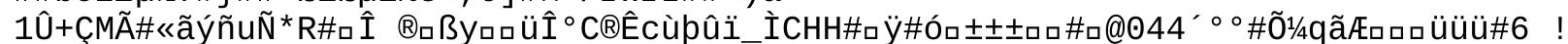

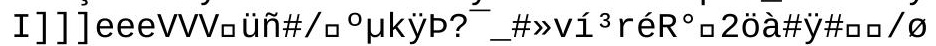

) Đåüù \#Ûm] ]prü\#ã\}\# i · "áX\#i\#3ú2, |\#ロ@ロXy\#Àa2ê3êÝ`1/2=öú4æ4 -

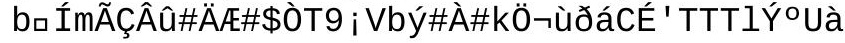




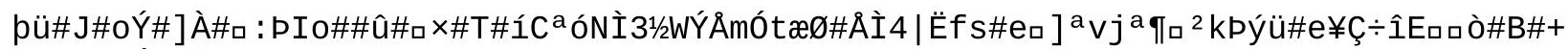

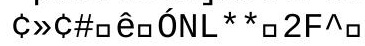




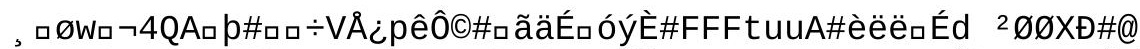

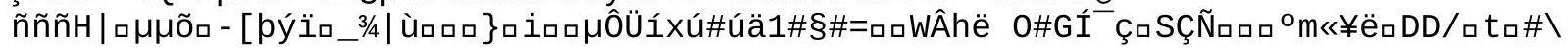




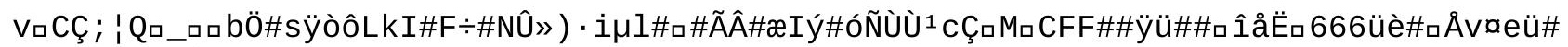

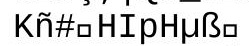

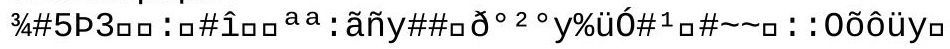




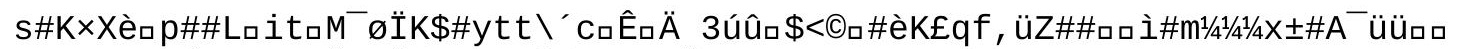

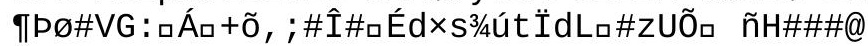

$\hat{\mathrm{I}}$

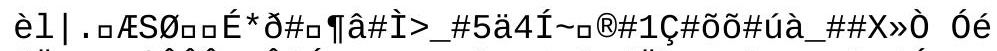

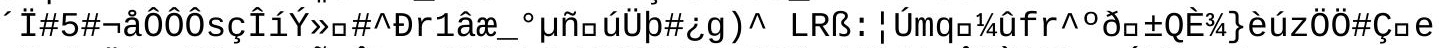

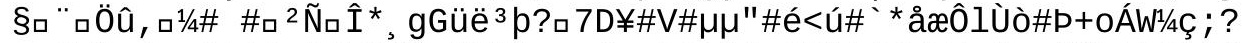

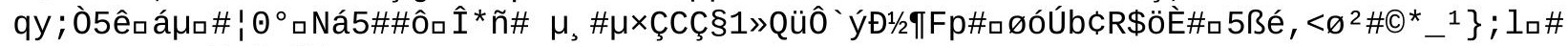
$\square \div$ Ryò;SI

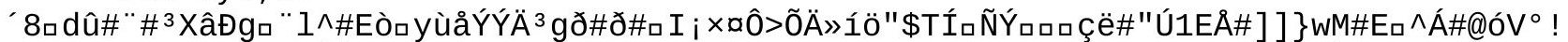

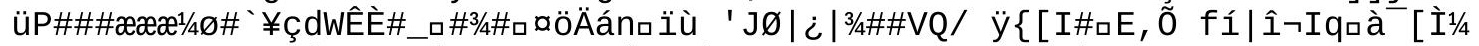

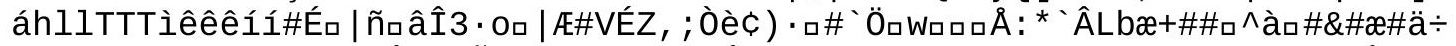

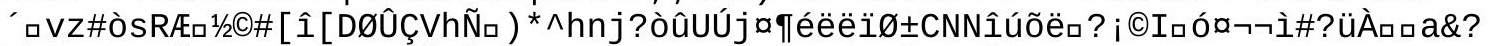

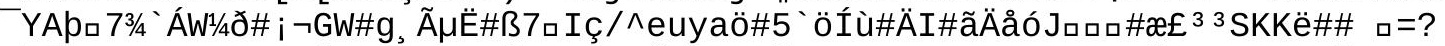

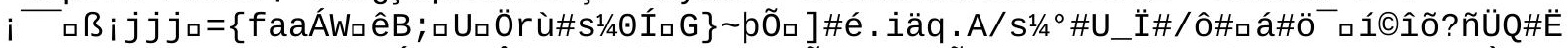

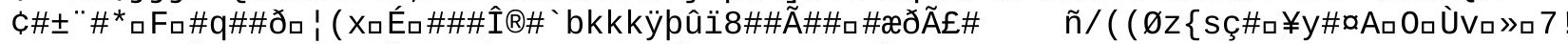

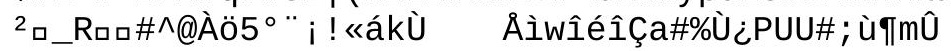






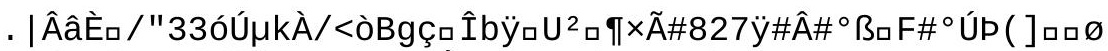

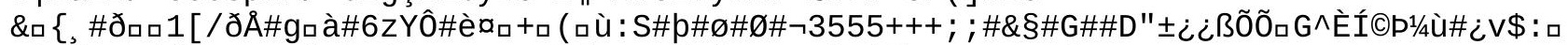

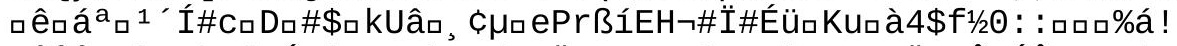

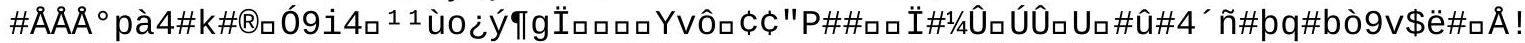

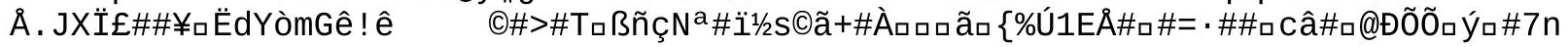


\#\#

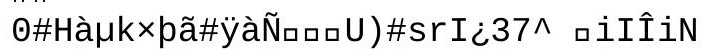

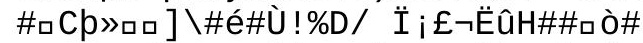

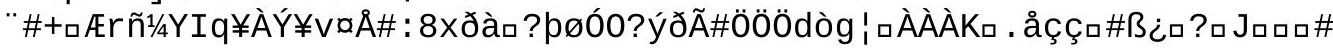

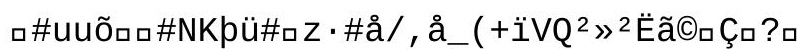

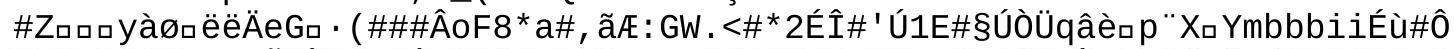

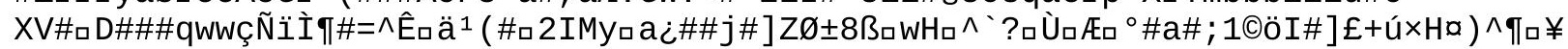

Õ\#口qïp=SSÓááá i i ! X\#

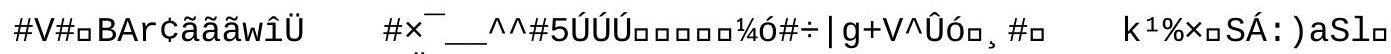
口\}_p $\left.{ }^{2} 8 Z A \ddot{1} 1 / 4 \square \square<H L U J u P A ̈ \#\right]$

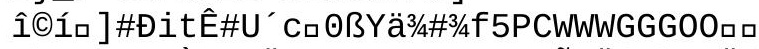

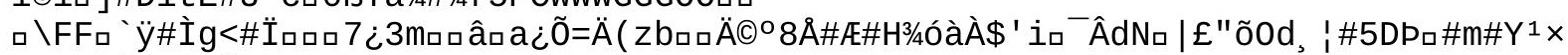
$i^{\wedge} \square \mathrm{mVİ} 1 / 4 \mathrm{PA} * \tilde{O} \mathrm{P} ; \# \square £ \backslash$ 
\#R077ロ\#口d.\#/\$

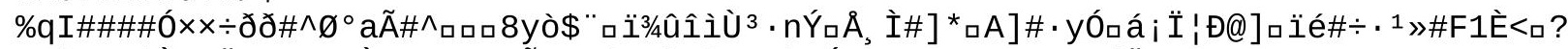

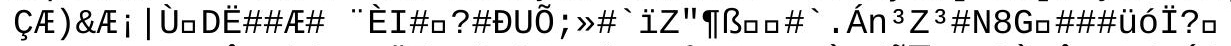

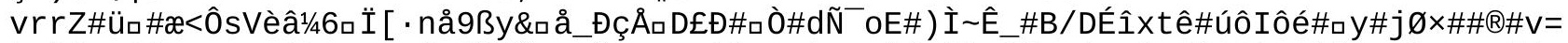

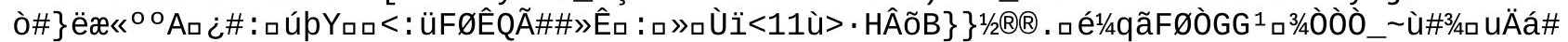

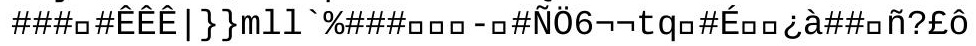




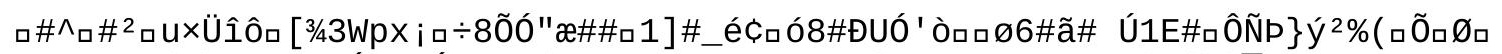

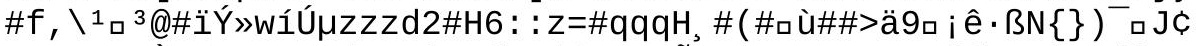

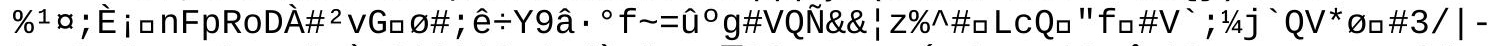

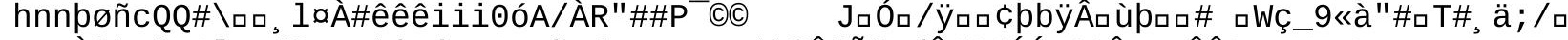

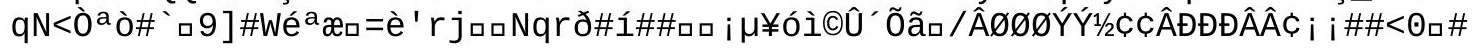

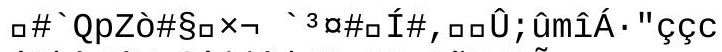

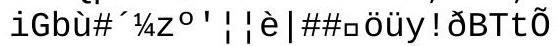

=j\&口व İUPÂFÜ 


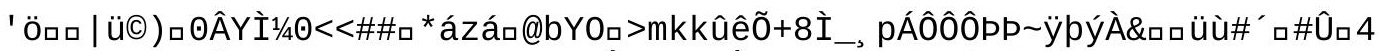

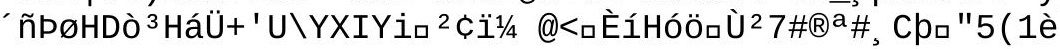


a "\#ÇDè_q"SVkÒıL\#\#ûÂp\#' \#Äロ\#r\#drMMÍouë@e_Ôa

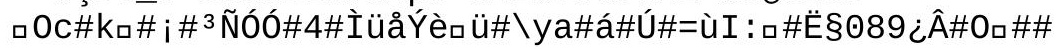




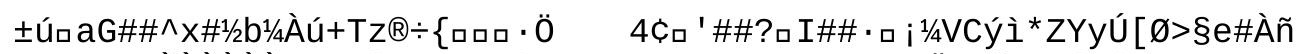

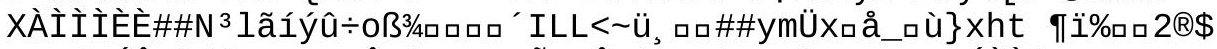

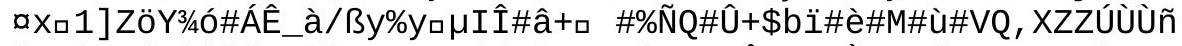

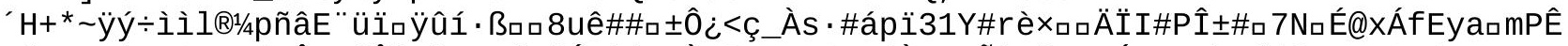

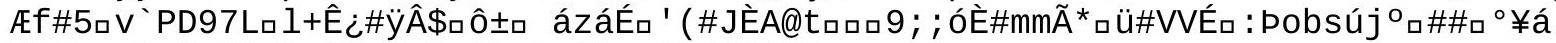

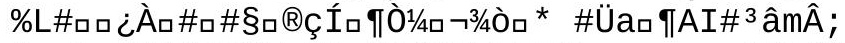

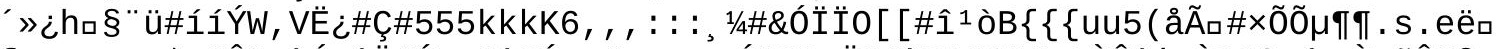

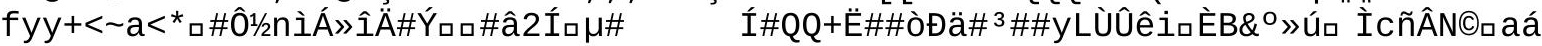
: vìXTTTè ' 
\#\#\#6\#_V\$-M»ÅùîิZْ İa 2 qup\#b¥ 


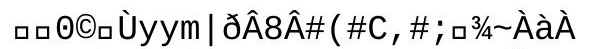

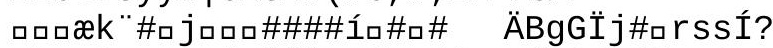

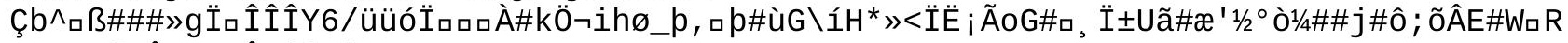
; 3-\#gf10̂ロ\#\#GÂBâ§\%ãı_

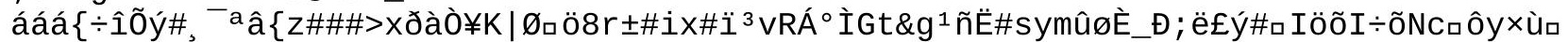

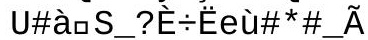

$\#^{-}$

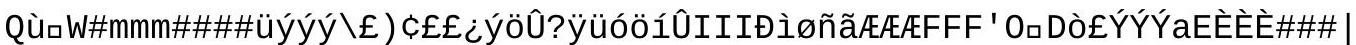

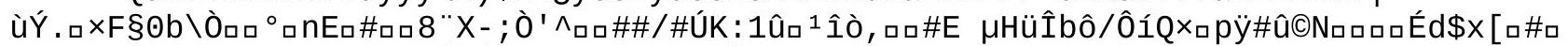

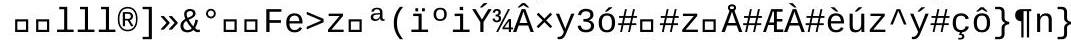

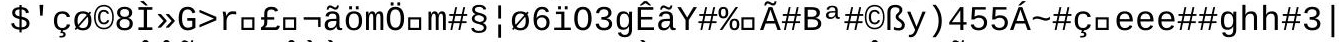

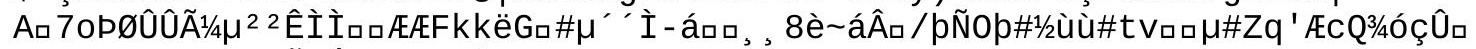

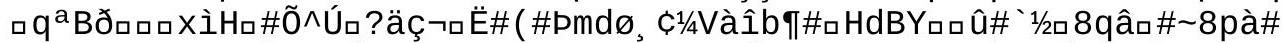

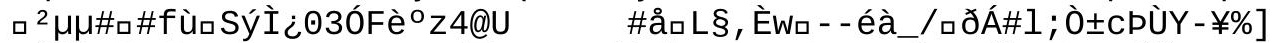

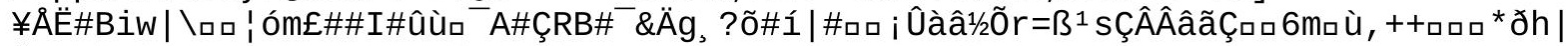

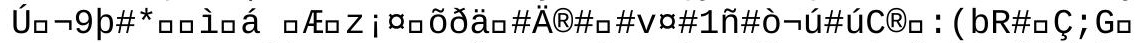

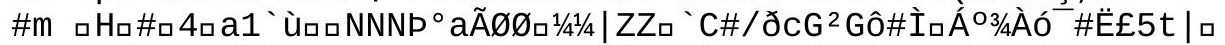

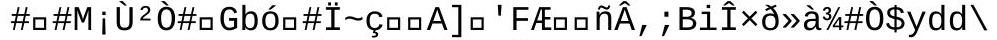

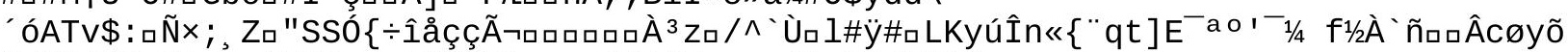

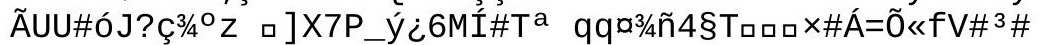

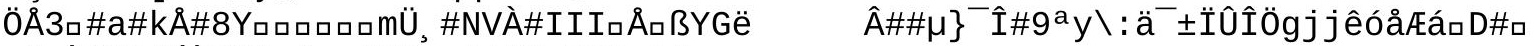

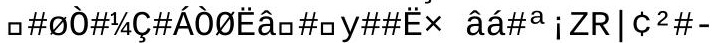

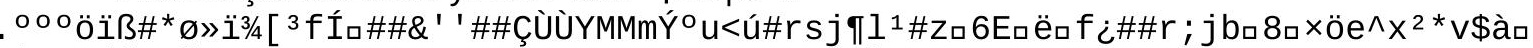

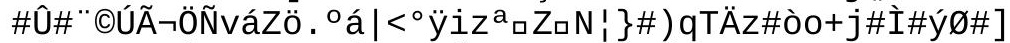

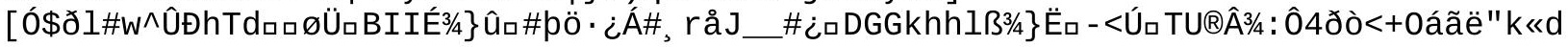
\#1/4§G\#ロBý| 1 ä_\#\#/D

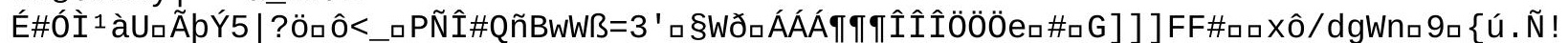

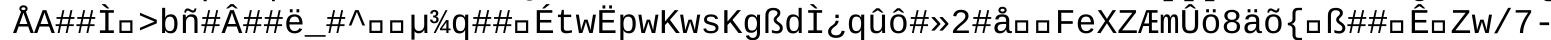

Ï॰äjÆ\#ßkêÜYıâç

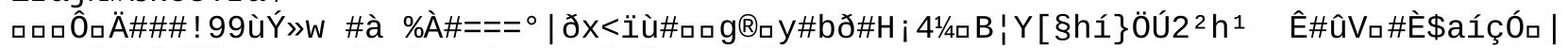

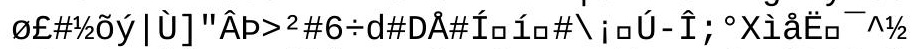

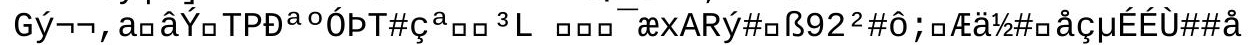

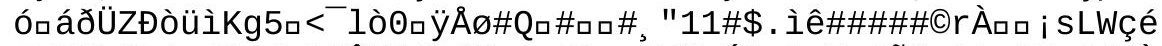

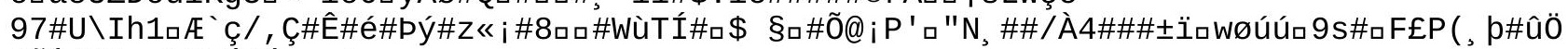

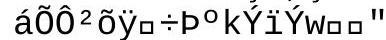

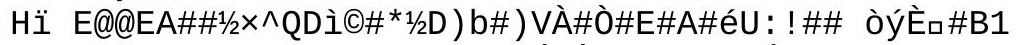

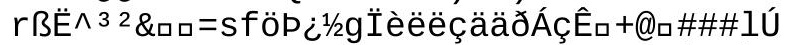

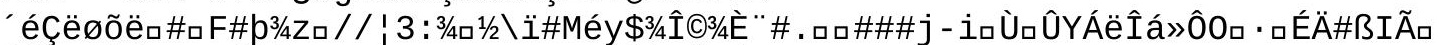

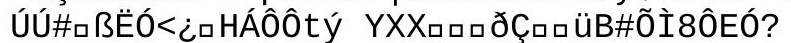

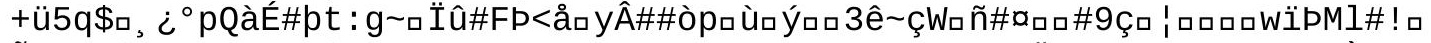

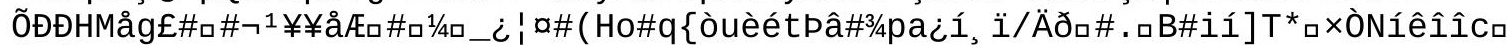

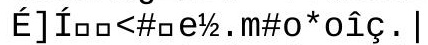

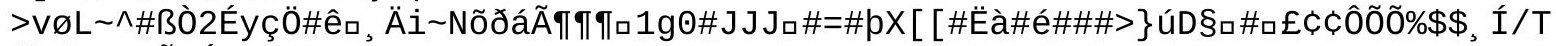

' h/ØухёÕpÁc 
\# . è\#PBCîr\#9\#ロ\#\#xÇם

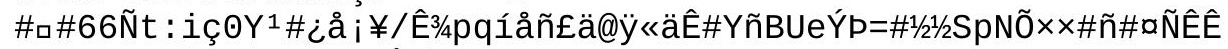

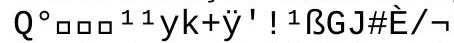

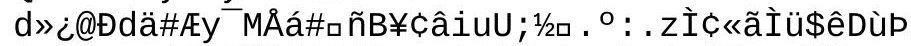

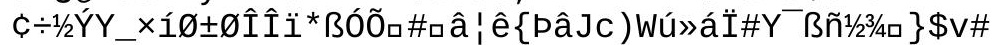

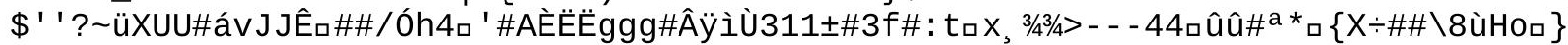

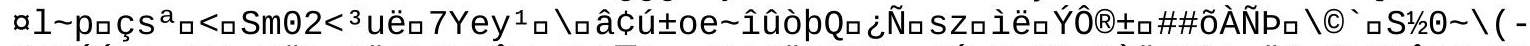

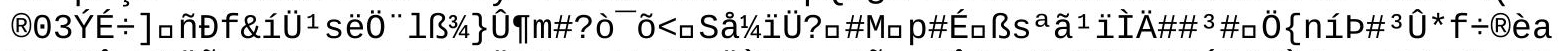

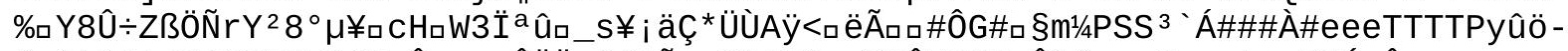

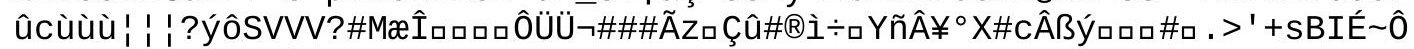

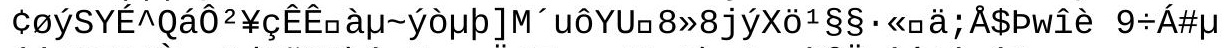

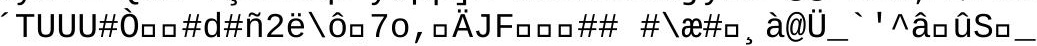

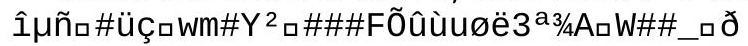

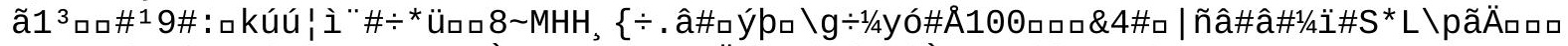

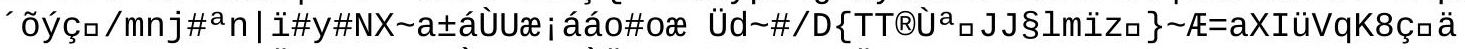

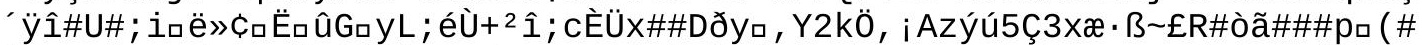

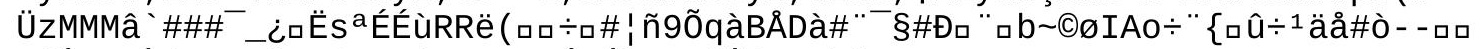

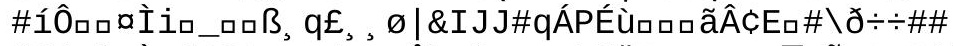

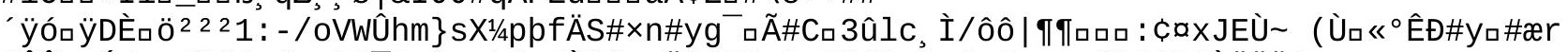

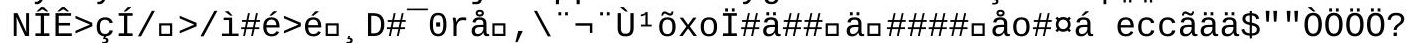

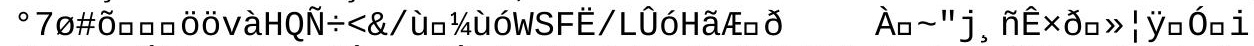

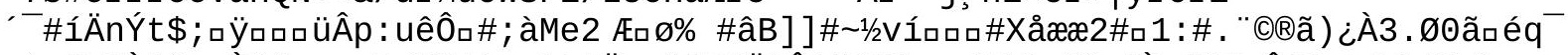

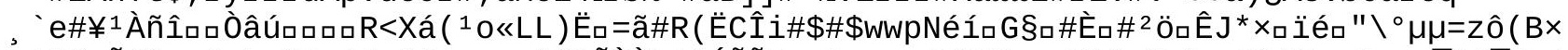

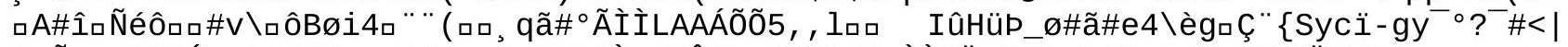

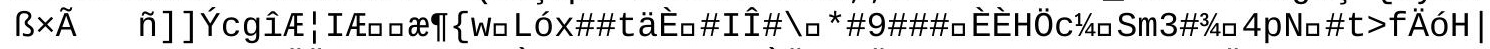

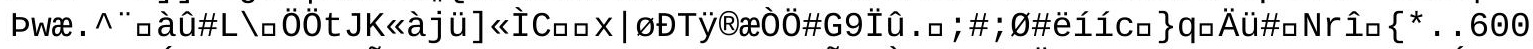

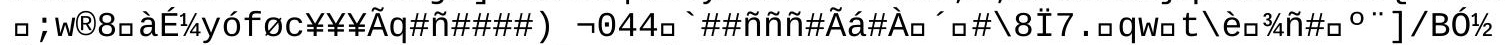

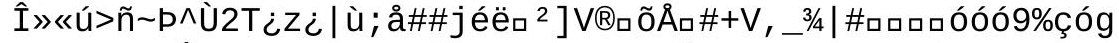

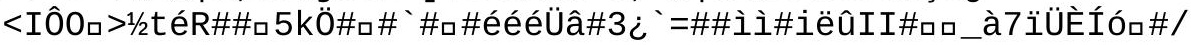




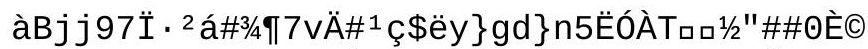

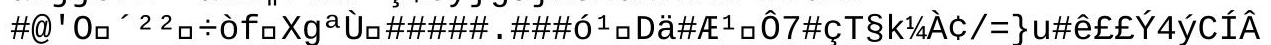

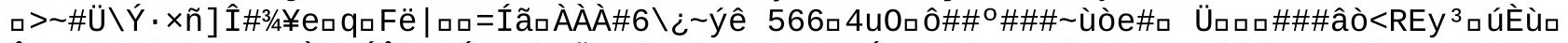

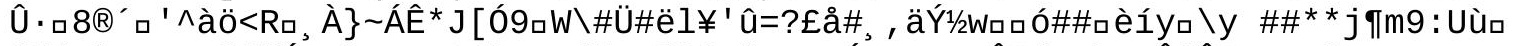

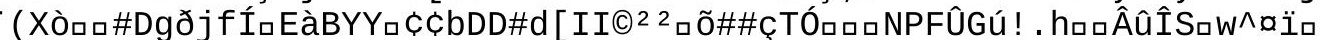

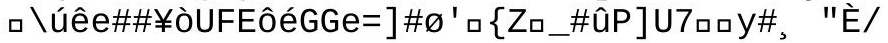




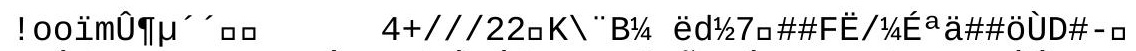

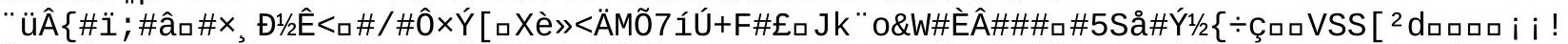

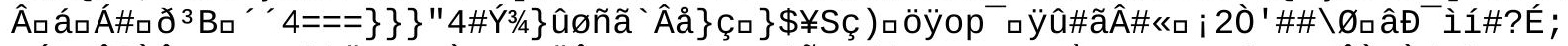

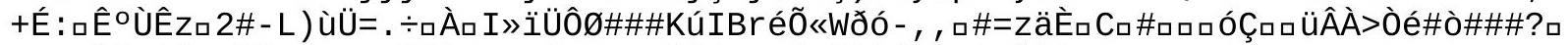

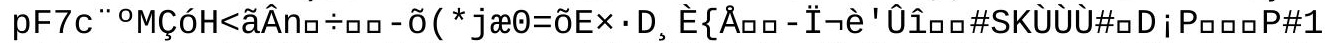

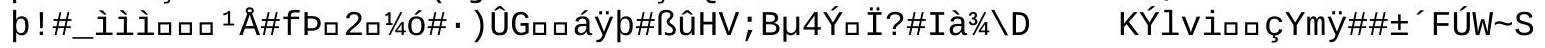




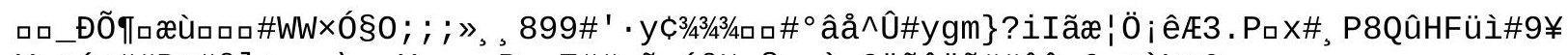

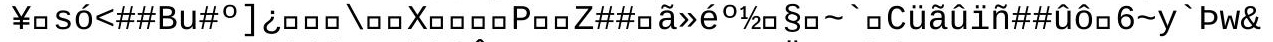

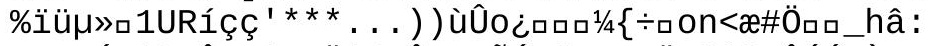

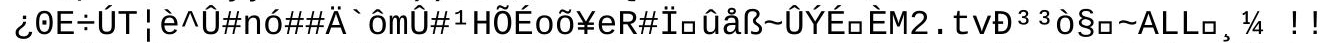

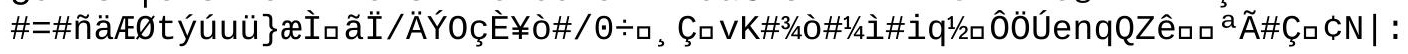




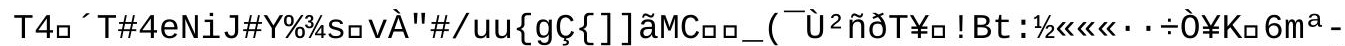

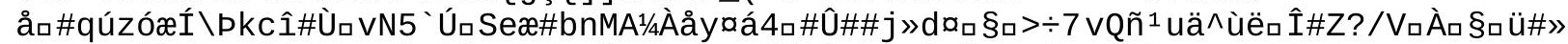

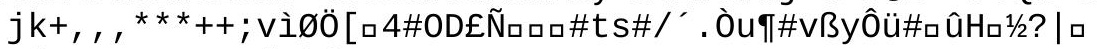

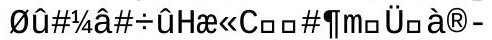

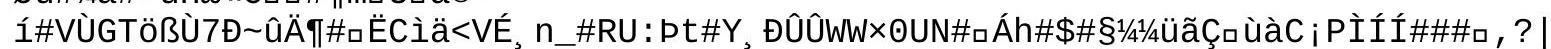

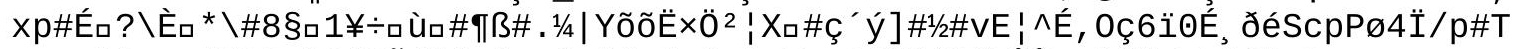

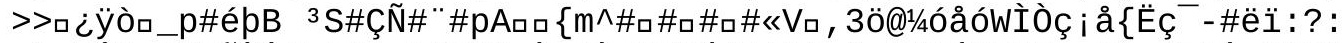

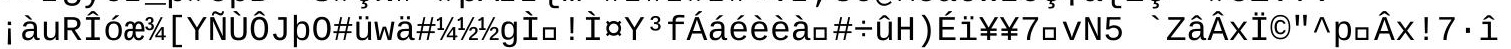

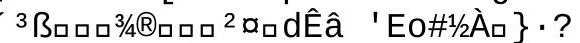

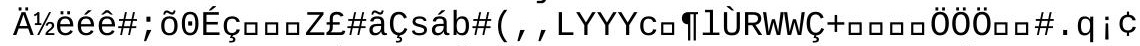

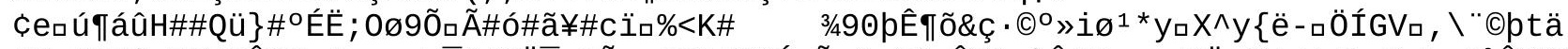

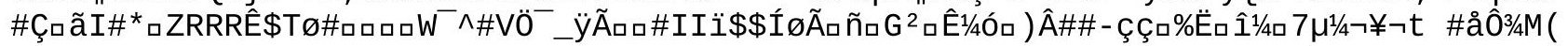

ođ̆óæ)Y07Mÿë [\#>\&\} ᄀ*o--m) -ýö 


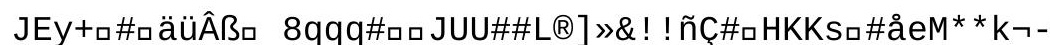

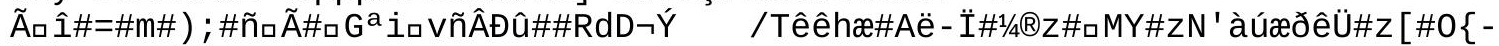
$\grave{A} \#] 2 p ; a, b \AA \AA^{2} m=d \_C ̧ æ a V$ 


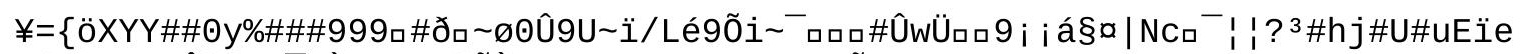

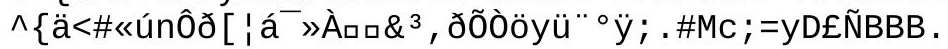




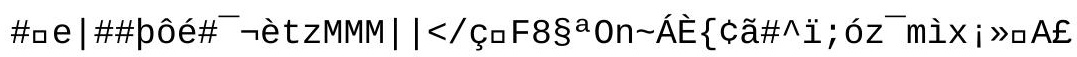




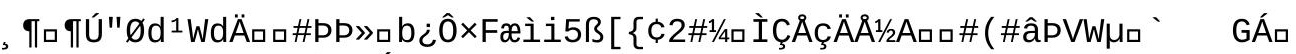
$a$ *ềGï口ÿrSâî¿NMp@\%Áò ${ }^{1} A$ :

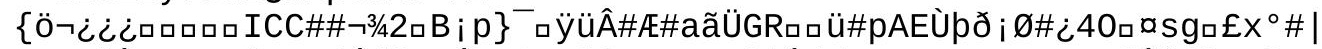

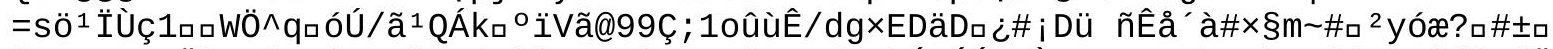

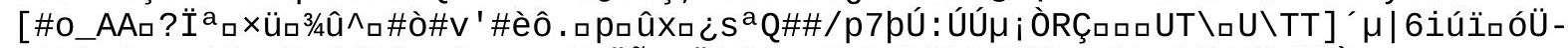

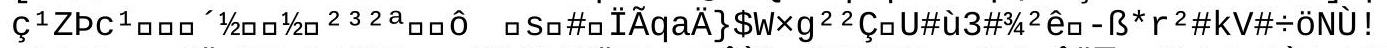

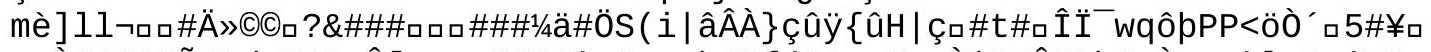

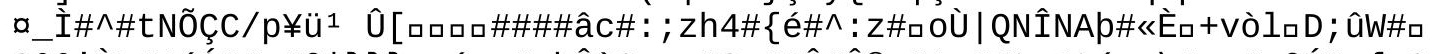

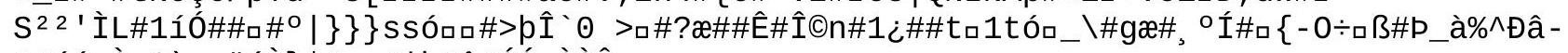

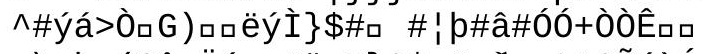

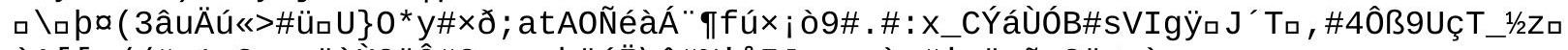

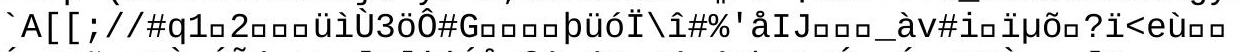

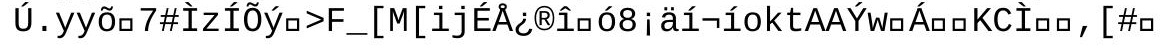




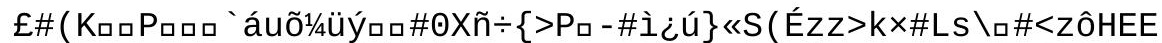

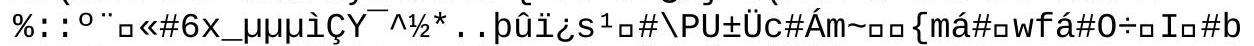

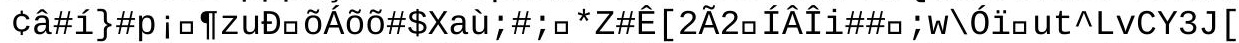

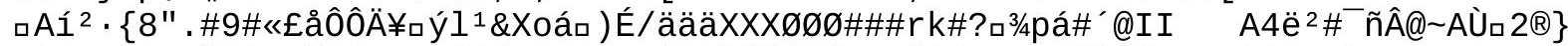

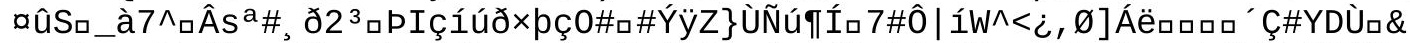

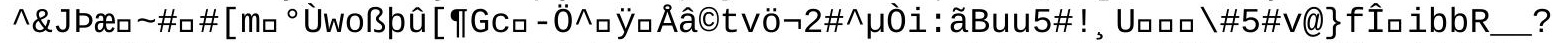

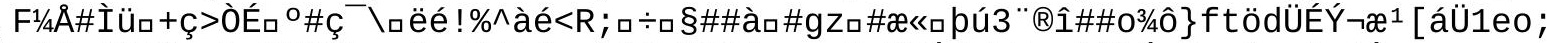

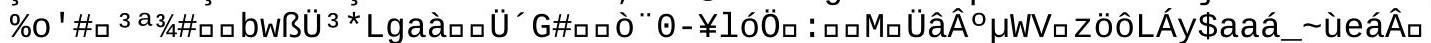

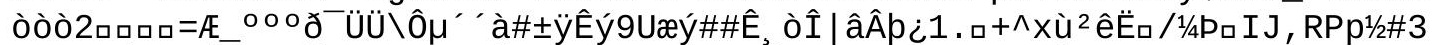

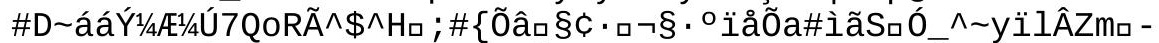

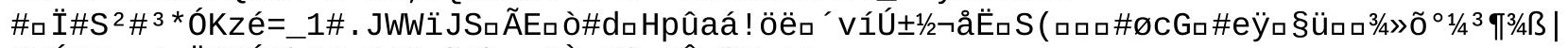

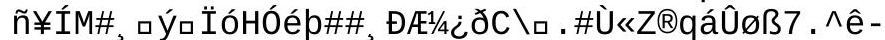

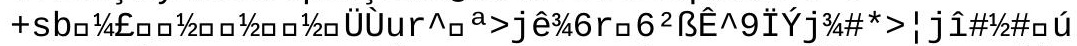

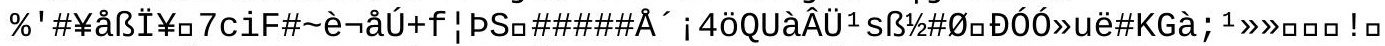

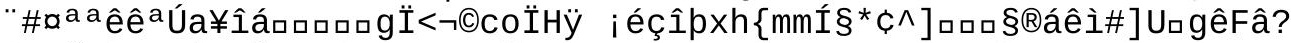

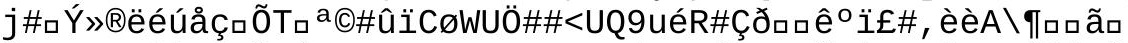

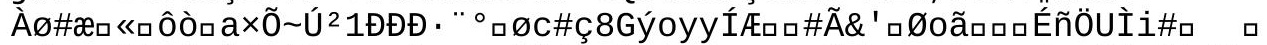

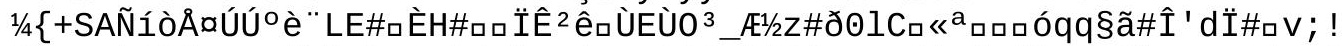

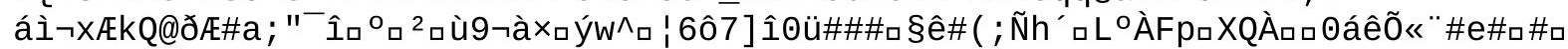

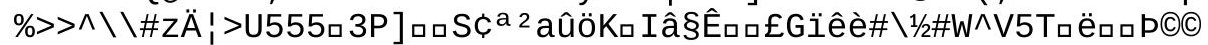

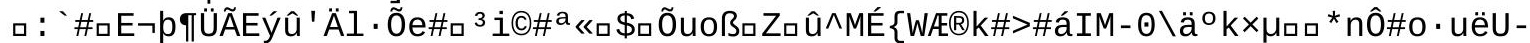
\#\#ñqù5ßÜ̈Ûh_@ [Ý⿴囗十

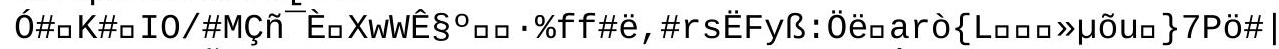

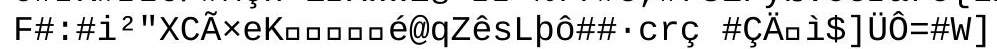


Ý\#\# ${ }^{2}-r^{\circ} U ̈$ 
Ý\#УロYं

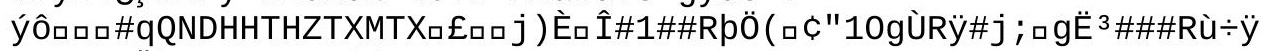

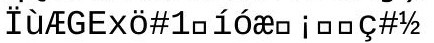


/"B

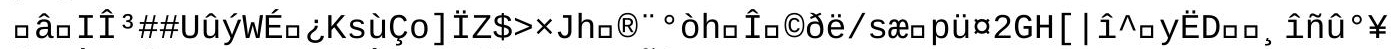

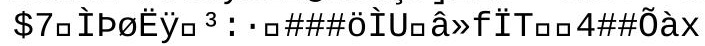





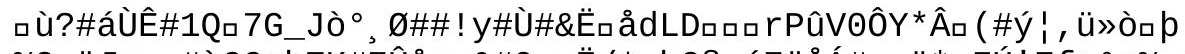

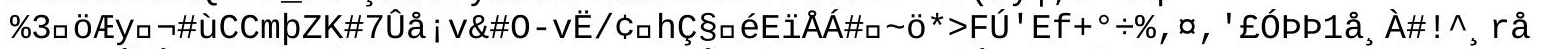

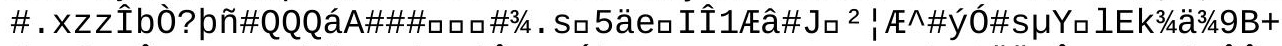

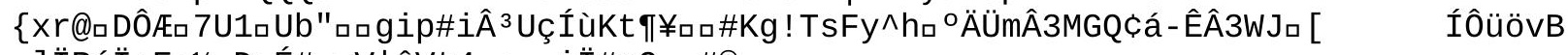

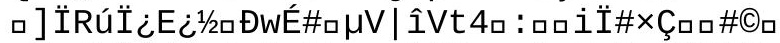

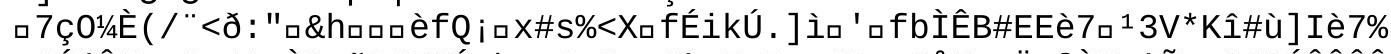

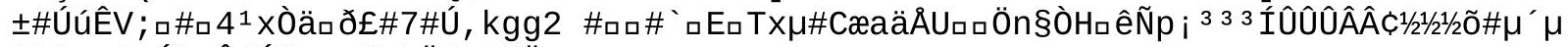

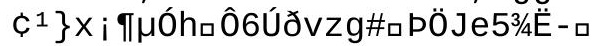

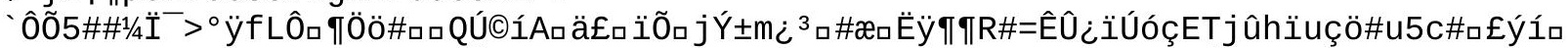

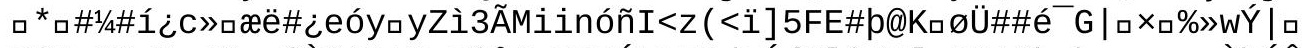

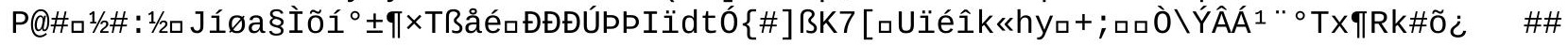


\#'\%11QBB\#

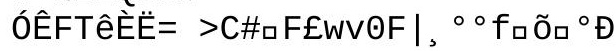




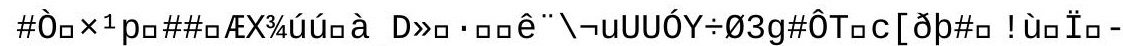

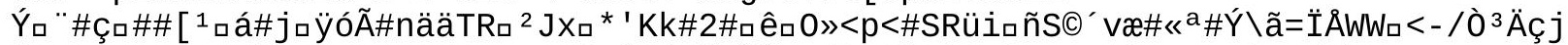

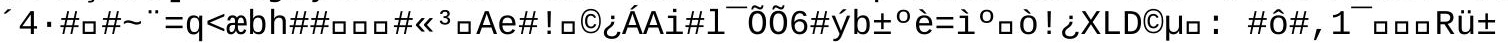




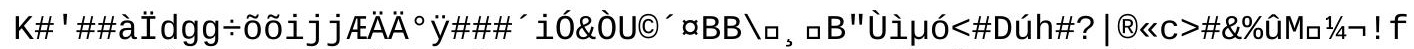

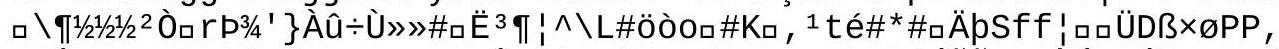

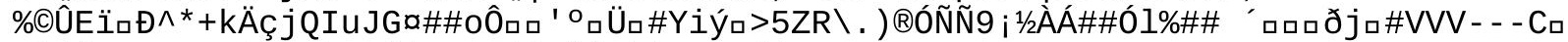

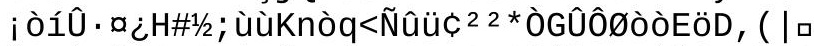

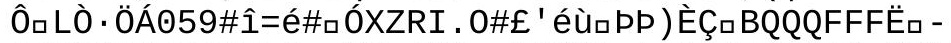

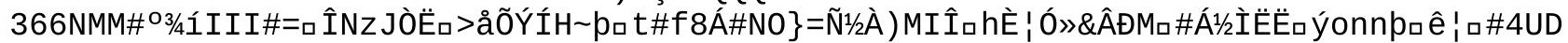

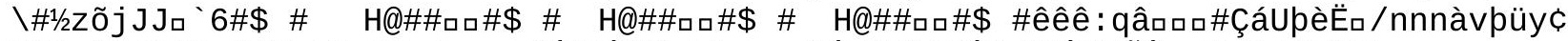

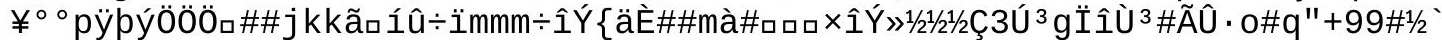

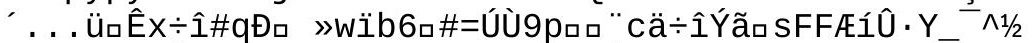

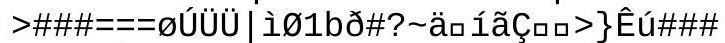

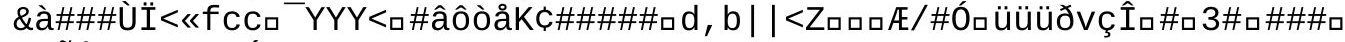

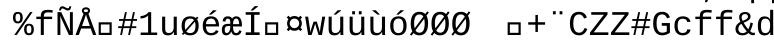




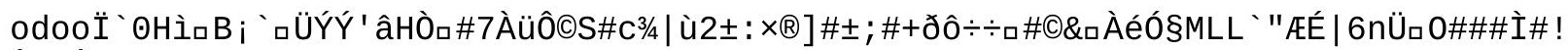
Ûsç⿳亠丷厂=sæ 


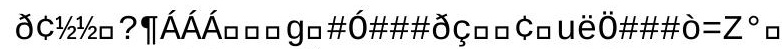

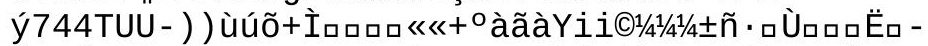

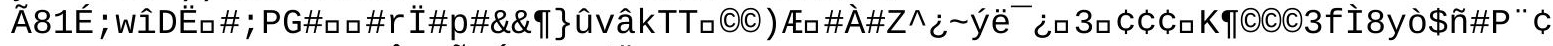

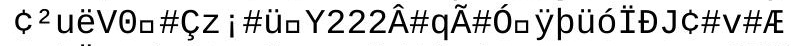

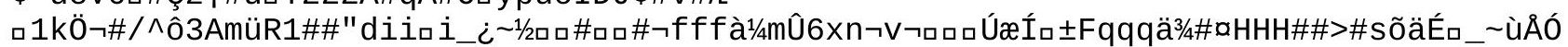

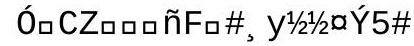

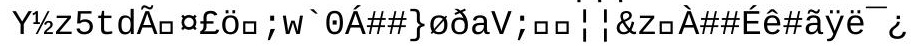




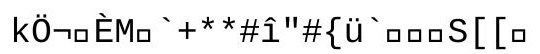

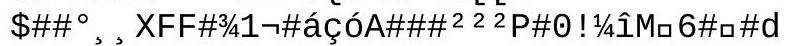




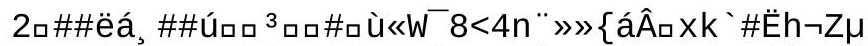

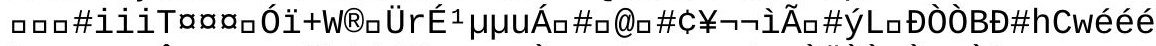

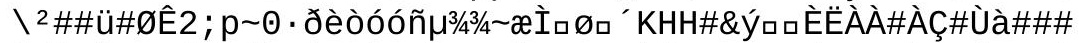

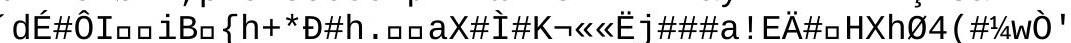

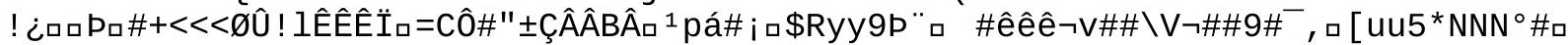

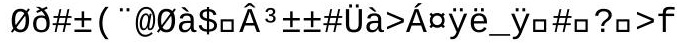




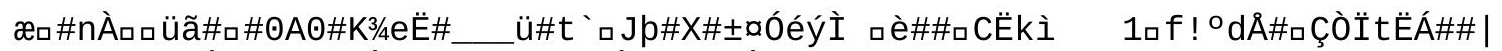

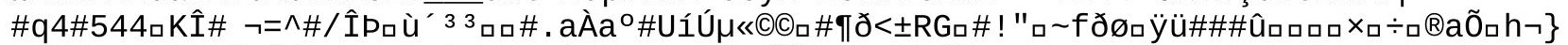

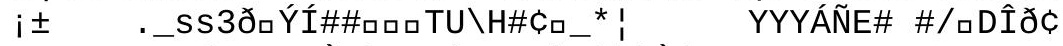

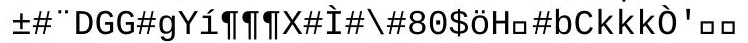

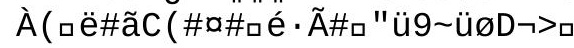




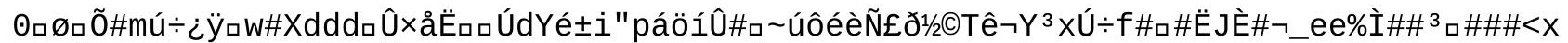

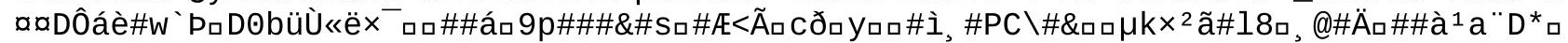
$\% \# \varnothing$

C(c) * *ê̈ÛÒäàÂcE $X i ́ \# \# \# \pm \pm \pm X \#$

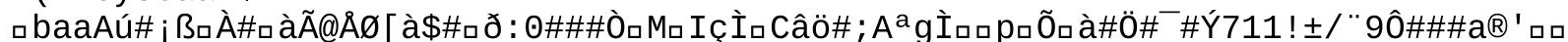

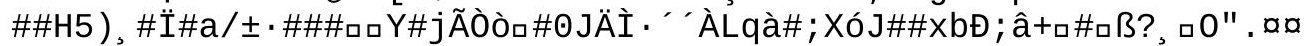

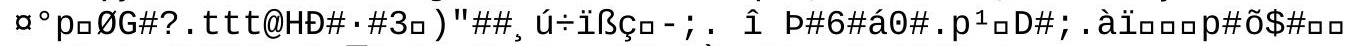

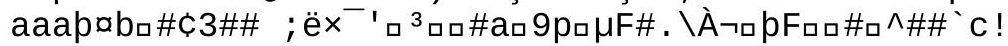




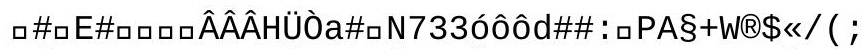

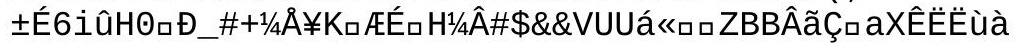

$\square \#>$ PRR\# | \{0๑-

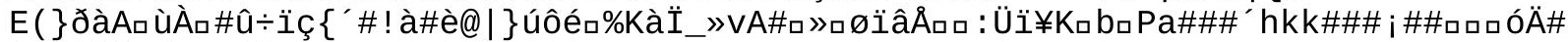

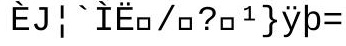


ฉロ०\# 
\#8khh ${ }^{\circ} /$ ú! a\&á1/2\#uGGGbbっ\#»wï口ZIIIÁ; MNN\#

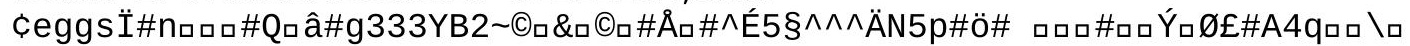




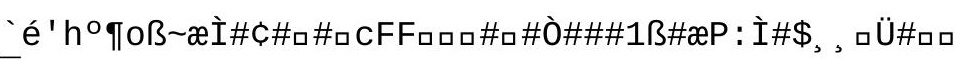




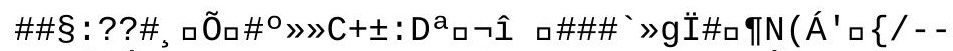

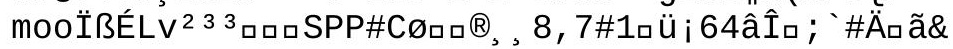




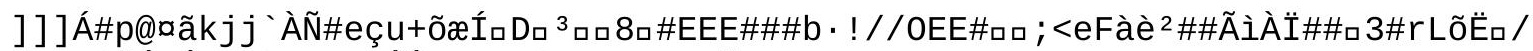

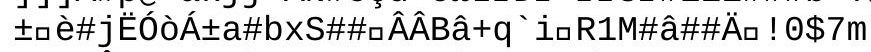

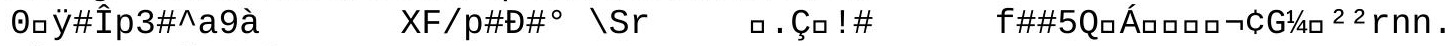

口\}\#\#\#BÆ\#[\#£JîaDFF\#

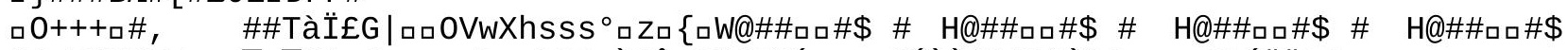

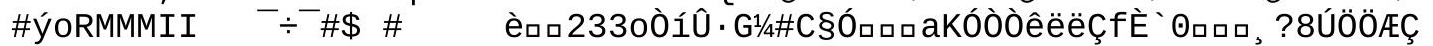

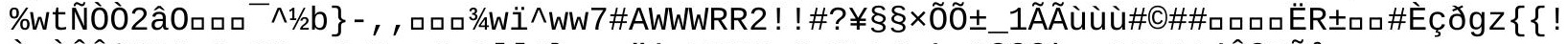

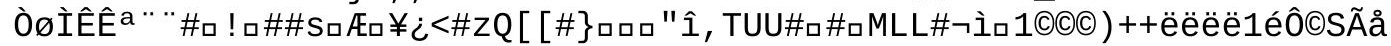

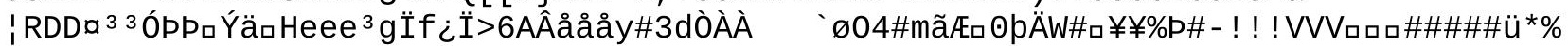
\%Е i РTTTX ¿уó': 


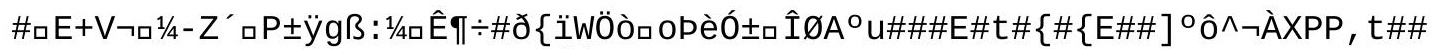

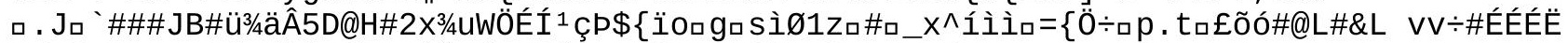

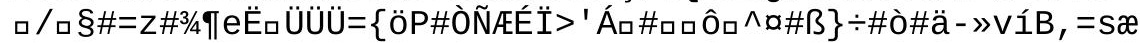




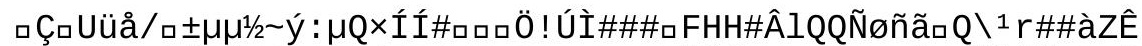

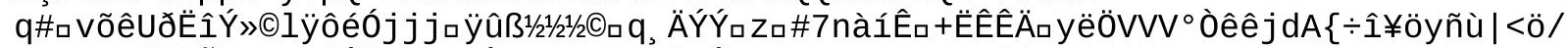

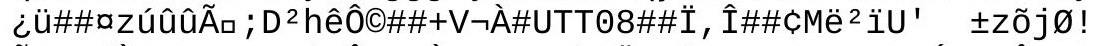

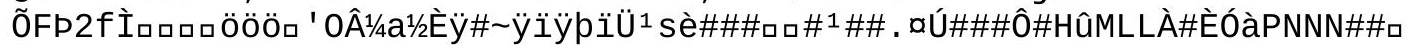




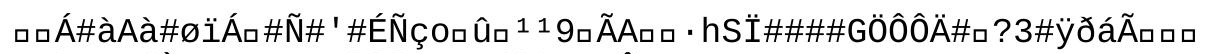

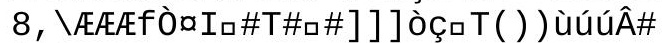

âââ\&0ロ 


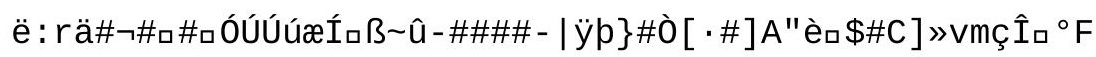


âéé@§§ロ\# 


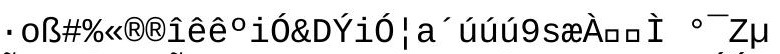

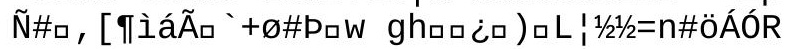

$\# \mid 9$ 
: Àô@1̂ิxr tF\#2çÇ\#ロÄÓâ 


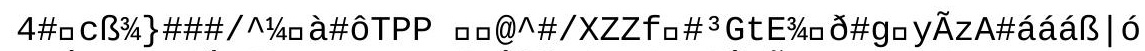

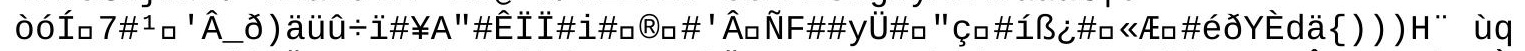

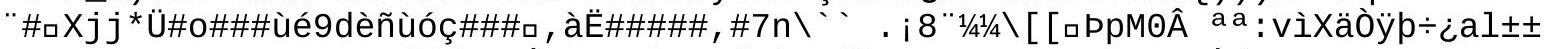

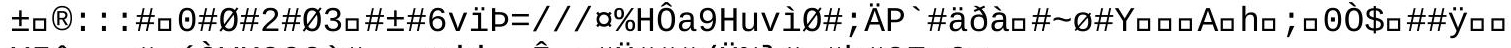

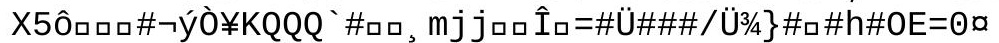

口 “ @\#口A \#6 


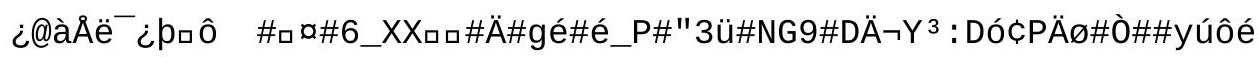




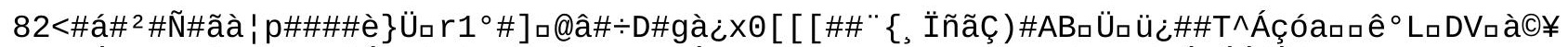

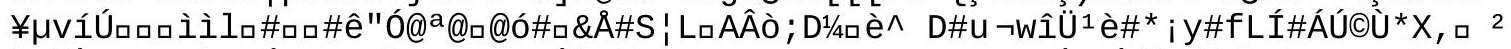

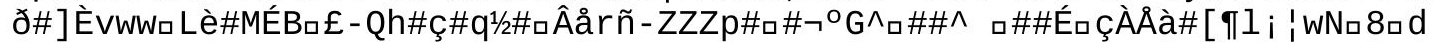


Ù\#ü\#ロ\#Í\#aaaHøè\#zänë× ${ }^{-}$Sロ\#\#\#CUèàAô\#\#" 


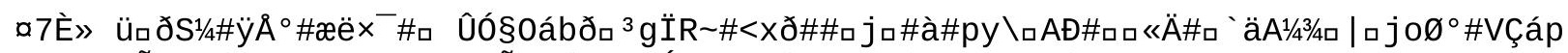

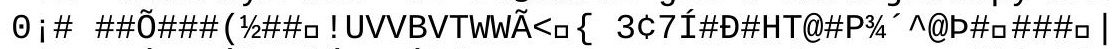

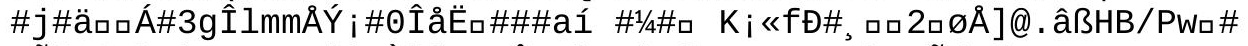

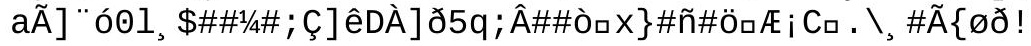


\#םロם1/4ר ר 


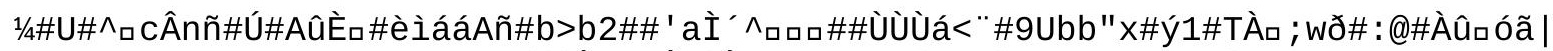

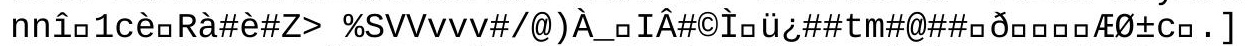

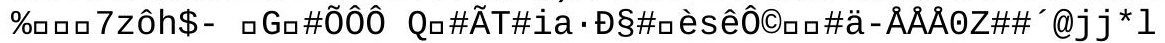




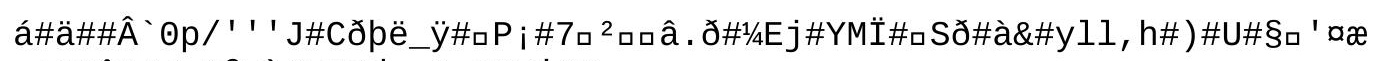
ฉ:D\#ê\#\#<ロA@Uà\#z\#ad a\#ロr\#5j\#\# 
‘n\#\#\#ѐollüÿEı\#\#\}\#æ

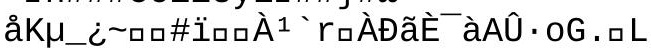




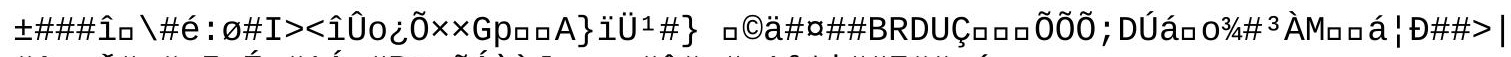

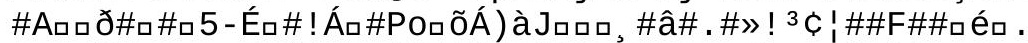

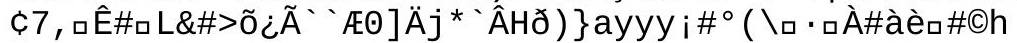




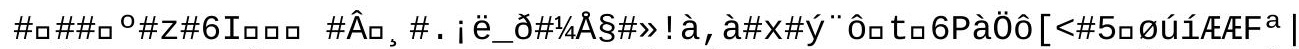

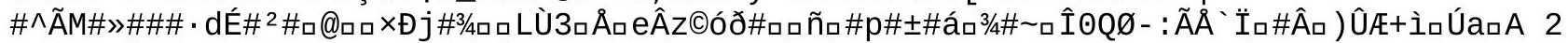

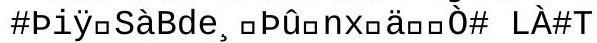

๑»P. ฉчÁ\#áÁð 
$\mathrm{H} \ll, \wedge 1 / 4 \mathrm{Hp} \backslash \# 9 \#$; \#e !

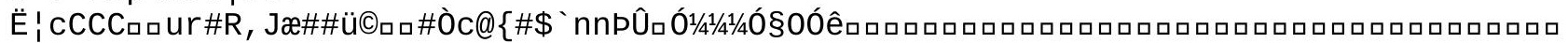

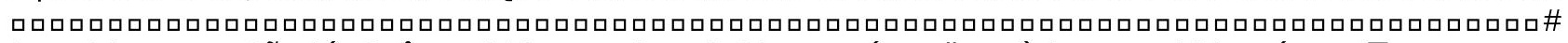

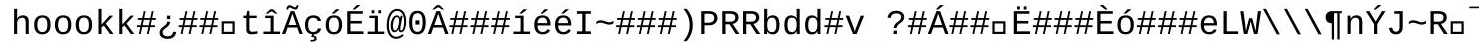

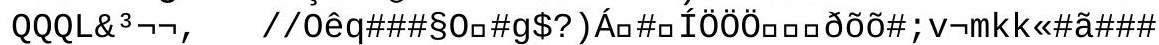

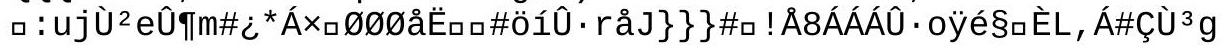





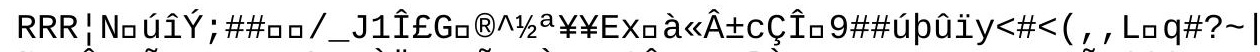

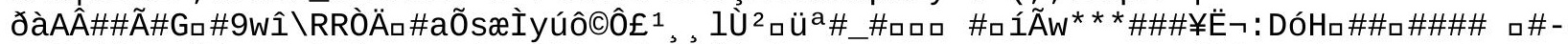

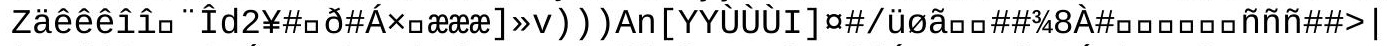

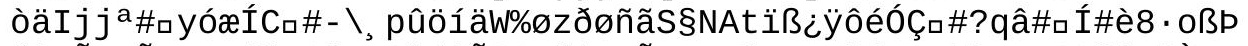

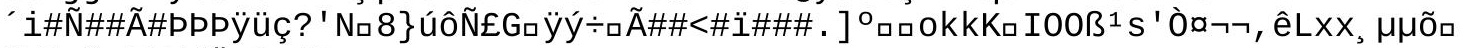

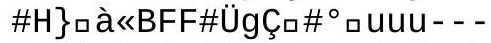

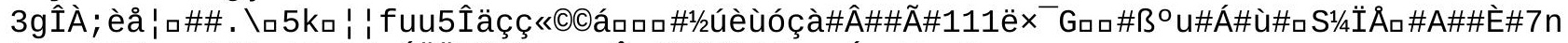

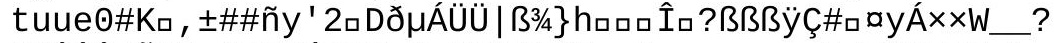

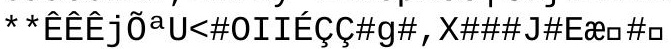

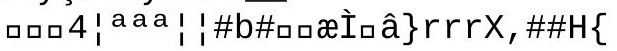






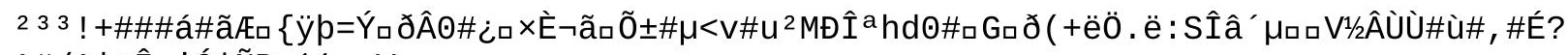
$\wedge \# / \wedge\left|c ̧ \hat{I} \_i O ́\right| \tilde{N} B$ 11 1 aV

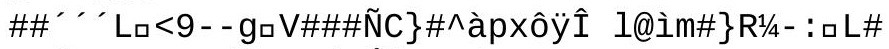

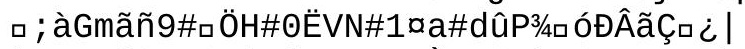

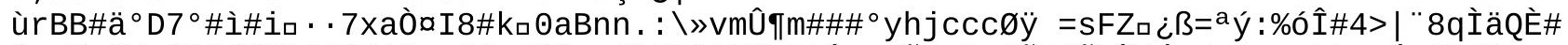

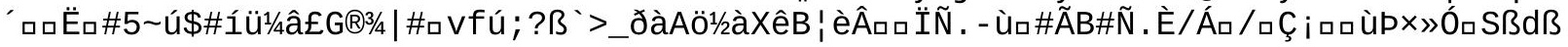




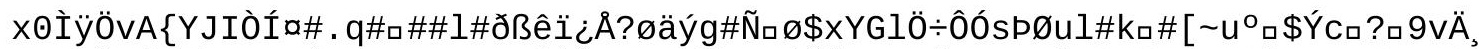

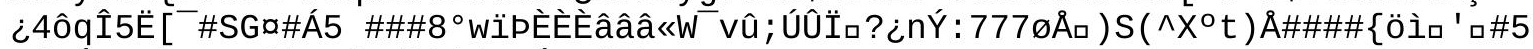

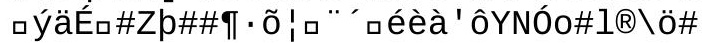


L/cTa?gåã\#\$qae\%ûøÑøÝpq] $\delta_{\square} \mu$

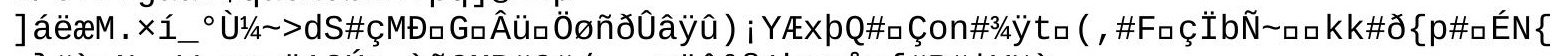

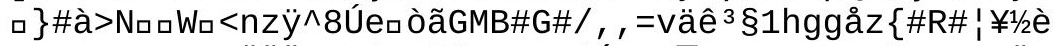

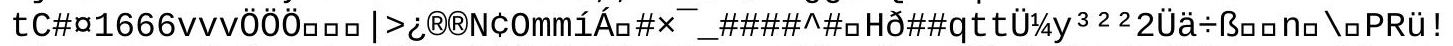

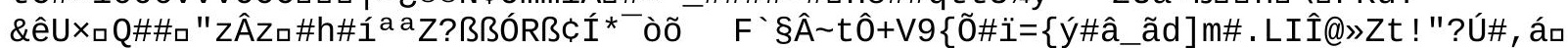

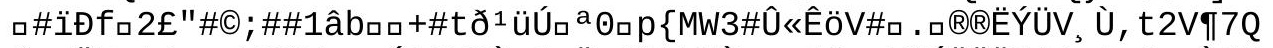

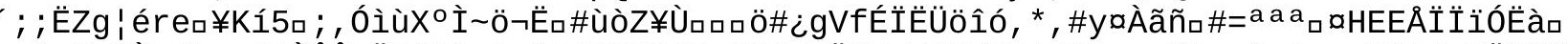

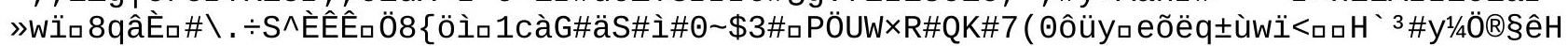

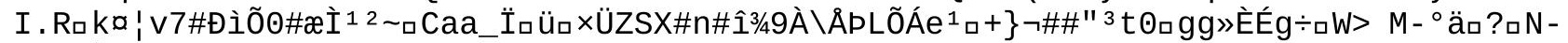
U\#íİH\#F 


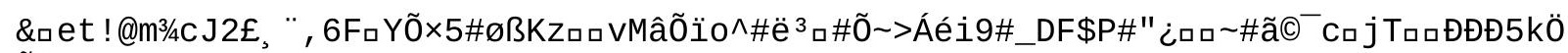
$\tilde{o} ß^{\circ} u+\wedge u t t$

>\%\#ha4ロóPóH \#j\# Bc(çロðã¹/4íl̂ãry² 


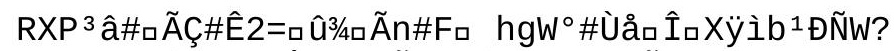

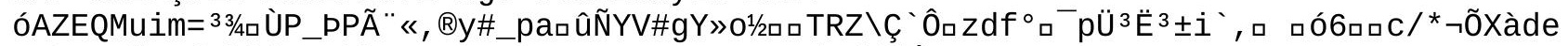

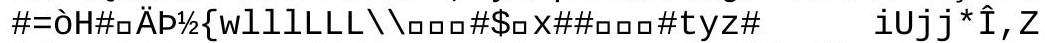

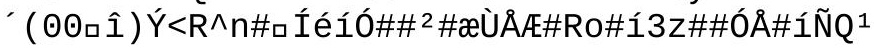

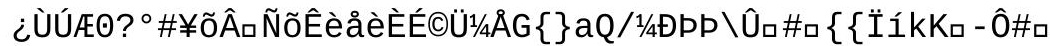

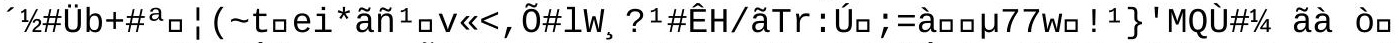

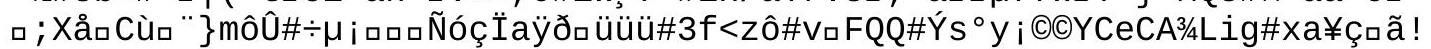

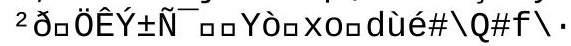




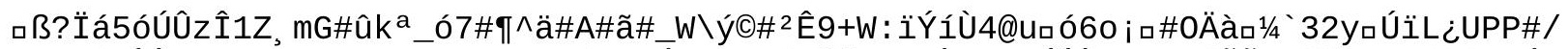

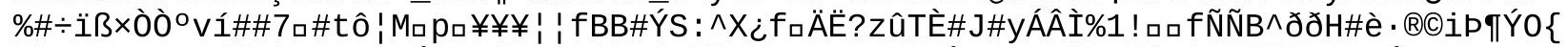

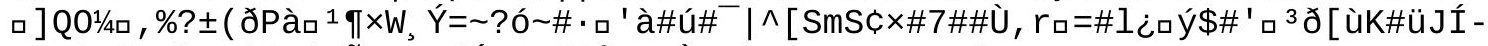

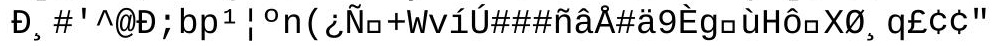


ロ2Úı/_* 


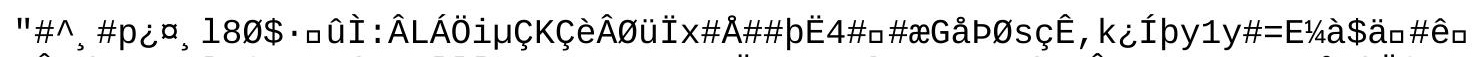

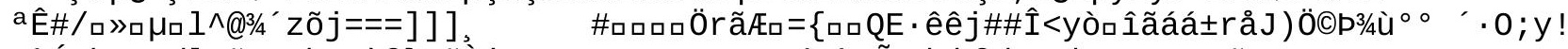

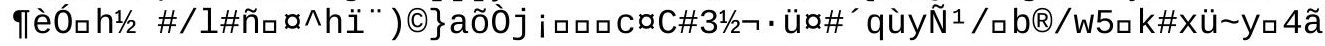




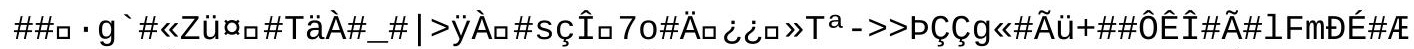

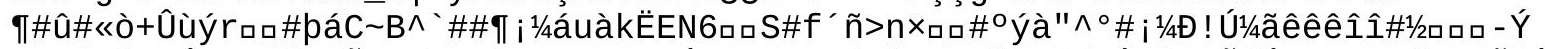

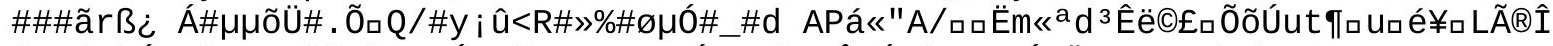
'2 2 áöòúquò\#\#《i $\left.{ }^{\circledR \circledR 1}\right\}$ \}

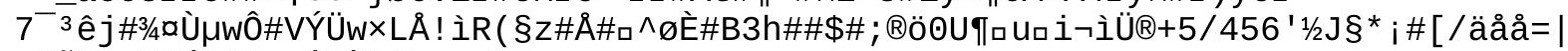
øðÑEGX¥á́RC ${ }^{\circ}$ yaô7Ù\} (høya\}» 


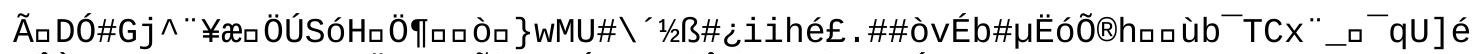

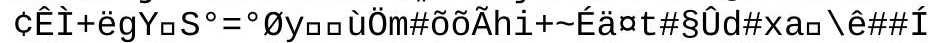

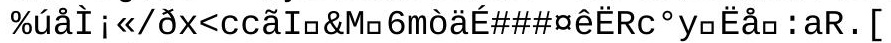
$\# 1 / 4 \mathrm{p}_{3} ; / \mathrm{P}^{`} 1 / 2 \mathrm{C} \# \#$ 


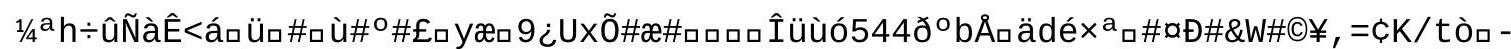

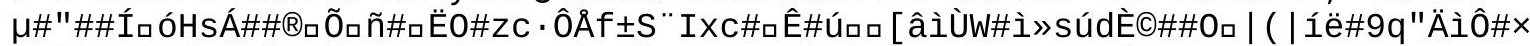

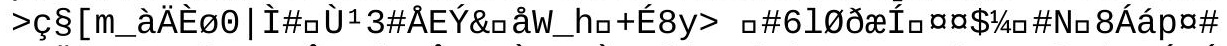

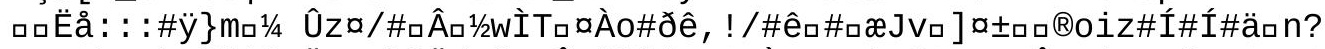

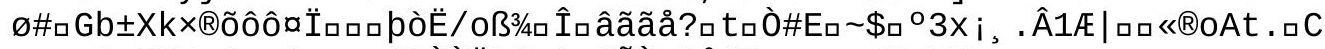

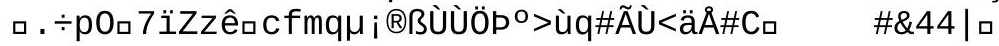


口AÎ॰ $3 c ̧ \mathrm{Ù}^{3} g R$ 
upüù\# øaôè̃̃ ; wî\#æßZ . zá\#C³/4" JH

$\mathrm{a}^{-}<$ãaû\#Ôa^è 
$P-) \div O ̀, T a k \#^{3} a ̂$

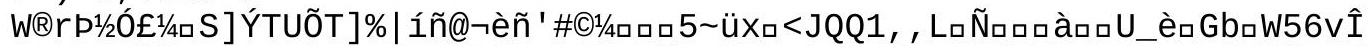

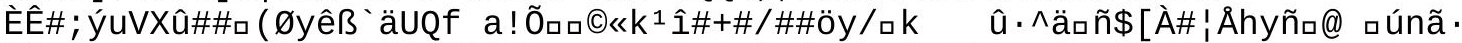

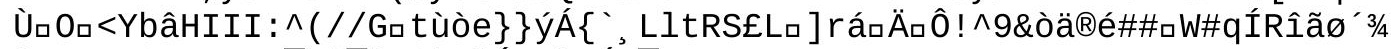

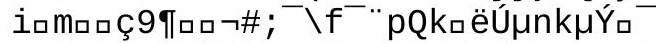

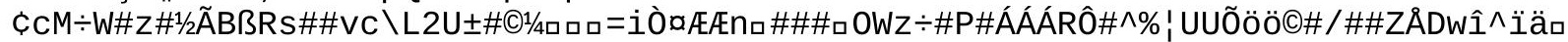

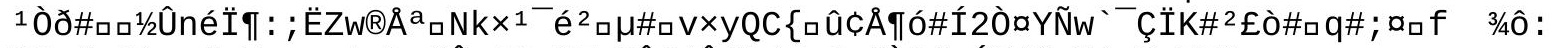

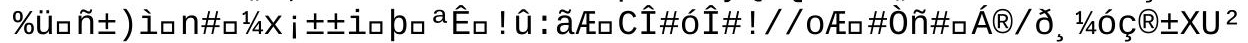




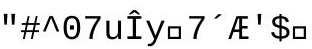

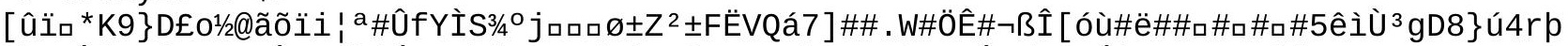

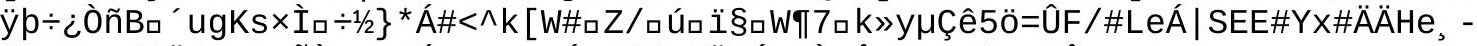

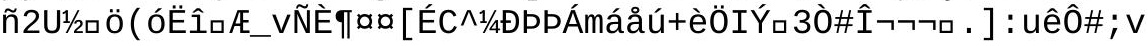




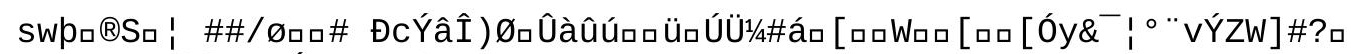

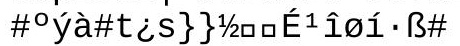




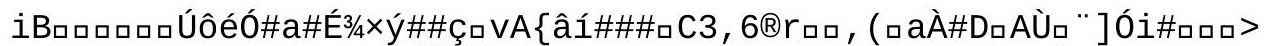

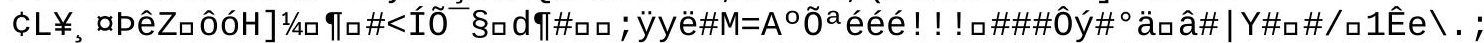

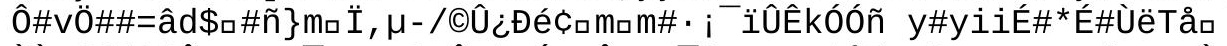

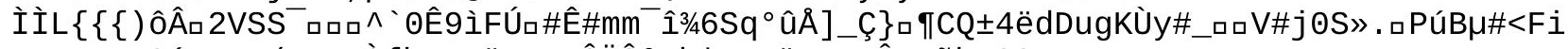

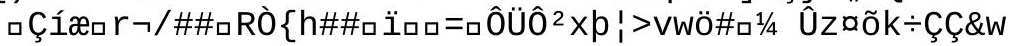


\#Ú(̂)»ö)Ø\#k

M<Ùıä $\mu$

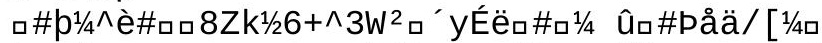

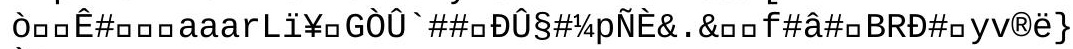

ò?

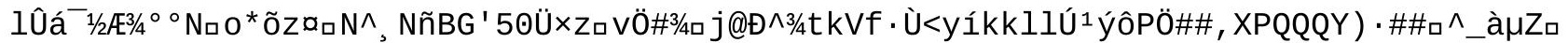

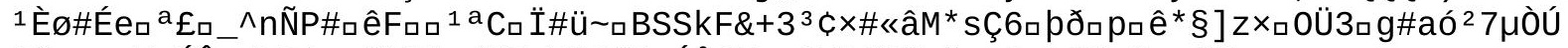

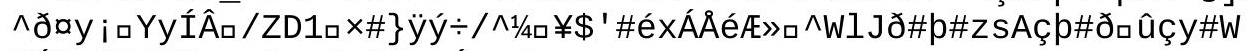

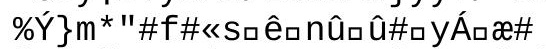

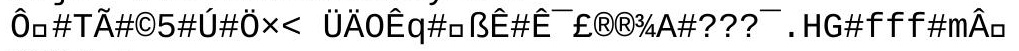

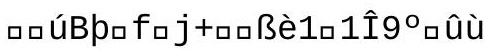
\%@õõ - G 


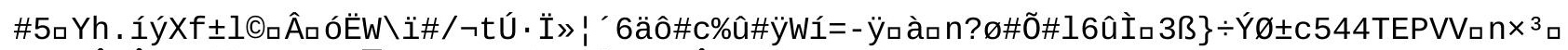

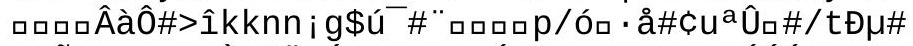

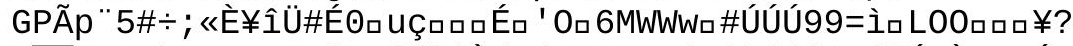

a

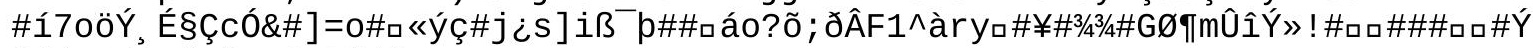

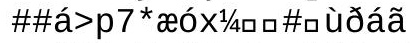

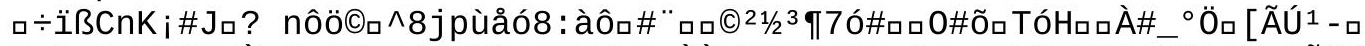

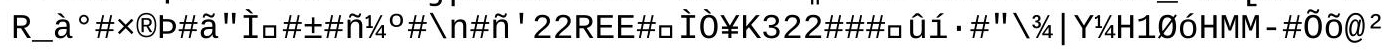




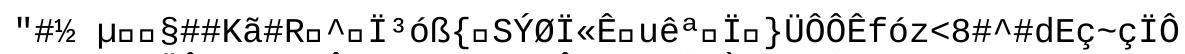

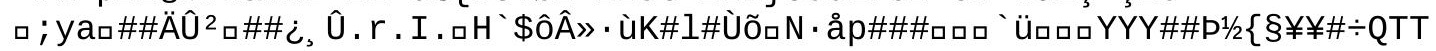




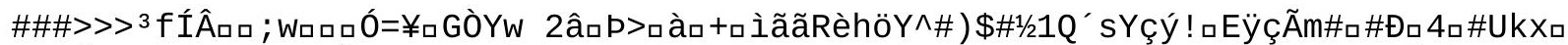

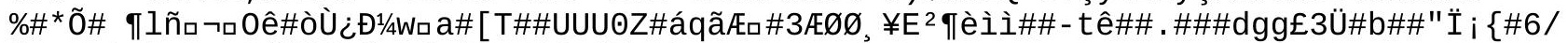
ๆ

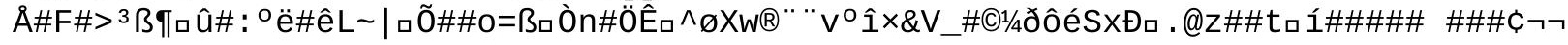




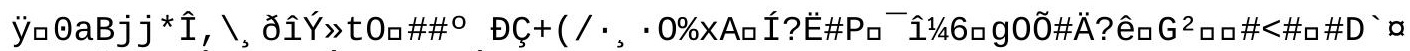

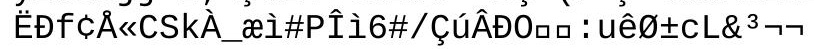




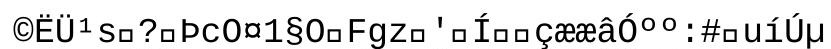

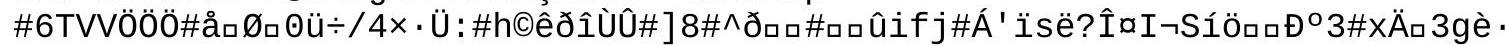

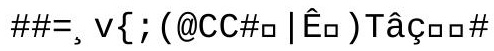

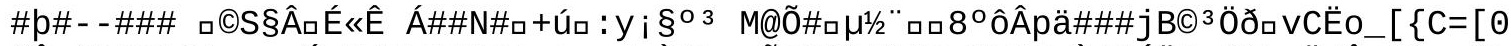

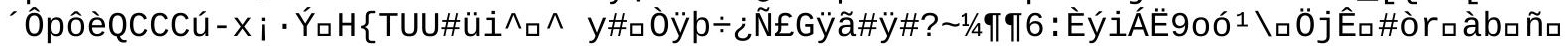

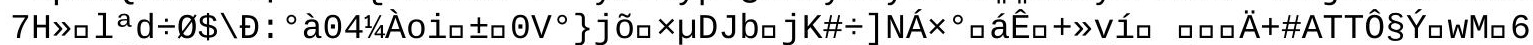

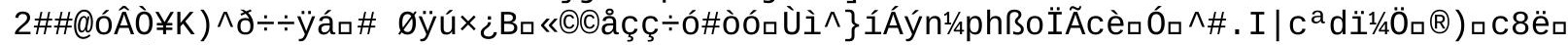

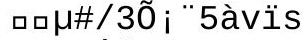

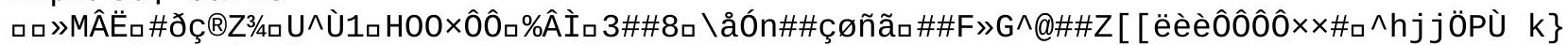

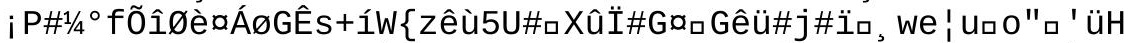

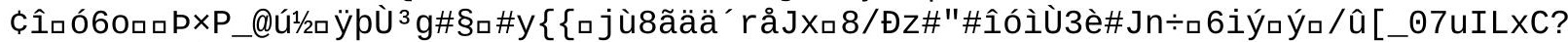
UL0 $1 / 4$ ĐÆ\#

në\}@

=\#ûQ/|aý\#ロá\#

Ôロ “ $w \times\left[\mu 5 \tilde{0}^{2}\right.$ 


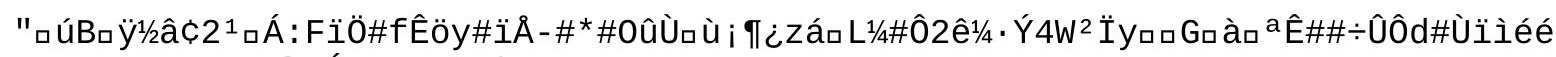
¿`仓\#ロÖT\}áÍ_7p¥\#\#\#|Z_\#è 


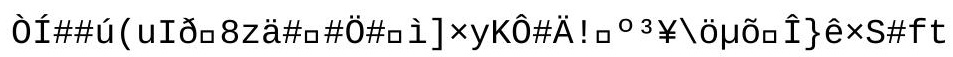


Úİ6pV/ロ»\#Ýם 


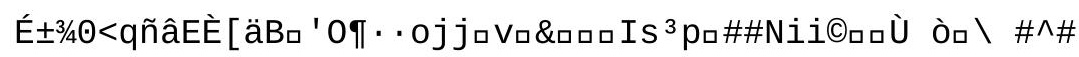




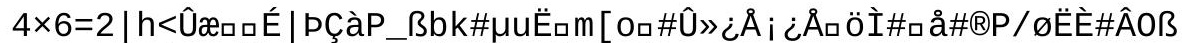

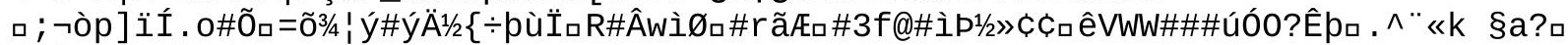
$\mathrm{G} \# ð \# \square \mathrm{W}^{\wedge} \# \mathrm{k}$

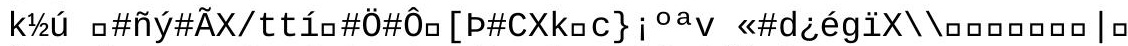

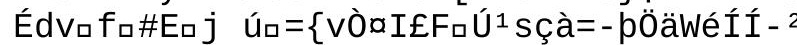




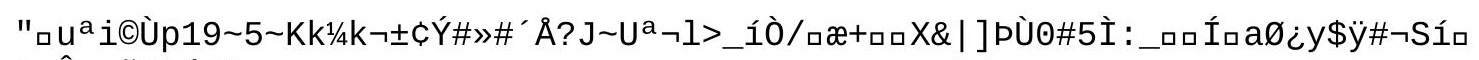
$\#$ \#僤<ãM\#í\#\#ロ

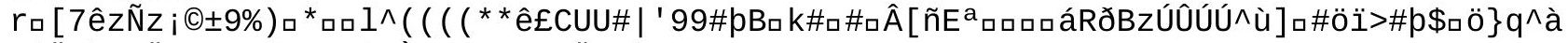

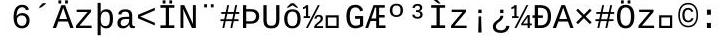




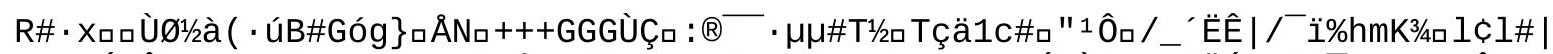

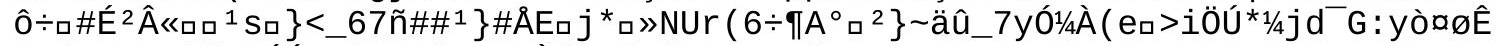

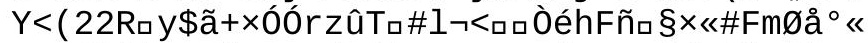

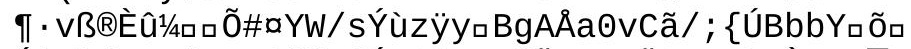

Ó' Mkda<\#(

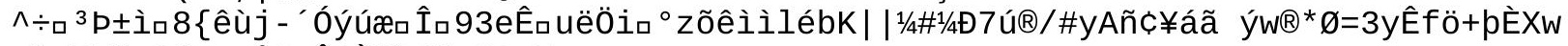
口\}=RE\#ロÇã $\div p \AA \AA \wedge » \hat{I} \mid$ Ėüà£ãm@\& $\neg 5$ 


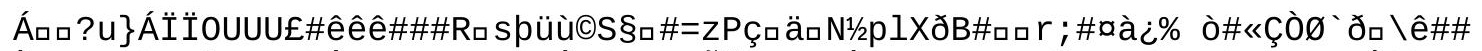

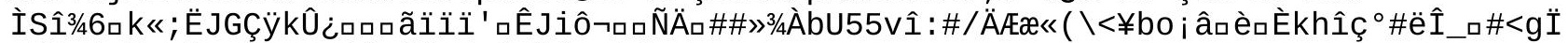

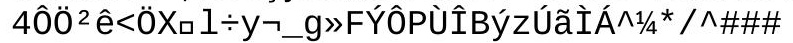




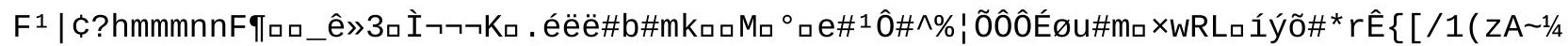

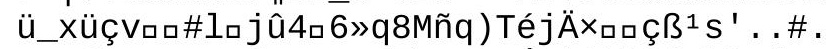

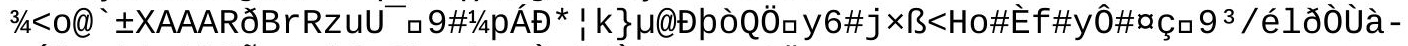

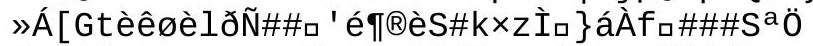

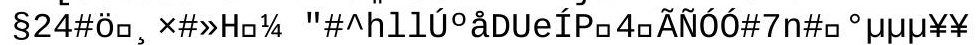

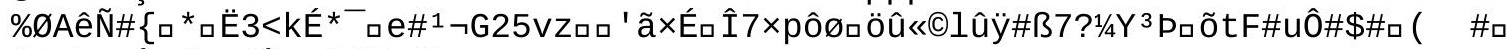

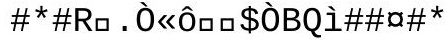

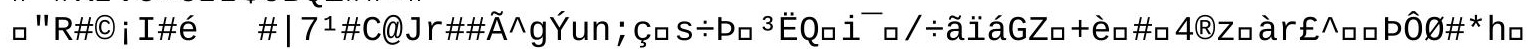

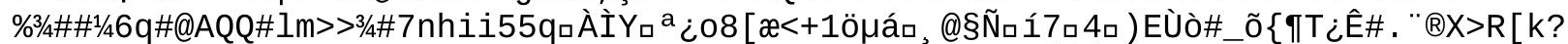

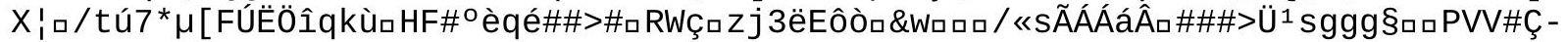
ñ\#\#P i i $\backslash H \backslash Y$ ? \#Õ \#\#口 (<Xö

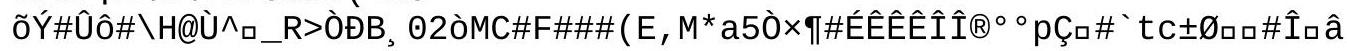


$\# \Re \S \# \AA ̊ \# \backsim K_{\square} £ \_C ̧ 80 ́ \# \hat{E}$

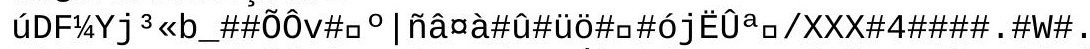

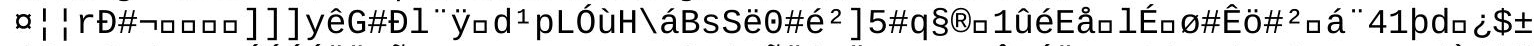

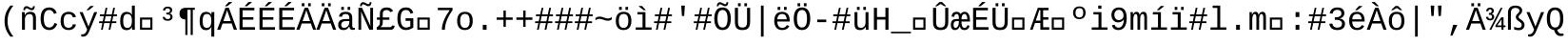




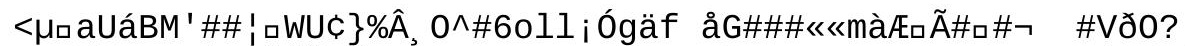

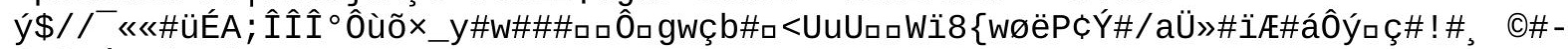
C\#ßRÚ\#\#ãa ${ }^{1} \mid$ Pö 
mxr\#ÕÚı/ 


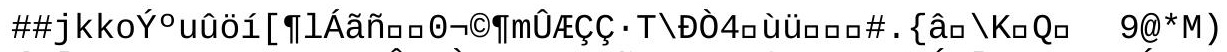

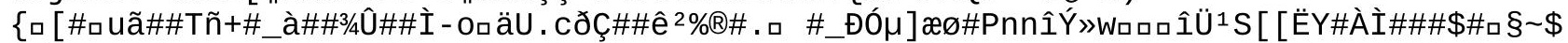
@d_\#2ロS॰J|! 
\#WUYÇÉDı〜Ë

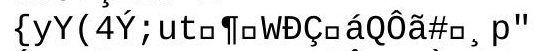

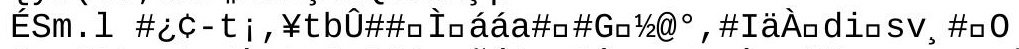

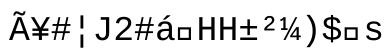

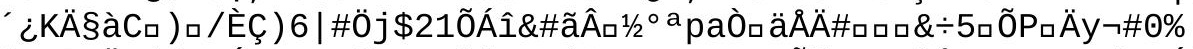

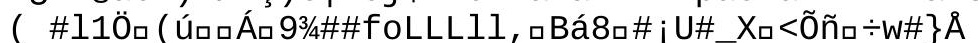

ú7\#Úú\#ù\#0â\#ロÇ\#\#Eûa $\{q \# x$ é\}\}

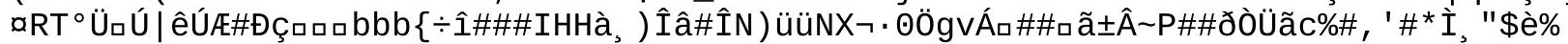

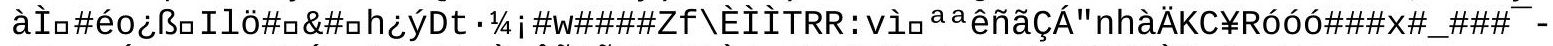

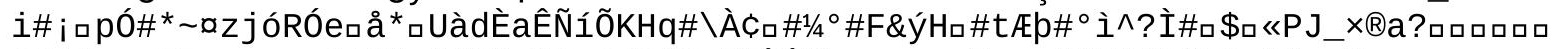

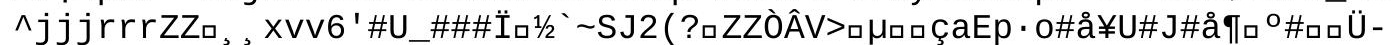

$+i^{1}\left\{\hat{u}^{1} / 2 \# \S \tilde{n} \# \$ \hat{i} \backslash C ̧ \# . D^{-}\right.$ 


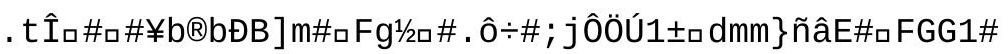


ðöìÇ\#\#\#\#<x\#æ 


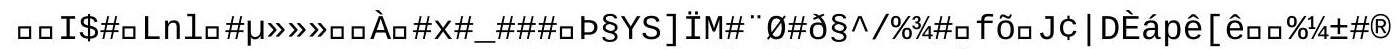

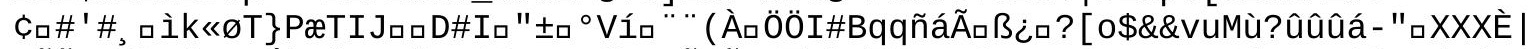

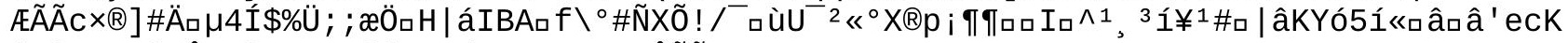

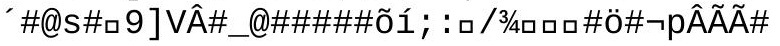






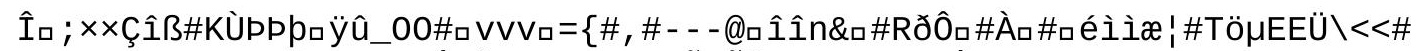

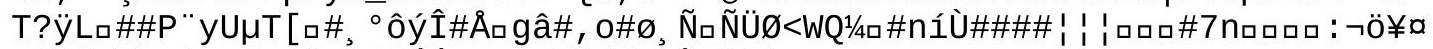

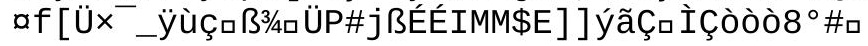


¿\#Hùwv 


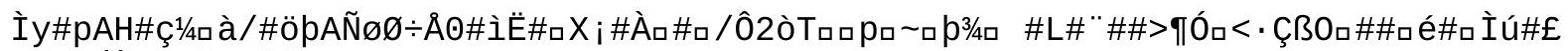
\$fo 


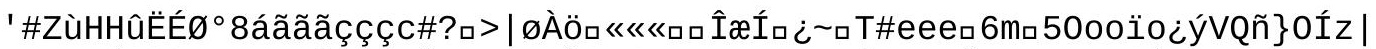

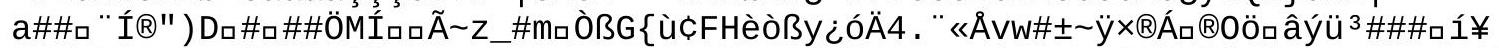

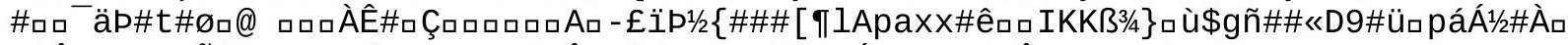

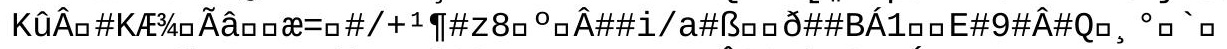

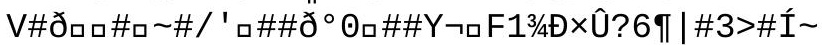




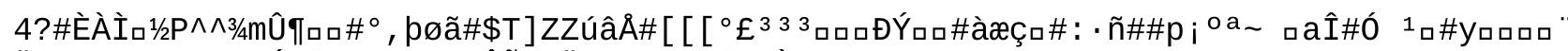

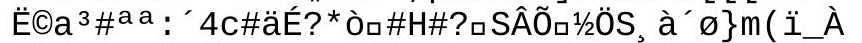

$\mathrm{y}^{*}($ _ $\mathrm{N}: \mid \odot \mathrm{dr \#}$

W\#IáıÄðÇ\#ః5\#£`Dı\#ャ\#í\#wt 


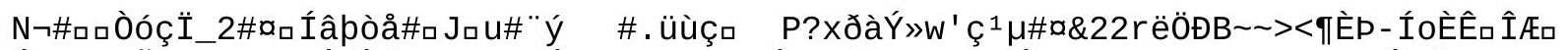

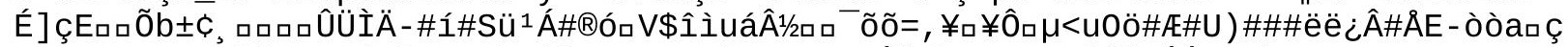

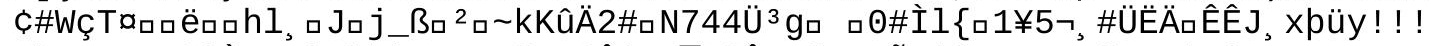

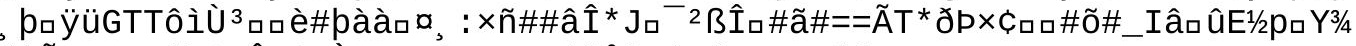

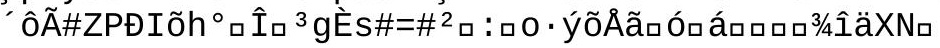




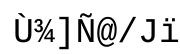




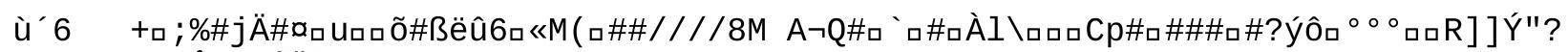
à̀ 


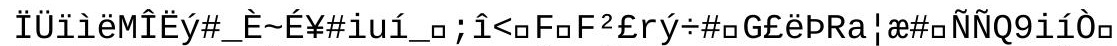

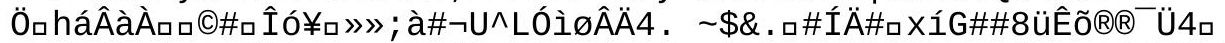

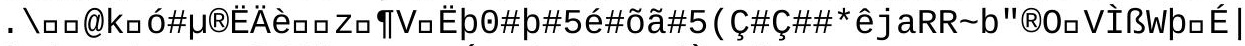

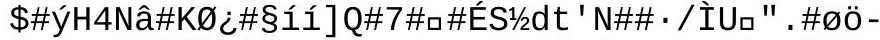

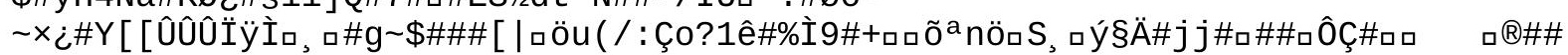




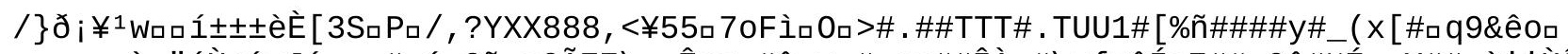

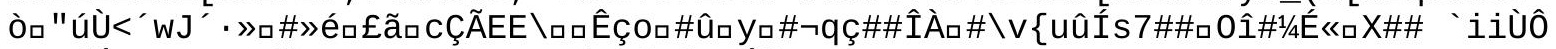

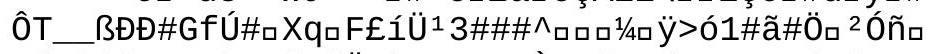

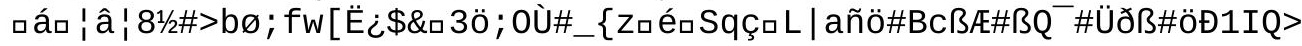




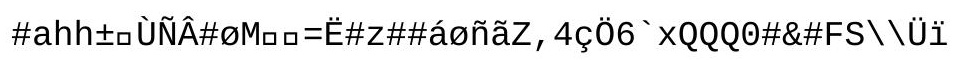


○Ví\#ÂA `Da¥¥\#Êèèèðîkar

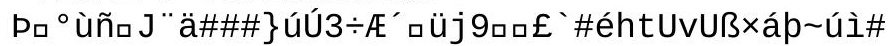




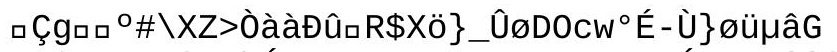

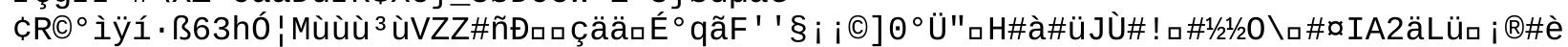




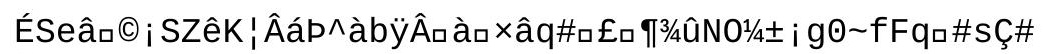
ß口 


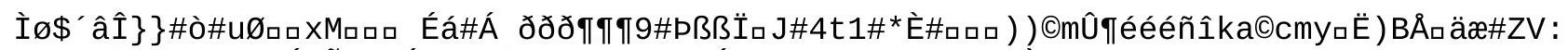

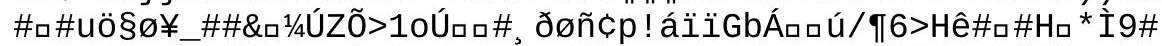

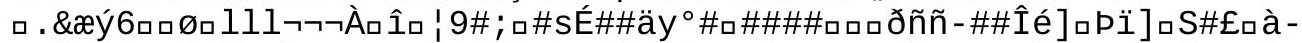

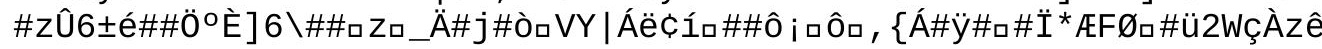




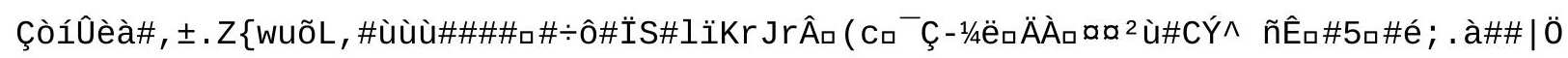




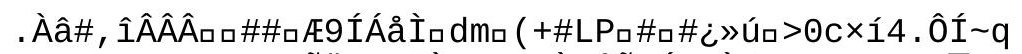

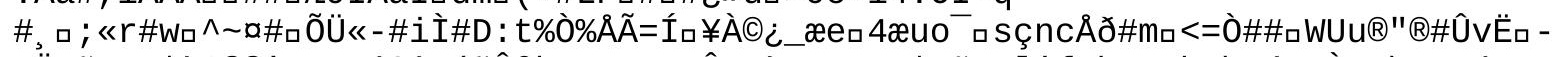

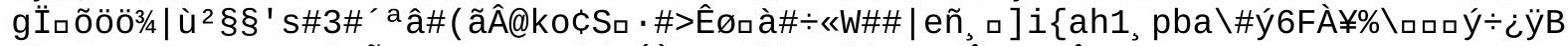

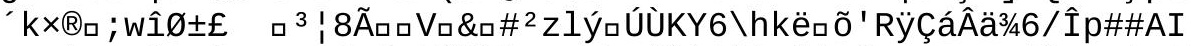

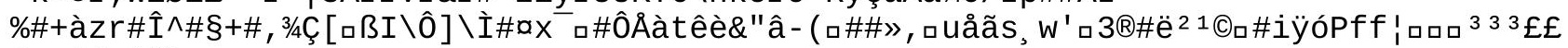

Łaロ\#\#g̈̈̈\ 


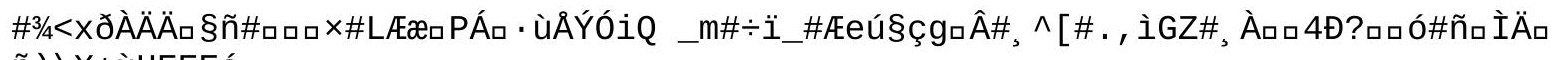
ñ ) \X+ùHEEEá 


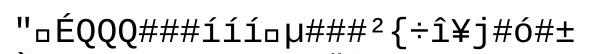

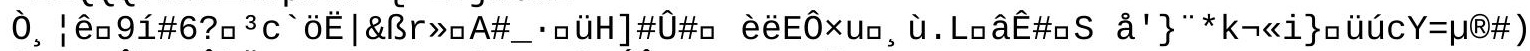

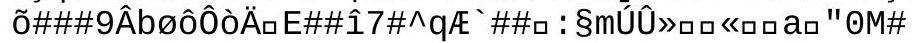




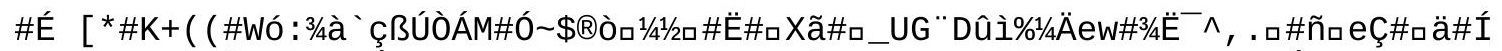

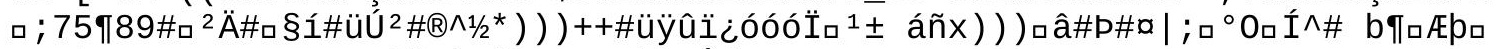

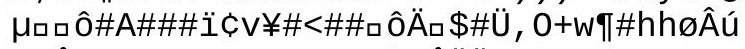

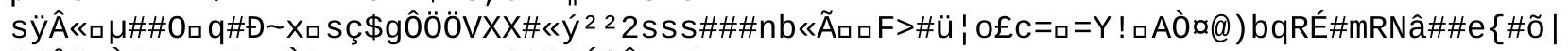

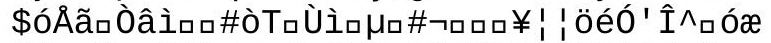

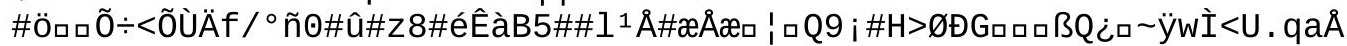

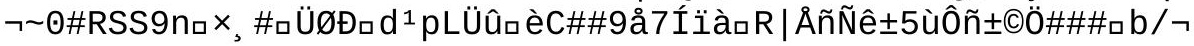




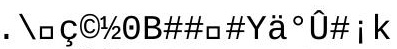

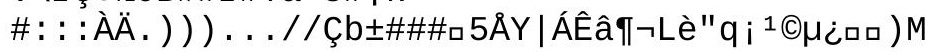




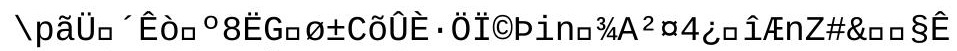

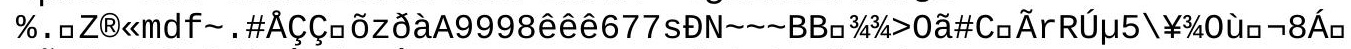

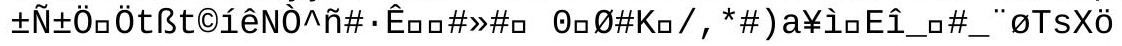




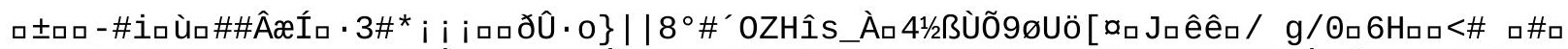

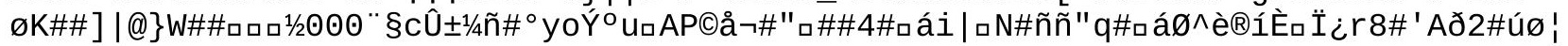




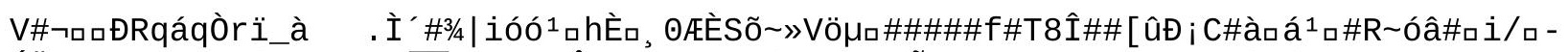

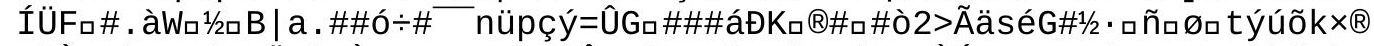

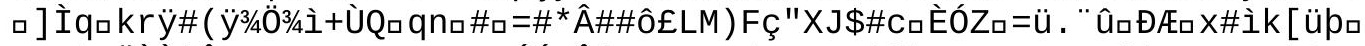

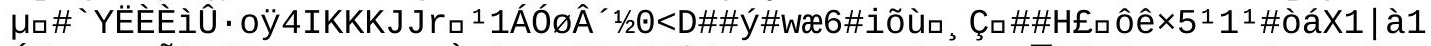

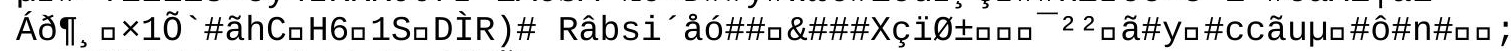

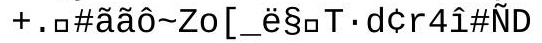




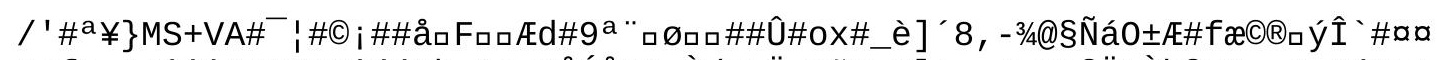

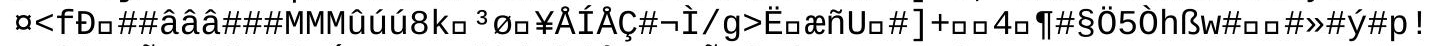

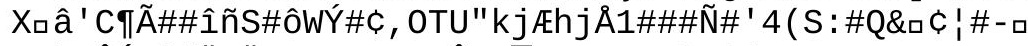

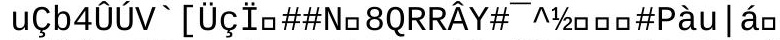

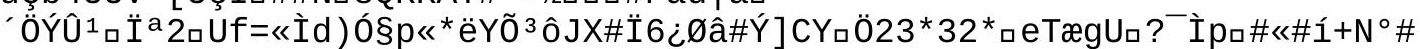


\pm 



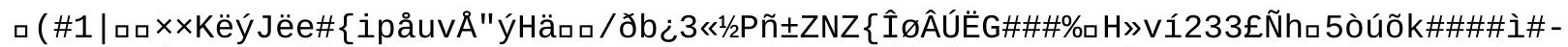

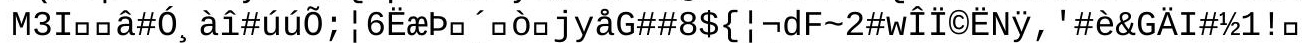

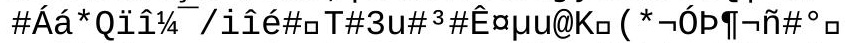

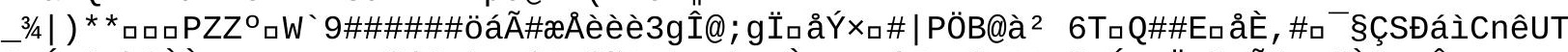

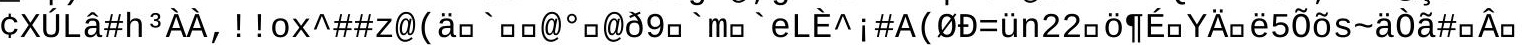

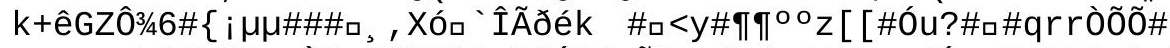

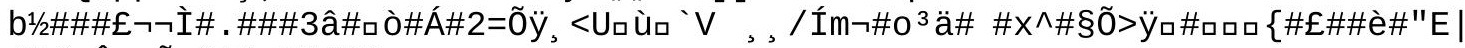

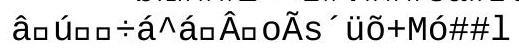

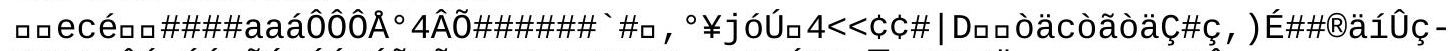

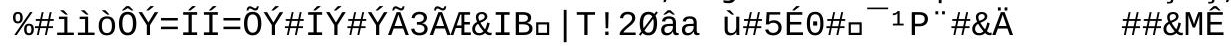




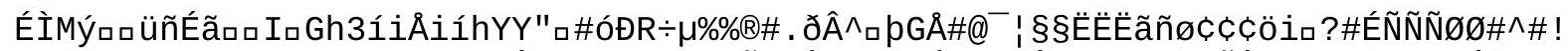

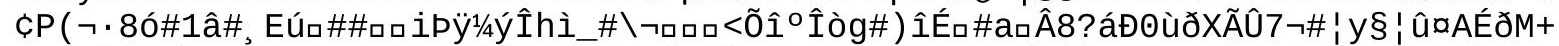

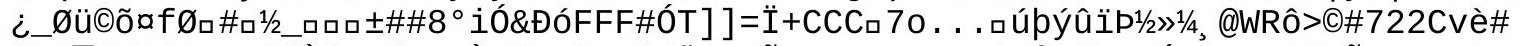

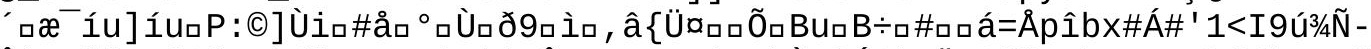

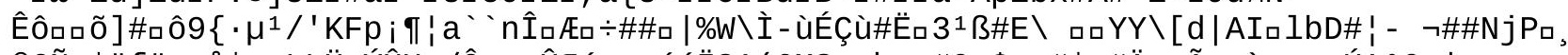

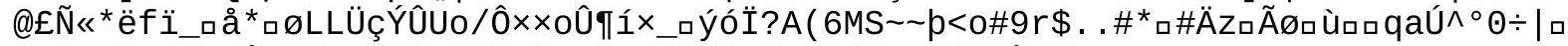

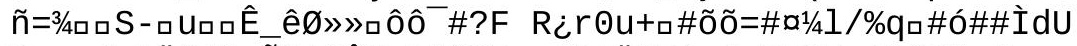

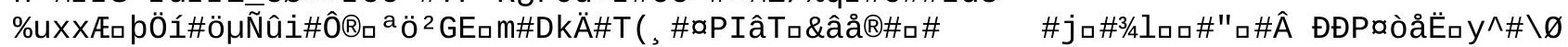

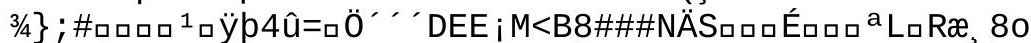

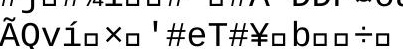

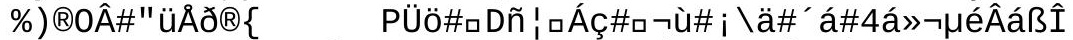

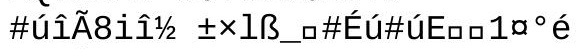

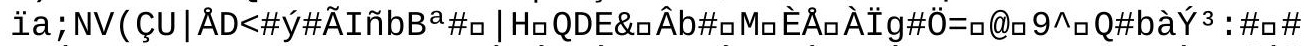

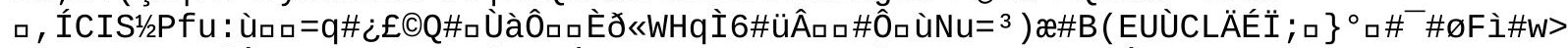

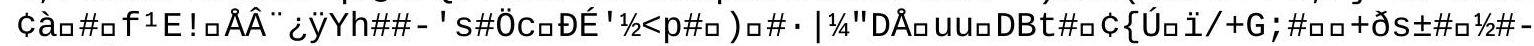

Ü»Óロ $\ddot{E}^{1} / 2 \square “ \# \square \emptyset m$ 
jロ \% ${ }^{3}$ ó

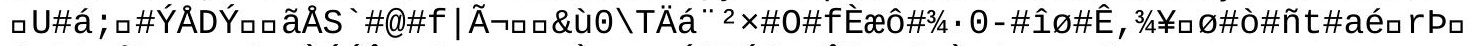

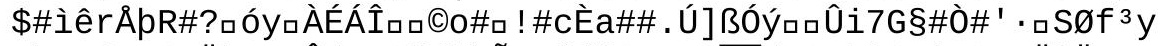

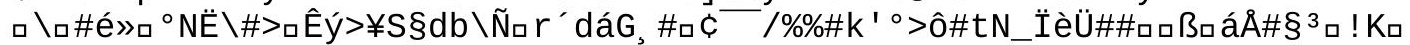

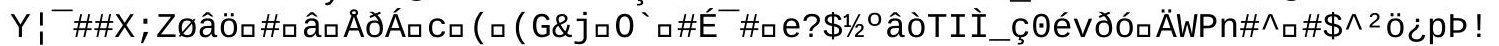

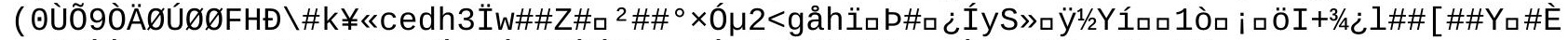

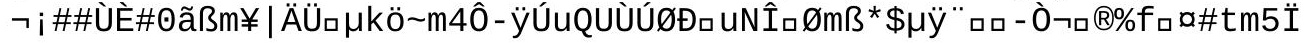




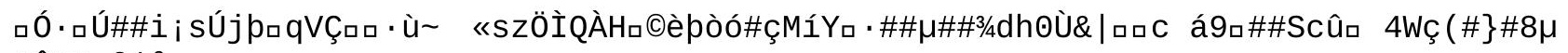
\pm ôuu $01^{2}$

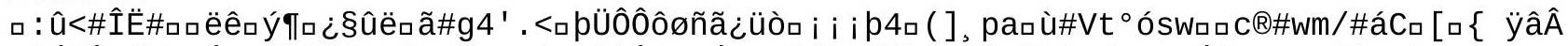

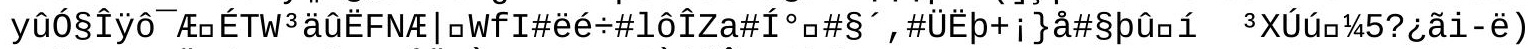

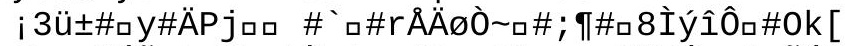

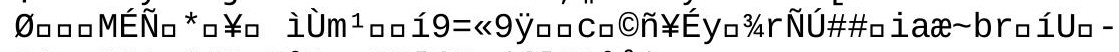

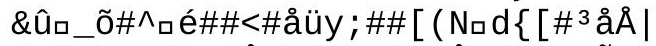

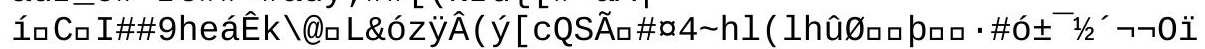

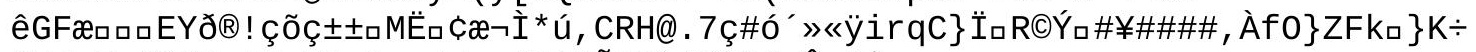

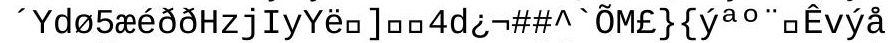

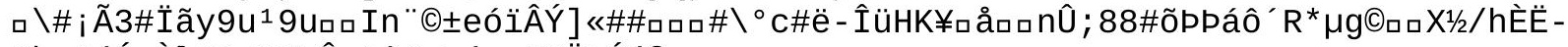

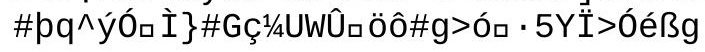




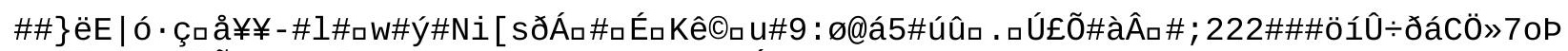

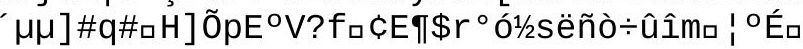




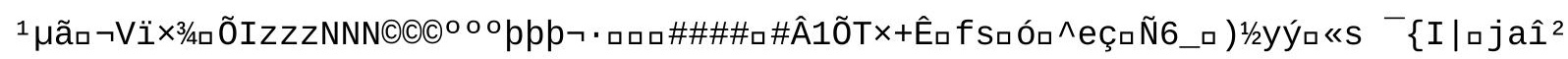




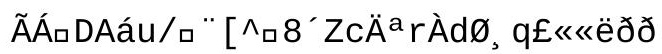




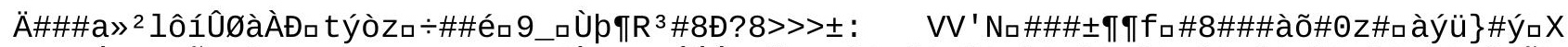

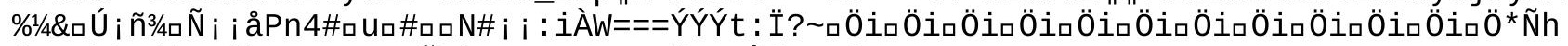

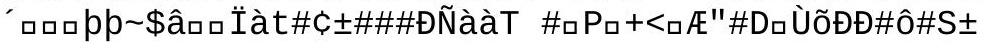


$\#><<$ 
\}1CüİïáõaÃ̈̈Ef\#9]ロÙı ) eÆgg $\div 8:$ :

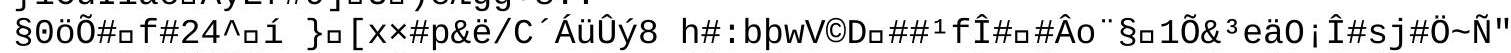

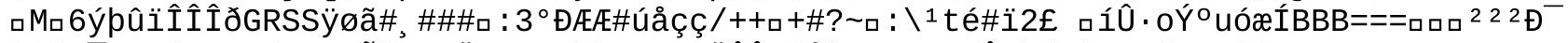

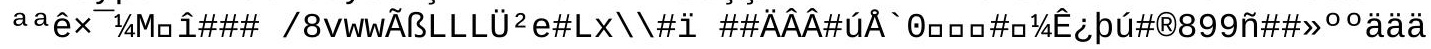

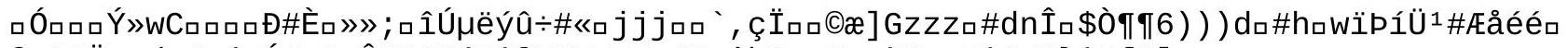

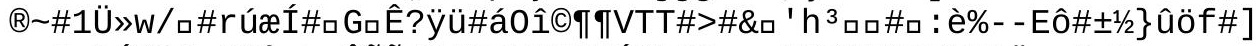

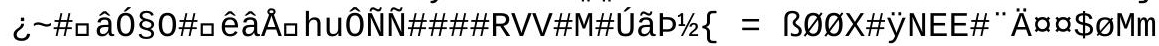


๑IËÏÏÿüùu( à

pqXLLquฉRuuõãçıUTTà

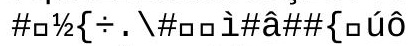

zÙ $\pm c C ̧$

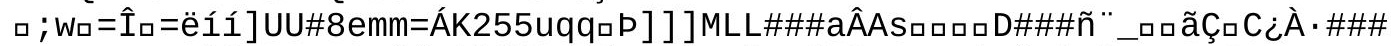

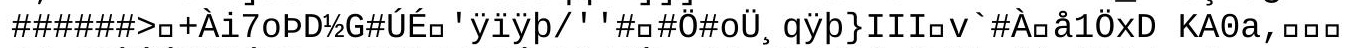

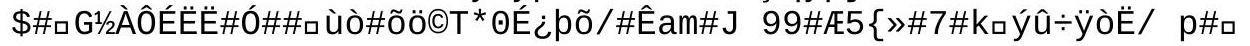




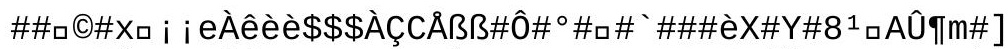

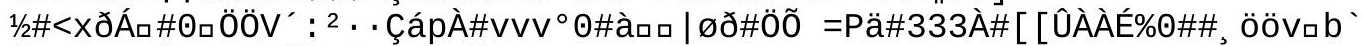

@ $\mathrm{p}_{\square} \# \emptyset \# g \hat{I}_{\square} \# \mathrm{t}_{\square} 1 \hat{A} \hat{E} \# \mathbb{R}_{\square \pm} \mathrm{è \#} \mathrm{N}$

口ÂSANI I\#İ\#ロ ${ }^{\circ} \eta \Uparrow v \times x 8 T .{ }^{3 / 4}$ 
\#ÂÓ \#ロ@\# \#G]]Ýqu\#ÀzXWםVa?[XXÈ£ AYÁlC\%+

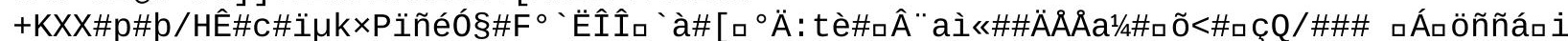

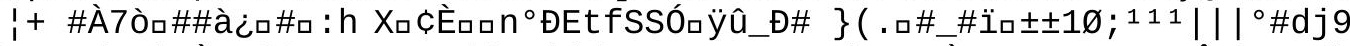

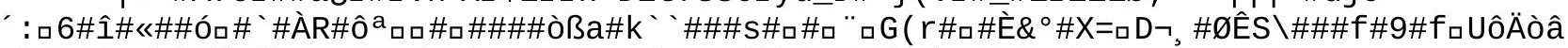

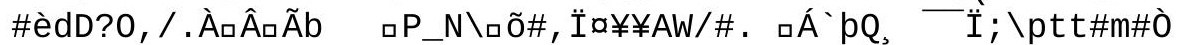

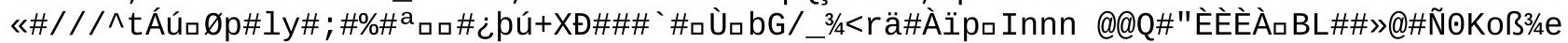

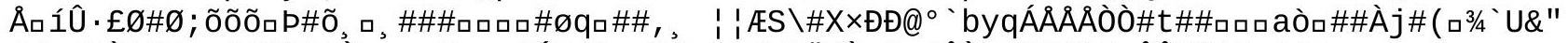

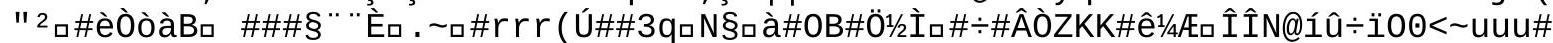




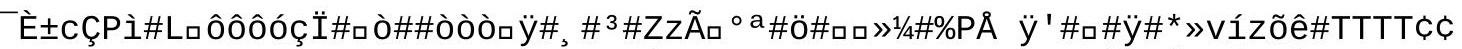

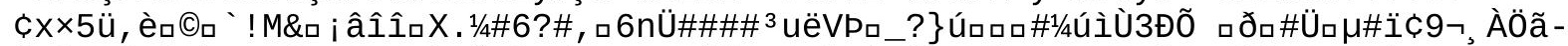

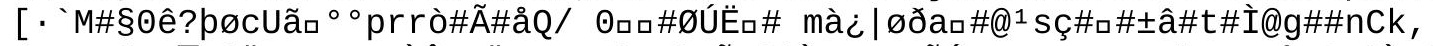

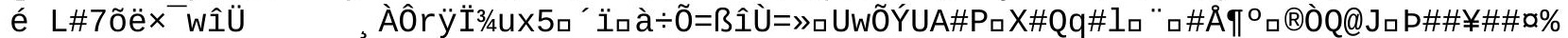

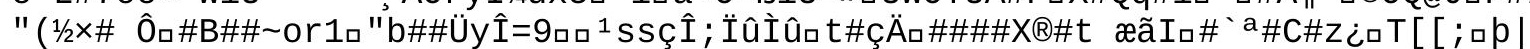

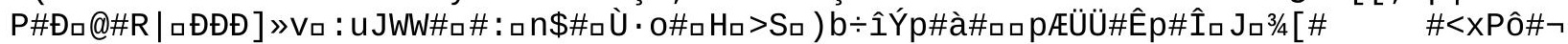




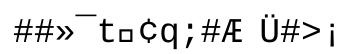


\ohh\#NæÉq ' AÂLG@Q4CÀK?\}ú4<, ¥¥\%๘Ç\#á\#B \{êéã\#`|

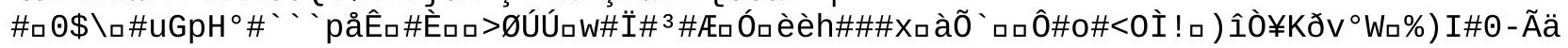


S4ロÁ

'X

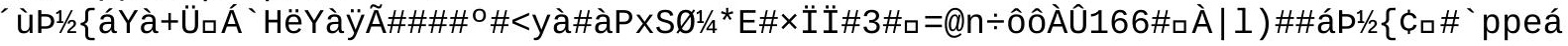


FLÓ

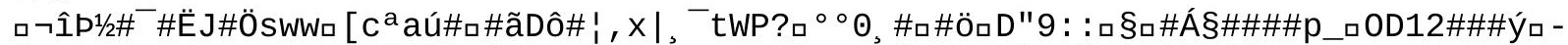

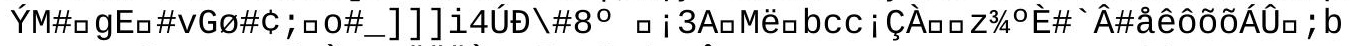

BıฉAøøñcQ : : p\#f\#ÒJR\#ÄÄÄÀnÇf3Lõ̃áC\#ÎH1G\#\#£\#\&.777x\#\#\#\#, x=**

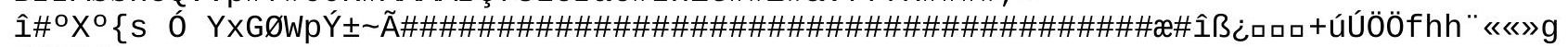
İ\#\#\#\#

口\#\#\# 


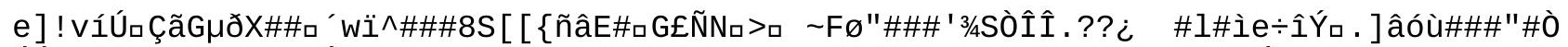

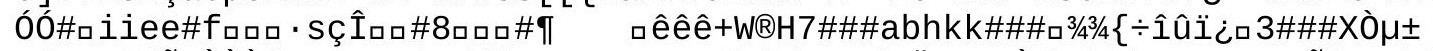

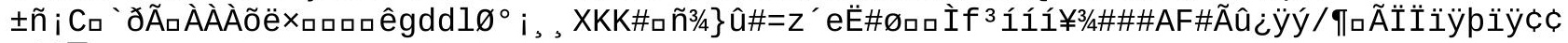
$\zeta^{`} \partial^{-} Z \mu$

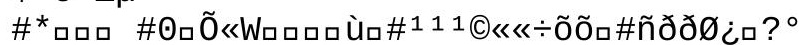

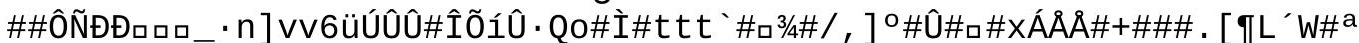

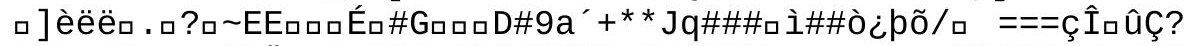

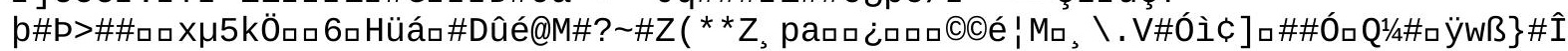
7MMMp\#| amÛđÝ\#\#Ô.è\#ロ

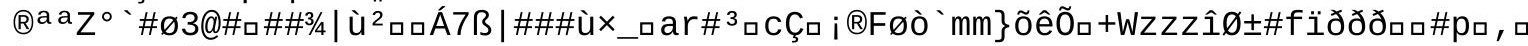

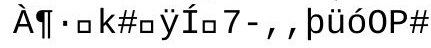




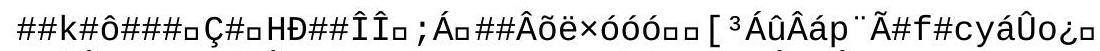

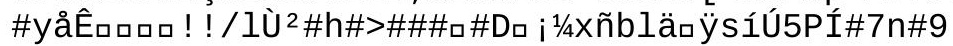




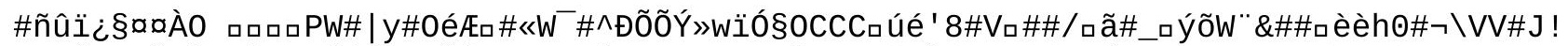

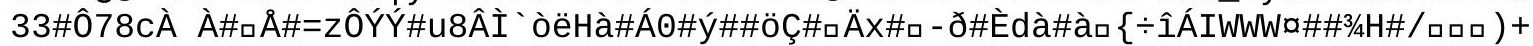

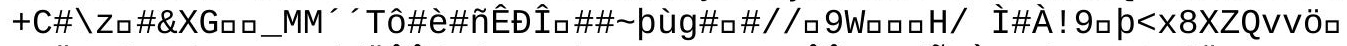

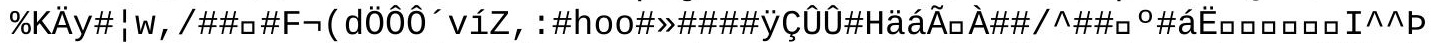

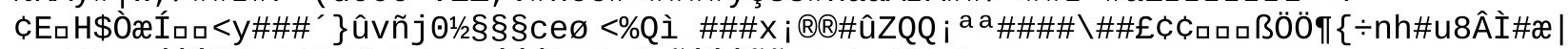

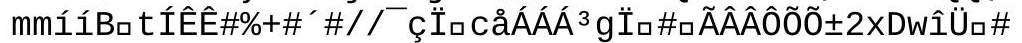




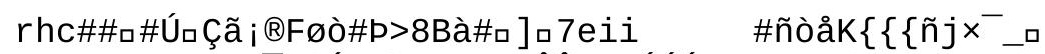

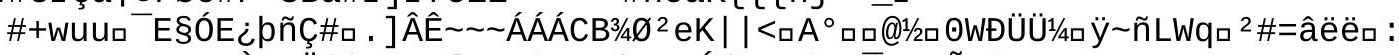

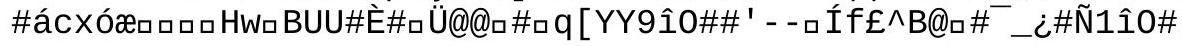

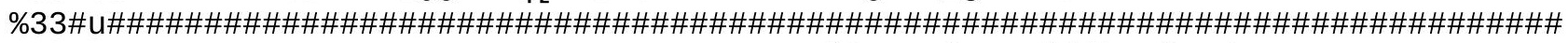

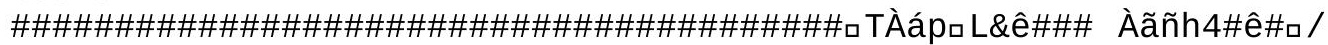


ฉロロ\#\#\#"\#\#p>\#\#ロÏÇ\# 

p10̈@4USSsòäIh

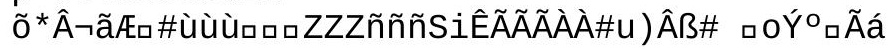

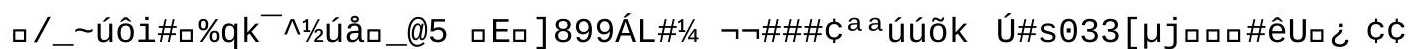

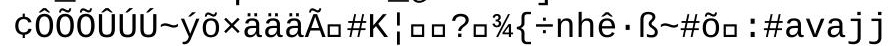

ó9ðÂ॰\%KØlöêÕ巛axKĐ\# 


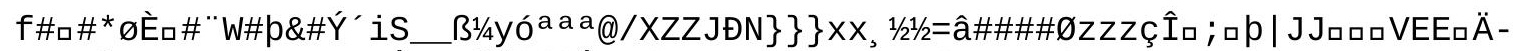

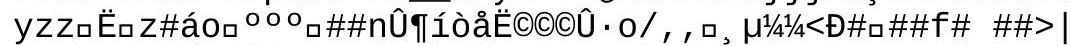

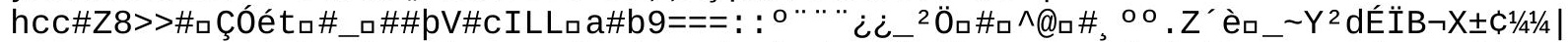

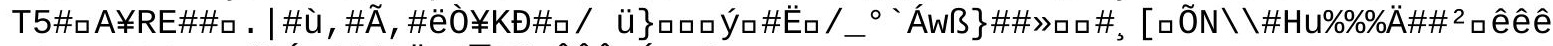

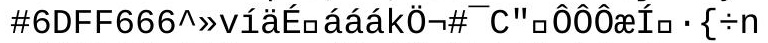

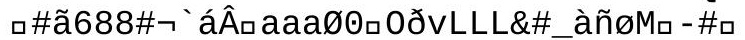

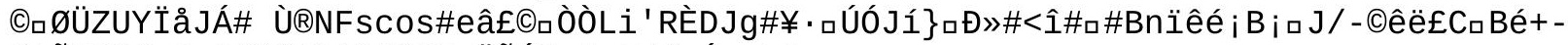

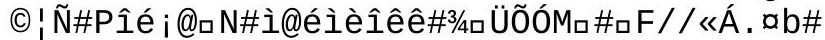

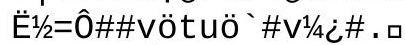

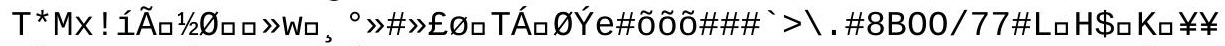

\%Ìù : : : àMÁn | i | Mø6Á\#

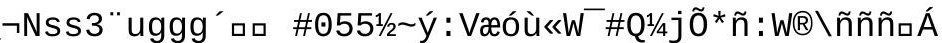




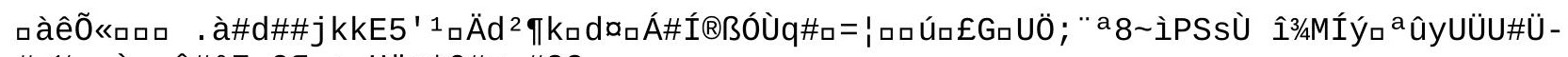

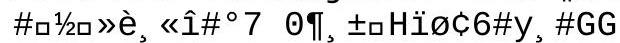

口

ÒSsTÖíËHËロòÓa 


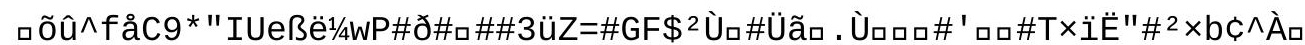

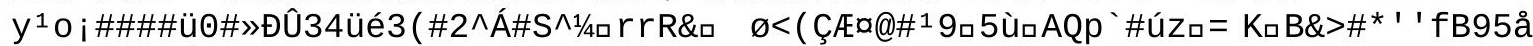

\%ם $\tilde{n} \#^{-} \mathrm{d} \S K A ́ / \#$.

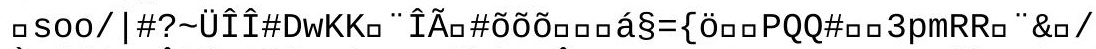

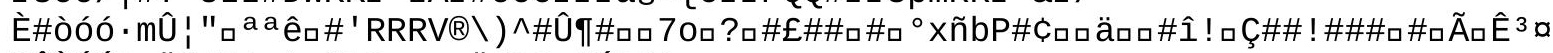

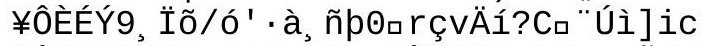

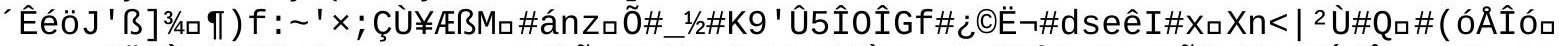

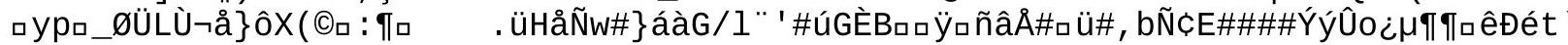
( "

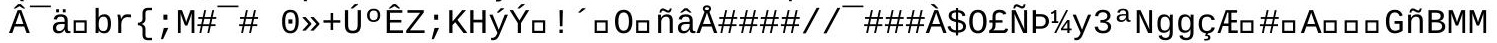
T\#_\#ロ\#\#ü·ß \#\#à\#\&םùt;

xáp "ÁáPà\#ì 


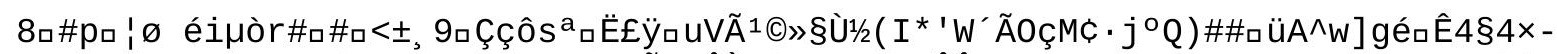

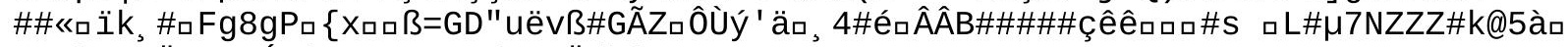

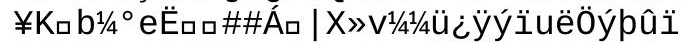

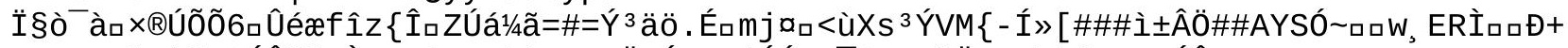

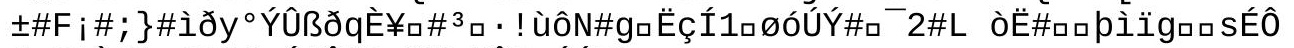

ëm\#kÀäìmGÇ\#\# i ÁÂ̂EŸr )¥·\#ÎßøÓÁĐà

口Î̃qråıÍñcxû

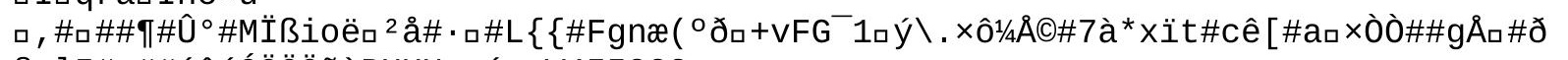
$r \S$ §gl5\#¿\#\#áôéóöööãòBUUUzzúa+W455322

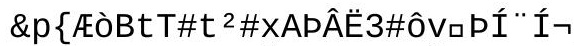

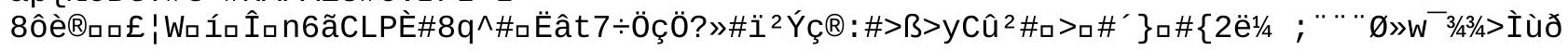
óyvvö, ${ }^{3}$ tppð口\#\#巴]»6./444ロ\{\#r\# 


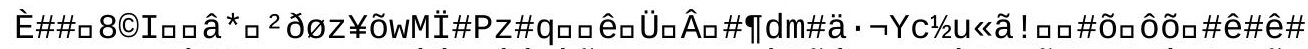

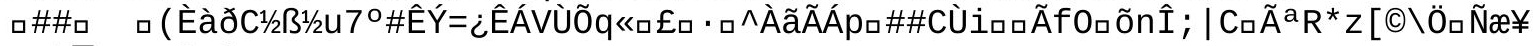

\%Uù ${ }^{-} \#^{1}$ å̊â

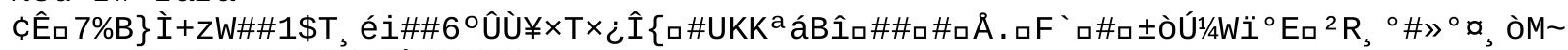
đ■ $\mathrm{Sô} @ 1 / 4^{\circ} \quad \# 1 / 4 \mathrm{~V}$, äĖáË-BV

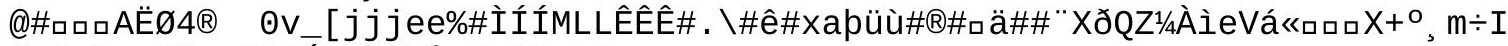

'JênìæaY\#ロa\#D\#ÍaqûûXÅq\%>û\#ロ ]\#

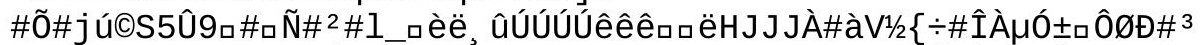

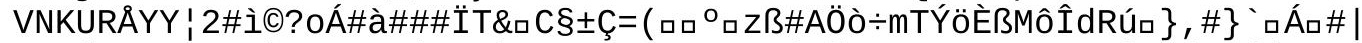

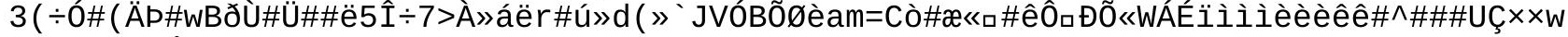

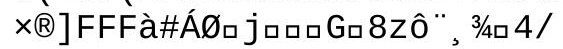




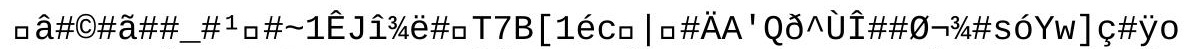

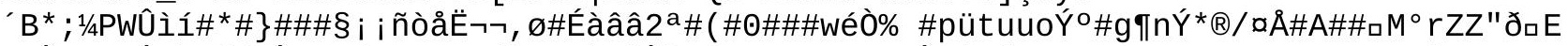

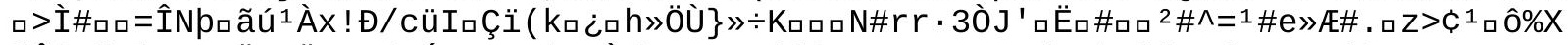
ðÂA

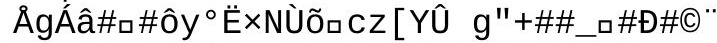

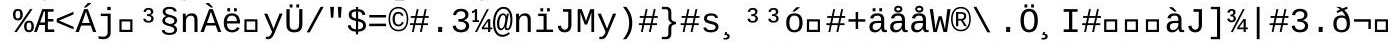

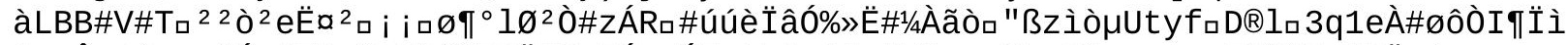

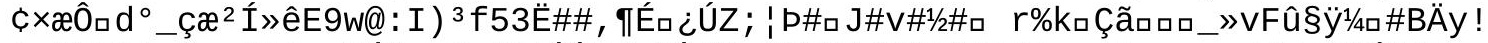

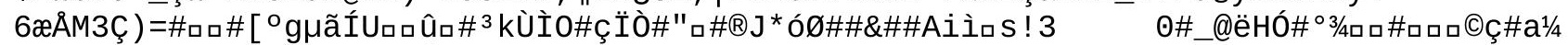
®\# :

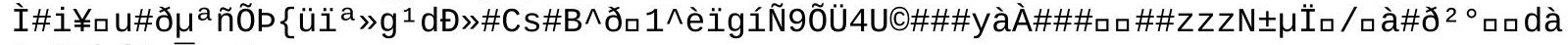
ò«ñ $5 p\{\# .-\operatorname{rr} 6$ 


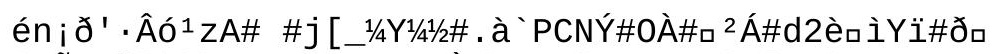

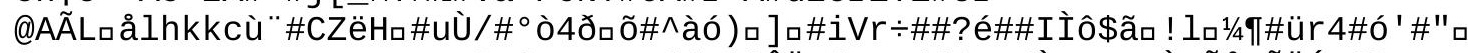

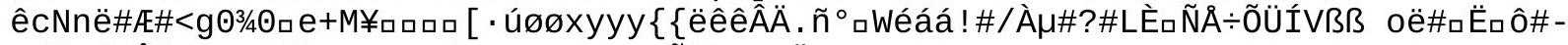

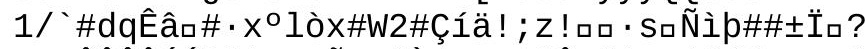

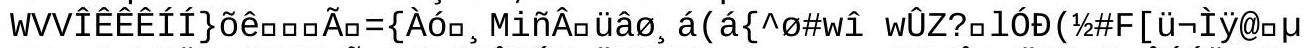

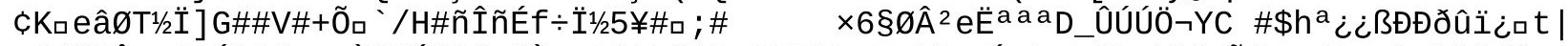

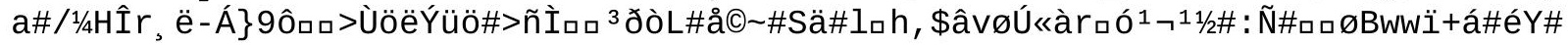




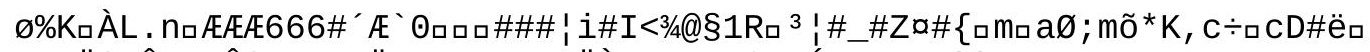

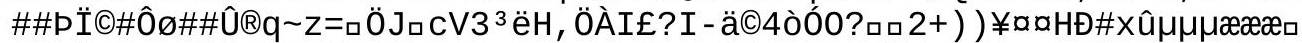

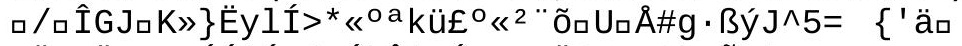

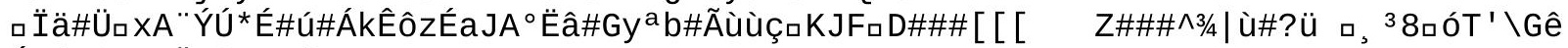
Ó«* ; G\#× $\times_{\square} \ddot{\mathrm{I}} \div \mathrm{GmM} \div$ Ó

\#\#ё \#|

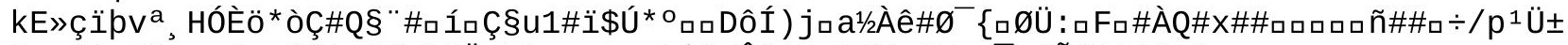

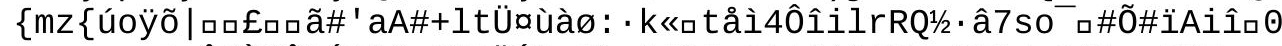

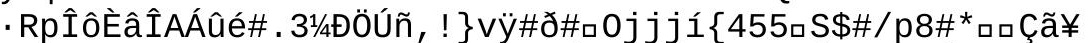

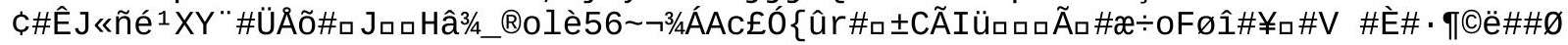




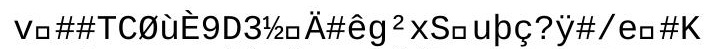

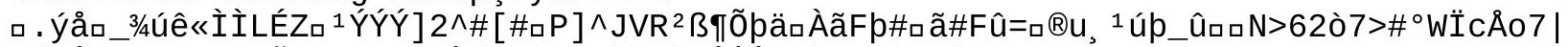

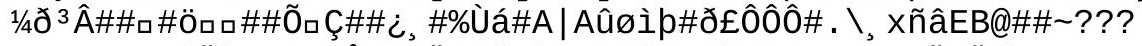

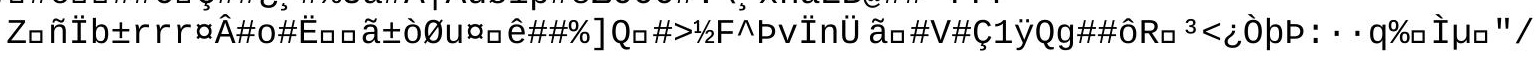




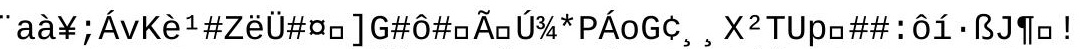

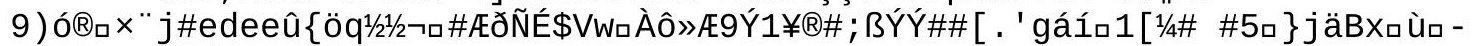

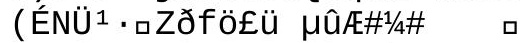

çty\#Àa

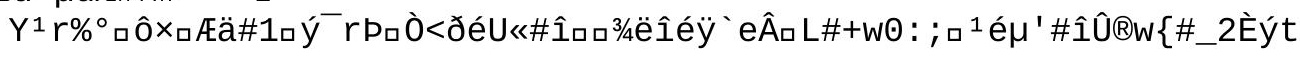




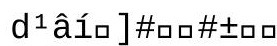

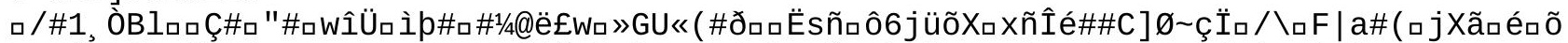

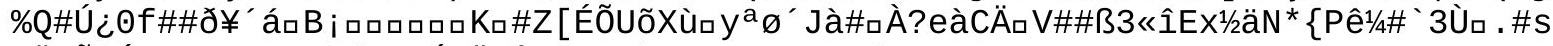

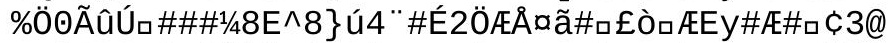

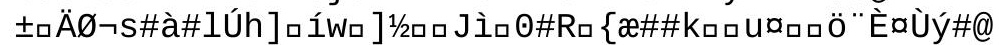

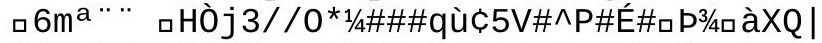

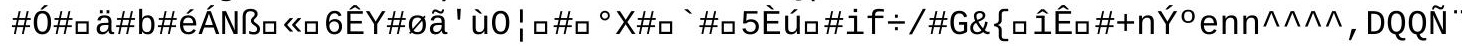




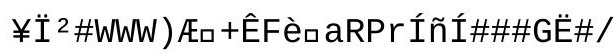




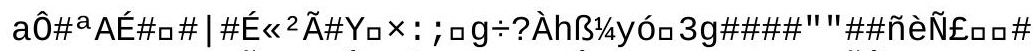

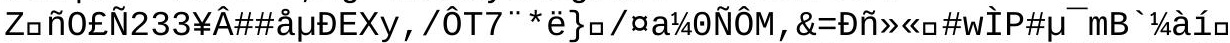

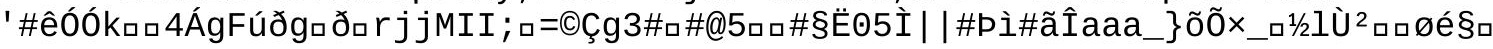

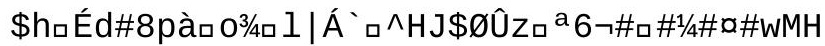

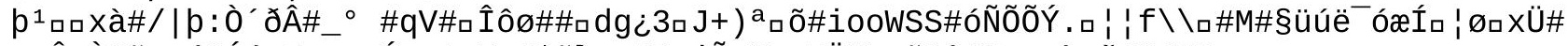

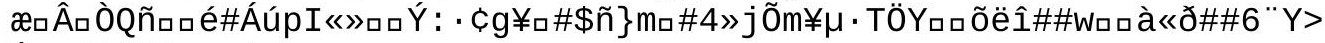

ÊtX\#A\#\#C, aì

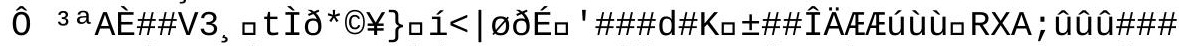

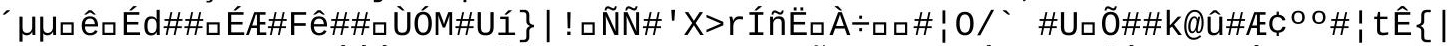

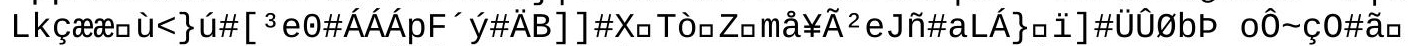

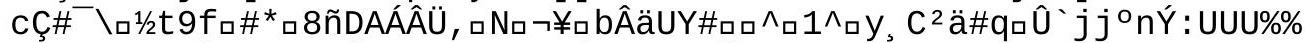

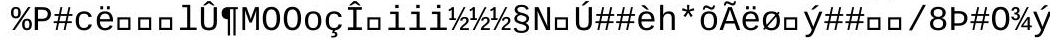




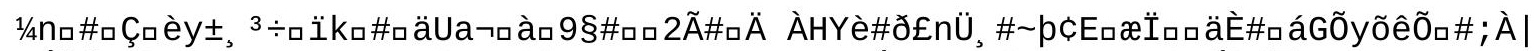

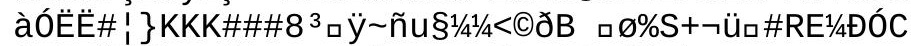
ð\#\#M\#\#ѐ\#b1É\} $\S^{-1 / 2}$

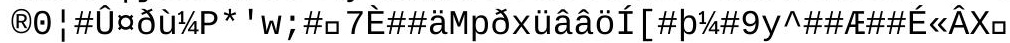




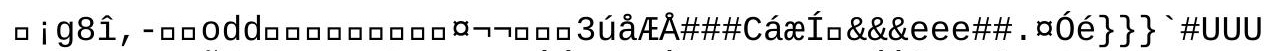

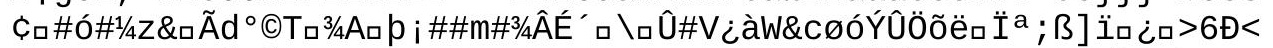

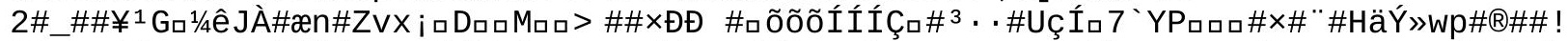

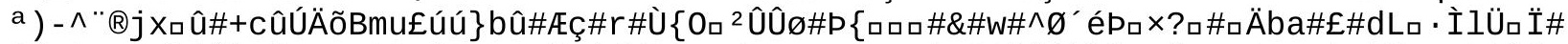

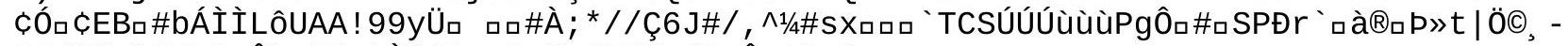

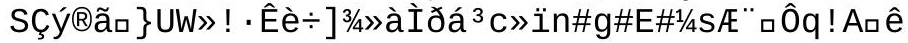




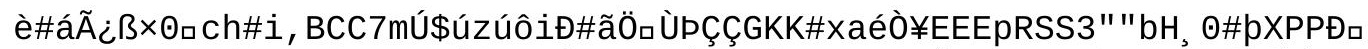

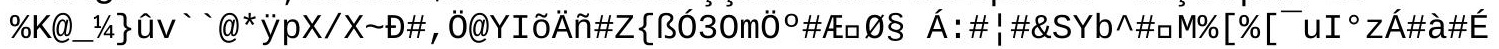




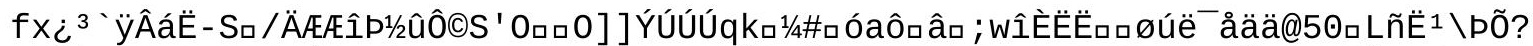

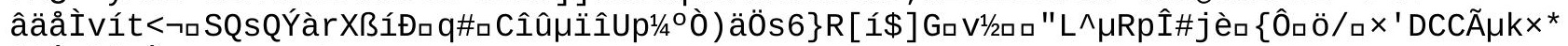
**Âç@]»À: :

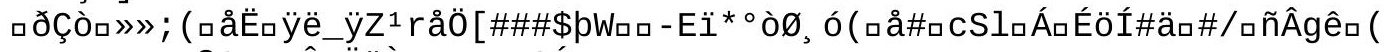
X\#ĐßÇZr1ÊnËëÀW $<\square 4 C \div S A ́$ 


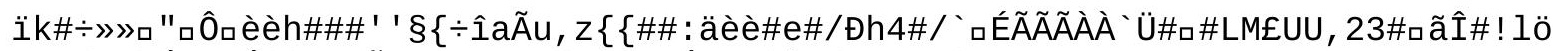

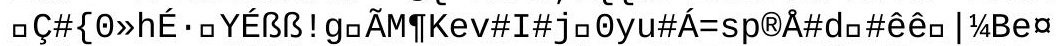

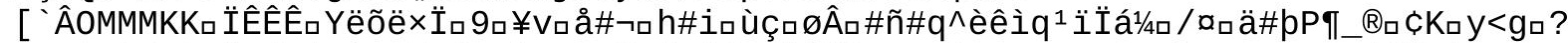

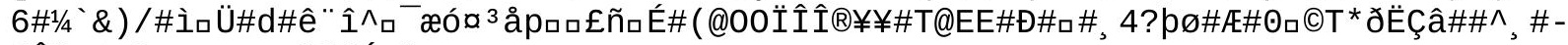

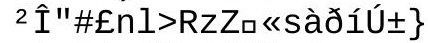

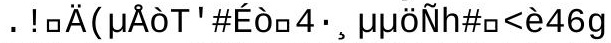

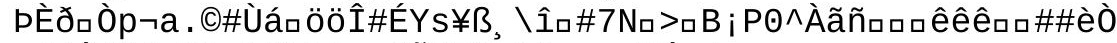

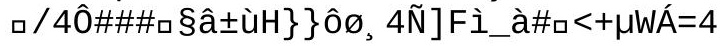




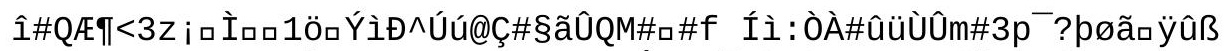

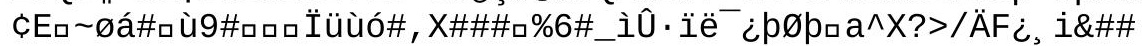




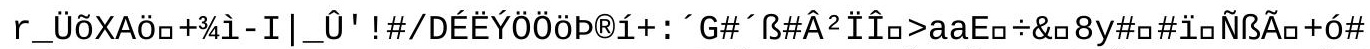

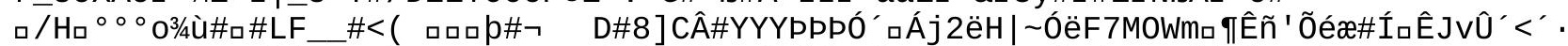

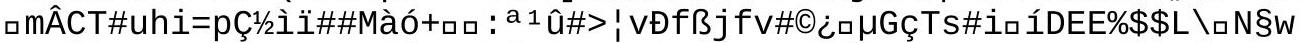

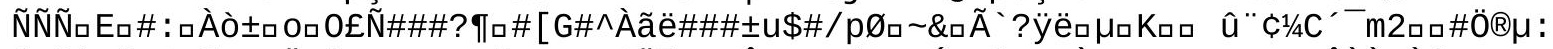

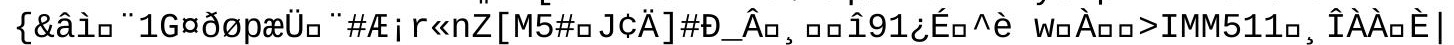

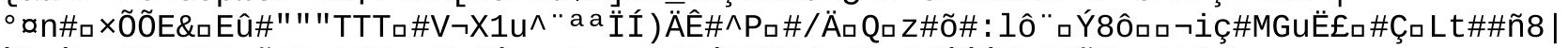

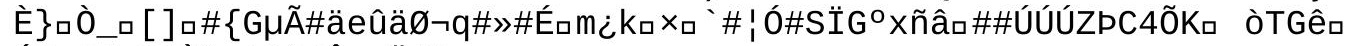
ÁQZ\#\#ロÇ\#İôzifìúÂ: Ë\#\$C 
ÏWVI॰äÝ\%Ý×ö\#ùHõ\#Jロ $\cdot R ! p \# Y=$ 
1/4S : PÃ£\#Á¥ ${ }^{2}$ ○ËM 


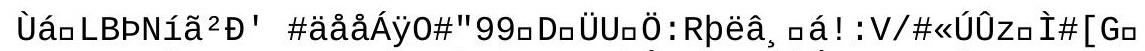
$-a^{1 / 2 \# k^{-}} a+a$

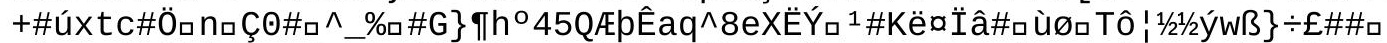

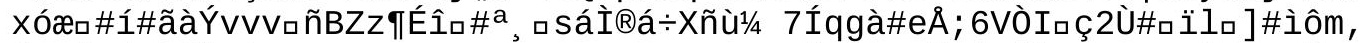

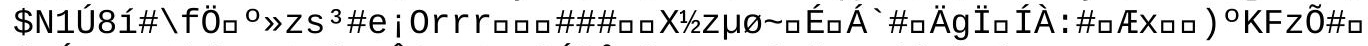

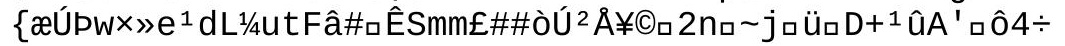




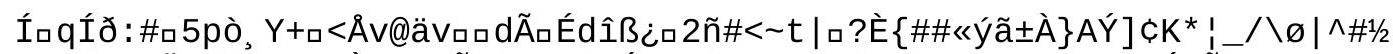

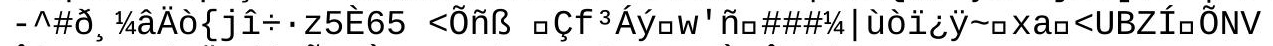

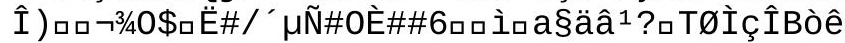


Ïkcõ\#ìÛs $\mathbb{1}^{1} \mathrm{I}$

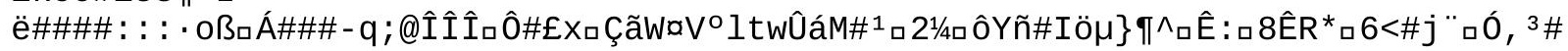




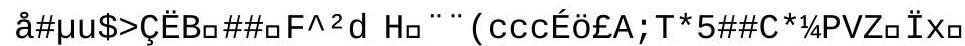

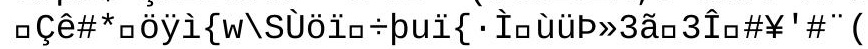

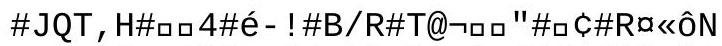

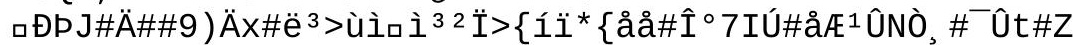

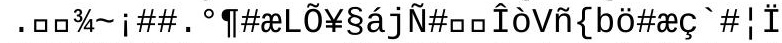

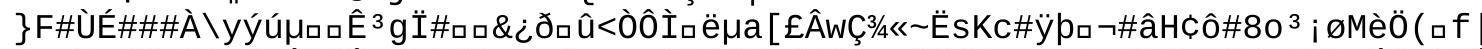

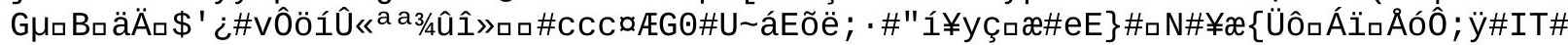

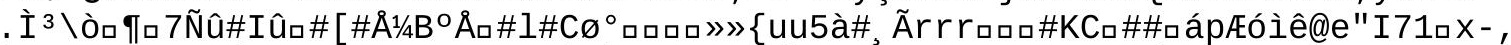

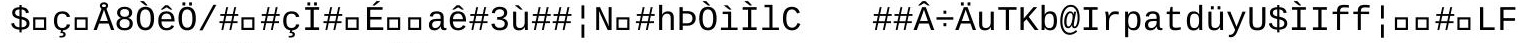

$<\neg \neg|| \mid \# \square \&$ \à| Eq i $\square$ \#T

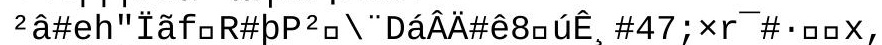

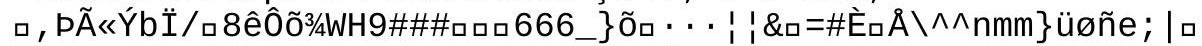

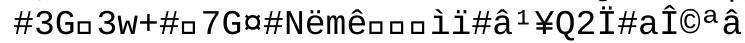





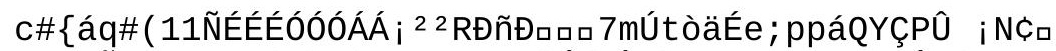

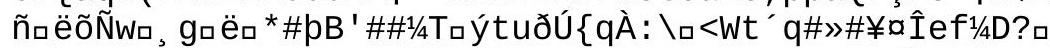

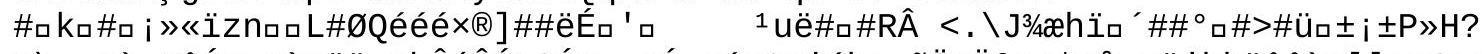

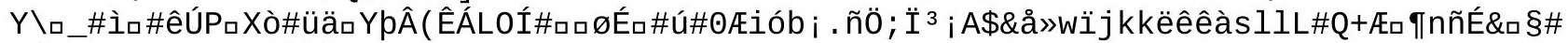

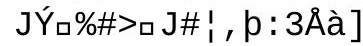




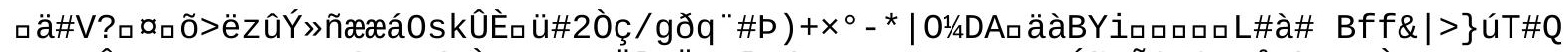

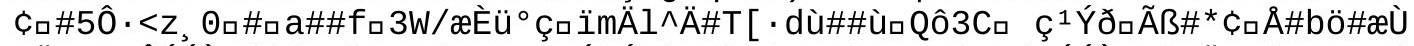

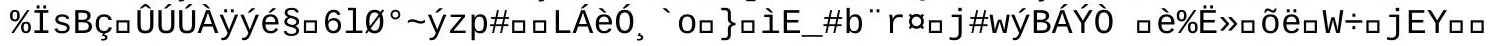




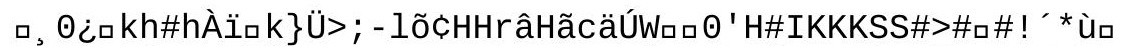

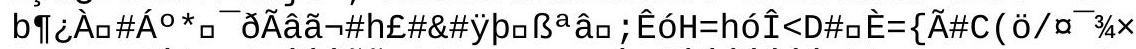

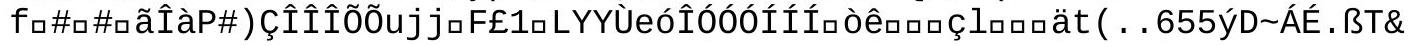




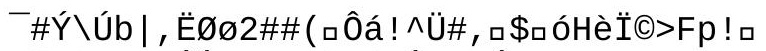

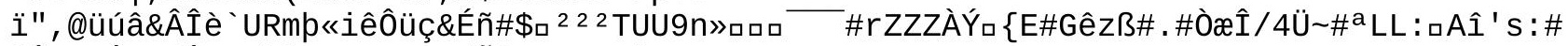

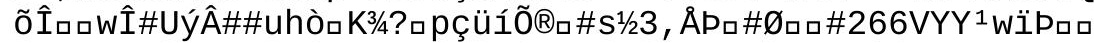

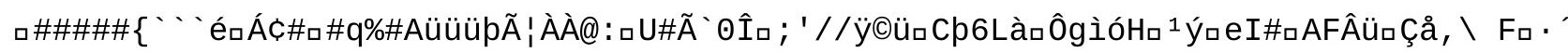




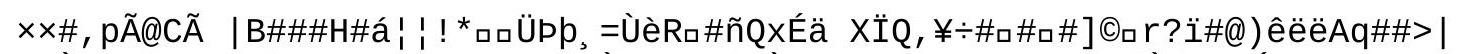

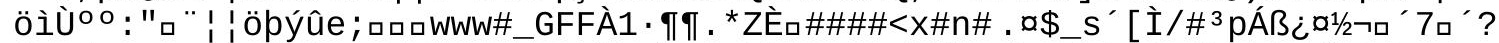

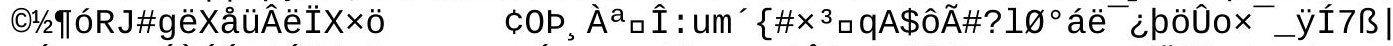

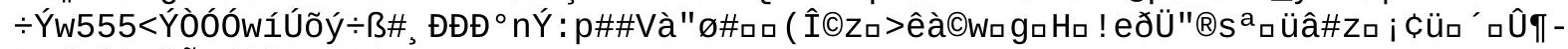

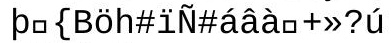

$=1 / 21 / 2 \ddot{I} \square \hat{E}$ 


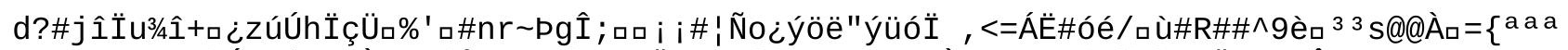

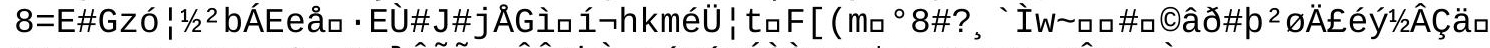

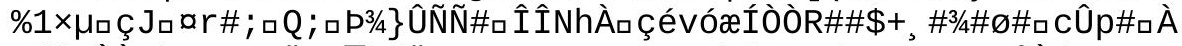

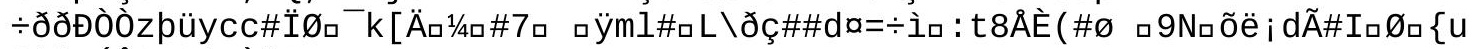
Cûû\#Ý̂ि9VQjAÈ\}?ם

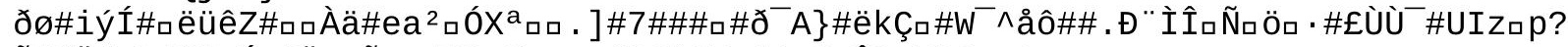

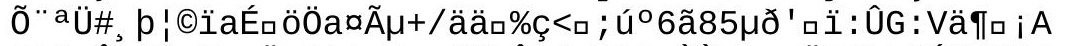

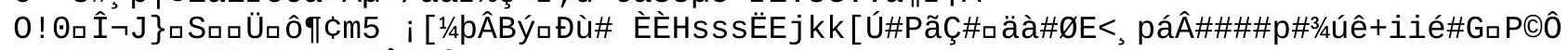
口ฉèão6ãıâHð8d2o 0"óîýüÅロØùn

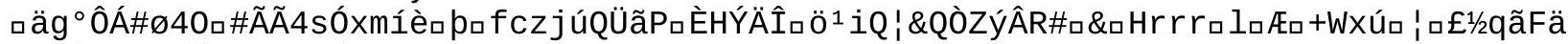

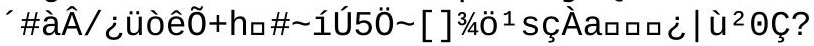

$\hat{1} 3 / 4$ TVòUV

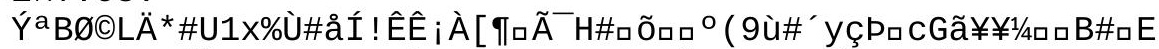

án

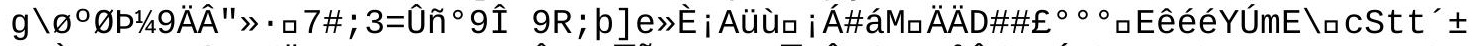

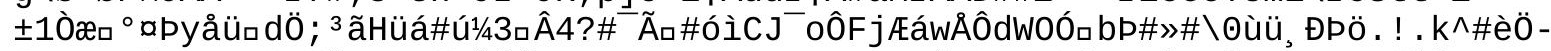

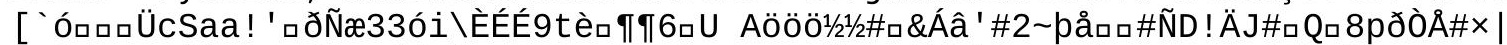

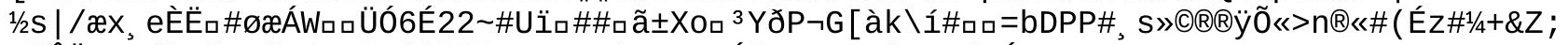

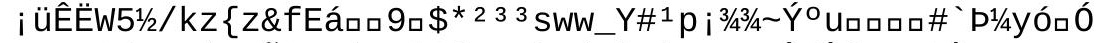

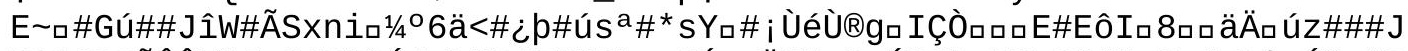

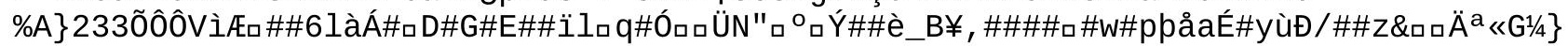
; FFx, ép

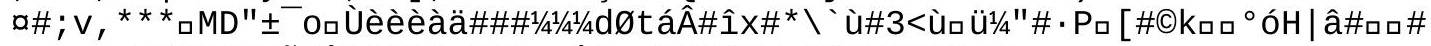

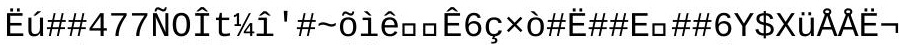

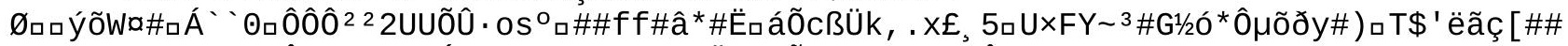

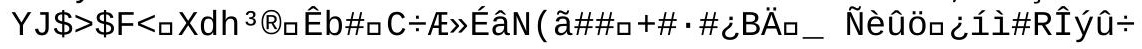





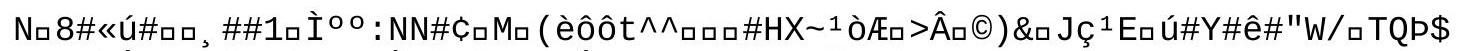

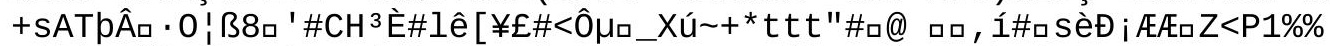

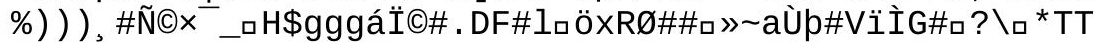




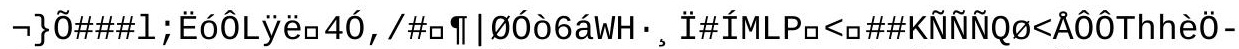

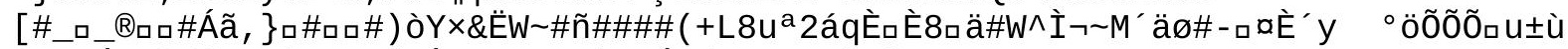

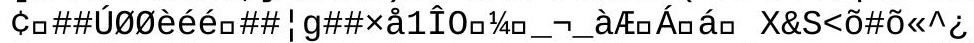

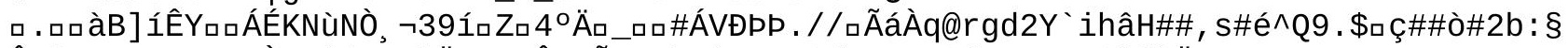

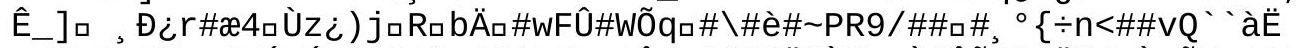

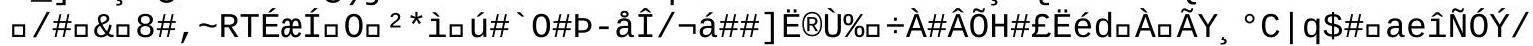

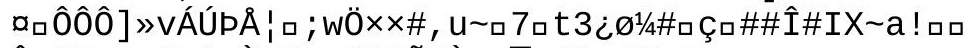

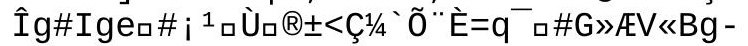

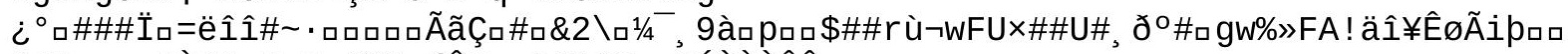

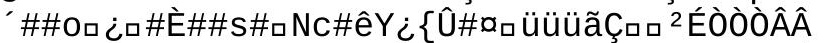

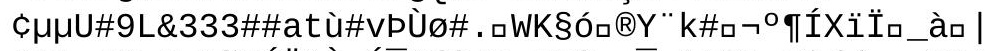

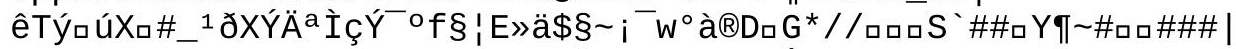

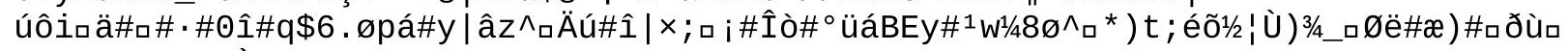

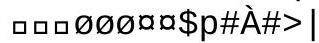

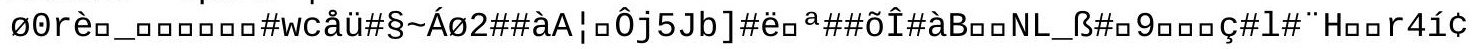




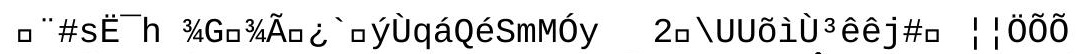

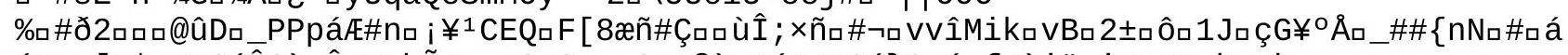

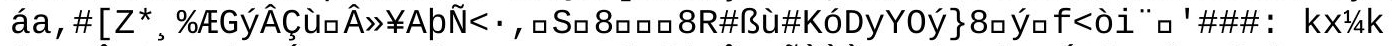

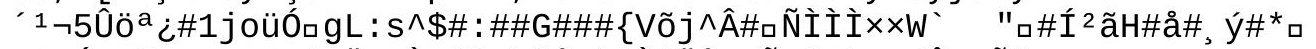

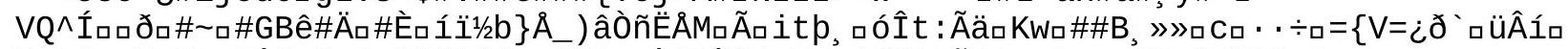

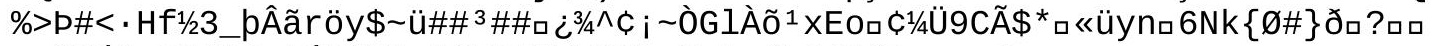

ÇaßUÓÅa\# [ [ ' 9q\$É ! ð\#@eö 2 I [ [ [WW\#@\#, MTùı ( b\#\#ёýGç XW\#

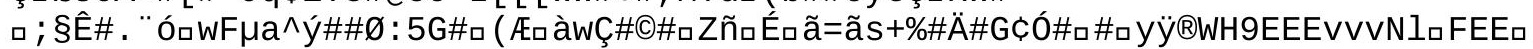

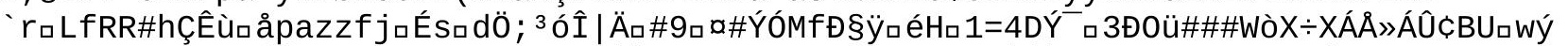

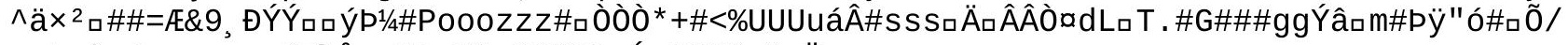

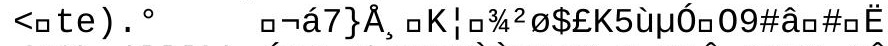

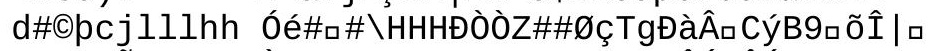

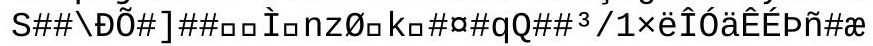




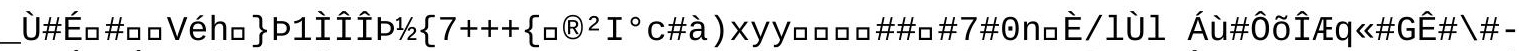

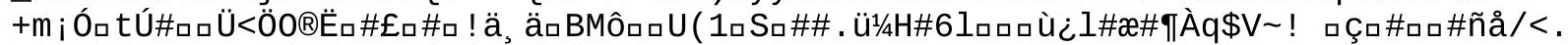

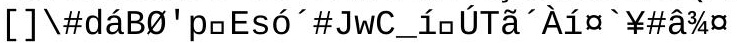




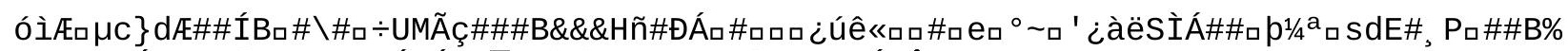

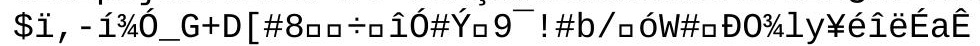




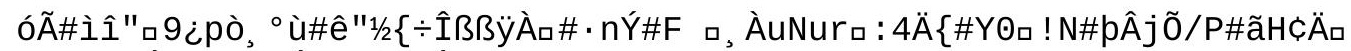

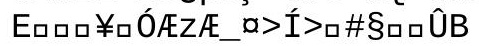




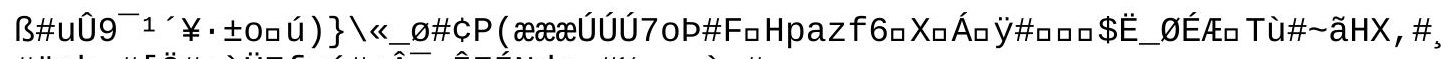

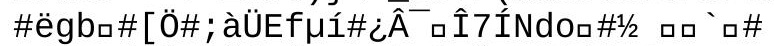

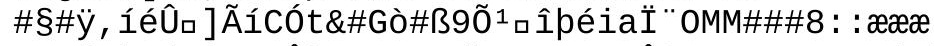

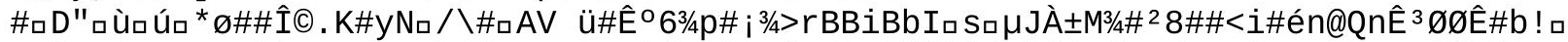

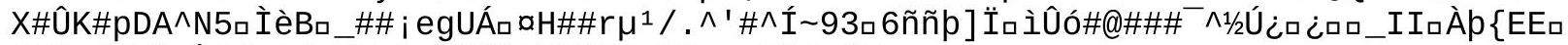

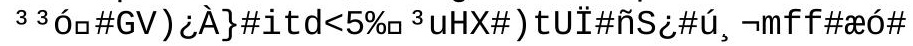




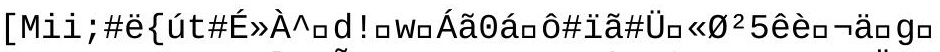

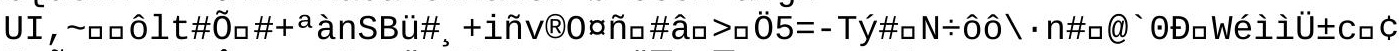

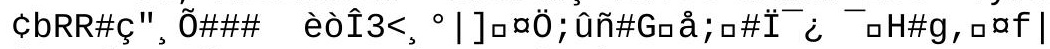

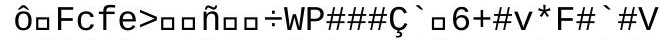

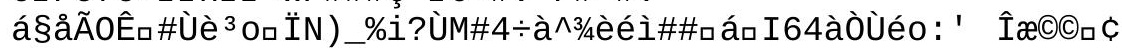

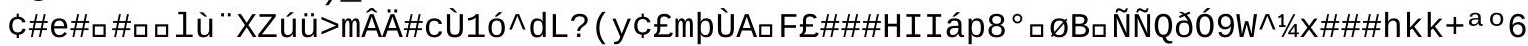




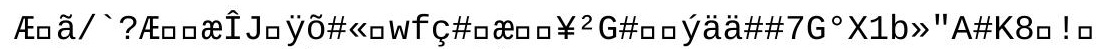

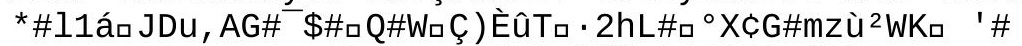

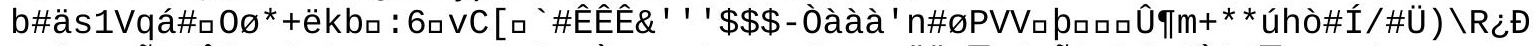
\# . ð\#G: ẪýÂêæ\#(

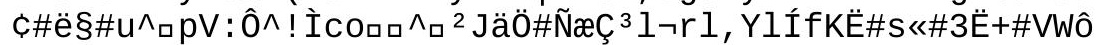




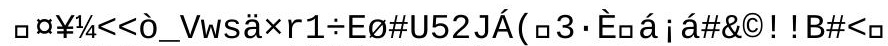

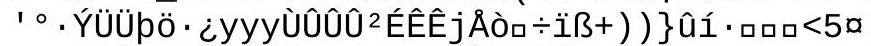

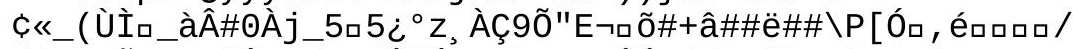

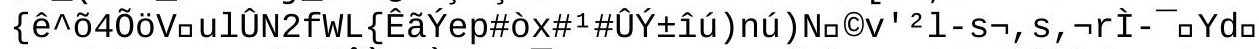

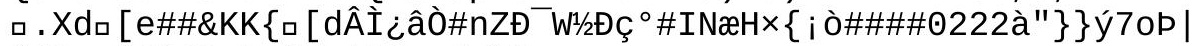

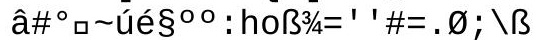

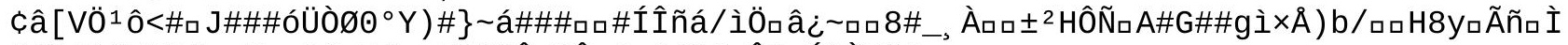

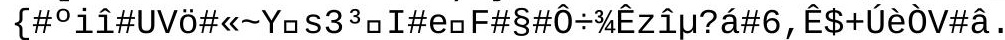

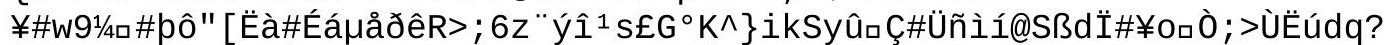

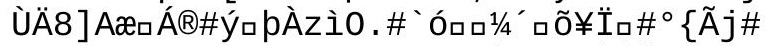

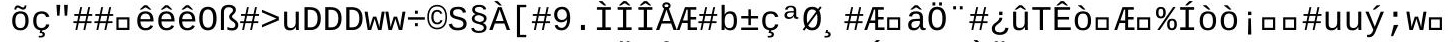

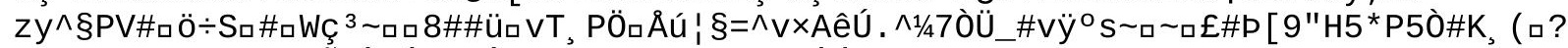

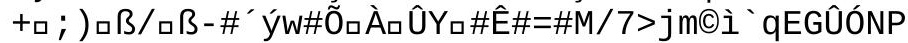

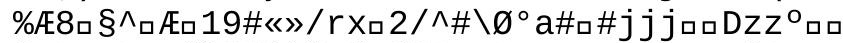

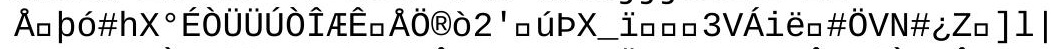

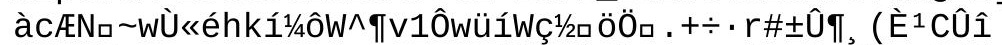

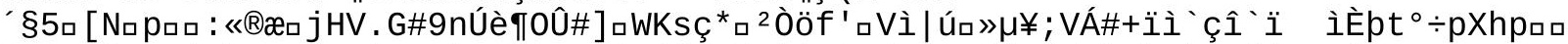

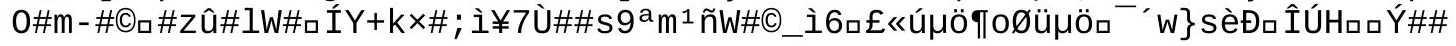




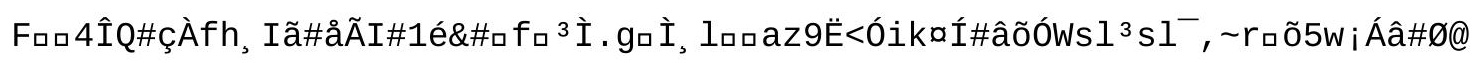


æÄ) \#W4S * 
$\left.\left[\mathrm{Z}{ }^{2} \#\right) \square j\right\urcorner \_\dot{\iota}^{\wedge} \mathrm{CC} \# \#$

$1^{2}$ ๆाæü

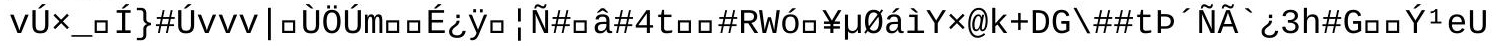




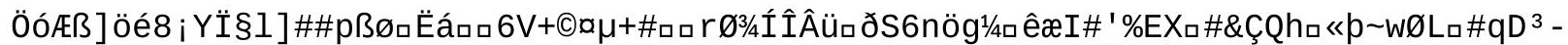

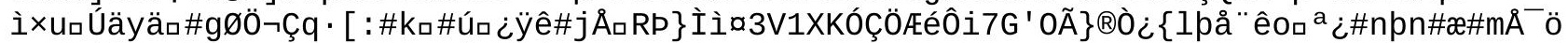

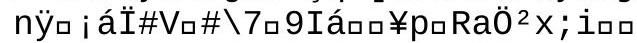




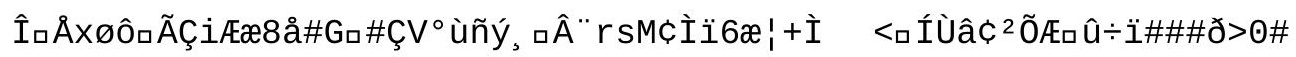




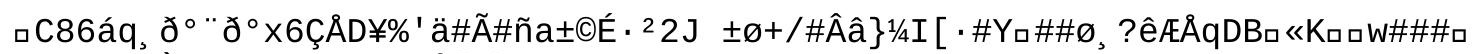

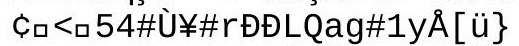

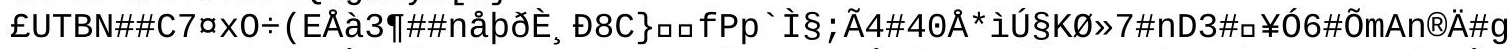

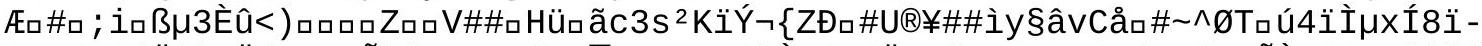

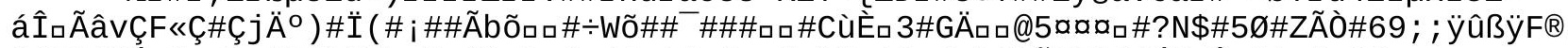

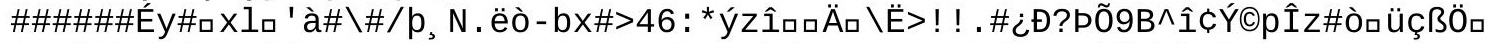
\#Gó@\#\1/4a\#Q^öËÊ"

$\$ b$

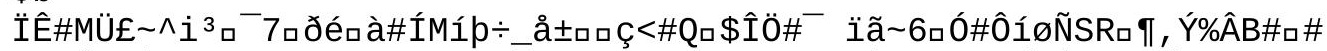

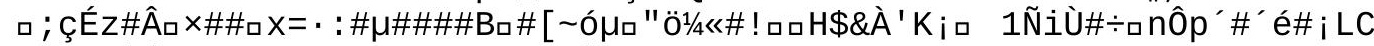

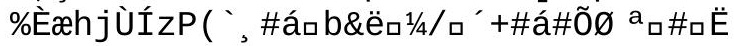




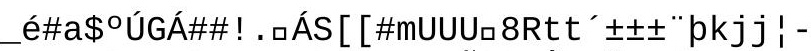

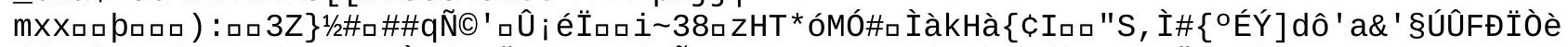

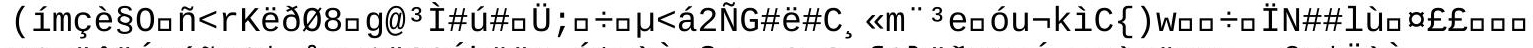

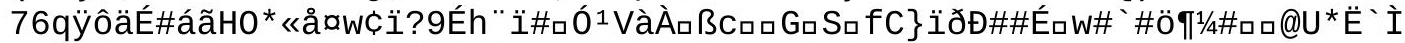

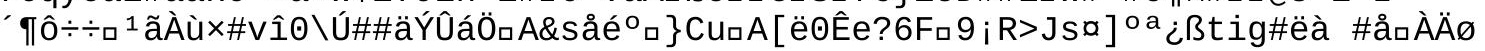


$1 / 2\} \#-\# T^{*} a_{\square}$

$\#$

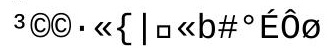




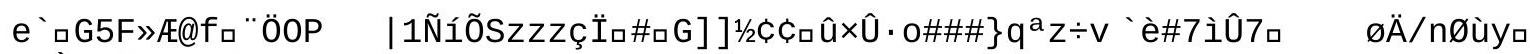
DBÒ\#71Q⿱ : :fJJ

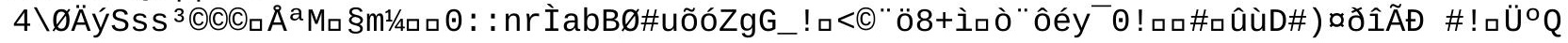




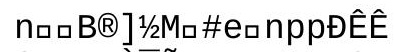

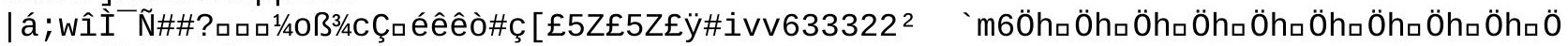
høםuLfkkkssóäää\#4l*a

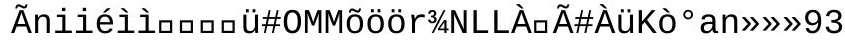


\#"

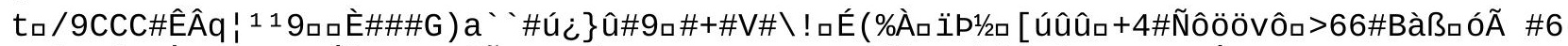

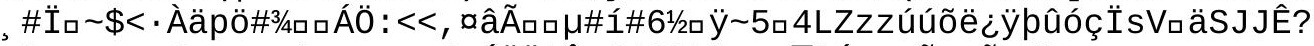

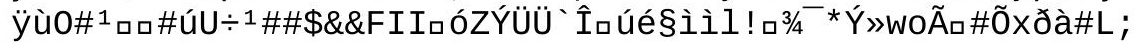




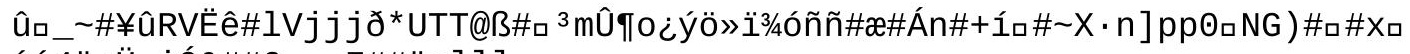

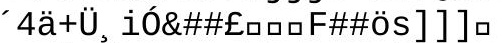

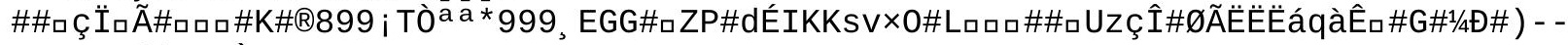
En122B@*11\#Ùs@\# 


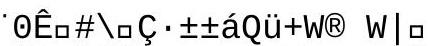

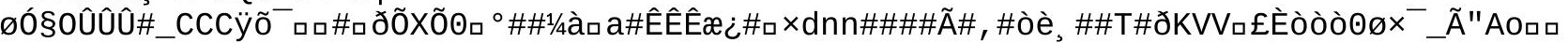
$\square$

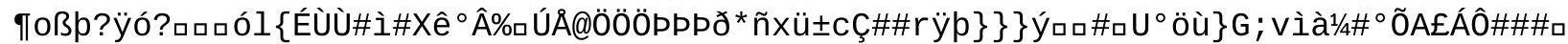

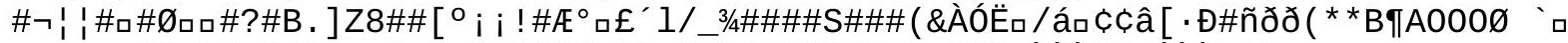

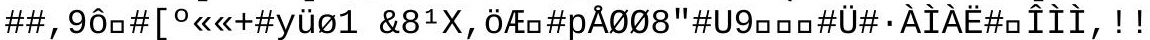

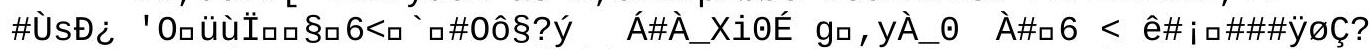

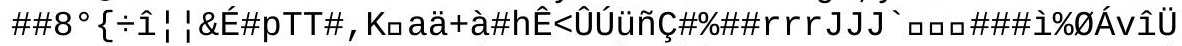

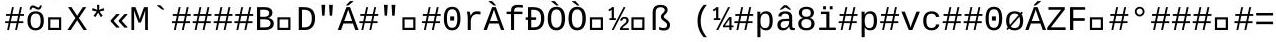
û\#r\#\#£ᄀᄀ 
úuâÂ\# \#\#, |0' 'ó\#q\#QÏ - [

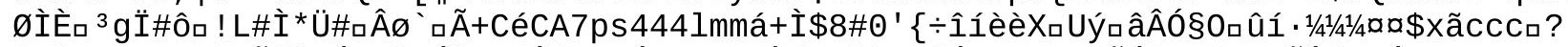

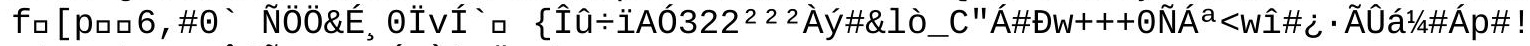
Øô8, \#ѐ\#\&7ロÎ́áÃロQ\#Ø\#Á\#À\$\#ËM\#\, sç\#Ø\#

Æ\#0qíú5ロ- 


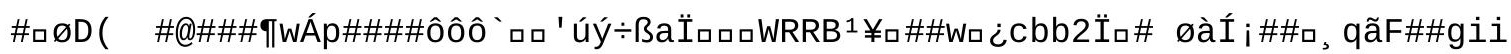

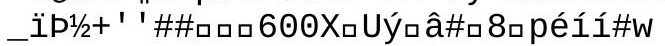




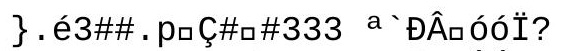

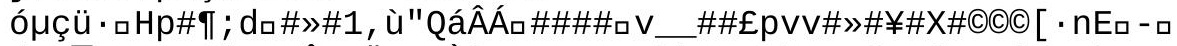

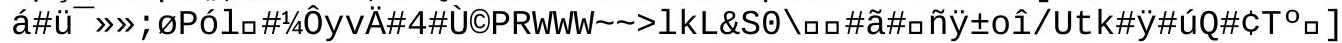




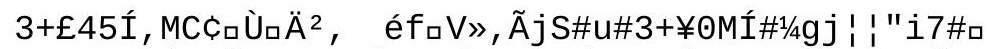

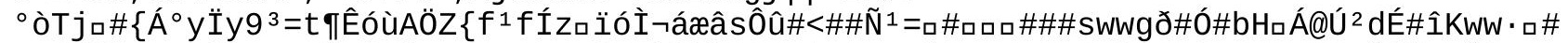

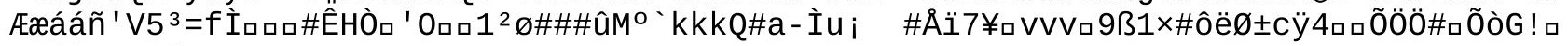
CN (àmHö\#ÖIFFם

ÉXÕ *。

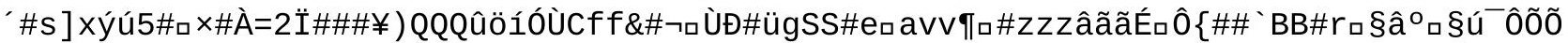

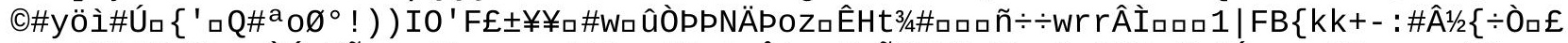

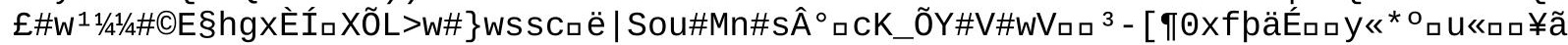

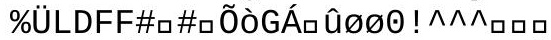

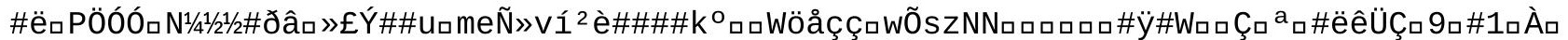

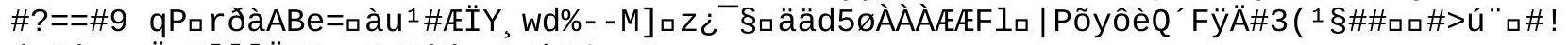

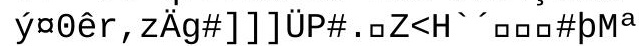

EYYYOU

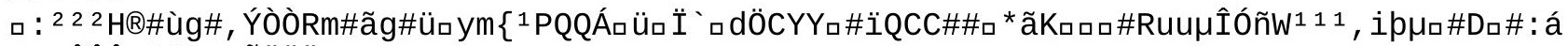

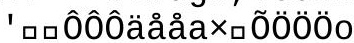

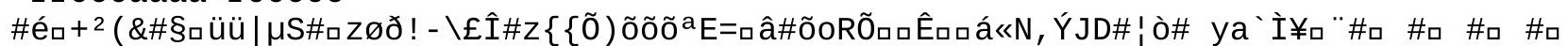

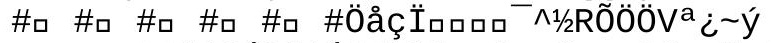

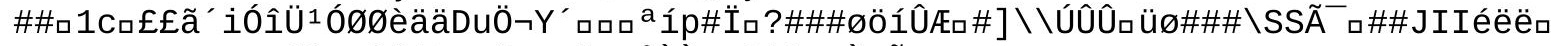

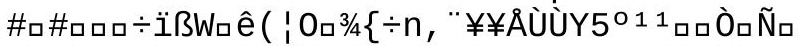

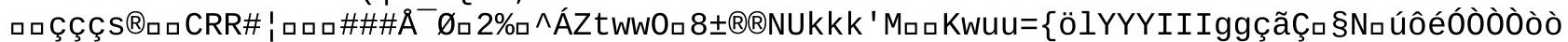
ò\#; Vıロ\#ÊEF/

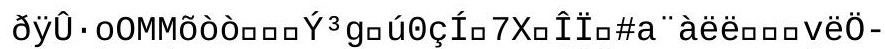

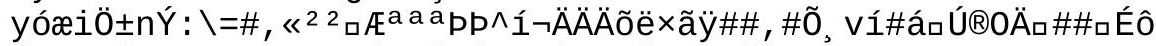

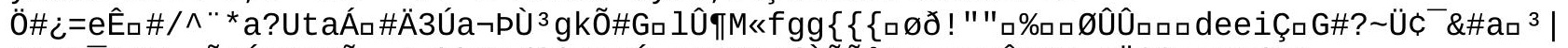

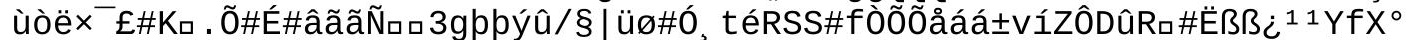

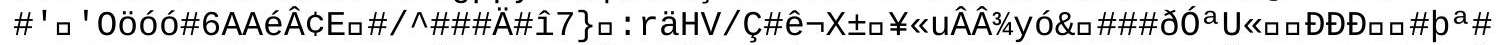

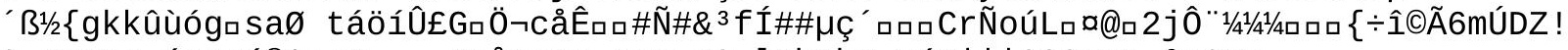

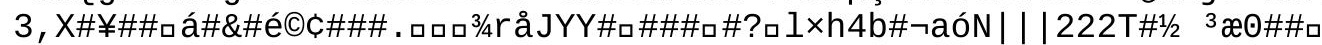

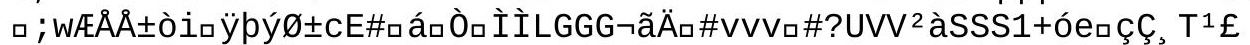

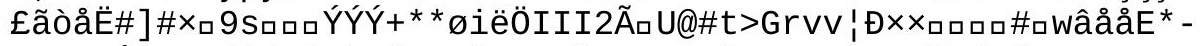

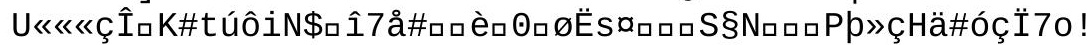

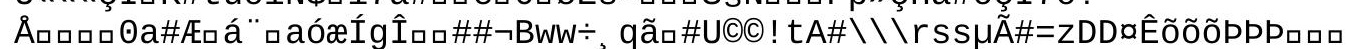

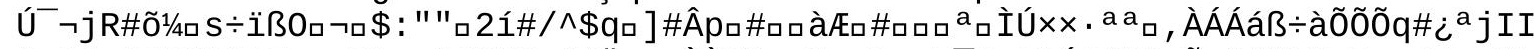

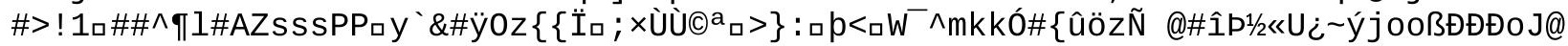




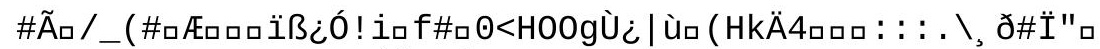

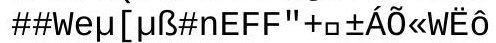

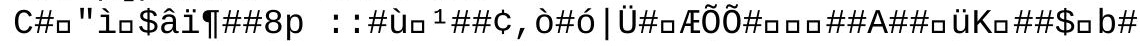

\section{endstream}

endobj

$33 \odot$ obj

$<</$ Length 3128

/Filter/FlateDecode

>stream

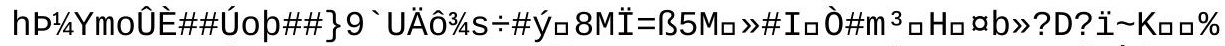

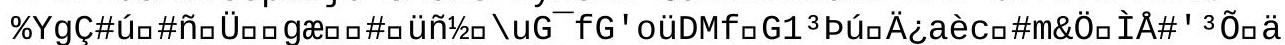

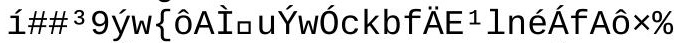

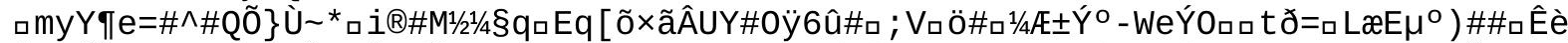

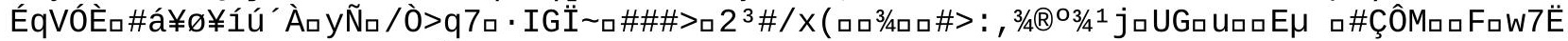

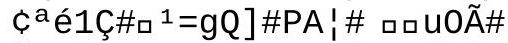

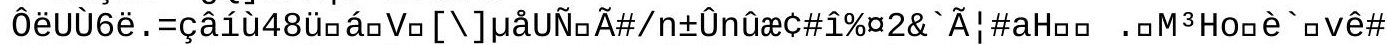

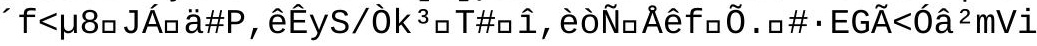




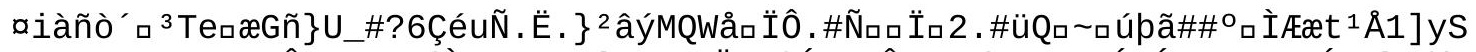

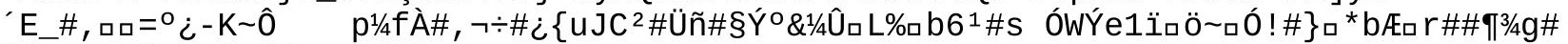

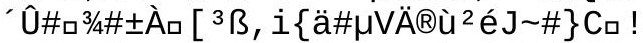

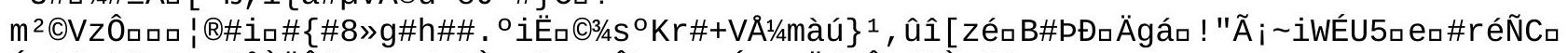

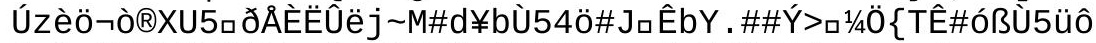

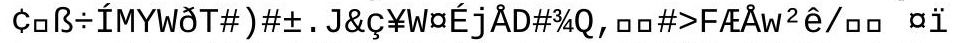

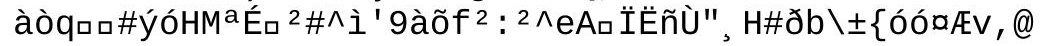




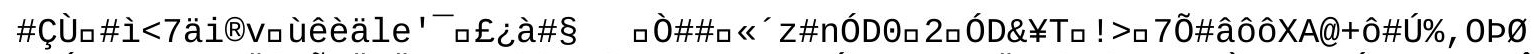

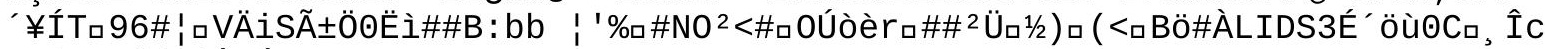
$V_{\square}{ }^{*} \mathrm{Na} \# \ddot{E}^{\prime} \sim \mid \hat{I}^{\circ} I^{\prime} \mathrm{CS}^{3 / 4}$ 


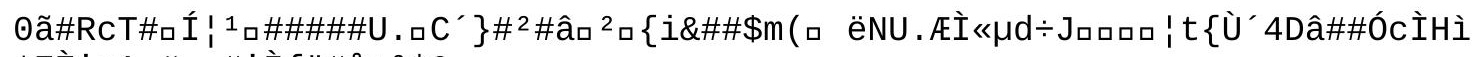
C7È ' UA口\#»ロ\#' I I $\left\{\right.$ ü\#å : ${ }^{2}$ * \& 


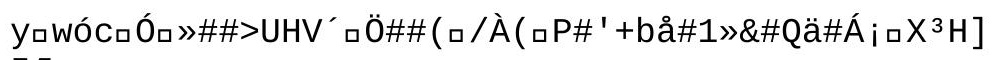

5A 


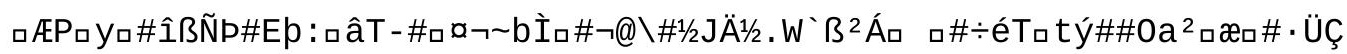




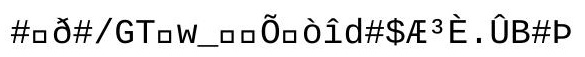




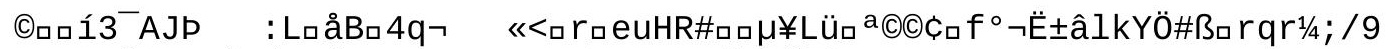

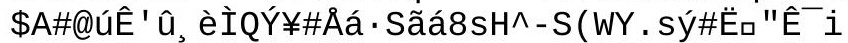

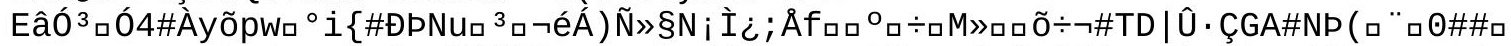

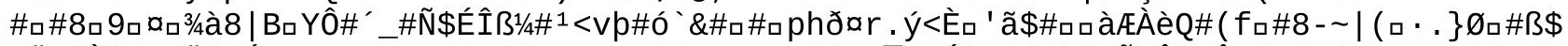

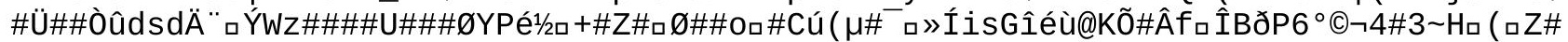

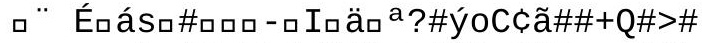

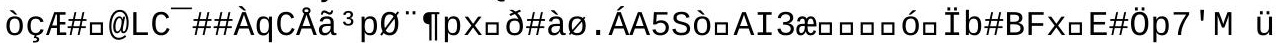

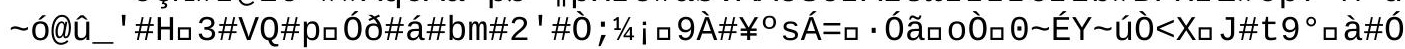

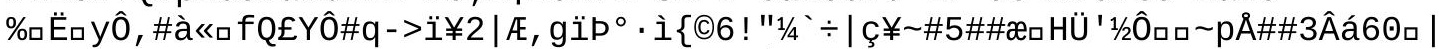

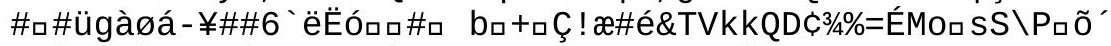

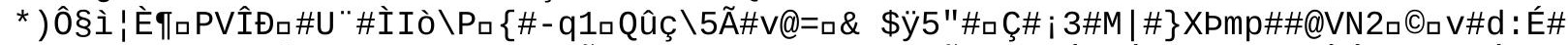

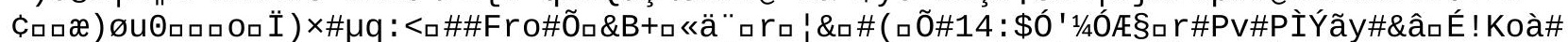

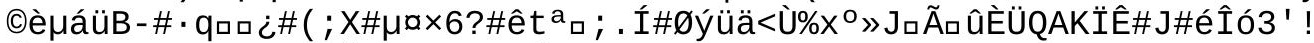

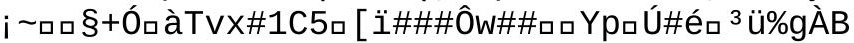


'éSa $\mathrm{y}^{1} \#$ 


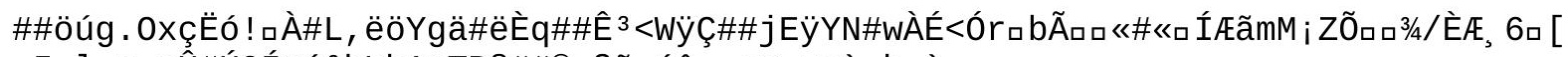

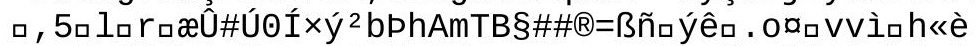

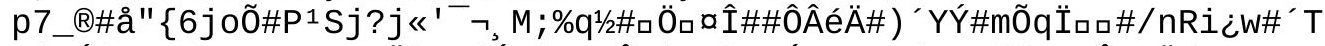

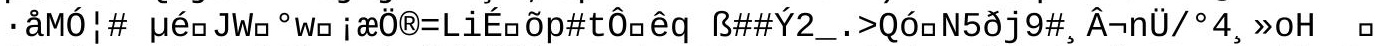

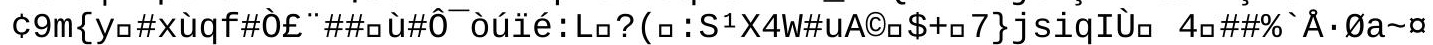

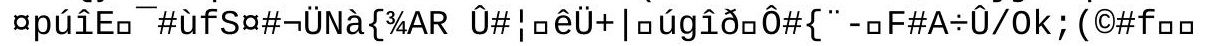




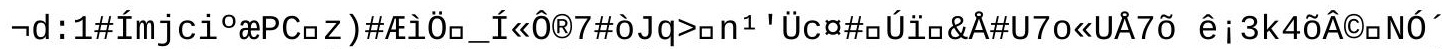

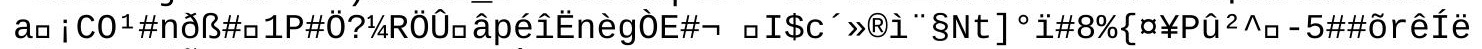

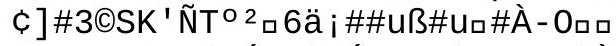

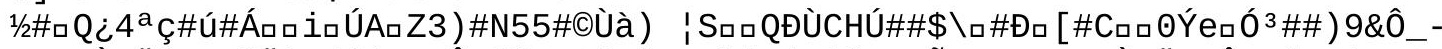

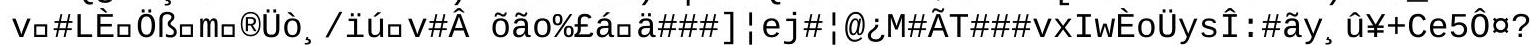

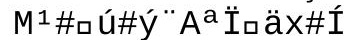




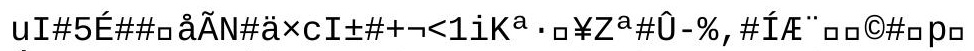

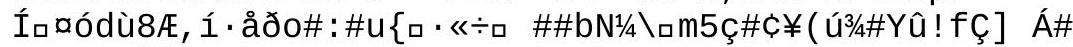
)/

"36\# $\left.{ }^{\circ} \# \neg Y 0 ̃ \#\right\}$ !3/4YÝ§õ \#8IB) ý<AôP

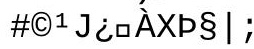




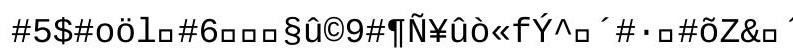




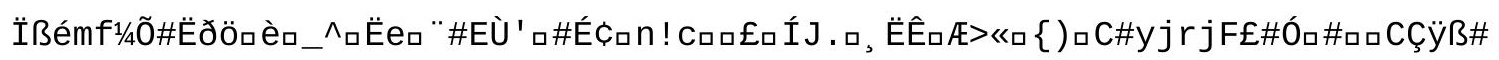




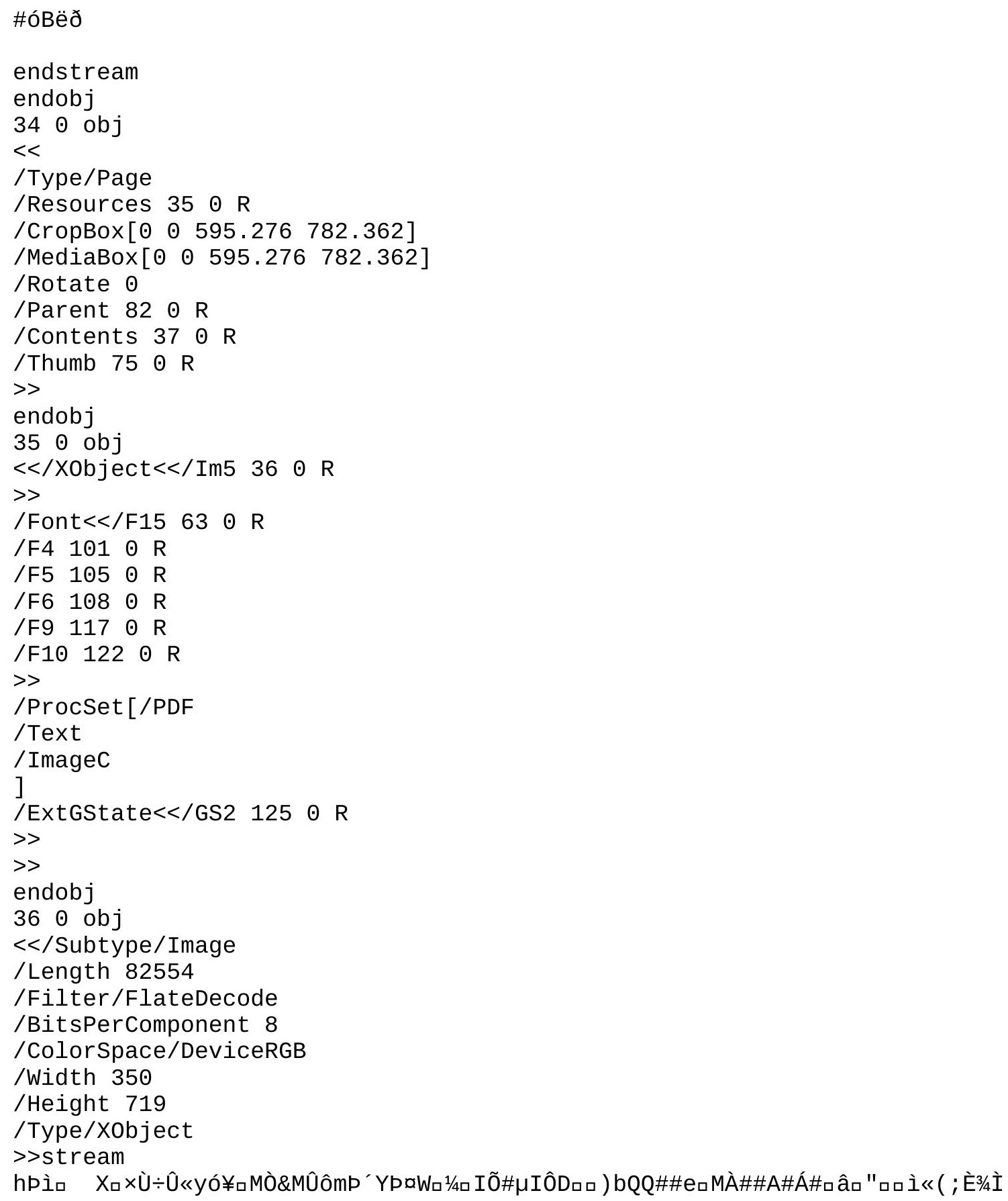




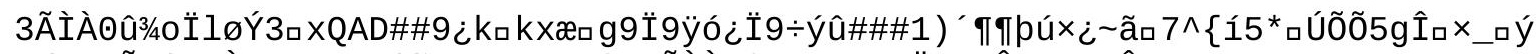

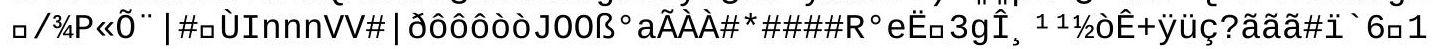


Óétı\#ø xëロロ \#Ï○ĐÒ\#\#\#=<< 


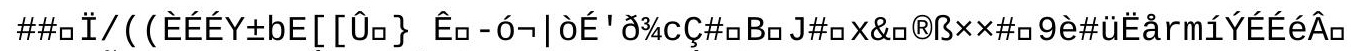

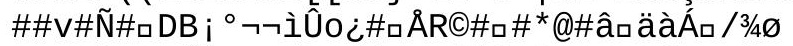

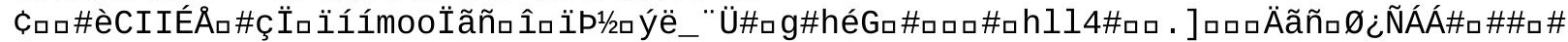


\#\#b(H\#\#\#ÄPロ2 \#ロÁ\# 


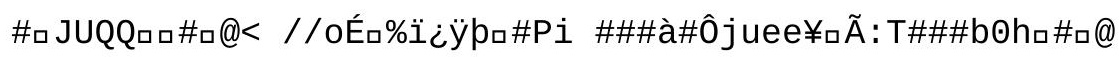


\#)\#\#ロ\#

R\#\#\#1\#a 
\#\#b ( H\#\#\#ÄPPD\#\#ロ\# 


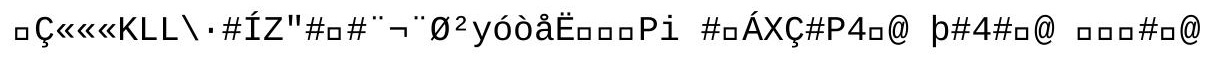


\#土\#ÍM \#ロロA॰\#ロ@ 
\#)\#\#\#

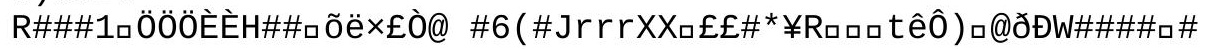




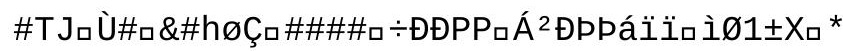




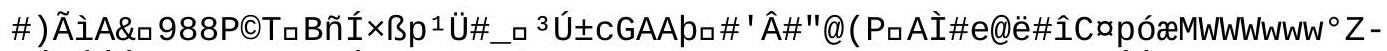

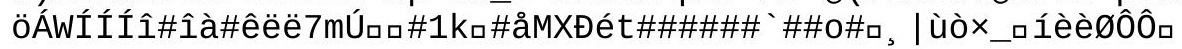

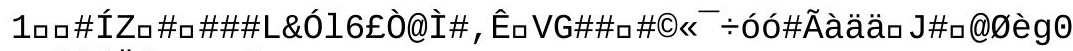

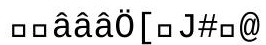




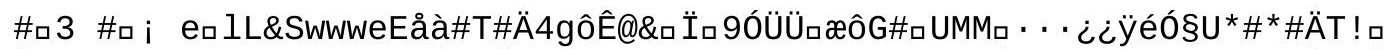

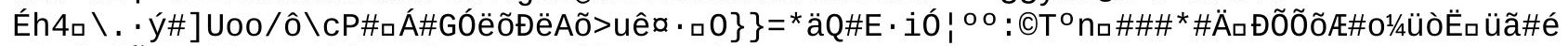

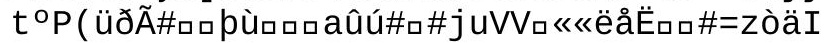


Ãàó ¡ $C_{\square} P 9 \square \# \# d^{\circ}$

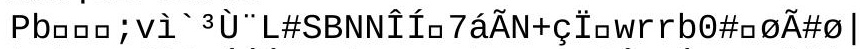

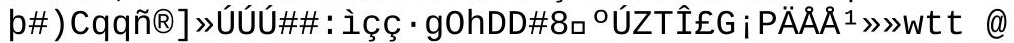




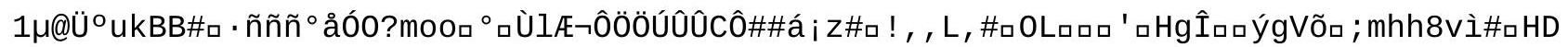
\#1ьฉö\#\#\# 


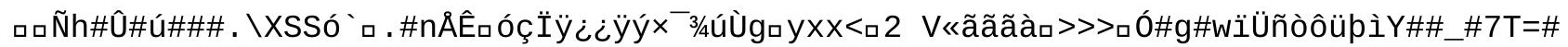
3\#èú\}\}\}A\#@\#à_@ 
\#\#èt ${ }^{\circ} \cdot \beta \sim \# b_{a} \# \gg \square I \#$

口\#ם' 'tõau' 


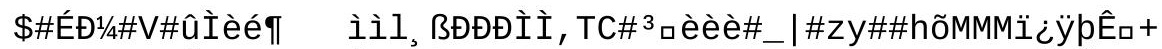

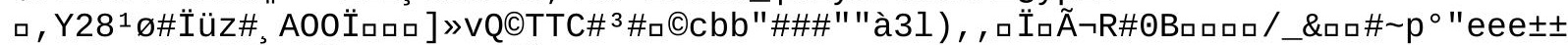

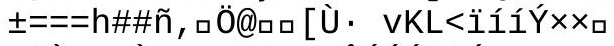

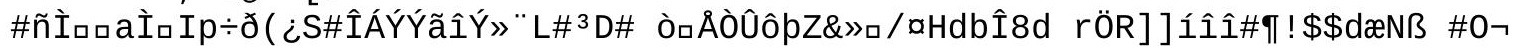


$\left.\div \ddot{1}^{3} \square^{a} \# \square^{\circ}{ }^{\circ} \AA\right) e ̈ i ́(E C e \#) T \square$ 
3/40̀\#ロ $\div \varnothing \backslash 1 / 4 \mathrm{PÌ}^{a}$ 


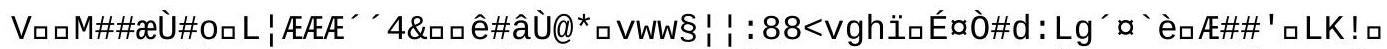

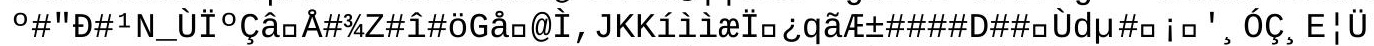

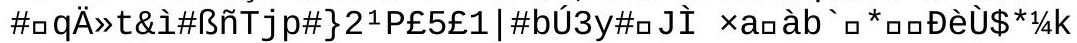




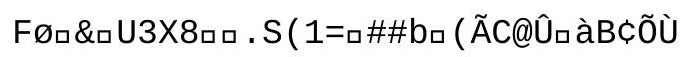




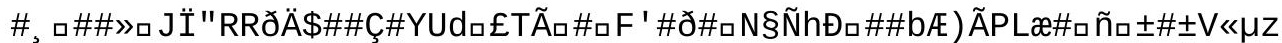

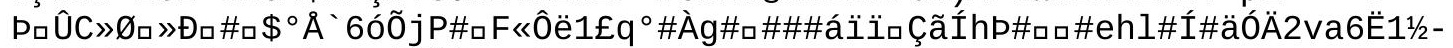
Î\#ÝĖí i ;\#Hé\#2Ä\#`3#"Ûİ)\#ロTC 
\#, võj Iqñ̃ - [Äb1z!\#Ä\#( $\tilde{A}$ 
Yé\#\#\#\$ * «uñE 
Zøe aG, MÅuq $\mid v m v U 5 \square A ̃ \mu^{3}{ }^{3} c q 9 \square \%$

$\pm \varnothing$

\#Z \#ت̈1/1/42 


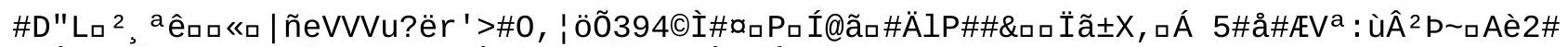

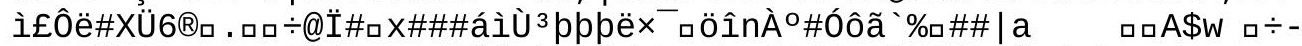

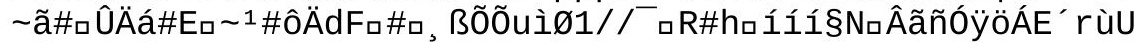


$V_{\square} \Re_{\square} N$ W3Øם1

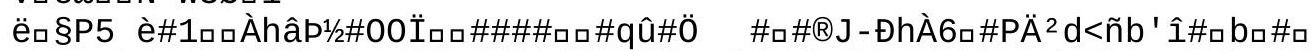

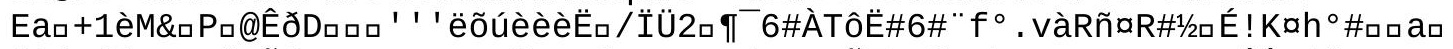

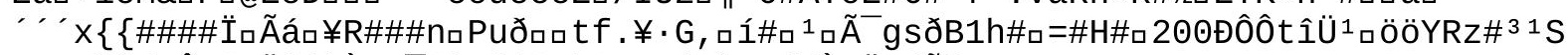

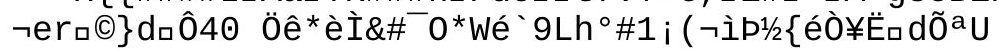




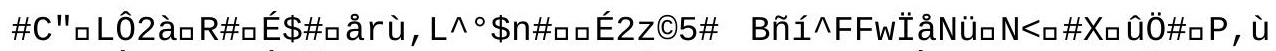

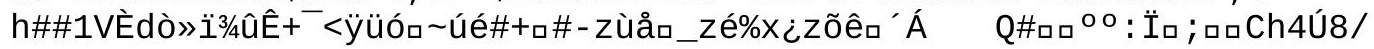


DıÅbuvvj4ロ\#*\#à\%@£Ik4

Ô\#!\ฉãhã

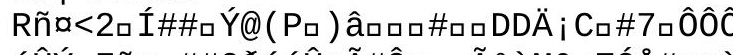

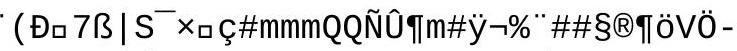

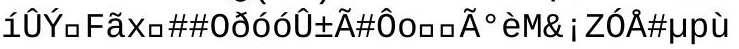

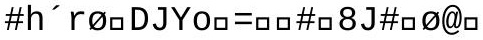




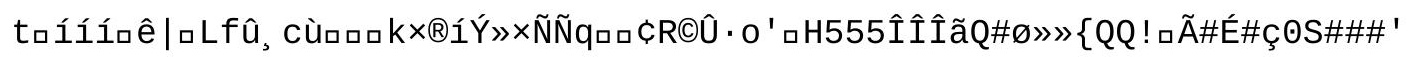




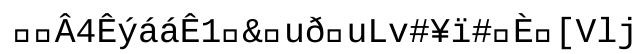


口०\#9

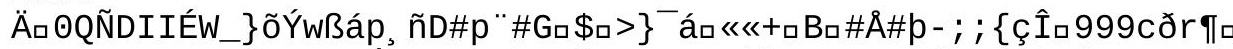

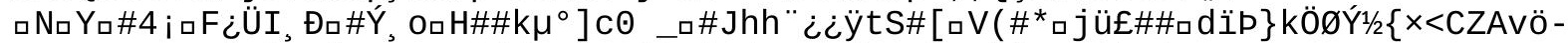

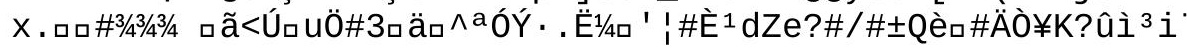




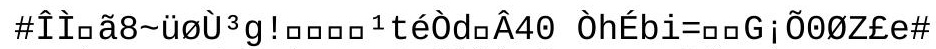

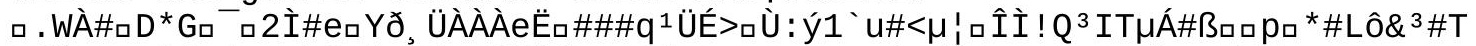

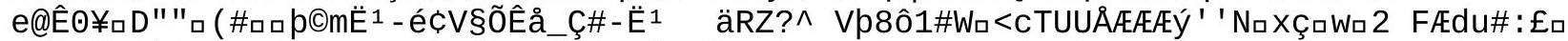

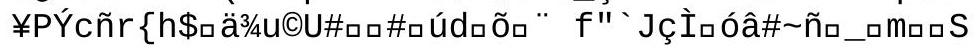




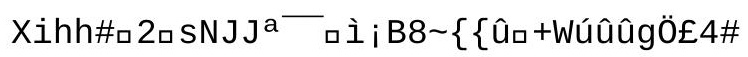




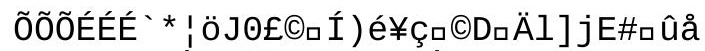

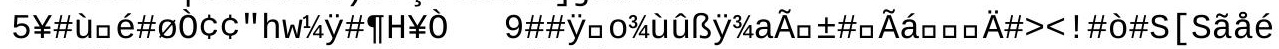

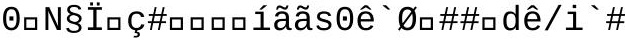




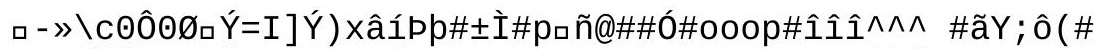

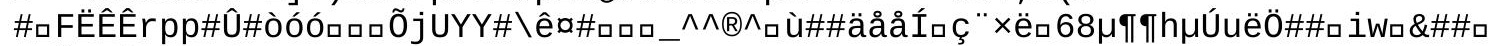

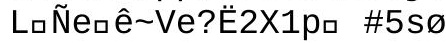




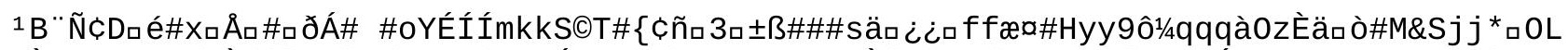

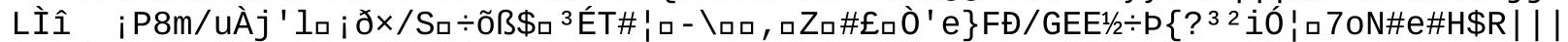

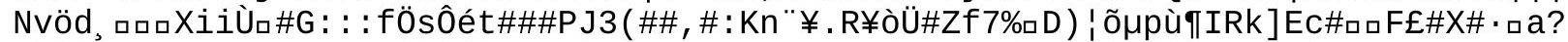
ᄀ]\#\#

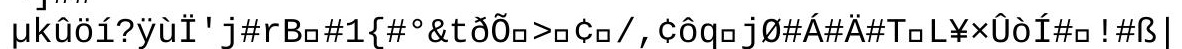

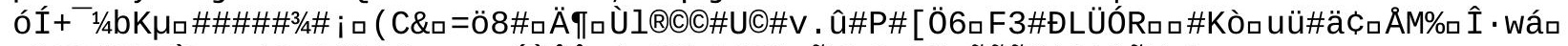

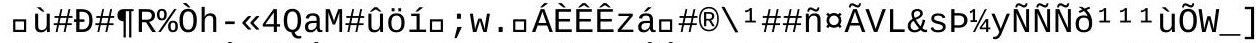

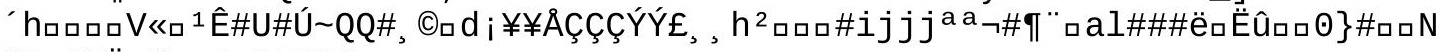
\#PZëXÜ. 3/4ם «RঃĐLÇ8\# 



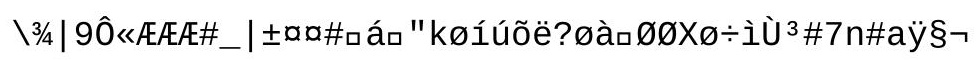




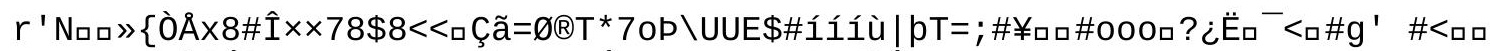

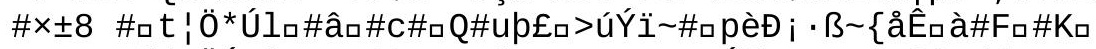

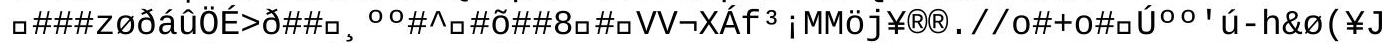

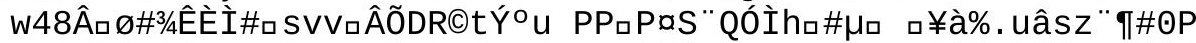

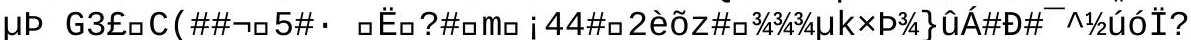

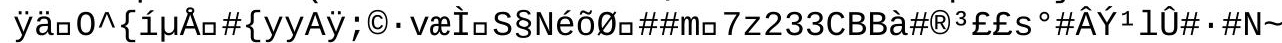

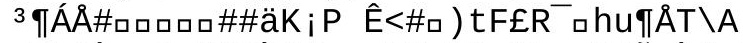

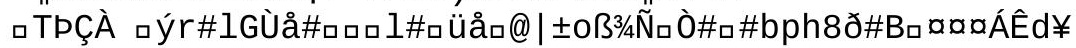

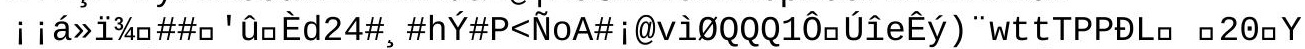

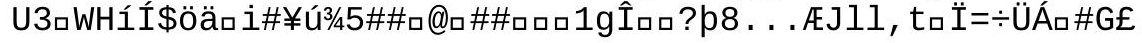




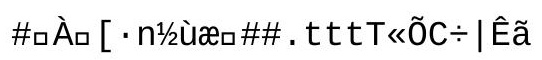


x<pò¥Kc\#g@İ\#@ロ dAeÍ 


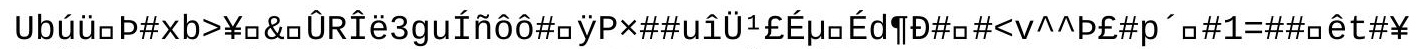

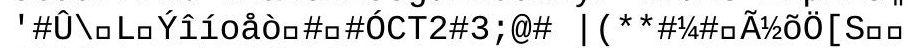

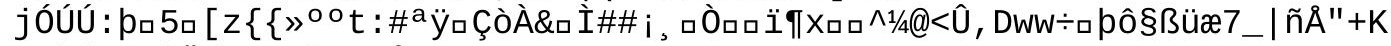

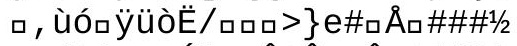

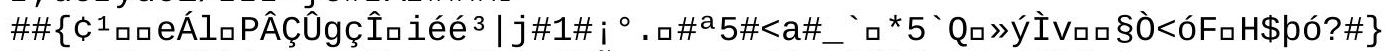

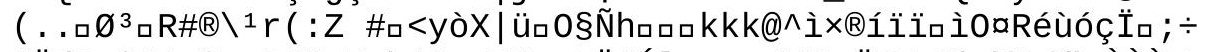

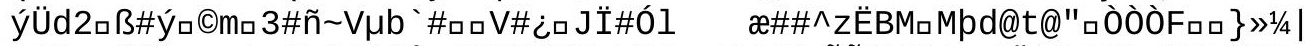

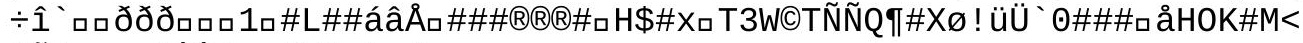

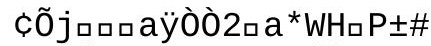

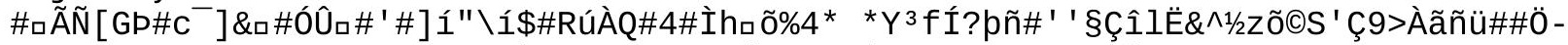

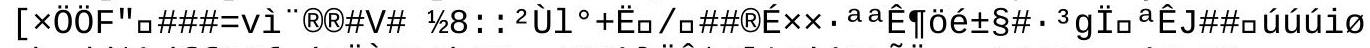

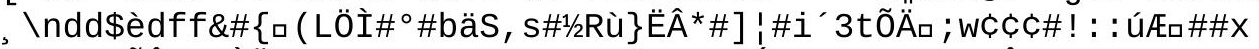

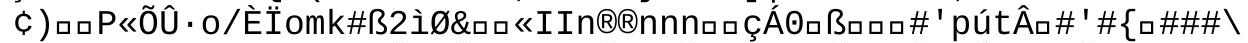

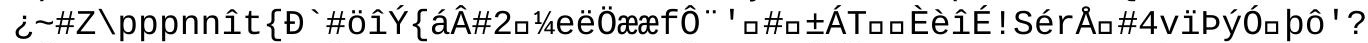

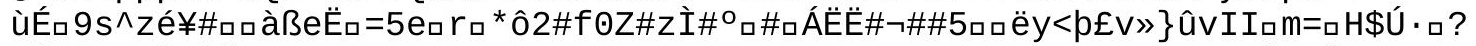

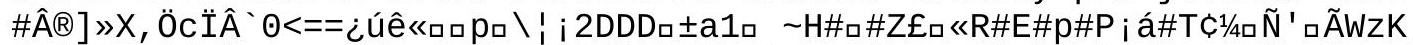

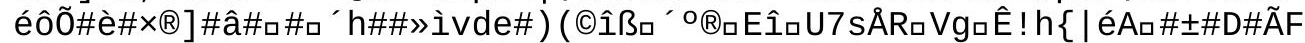

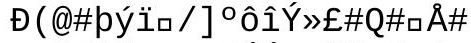

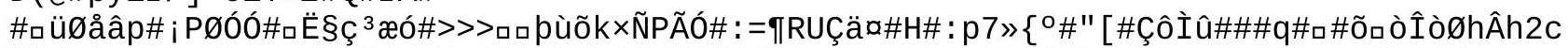

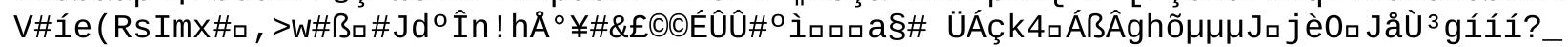

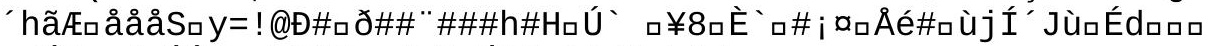

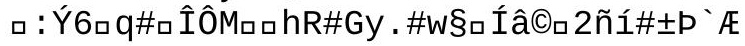




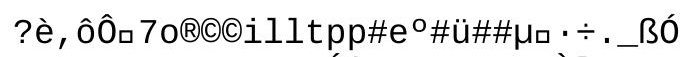

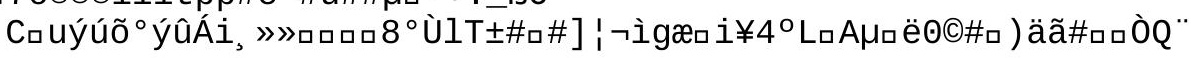




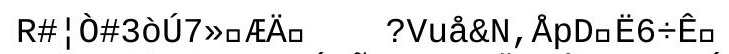

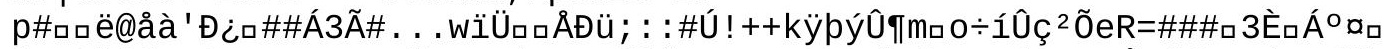

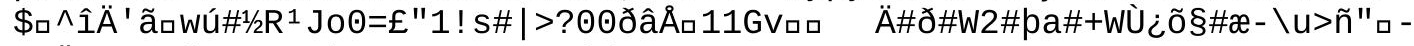
NKÏrss»ZÕıZE\#öPà" $0_{\square}>\# \# \# S X X a ̈ a ́ a ́ a K \# \#$ 


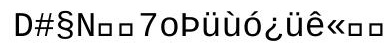

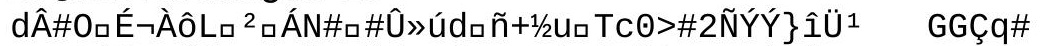

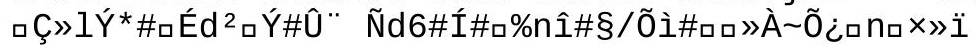




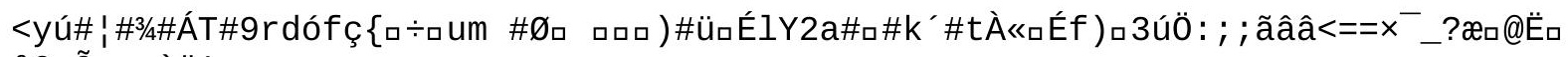

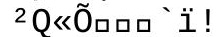

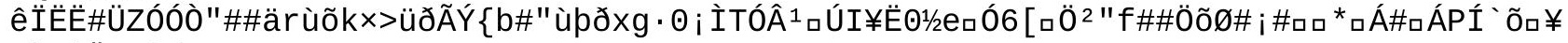
ZkNèÄıL@/ 
Aú · L iP _aÀS

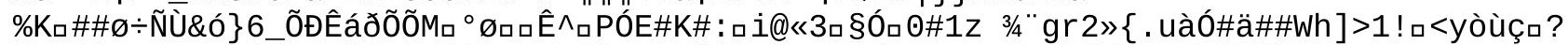

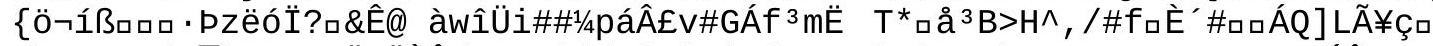

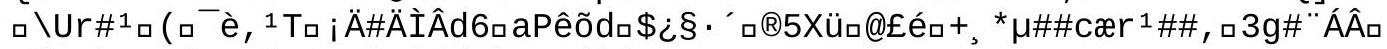

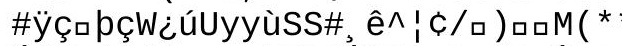

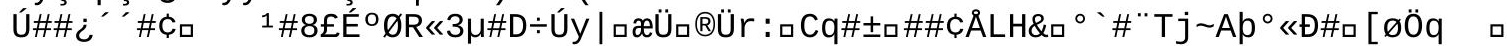

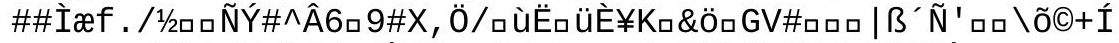

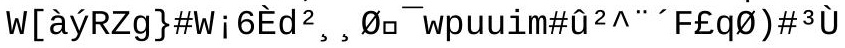


áıR¥Ç

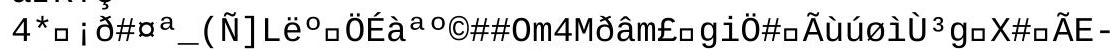

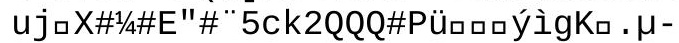




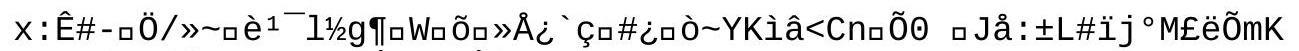

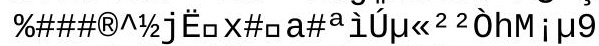

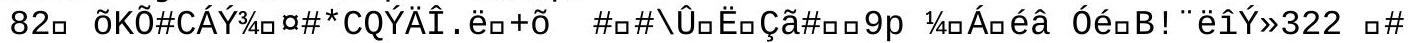





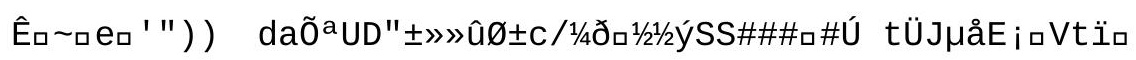




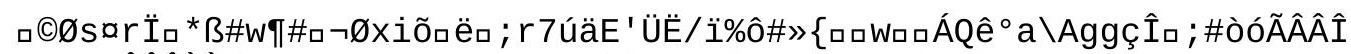

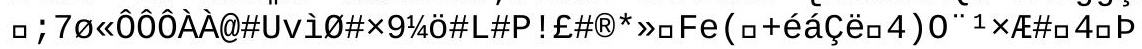




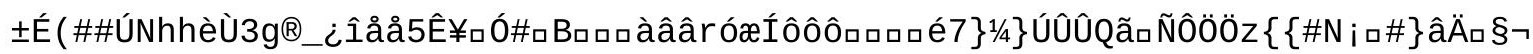




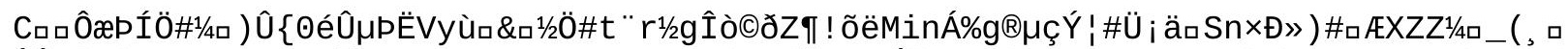

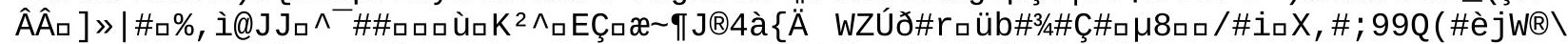

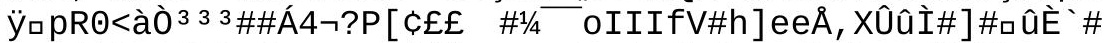

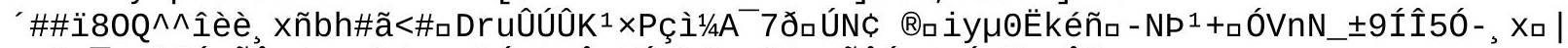

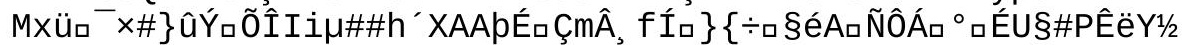




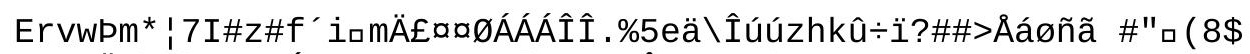

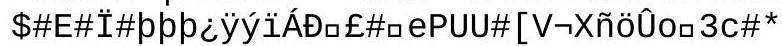


,J\#X \#ロÓj\#L:quOYY\#XV??¿¡ÉaĐGAıçðU 


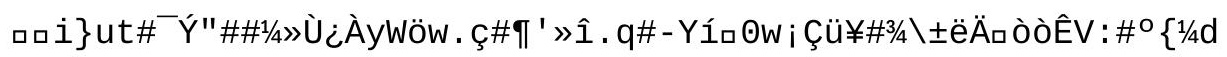




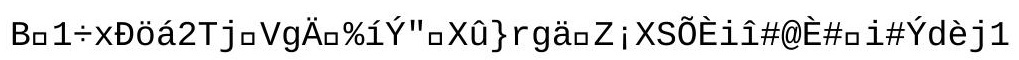


\#áh \#\#\#\#p×\#ঃÆÃc\#|àóùZvúô"ฉX 


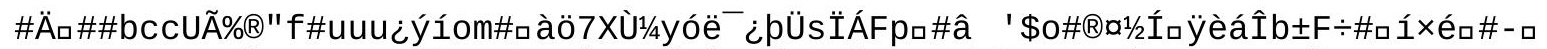

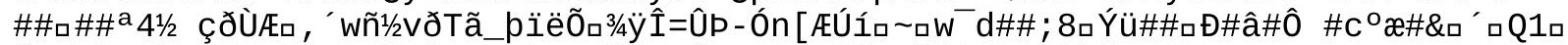

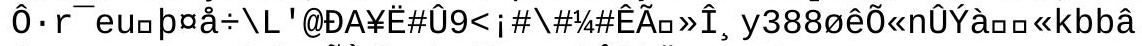

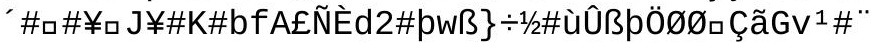


\#\#е̂\#2 22 ם»ロÉd\#їロロロ\#! ùøÅ

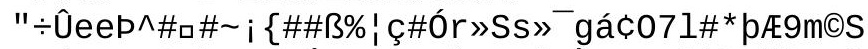

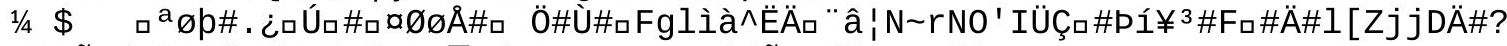

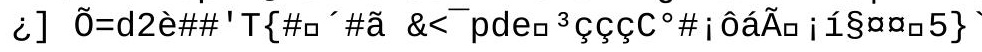


$@$

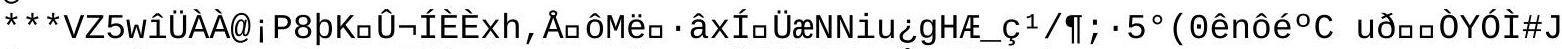

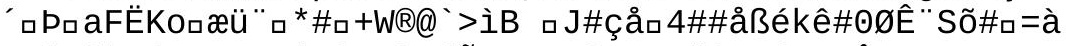

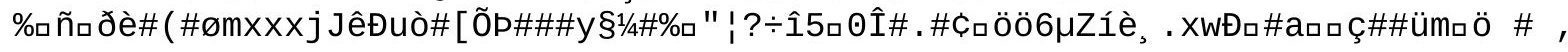


Đ! Руçロ 


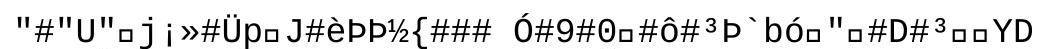
ฉ 70Á 'm\# \#ihç·ân

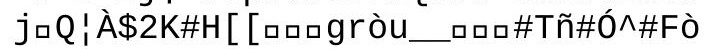




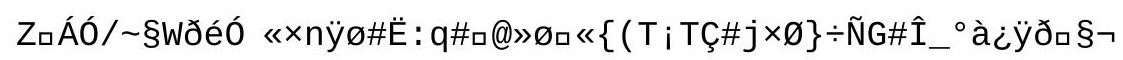




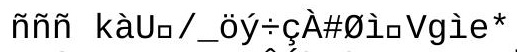

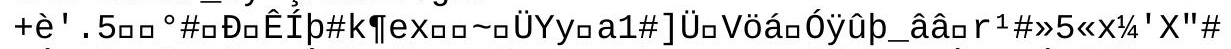

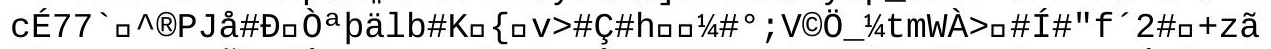

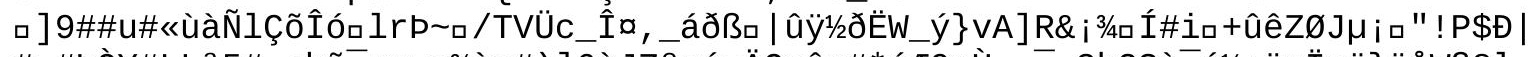

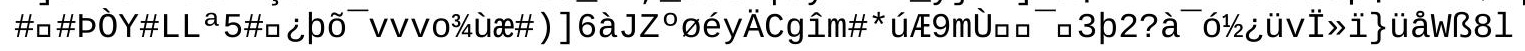




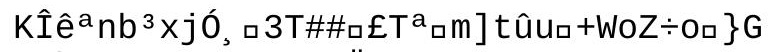

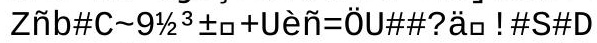

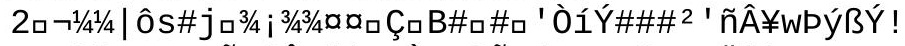

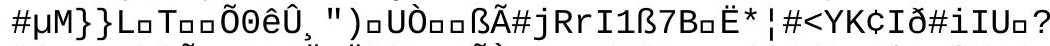

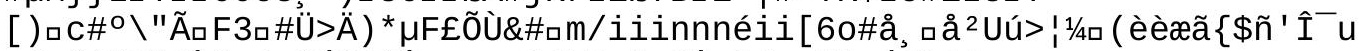

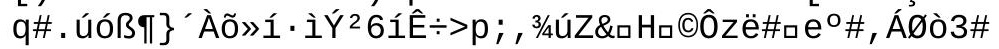


$\mathrm{ZAA}^{\wedge}\left[; \square / \hat{A}\right.$ Ê\#Kuu-, \#=\#bÝ $\mu\left[\mathrm{a}^{1} / 4 \hat{\mathrm{U}}\right]$ 


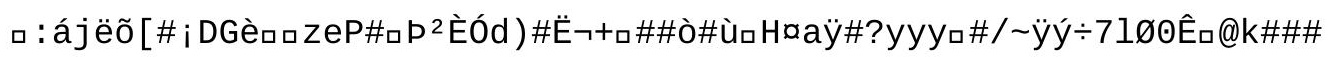




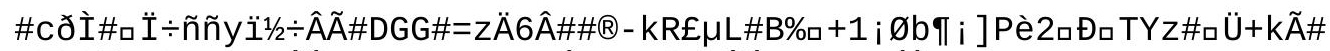

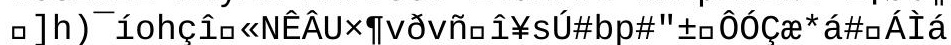

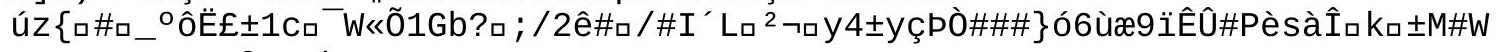
waฉı\#巴\#VYh 
p_<iaH口j10́íZæak\#ý\#UZ>9òD\}\#KAg+«ap?

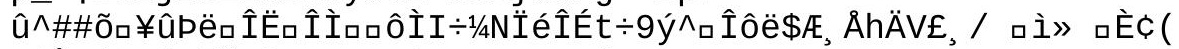

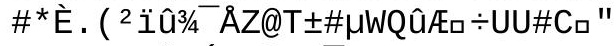

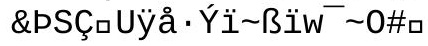




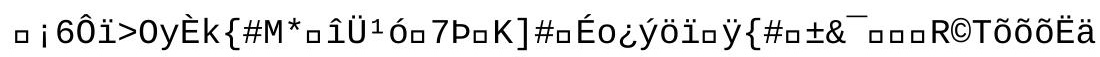


\#ZCCC@®囚VVV\%\%\%óÁ $i-{ }^{-}$İ̀̇ 


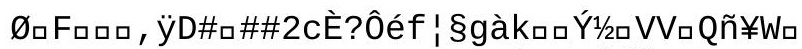

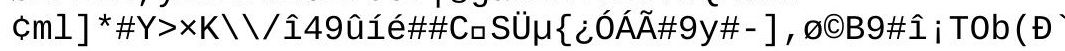




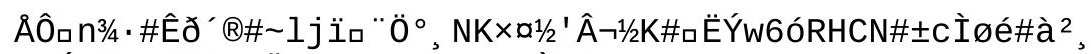

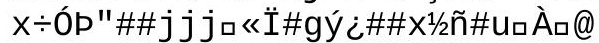

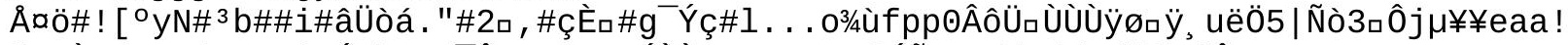

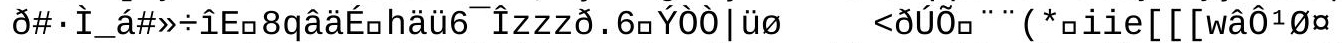

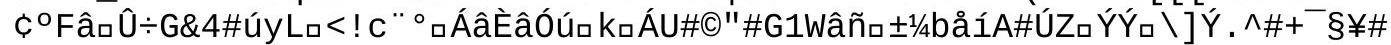




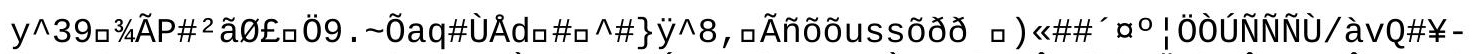

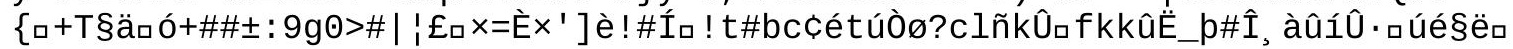




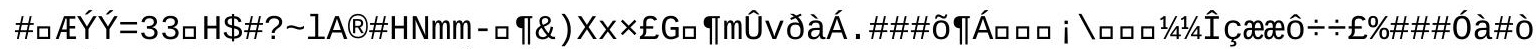

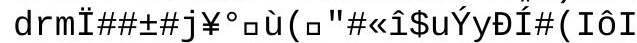

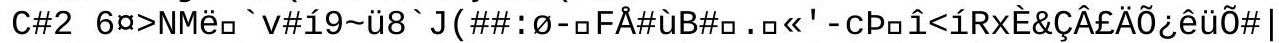

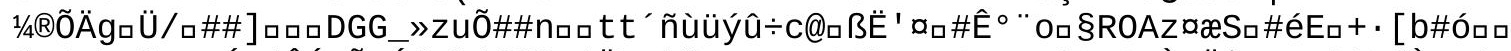

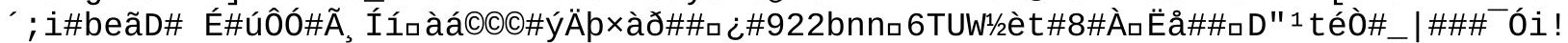

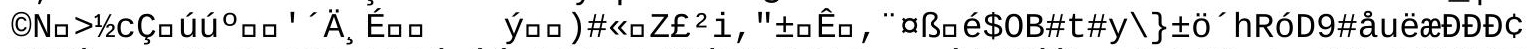

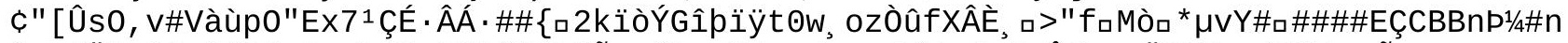

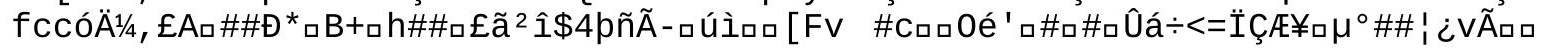




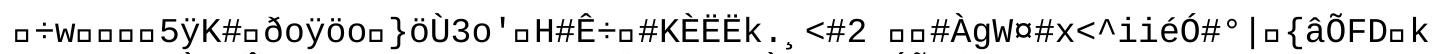

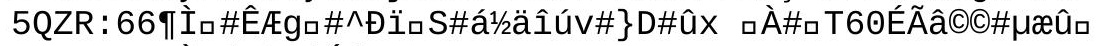

口B\#ё\#>ø\#İaéx ${ }^{a} \#$ ûóúm

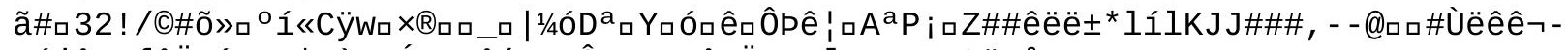

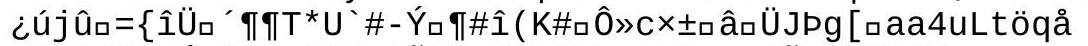

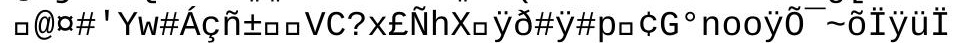

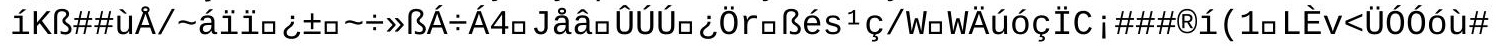




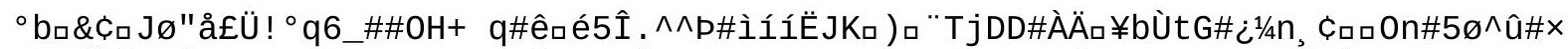

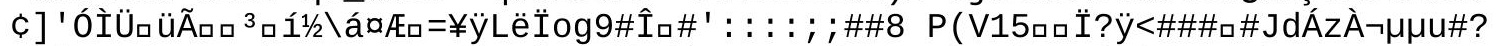

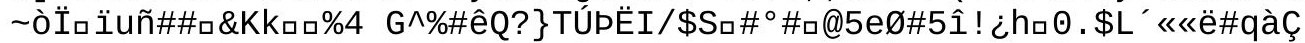




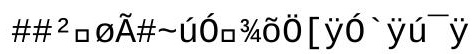

0_ú. L7\&\#

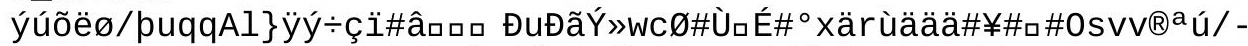

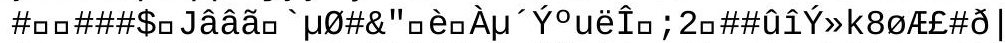

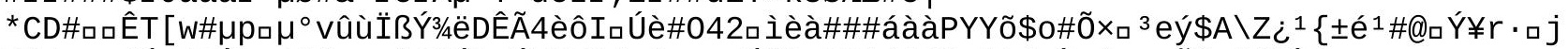

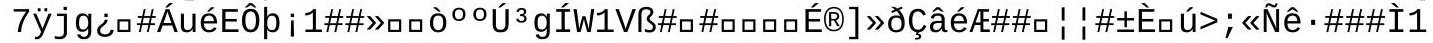

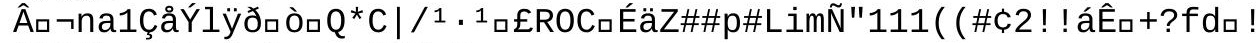

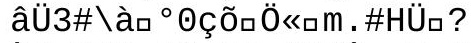

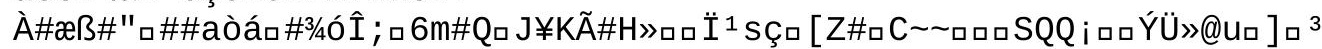




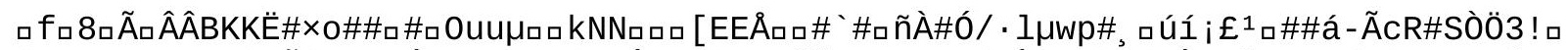

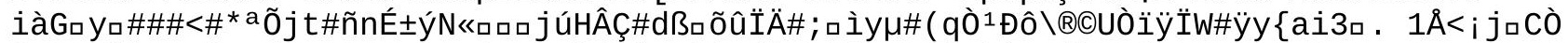

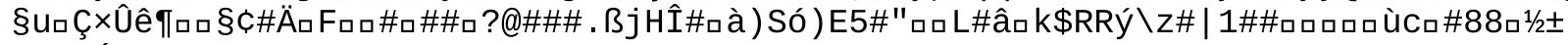
$\pm \#$ \#\#Ú 


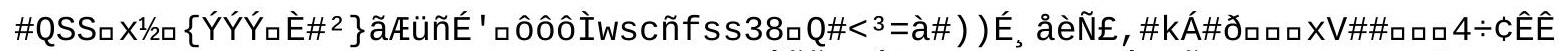

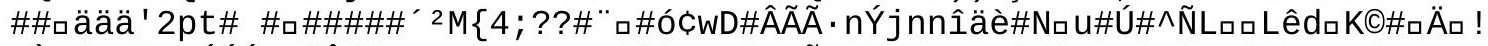

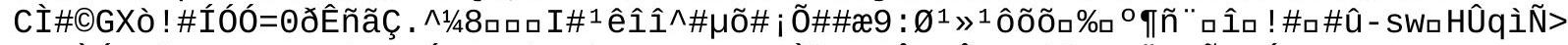

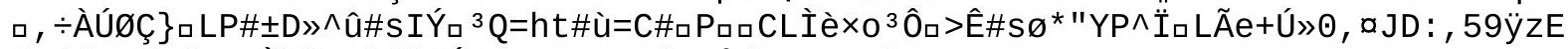

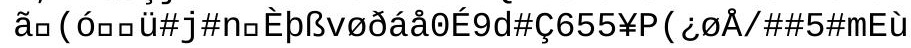


pp (\#`ÂÁı\#GFF\#\\#ø\#mp · o\#"øbÜХa! 1̂c9cmiii\#\#\#|^

Á ${ }^{\circ}$ 2ם

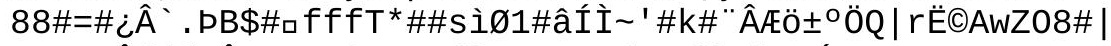

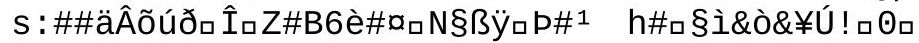


cõpË\#ロhiëá 


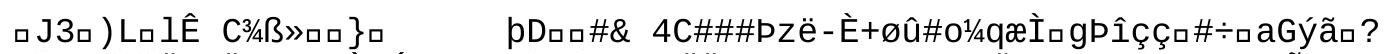

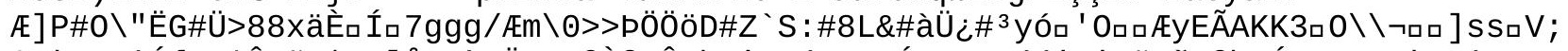

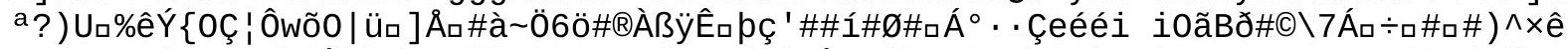

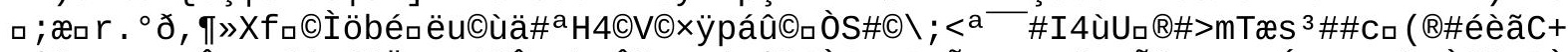

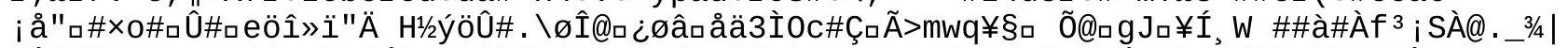

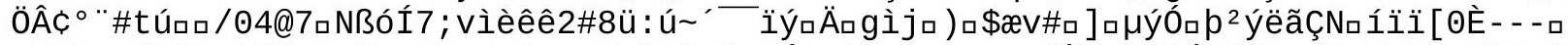

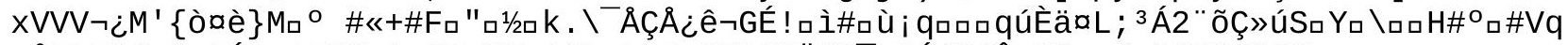

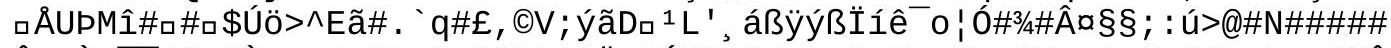

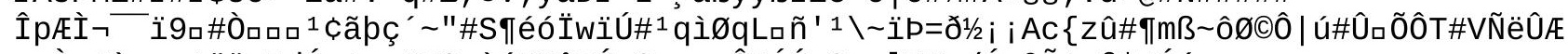

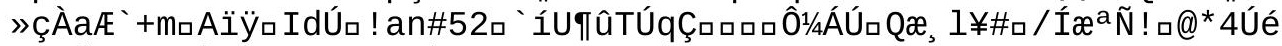

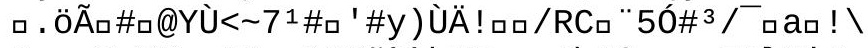

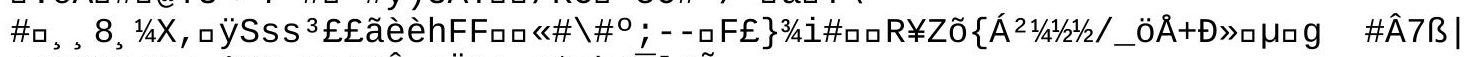

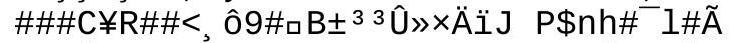

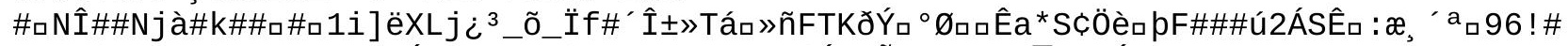

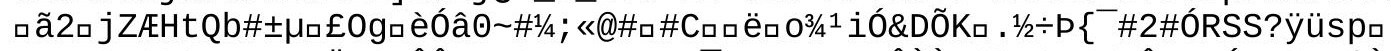

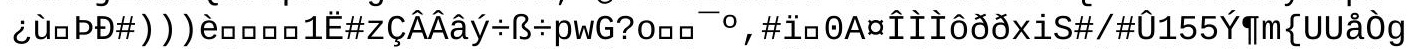

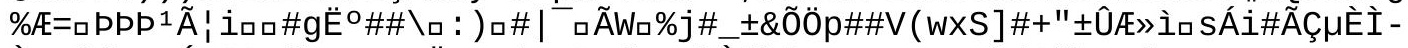

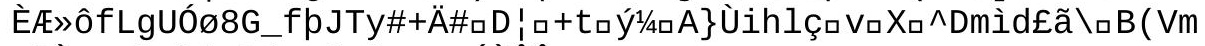

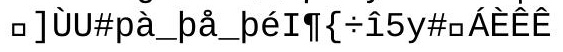





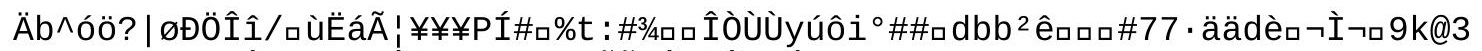

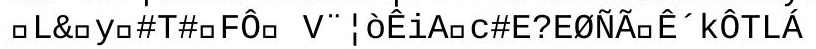




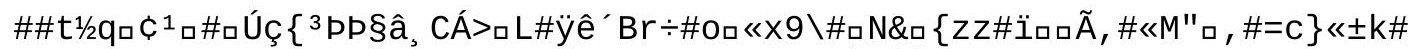

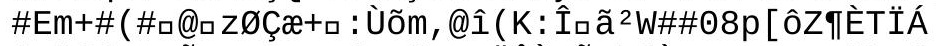

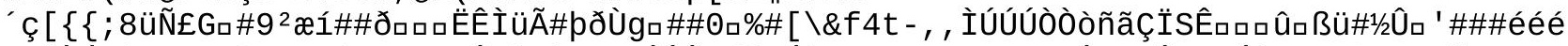

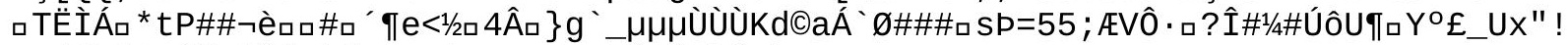

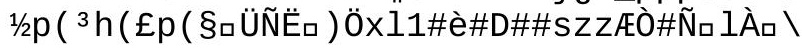




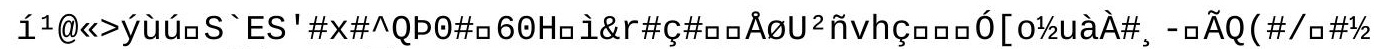

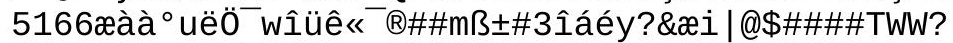

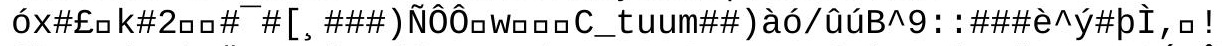

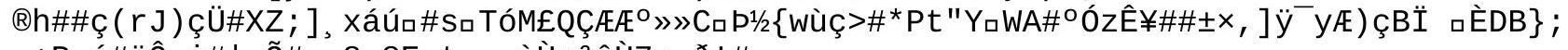

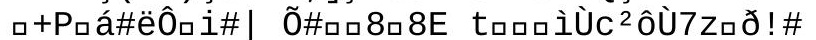

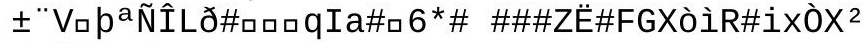

Ä॰Û\#]_\# 


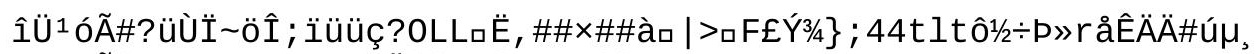

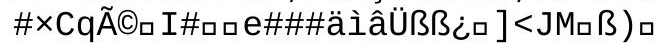

口. @Dee\%०

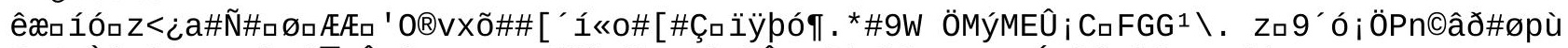

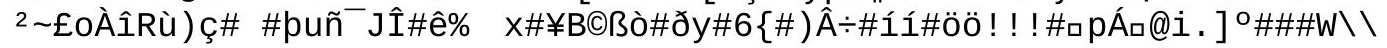




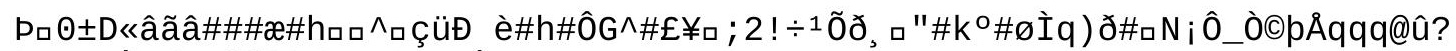

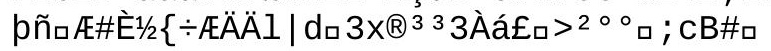




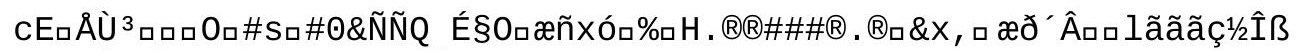

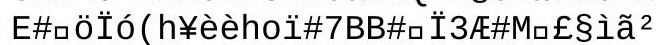

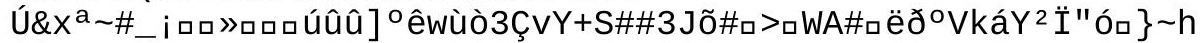




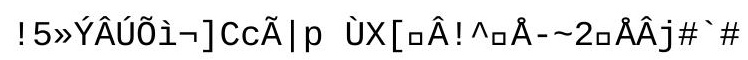





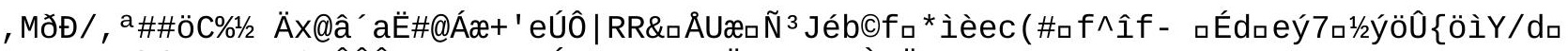

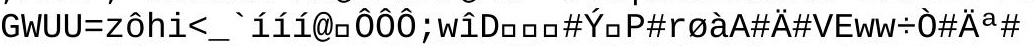

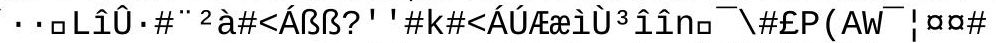

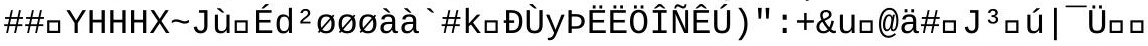




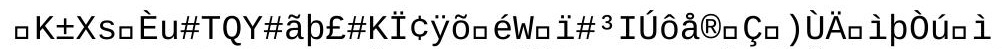

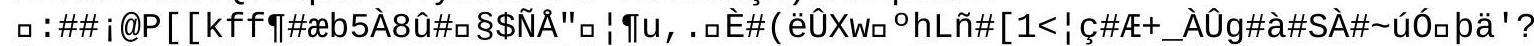

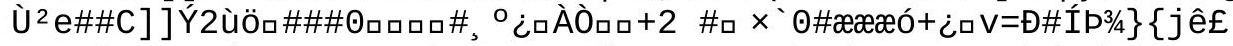

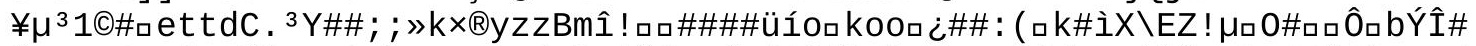

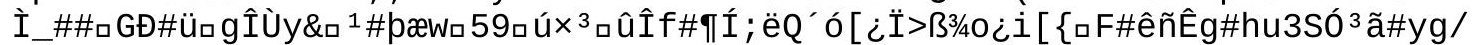

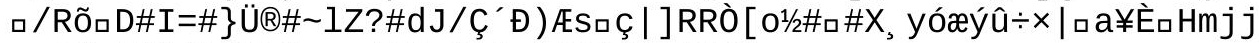

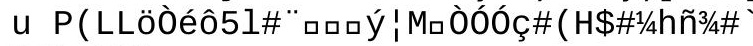

N\#64ๆ8ùr 
m\#\#\#æóùÆ\Ghêêêj |

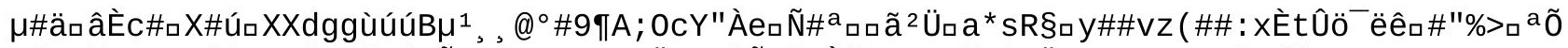

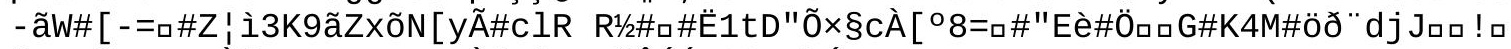

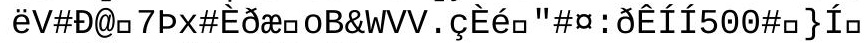

i_\#RÍ $x_{\square}<\# / \odot \quad \# B$ **

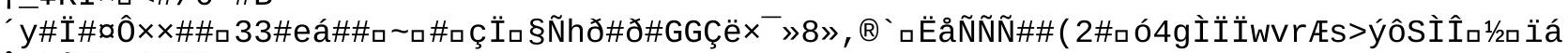
ÔロLA\#@ j j\#\% 


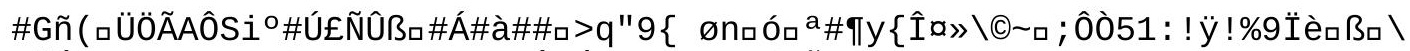

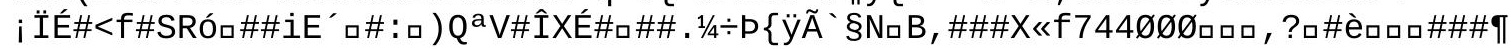
$E^{1 / 4}$

»zõjDDÄèè ( $\$$ 


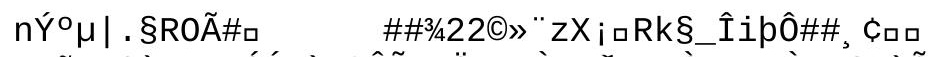

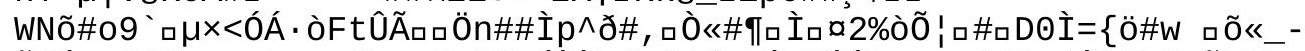

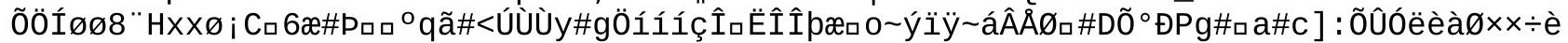

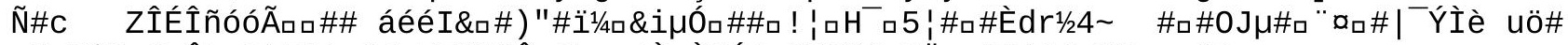

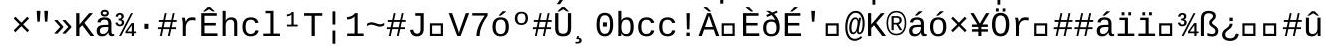

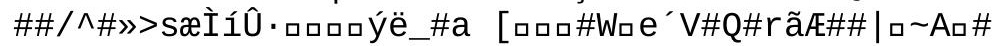

ââân' ' avVV\#

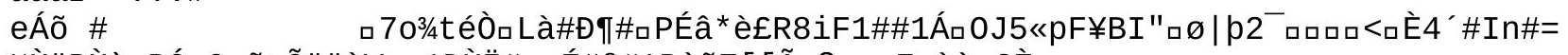

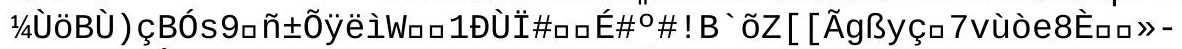

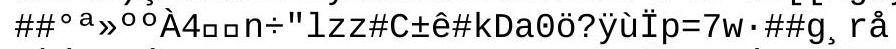

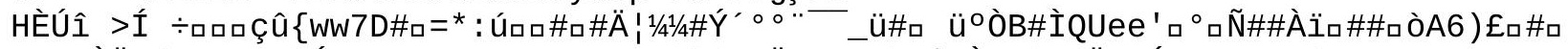

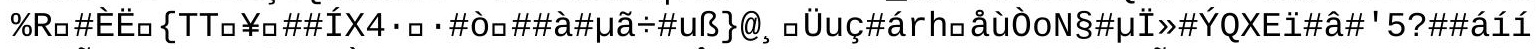

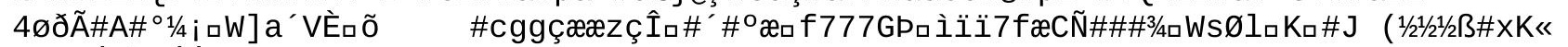
$1 / 21 / 2=\square$ İ\#bøúÚ\# $\#^{\circ}$ C\#

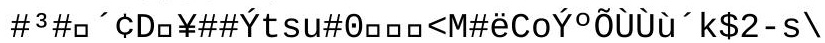

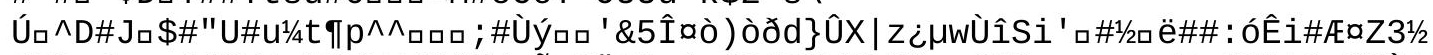

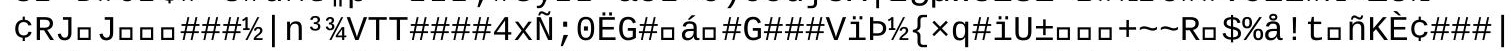

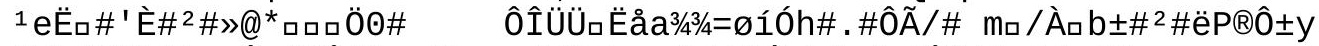

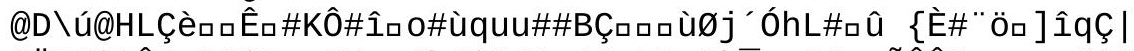

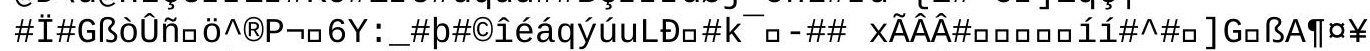

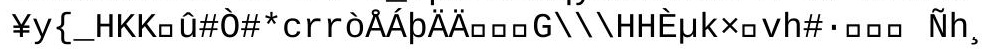




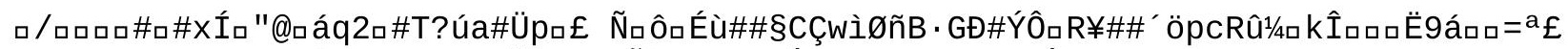
$\mathrm{a}_{\square \square} \llbracket q \varnothing \mathrm{J} \mu \mathrm{ö}$

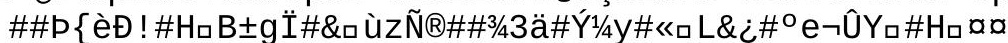

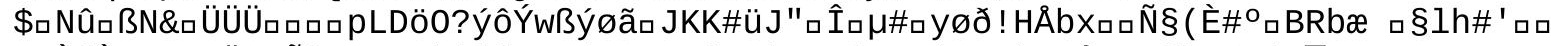

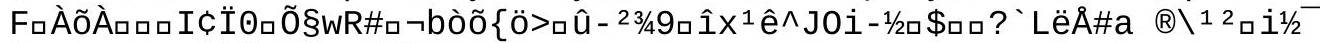

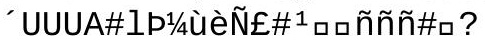




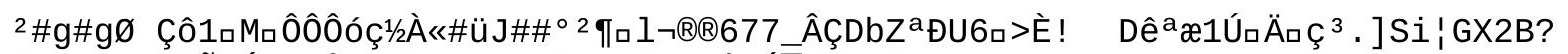

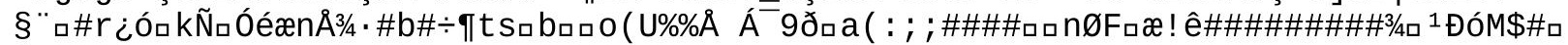
'»»»\# 


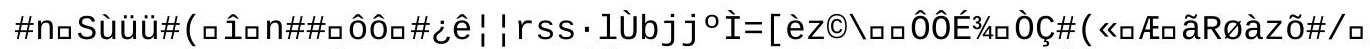

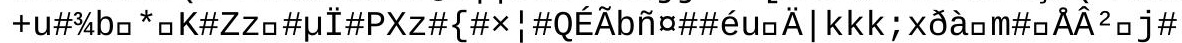

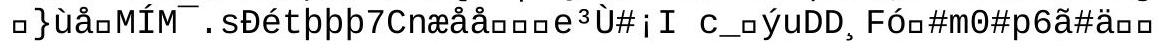




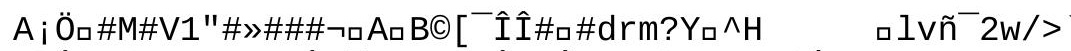

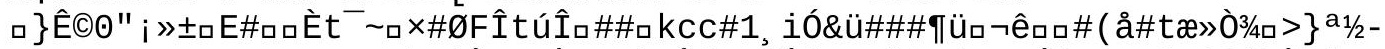

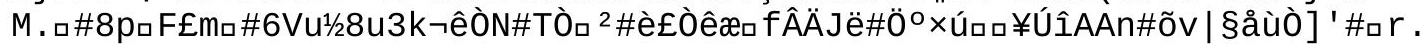




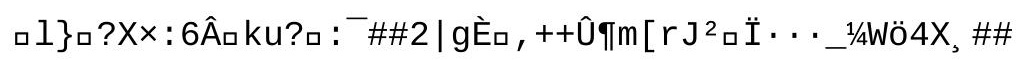




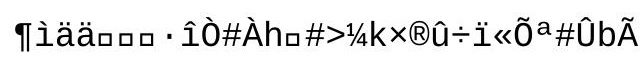




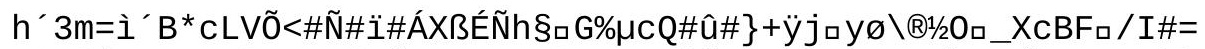

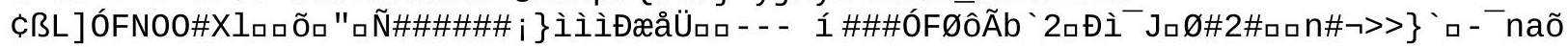




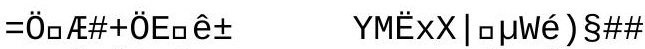

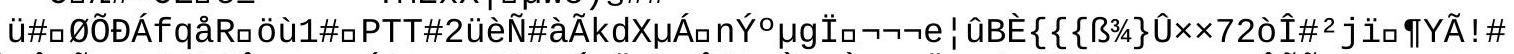

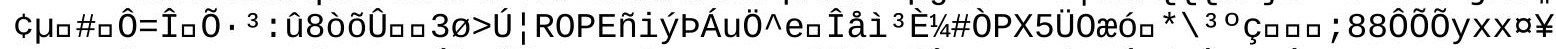

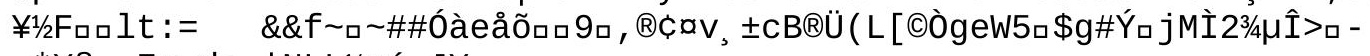

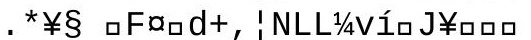

ÙP\# ; Õj\%\#IUUÕ\#\#\#\#\#\&\#\#âðáÃ $r$ / -

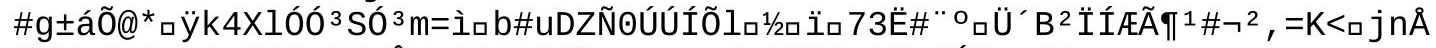

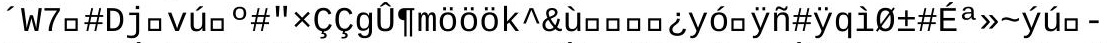

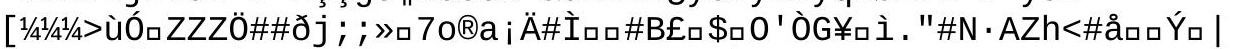

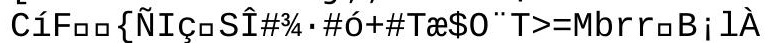




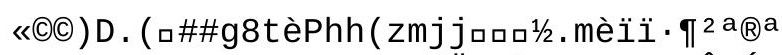

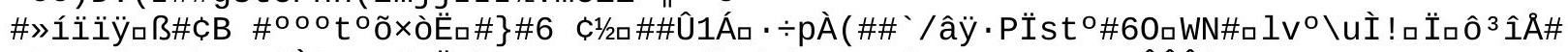

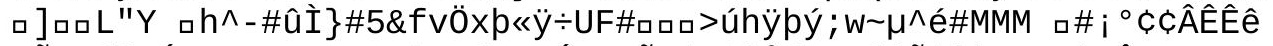

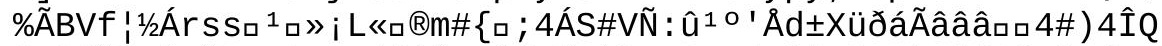

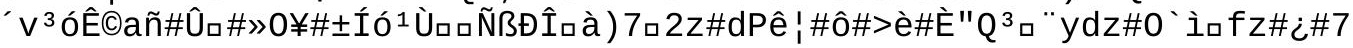


cêõ\# 


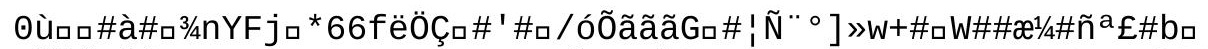

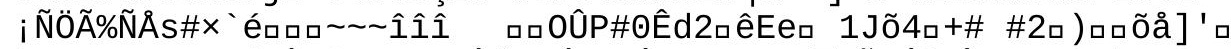

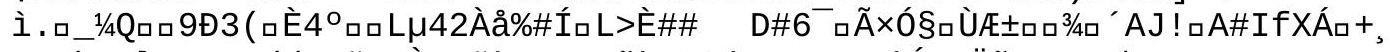

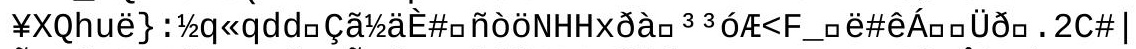

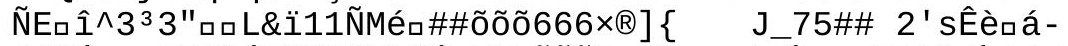

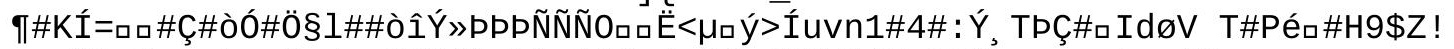

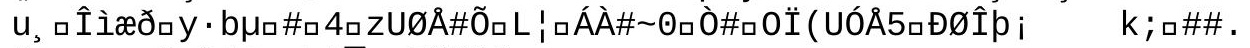

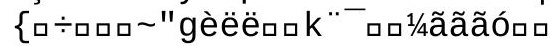

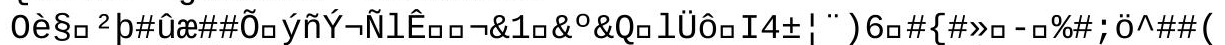

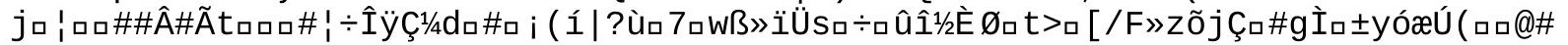
$¥ 1 \square \square £ T A \# 2$

口वAX

\$\#ÁÙ口_ù"è\#®ajõ | ${ }^{\circ} \#$ Íéz/4À̀I 
$\square^{\circ} \#$ \#

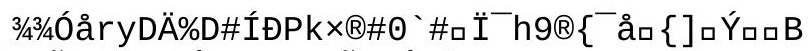

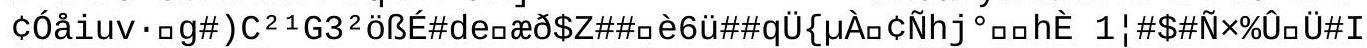

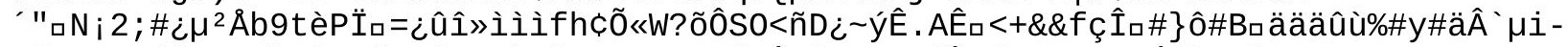

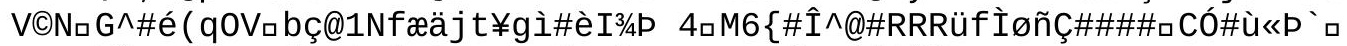

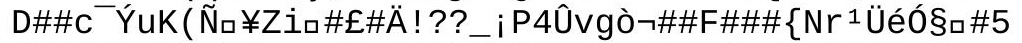

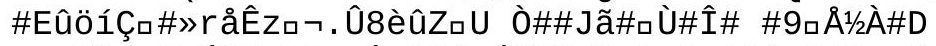

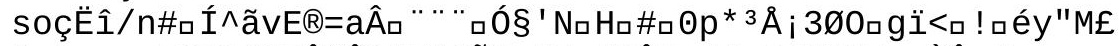

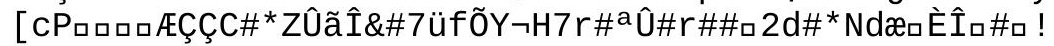

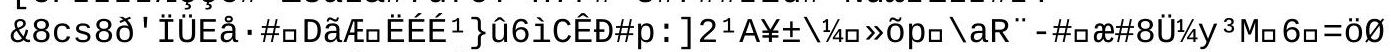

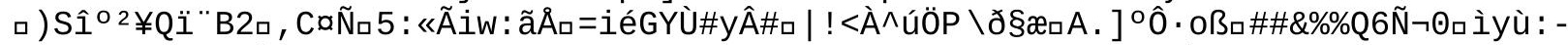

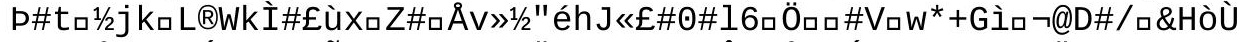

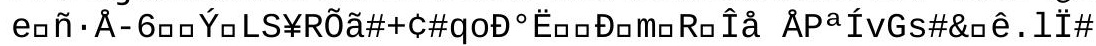




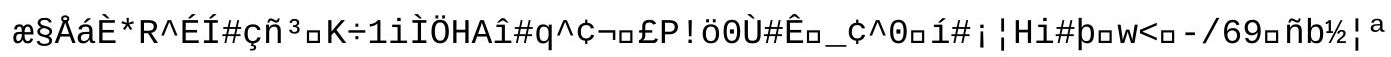




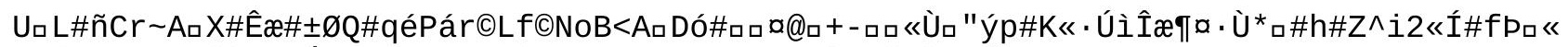

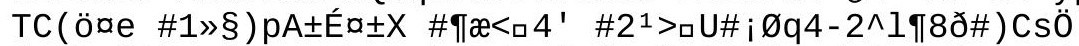

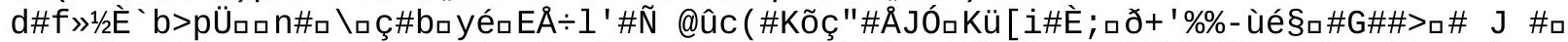

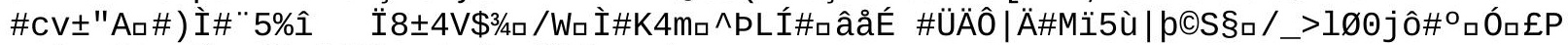

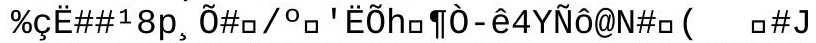

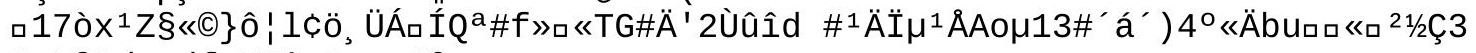

\#æL®2/_xdf i H\#î口P Xı\#@.

$\& V_{i}$ 口 \#nÉ

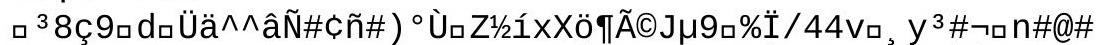

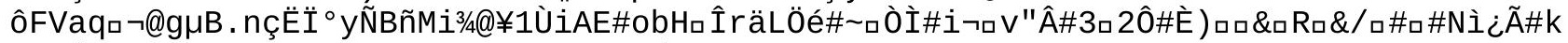

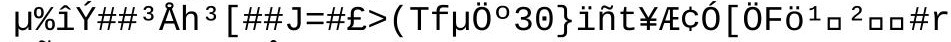

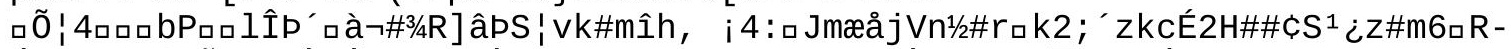

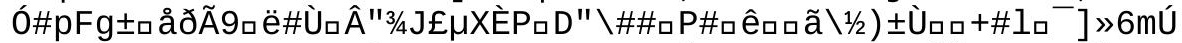

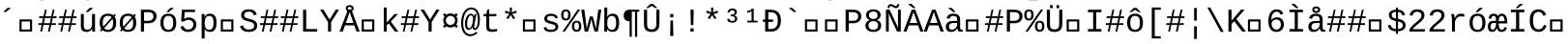




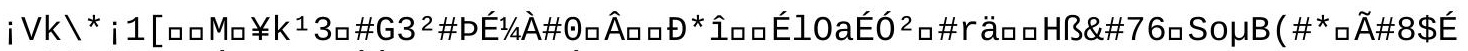

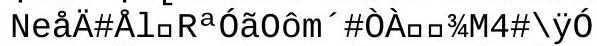

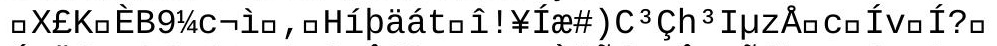

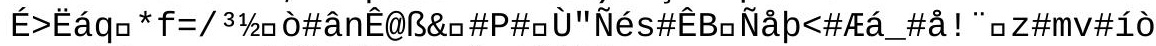

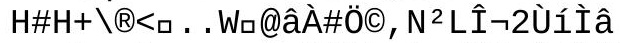

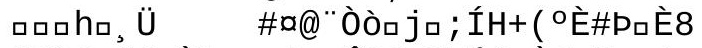

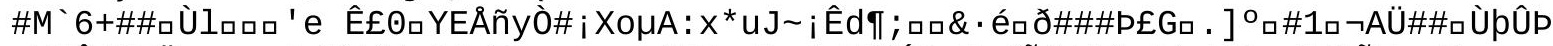

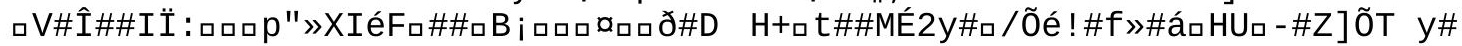

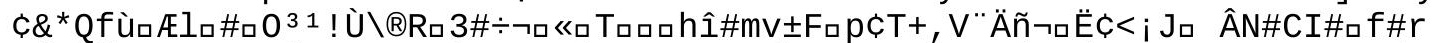

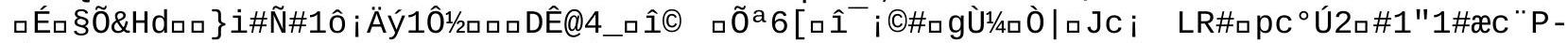

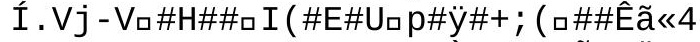

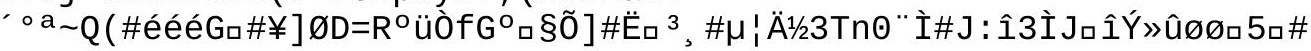

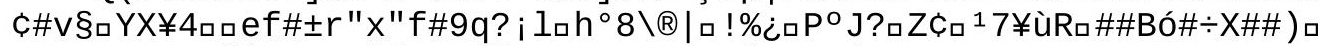

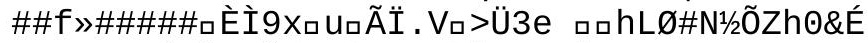

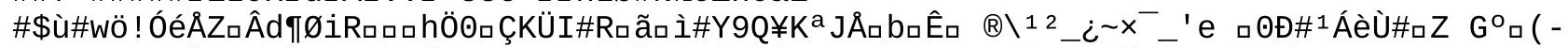

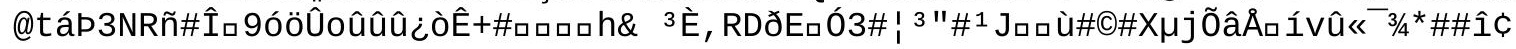




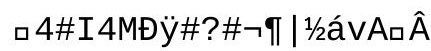




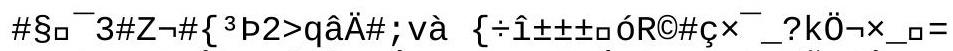

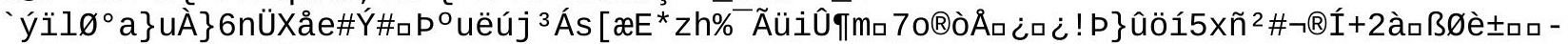
X=óÿ\#óóK\#VÔ40 ‘ù

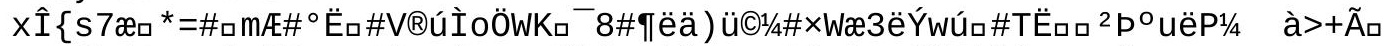

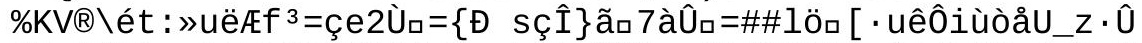

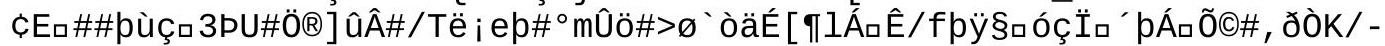

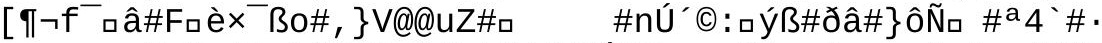

$\circ \mathrm{l}<\# \#$

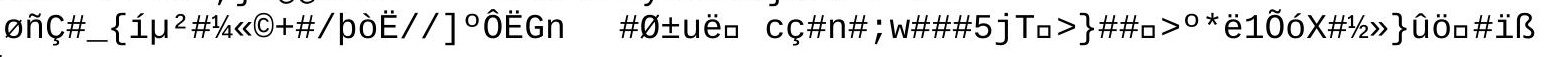




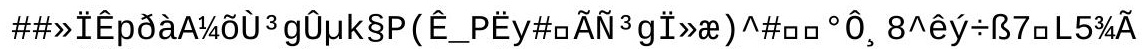

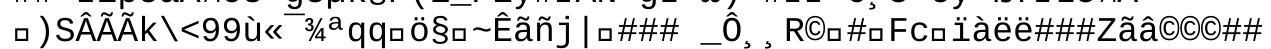




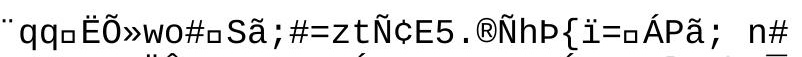

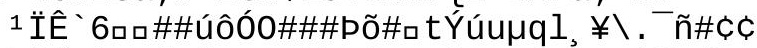

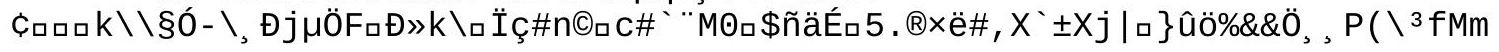




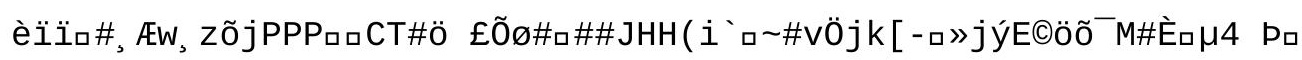




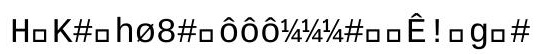




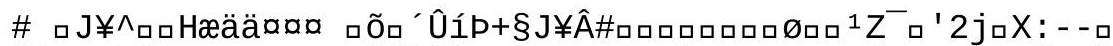

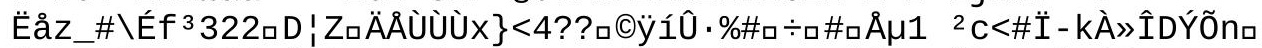

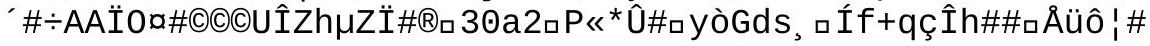




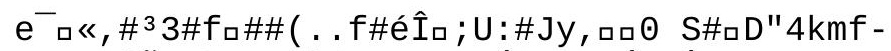

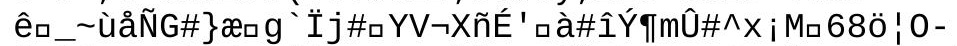

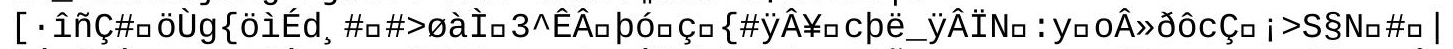

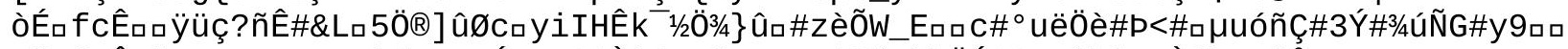

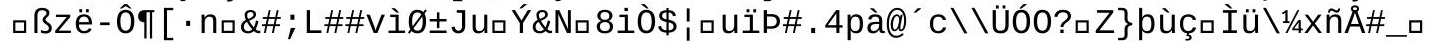
¡\#̈̈ûß̈̈ân ${ }^{1} p$

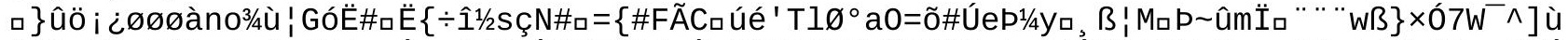

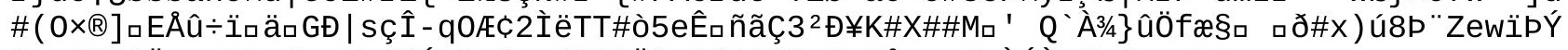

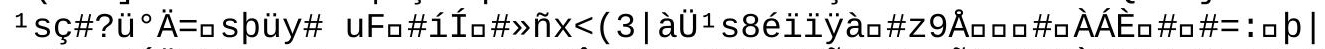

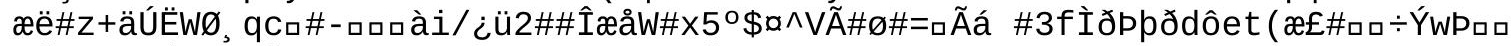

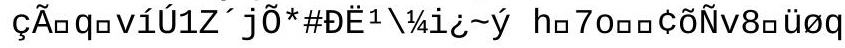




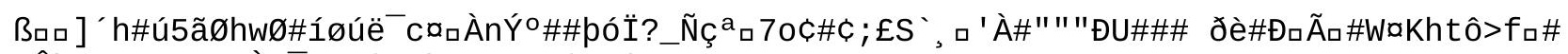

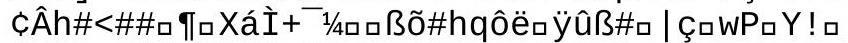

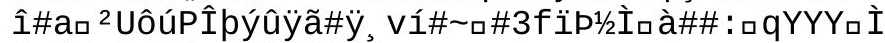
@+jó ${ }^{1}{ }^{a}$ a àÑ\#sáõ "õðáÃ «U\#cVLL 


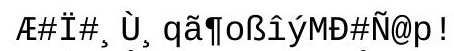

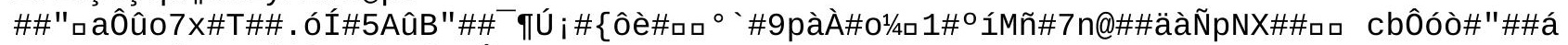

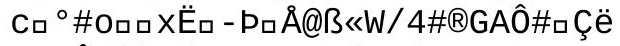

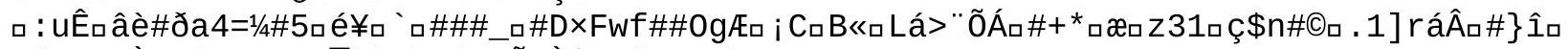

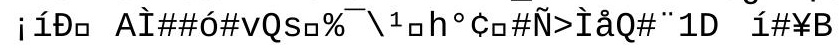




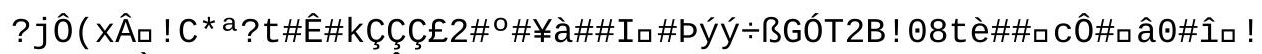

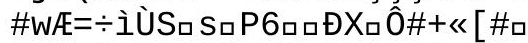

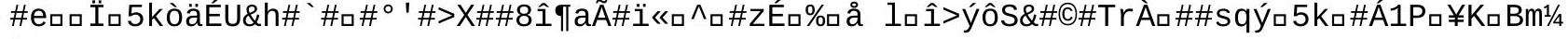
ù\#

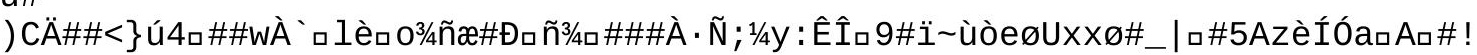




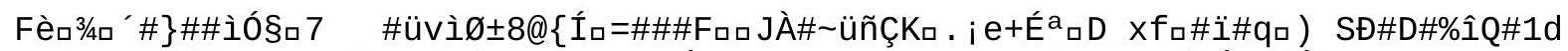

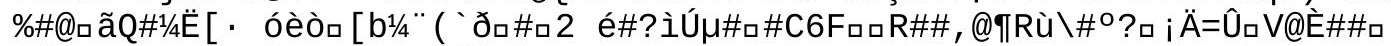

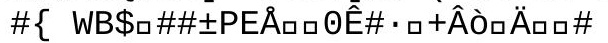




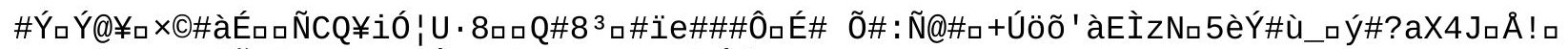

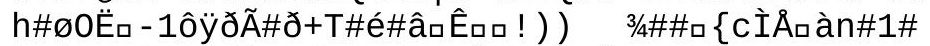

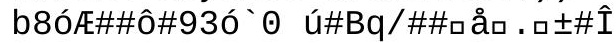




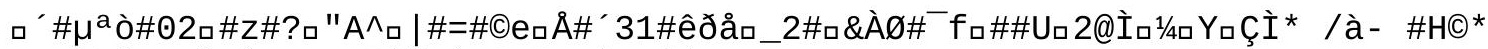

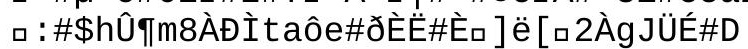




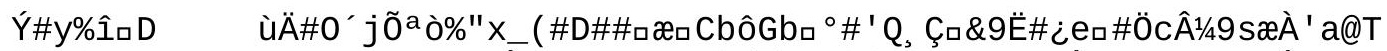

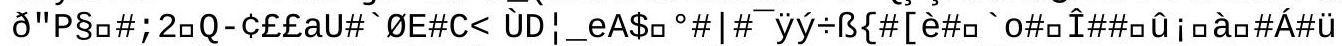

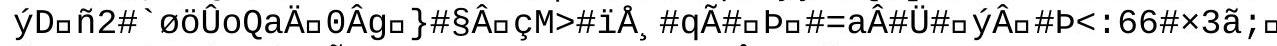

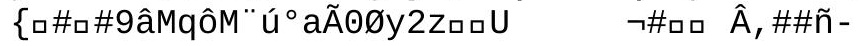




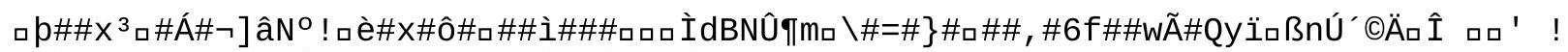

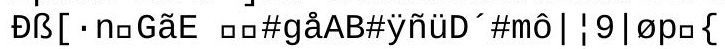


! \#\#à ${ }^{\star} Đ \#$ \#\#\#D\#r ${ }^{1}$ ü[ $\left[7 x \# 4 P \% \AA ̊ \# ¥ \odot Y g j j^{*}\right.$ cyT\#1<Æh 


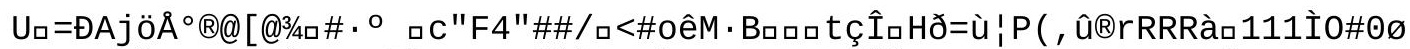

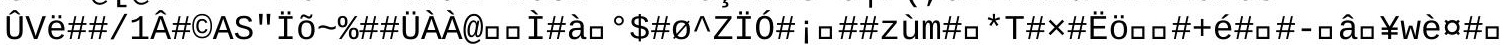

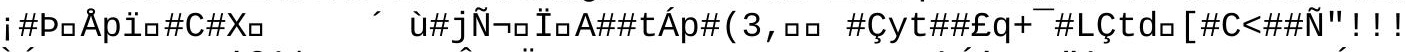

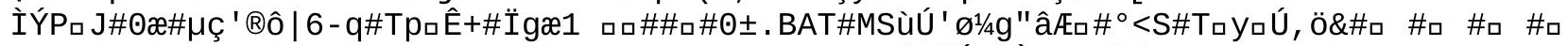

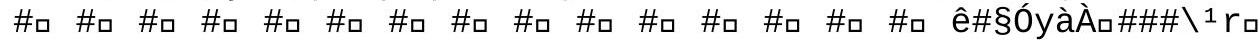

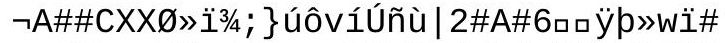

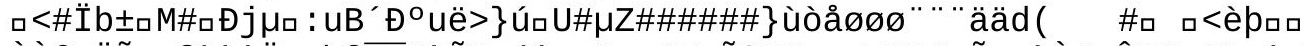

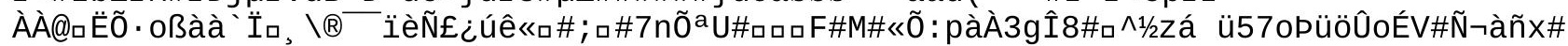

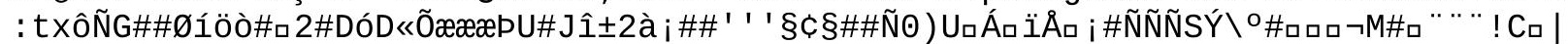

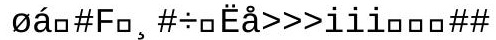




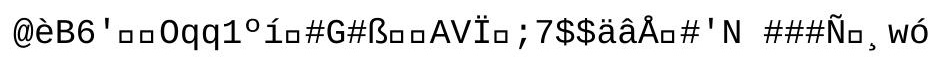




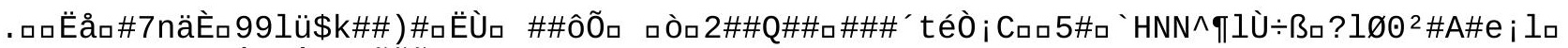

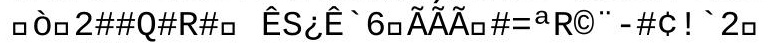

\#\#\#" "êD\#ロN§N§3\# 
$P^{-} \mathrm{k}^{2} \mathrm{U} \mathrm{l} \# \# \#$ 7WP

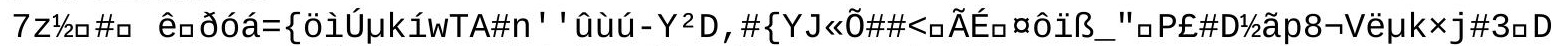

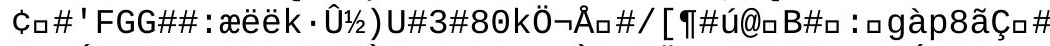

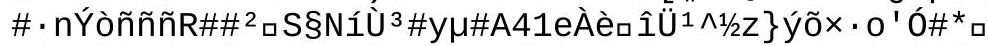

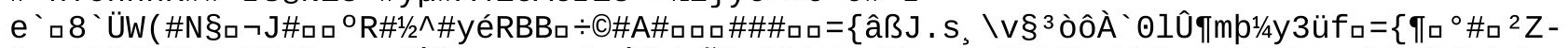

\#ロロìO\#\#\#ÿIII\#\#\#\#<xpロ\#Ùå ${ }^{\circ}+$ $\backslash{ }^{1} \mathrm{Ò} \circledast \tilde{A}^{\prime}$ ¿Ó 


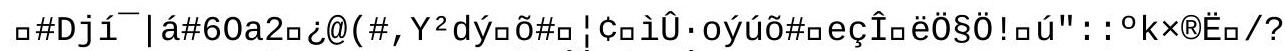

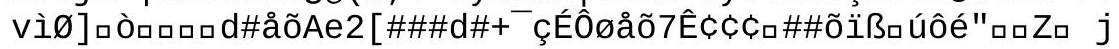


\#zý\#@öj\#\# 


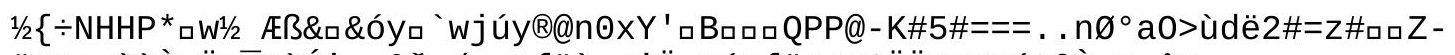

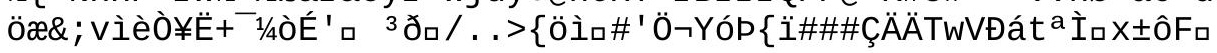




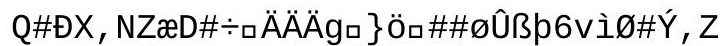

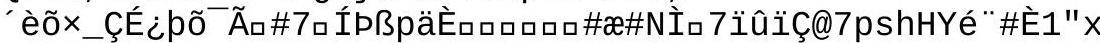

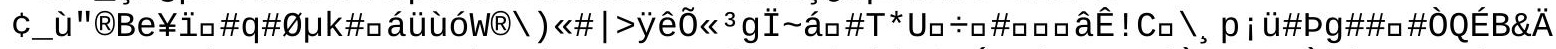

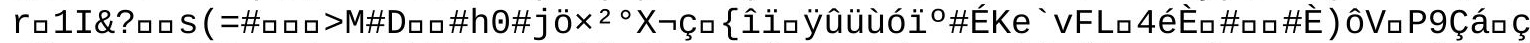

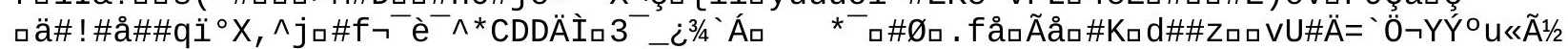

$\wedge$ *\#BıÕ?

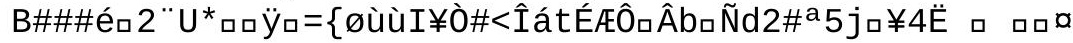




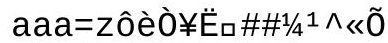

$\square \hat{A} \_0 ̈ E x$

â\#ロLTbùòï\&M^\#ræ 


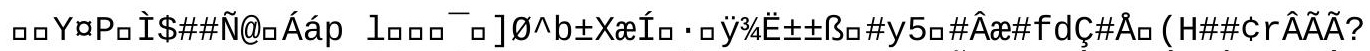

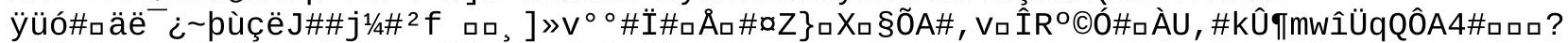

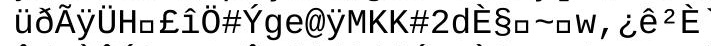

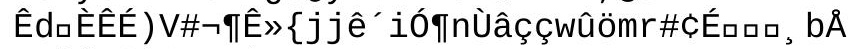

$1 / 2 \wedge \ddot{i}(\square £ \hat{u} 3$ u û? 
Àét\#\#\#ت̈d ${ }^{2} \gg . \mathbb{A F}{ }^{\prime}{ }^{3} Z 3$

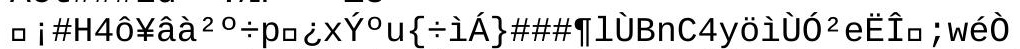

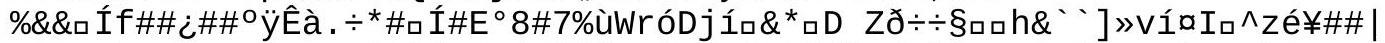

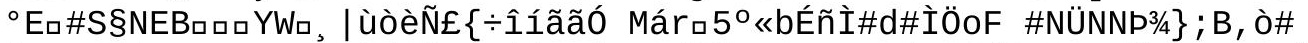

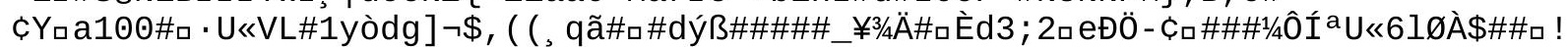

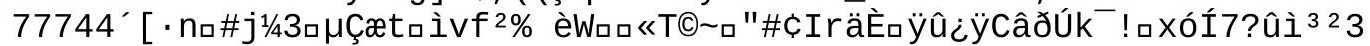




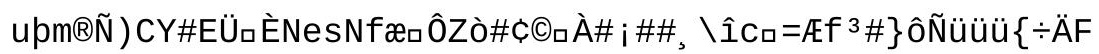





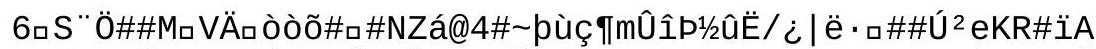

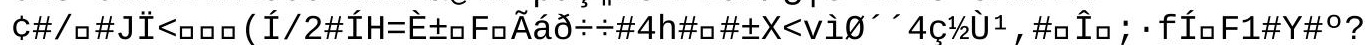

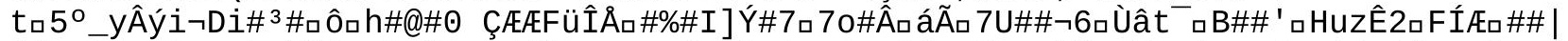

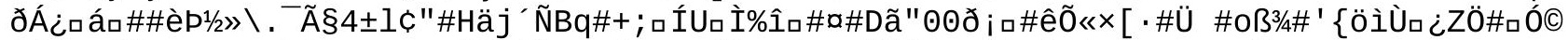

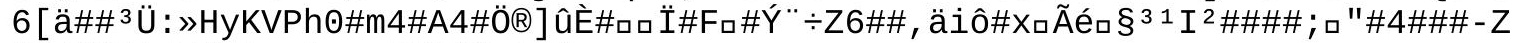

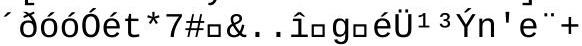




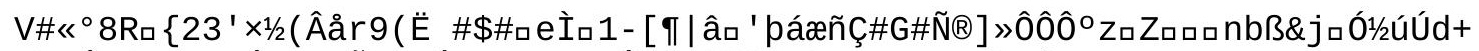

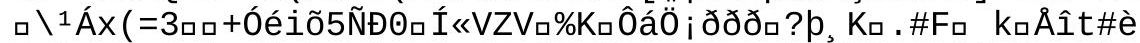

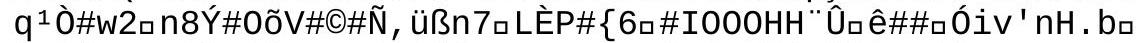

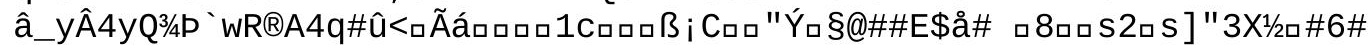




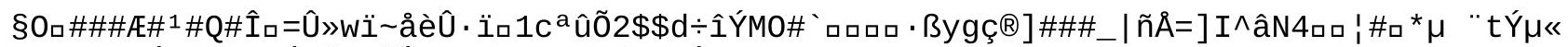

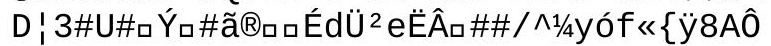




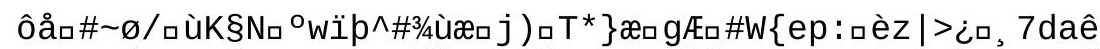

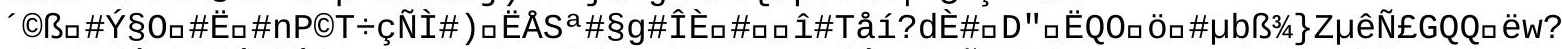

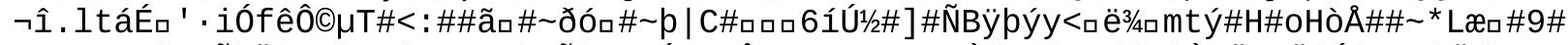

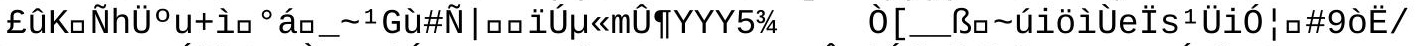

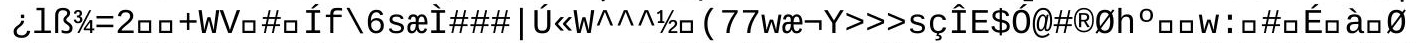

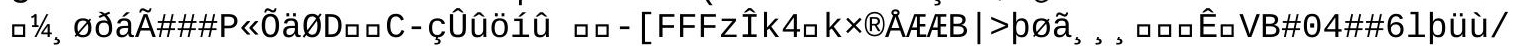
¿ü2zeCOロX, 3/4zõjn®” i 5aÆb\#k

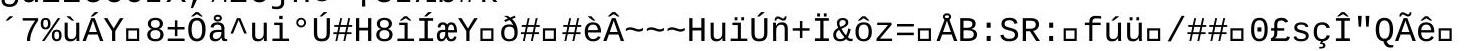

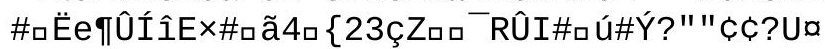




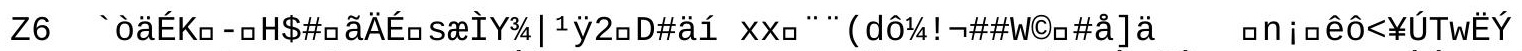

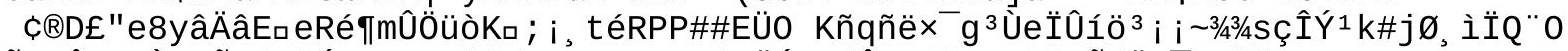

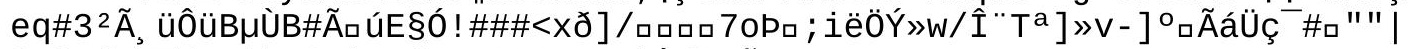

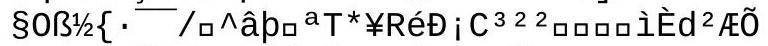

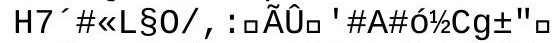

i Ếqu »nÝ: \#\#Gâıt\&, , 


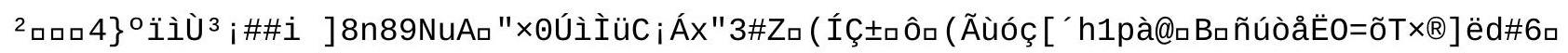
ý+! ! ¡ व\#H 


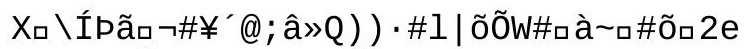

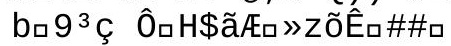




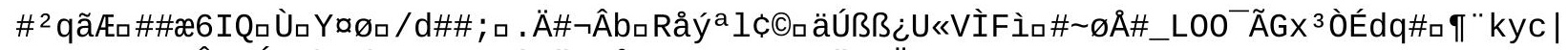

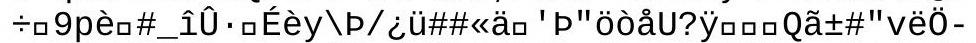

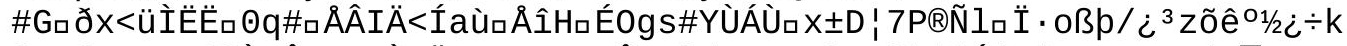

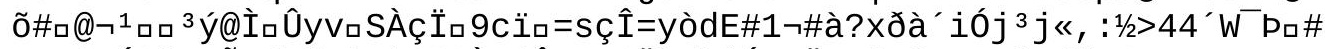

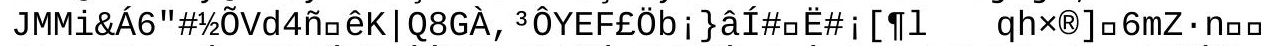

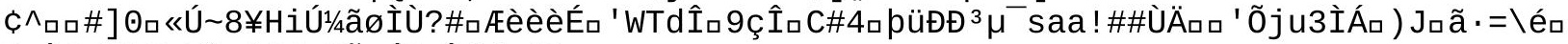

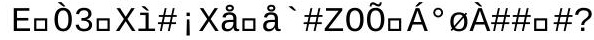

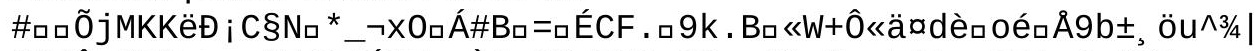

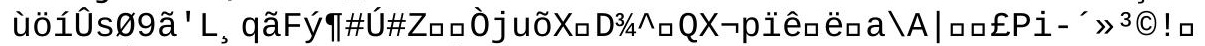

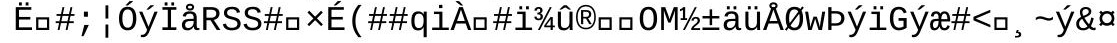




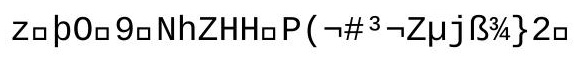




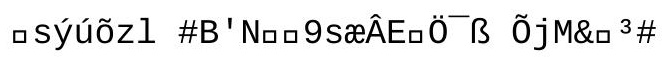




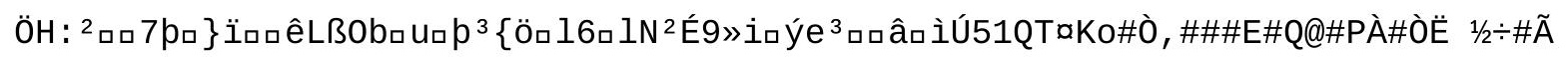




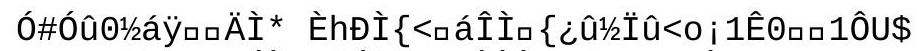

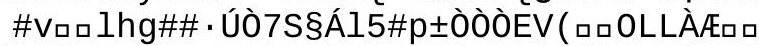

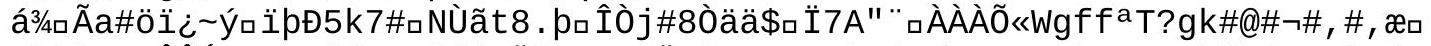

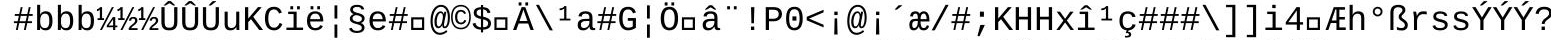

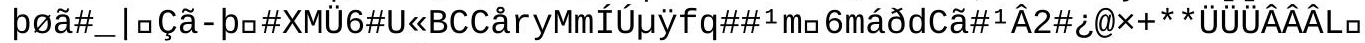
口ÛÆÄ $\neg$ LÓpÜMÅpm@Ciı\#\#>sæ 


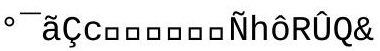




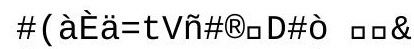

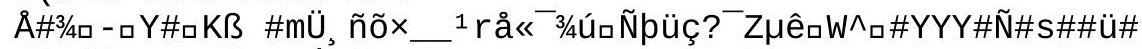

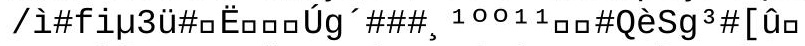

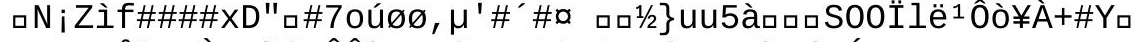

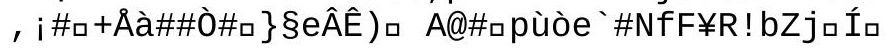




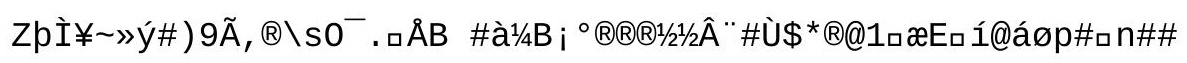


7 ( JMロ $\} 11$ 


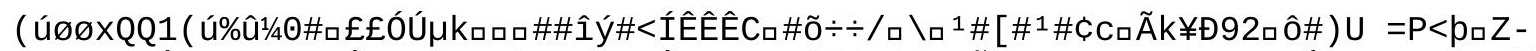

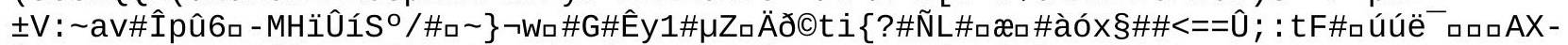

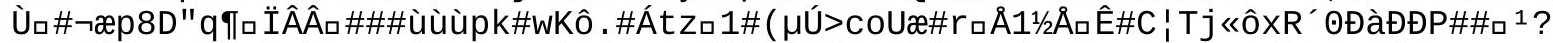

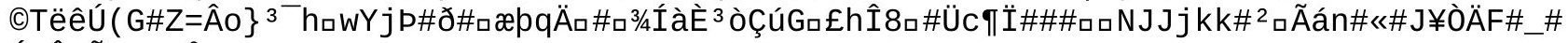

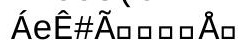

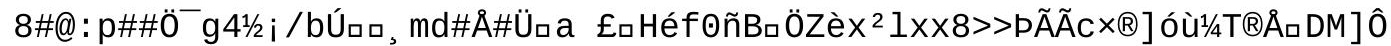

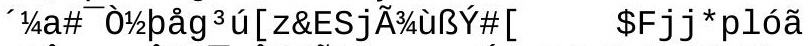

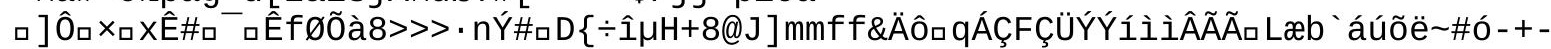

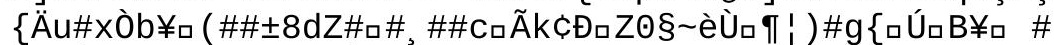

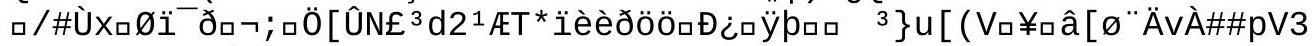

\#\#\#31aoo¿fí 


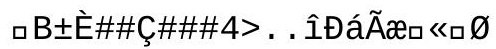




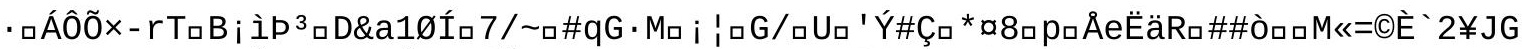

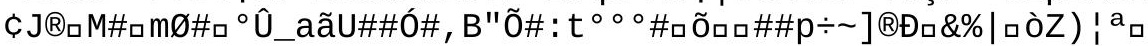




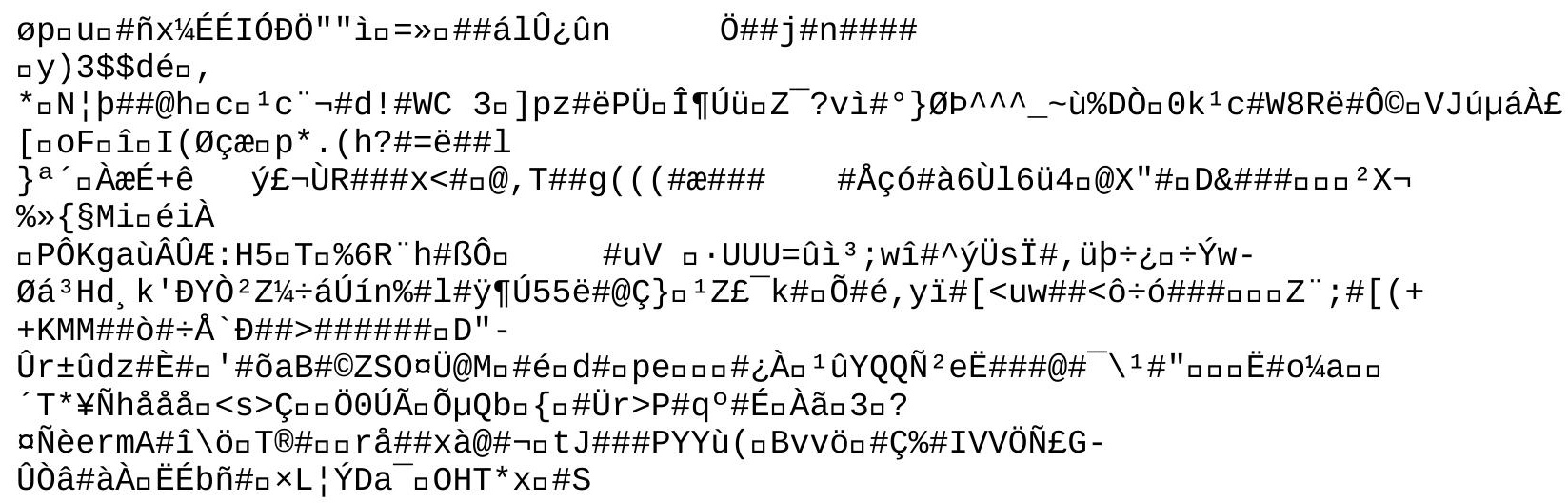


๑9XøHaRঃZ *LTÄ 
İ<óL 3/4- ̈̈a U«ètún - [ , a 


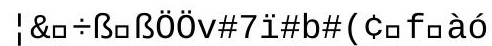

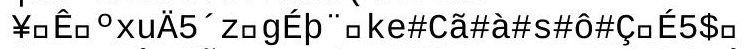

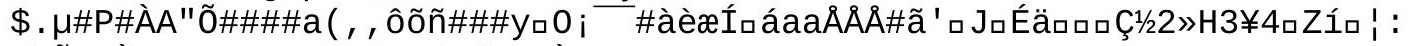

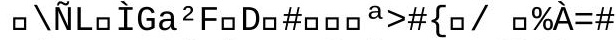

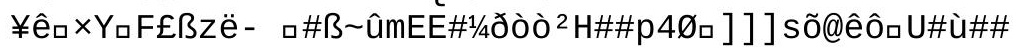




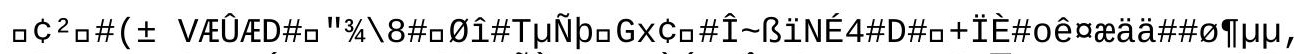

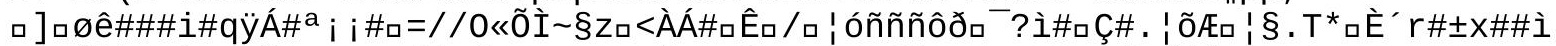

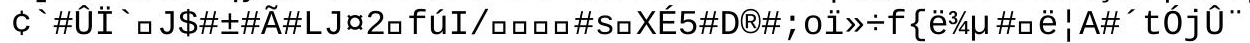




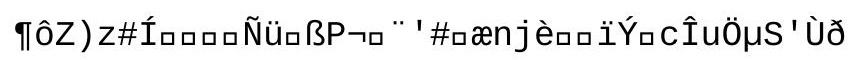


ÕSロ\#cÇ8ロ7ฉâ28òü\# 


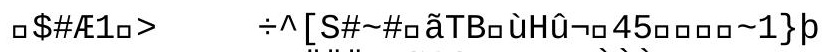

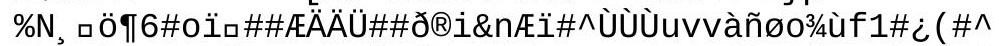

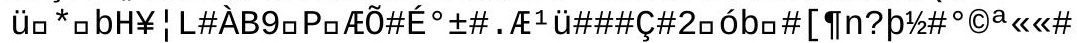

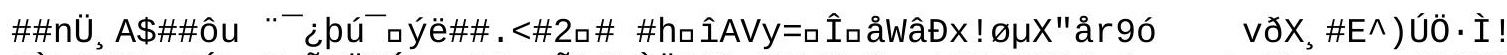

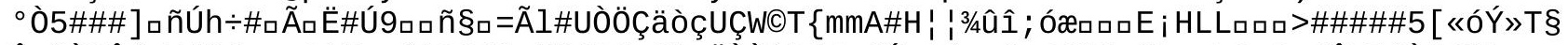

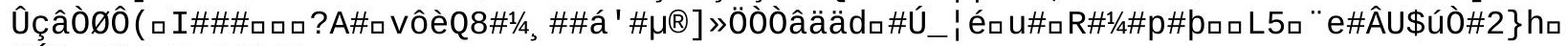

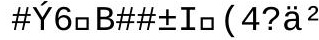




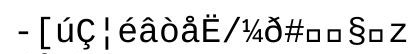

dÅ?pñ̄a\#

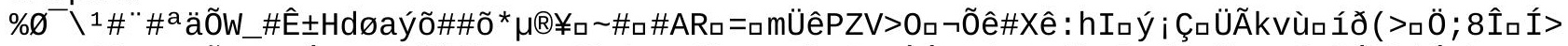
3/4m" ${ }^{3}$ é $^{1}{ }^{1} \#$ eNÑXA\% Í́

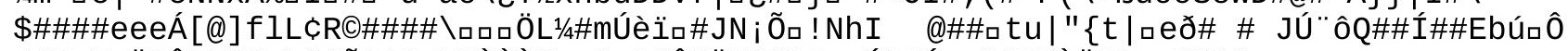

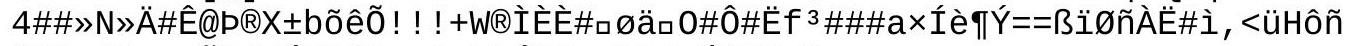

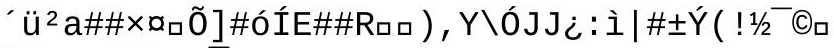

î?ฉฉषPBbxç 


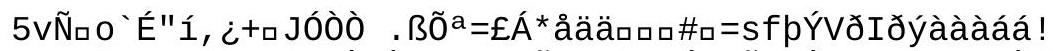

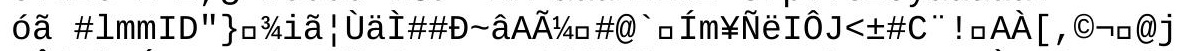

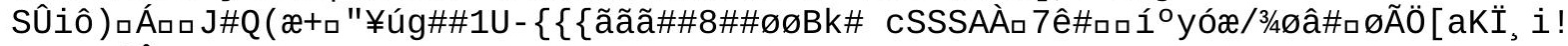

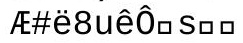




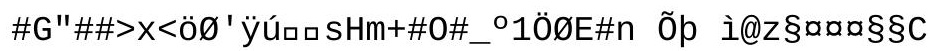




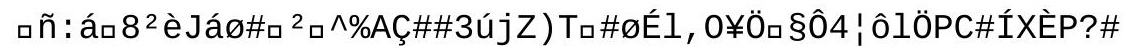

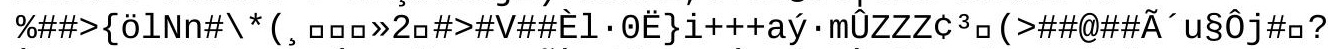

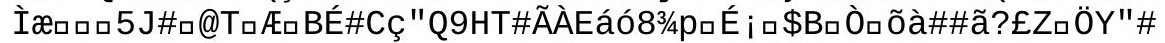

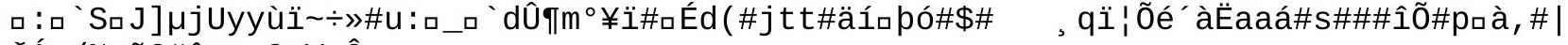

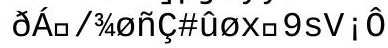


9\#\$\#0^XM\#\#\#\#æDGG $<$ $\#^{3} \# 0 \#$

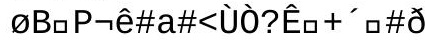

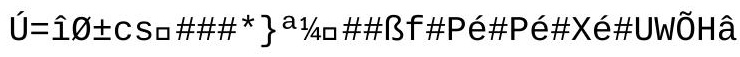




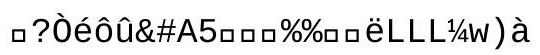




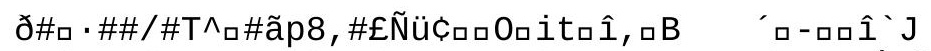

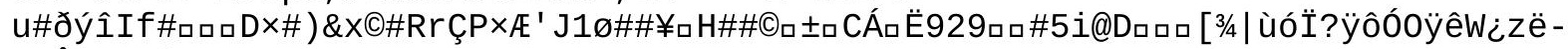
- \#Ôü\#ロüûï¿ONN\#2\#ロ

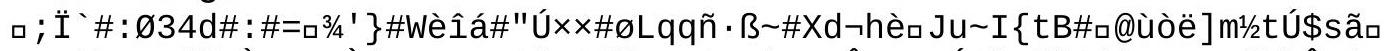

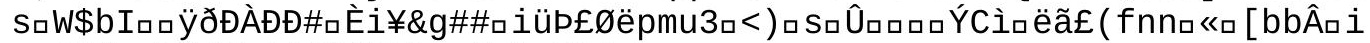

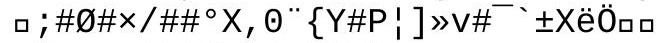

口0̃ !

${ }^{2} \mathrm{HB} \#$ KFƯ $1 / 4 \mathrm{~N} \# £ \_\hat{\mathrm{I}} \hat{\mathrm{E}} \mathrm{E}$ 


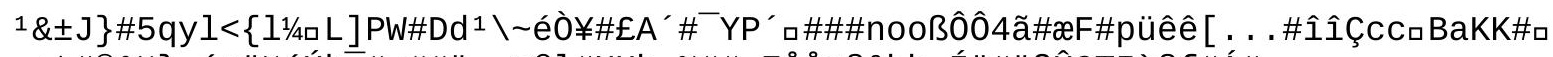

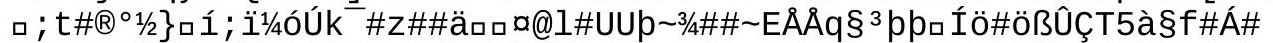

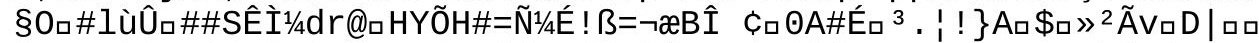

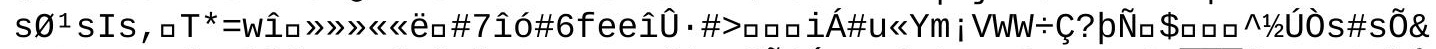

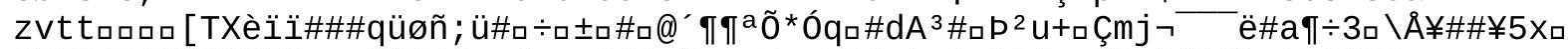

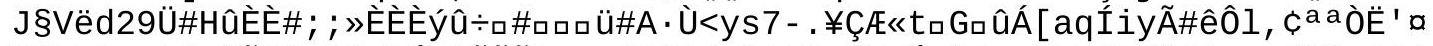

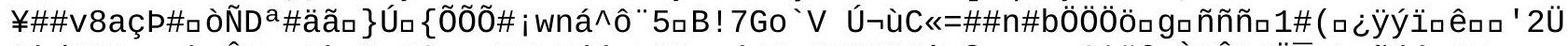

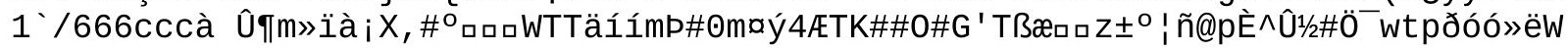




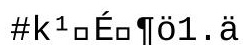




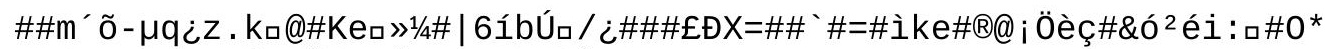

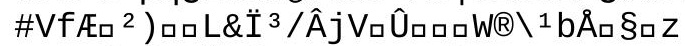

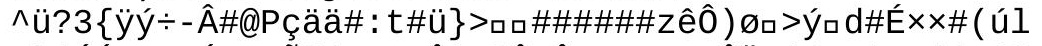

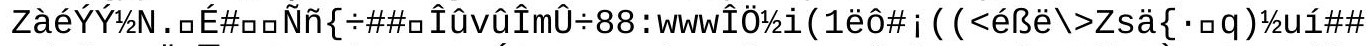

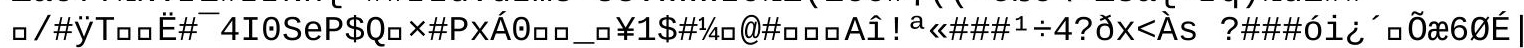

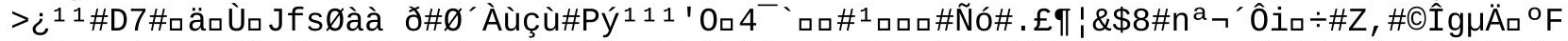

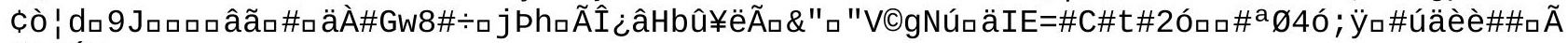
á|óí7 


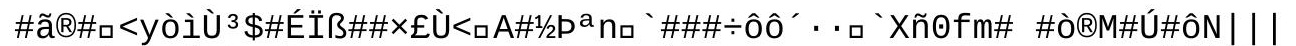

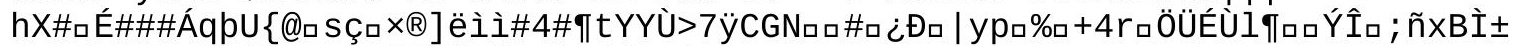

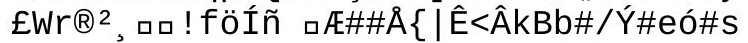


๑\%mí¥\#iSõíøË7Çœ 


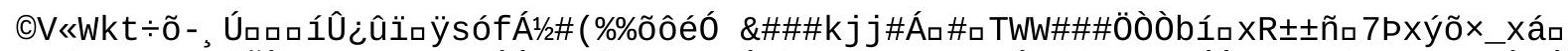

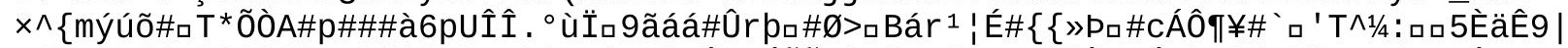

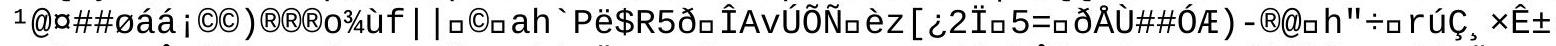

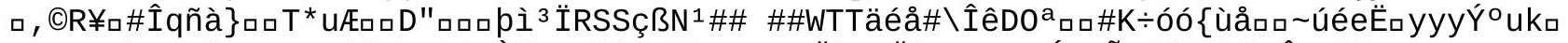

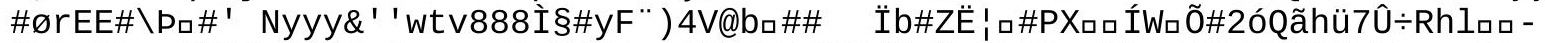

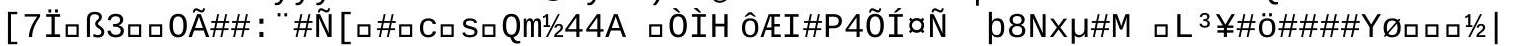

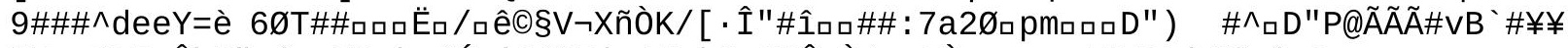

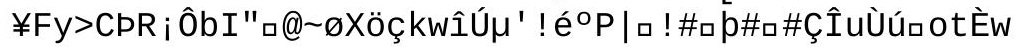
FD\&Tg\\#囚O§口ĐャC 
$\backslash<\#$ nè $` I B{ }^{\prime} \#$ a $\left.\#\right\}=C$ 


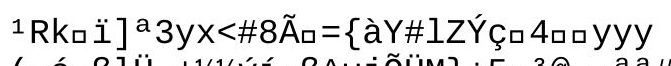

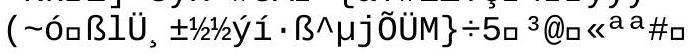

口\# 


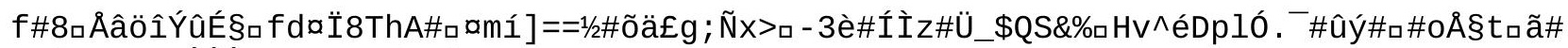

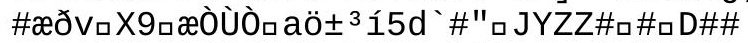

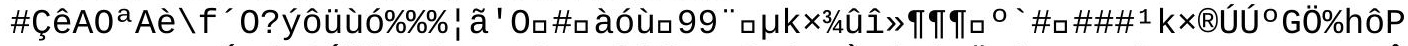

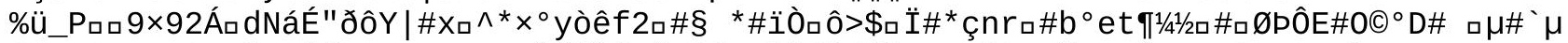

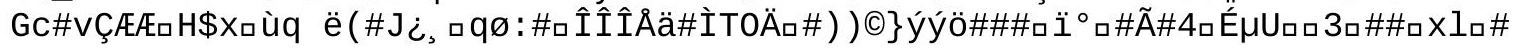




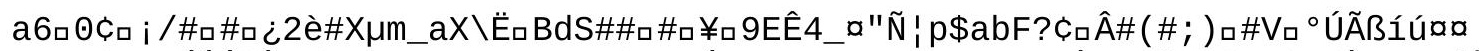

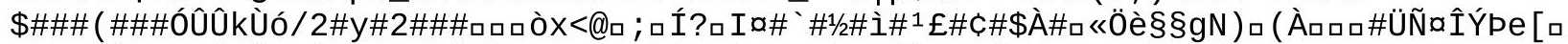

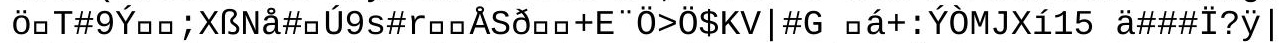

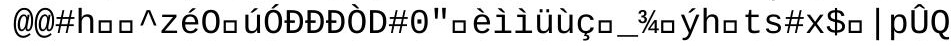


?ùÊ@

Çï\#å 
$£$,

$¥ \ddot{O} \# \# \mathrm{~d} 2 \mathrm{~g} \_\mathrm{E} \square \mathrm{CB} \#$ 


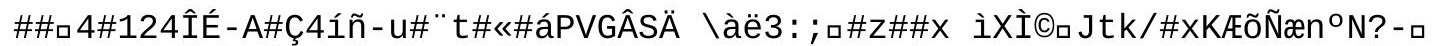

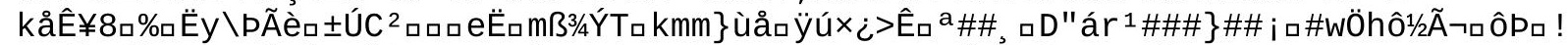

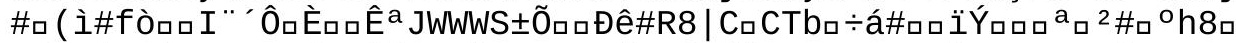

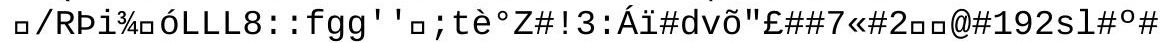

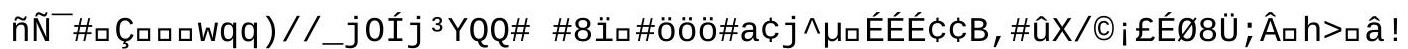

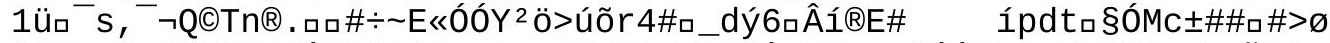

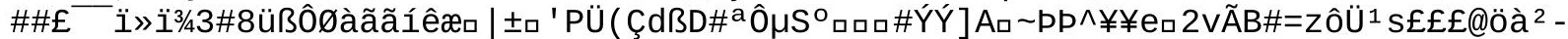

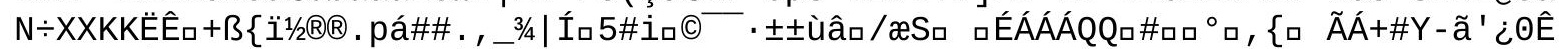
\#Iロ $\left.Z^{1}\right\}$ ÛvÇõ\#ๆ $8^{\circ} \# \%$ SùÀüçÈ 


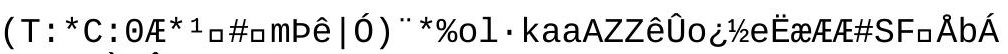

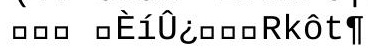


ÈCKÏd \% Ã\#oØ1/4\{ÿ i ëÝ\} "o ¿ýfr

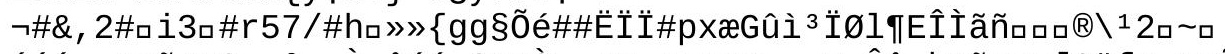

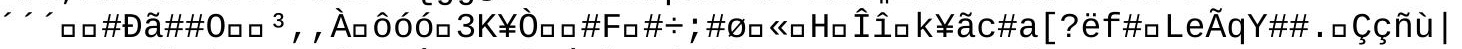

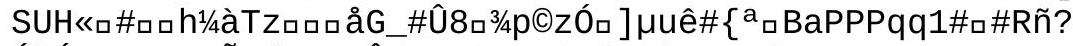

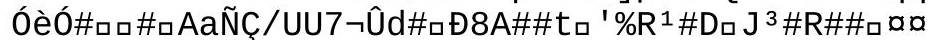

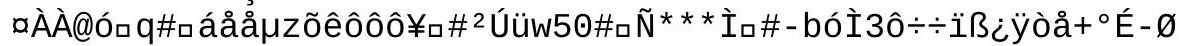

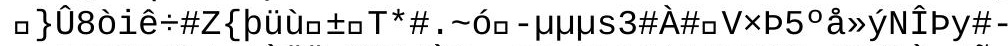

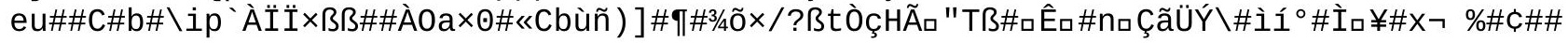


) PロロI\#ZÜロ ÎÎA\&

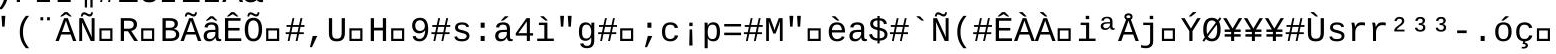


\&

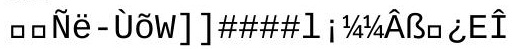

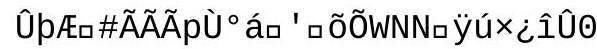




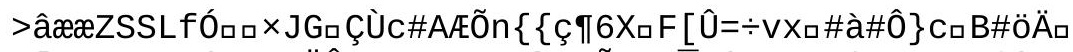

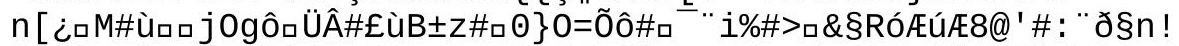

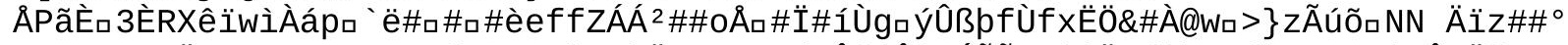

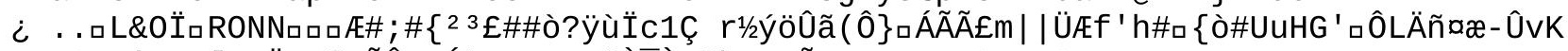

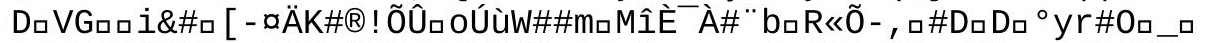

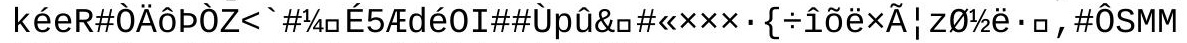

èß]»v[<=õKTा ०००åËquúéSh4zbbâúõë«V-

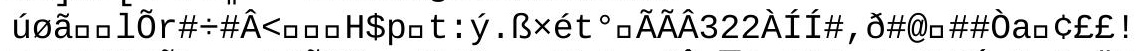

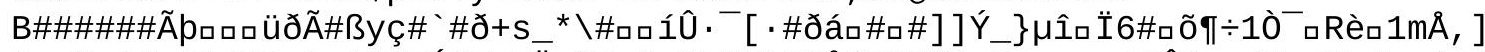

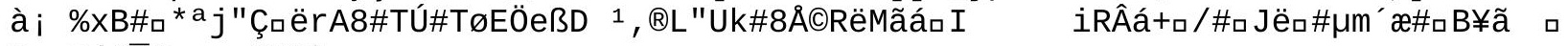
UaZPj\# ${ }^{-} M \quad \# X 0^{*}$ व

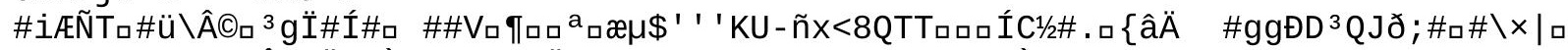

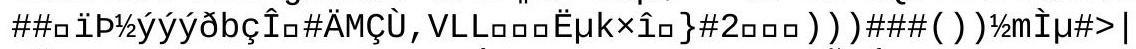

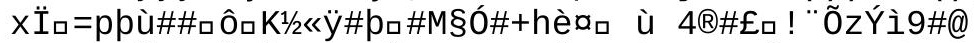

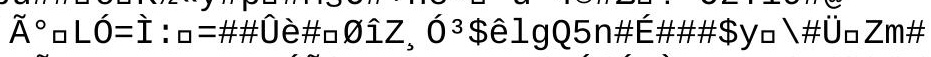

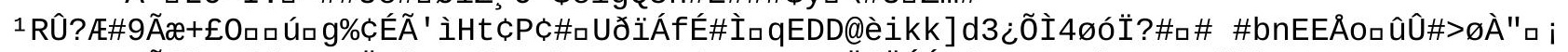

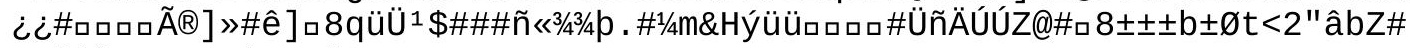

\#\#їèè, $\div \# A 8 \# a ̂ \# \# \#$ ët\#1/2\#

$\#$ ¿@ø6ıâ 


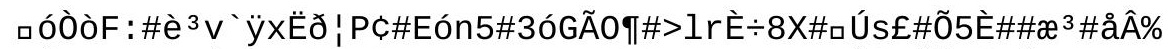

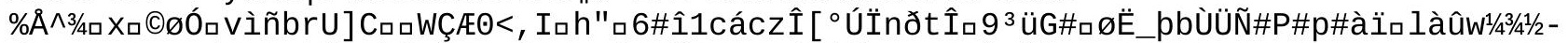

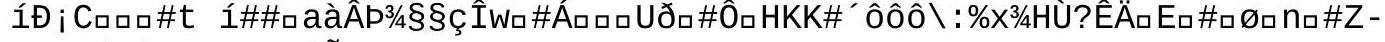

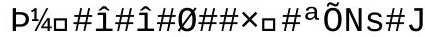




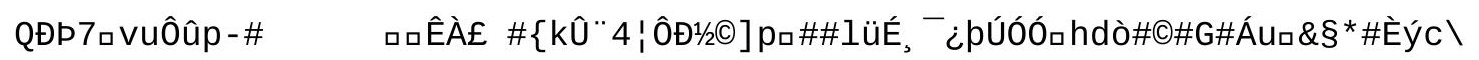




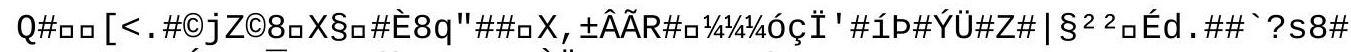

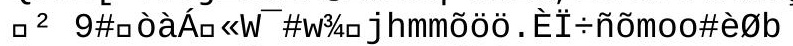

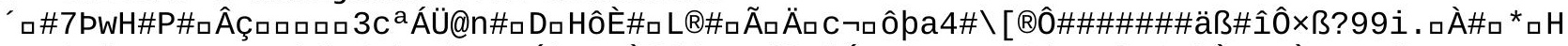

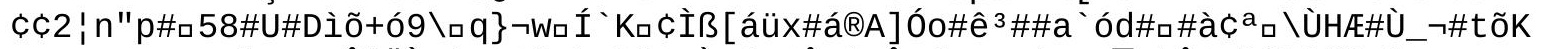

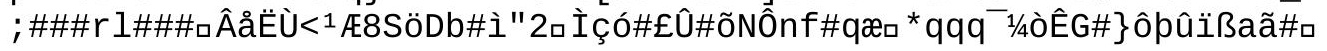


p6\#

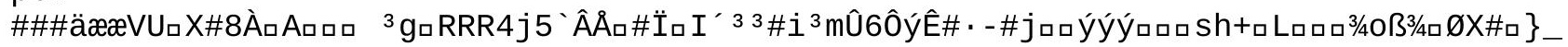


\#\#\#]Cロ\#SÓä\#

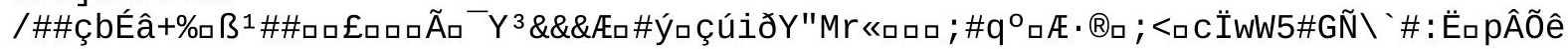




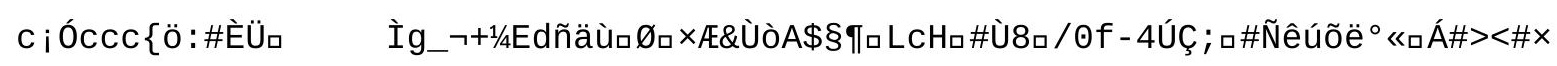




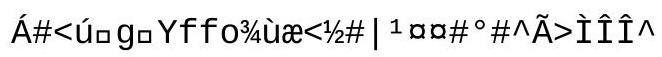




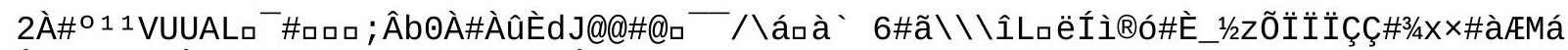

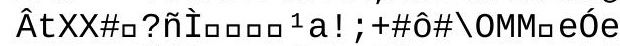

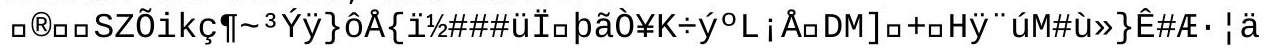




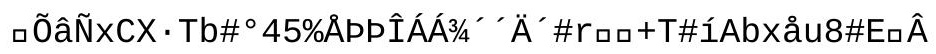




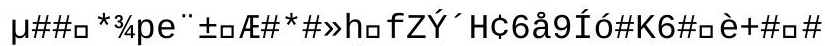

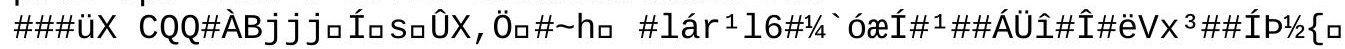


/ô2ロ 


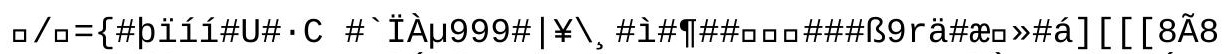

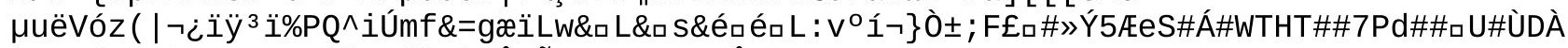

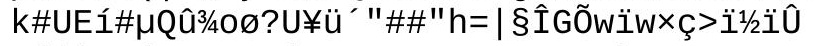

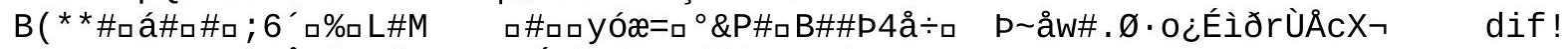

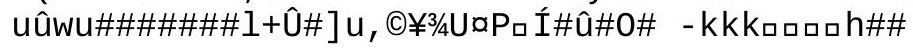


वर्

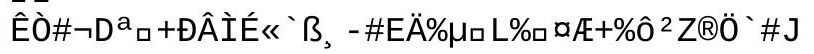




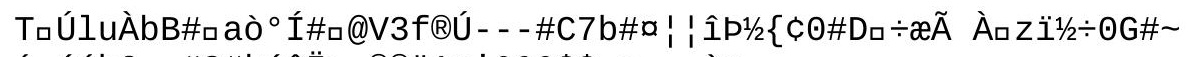

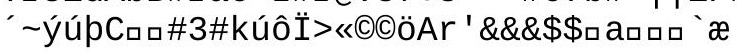

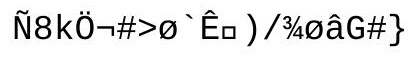




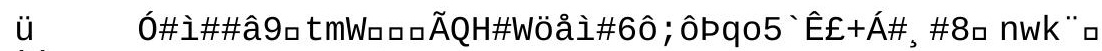

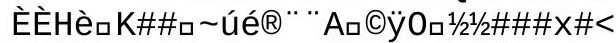




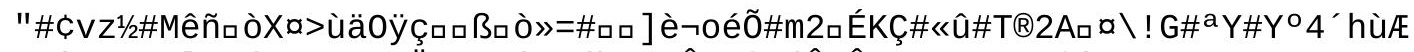

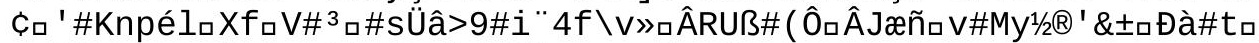

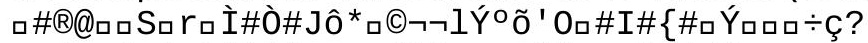

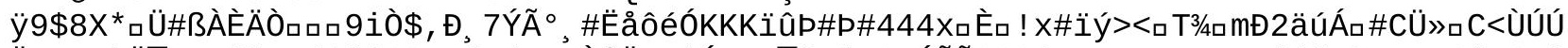

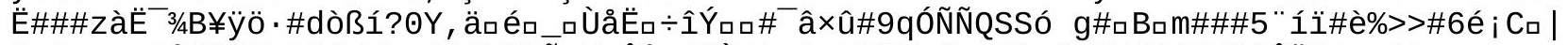

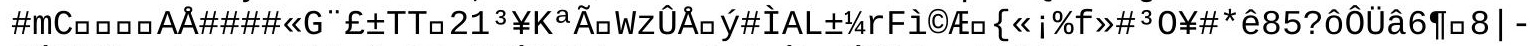

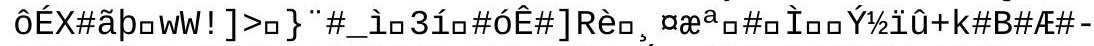

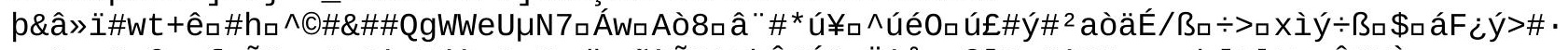

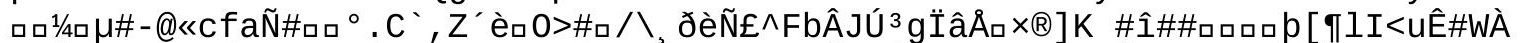

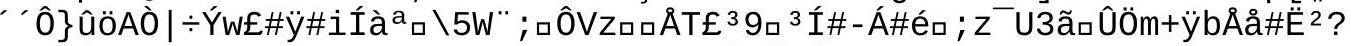

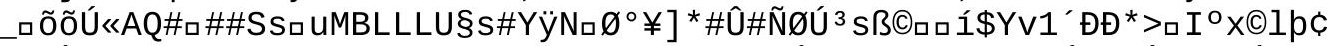

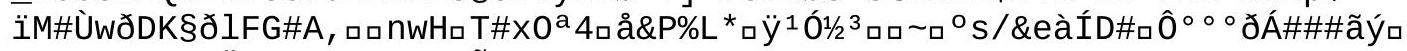

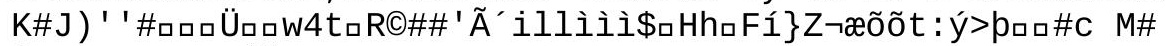

Ån9sølöp+røúî\#; )\#

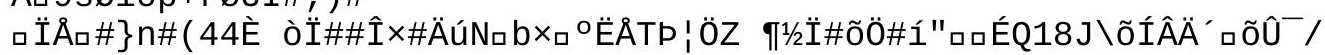




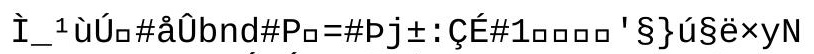

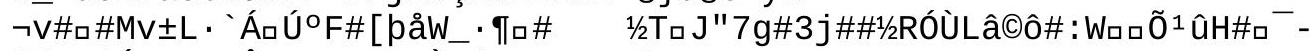

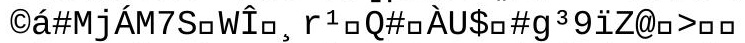

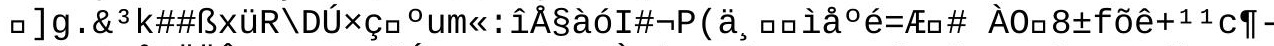

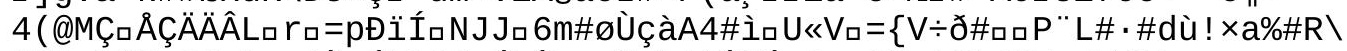

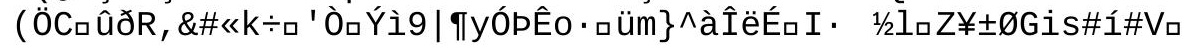




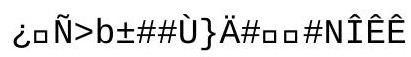

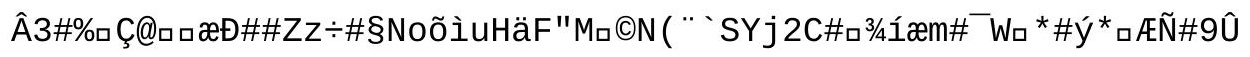




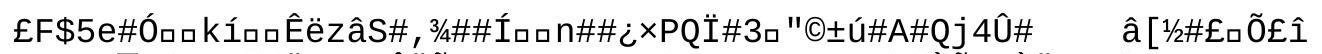

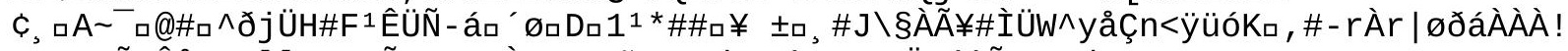

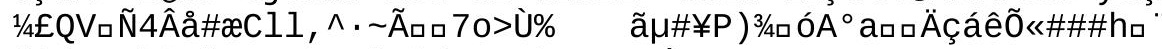

$\mathrm{h}-$

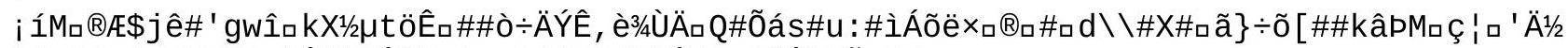

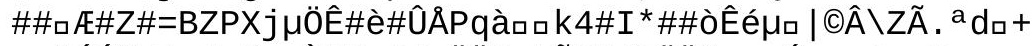

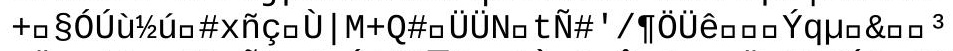

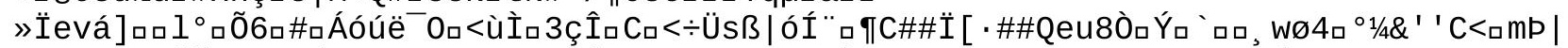

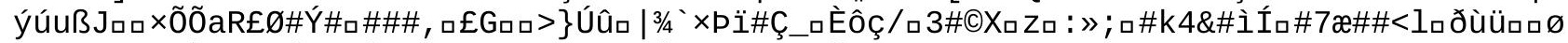

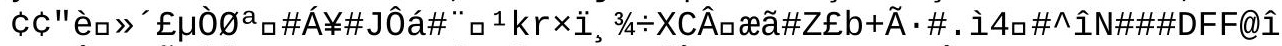

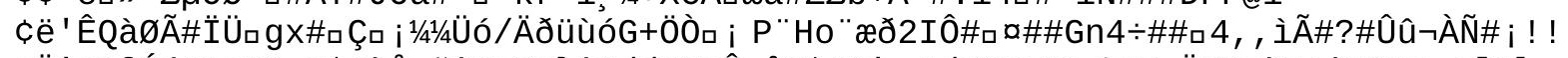

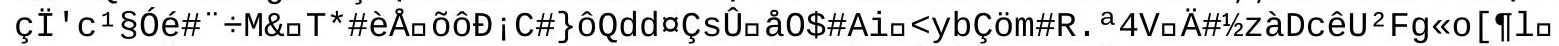

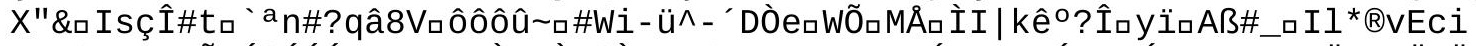

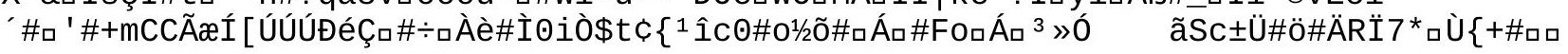
$\% \mathrm{r} \$$ 口 


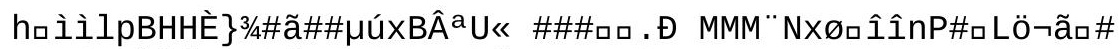

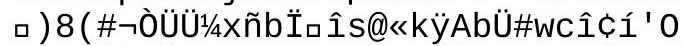

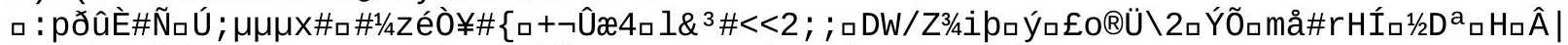

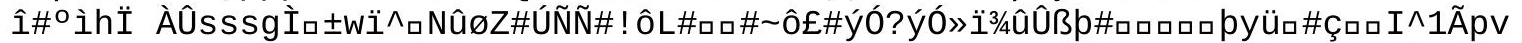

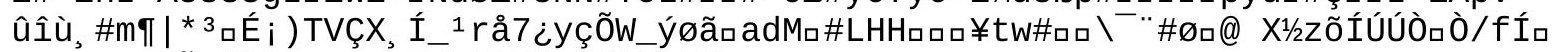

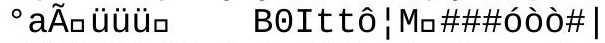

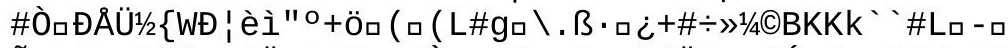

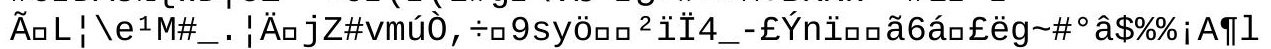

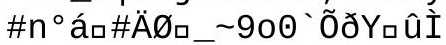




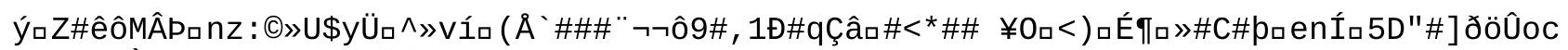
\#ç ${ }^{\circ}$ SææİyXT, ฉםPøêêj»\} ( s\#\#!

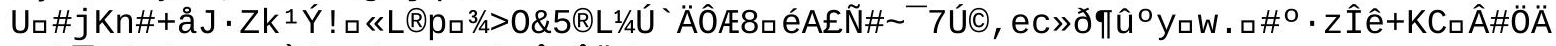

$\% \cdot \backslash$ ëé\#é-\#»Ç=ÒjuØí£9R ロ`cÂDÊİ/\#

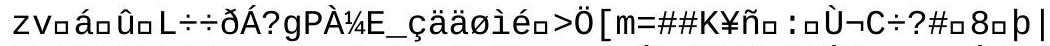

LLLKKKhh(

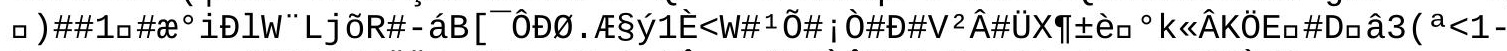

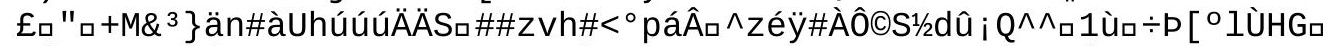

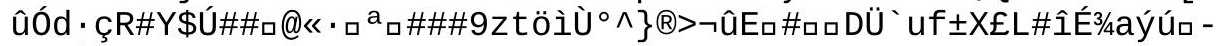

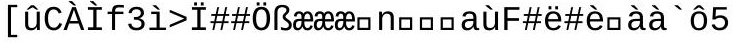




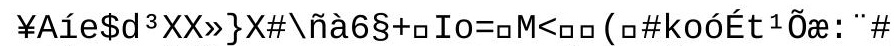

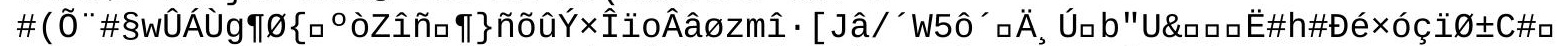

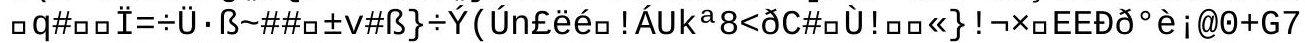

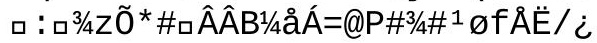




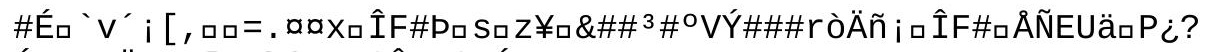

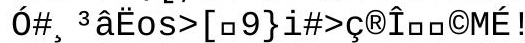

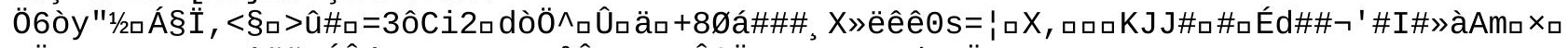

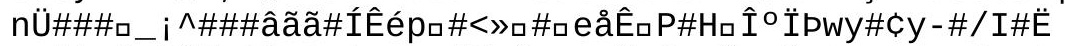

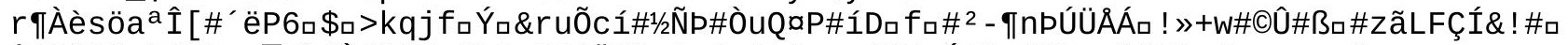

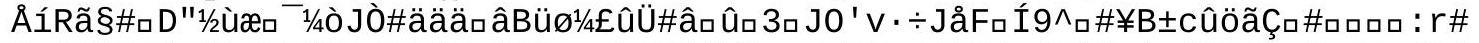

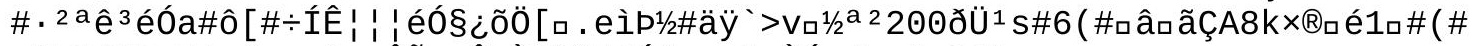

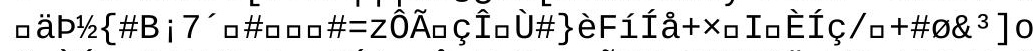

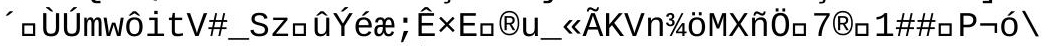

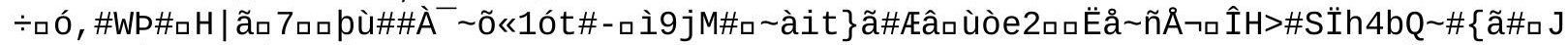

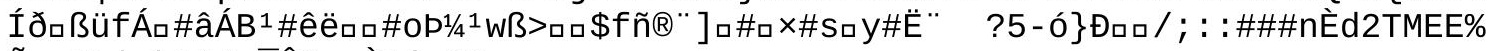

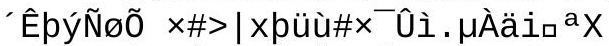

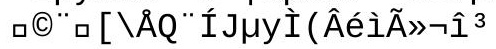




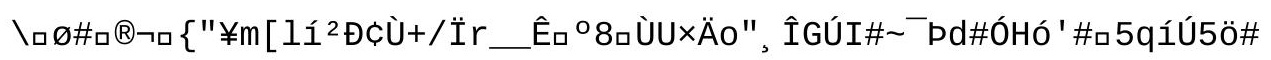




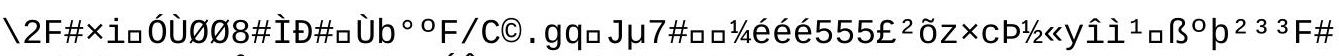

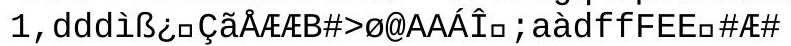




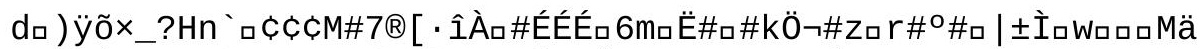

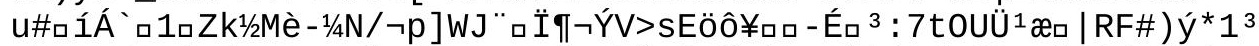

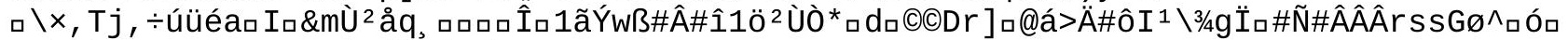

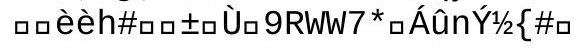

Lø\#\#. 


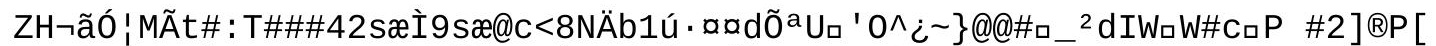

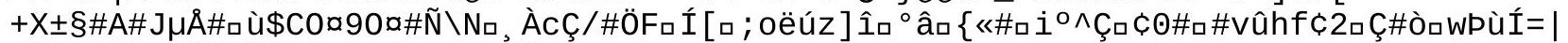

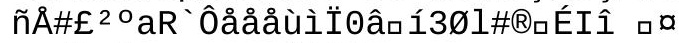

ODí\} $1 / 20 \tilde{2} Đ \bigoplus \# \#, A £ \tilde{N}$ 


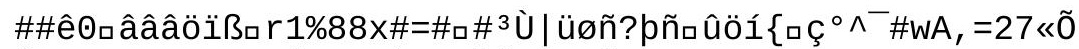

Í \#\#\#\#\#ѐeĐ x\#Ê022òÒ¥KøÄÃ̂ि\#æä̈

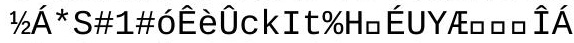

$£ O ̀$ å $£ £ c ̧ a ̂$

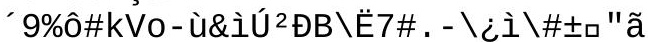

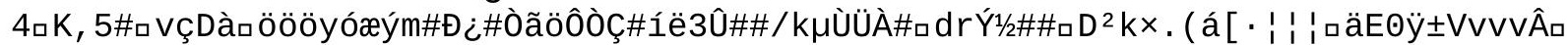

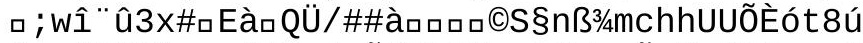

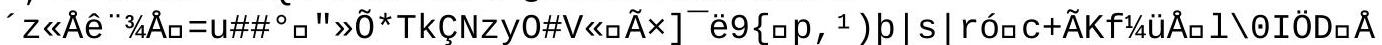

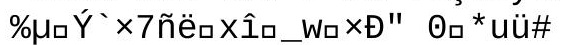




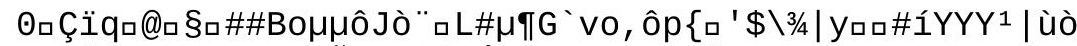

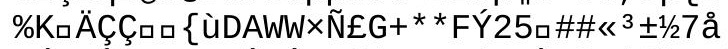

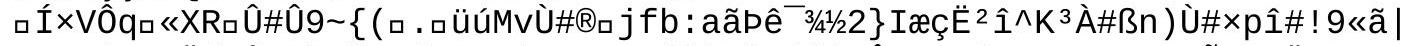

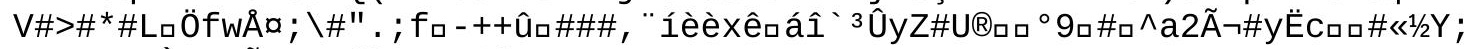

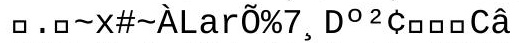

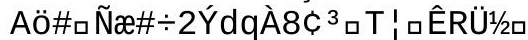

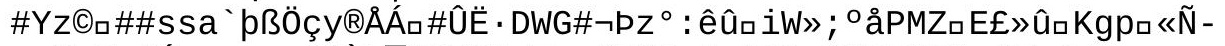

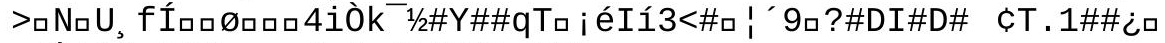

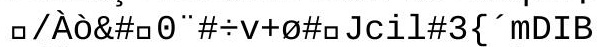

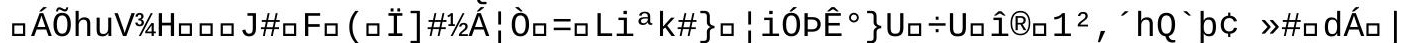

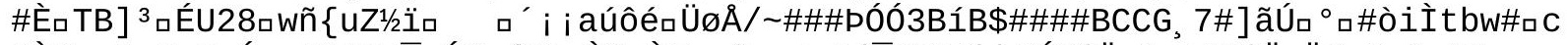

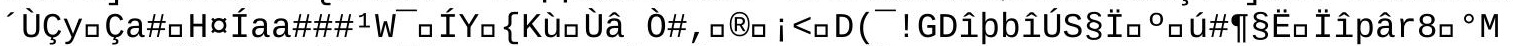

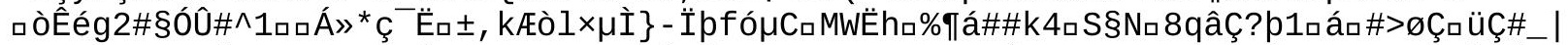

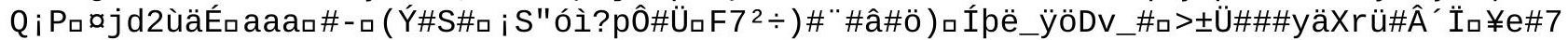

IaJ\#: ðっlJaL! üüü ${ }^{3} g$ İzs 
äã+İ · g/Evau\# · ú\# · æbUYÏI³/4L 


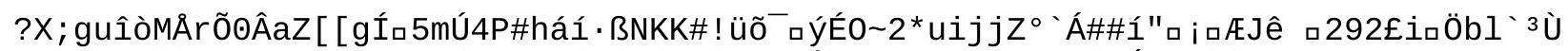

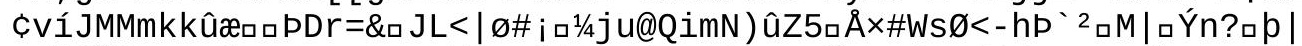

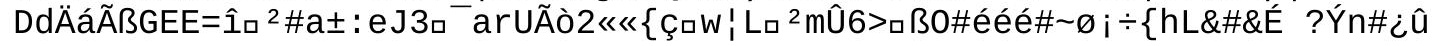




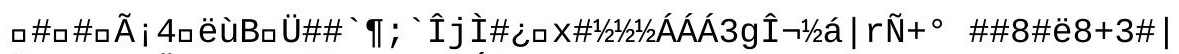

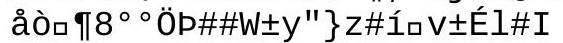


øpก̃̃Äh4bYìî̂ิ6\# 


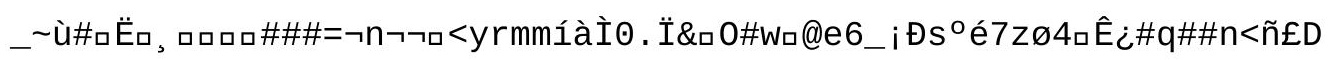




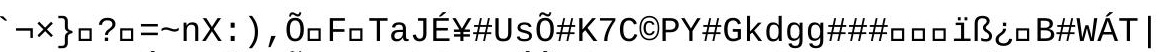

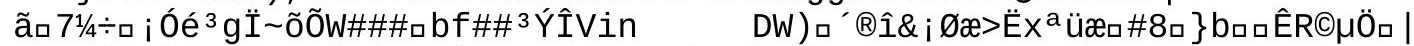

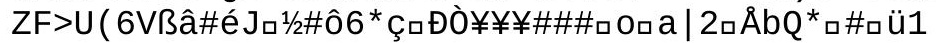




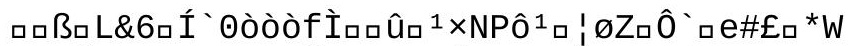

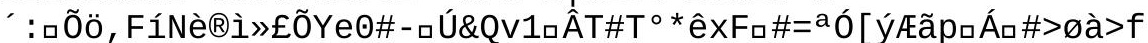

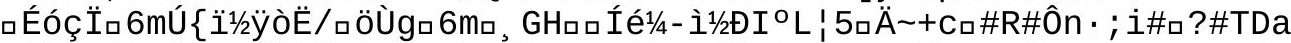

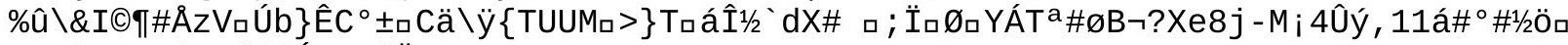

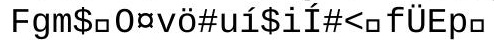

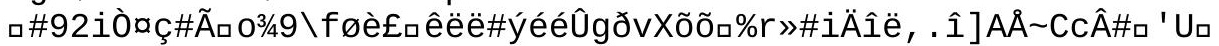




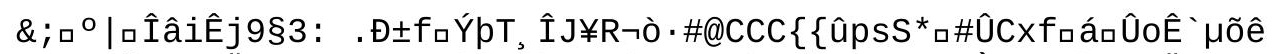

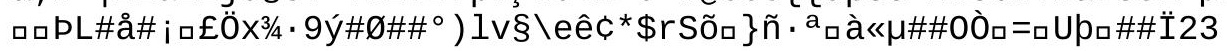




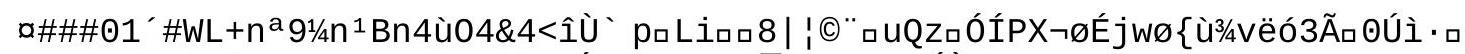
1/2ù4f6rpa@ä' '

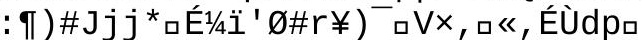

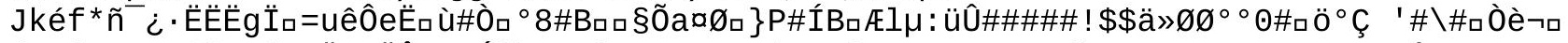

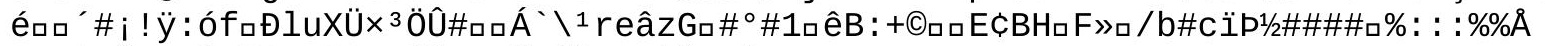

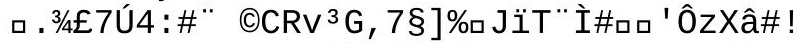

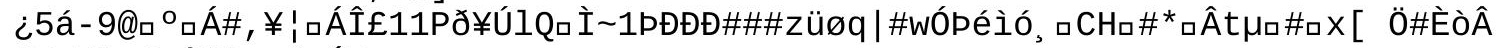

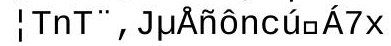




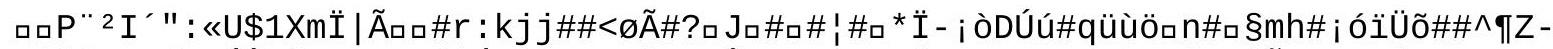

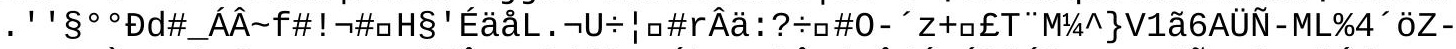

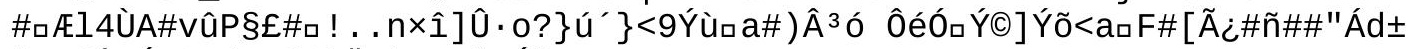
$\left[\neg \#^{3}\right.$ ÅPÍTQXj\#_ ${ }^{3}$ ?pö\#|ฉJc) ¿Éëo

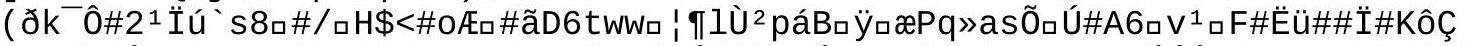

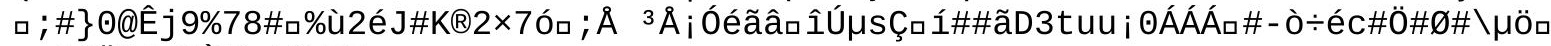

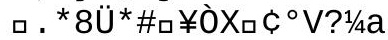




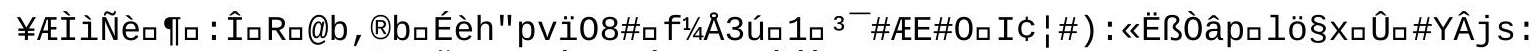

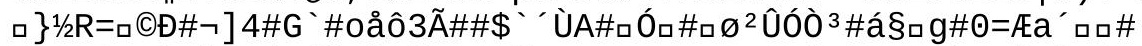

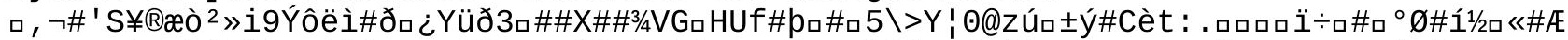

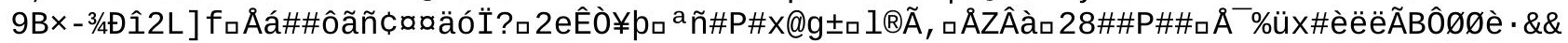
$\mathrm{QH}$ 
Æ\&ao1っårı Ó`nょípóo?\#\#üû 


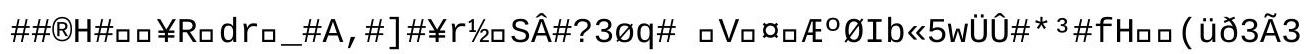




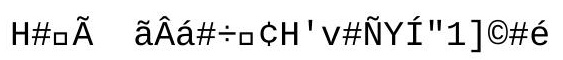




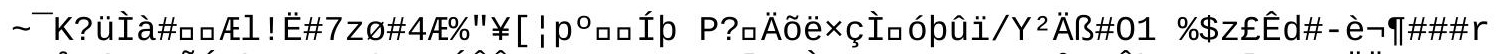

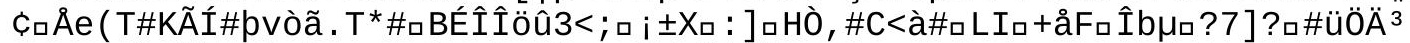

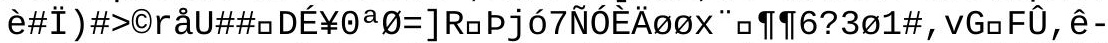

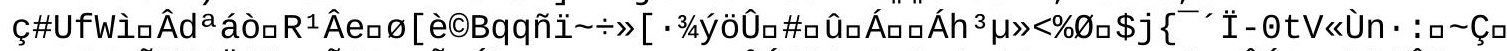

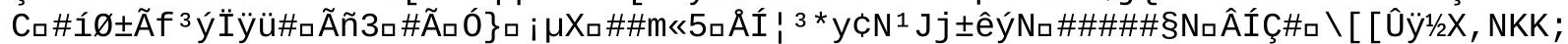

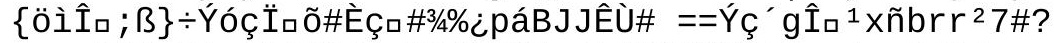

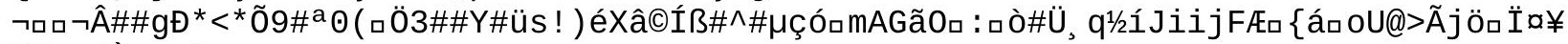
$¥ \hat{U}<\square \# \bar{I} \quad \square \square U \hat{~}$

$\odot \neg \# \AA^{-}---C \notin$ 


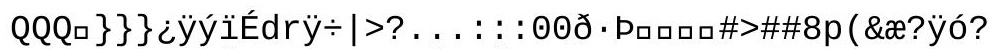

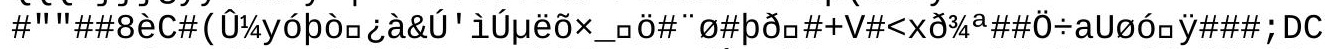

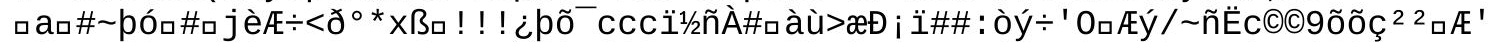




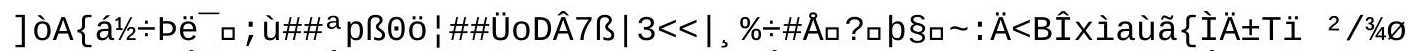

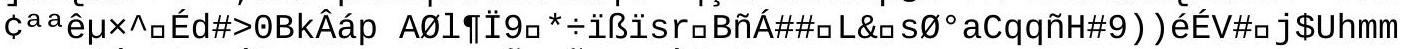

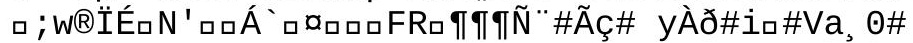


3 fízán\#@\#\#\}ロJ¥ロÉFòa\# 


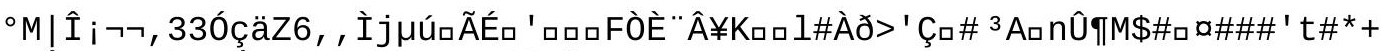

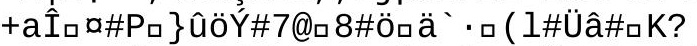

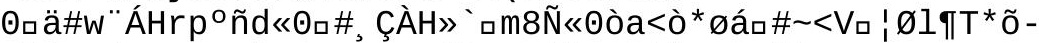

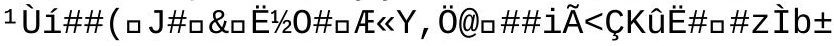




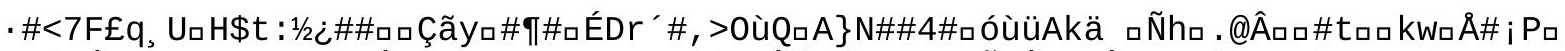

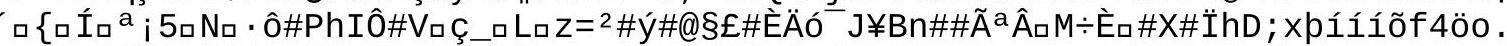

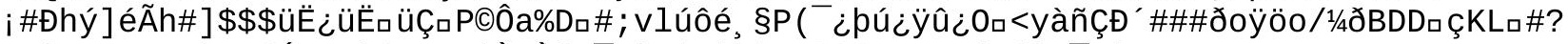

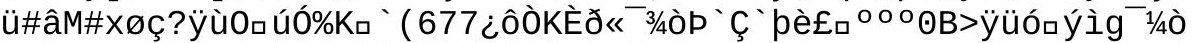

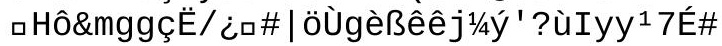

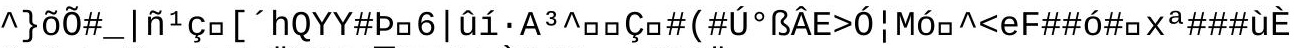

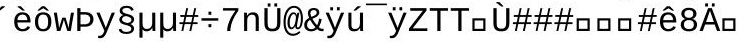




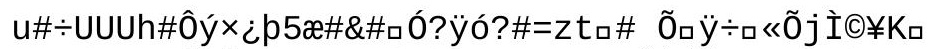

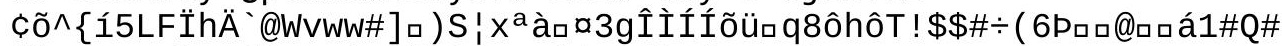

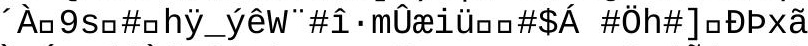

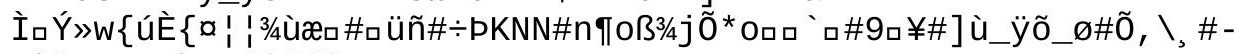

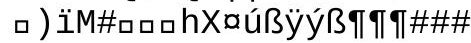


è 


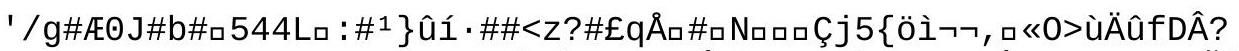

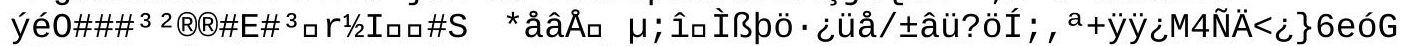
口\}bİnÖロ»É\#£1VaUaM, QTb4k\#El bÁN\#MV\#\#\#०`G)

口 (U\#\#· ) İÀĐ

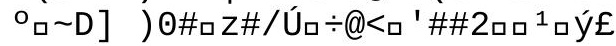




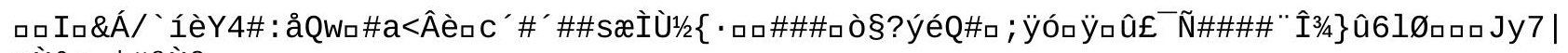
$m U^{2} e_{\square}{ }^{*} \#^{a} \bigcup^{\circ}$

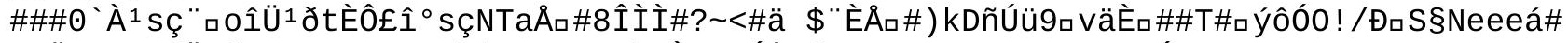

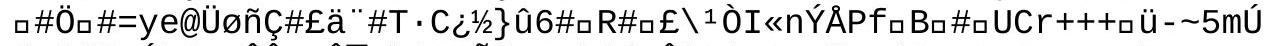

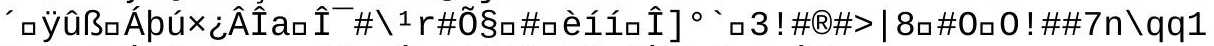

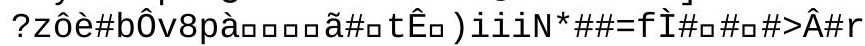

BË<öçX\#ロĖ̃̃a\#Ãû@tâ>h'! â@tîc\#6 
ë1/2Sç\#ロ\#\#ロuAÒqFV\#9r\#e@ã \#xÔ\#nÝouì

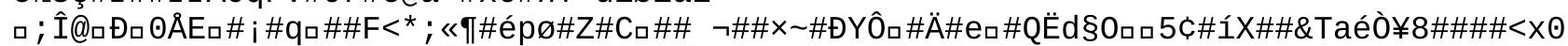

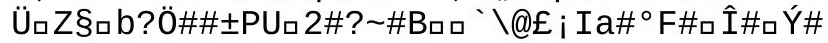

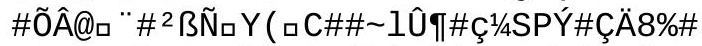

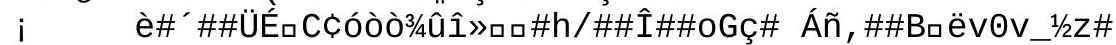

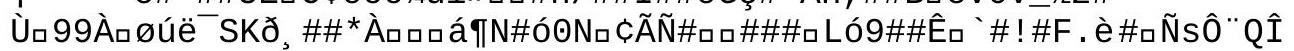

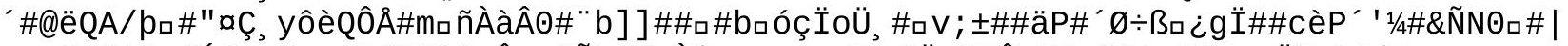

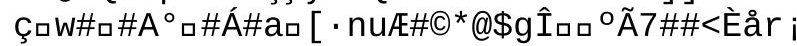

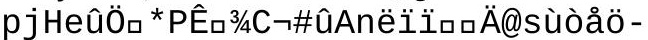

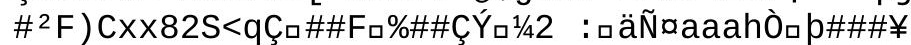




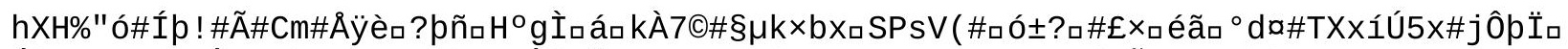

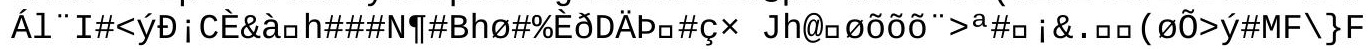


口V@ðÃãñĐ2ígè\#x (Ü

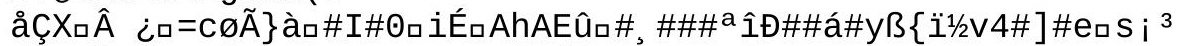

(\#ロ\#\}ロó">\#Ö\#kaa 


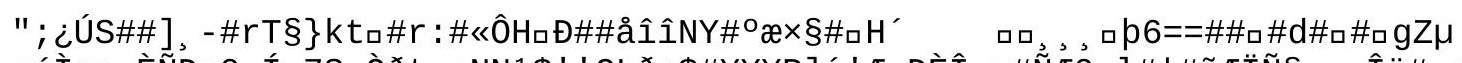

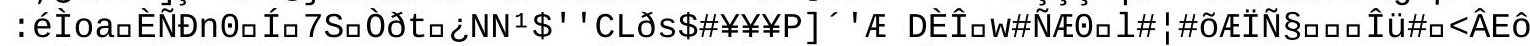

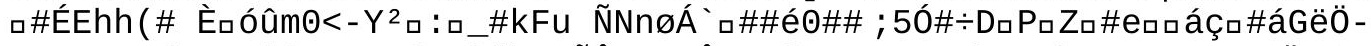

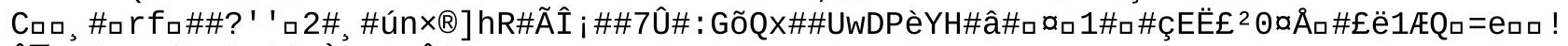

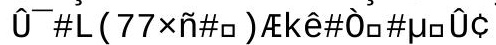

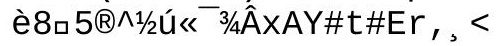




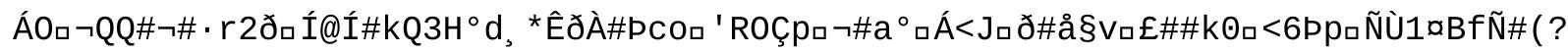




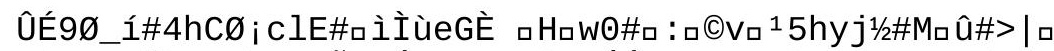

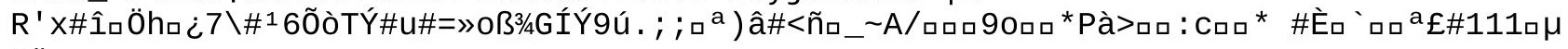

Fü

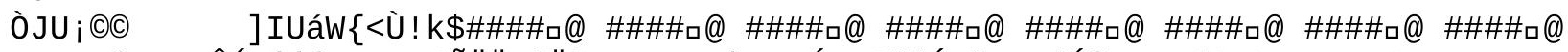
\#\#\#\#@\#\#\#ÂÓ\%' ' § \#\#F£ÑÏÏô̂Ü1

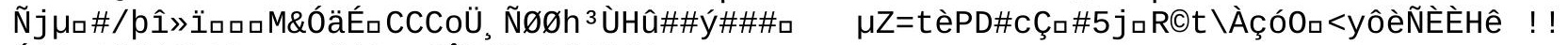

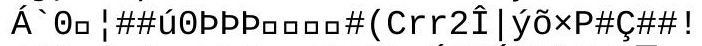

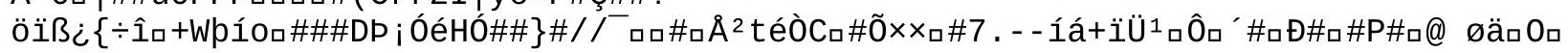




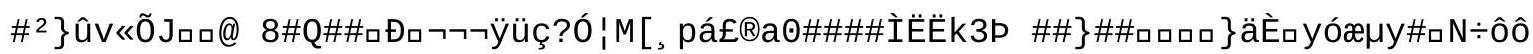


\#\#[ [ ${ }^{3}$ Ví 


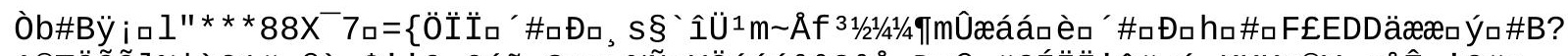

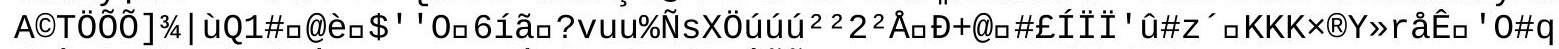

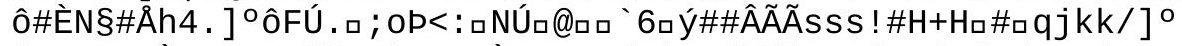

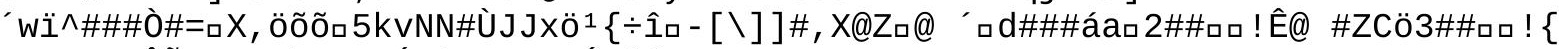

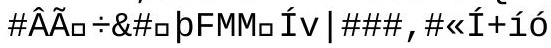




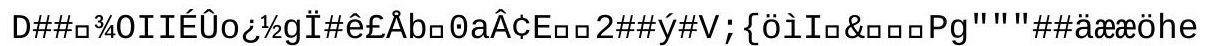

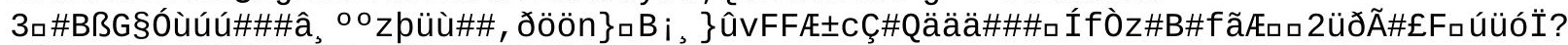

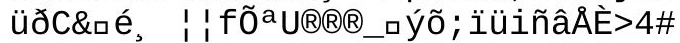

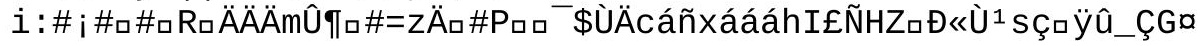




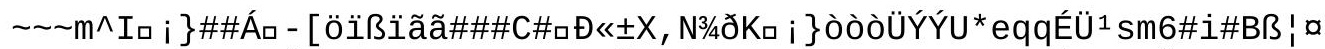

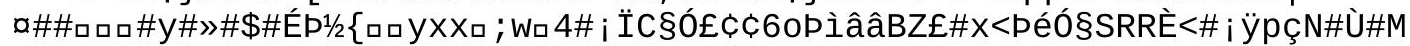

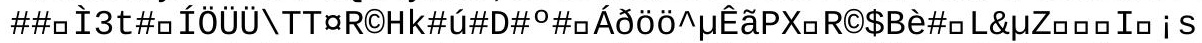

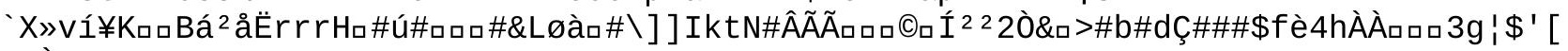
, \#Oे 口>\#口g

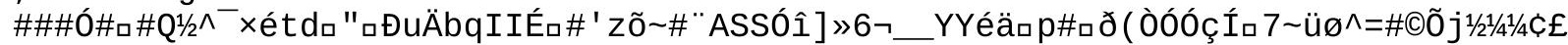

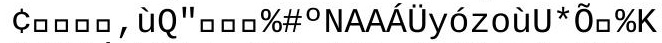

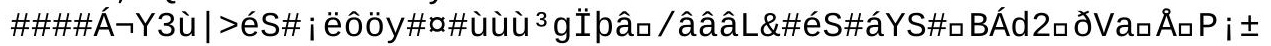

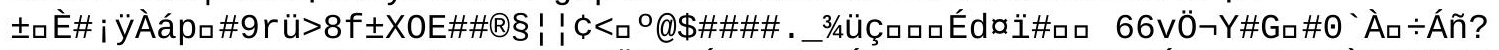

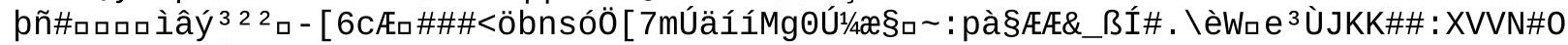

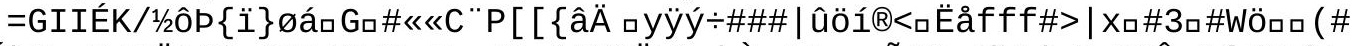

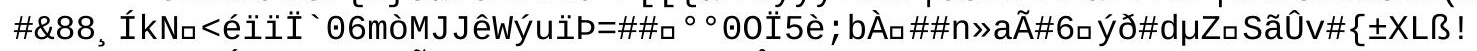

õNII9Wî\#T¥Í+\#ロMXm6 ${ }^{3}$ ÕZaqga $\div$ Æ«

ÛđlIJLlóJ -

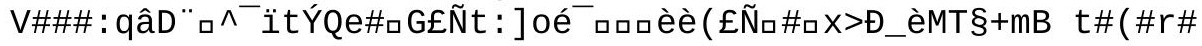




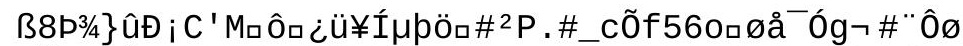

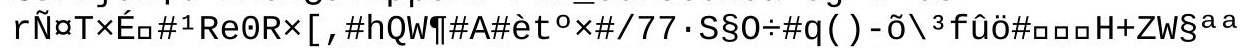

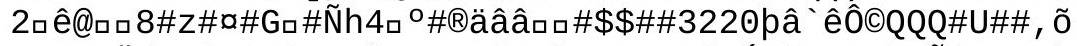

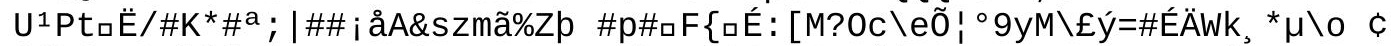

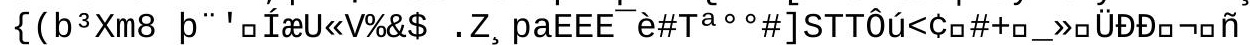

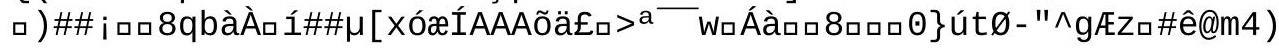


$\#^{1}$ Á 5 a [îiq4@ÔJe-

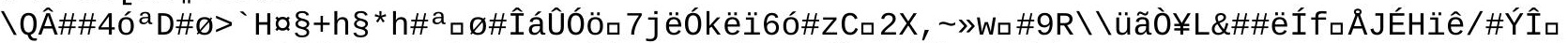

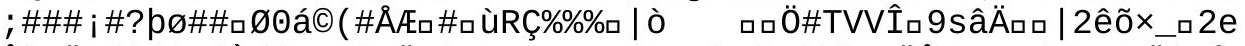

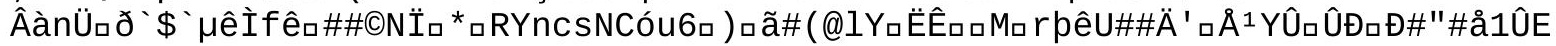




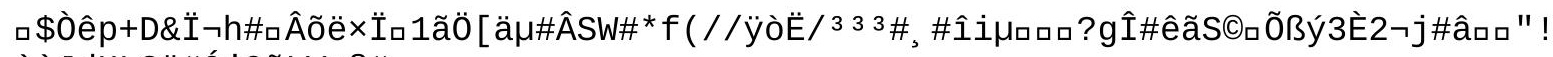
JdHL2ë\#Ój 9ñLV: §\#

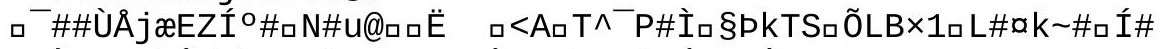
$\mathrm{qF} \#|| \mid$

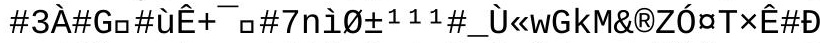

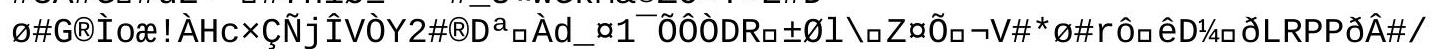




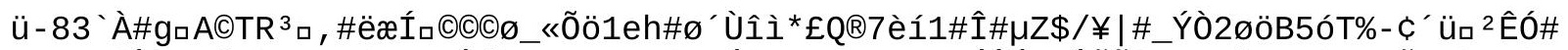

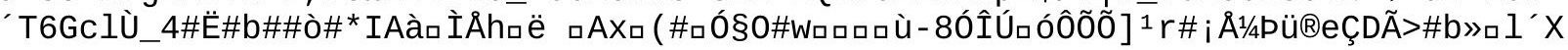




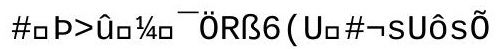


JX 'ñtVf\}SNCSı@T\%\#\#5Z»

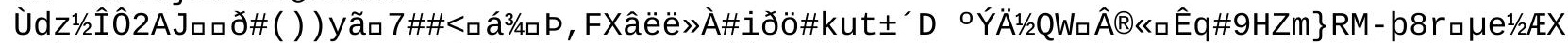

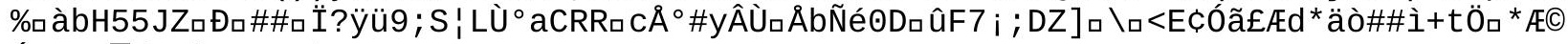
Ú\%: So $/ 0 ¥) æ 9 r \% a ̂ ı 1 / 2 \#-$

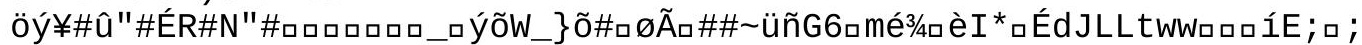

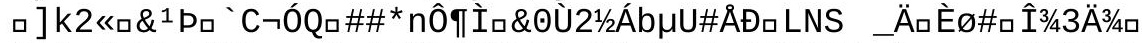

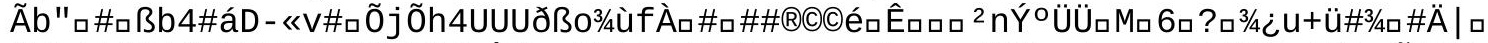

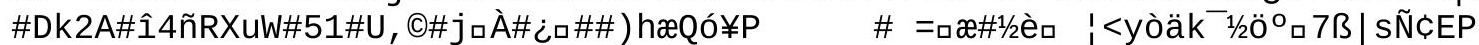

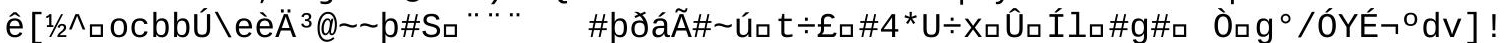

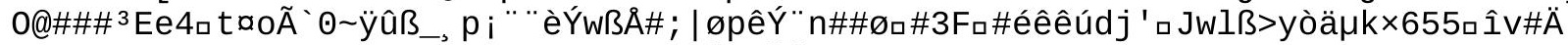

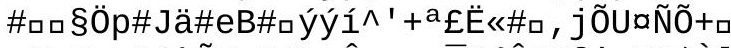

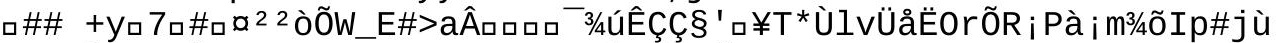

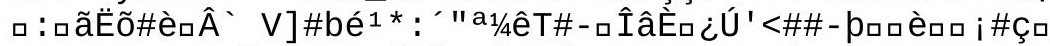

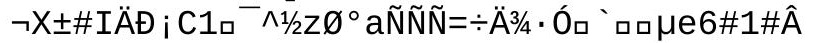




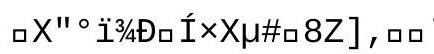




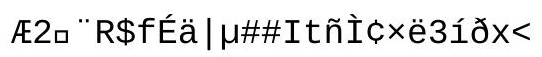




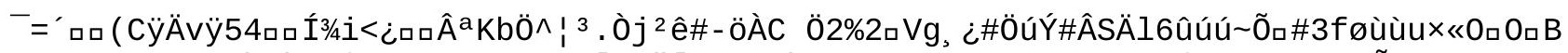

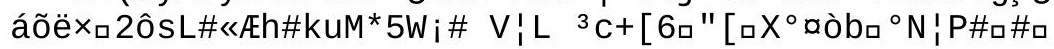
\#ロロ\#\#0\#*\#8p ra01̂óÑG\#á 


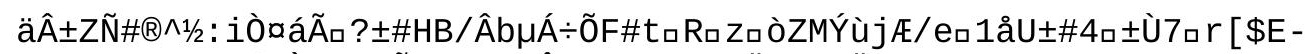

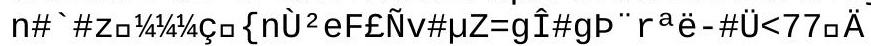




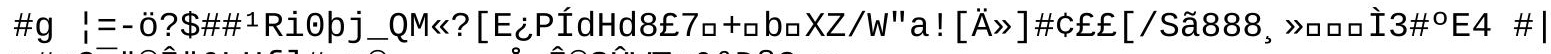

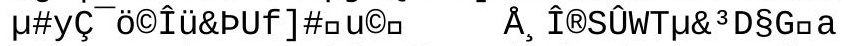

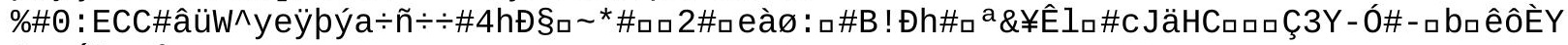

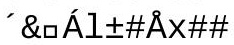




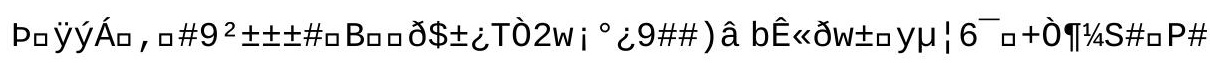




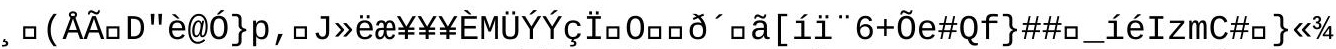

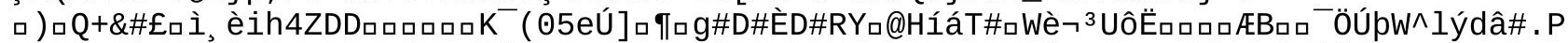

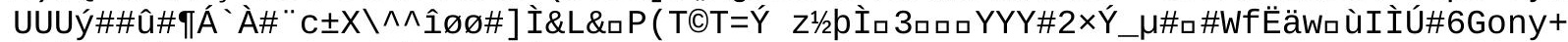

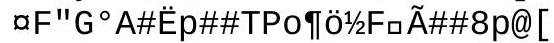




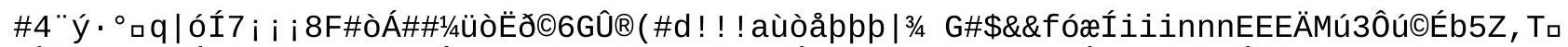

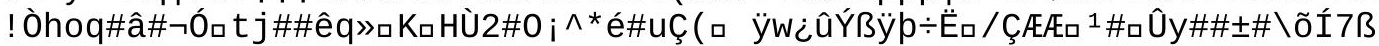




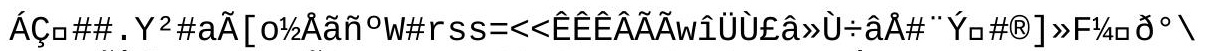

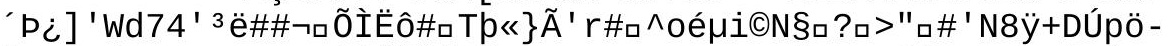

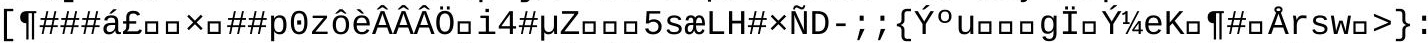

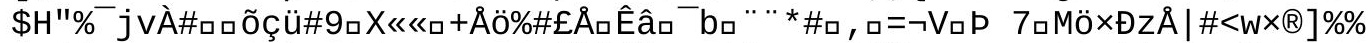

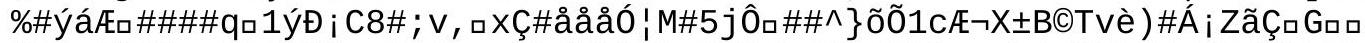

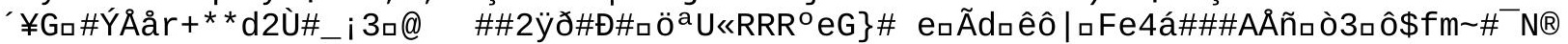

$8 \&+u ́ 0 ̈ 1 / 4: \cdots 7 ¥$ 
i i i kÖ $\neg A<0$ tèĐÖ\#! àDHX \#\#\#\#ß û-\$\#!

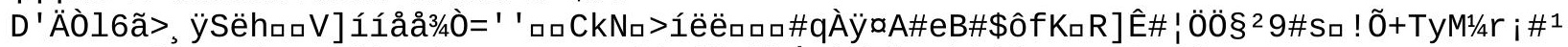

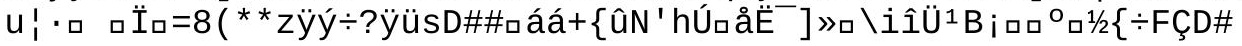




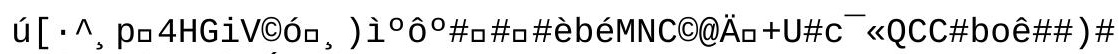

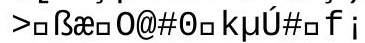




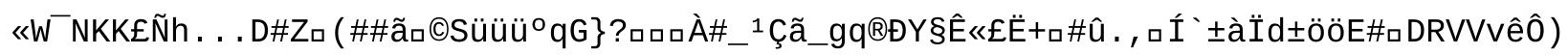




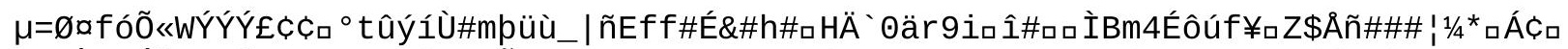

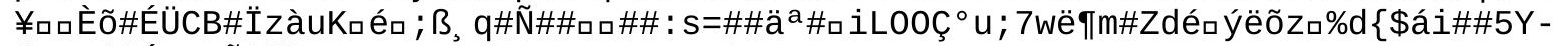

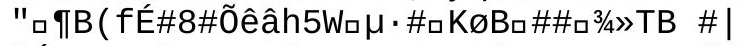

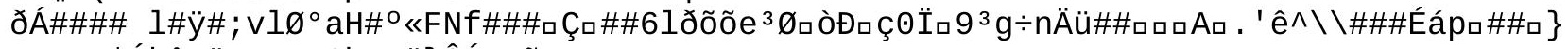

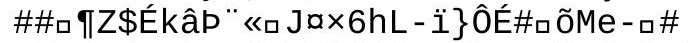

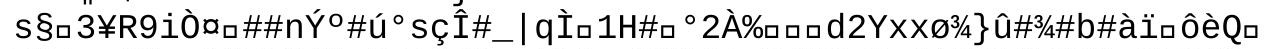

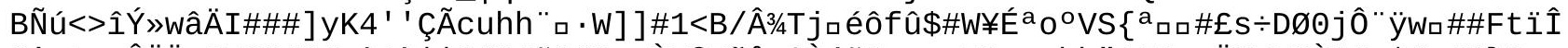

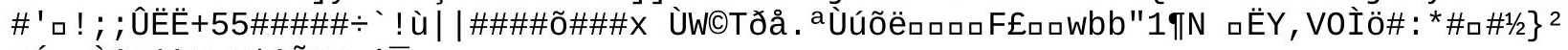

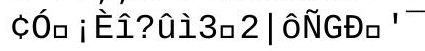




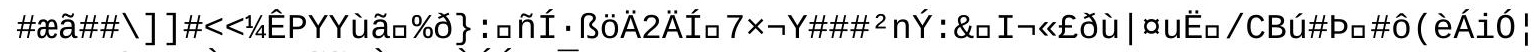

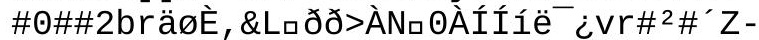

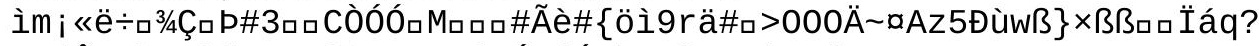

ÿüóర̂o¿]_ßõa777ßºuëĐiCóæİëúÝ b·oß., , |\#wá\# 


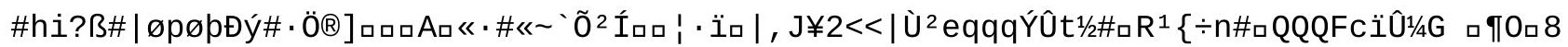
$1++K, \# \cdot \hat{I} G$ 


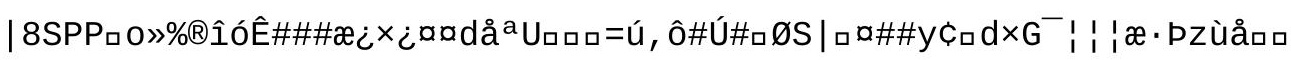




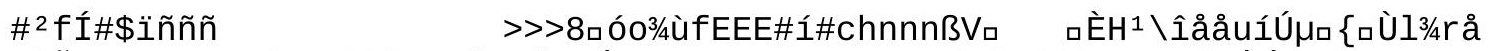

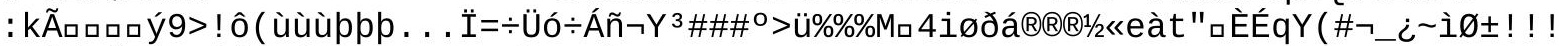

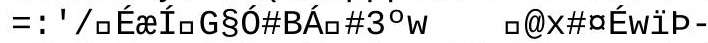

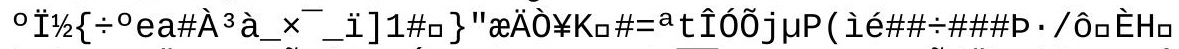

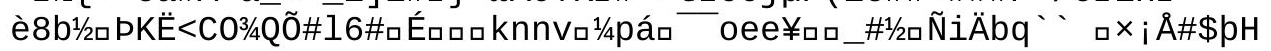


口૧口Ëё॰\#C 


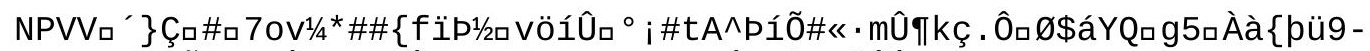

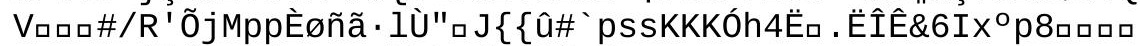

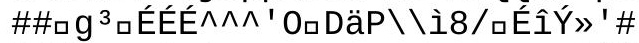

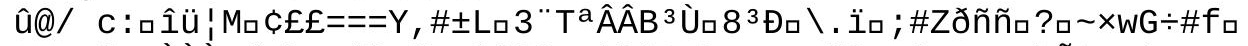

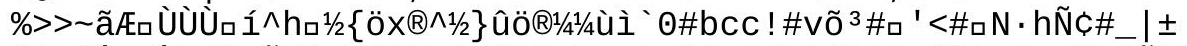

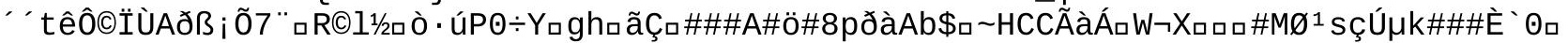

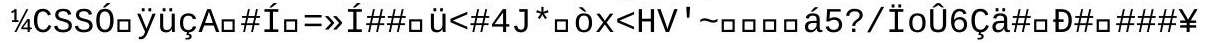




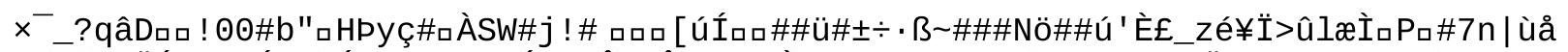

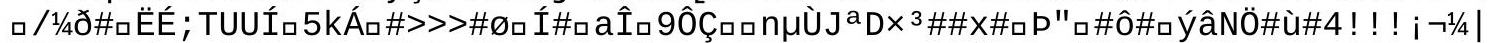

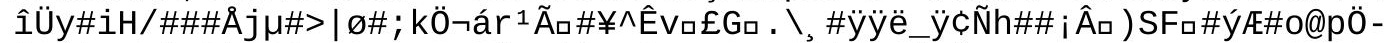

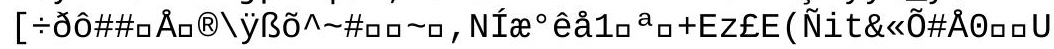




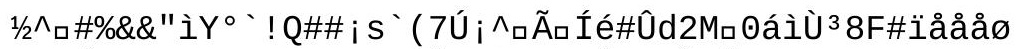

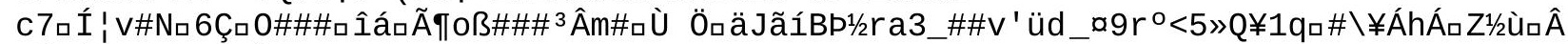

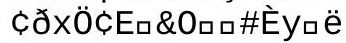

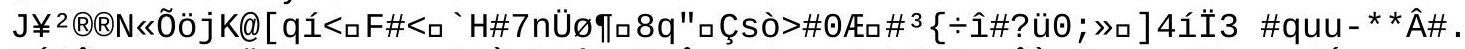

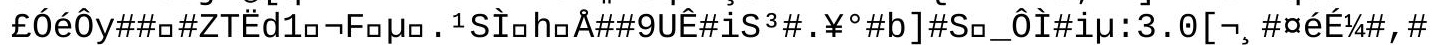

ýýýı - ]\#\#\# i V«\{ , äåå\#\#«ÑeäMêpᄀ 


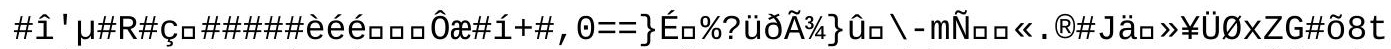

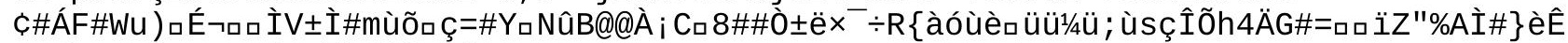

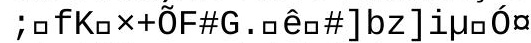

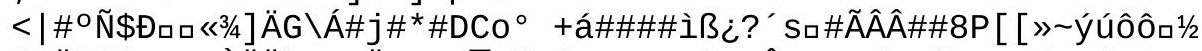

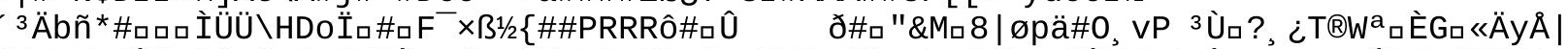

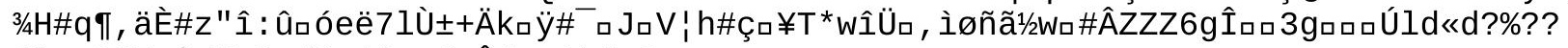

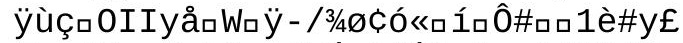

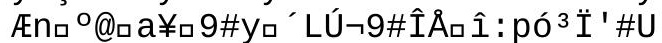

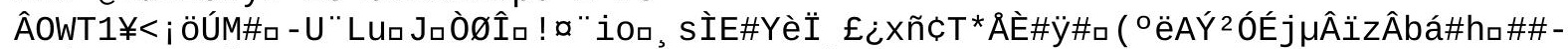

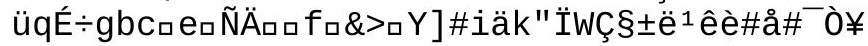

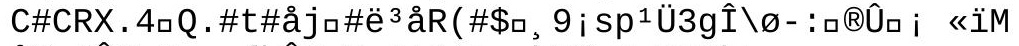

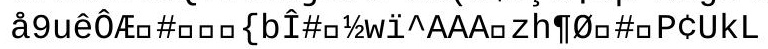




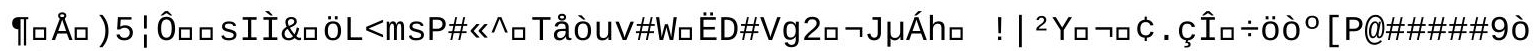
口) Sa $\left\{\hat{1}^{1} \# \# 4 \mathrm{hPMMM} \cdot\right) \mathrm{C} \times \mathrm{b} \#$ 


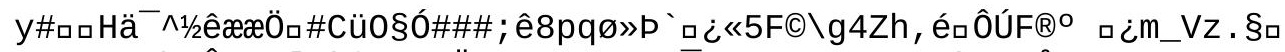

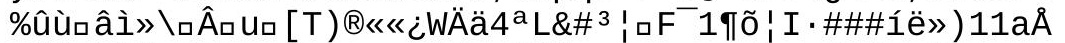

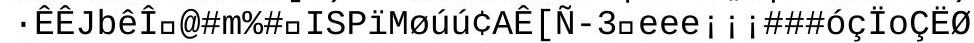

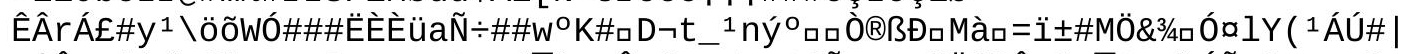

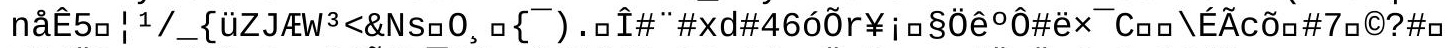

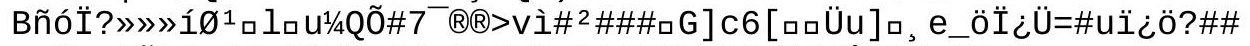

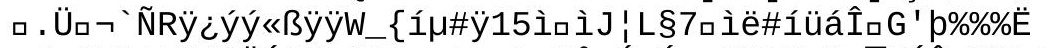

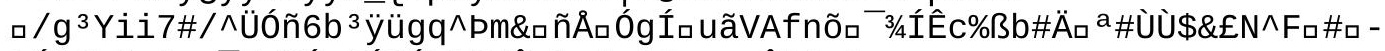

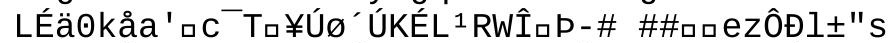

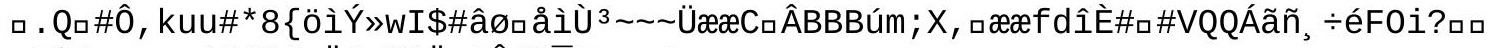

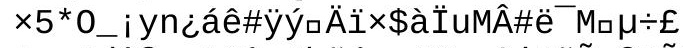

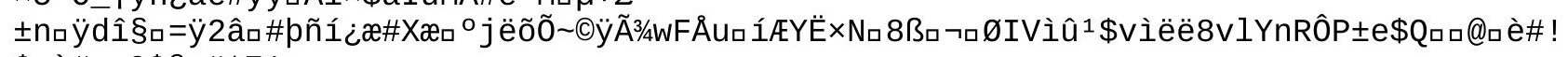

\$っè\# Q\$@ロ\#C71 


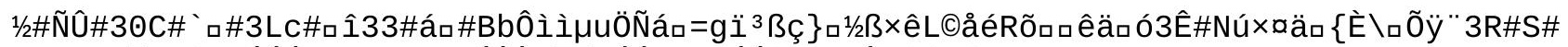

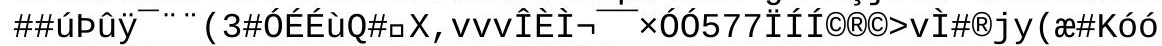

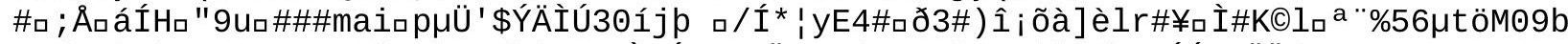

$\#_{\square} / E_{\square} a ̀ 3 h V 1 / 23 \ll X$,

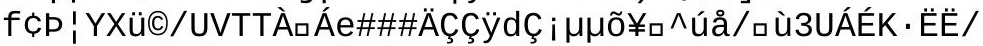

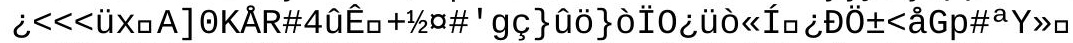

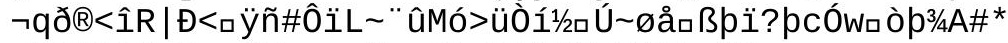
G

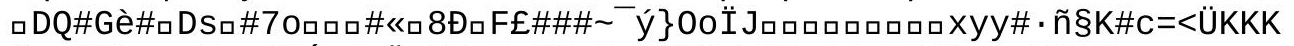

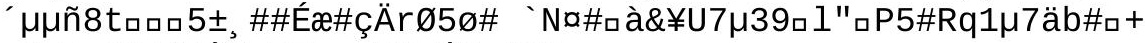
o\#akcó\$é\#jĖ¥vBIs qWêuÚ\#u\#a §

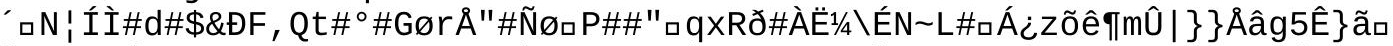

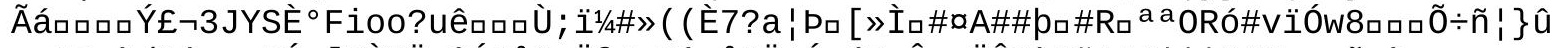

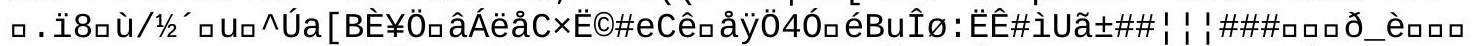

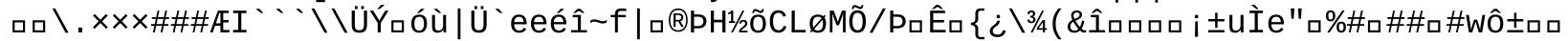

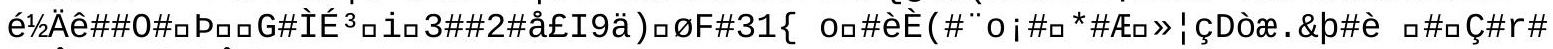

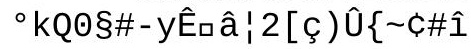


öÛòÒı£\#Ù\# 


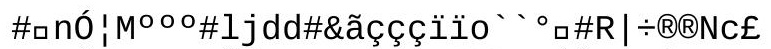

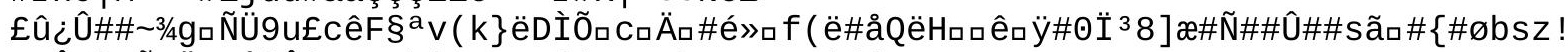

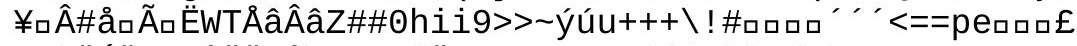

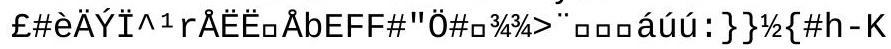




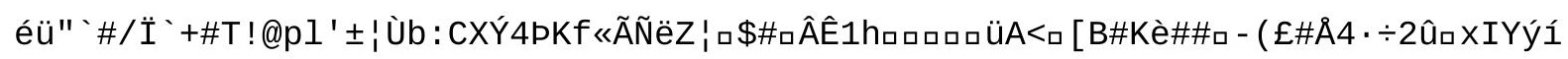




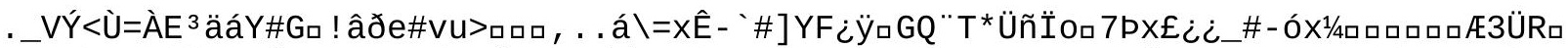

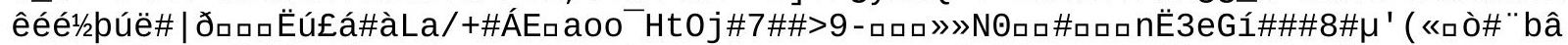

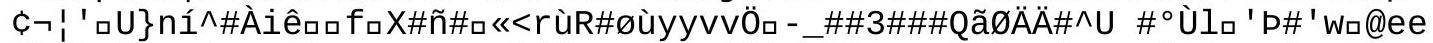

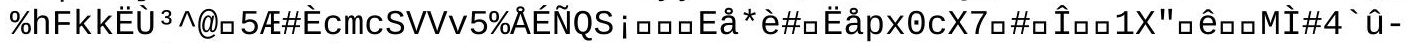

$=$, :C\#á $[2 \backslash \hat{Y}<5+\square$ 'ç 


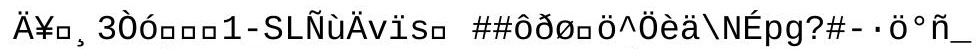

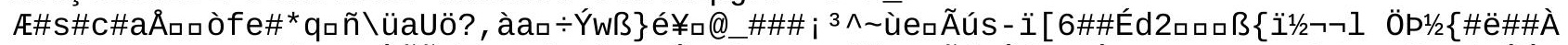

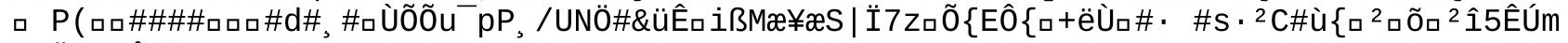
口Ẍ̈口\#\#ÛV 3

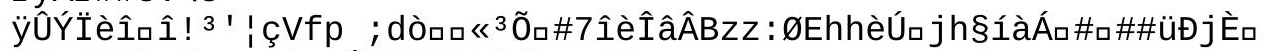

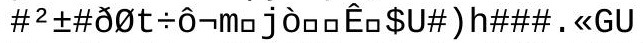

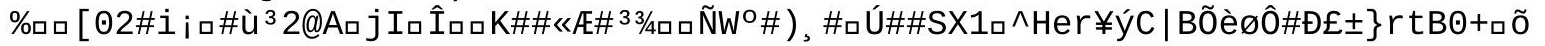




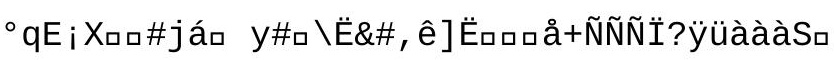




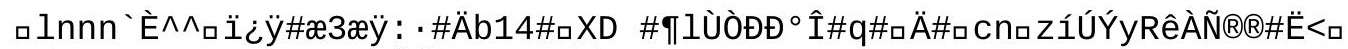

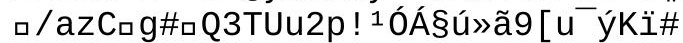

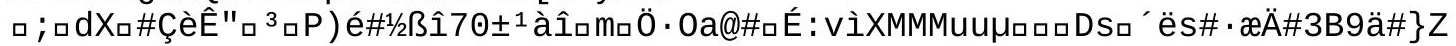




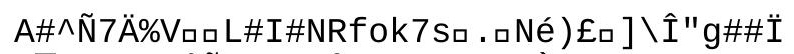

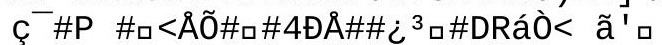




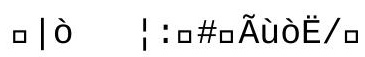




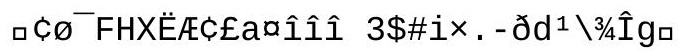




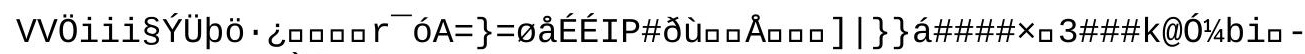
é\#ävôNWqu" \#\#ロÈfm ${ }^{3} \backslash 0$

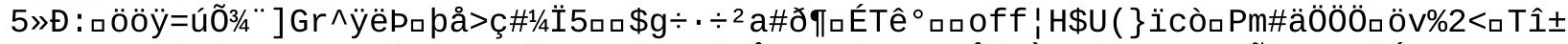

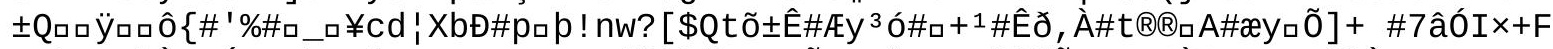

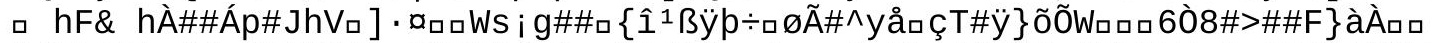

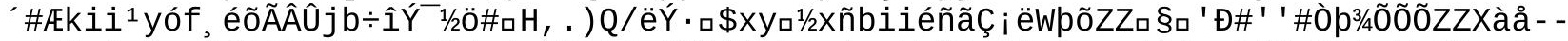

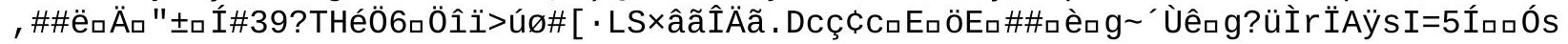




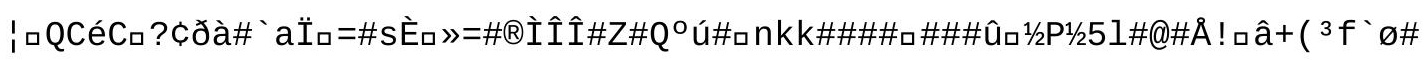




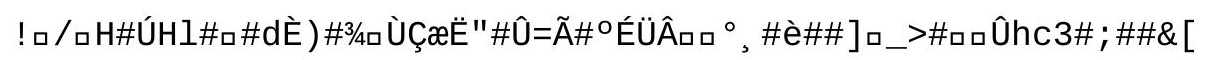




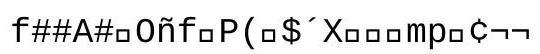




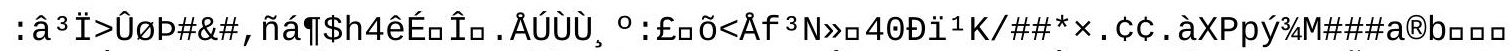

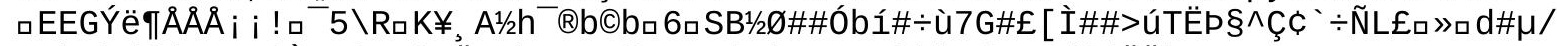

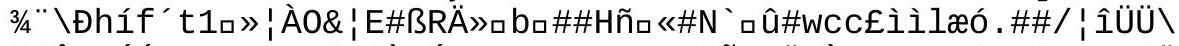

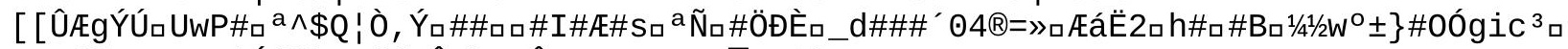

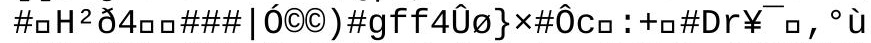

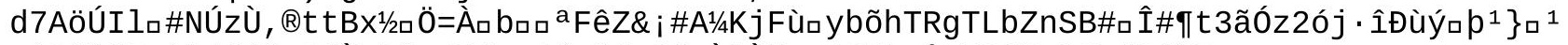

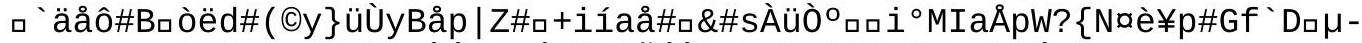

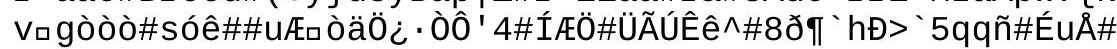




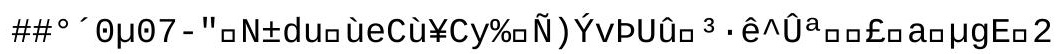




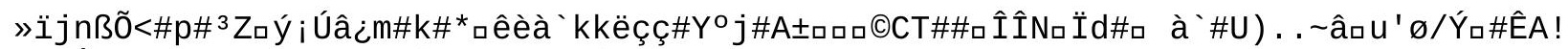

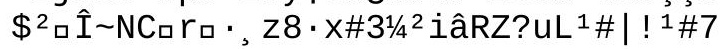




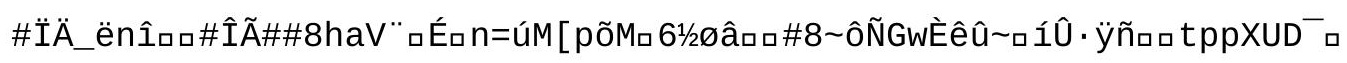




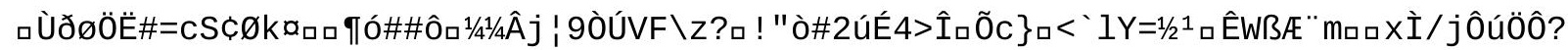
$D G ß, \& i \# \square$ 


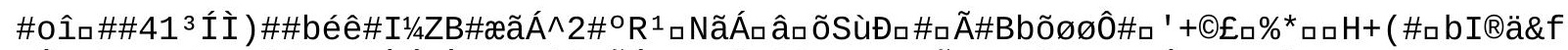

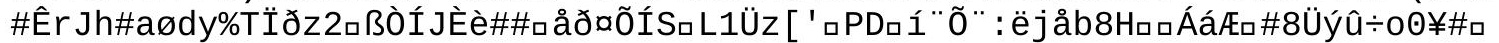
$i\left\{; \# \square j a \# A \_\tilde{N} \div \wedge\right.$

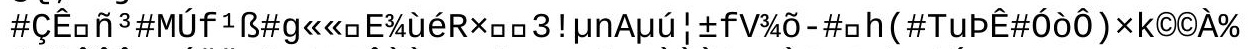

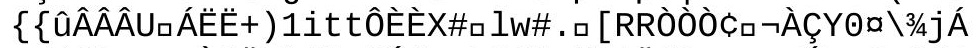

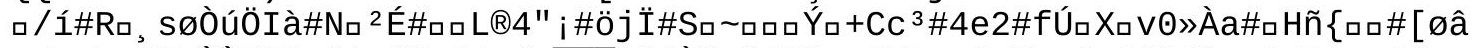

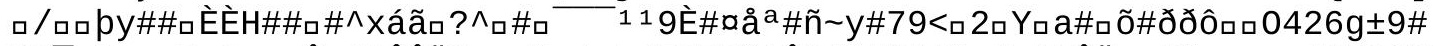

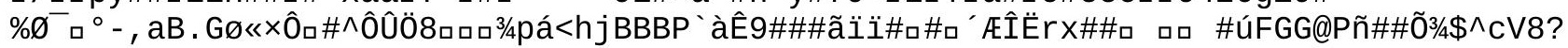

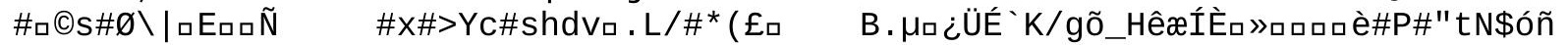

\#ロ ( .\&ua iv3qíc"i

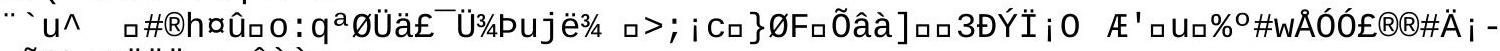
uÕßb \pm XÖÖÖロ 70Îİ̇̇̀<2 


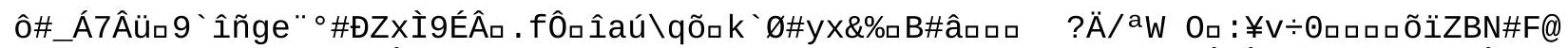

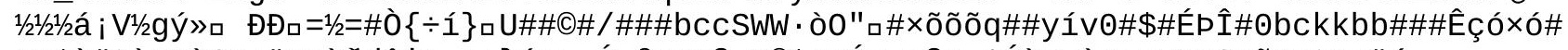

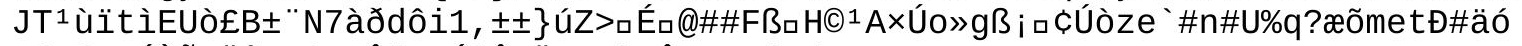

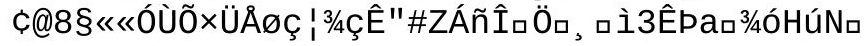




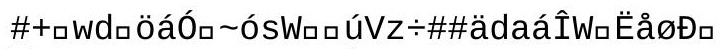

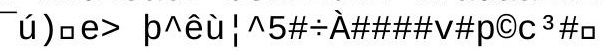




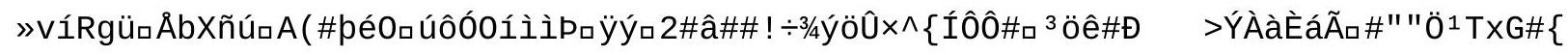

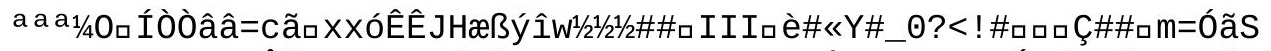

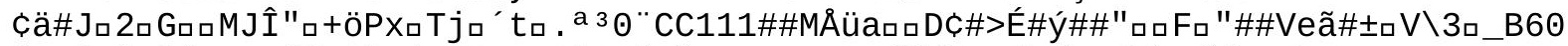

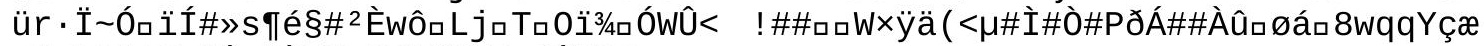

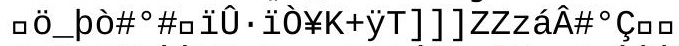

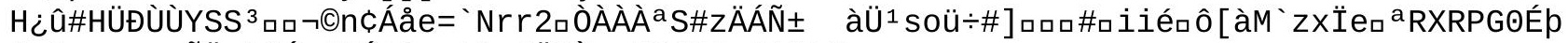

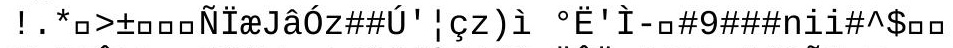

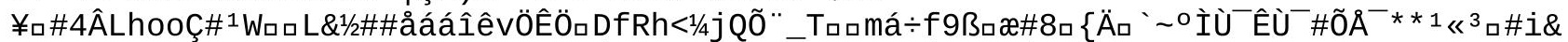

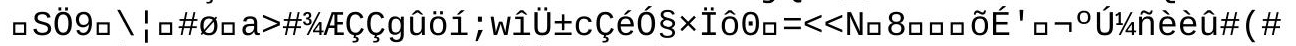

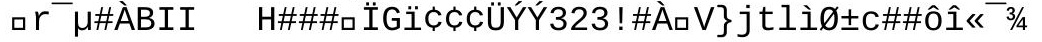

\#\#^1ロロSücf£\#\# 


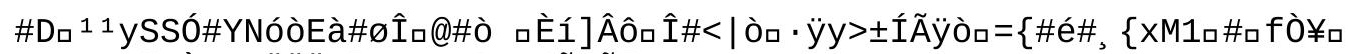

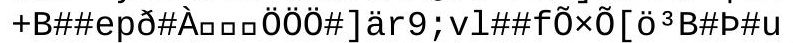

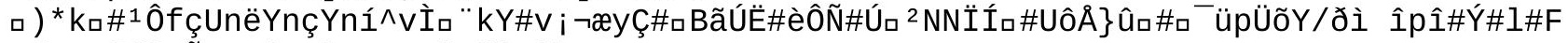

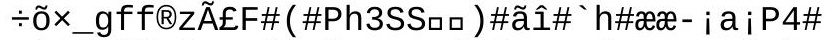

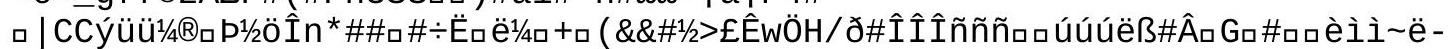

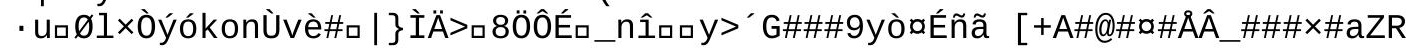

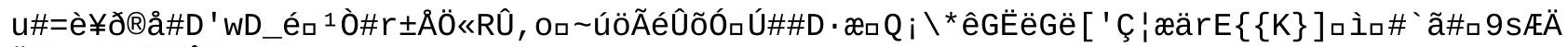
Ä\#Lø^|éG\Ôë 
çİ॰ロ\#ロÃ॰๑o\#\#\#4ロ 


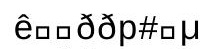

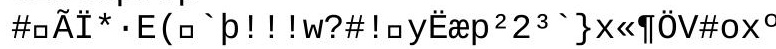

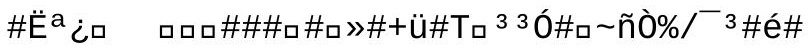




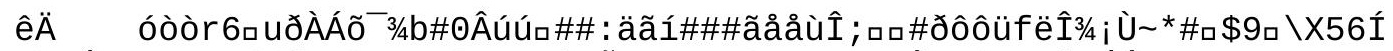

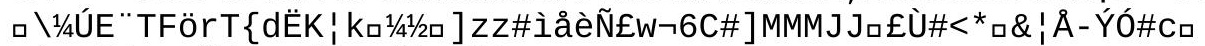

cåuモñé $1 / 2 \#>0 \tilde{0}\left\{a^{2} \cdot\right.$ é§mWÁÅ $>$ ãlka

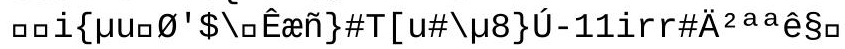




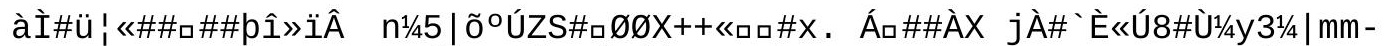

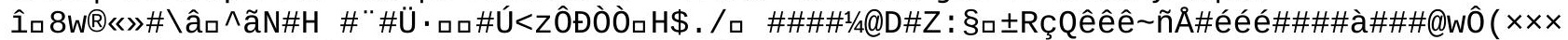

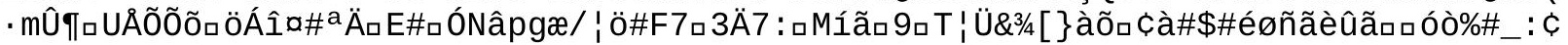




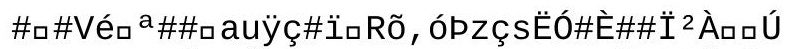

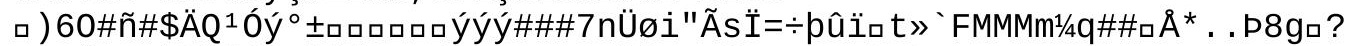

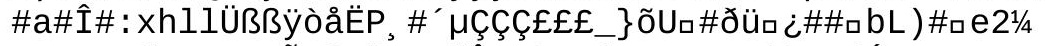

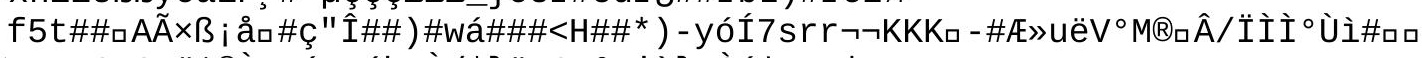

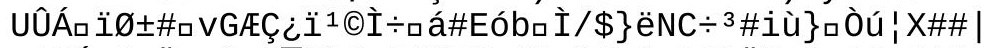

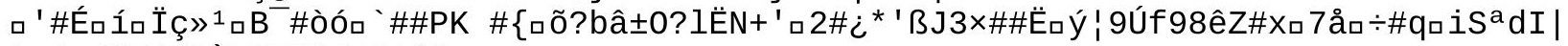

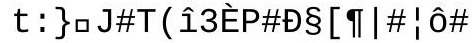




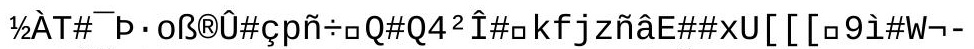

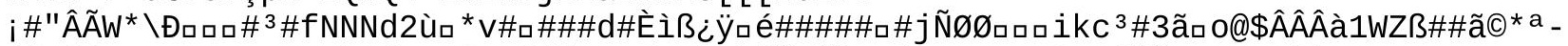

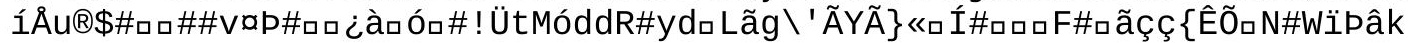




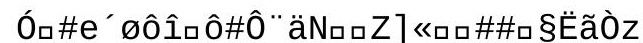

口’ÄÔúouÍwì5IN-

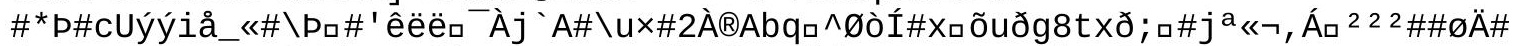

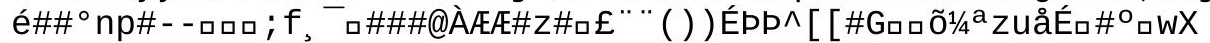

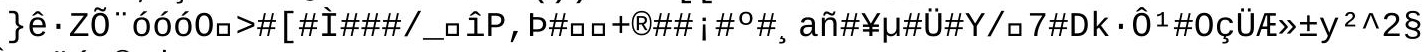
ca<øbÛ!ఃüíz@ロ|

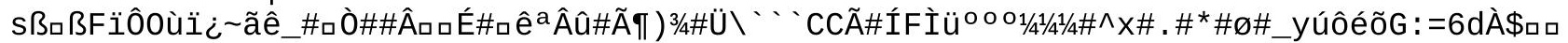

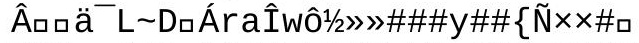
口1/4p\#\}ôÑ̃̃á́ßíO!

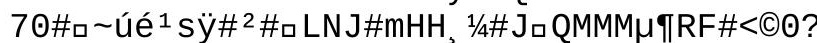




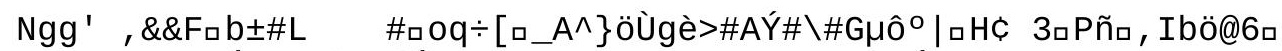

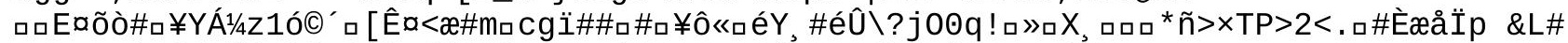

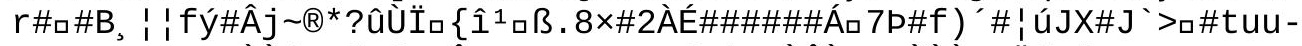

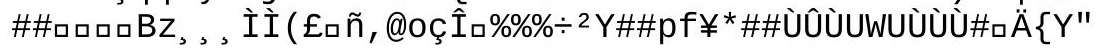


$\left.a_{1 / 21 / 2}\right] \$ \# \% \& \& \square: u^{a 1 / 41 / 4}$ 


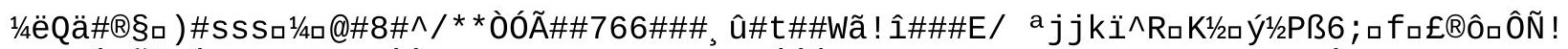

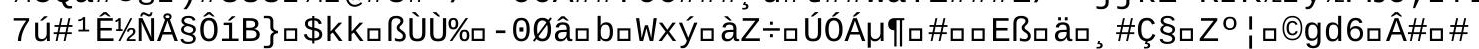

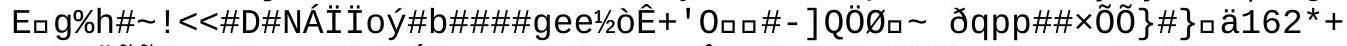

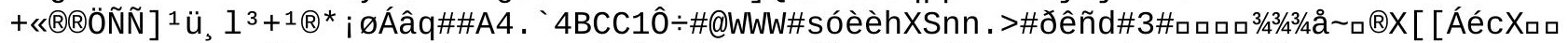

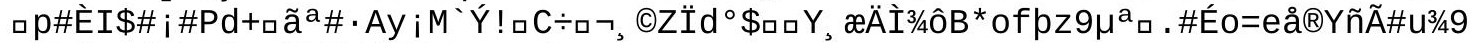

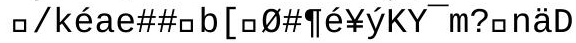




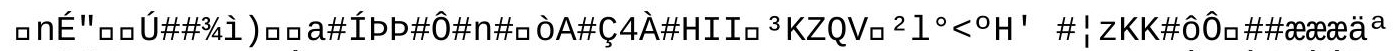

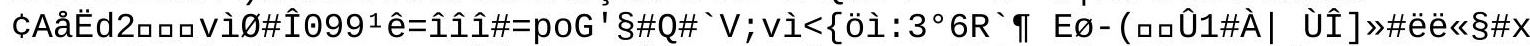

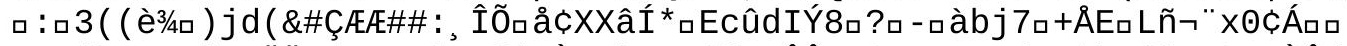

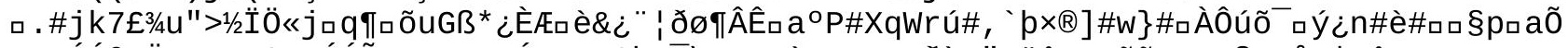

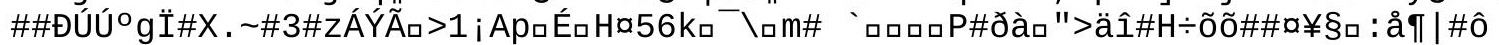
$\mathrm{h} \# £ \mathrm{~V}^{-}$«c \#kýýý-

ëZYOtCCT $\}=1 / 2-3 / 4$ Ú

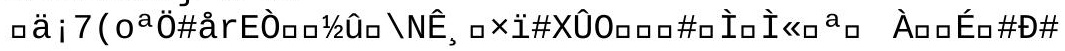




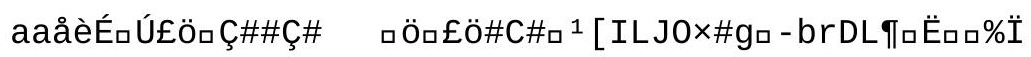




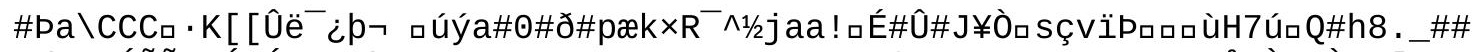

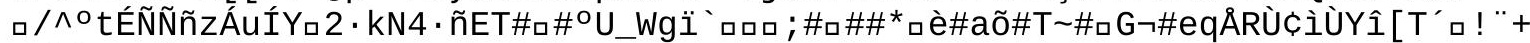
๑/kh॰3/4ロÖã\#ZgâR 


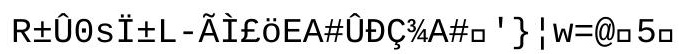




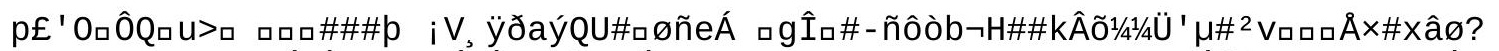

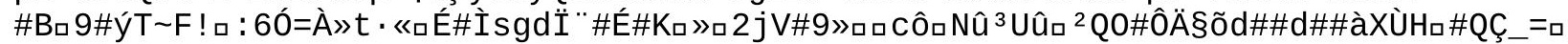
$\#>W l E \# \ddot{y}$ 
Õ\#2ÀHóòòüüü\#\#8םû\#

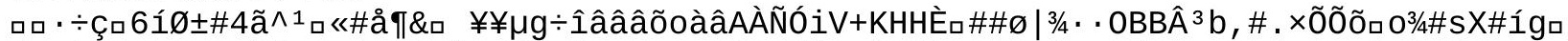

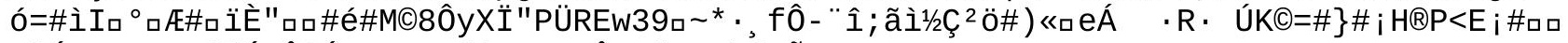

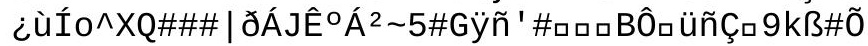




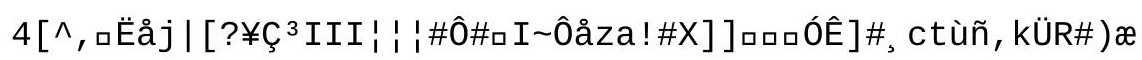




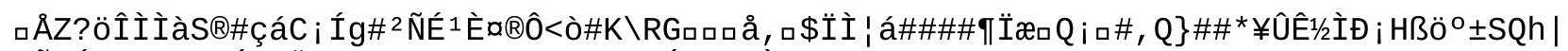

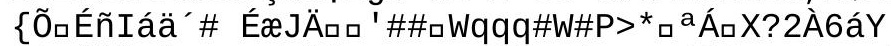

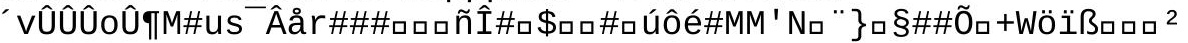




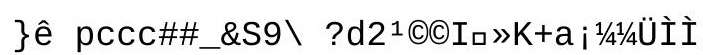




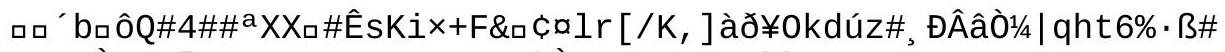

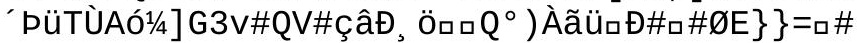

$E^{3}$ व?Đ

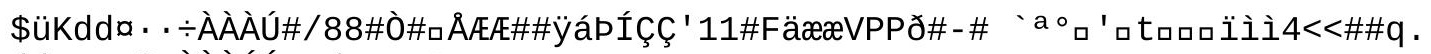

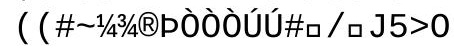


»००JJ 



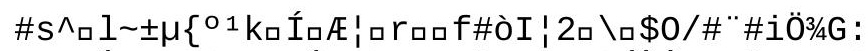

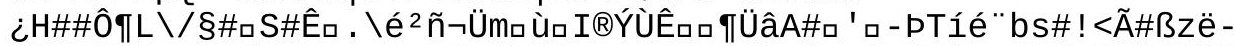

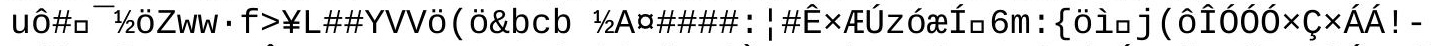

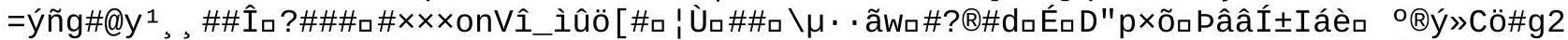

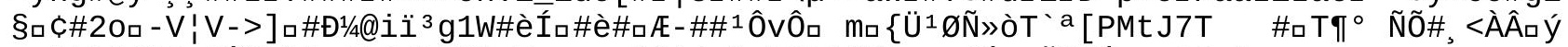

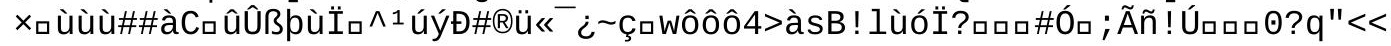


1́88: 2 ¿_\#tËÄÄD \#tuwk ${ }^{-}$ýøga: öõ

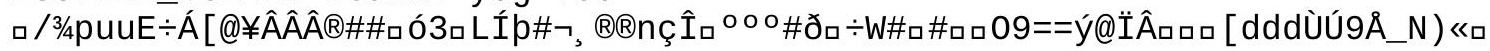




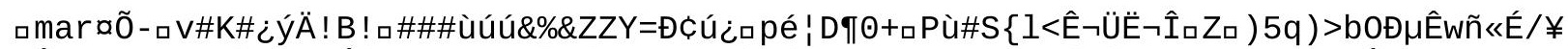

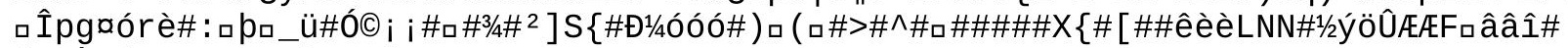

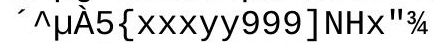

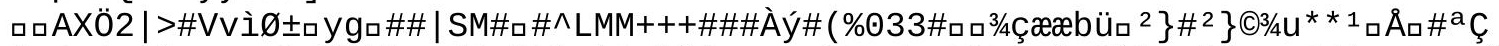

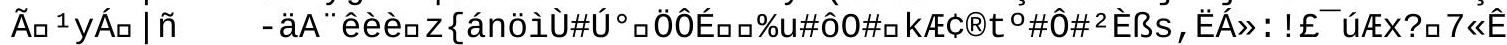

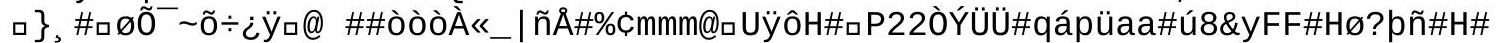

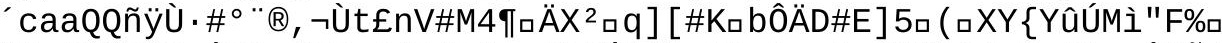

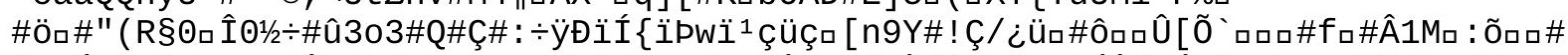

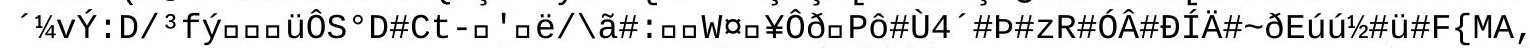

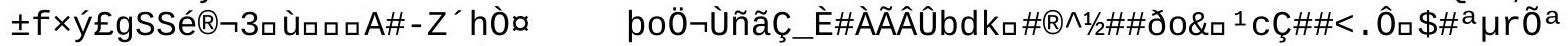

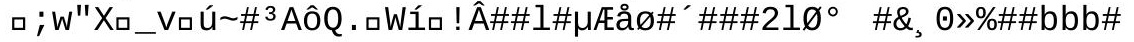




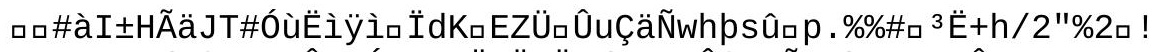

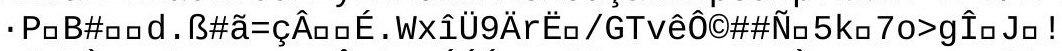

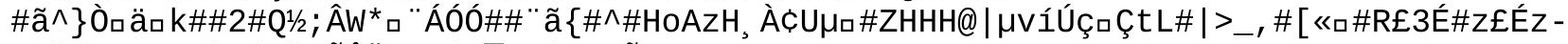

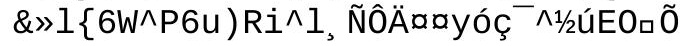




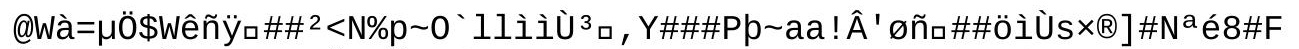

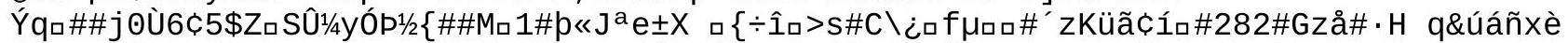

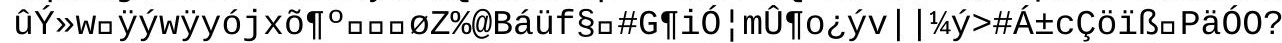

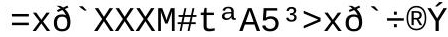




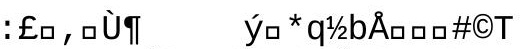

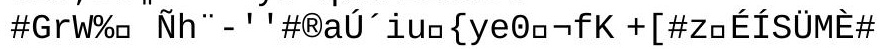




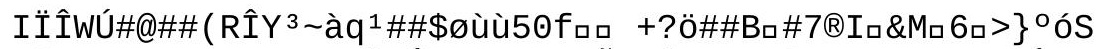

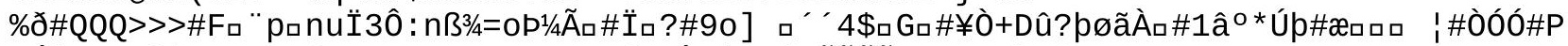

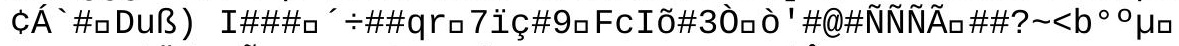

c J\#\#<qâÄ $\left[01 / 20 \tilde{2} ¥ K_{\square} 3 g^{\circ} \Theta_{\square} \# / \wedge<\sim u ̈ X q u \#<3,-\pi\right.$, âÂ\#X

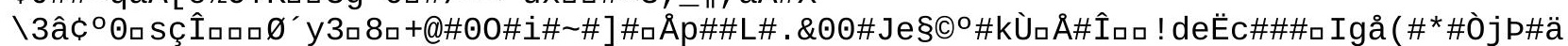
口Hd2Y\#Ë=íÀa ", ôðð@ "Đ《W ÜÜÜ * 'À\#̈̈ 


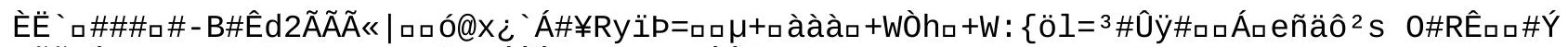

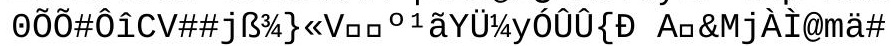

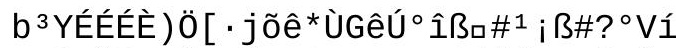

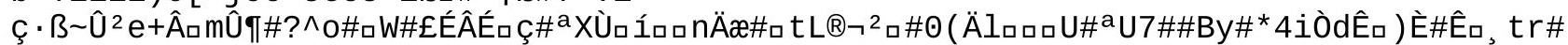

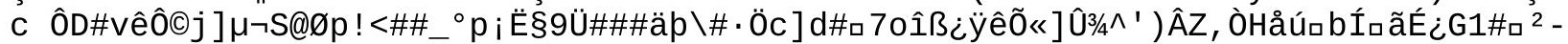

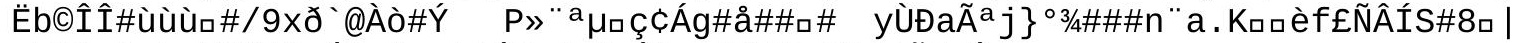

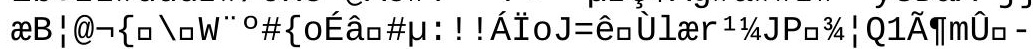

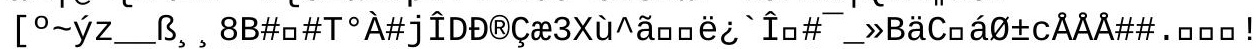

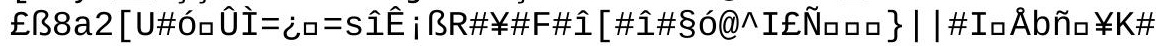




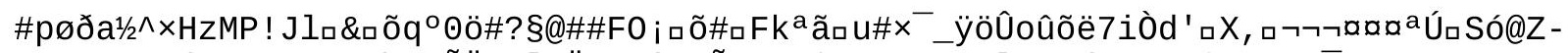

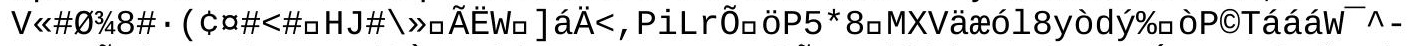

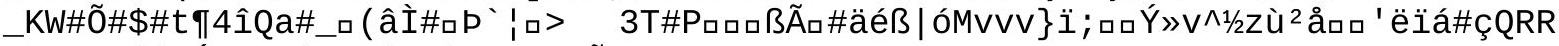

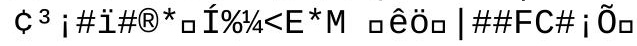




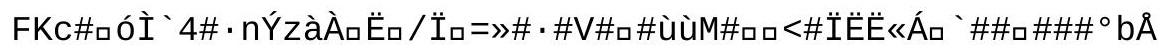

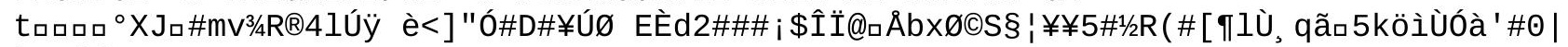
psç"\{

\#\#\#-7È= 
õ

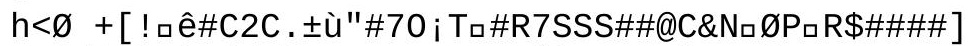

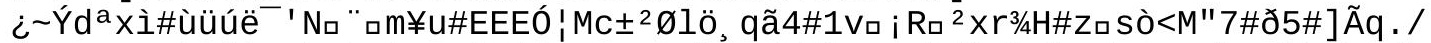

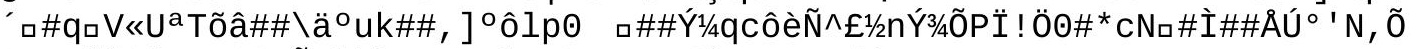

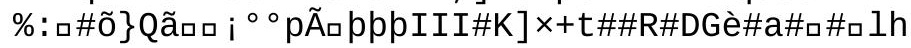


oÀİ `Đ\#à|0๘ 


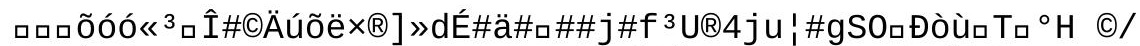

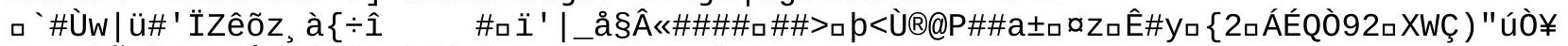

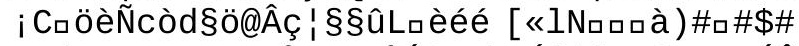

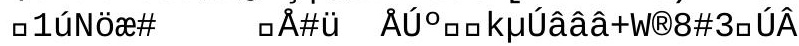




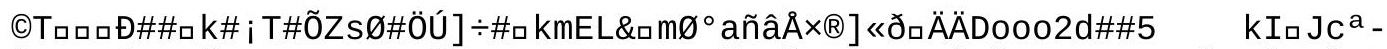

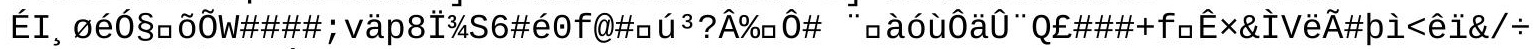
- )):\#Án)\#se

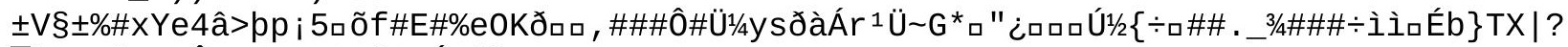

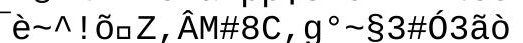

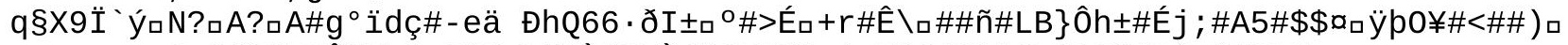

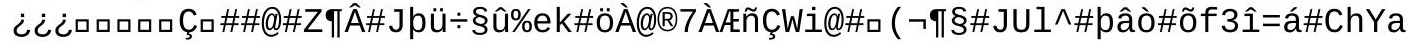




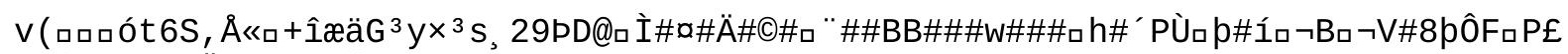
+Ripっûrq\#Ä\#` ฉÇüâ" $\mu \#$ 
ฉûqùEQYÜp\#\#qHp\&3ロÅóÙ\# 
ITHÍââ\#dロ\#\#/ËÔa|¥

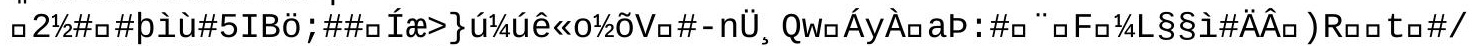

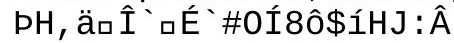




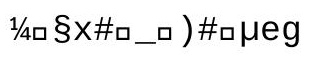


ロ\#! ÂQ\# 


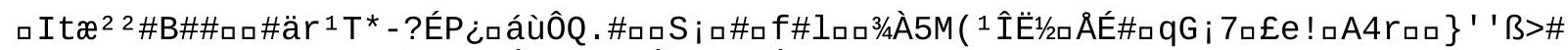

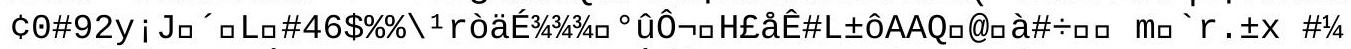

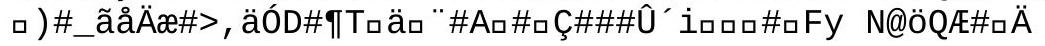




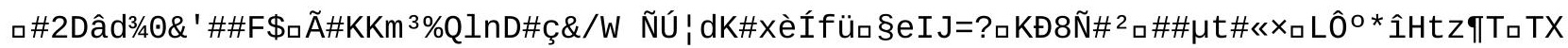


Ö\#-H`o P\#ёHJúロ 


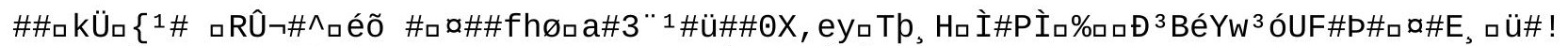

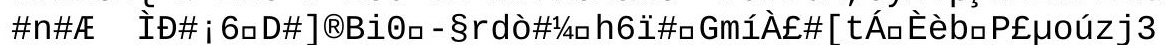

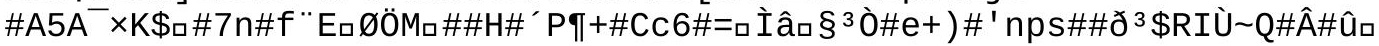




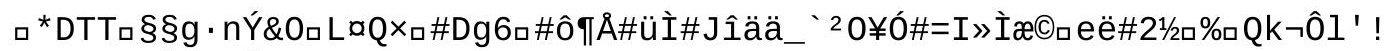
$\# W ` \pm \# 6 \ddot{Z} \dot{\check{a} \square \mathrm{A}}$ 


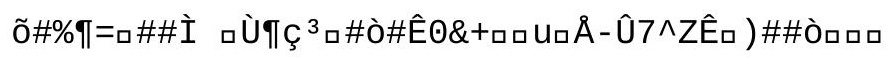




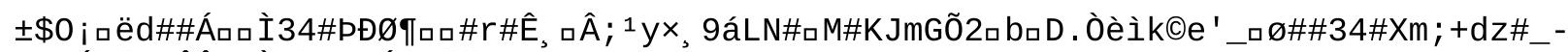

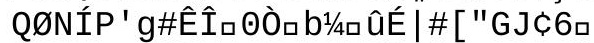

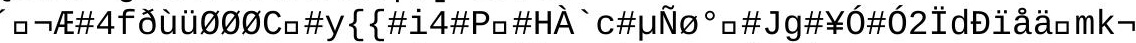

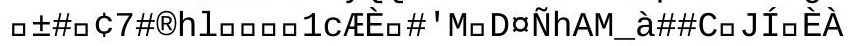




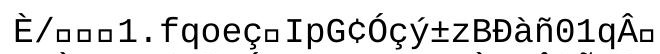

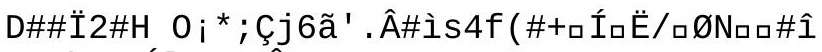

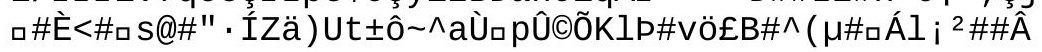




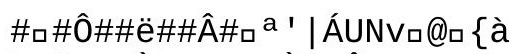

\Çå\#1\$Ò\ロ ${ }^{2}$ XQ\#Ė/OÂ 


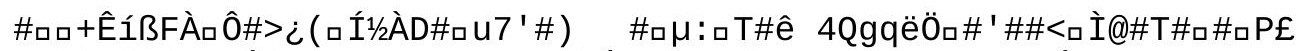

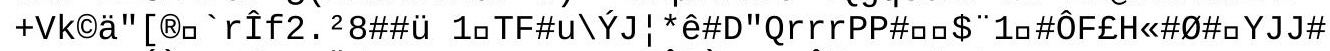

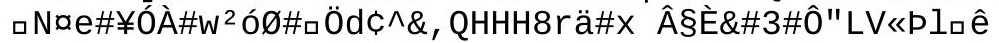




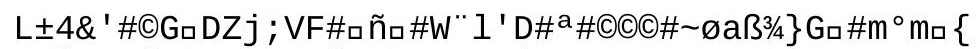

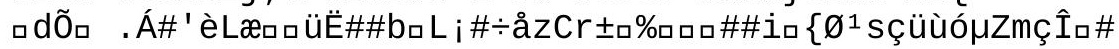

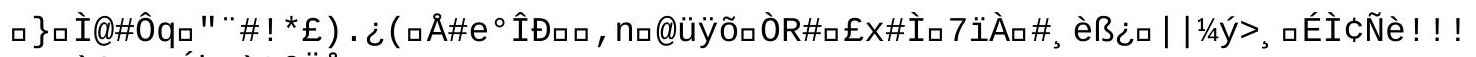

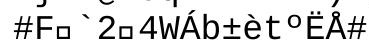




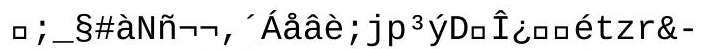

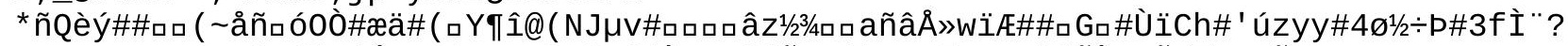

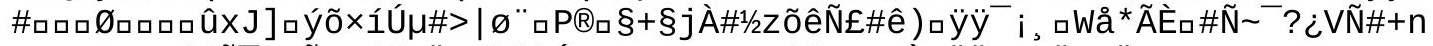

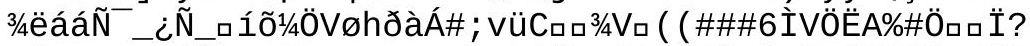

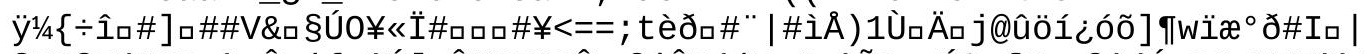

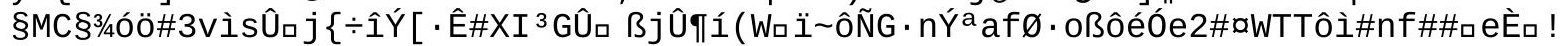

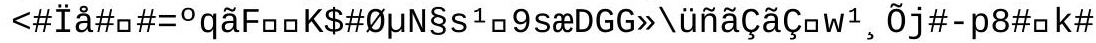





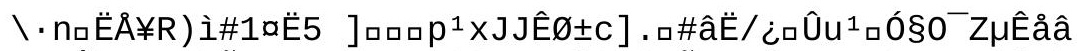

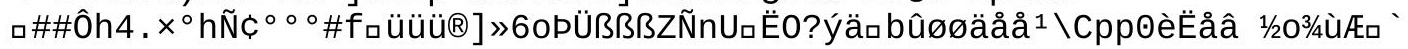




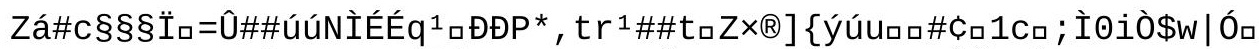

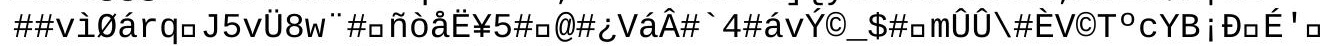
口; $\ddot{B} B \# \# \#^{\circ} \mathrm{C}, \& \square E ́ M \# \square A ̊ b W \# \# € ® \times \# \$$

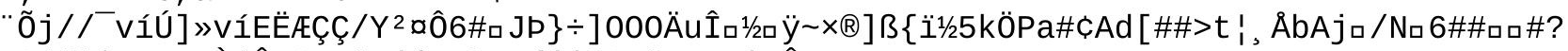

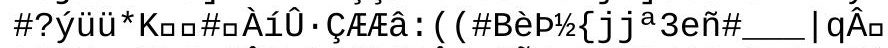

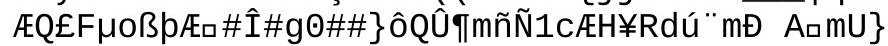

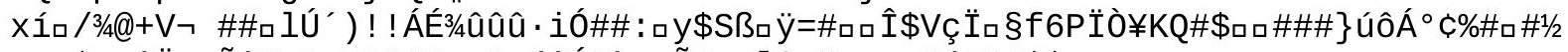

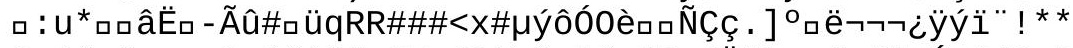

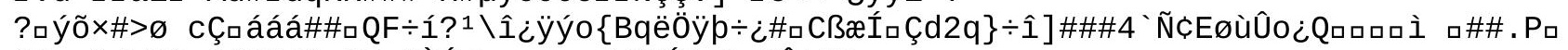
@* : yòdH\#Òa"\#\#\#ýóaÿDmî̀̇ÉW vìØ\#ÝFwĐ»\#Êt\#?

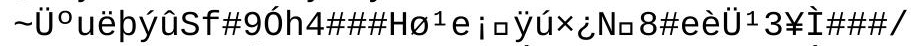

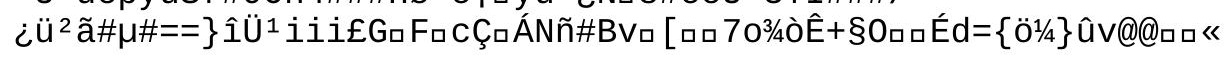




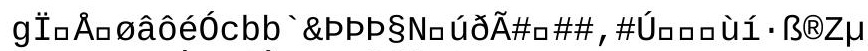

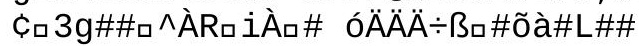




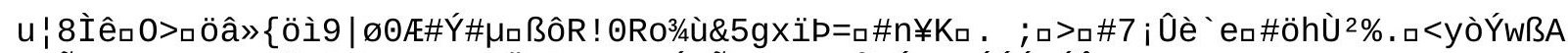

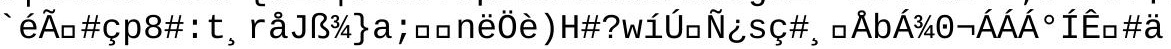


i i ûö

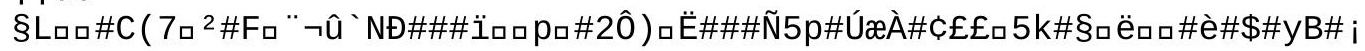




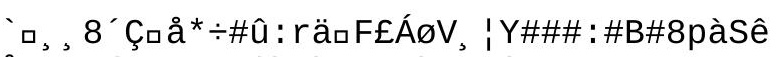

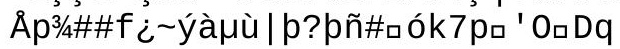




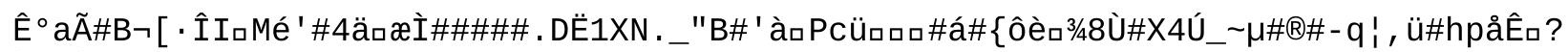
pø\#|>

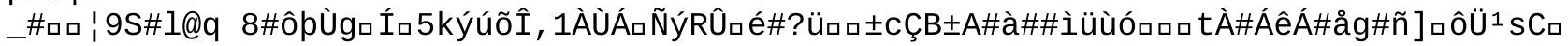




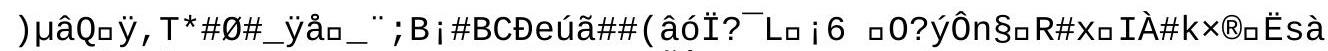

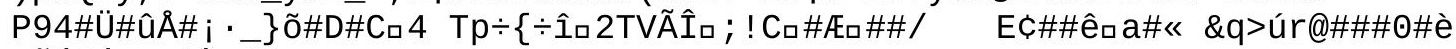
tÃÉ@Ú\#, ฉòİpñâE 
nqq土ó_\#/ロ\$\#\#ÂýÁwCJロya

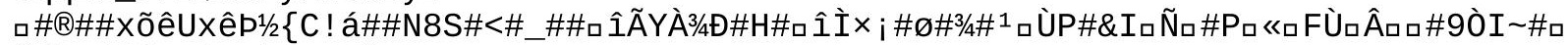
ÂÛpüóİð_qáaJh91/4aı0̂

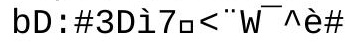




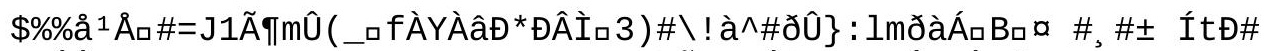

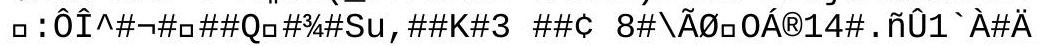




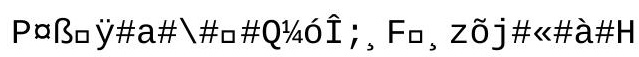




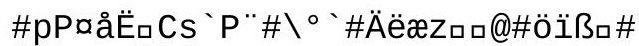

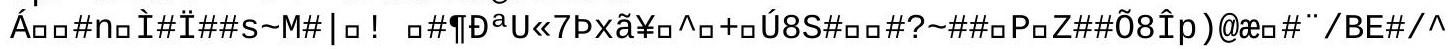




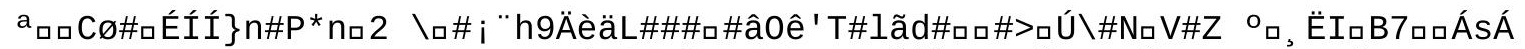

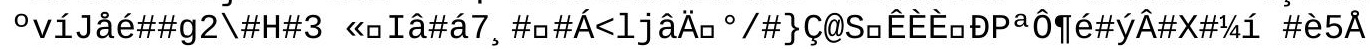




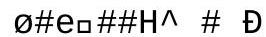

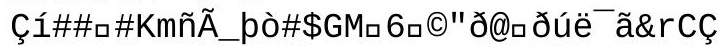

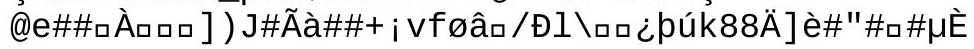




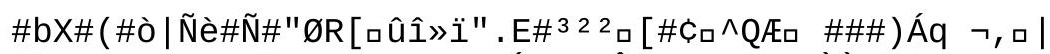

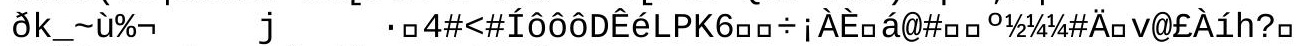

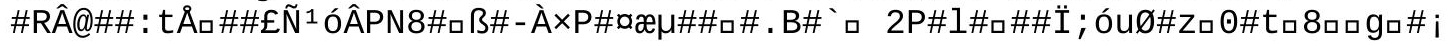




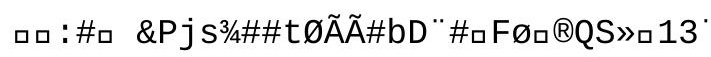




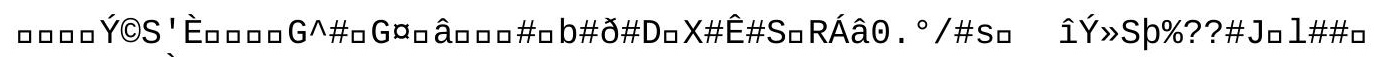

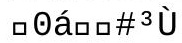

äää $N^{1}{ }^{1} \tilde{=}=$ 
$\mathrm{U}^{\circ} \mathrm{u}+\mathrm{x}$ 


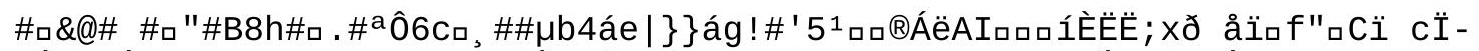

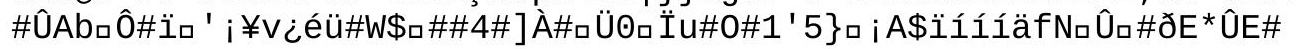




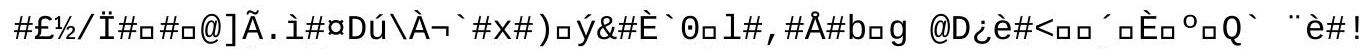

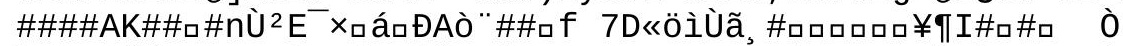

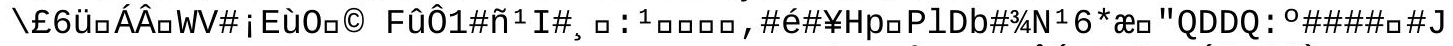

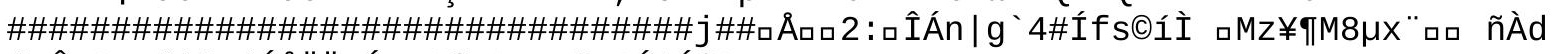

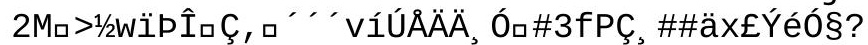

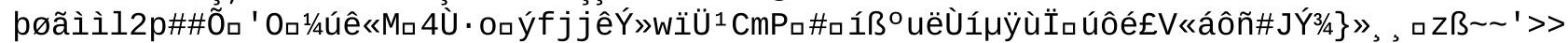

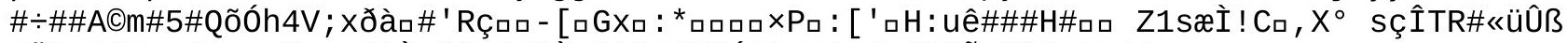

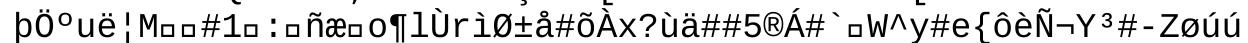

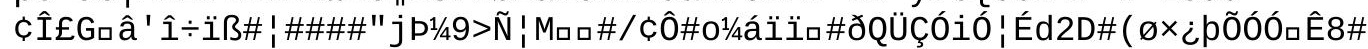




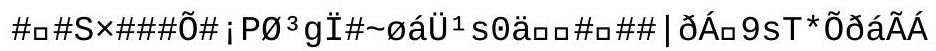




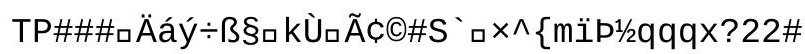


\#»Fıòúë \#8p

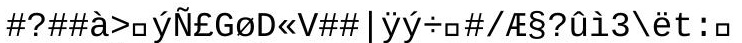

8'77\#e\#WĐéôÍ_7SS\#

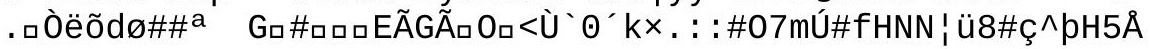




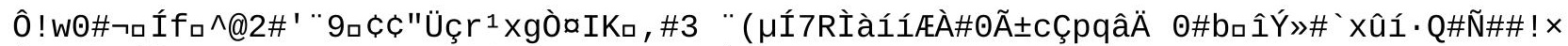

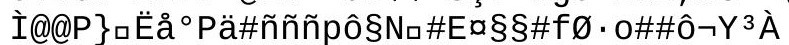




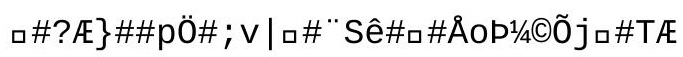




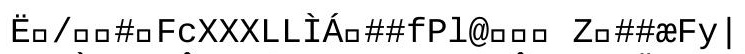

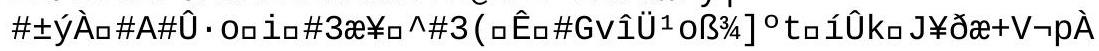




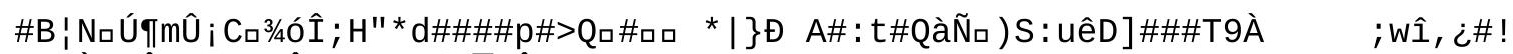

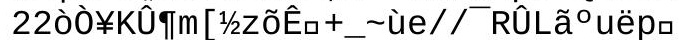

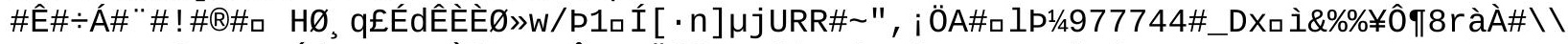

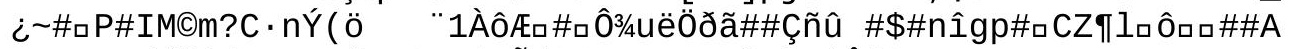

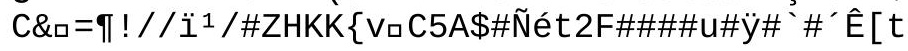

endstream

endobj

$37 \odot$ obj

$</$ Length 4375

/Filter/FlateDecode

>stream

hDÄZínãÆı\#vÿõC\#ú\#ロ

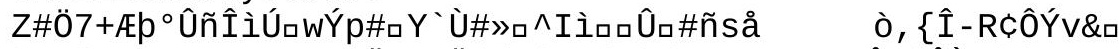

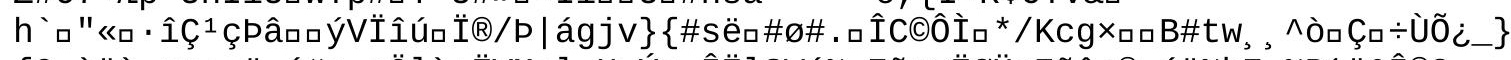

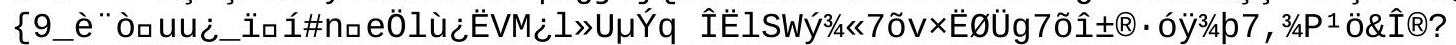

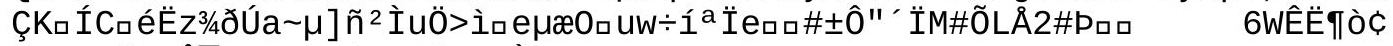

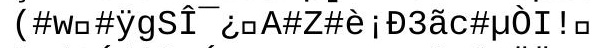

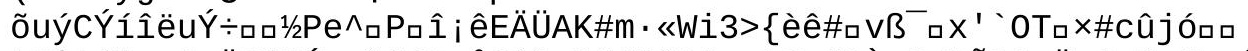

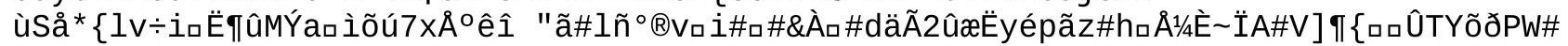

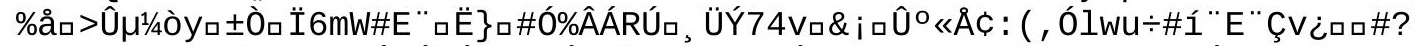

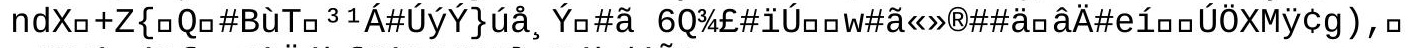

口\#\#I , úGf_v\&ïÄúhf\#î”\#\#8ö\}vMóh/áÑö 


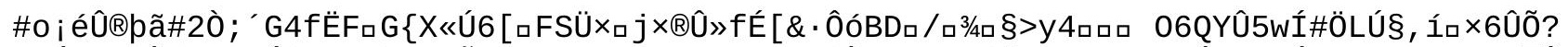

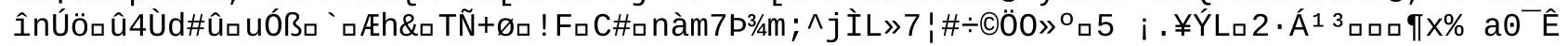
rFi5\#\{ 


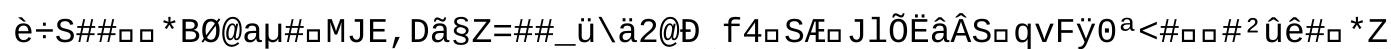

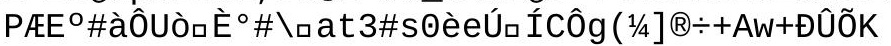

\#+๑Önë×ÃÃ\#\#DÛ 


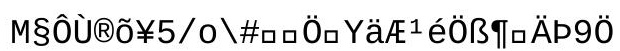


Ù้อฉ $\odot S \div 5$ ฉ 


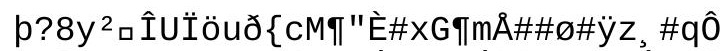

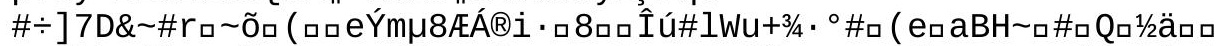

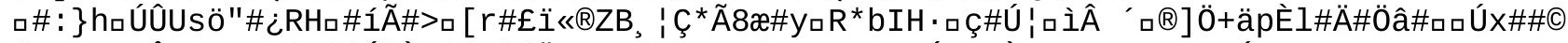

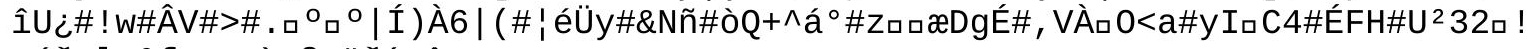

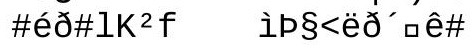


\%H\#lòbB\#C\#ÝÖロ, 'Å4\#Zc\#4\#囚hsf\#W\#; y ${ }^{\circ} \#$ 


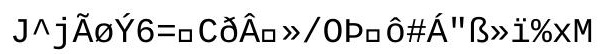




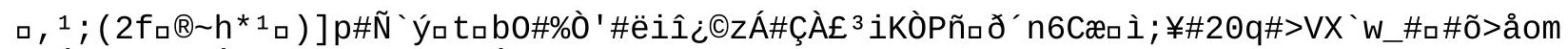

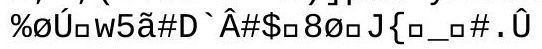




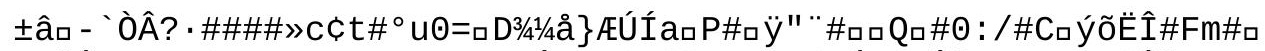

ù-ÄÂ¥ä?Bı ( 


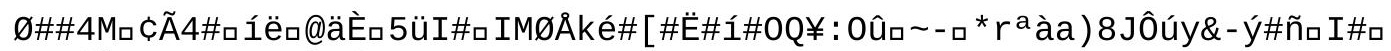

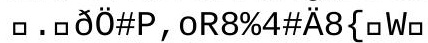

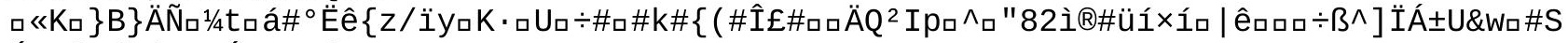
Áı\#"\#/bjađıÁ \#D7íPıם

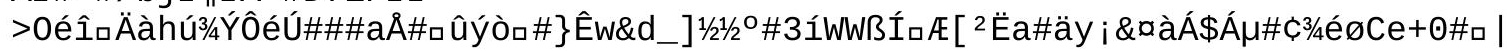




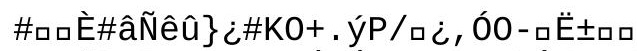

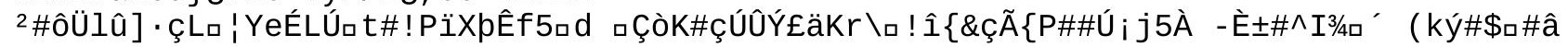
व๑๐०Lå, 


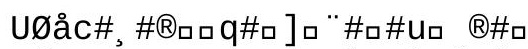

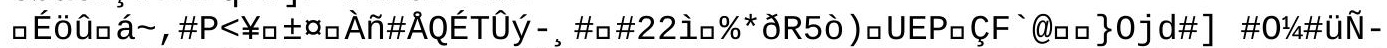

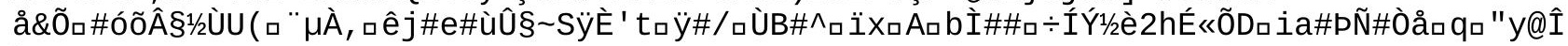

\}口\#口\#

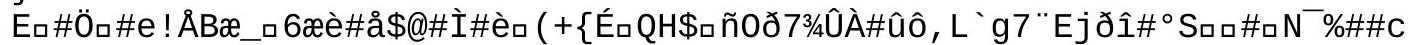

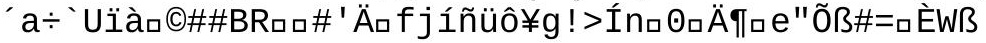




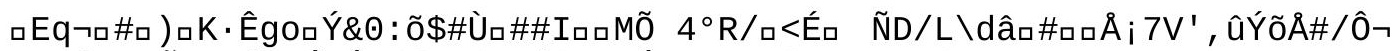

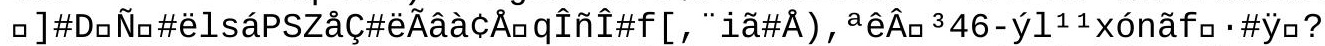

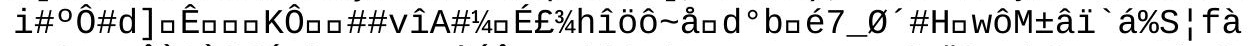

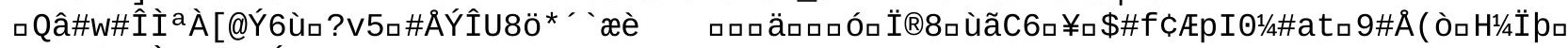

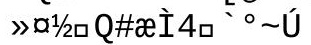




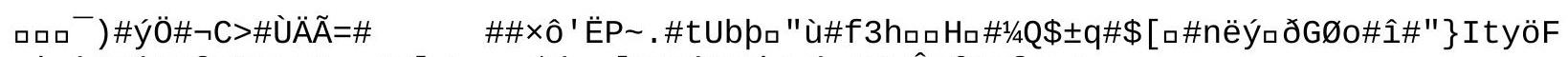

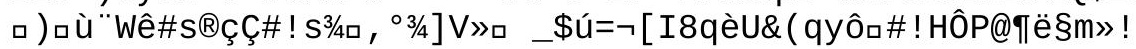




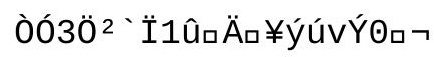




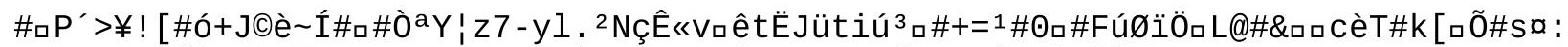

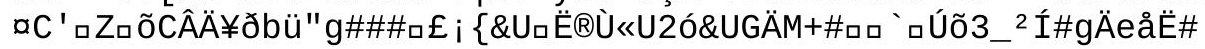




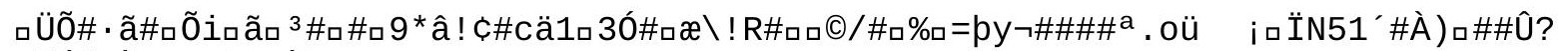
8 ÉËBÙä $1 / 2 C \# p$ ' 3È \QcC ! + 
åaã3é $>2 \# Z\left\{5 \# X^{\circ} \mathrm{N}-\right.$

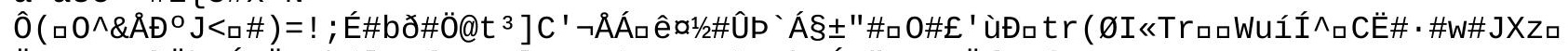

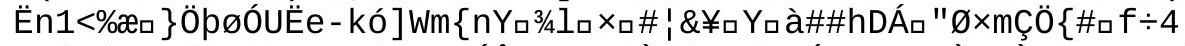

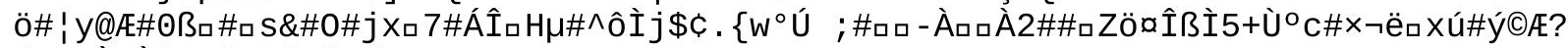
$\mathrm{d} \pm \varnothing u O ̀ y \grave{I} \otimes P A \circledR a \# \ddot{i}, “ \times d$

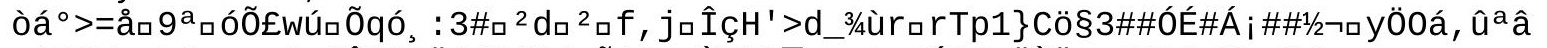

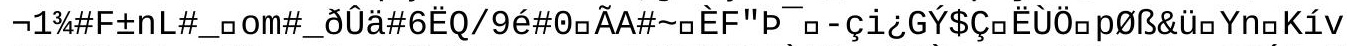

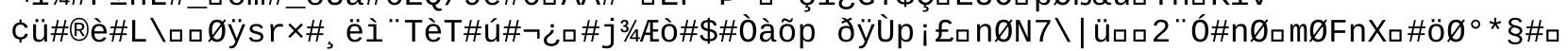

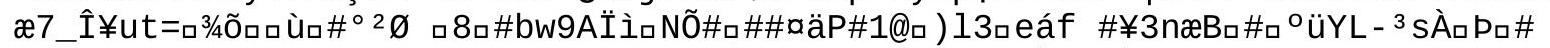

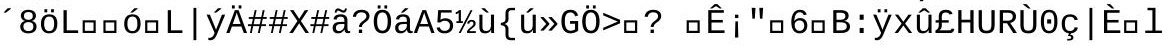




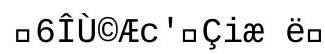




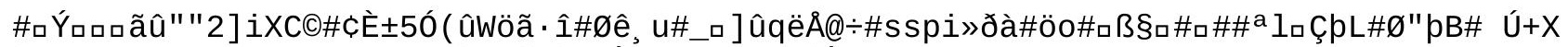

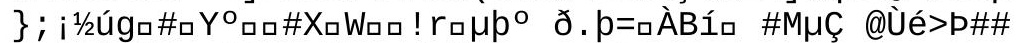

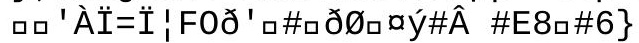


\#Ôa ${ }^{1}$ ù [\#À

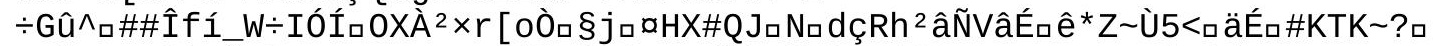

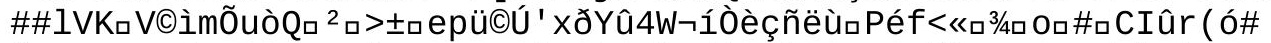

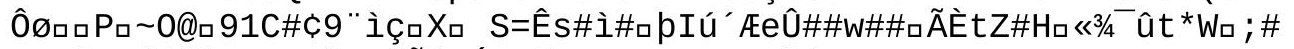

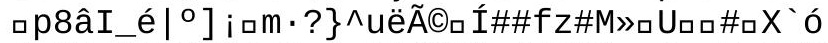

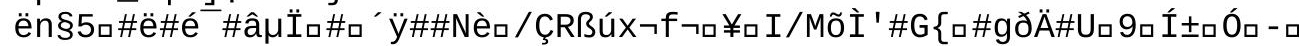

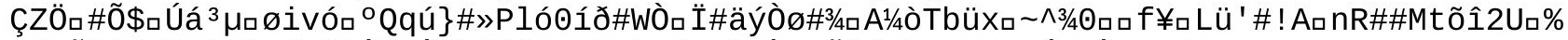

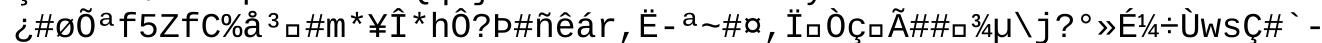

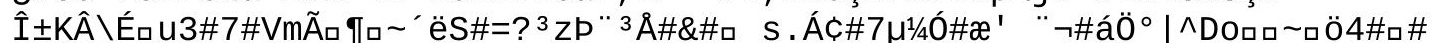

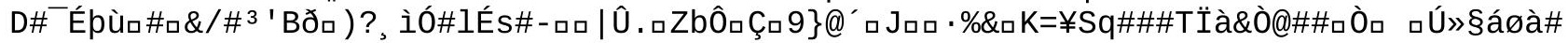
口, \#DヶVð ĐÆôñ 


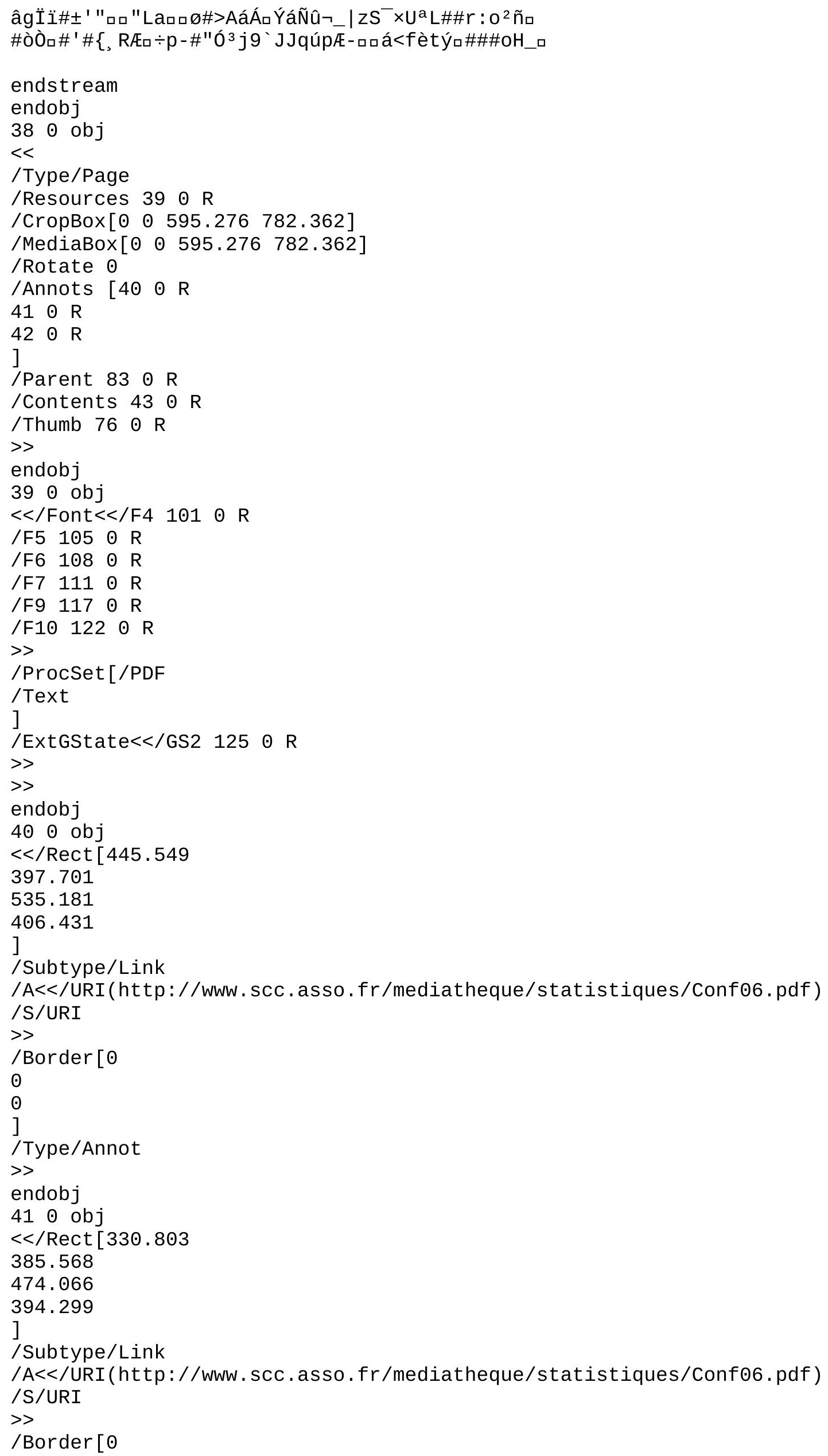




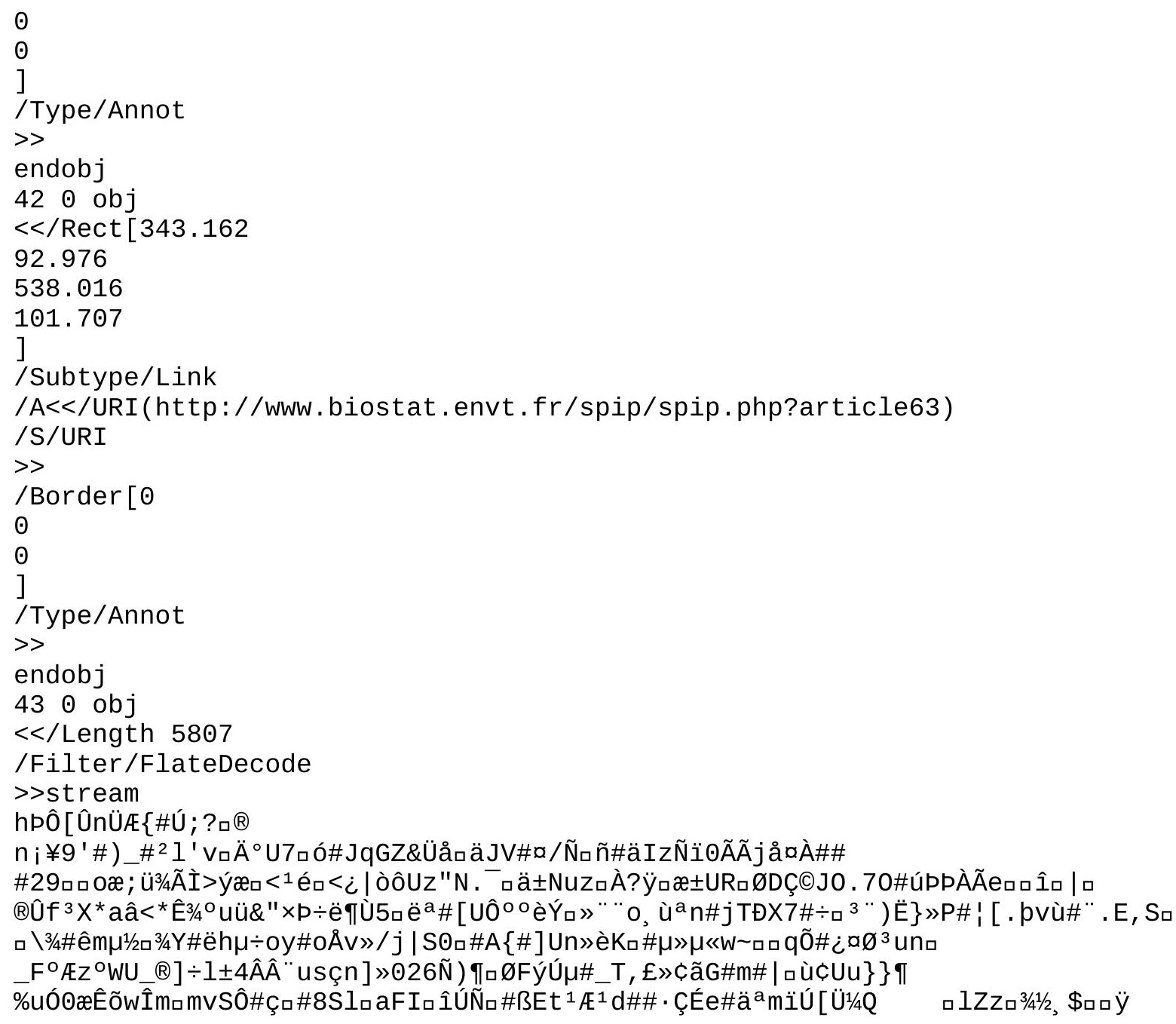




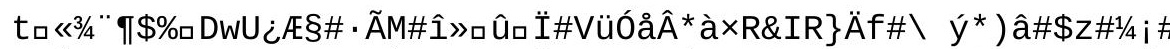

3\#Ûロロロ=\#๑Áùêף *è\%\#29

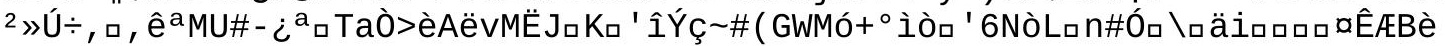




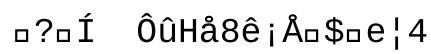




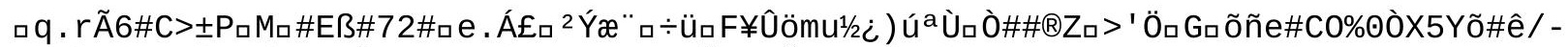

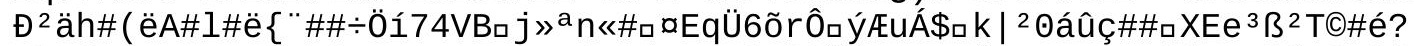

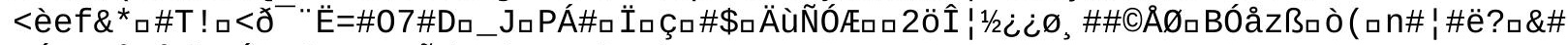

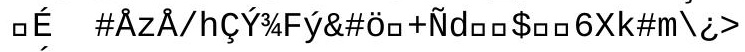

口í

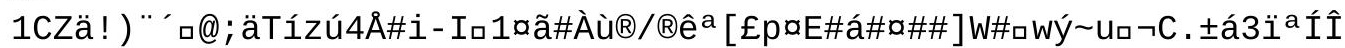

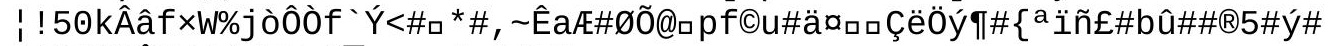
$5 A 9$ a"öÊ"Pí/\&Ihp§ ${ }^{-} r V_{\square} \# \square J \# Æ \#$ 


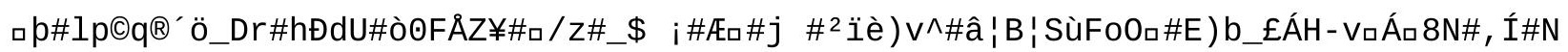




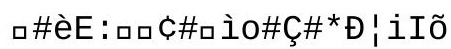


$\square^{1} \mathrm{u} ¥ \mathrm{~g} 6 \sim 0 ́ £ V a q V$

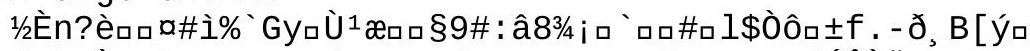

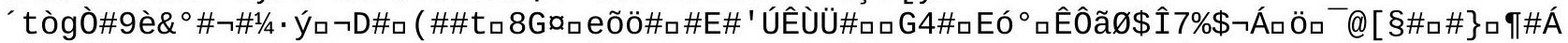

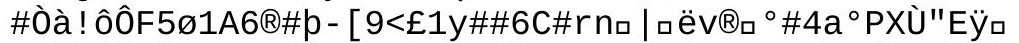
ææ] $1 \frac{1}{2} 4 \& \square \AA ̊ G \# \ll \square 5 n A \# D \# p J$

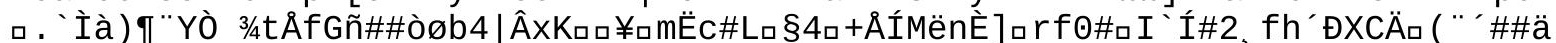

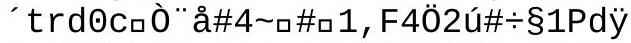

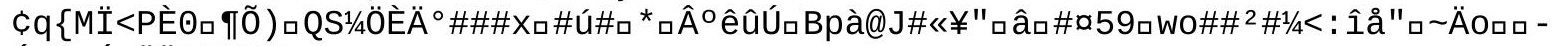
Á=ð, Á=ÄÜCॄ£\#®\#

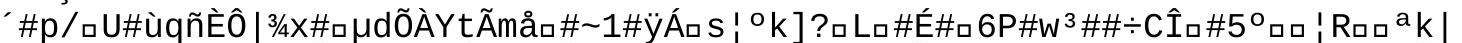

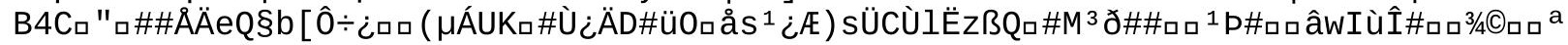




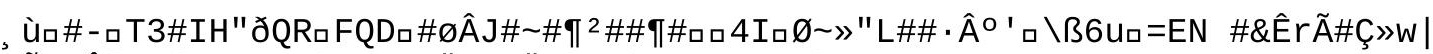

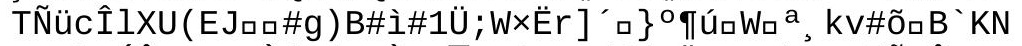

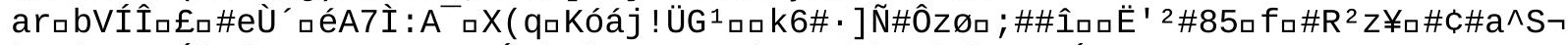

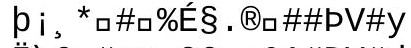

$\ddot{I} \backslash 6 \square \# ; x_{\square} 06 \neg \div \& A \# D Y \# d s Z a ̃$

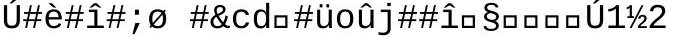

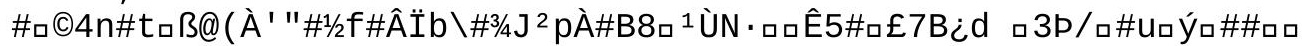




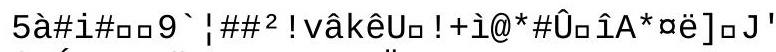
§PÉ\$éô\#/h_\#S ÖP $5 ¥ \# C$

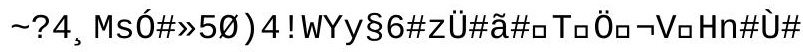

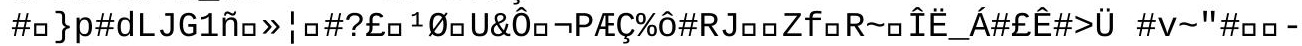

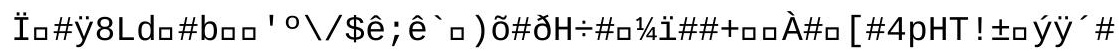


ýıQ. 1 /ä॰ JD\#Ú+',

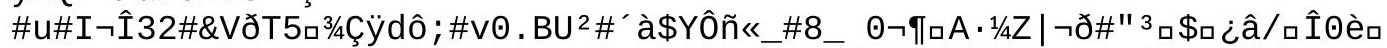

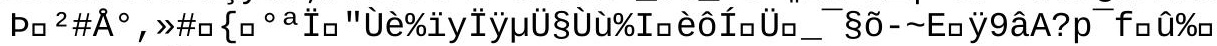
+faky\# \#=ÿçם ¿êù\#:5ם

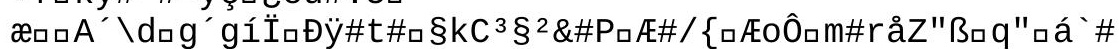




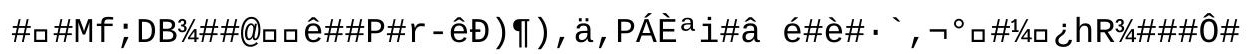




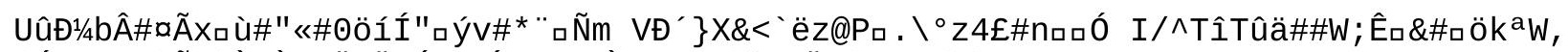

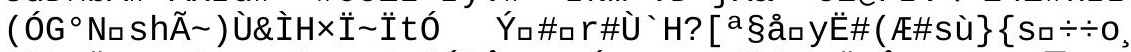

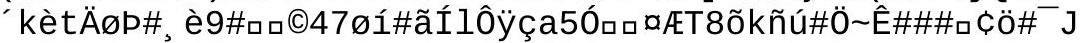




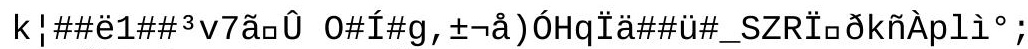

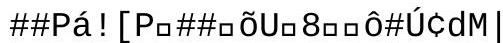

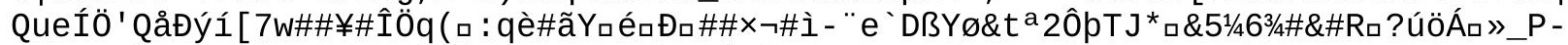

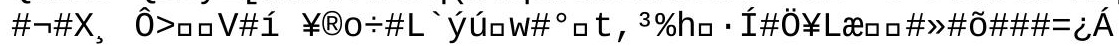




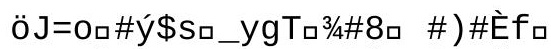

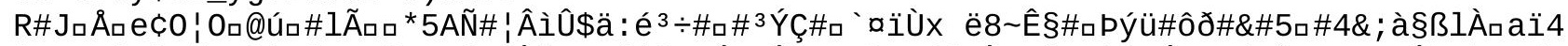

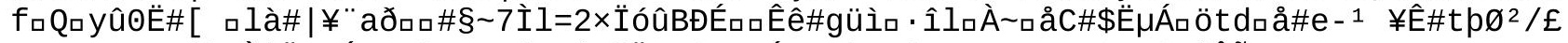

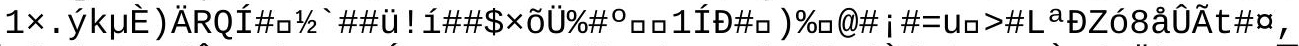

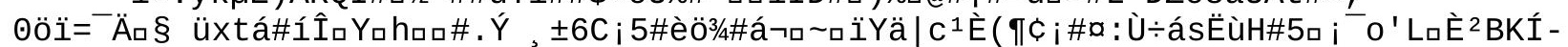

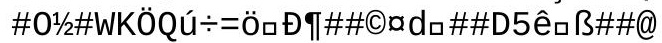




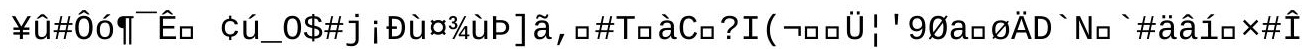

\#'0ิ

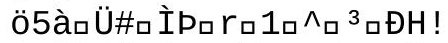

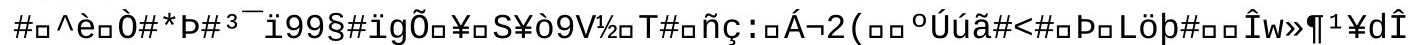

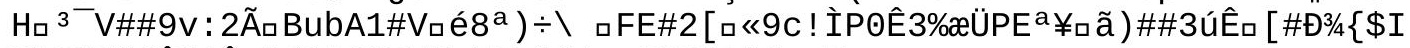

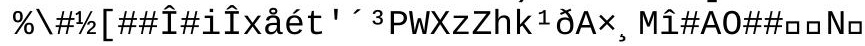




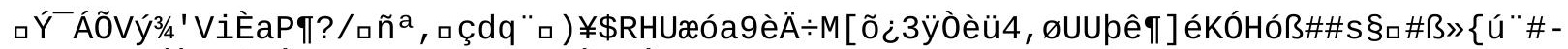

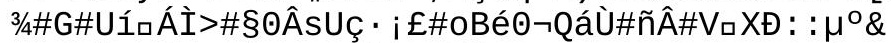
Ó ᄀùX\#1/4ç IÇa

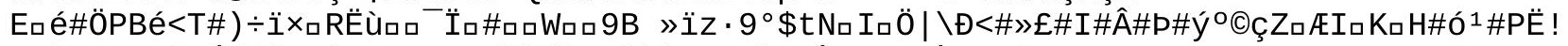

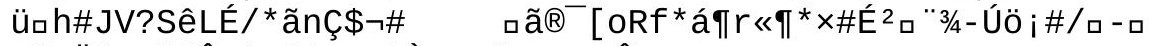

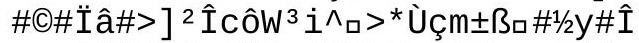

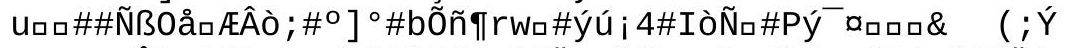

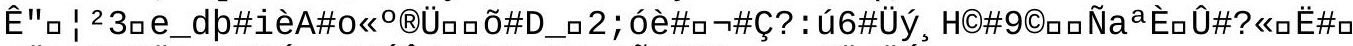

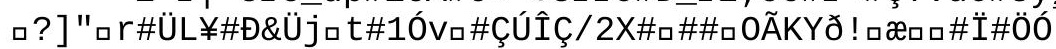

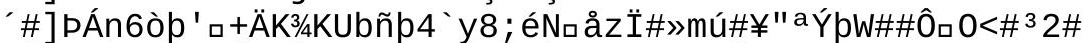

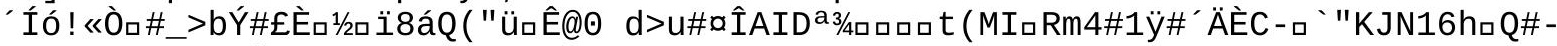

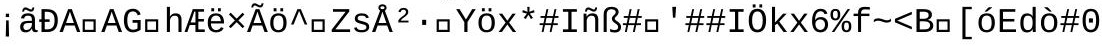




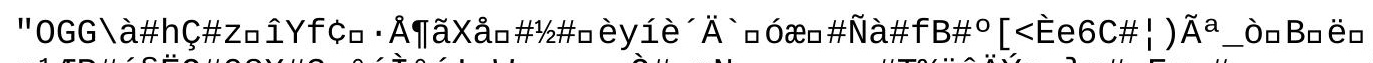
$\square 4 \mathrm{FgÃ} a$ $i^{1}$ ÆEP\#ý§̈̈Q\#08X\#3ロ ${ }^{\circ}$ áI ${ }^{\circ}$ ! $\square W$ Oे\#口mna

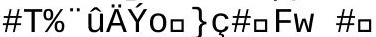

$\hat{A} J \hat{O}^{2}<\# \square ?$

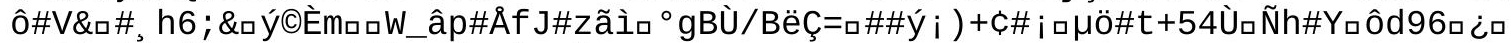

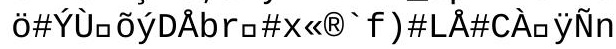
口\# 7 
ÀP\} ฉñZロ $\hat{e}^{1} h^{*} \# \# U ̂ j 7$ 


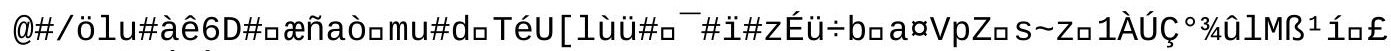
tL\#õp*\#Ú8Ù_,\#1C ]7 


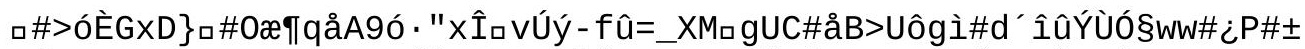

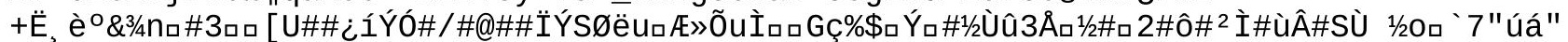

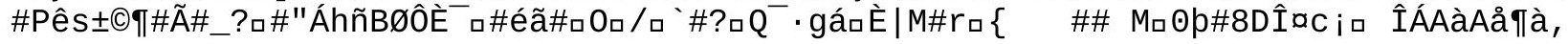




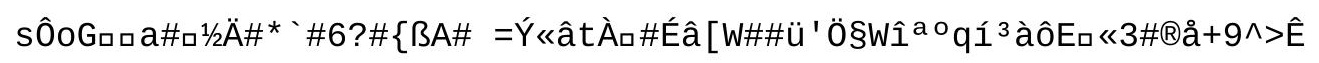




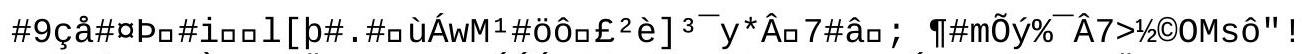

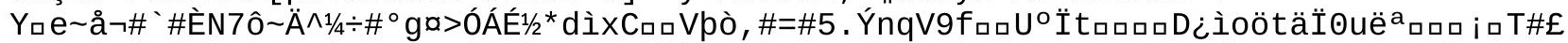

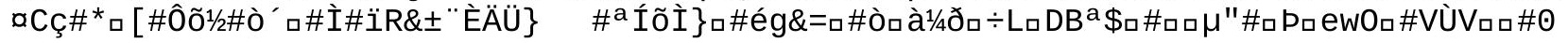




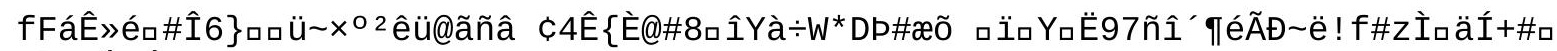
口\}X\#ÂıÓâĐì

ö $1 / 4 \#$ \$Í 


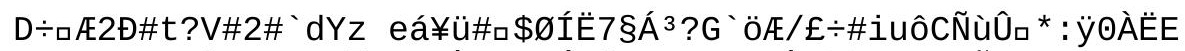

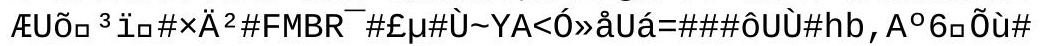




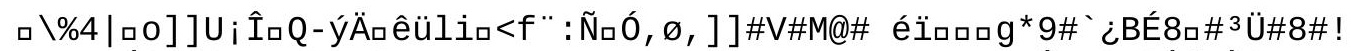

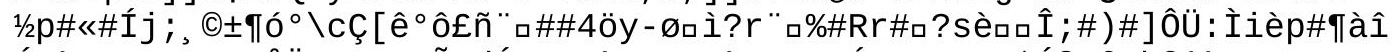

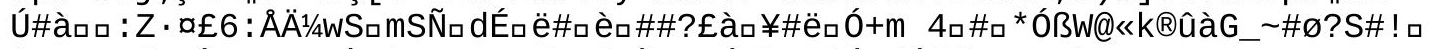

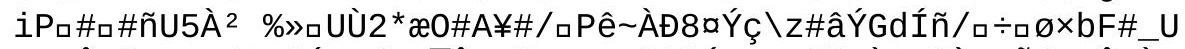

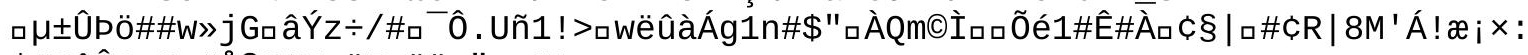

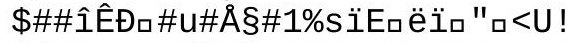

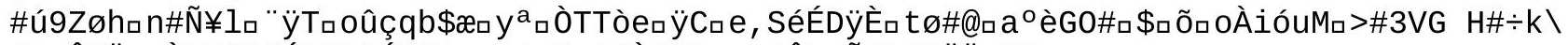

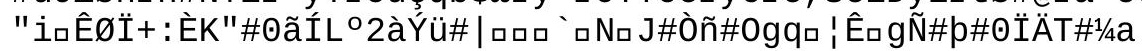

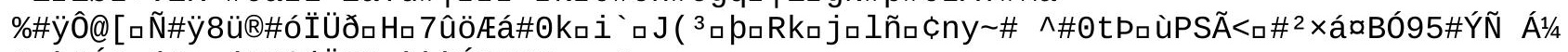

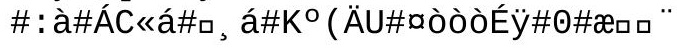

endstream

endobj

$44 \odot$ obj

$<$

/Type/Page

/Resources $45 \odot \mathrm{R}$

/CropBox $\left[\begin{array}{llll}\odot & \odot & 595.276 & 782.362\end{array}\right]$

/MediaBox[ $\left[\begin{array}{llll}\Theta & \odot & 595.276 & 782.362\end{array}\right]$

/Rotate 0

/Parent $83 \odot \mathrm{R}$

/Contents $46 \odot \mathrm{R}$

/Thumb $77 \odot \mathrm{R}$

$>>$

endobj

450 obj

$<</$ Font $<</ F 4101 \odot \mathrm{R}$

/F6 $108 \odot \mathrm{R}$

/F7 $1110 \mathrm{R}$

/F9 $1170 \mathrm{R}$

/F10 $122 \odot \mathrm{R}$

$>$

/ProcSet [/PDF

/Text

]

/ExtGState<</GS2 $125 \odot \mathrm{R}$

$>>$

$>$

endobj

$46 \odot$ obj

$<</$ Length 4579

/Filter/FlateDecode

>stream

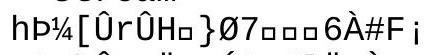

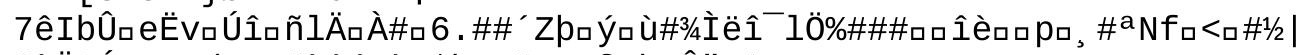

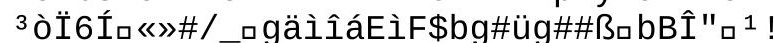

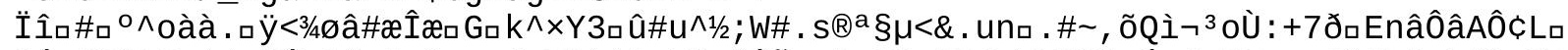

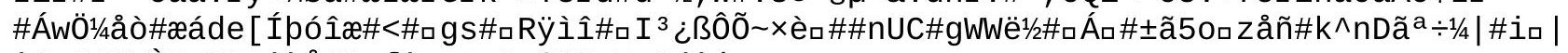

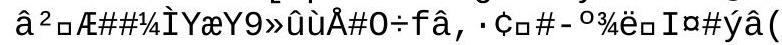




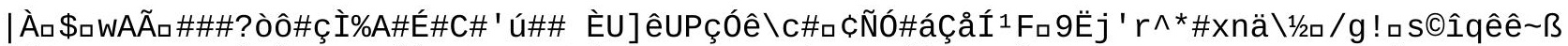

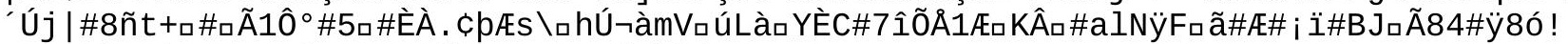

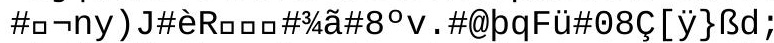

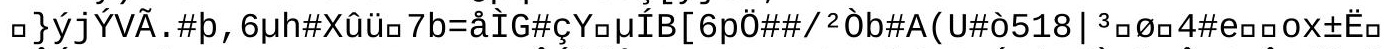

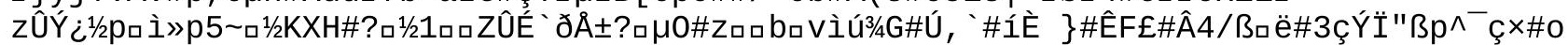

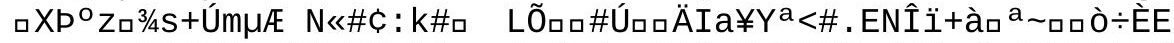
$\# ? £$

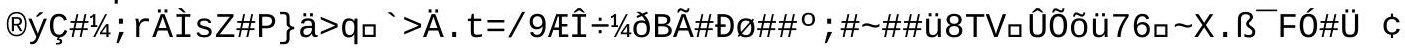


ßÚ\#G\#םe\#ロ

| $U_{\square} \#$ ì; ãa $: v^{\wedge} B \_J ?$

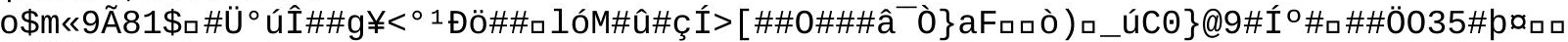
$¥ \hat{I} \S \#$ Dq\#Dè9\#t 


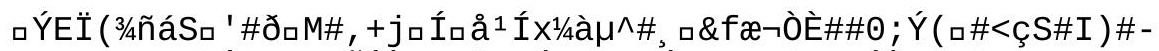

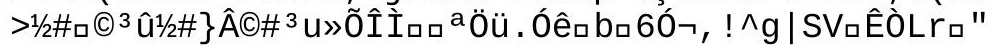




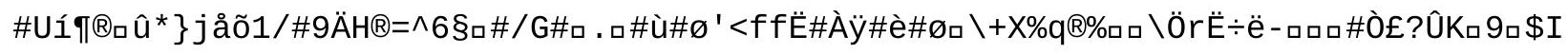




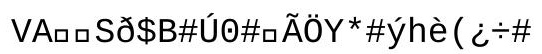




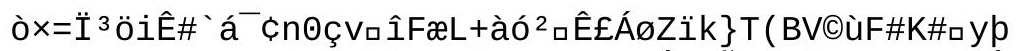

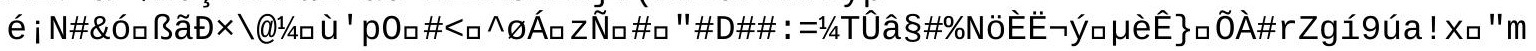




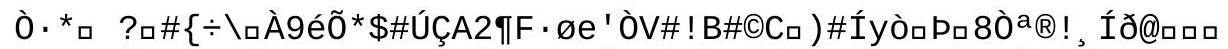

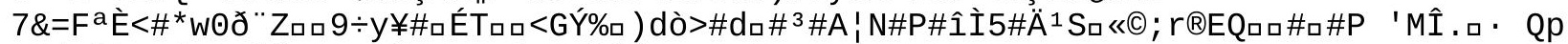

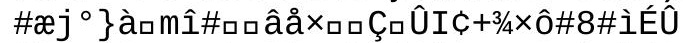

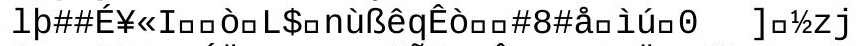

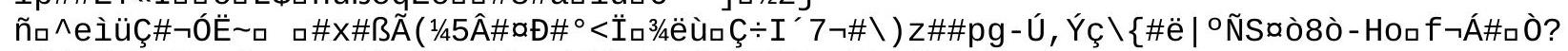

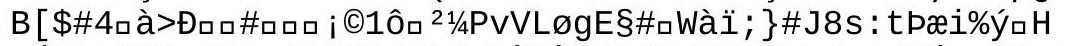

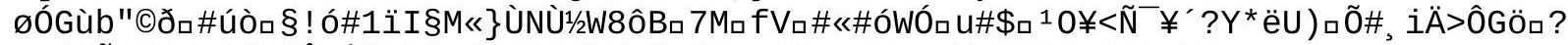

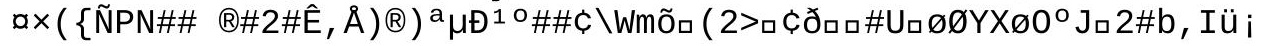




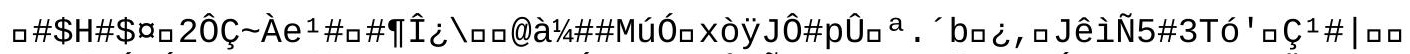

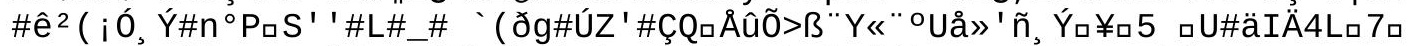

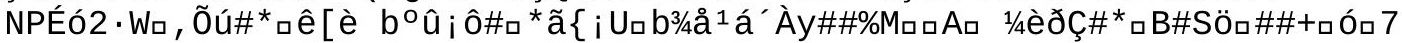




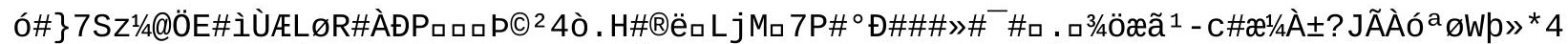

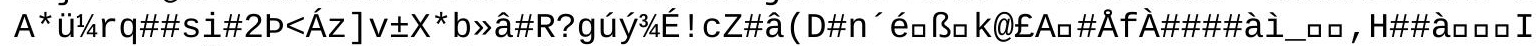

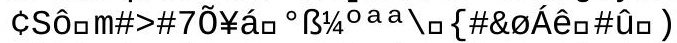

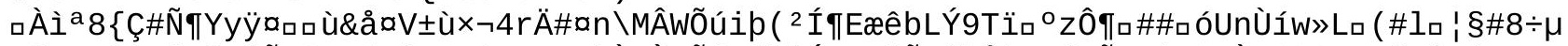

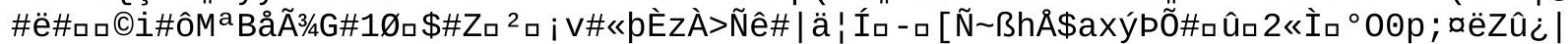

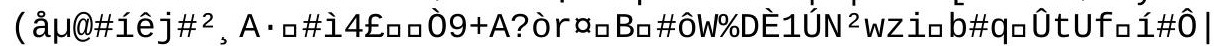

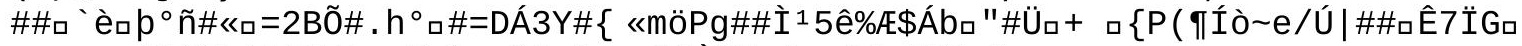

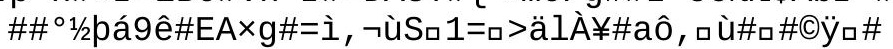

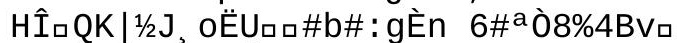

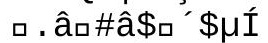

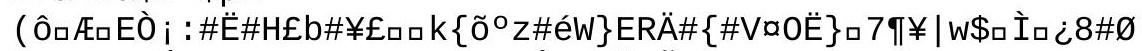

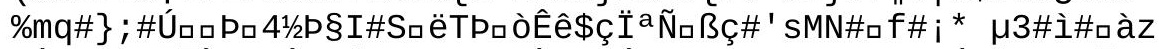

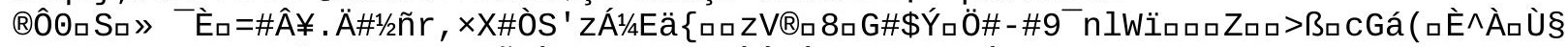

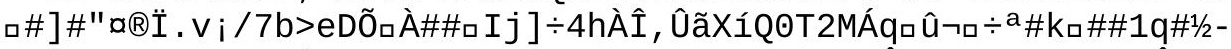

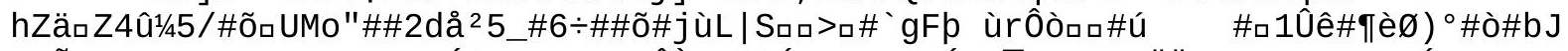

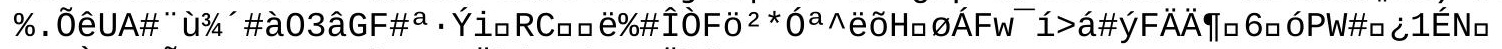

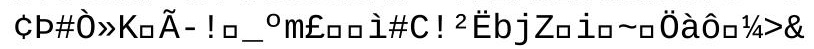




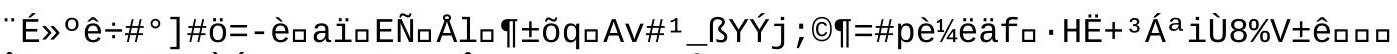

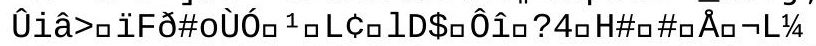

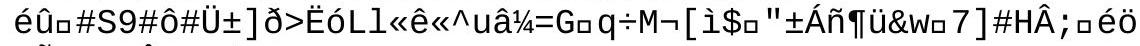
$\# \tilde{0}=n ¥ \div \hat{E} M " \#$ é.

Ø\Tà·õnıåıÝıíSdõú- 


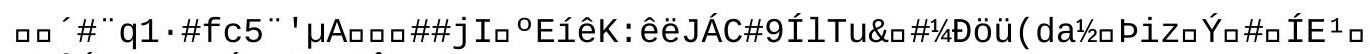
èbÅóscHú\#ôÝaUjóøîิ瓜 $f \backslash Z \#$

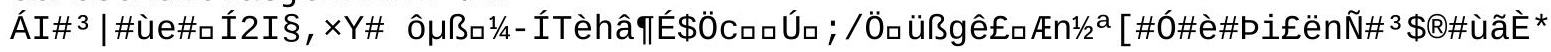




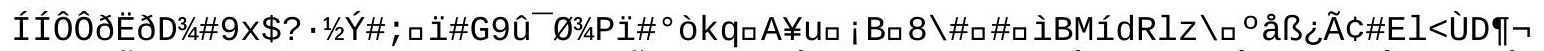

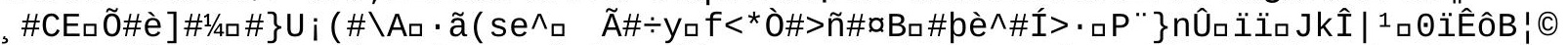

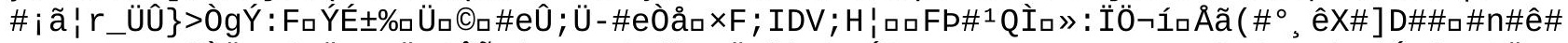

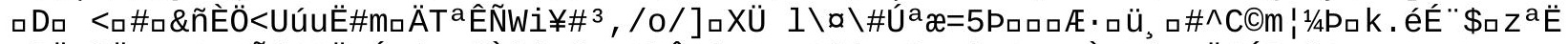

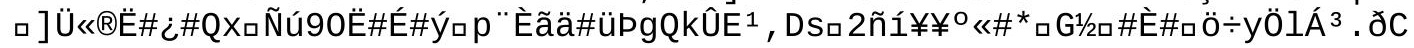




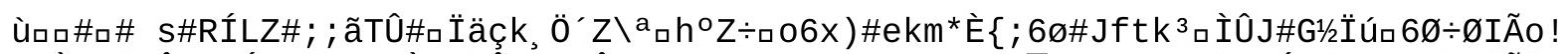

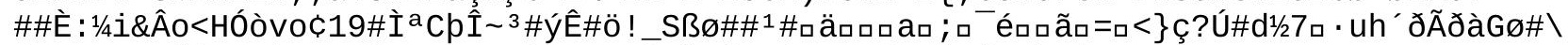

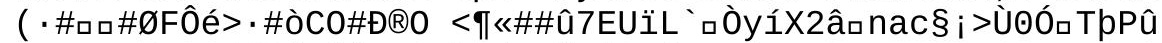

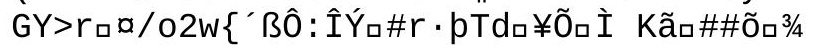




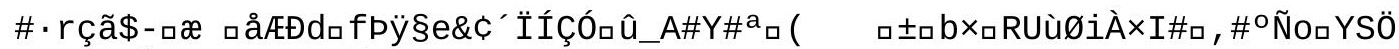

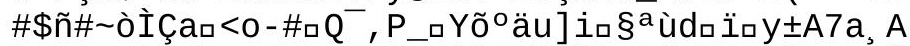

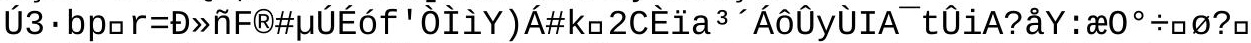

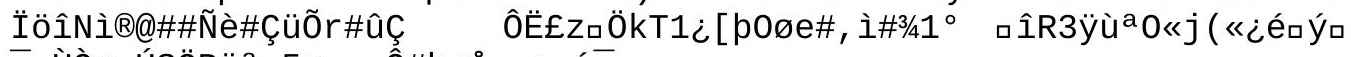

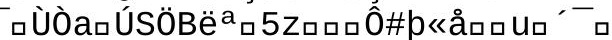

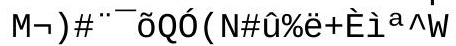




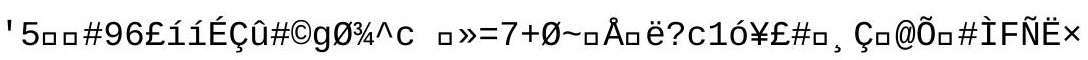


ฉ3N -

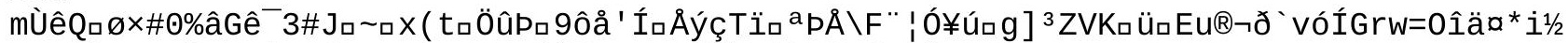

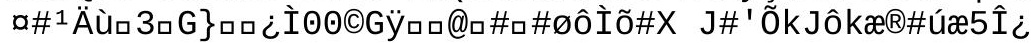




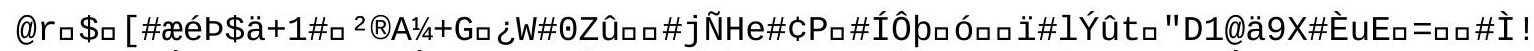

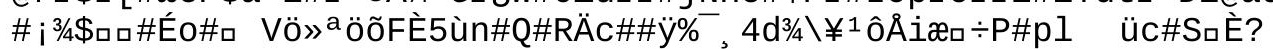




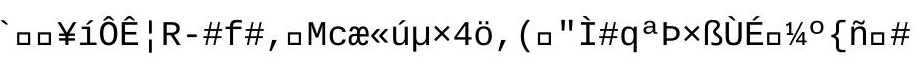


\#అXîç

endstream

endobj

$47 \odot$ obj

$<<$

/Type/Page

/Resources $48 \odot \mathrm{R}$

/CropBox[ $\left.\begin{array}{llll}0 & 0 & 595.276 & 782.362\end{array}\right]$

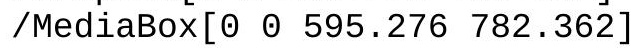

/Rotate 0

/Parent $83 \odot \mathrm{R}$

/Contents $49 \odot \mathrm{R}$

/Thumb $78 \odot \mathrm{R}$

$>>$

endobj

$48 \odot$ obj

$<</ F o n t<</ F 4101 \odot \mathrm{R}$

/F6 $108 \odot \mathrm{R}$

/F7 $1110 \mathrm{R}$

/F9 $1170 \mathrm{R}$

$/ \mathrm{F} 10122 \odot \mathrm{R}$

$>$

/Procset [/PDF

/Text

]

/ExtGState $<</ G S 2125 \odot \mathrm{R}$

$>>$

$>>$

endobj

$49 \odot$ obj

$</$ Length 1056

/Filter/FlateDecode

>stream

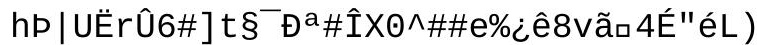

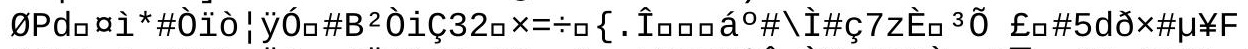

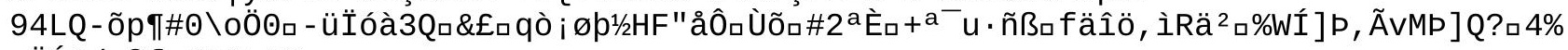
¿ÖÍ6/ロßfW\#N ${ }^{3} \mathrm{~L} \# \mathrm{G}$ 口

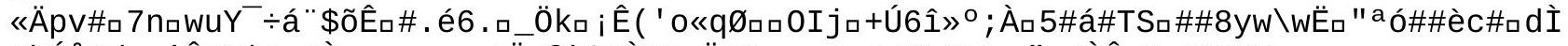
\#hÓÅ\&/. व'ÊPK|\#ロBÈq

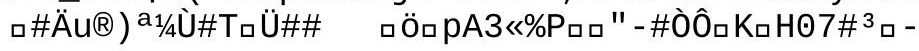

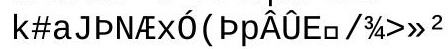




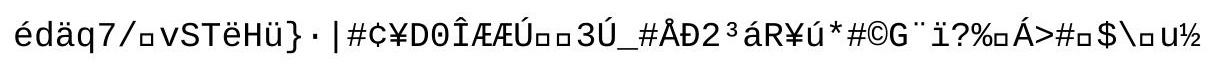




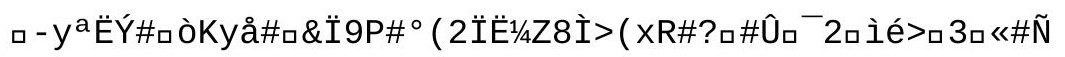




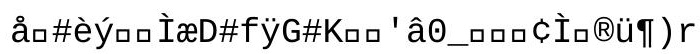


mÈc\#É

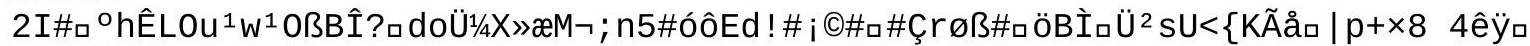
口; $\times$ Öß3§\&口\#J (ËP \#2|

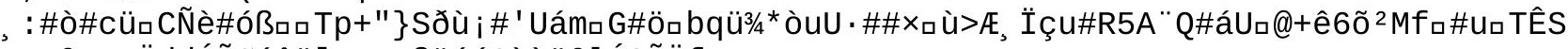

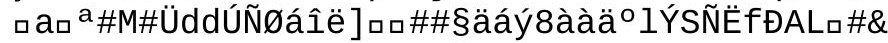




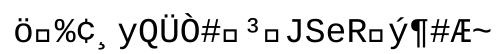




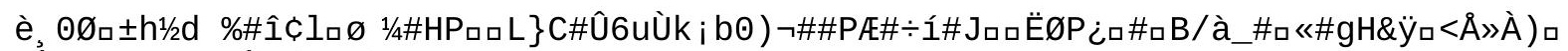

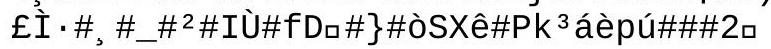




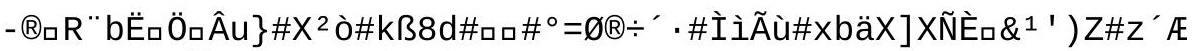
$\tilde{n} 0 \# \# S \# \square=\tilde{a}-\mu \#$

$\{: / \# \square \tilde{n}$ 
0ỗ̃̃̃k

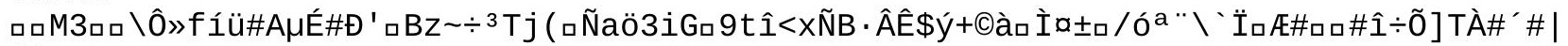
$\$ \mathrm{P}^{\circ} \mathrm{B}$

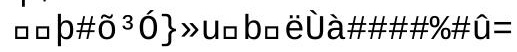

endstream

endobj

$50 \odot$ obj

$<</$ Subtype/Type1

/FontDescriptor $51 \odot \mathrm{R}$

/LastChar 90

/Widths [385

0

0

0

0

0

0

0

0

$\odot$

0

0

0

0

$\odot$

$\odot$

0

0

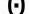

0

$\odot$

$\odot$

$\odot$

0

0

$\odot$

$\odot$

$\odot$

0

$\odot$

$\odot$

0

0

0

0

0

$\odot$

0

0

0

979

]

/BaseFont/KJMHFF+AdvEls-ent5

/Firstchar 49

/Encoding/WinAnsiEncoding

/Type/Font

$>>$

endobj

$51 \odot$ obj

$<</$ StemV 0

/FontName/KJMHFF+AdVEls-ent5

/FontFile3 $52 \odot \mathrm{R}$ 
/Flags 34

/Descent - 98

/FontBBox [9

$-343$

1166

791

]

/Ascent 528

/CapHeight 714

/XHeight 634

/Type/FontDescriptor

/ItalicAngle $\odot$

/CharSet (/Z/one)

$>>$

endobj

520 obj

$<</$ Subtype/Type1C

/Length 168

/Filter/FlateDecode

>sstream

hpbd`ad `dd\#ööòõpsóvL) sÍ)ÖMÍ+1\#

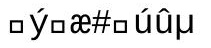




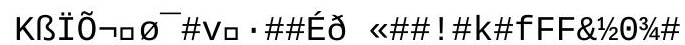

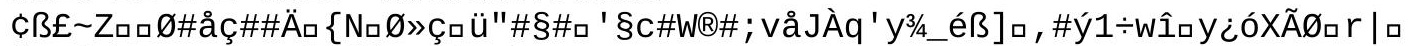

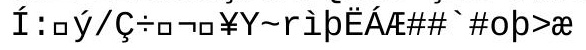

endstream

endobj

$53 \odot$ obj

$<</$ Subtype/Type1

/FontDescriptor $54 \odot \mathrm{R}$

/LastChar 52

/Widths [1000

]

/BaseFont/KJMHJE+AdvPi1

/Firstchar 52

/Encoding/WinAnsiEncoding

/Type/Font

$>>$

endobj

$54 \odot$ obj

$<</$ StemV 0

/FontName/KJMHJE+AdvPi1

/FontFile3 $55 \odot \mathrm{R}$

/Flags 34

/Descent - 207

/FontBBox[13

$-323$

958

824

]

/Ascent 712

/CapHeight $\odot$

/XHeight 714

/Type/FontDescriptor

/ItalicAngle $\odot$

/Charset (/four)

$>$

endobj

$55 \odot$ obj

$<</$ Subtype/Type1C

/Length 122

>stream

\#\#\#\#\#\#\#\#\#KJMHJE+AdvPi1\#\#\#\#\#ø\# 


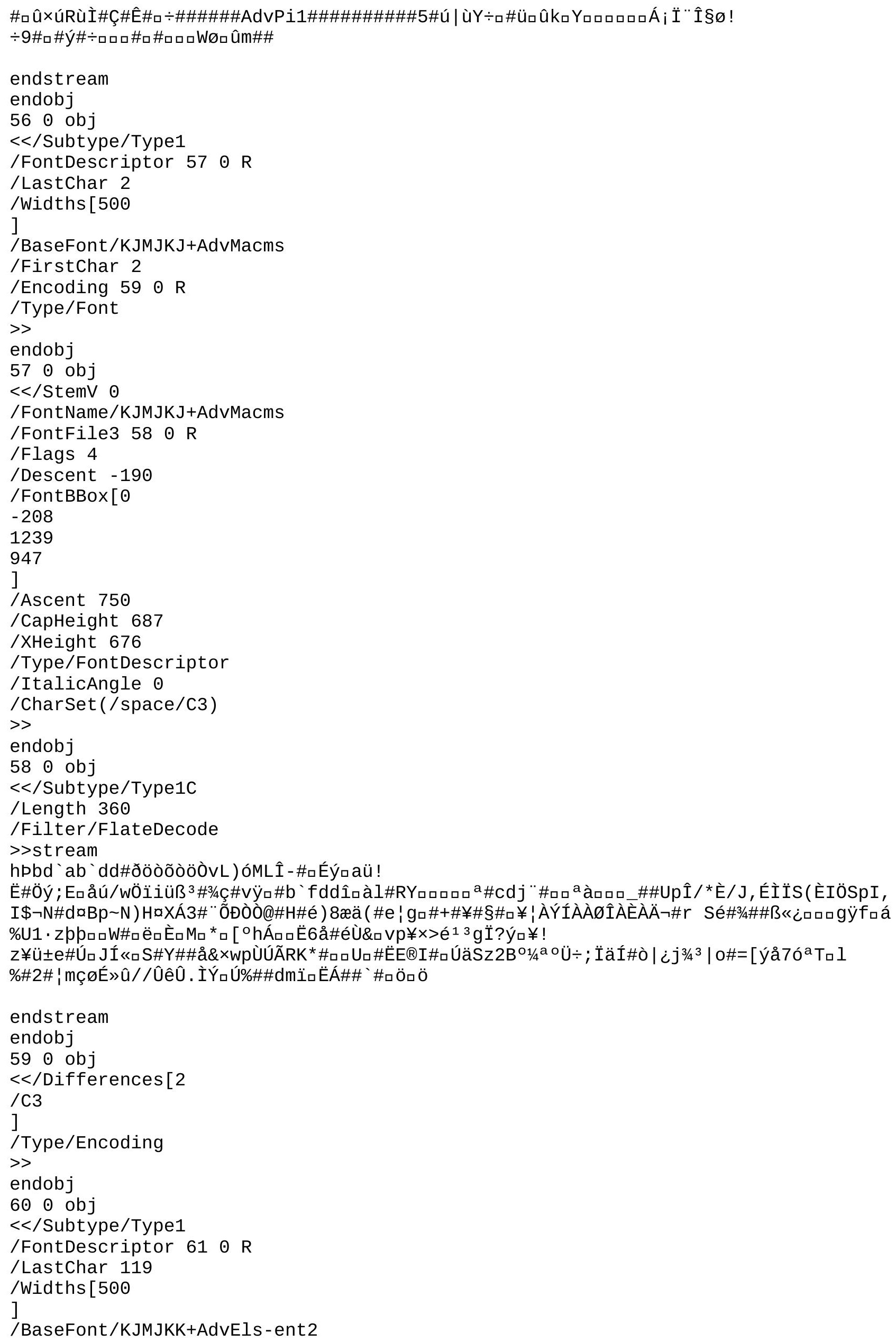


/Firstchar 119

/Encoding/WinAnsiEncoding

/Type/Font

$>>$

endobj

$61 \odot$ obj

$<</$ StemV 0

/FontName/KJMJKK+AdvEls-ent2

/FontFile3 $62 \odot \mathrm{R}$

/Flags 32

/Descent 2

/FontBBox [9

$-322$

979

854

]

/Ascent $\odot$

/CapHeight 697

/Type/FontDescriptor

/ItalicAngle $\odot$

/Charset (/w)

$>>$

endobj

$62 \odot$ obj

$<</$ Subtype/Type1C

/Length 187

>stream

\#\#\#\#\#\#\#\#\#KJMJKK+AdvEls-ent2\#\#\#\#\#ø\# 
\#ロûöúgùê\#Ñ\#0̂\#ロ $\div$ N\#\#\#\# 


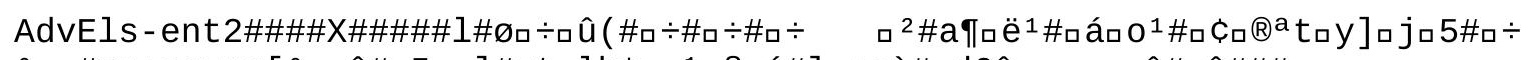

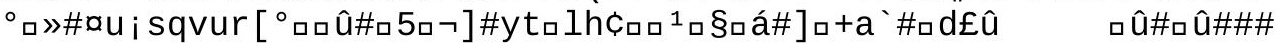

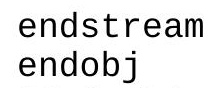



]

/BaseFont/KJMJKL+AdvFrutiger -L

/Firstchar 30

/Encoding $104 \odot \mathrm{R}$

/Type/Font

$>$

endobj

$64 \odot$ obj

$<<$ StemV 0

/FontName/KJMJKL+AdvFrutiger - L

/FontFile3 $65 \odot \mathrm{R}$

/Flags 32

/Descent - 215

/FontBBox [-145

$-239$

1000

770

]

/Ascent 750

/CapHeight 698

/XHeight 510

/Type/FontDescriptor

/ItalicAngle $\odot$

I

CharSet (/space/A/n/a/l/y/t/e/s/slash/i/d/c/m/u/r/p/v/o/f/b/period/M/quoteright/R /B/C/hyphen/0/S/x/w/h/g/two/parenleft/colon/comma/W/one/seven/six/eight/zero/nin e/L/parenright/semicolon/three/four/equal/endash/D/P/I/fi/F/five/V/T)

$>$

endobj

650 obj

$<</$ Subtype/Type1C

/Length 2801

/Filter/FlateDecode

>sstream

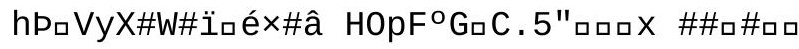




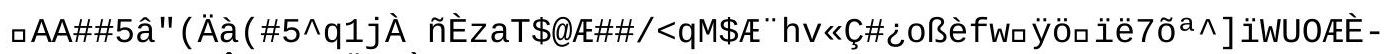

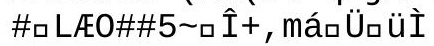




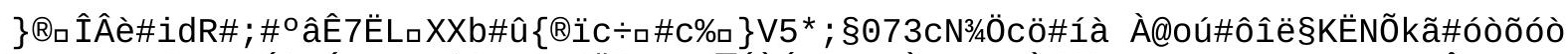

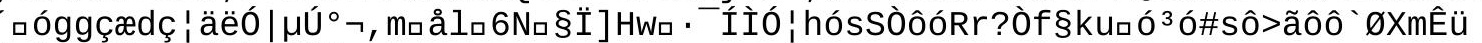

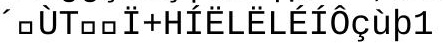


FFøฉÂaénÏ\# 
ãbÍ, 3ロ 'ÃøÈロA2fa\#\# cÂ\#LıquÇםy 


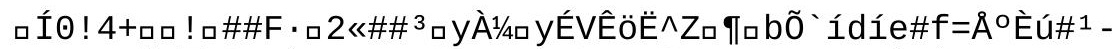

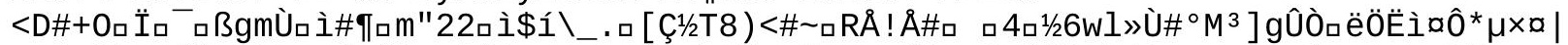

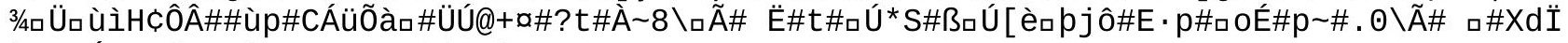

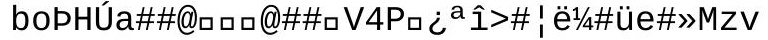


$\mathrm{QB} \_\bigcirc 7 \# \circledast\left[\mathrm{q} \#{ }^{\star} \mathrm{P} \pm \mathrm{q} \cdot 囚 \mathrm{è}^{\circ}\right.$ 


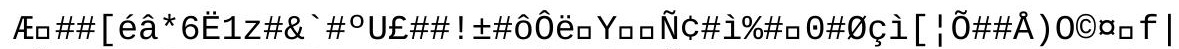

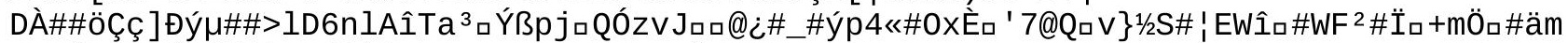

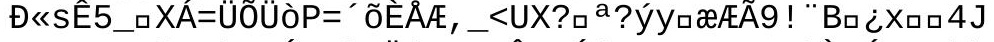

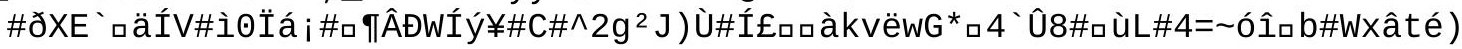




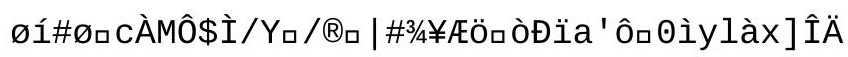




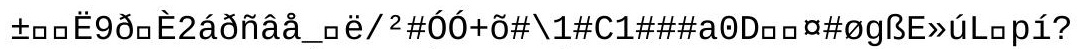

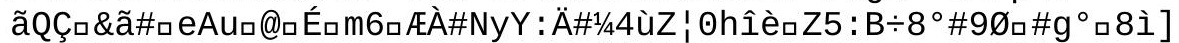

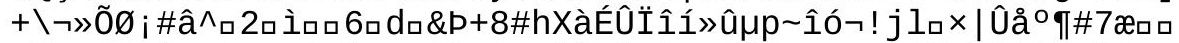

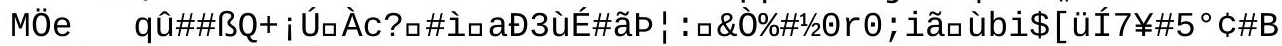

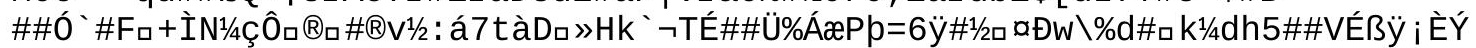

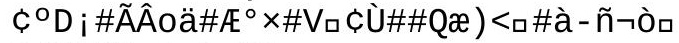

D\#®Đã\#x?םù È6

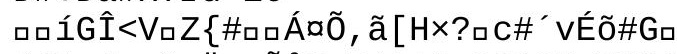

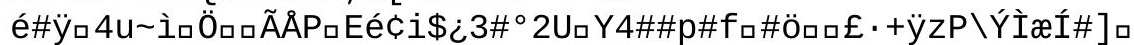

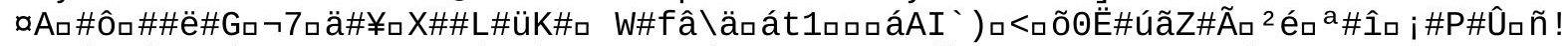

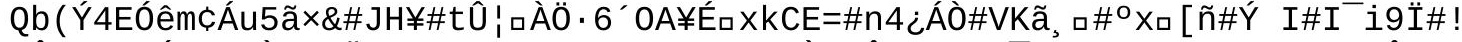

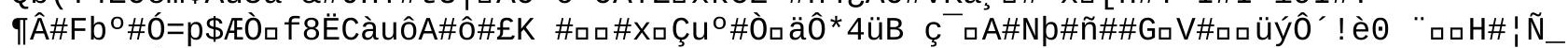
\%Oїロ ñôÍ

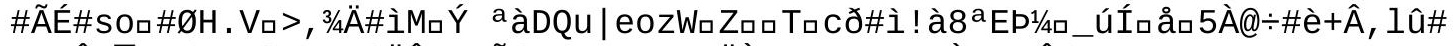

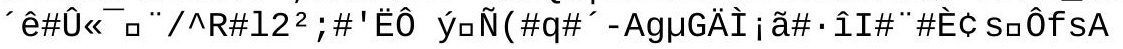




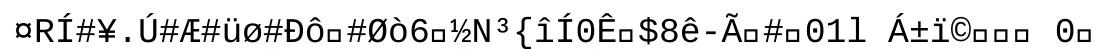

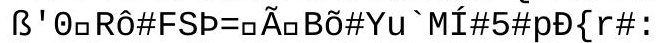

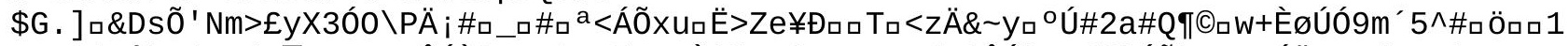

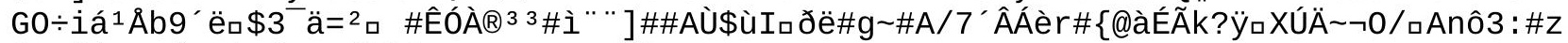

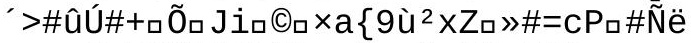

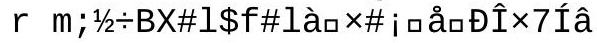




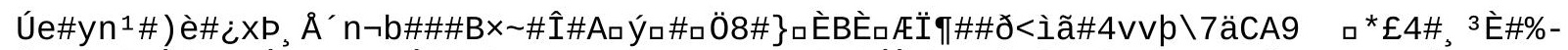

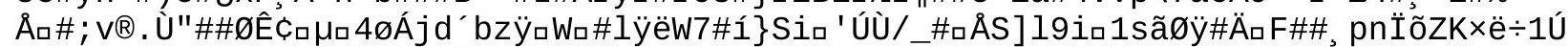

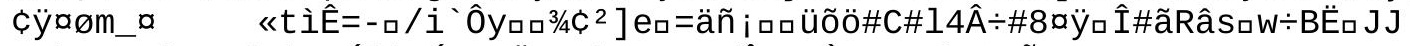

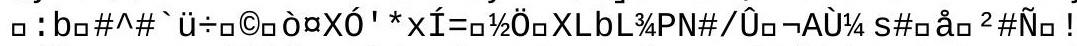

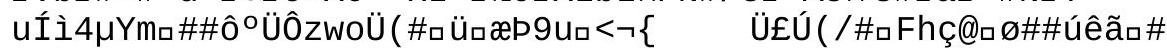

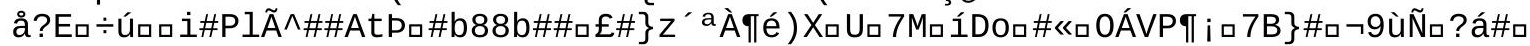

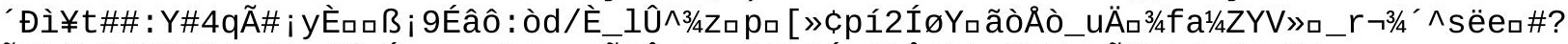

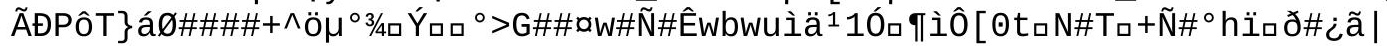

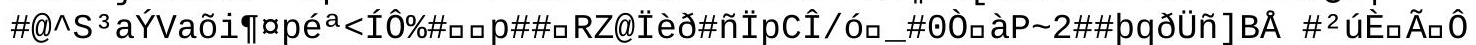

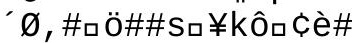


Âİ

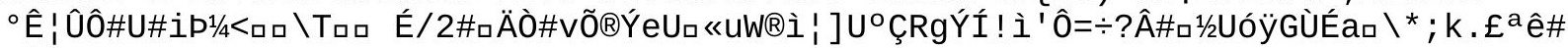
$\%$ 'p_\# 
\#ロCíK

endstream

endobj

$66 \odot$ obj

$<</$ Length 438

/Filter [/ASCII85Decode

/FlateDecode

]

/BitsPerComponent 8

/Colorspace $79 \odot \mathrm{R}$

/Width 74

/Height 97

>sstream

$8 ; Z \backslash 7 \$ P n C<\#$ itt $\$$ j>u\$c/JA[hm 'BE )h5qB ' - =mYV_n? * "JM=JCReciQ\&@q@j; EX\#* noV , 2/L_1S>N] ON1T, =G\$Zr/SS? LNEe* >aop\#IqWp . Xt 1\%=mQqBUe2s5N*XtQ९"_d @oLF-_K(ZX\&Lot5He* =e69_K04N?.kK-\#d5, *hFI(5.10-^pGkZCIqWE\&SpXF_, 7R

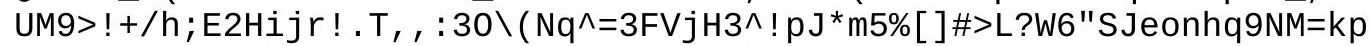
M! Ea7WF\$of \7bXpT\$p5, b\%/oCs^??jL1g, Bkcs3Yr, fIX-; H"^Q@IOTB5, uBVoVuD /piL:Ggr'g,F(-S=3!9q; (ogBigh2>'o<hQAcL], pAQu8G@AO@DL j ] ; s<;aT' ohu j! du ' 9) II $>>q B Q) 1 J @ N K c K p<C E \# c t 1 H i+$ JA(DXA > endstream

endobj

$67 \odot$ obj

$<</$ Length 486

/Filter [/ASCII85Decode

/FlateDecode

]

/BitsPerComponent 8

/Colorspace $79 \odot \mathrm{R}$

/Width 74

/Height 97

>stream

8 ; Z] "\$aRYm\#Y! SL\#2) c0.GtAo8CEo $\backslash H^{*}>$ IlKm0pS6hCW7Sq\$M7p9<L\%_ila[D/TVC <SOLf\\%HPUYK516\&1k_j[a?RG1\$\$Z, ' (f_V28MoLI!Z5, \4WJ*AarinIB. obra9V $1 ; ; \mathrm{R \# Qd3*88"1; \% -8,} \mathrm{d=;} \mathrm{fMG"9Ko"RPDtGu} \mathrm{[/"} \mathrm{;} \mathrm{CK>a9j} \mathrm{*8ZTK18RKKqKnX \% !} \mathrm{:} \mathrm{,} \mathrm{q \$}$

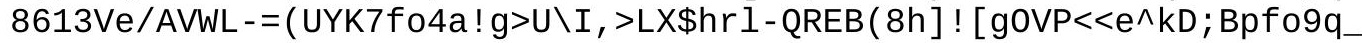

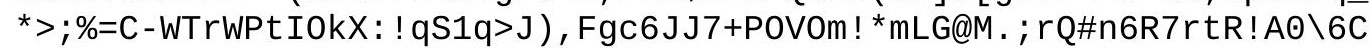
JTN6 (o?N3ajNtFX@h\%7]VEr2cF/E\\&8Pe5]_] :d [; aDbT.U/]?Y3L=iSlDduBn>ql

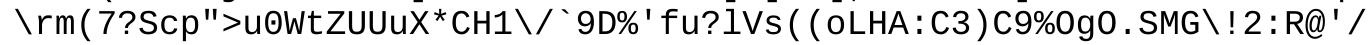
P; lAIIW;\%obG<qu ! : eI^1B >

endstream

endobj

$68 \odot$ obj

$<$ /Length 542

/Filter [/ASCII85Decode

/FlateDecode

]

/BitsPerComponent 8

/Colorspace $79 \odot \mathrm{R}$

/Width 74

/Height 97

>stream

8;Z\6\$a' OS\%-7C( luen<@JQ . :Tr ]TpBJ >Z1IDNXn . h' IYQ\$;Y/80\%80+LJbU>0Pm 9=ji"m\:N]K\$"rK@:SVg3Y\$; ]i]G1d[Fg) (Z; .\#kV`K9TR5\%\%\#ZYGt3_Pr/4XDuAi X7\$*; ! eH\%m\&?KJaN4bBQ \q) po=HJj J?3X1C! 1KQA; a"Qc ! s+u\$]PggqK6p?A"tjK

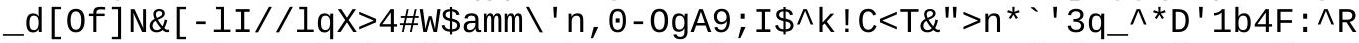

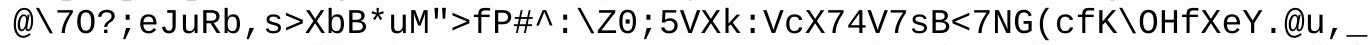
CMC4EOHO\&CL; Z\& [ ' IVCY4K! IArrEFseA84hreNaHZ 9\%/6E9WhFXB\#@Q=KQPE2f\%' Ua $\backslash I G(d E p<e G 8 r 49 T 2 r+k C a X ! n J ? E \odot j A V, 6 N q S M b K R=X-H o L 5 p l L m 5 \backslash 8 \% \& \wedge 1 E \$ B 7$ :

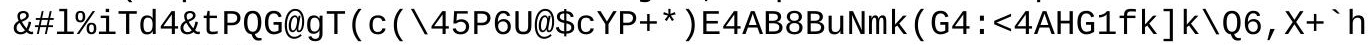
(0c2j ! \#\&\$@"9 >

endstream

endobj 
$69 \odot$ obj

$<$ /Length 562

/Filter [/ASCII85Decode

/FlateDecode

]

/BitsPerComponent 8

/Colorspace $79 \odot \mathrm{R}$

/Width 74

/Height 97

>sstream

$8 ; Z \backslash 7 J T 8 R] \#$ h2\&p-i7GHm) ( $1 \$>$ hMH27j (HEHhCaoCE2 [9\#=N*sIt 4o?^>J/apCd5F

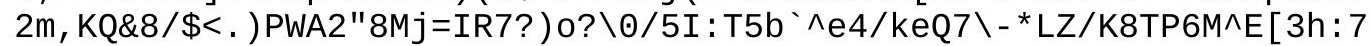
(hoZnKOcc"X/j. \qp[J, be980: l=7X12^8QD; U1"P"u`a<1geoY7A*_!f/Qf\%A( : 4 bK@7_P5j`sf>;0Y\%q[] L<\&H7f?dZ=D`bsor [XW\$jaW>Xtg_VWF1IEZm\%\&; NK` ]\#\(

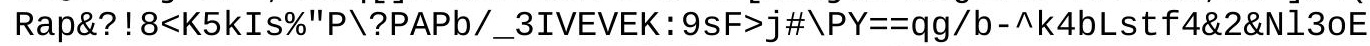
U4\&\#93\$9R3]XpGr : r I ; sm\%Eo9rmR=4lD [8cNQuXd4\$) b3hBko2E45MA\$: ] 八jq)_ Q709M-F [V<BIHNKh/Pr-Vk26\*m\pTdT4/JJBS\$W; t ; 1YR-r+"KRF=6D9Q ' pbi

] IM7>r\$Zic<u2jU*UYa09 [cL?jZ( J; q [^sNaXGP\$\&dNZr\#'s! d9t\&bkgt=uE $\backslash,>$ RB ?t+IC2X, s?mH4/]62?10c==\#! $1 \mathrm{HJ} \% \mathrm{~d} / \sim>$

endstream

endobj

$70 \odot$ obj

$<</$ Length 340

/Filter [/ASCII85Decode

/FlateDecode

]

/BitsPerComponent 8

/Colorspace $79 \odot \mathrm{R}$

/Width 74

/Height 97

>stream

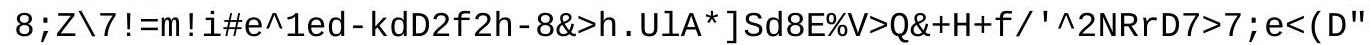
\&K9\%>"t ! e\#"HHpmK6*\#3(T33ALt */! ${ }^{*}$ FBn! NN7B"R5ggDEp)df`]/5u+] [s7FMocC

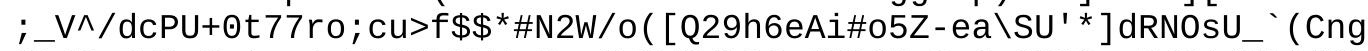
Q] d3+REC-\$g^ . c ! nW322h9FdgU, 0378ZGWkDCn' "9QHe601; 80G@usMd\&1/NJ^\#Qb hPI]/7^Rs\%:@X=V, J\#XQ7i. [10AoB!W7]8BgAFfe\%lHqInr * SL<ds76\$b92a_b[; \$ioLaolp >

endstream

endobj

$71 \odot$ obj

$<</$ Length 565

/Filter [/ASCII85Decode

/FlateDecode

]

/BitsPerComponent 8

/Colorspace $79 \odot \mathrm{R}$

/Width 74

/Height 97

>stream

$8 ; Z \backslash 7=d H r$ J\%. jENaE9NWG ._2d) Eo (SBd [ JS - (A9Ji<P=E^V : <XRsU\$>n7rCiM/c -S 8HiC6]RK\# AP9r\#Z^<hK-F6Z`1SiDDb10 ( , ( fqTPh/nNnij^-=o/Ld \"1<gsC>K_i

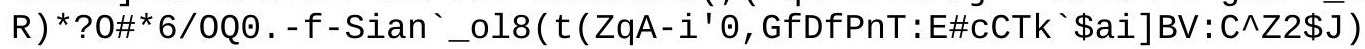
;/J^\&Vk\&L'7K3nl; eLk\$kYUDM, \&ZJp6@olVM>r/g\#\fcoD<ceF2Dnu8P?26omp)DC 7T! eAkp)d! BCF\, VI; ] tE`iI : : > jXm\>'Oldlmla;-3P]+??ItKBh`9X; l\%f : )<fB

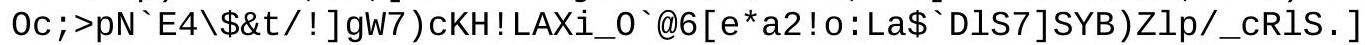

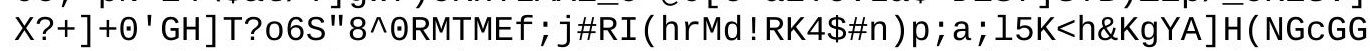
o\&ULe/_fPbZYZ)1hqV6UFi8=Q\r\.Ur (Q95M\&A; <\%ekBobdXH`>ceX\%VXN/_HI\&\8 $\$ n>-1$ ! ekQHY?b 'k\$ $\$>B i B ` X 1 p \% B 7 '>2 q Y R \sim>$

endstream

endobj

$72 \odot$ obj

$<</$ Length 568

/Filter [/ASCII85Decode

/FlateDecode 
/BitsPerComponent 8

/Colorspace $79 \odot \mathrm{R}$

/Width 74

/Height 97

>stream

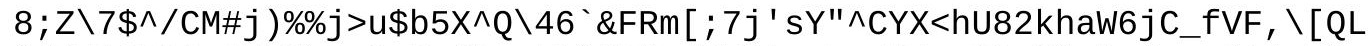
f/1W(CPBA_HZ74 \a=0eRe [P=gLk) '\&@s4RWSi>uh-dU4 - -@Qs\%)_tq(aqr (i! - 7)+

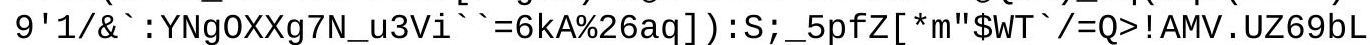
*\%1NTqs"J700[if2, \$MueKfQm`9Y^(, JHop"\#kFC+1n5\&Vk)PGB: bEWDS^46: T>dK hQR+\$\%] iA 4 46V [6PbQON (=TNOF . K8nos*7R: NGsB] 5teb $7 \mathrm{~N}^{*}$ j 4\&QMV ( gMnnesNM9 :S-fIs1o"D"<D3HAdA+ (H5_\%c@5LDq=nbB\&HO\$HVIK<cJU, Te-Ftb) 2.p3d4F?\$N6

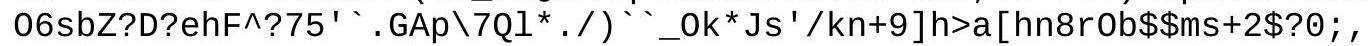
EMo.g [027R^k* WiB?P^5AL"UsC $\backslash$ ) Y6>lg $>$ pm : hNcMrU; [VTY? lOtFVOh<_? [a\&rcD m\#\&>("ac26Bq6nmp1\$uo:@4/8Mtj42DZH8\&?@ ’>

endstream

endobj

$73 \odot$ obj

$<</$ Length 715

/Filter[/ASCII85Decode

/FlateDecode

]

/BitsPerComponent 8

/Colorspace $79 \odot \mathrm{R}$

/Width 74

/Height 97

>stream

$8 ; Z \backslash 74 a P ! g \%-$ ?etYj^!mOK\#_Qe-Smu.?f.H: : a1T\#dBF_6? (C3c22=9q0>Nn+Y_n?

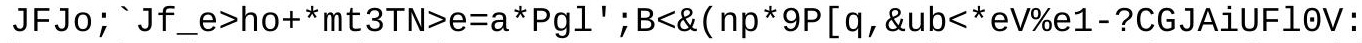
VV3CX)PF[QgF; 81.d1>Fj?4.rY@SVM2L+UQC40<d3`F9]FagD_9: ; s/FoP9@:Mgd/ ] $1 r^{*}, 3, S^{\prime} d+I f a M L=81+\& N u 86 V " E A Y 6 U ? F Z V E$ (3ip4! [hHtd] e $\$ \$ 55 q$ (Gf "SW103P V_PM)VJ38QD*/_E>C9Y<Bfk\%np, *i[?u[\%(1j dofToFmpD.,, OCqZlg8! XpeBdo/b 6]AfUhgPsA<V*a;4]DVFd@i eX__37m=BpfBIr $=5 \mathrm{~m}$ ( 9h<fdOMUU3u\%o20dq *\$] :pn"

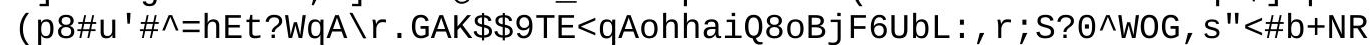
jt ( . \&YLTTIM(Aln"\&P/?ClC - jMuP\%p08; !+>^Qg\&d08\&4YM\#X^ ' "neTZf7Cgc64a\&

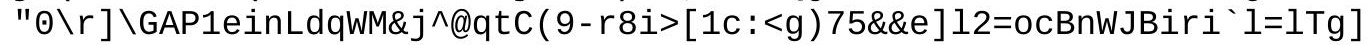
_W: I^k-_J5[+\#"fK?Cl509_)2gA; .f)\$5M2CFLUuiCi; 96Q\&d)cGl0/.FCutV\#J;\$ r</CB1 ${ }^{\circ} \mathrm{e} \& \&=s 9 V c \backslash 9 M<+;$ LKg: $i$ (C5q>ZRt (bi'"jHshr; !<<lu` $7 Y \sim>$

endstream

endobj

$74 \odot$ obj

$</$ Length 628

/Filter[/ASCII85Decode

/FlateDecode

]

/BitsPerComponent 8

/Colorspace $79 \odot \mathrm{R}$

/Width 74

/Height 97

>stream

8;Z\79q\$\W-9=P;\%D\&Lm7"U, Knnn"n 'YA\#3huUE, !\%U_A\&\%QA<Vab [98jPcrWUsH SIma.r\#uSF] GIjZ9*; sgW\%VjY! [NQ(\%?+gGjVq61.ab\&+;MLj5EafAih; lt ] - Ir , \# $\mathrm{fld}, \mathrm{t} \backslash ; \mathrm{E}: \wedge \mathrm{VXmE} . ` \mathrm{Bd} \$<\mathrm{I}$ : GduPgOdW4?GbP"\$@2Hb"G.cpQ, $1 * / .2 \mathrm{t} @ 0 f$ ! SnPuLN`

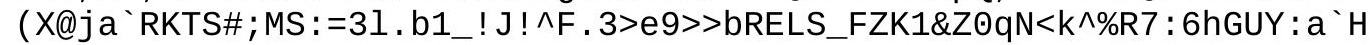

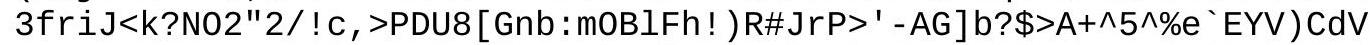

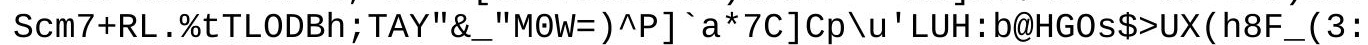
0>;A. /KIP^ [?t : Z\%2oIC! Si3sZV2m5g+>"\&! 9+5VA [ JulWg*\$7^i\$CaAdXtFTU+IX '@BWKE2f; K+ [<P_9h3EP3Fk05k! QXE1Rsp ' UM21_' \$h/a_lFZ5cr : L\#d3Ur IWV)MJ ( I15k ) I_\%tRU [WKJ j>iPDrYO.2S<+HEJaG\#-Dt ' l fbcEI) \$s3or L5UI - acqMTO5d( ) $q \mathrm{k}+8^{\wedge} 4 \mathrm{k}$. Ul4LfJH\Uu\&H, @VJ!3<g!=9 >

endstream

endobj

$75 \odot$ obj

<</Length 753 
/Filter [/ASCII85Decode

/FlateDecode

]

/BitsPerComponent 8

/Colorspace $79 \odot \mathrm{R}$

/Width 74

/Height 97

$>$ stream

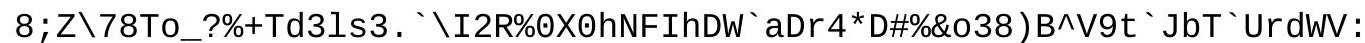

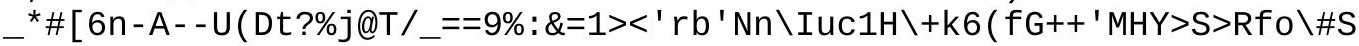

V^b\%-\BRB! Iln; 2UOc\%M5HKaVQgKO+; Mpk>2M`V?\&! 0l4d_Y;PboF2">619jYoCl"

N?_(3dTOUY : loWS ["OOB . j : de71\$KaE]1KZ(g\&8A`s0C+/dKYMMWAdJ>\$, - kL ๑er

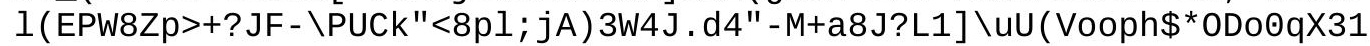

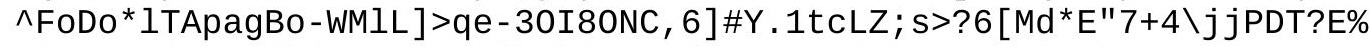
5j7RcVCjoEZ7ib(oVoq@+qLj\Ak@MY : ?d>UK\#t4s (YP`tVGs3*meh+38s.R^M5?02

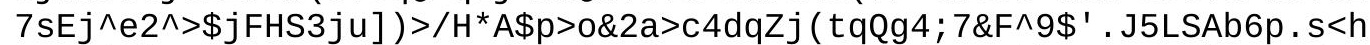
$6 ; \operatorname{Sr}(4 \mathrm{kF} ; \mathrm{GK} ? f) @ g / 0 \& I Y \& m / g E R ` e H \$ R H ? 7 E V k>h 1 G Q$ ! BZn>kb+B=X^GI ) L_A- /; $\odot$

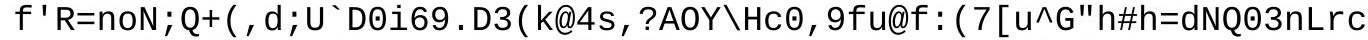

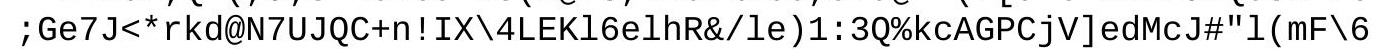
DmJeL6Z?9fP5 '\%lHgs/t=*/r< >

endstream

endobj

$76 \odot$ obj

$<$ /Length 543

/Filter [/ASCII85Decode

/FlateDecode

]

/BitsPerComponent 8

/Colorspace $79 \odot \mathrm{R}$

/Width 74

/Height 97

>stream

$8 ; Z \backslash 7 d>1$ *u\#j_Nj@")a4>,+A;"sVK^\%Ga o6] LK/\#ohnTiqiQL0\&Y9?T7\#Q5LZ>tD

! l] NA+' XkHi_7 $\backslash X o V t h @ e<Q 1+Y \$ H \odot N a<A i p=X \$ d b E o b ' *$ ")AhplF]WOjVaGD/K/84 a/?W_-JtGel\&: gORYdIDWRDp; 3dm0H5\$; Kenc2k78T\# ' HRRIB"D7=h>W1mMuPVPA7 $\$=r-J G Q ; 1 \& 05+p "[\& ? U)$ ' - s\&o>"Pg@E .V, \#H) rZd : er1tQF, d=Do>9^, ) Oh@>*qA\& E5700_9M 'AksSOtAHBh-8: Foi5b+02D12"j ! Uc>1 [\&hUfRn: ? 'A) aNo*VF - 3Y" “ 3 KJEq ) IU ! Q\$Hc\&! - bK>0+IuX603] f ' GI - 1aQ-DqL>NCnN [fg7 . >UkQB^ " ; =FL6^\%DL :aUVJmBh.@TA 'A:KXUQ.fi^e4dlLi>SEJgjmRh8N-3g*JYr\_\#W, m=] )8]K-H"1,6

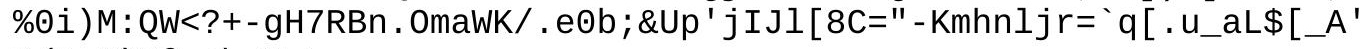
$\mathrm{P} / \mathrm{l}+\mathrm{K})$ ?@_c $\backslash<? \sim>$

endstream

endobj

$77 \odot$ obj

$<</$ Length 570

/Filter [/ASCII85Decode

/FlateDecode

]

/BitsPerComponent 8

/Colorspace $79 \odot \mathrm{R}$

/Width 74

/Height 97

>stream

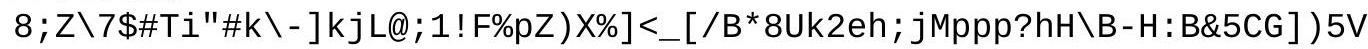
1YU p2ap6uH_q148seaVS1; KXU9"4<jC2\m>]\#W: InUlWo>WUtge-A"6[Z:S3Ysr+ eF=9g1NaOL *nIM [E=QlIEP+nC_o! e>WlSOgnsm?oJ+An8I * +\@F<mm8RBaIrQY12i b0 ! aOok* 'n^3bF?YmHr 75+)R4IFSf\%P - ibV] hWrRIR9Ii *0Thb1oeEq . c6kFY8\&5

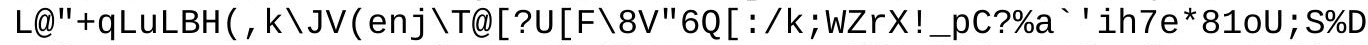
\&E'T^OLsJa, uKqsI\#VZ2iAceH6"W] ]?0\#_ZnXoYff9<')/=<?2fXX@8sN, G?d(sX @ ) - L, 'CTFUX-qg!0.Ppa9I9*?]-B^?pZQstKl^>:fP-A\#o\&( . J<]@\&>k7L_se3)c9 E1c\&; =eFI , XPUuiSZ[>DnOpd\&_2A\&\&; 3s \IFX\%)\&<<h18.fOjiQ5*ldm9J * $0 i u 5 / \wedge$ \#b'\%\#: e@9k_l.\&;R, fn/a\%c'5K\$F85p\$js@51130 >

endstream

endobj 
$78 \odot$ obj

$<$ /Length 150

/Filter [/ASCII85Decode

/FlateDecode

]

/BitsPerComponent 8

/Colorspace $79 \odot \mathrm{R}$

/Width 74

/Height 97

>stream

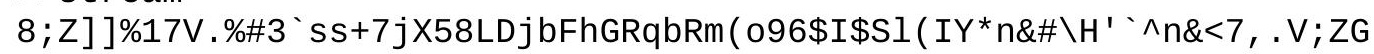
$; r-10=\wedge J 5 M 5 . Q / /] V k \wedge K Q R I l c I h F,+k t g]$ OL ' NNMPtK=Nhp; 6DdhcHnz!! 'egEh^U@ B]6I_YbhF\$?f7sQ >

endstream

endobj

$79 \odot$ obj

[/Indexed

/DeviceRGB

255

$80 \odot \mathrm{R}$

]

endobj

$80 \odot$ obj

$<</$ Length 428

/Filter [/ASCII85Decode

/Flatedecode

]

>stream

8; X]0>EqN@\%' '0_@\%e@? J;\%+8 (9e>X=MR6S?i^YgA3=].HDXF.R\$IIL@"pJ+EP(\%०

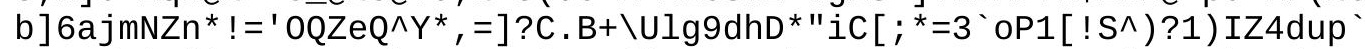

E1r! / , $\odot[* 9 . a F I R 2 \& b-C \# s<X 15 F H @[<=! \# 6 V)$ uDBXnIr .F>oRZ7D1\%MLY $. ? d>M n$

6\%Q20YfNRF \$+ON<+] RUJmCOI<jlL . oXisZ; SYU [/7\#<\&37rclQKqeJe\#, UF7Rgb1

VNWFKf>nDZ40TsOS! saG>GGKUIQ ${ }^{*} Q ? 45$ : CI\&4J ' 2 j<etJICj7e7nPMb=06S7UOH<

P07r $\backslash I$. Hu\&e@d\&E< . ' ) fERr $/ 1+{ }^{*} W$, ) $q^{\wedge} D^{*}$ ai $5<u \bar{u} L X .7 g />\$ X K r c Y p \odot n+X 1 \_n U^{*} 0($

1 [\$6Nn+Z_Nq0]s7hs] 'XX1nZ8\&94a\ >

endstream

endobj

$81 \odot$ obj

$<</$ Type/Pages

/Count 13

$/ \mathrm{Kids}[$

$82 \odot \mathrm{R}$

$83 \odot \mathrm{R}]$

$>$

endobj

$82 \odot$ obj

$<</$ Type/Pages

/Count 10

/Parent $81 \odot \mathrm{R}$

/Kids [

$90 \odot \mathrm{R}$

$1 \odot \mathrm{R}$

$4 \odot \mathrm{R}$

$7 \odot \mathrm{R}$

$11 \odot \mathrm{R}$

$14 \odot \mathrm{R}$

$22 \odot \mathrm{R}$

$25 \odot \mathrm{R}$

$3 \odot \odot \mathrm{R}$

$\left.\begin{array}{lll}34 & \odot & \mathrm{R}\end{array}\right]$

$>$

endobj

830 obj

$<</$ Type/Pages 


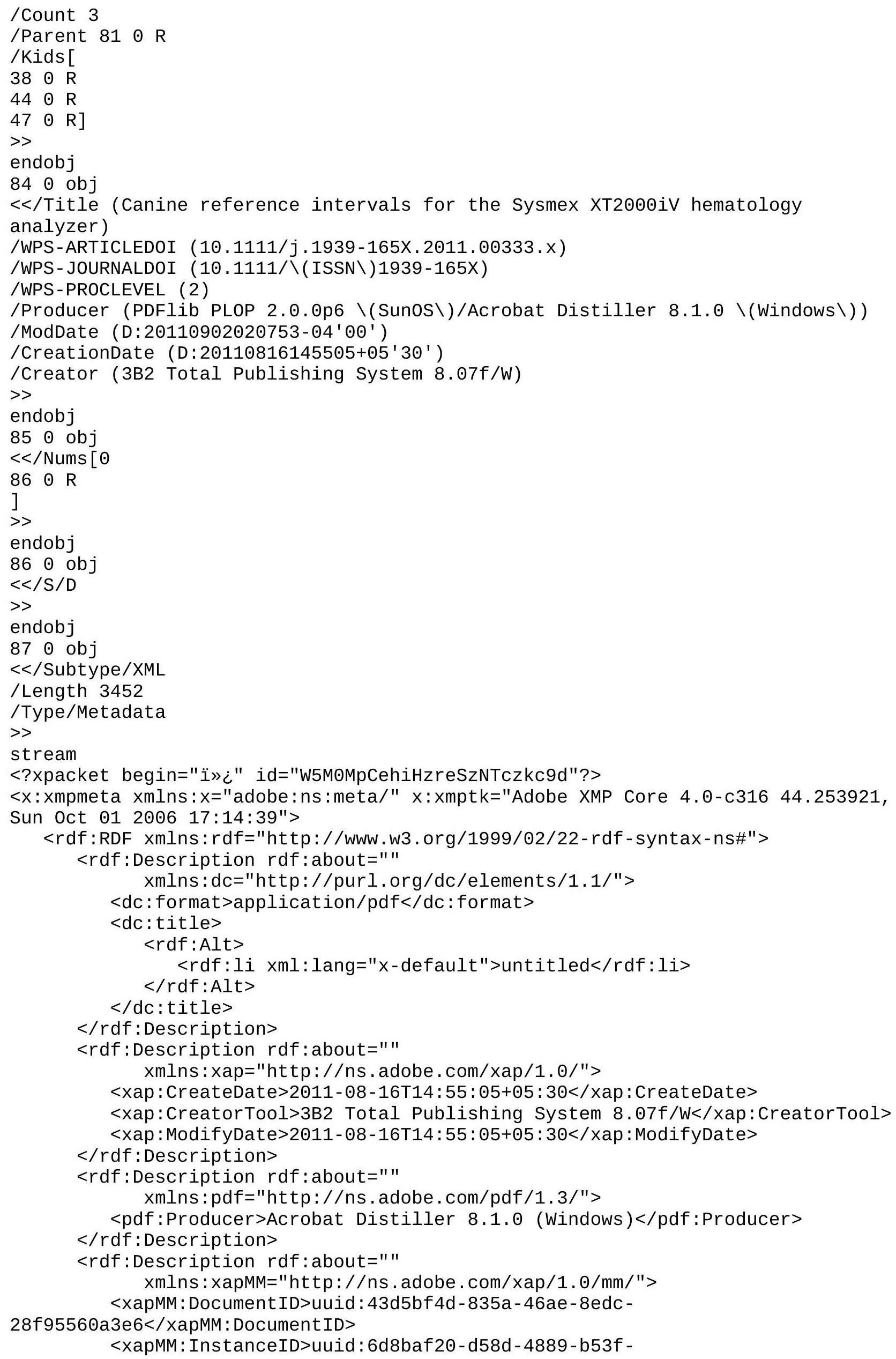




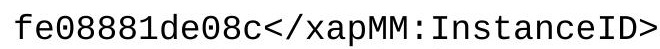
$</$ rdf:Description $>$

$</$ rdf:RDF $>$

$</ x$ : xmpmeta $>$

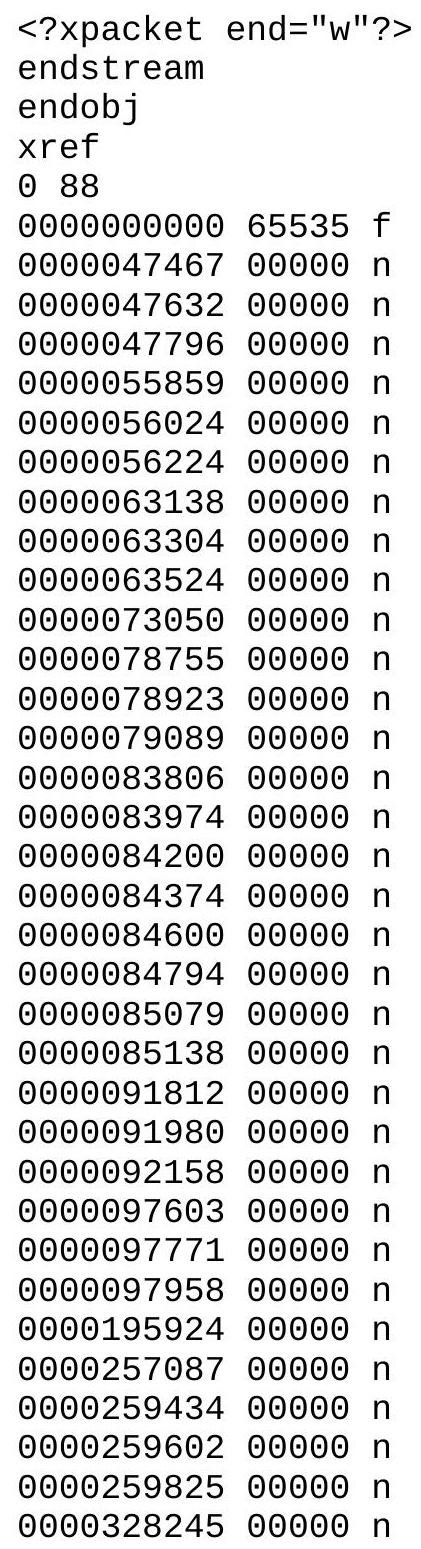




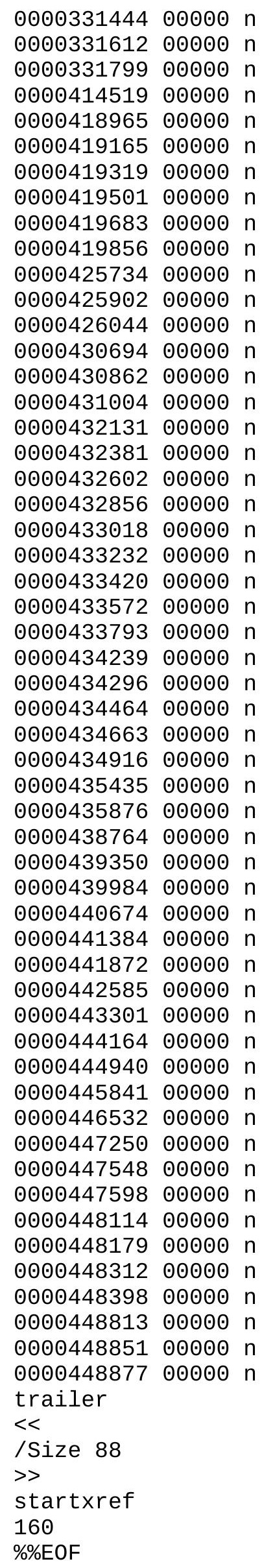

